Florida International University FIU Digital Commons

$11-13-2000$

\title{
Health care utilization behavior of elders in a multicultural urban environment
}

Katherine Marie Condon

Florida International University

DOI: $10.25148 /$ etd.FI14060890

Follow this and additional works at: https://digitalcommons.fiu.edu/etd

Part of the Sociology Commons

\section{Recommended Citation}

Condon, Katherine Marie, "Health care utilization behavior of elders in a multicultural urban environment" (2000). FIU Electronic Theses and Dissertations. 2420.

https://digitalcommons.fiu.edu/etd/2420

This work is brought to you for free and open access by the University Graduate School at FIU Digital Commons. It has been accepted for inclusion in FIU Electronic Theses and Dissertations by an authorized administrator of FIU Digital Commons. For more information, please contact dcc@fiu.edu. 


\section{FLORIDA INTERNATIONAL UNIVERSITY}

Miami, Florida

HEALTI CARE UTILIZATION BEHAVIOR OF ELDERS IN

A MULTICULTURAL URBAN ENVIRONMENT

A dissertation submitted in partial fulfillment of the

requirements for the degree of

DOCTOR OF PHILOSOPIIY

in

SOCIOLOGY

by

Katherine Marie Condon 
To: Dean Arthur W. Herriott

College of Arts and Sciences

This dissertation, written by Katherine Marie Condon, and entitled Health Care Utilization Behavior of Elders in a Multicultural Urban Environment, having been approved in respect to style and intellectual content, is referred to you for judgment.

We have read this dissertation and recommend that it be approved.

Lilly Langer

Betty Mortow

Hugh Gladwin, Major Professor

Date of Defense: November 13,2000

The dissertation of Katherine Maric Condon is approved.

Dean Arthur W. Herriott College of Arts and Sciences

Interim Dean Samuel S. Shapiro Division of Graduate Studies

Florida International University, 2000 
(C) Copyright 2000 by Katherine Marie Condon

All rights reserved. 


\section{ACKNOWLEDGMENTS}

In completing any task as monumental as a dissertation there are many individuals who have played a part in the process. Through all the turmoil of life that has taken place on the road to getting this dissertation completed, I have been the recipient of much love, support, and encouragement which has made it possible for me to complete this goal.

First and foremost I could not have done this without a sense of my own self worth and self-confidence. This I owe to my parents, Paul and Carol. You didn't smother me, but were there when I needed you. You were al ways confident that I would succeed even when I wasn't so sure about the road I was travelling. Thank you! I also owe a great debt to my two sisters, Francesca and Rachel. You have had to be there when family emergencies have arisen and I haven't been able to take my share of the load because of my physical distance from y'all during this pursuit. To Aunt Janet, thank you for keeping my eyes fixed and not wandering from this goal. To Aunt Carol and Uncle Joe, thank you for your encouragement and offer of support when I needed it most.

Of course I couldn't have done the dissertation without my committee. I wish to thank the members of my committee for their support, commitment, and dedication to this process. Their encouragement and expertise has been most appreciated. To l lugh Gladwin for agreeing to take on the responsibilities of chairmanship of my committee. Fortunately for me you agreed before you knew about your "additional" responsibilities to the Institute for Public Opinion Research, as well as my "fast-track" expectations for this dissertation. To Lilly Langer, your encouragement in classes, as well as with this dissertation, has been infectious and highly motivating. To Betty Morrow, I gained 
invaluable experience in your qualitative methods class, particularly for this dissertation. I also appreciate your stepping in at short notice to serve on my committee. I am also grateful to the faculty at large in the Sociology/Anthropology Department, through the program and coursework I have gained more than I ever imagined.

I wish to thank Max B. Rothman, Executive Director and Burton D. Dunlop. Director of Research at the Center on Aging for giving me the flexibility in my work schedule to pursue this goal and make this happen. I would also like to thank the many past and present colleagues at the Center on Aging for their generosity in the day to day conversations we have shared, allowing me to expand my "consultable record."

Last but not least, thank you to my friends who have been understanding and supportive. In particular, I would like to thank my pre-graduate school friends - Hallie, Cathy, Nancy and Heidi - who have kept me grounded, even while I have resided within the "academic ivory tower." To my "Princeton" friends - Deb Garvey, Fadi Maamari, Dexter Chu, West Addison, and Charles Nagatoshi - 1 will always be grateful for your friendship and generosity in some very trying times. I am also grateful to my "Florida" friends - Bob Mooradian, Gail Silverstein, Howard Frank, Ana Carazo-Johanning, Elena Sabogal, Alma Cirugeda Suarez, and Iveris Martinez - your encouragement and support on this last leg of my journey to complete the $\mathrm{Ph} . \mathrm{D}$. has been appreciated beyond mere words. 


\title{
HEALTH CARE UTIL.IZATION BEHAVIOR OF ELDERS \\ IN A MULTICUL.TURAL URBAN ENVIRONMENT
}

by

\author{
Katherine Marie Condon \\ Florida International University, 2000 \\ Miami, Florida \\ Professor Hugh Gladwin, Major Professor
}

The objective of this research is to determine the influences of social, environmental, behavioral, and economic forces on the health care service utilization of four racial/ethnic groups of non-institutionalized elders in a multicultural urban environment. To address these issues this dissertation examines three intertwined themes of culture, aging, and hcalth, using a sample of elders residing in Miami-Dade County, FL in four racial/ethnic groups: white non-Hispanic; black non-Hispanic English speakers; Cuban; and non-Cuban Hispanic.

The research questions were analyzed using both quantitative and qualitative data. Data for the quantitative component uses telephone survey data from the Dade County Needs Assessment. The purpose of this component is to develop a more comprehensive model of elder health care utilization behavior. The qualitative component uses data from focus groups from Dade County Needs Assessment, archival data and a literature review of previous ethnographic research. The purpose of this component is to gain a better understanding of the social construction of the terms "age" and "aging," as well as to place issues of health and health care in the lives of elders. 
The findings raised several important issues. First, just because people share a common chronological age does not mean that they are the same in every other respect. Examining elders as a homogeneous group of users of formal health care services in a community is simplistic. Placing "aging" and "health" in a cultural context is important. My findings confirm that the meaning of "aging" and "old" are socially constructed. Further, the term "aging" is NOT synonymous with ill health or frailty. This was a consistent finding in both the quantitative and qualitative results.

While all aging individuals share a mutual orientation toward aging (i.e., biological process), they do not age the same way (i.e., social construction of "aging"). Thus, policymakers and others serving the elder population must be aware of the particular cultural context, as well as the previous life experiences of the individuals that they serve. This analysis documents the importance of culture and geographic community in understanding health care service utilization of elders. 


\section{TABLE OF CONTENTS}

CHAPTER

1 INTRODUCTION AND STATEMENT OF PROBLEM ..............................

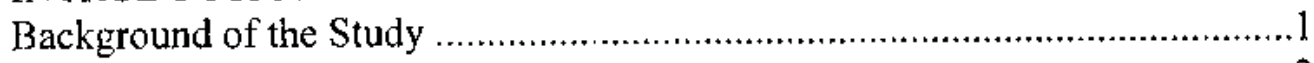

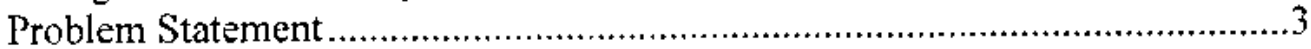

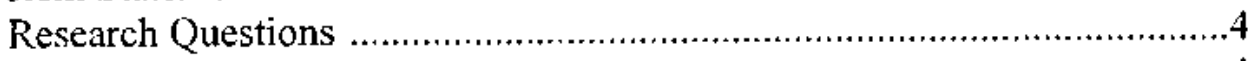

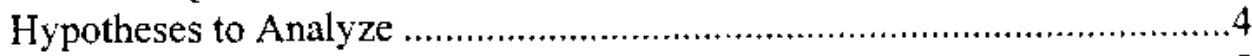

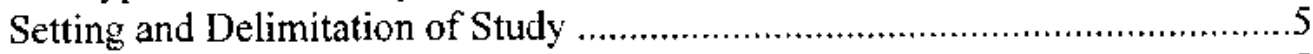

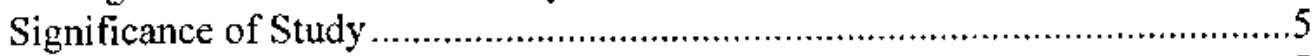

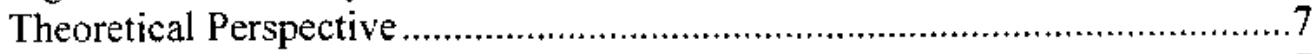

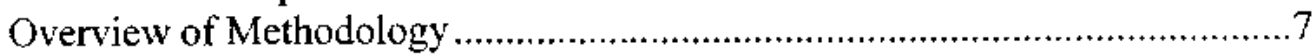

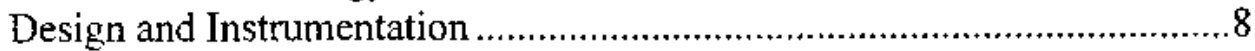

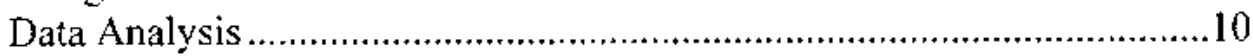

Organization of the Dissertation ..............................................................

2 LITERATURE REVIEW ON HEALTH CARE UTILIZATION ..................13

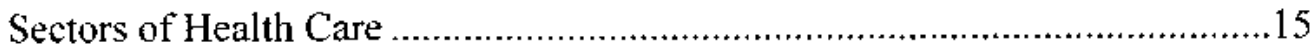

Determinants of Health and Illness Behaviors.............................................18

Caring and Curing: The Therapeutic Process and Networks...................18

Health Care Utilization: ..................................................................23

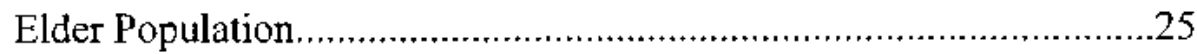

Minority Elder Population ...........................................................28

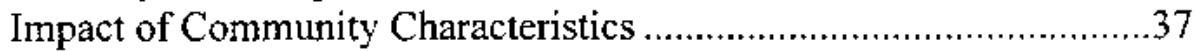

Health Care Belief Systems: A Case Study of Cuban Elders in

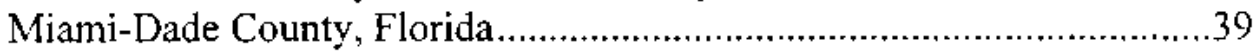

Historical Experiences of Cuban Medicine and Health ........................40

Health Beliefs and Practices of Cuban Elders in Miami-Dade

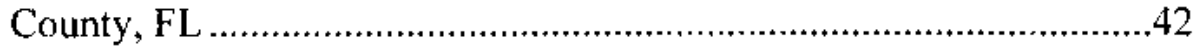

Summary and Future Research Needs ...............................................48

Approaches to Health and Utilization of Services ......................................50

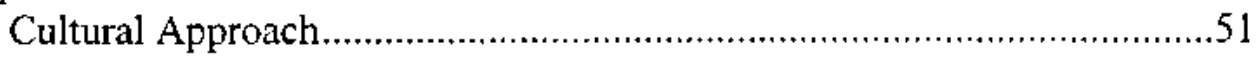

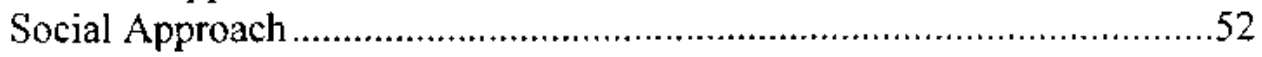

Social-Psychological Approach .........................................................52

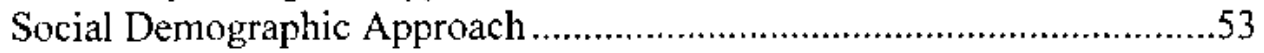

Socio-Behavioral Model of Health Care Utilization .........................54

Conceptual Framework ........................................................54

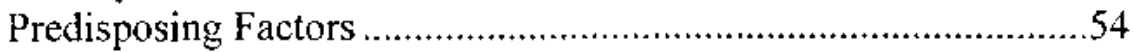

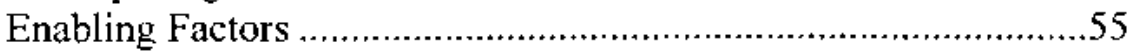

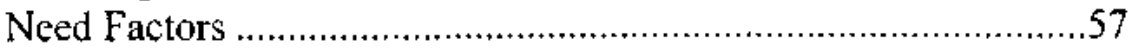

Health Care Service Utilization ...........................................57

Application of the Socio-Behavioral Model ....................................59

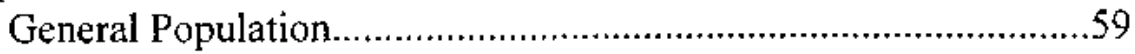

Elder Population ........................................................................60 
3 LITERATURE REVIEW ON AGING IN THE UNITED STATES ............67

Social Theoretical Perspectives on Aging Research.....................................70

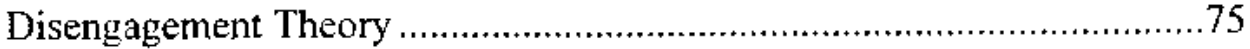

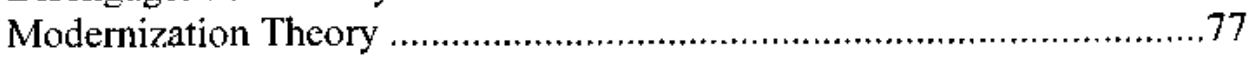

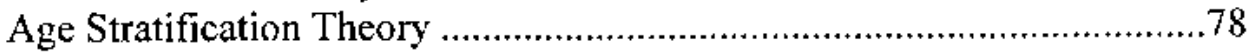

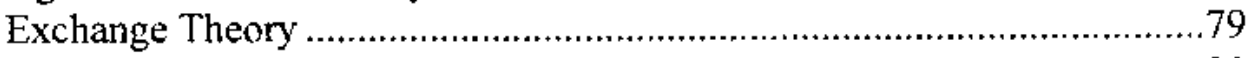

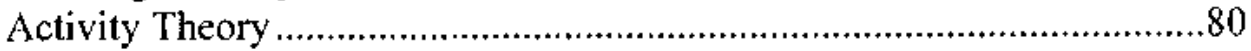

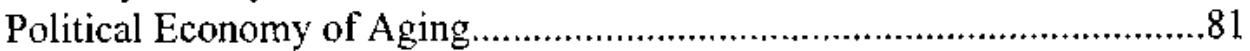

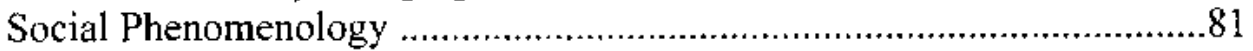

Recent Combining of Theoretical Approaches.......................................83

Literature Review of Aging Research......................................................84

Vesperi's City of Green Benches (1985) .............................................8 87

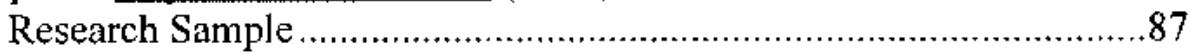

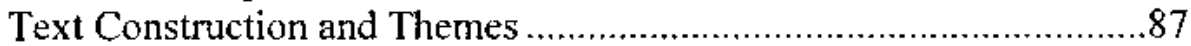

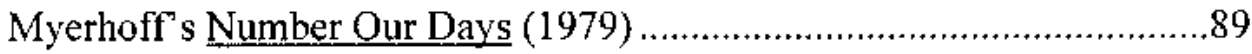

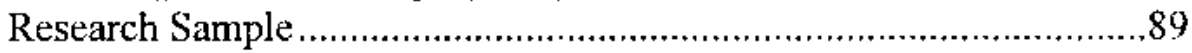

Text Construction and Themes ......................................................89

Erikson, Erikson and Kivnick's Vital Involvement in Old Age

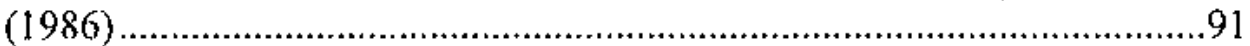

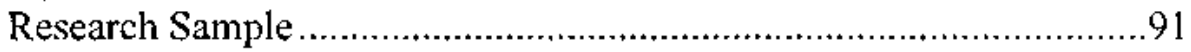

Text Construction and Themes ......................................................91

O'Reilly's Decoding the Cultural Stereotypes About Aging (1997) ........94

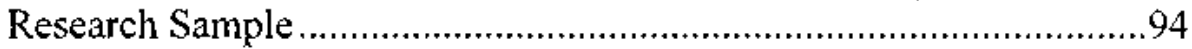

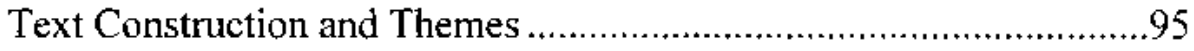

Berman's Interpreting the Aging Self (1994) ...........................................96

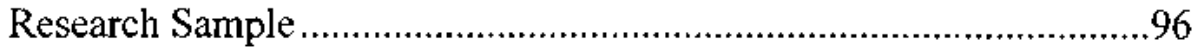

Text Construction and Themes ......................................................99

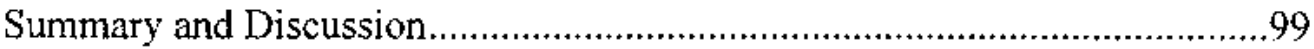

4 HISTORICAL DEMOGRAPHIC TRENDS OF ELDERS IN

MIAMI-DADE COUNTY, FLORIDA, 1950-1990 ................................104

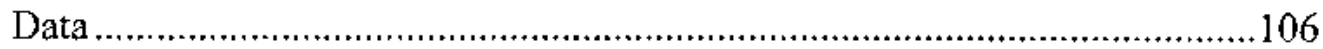

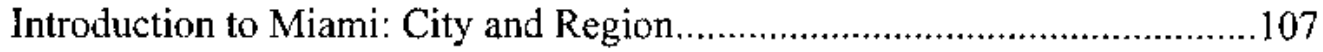

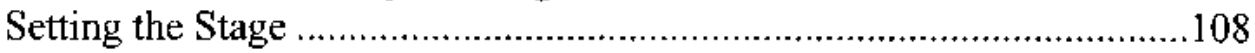

Demographic Characteristics..........................................................110

Economic Development .......................................................................112

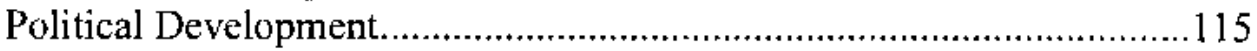

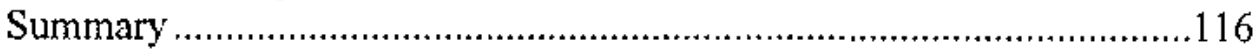

Growth of Elder Population in Miami-Dade County ................................116

Basic Demographic Characteristics of Elders.....................................118

Poverty and Economic Status of Elders..........................................119

Social Support for Elders ..........................................................................120

Geographic Residency of Elders within Miami-Dade County ................123

Racial/Ethnic Differences of Elders in Miami-Dade County ..................124 
Summary and Discussion

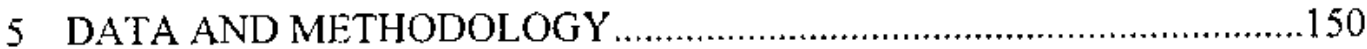

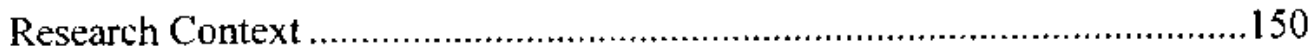

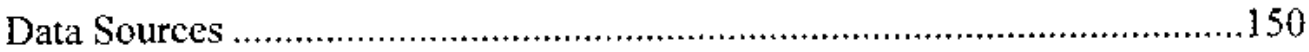

Dade County Needs Assessment ......................................................151

Telephone Survey Sample ...........................................................152

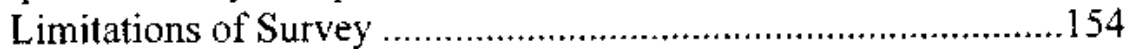

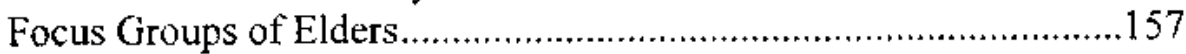

Contextual Data Sources.............................................................. 160

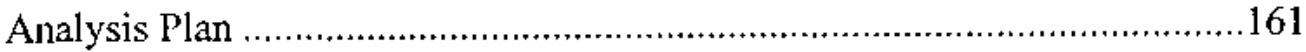

Instrument Used in Data Collection and Measurement of Variables ............165

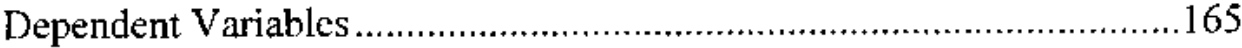

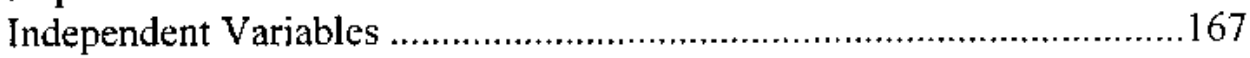

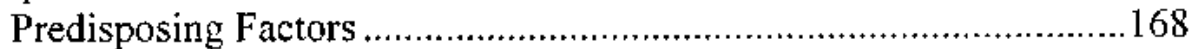

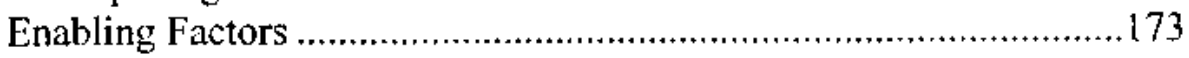

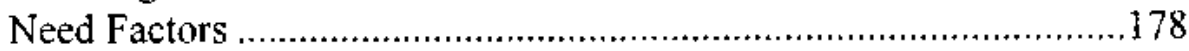

6 QUALITATIVE ANALYSIS OF HEALTH IN THE LIVES

OF ELDERS IN MIAMI-DADE COUNTY, FL …...............................192

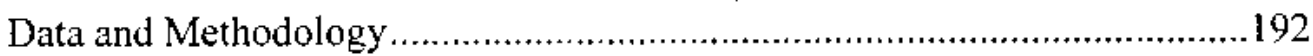

Identification of the Focus Groups and Entry into the Field .................194

Descriptions of Focus Groups.........................................................195

Description of Dade County Elder Population .....................................196

Seriousness of Current Problems and Concerns for Future ...............197

Health Status and Functional Ability ................................................199

Findings from Focus Groups of Elders in Miami-Dade County....................201

Living in the community and Dade County.......................................202

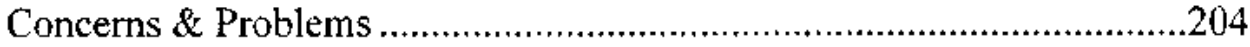

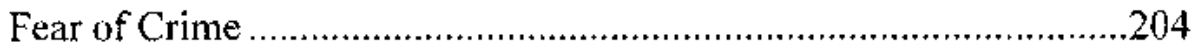

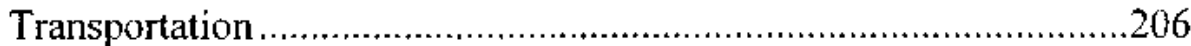

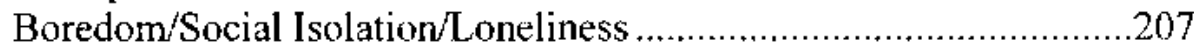

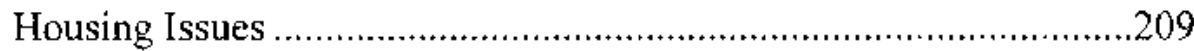

Health and Health Care Issues ...................................................210

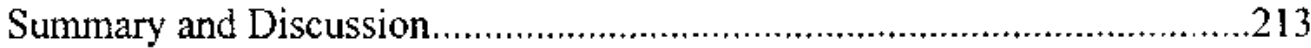

7 QUANTITATIVE ANALYSIS OF HEALTH CARE

UTILIZATION BEHAVIOR OF ELDERS IN MIAMI-DADE

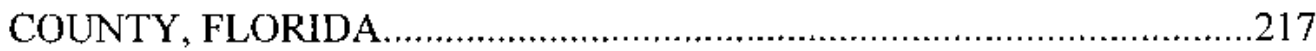

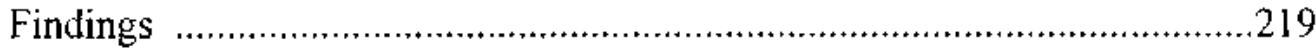

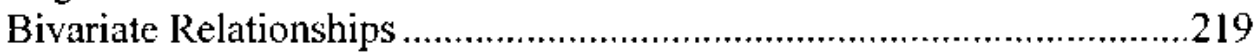

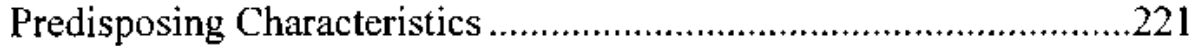

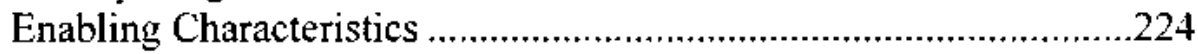

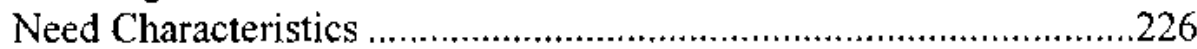

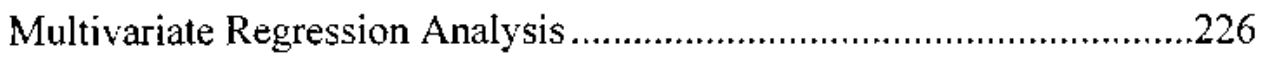


8 SUMMARY AND DISCUSSION

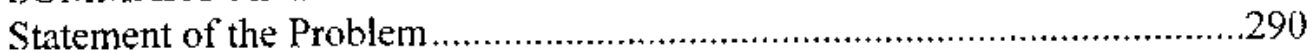

Review of the Methodology ...............................................................292

Summary and Discussion of the Results................................................295

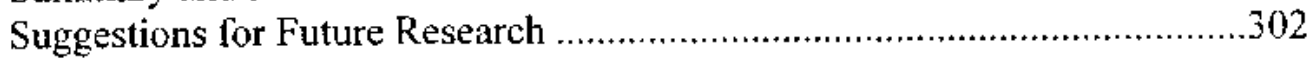

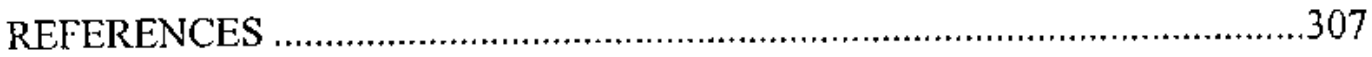

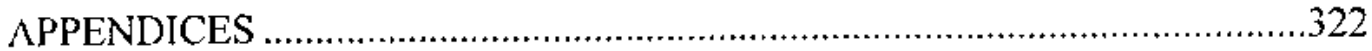

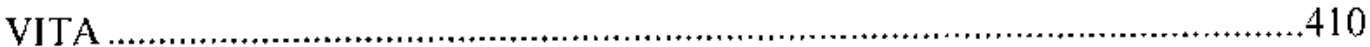




\section{LIST OF TABLES}

\section{TABLE}

1.1 General Socio-Behavioral Model Framework proposed by Andersen (1968).

2.1 Comparison of Ethnic Group Value Oricntation Profiles with the Core American Medical Value Orientation Profile

4.1 Elder Population Residing in Miami-Dade County, FL 1950-1990 ........130

4.2 Comparison of Elder Populations Residing in Florida, South Florida, and the individual counties of South Florida 1980 and $1990 \ldots . .130$

4.3 Percentage change in the Elder Population and Total Population between Censuses 1950-1990

4.4 Demographic Characteristics of Elders in Miami-Dade County, FL 1950-1990.

4.5 Comparison of Demographic Characteristics of Elders in South Florida, 1990

4.6 Selected Socio-Economic Characteristics of Elders in Miami-Dade County, 1950-1990

4.7 Comparison of Selected Socio-Economic Characteristics of Elders in South Florida, 1990

4.8 Selected Social Support and Household Characteristics of Elders in Miami-Dade County, 1970-1990.

4.9 Comparison of Selected Social Support and Household Characteristics of Elders in South Florida, 1990

4.10 Selected Household Composition Characteristics of Elders Not Living Alone in Miami-Dade County, 1970-1990

4.11 Selected Household Composition Characteristics of Elders Not Living Alone or in Group Quarters in South Florida, 1990

4.12 Selected Demographic Characteristics of Elders in Miami-Dade County by Racial/Ethnic Group, 1970-1990

4.13 Selected Socio-Economic Characteristics of Elders in Miami-Dade County by Racial/Ethnic Group, 1970-1990

4.14 Selected Social Support and Household Characteristics of Elders in Miami-Dade County by Racial/Ethnic Group, 1970-1990

4.15 Selected Household Composition Characteristics of Elders

Not Living Alone in Miami-Dade County by Racial/Ethnic Group, 1970-1990

5.1 Population Estimates and Survey Sample Numbers

5.2 Coding Algorithms for Categorical Variables and Ranges for Continuous Variables Used in the Study

5.3 Descriptive Statistics on Formal Health Care Service Utilization Measures for Four Racial/Ethnic Groups of Elders Residing in Miami-Dade County, Fl 
5.4 Descriptive Statistics on Predisposing Measures for Four

Racial/Ethnic Groups of Elders Residing in Miami-Dade County, FL ...187

5.5 Descriptive Statistics on Enabling Measures for Four Racial/Ethnic Groups of Elders Residing in Miami-Dade County, FL ...189

5.6 Descriptive Statistics on Need Measures for Four Racial/Ethnic Groups of Elders Residing in Miami-Dade County, FL

6.1 Top Four Ordered Responses of Elders on Perceived Potential Problem Areas as Very or Somewhat Serious by Race/Ethnicity of Respondent (Percentage)

6.2 Top Five Ordered Responses of Elders Who are Very or Somewhat Worried About Problems in Their Future by Race/Ethnicity

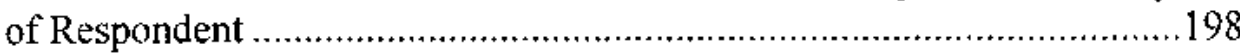

6.3 Self-Reported Health Status of Telephone Survey Respondents by Racial/Ethnic Group.

6.4 Self-Reported Functional Ability of Telephone Survey Respondents by Racial/Ethnic Group..................................................................200

7.1 Zero-Order Correlations by Four Racial/Ethnic Groups with Self-Reported Physician Visit in the Last 12 Months.

7.2 Bivariate Associations with Percentage Self-Reported Physician

Visit in the Last 12 Months

7.3 Zero-Order Correlations by Four Racial/Ethnic Groups of Elders with Six or More Self-Reported Physician Visits in the Last 12 Months

7.4 Bivariate Associations of Selected Independent Variables with Six or More Self-Reported Physician Visits in the Last 12 Months........253

7.5 Zero-Order Correlations by Four Racial/Ethnic Groups with at Least One Overnight Hospital Stay in the Last 12 Months .256

7.6 Bivariate Associations of Selected Independent Variables with Overnight Hospital Stay in the Last 12 Months .258

7.7 Zero-Order Correlations by Four Racial/Ethnic Groups with a Complete Physical Exam in the Last 12 Months .261

7.8 Bivariate Associations of Selected Independent Variables with a Complete Physical Exam in the Last 12 Months

7.9 Explained Variance $\left(\mathrm{R}^{2}\right)$ Resulting from Separately Entering the Three Major Blocks of Variables and Increments of Explained Variance within Major Blocks for Self-Reported Physician Contact in Last 12 months for Four Racial/Ethnic Groups of Elders 266

7.10 Explained Variance $\left(\mathrm{R}^{2}\right)$ Resulting from Separately Entering the Three Major Blocks of Variables and Increment of Explained Variance within Major Blocks for High Volume Utilization of Physician Contact in Last 12 months .266

7.11 Explained Variance $\left(\mathrm{R}^{2}\right)$ Resulting from Separately Entering the Three Major Blocks of Variables and Increment of Explained Variance within Major Blocks for Overnight Hospital Stay in Last 12 months 
7.12 Explained Variance $\left(R^{2}\right)$ Resulting from Separately Entering the Three Major Blocks of Variables and Increment of Explained Variance within Major Blocks for Iaving a Complete Physical Exam in Last 12 months .267

7.13 Regression Models Explaining Self-Reported Physician Contact in the Last 12 Months for Four Racial/Ethnic Groups of Elders When Three Major Blocks of Variables Are Separately Entered 268

7.14 Regression Models Explaining High Volume Physician Contact in the Last 12 Months for Four Racial/Ethnic Groups of Elders When Three Major Blocks of Variables Are Separately Entered .270

7.15 Regression Models Explaining Overnight Hospital Stay in the Last 12 Months for Four Racial/Ethnic Groups of Elders When Three Major Blocks of Variables Are Separately Entered .272

7.16 Regression Models Explaining Self-Reported Complete Physical Exam in the Last 12 Months for Four Racial/Ethnic Groups of Elders When Three Major Blocks of Variables Are Separately Entered .274

7.17 Increments of Explained Variance $\left(R^{2}\right)$ Resulting from Entering the Three Major Blocks of Variables for Best-Fit Regression Models Explaining Self-Reported Physician Contact in Last 12 Months For Four Racial/Ethnic Group of Elders .276

7.18 Increments of Explained Variance $\left(R^{2}\right)$ Resulting from Entering the Three Major Blocks of Variables for Best-Fit Regression Models Explaining High Volume Physician Contact in Last 12 Months For Four Racial/Ethnic Group of Elders .276

7.19 Increments of Explained Variance $\left(\mathrm{R}^{2}\right)$ Resulting from Entering the Three Major Blocks of Variables for Best-Fit Regression Models Explaining Overnight Hospital Stay in Last 12 Months For Four Racial/Ethnic Group of Elders .

7.20 Increments of Explained Variance $\left(\mathrm{R}^{2}\right)$ Resulting from Entering the Three Major Blocks of Variables for Best-Fit Regression Models Explaining Self-Reported Complete Physical Exam in Last 12 Months For Four Racial/Ethnic Group of Elders

7.21 Best-Fit Regression Models Explaining Self-Reported Physician Contact in the Last 12 Months for Four Racial/Ethnic Group of Elders

7.22 Best-Fit Regression Models Explaining High Volume Physician Contact in the Last 12 Months for Four Racial/Ethnic Group of Elders

7.23 Best-Fit Regression Models Explaining Overnight Hospital Stay in the Last 12 Months for Four Racial/Ethnic Group of Elders 282

7.24 Best-Fit Regression Models Explaining Self-Reported Complete Physical Exam in the Last 12 Months for Four Racial/Ethnic Group of EIders. 
7.25 Summary of Findings for Research Question 2 Hypotheses Across by Racial/Ethnic Groups....

7.26 Summary of Findings for Research Question 3 Hypotheses Across Health Care Service Variables. 


\section{LIST OF FIGURES}

FIGURE

PAGE

3.1 The generation of theories in social gerontology as organized by Bengtson, Burgess \& Parrott (1997) ....................................................103

4.1 Population Distribution in Miami-Dade County, FL 1970-1995 .............136

4.2 Population Distribution in Miami-Dade County, FL 2000-2015 ............137

4.3 Distribution of Proportion Black in Total Population, 1990 Miami-Dade County by Census Tracts...............................................138

4.4 Distribution of Proportion Hispanic in Total Population, 1990 Miami-Dade County by Census Tracts.

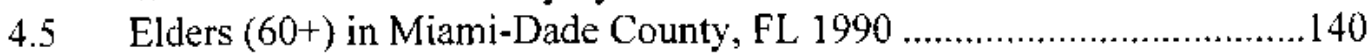

4.6 South Florida Counties per Capita Incomes 1969-1997 (\$1995) ............141

4.7 Distribution of Percentage of Total Population Living Below Poverty, 1990 Miani-Dade County by Census Tracts

4.8 Elder and Total Population in Miami-Dade County, FL (1900-1990)

4.9 Distribution of Percentage Elders $(60+)$ in Total Population, 1990 Miami-Dade County by Census Tracts .........................................144

4.10 Distribution of Proportion 85+ of Elder Population, 1990 Miami-Dade County by Census Tracts.

4.11 Percentage of Blacks in the Elder Population in Miami-Dade County, FL 1990. 146

4.12 Proportion of Hispanic Origin in Elder Population in Miami-Dade County, FL 1990

4.13 Distribution of Proportion Living Below Poverty of Elder Population, 1990 Miami-Dade County by Census Tracts

4.14 Distribution of Proportion Living Alone of Elder Population, 1990 Miami-Dade County by Census Tracts

5.1 Location of Area Agency on Aging Sub-areas within Miami-Dade County, Florida 


\section{CHAPTER 1: INTRODUCTION AND STATEMENT OF PROBLEM}

Issues of population aging and access to health care services for elders are not new problems that face society. However, the visibility of these issues has grown due to increasing numbers and cultural diversity of older individuals $(60+)$, as well as the skyrocketing costs of care. Thus, the three themes that will be examined in this research will be culture, aging, and health. Yet, each has an impact on the other, such as culture has an impact on how the terms "aging," "illness" and "health" are defined, both socially and individually. Aging increases the risk of illness/ill-health. For the purposes of the research, the focus will be on health care utilization of elders in the multicultural urban environment of Miami-Dade County, Florida.

\section{BACKGROUND OF THE STUDY}

Many factors influence the health status and use of health services by older Americans. It has been posited that the utilization of health services by an individual can be viewed as a behavior pattern (Andersen, 1968; Andersen \& Newman, 1973). As such, there have been numerous attempts to explain "individual behavior as a function of characteristics of the individual... characteristics of the environment in which [the individual] lives, and/or some interaction of these individual and societal forces..." (Andersen \& Newman, 1973).

Helman (1994:63) wrote "society's health care system cannot be studied in isolation from other aspects of that society, especially its social, religious, political and economic organization." In general, medical anthropology literature notes that cultural 
factors determine what an individual perceives to be an illness and where/who that individual will go to for healing/curing of that "illness." Csordas \& Kleinman (1996:3) write that, "therapy, treatment and healing are terms that define the domain of active response to illness, disease, pain, suffering, and distress." Further, it is important to recognize that in any given society or culture, there are a number of ways that individuals within a society can help themselves, or seek help from those around them (Helman, 1994). Empirical studies have documented that elders are disproportionately heavy users of health care services (Lubitz \& Prihoda, 1984a, 1984b; Soldo \& Manton, 1985; Fisher, 1980; Waldo \& Lazenby, 1984). Wolinsky, et al. (1989) report that 12 percent of the population is elderly, however they account for 31 percent of total health care expenditures.

Like other socially constructed terms, "illness" and "health" depend upon the "cognitive frames in which they are placed and these in turn are products of prior social interactions" (Portes \& Stepick, 1993: 9). Portes \& Stepick (1993) go further to state that, "common meaning are arrived at when relevant audiences agree to stress certain aspects of a given phenomenon and interpret them on the basis of shared past experience" (Portes \& Stepick, 1993: 9). However, projected increases in the cultural diversity of the elder population will have important implications for the provision of bealth and social services (Nelson, 1993; Angel \& Hogan, 1992; U.S. Bureau of the Census, 1992; U.S. G.A.O., 1992; Kravitz, et al., 1989; Wolinsky \& Arnold, 1988). Thus, an understanding of the unique factors and determinants that influence the health care utilization behavior 
of racial/ethnic groups of elders has broader connotations beyond just an academic pursuit.

The objective of this research will be to determine the influences of social, environmental, behavioral, and economic forces on the health care service utilization of four racial/ethnic groups of non-institutionalized elders in a multicultural urban environment.

\section{PROBLEM STATEMENT}

In a fair world, health care services would be distributed equitably according to need, however, enabling characteristics such as availability of health care services, as well as income, transportation, community ties, knowledge of community services, insurance and common language between patient and physician are potentially important in determining utilization by elders. This research has two major goals. They are as follows:

1. Examine the sociodemographic, cultural, and community availability factors, which affect health care utilization behavior.

2. Investigate the ways in which elders of the four racial/cthnic groups talk about issues of health and health care in their lives.

Finally, this research proposes two arguments. First, this research argues that elders of different racial/ethnic origin use health care services differently. Second, this research will argue that the specific local environment (i.e., contours of the available health care services market), is an important factor on utilization decisions of elders. 


\section{Research Questions}

The questions under investigation for this research project are:

Question \# 1: $\quad$ Are there differences in perception of the importance of health and health care in the lives of elders by cultural groups?

Question \#2: What factors predict utilization of health care services?

Question \# 3: Do these factors vary among racial/ethnic sub-populations?

\section{Hypotheses to Analyze}

The following hypotheses attached to each of these research questions are:

Hypothesis \# 1: Discourse on health and health care issues are significantly different by racial/ethnic group, because of significantly different concerns in their lives and previous life experiences.

Hypothesis \# 2a: Need factors will not be the strongest predictors of formal health care service utilization of elders.

Hypothesis \# 2b: Among enabling factors, community resources will play an important rolc in inhibiting or enabling use of formal health care services.

Hypothesis $\# 2 \mathrm{c}$ : Among predisposing factors, social structural variables will play an important role in inhibiting or enabling use of formal health care services.

Hypothesis \# 3a: Factors predicting health care service utilization will vary among subpopulations. Need factors, such as health status, will be a stronger predictor for white non-Hispanics, while enabling factors will be stronger predictors for black non-Hispanics, Cubans, and non-Cuban IIispanics.

Hypothesis \# 3b: Enabling factors will be stronger predictors of certain types of health care service utilization, (i.e., physician utilization), than need or predisposing factors for minority elders compared to white nonHispanic elders.

Hypothesis \# 3c: Among enabling factors, social support networks and family ties will be stronger predictors of health care service utilization with Hispanic elders. For black non-I Iispanic elders, structural barrier factors, (i.e., availability of services), will be stronger predictors of health care service utilization. 


\section{SETTING AND DELIMITATION OF STUDY}

To address the issues of health care seeking and utilization behaviors of elders in a multicultural urban environment, this dissertation focuses on elders in Miami-Dade County, FL. Four racial/ethnic groups of elders will be analyzed: white non-Hispanic, black, non-Hispanic (English-speaking only), Cuban, non-Cuban Hispanic. ${ }^{1}$ These four racial/ethnic groups of elders have had different life experiences, as well as different expectations of the aging process. Unlike other areas in the United States, Miami-Dade County already has a very culturally diverse elder population $(60+$ years). Thus, MiamiDade County provides a unique opportunity to study in microcosm the future complex interaction of age structure and racial/ethnic diversity on health care service utilization that will come to characterize the aging population in the United States.

\section{SIGNIFICANCE OF STUDY}

Most of our knowledge concerning factors which influence the utilization of health care services and health care seeking behaviors by elders is derived from somewhat homogeneous studies, i.e., mostly white non-Hispanic elders. Those studies that have explored minority elder health care seeking and utilization behaviors have been hampered by small population sizes.

\footnotetext{
' The four significant racial/ethnic groups in Dade County, FL. (with the respective percentage distribution) are white non-Hispanic (42.8 percent); black non-IIispanic ( 9.7 percent); Cuban (38.2 percent) and nonCuban Hispanic (8.8 percent) (Ruggles, Sobek, et al., 1997). The elder population in 1990 is 18.5 percent of the total population in Dade County. Chapter 4 has an in-depth analysis of sociodemographic characteristics of elders in Miami from the 1990 U.S. Census.
} 
This dissertation research is unique with respect to five aspects. First, the heterogeneous nature of the elder population in Dade County, as well as the large percentage of the population aged 60 years or more will allow this study to investigate the health beliefs and preventive health care practices of four racial/ethnic groups of elders in one multicultural setting with its specific health care resources. This has not been previously done. Second, the study has a neighborhood focus within a specific county. At the county level, more local community data is available on health care services actually available to elders in their neighborhoods and not just those services available in the same SMSA or same county. ${ }^{2}$ Third, this study will be able to analyze the equitability of access to health care delivery system for particular racial/ethnic groups of elders within a community. In terms of policy implications this research will allow policy makers to better understand how barriers to access confront particular racial/ethnic groups of elders and possible solutions for overcoming these barriers. One such hypothesis will be federal entitlement programs have "leveled much of the discriminating ability of socioeconomic status" (Wolinsky, 1990). Information is available on the type of Medicare coverage (Part A only, Part A and Part B, or no Medicare coverage), as well as other types of health insurance coverage (Medicaid, Medigap or a Medicare supplemental policy, an HMO plan, private insurance other than Medigap, long-term care insurance, or no health

\footnotetext{
'As noted earlier, Wolinsky, et al., (1989) found that there was little effect of enabling factors. They thought that this was in part due to "the crudity with which participation and concentration measures[had] been constructed... That crudity lies ... with the catclıment area ... at the SMSA level..." (Wolinsky, et al., 1989). They suggested that a more accurate assessment would involve the construction of these measures at the neighborhood level, which is possible when studying a particular county. This study will endeavor to bring the catchment area of access to health care services to the neighborhood level.
} 
insurance coverage). Fourth, there is an ability to analyze preventive health care service utilization separately by racial/ethnic groups that has not previously been done. Fifth, the data that will be used in this research has not previously been analyzed.

\section{THEORETICAL PERSPECTIVE}

The theoretical perspective for the proposed study is most commonly referred to as the Socio-Behavioral Model. The Socio-Behavioral Model (SBM) is the most widely used approach and was originally developed by Andersen (1968). Andersen with other colleagues further refined it. The SBM proposes that utilization of health care services by an individual can be viewed as a behavior pattern. As such, there have been numerous attempts to explain "individual behavior as a function of characteristics of the individual... characteristics of the environment in which [the individual] lives, and/or some interaction of these individual and societal forces..." (Andersen \& Newman, 1973). In SBM, health care service utilization "is seen as a joint function of the "need," 'predisposing,' and “enabling' characteristics of an individual" (Soldo \& Manton, 1985). A more detailed explanation of the theoretical perspective will be found in the literature review in Chapter 2.

\section{OVERVIEW OF METHODOLOGY}

The data for this research was collected as part of a needs assessment for the Alliance on Aging, Inc., the Area Agency on Aging for Dade and Monroe Counties (Alliance) between August 1993 and October 1994. There were two purposes for this needs assessment. The first purpose was, "to document the needs of citizens 60 years of 
age and older to provide data for use in planning services for elders" (Rothman, et al., 1994). The second purpose was "to estimate the impact of Hurricane Andrew on older persons living in Dade County" (Rothman, et al., 1994). Chapter 5 will have a more detailed examination of the needs assessment and the methodology used in the analysis of the Research Questions and Hypotheses.

This data was originally collected for the Alliance for the purpose of giving them the ability to write a 5 - and 10 -year pian for services in the county. However, other than this descriptive analysis the data has not been analytically analyzed with respect to health care utilization behavior of elders.

\section{Design and Instrumentation}

The research design has both a quantitative and a qualitative component. The quantitative component of this research uses a cross-sectional design. The data collection method was a closed-ended survey instrument designed to measure the variables that are being examined in this study and conducted by telephone. The target sample size for the needs assessment was 1,800 non-institutionalized elders (adults aged 60 years or older) in Miami-Dade County, Florida. The overall sample was designed to be directly representative of non-institutionalized elders living within Dade County with either a listed or unlisted telephone number. It was conducted between January and March 1994.

The survey instrument used collected information on each of the three factors:

predisposing, enabling, and need, as well as the dependent variables measuring utilization of physician visits and hospitalization within the previous 12 months. 
In addition, contextual community variables, such as availability of physicians and hospital beds, were collected from other sources such as the Dade County Medical Association Directory, the Florida Medical Association Directory and the American Hospital Association Guide. Because direct measures of barriers to accessing health care services are usually unavailable, indirect measures of whether the neighborhood (service environment) that an elder lives in is "service rich" or "service poor" can be gathered from data collected by zip code on the U.S. Census Bureau's Summary Tape File 3B. Items that would indirectly indicate service richness are: median household income, per capita income and percentage of population living below poverty in each zip code, as well as the percentage black, Hispanic and elder within each zip code. A separate chapter of this dissertation will describe the historical demographic trends of elders in MiamiDade County and the overall population growth of the county since 1950 , as well as a comparison of Miami-Dade County to its neighboring counties of Broward, Palm Beach and Monroe, and the State of Florida (Chapter 4).

The qualitative component of this research will be in two parts. The first part will be an analysis of the social construction of the terms "aging" and "age" in the United States by examining the theoretical approaches of studies on elders and aging and more in depth examination of five ethnography's focused on elders (Chapter 3). The second part of the qualitative component of this research will analyze health and health care issues in the lives of elders residing in Miami-Dade County, Florida using focus groups conducted in Miami-Dade County as part of the same needs assessment already mentioned. The qualitative data collected in the needs assessment consists of ten focus groups of elders, 
informal caregivers, volunteers, and service providers throughout Miami-Dade County completed in October 1993, of which six will be used.

Table 1.1 General Socio-Behavioral Model Framework proposed by Andersen (1968)

\begin{tabular}{llll}
\hline Predisposing & Enabling & Ned & $\frac{\text { Utilization }}{- \text { Self-Perceived }}$ \\
\hline- Demographic & $\bullet$ Family & $\bullet$ Discretionary \\
- Social Structure & $\bullet$ Community & - Professional diagnosis & - Non- \\
& & & Discretionary
\end{tabular}

- Health Beliefs

\section{Data Analysis}

The data analysis for the quantitative component of this research will be conducted by using the computer package SPSS for Windows (SPSS, 1999). Four statistical procedures will be used to analyze the data: frequency distribution, reliability procedure, correlation analysis, and multiple regression analysis, specifically logistic transformation, to develop a more comprehensive model of elder health care utilization behavior.

For the qualitative component of this research, data from focus groups will be qualitatively analyzed. Archival data and a literature review of previous ethnographic research will also be examined to gain a better understanding of the social construction of the terms "age" and "aging." Further, qualitative data will be used to place issues of health and health care in the lives of elders using focus groups conducted in Miami-Dade County.

Together, both the qualitative and the quantitative components will allow the researcher to gain a better understanding of the determinants of health care services 
utilization by elders in a multicultural urban environment. A more in-depth description of data and methodology used to analyze the data will be provided in Chapter 5 .

\section{ORGANIZATION OF THE DISSERTATION}

The organization of this dissertation will be as follows. Chapter 1 has been an introduction and overview of the research. In the second chapter, there will be a discussion of the literature on health care utilization, particularly the conceptual frameworks that have been established on health care utilization and health care seeking behaviors, as well as, ethnomedical beliefs and practices. While the primary focus of the analysis in this dissertation will be on formal health care utilization, since biomedicallytrained physicians are the primary providers of formal health care. Further, biomedicine is a very powerful system of knowledge in the United States. Kaufman (1994) wrote that, the role of medicine in contemporary society has on,

...the problems of old age is not unexpected considering the fact that medicine has been an extrcmely powerful force in shaping knowledge in late $20^{\text {th }}$-century life.

Thus, it is for this reason that third chapter will step back from hcalth and health care utilization to examine the literature on aging, particularly the dominant theoretical approaches used to examine the social construction of the terms "aging" and "old". Recent research suggests that "old" is socially constructed and not just a universally clear biological process. Thus, in this chapter there will be an examination of ethnographic literature and theoretical perspectives with regard to the meaning of aging for elders in the United States. Chapter 4 will begin to set the stage for the analysis in this dissertation by placing the research within an historical and cultural environment. This chapter will 
examine the historical demographic trends of elders in Miami-Dade County, FL, as well as an overall description of the community, i.e., the economy, geographical location of elders within the community, and health care service availability. There will also be a comparison of Dade County with its neighboring counties of Broward, Palm Beach, and Monroe and the State of Florida. Following this background, Chapter 5 will focus on the data and research methods that will be used to address the research questions and hypotheses. There will also be a discussion of the concerns and limitations of the data collected. Following this chapter will be two analytical chapters. In Chapter 6 there will be an analysis of the different discourses that elders use to communicate the importance of health using six focus groups of elders residing in the Miami-Dade County community conducted in October 1993. This will also allow for a discussion and placing of the issues of health and health care utilization in the lives of elders and answer Research Question 1. In Chapter 7 there will be an analysis of the quantitative data gathered using telephone survey techniques and an examination of Research Question 2 and Research Question 3. The final chapter of this dissertation will be a synthesis of the threads of culture, health/medicine and aging. From this synthesis, recommendations will be produced on constructing culturally oriented health care strategies for elders. Although not the focus of this research, the need for better culturally oriented health care strategies will be urgently needed. This will be useful to alert planners and provider agencies to elder health care issues. Further, improving culturally oriented health care strategies will enhance the promotion of health care prevention with sensitivity to traditional ethnohealth and ethno-caring modes within a variety of cultural settings. 


\section{CHAPTER 2: LITERATURE REVIEW ON HEALTH CARE}

\section{UTILIZATION}

Andersen (1968) originally wrote, "use of health services can be viewed simply as another form of human behavior." Those behaviors related to health, however, encompass a variety of activities, from restoration of health, to maintenance, and on to improvement of health (Gochman, 1988; Bradsher, 1992). Further, Kleinman (1980) writes that,

Patients and healers are basic components of such [health care] systems and thus are embedded in specific configurations of cultural meanings and social relationships. They cannot be understood apart from this context. Illness and health also are part of the system of health care. Within that system, they are articulated as culturally constituted experiences and activities, respectively. In the context of culture, the study of patients and healers, and illness and healing, must, therefore, start with an analysis of health care systems (p. 24-25).

Health-related behaviors may be broken into three sub-categories: health behaviors, illness behaviors, and sick-role behaviors. Kasl \& Cobb (1966) defined health behaviors as "those activities undertaken by a person who believes himself or herself to be healthy for the purpose of preventing disease." The World Health Organization (WHO) defines health as a state of physical, social and psychological well-being or free from disease, and having the capacity to function optimally and perform one's roles in society (Cockerham, 1995:2). This concept of health, as well as its counter-part - nonhealth - depends on a cultural construction of "normality." In a forthcoming article on large scale health systems, Kincaid states, 
Health is a state of being embedded in complex relationships relationships not only with nature and the nature of one's body, mind, and soul but also with family, friends, neighbors, and even polity. These relationships shape how illness is understood, how it is treated, and how the person with an illness can choose to respond to it.

Medical anthropologists distinguish between etic and emic definitions of health, i.e., health as etic and ethnohealth as emic.

In contrast, illness behaviors are those "activities undertaken by a person who believes himself or herself to be ill" (Bradsher, 1992: 8). Further, the purposes of these activities are first to define "non-health," and second to seek relief from "non-health" (Kasl \& Cobb, 1966). There is also an emic and etic distinction for non-health. The etic term-disease-is a professional construct determined by the professional knowledge of physicians. Further, the medical definition of "ill-health" or "dis-ease" is, "... based on objectively demonstrable physical changes in the body's structure or function, which can be quantified by reference to 'normal" physiological measurements" (Helman, 1994:

104). The emic term-illness - is culturally constructed also, yet it is less precise and cannot always be quantified. Helman (1994: 107) described the difference between the two terms as follows:

... word 'illness' [stands] for "what the patient feels when he goes to the doctor', and 'discase' [stands] for 'what he has on the way home from doctor's office.'

Finally, "sick-role behaviors" are activities undertaken by an individual defined as being sick (Kasl \& Cobb, 1966; Bradsher, 1992; Parsons, 1951). The stability of a group, whether it is a family, community, or culture, can be threatened when members of the group are not health (sick). The sick members of the group are not able to fulfill their 
normal social obligations whether it is within the family or more generally in the community (Parsons, 1951).

\section{SECTORS OF HEALTH CARE}

The majority of researchers studying health and health practices in terms of the cultural perspective tend to examine health care not as one sector but three sectors. Depending on the researcher, these three sectors are the popular sector, the folk sector, and the professional sector. Starr (1982) denotes these three spheres of practice as, "the medicine of the domestic household, the medicine of the physicians, and the medicine of lay healers" (p. 32). Regardless of the naming convention, an individual moves,

... freely between the popular and the other two sectors, and back again, often using all three sectors at once, especially when treatment in one sector fails to relieve physical discomfort or emotional distress ( Helman, 1994: 67).

Similarly Starr (1982) wrote,

Medical care is never solely a matter of professional practice. Even today, much treatment of the sick takes place outside of the doctor's sphere in the home or under alternative practitioners (Starr, 1982: 32).

Kleinman (1980) stated that there is a need for comparative research that appreciates the social and cultural construction of the health care system in a particularly society.

They are forms of social reality. Social reality signifies the world of human interactions existing outside the individual and between individuals. It is the transactional world in which everyday life is enacted in which social roles are defined and performed, and in which people negotiate with each other in established status relationships under a system of cultural rules (Kleinman, 1980: 35-36) 
Regardless of culture, the popular sector of health care is usually the largest.' It is the non-specialist, non-professional, lay domain that an individual who believes he/she is in ill health will turn to first. This sector is usually where "ill health is first recognized and defined and health-care activities are initiated" (Helman, 1994: 64-65). The relationship between "patient" and "healer" from this sector is usually informal, unpaid, and "of variable duration" (Helman, 1994: 66). The "patient" and "healer" are usually "linked to one another by ties of kinship, friendship, neighborhood, or membership of work or religious organizations" (Helman, 1994: 65-66). The main providers of this type of health care are usually women (Helman, 1994). Historically, this was due to the fact that "care of the sick was part of the domestic economy," thus the responsibility of the wife (Starr, 1982). The practice of domestic medicine has been passed down by oral traditions from mother to daughter (Starr, 1982). Overall, this sector is the largest, yet, "it is the least studied and most poorly understood" (Kleinman, 1980: 50).

The next sector of health care that will be discussed is the "folk" sector. In comparison, it is of varying size depending on the culture. Non-Western societies usually have larger folk sectors than Western societies. The type of medicine practiced in this sector is usually identified as "Ethnomedicine." The practitioners in this sector "... are not part of the 'official' medical system and occupy an intermediate position between the popular and professional sectors" (Helman, 1994: 67). However, they are distinguished from the popular sector practitioners, particularly in non-Western societies, by the fact

\footnotetext{
${ }^{1}$ Helman (1994) estimated that "...70-90 percent of health care takes place within this sector in both Western and non-Western societies" (p. 65).
} 
that "...certain individuals specialize in forms of health which are either sacred or secular or a mixture of the two" (Helman, 1994: 67). Yet, the practitioners usually share "basic cultural values and world views, of the communities in which they live, including beliefs about origin, significance and treatment of ill-health" (Helman, 1994: 68-69). The relationship between "patient" and "healer" usually includes the family in the treatment decision. Finally, some societies view health "as a balance between [the individual and his/her] social, natural and supernatural environments." In these societies, an imbalance between any of these aspects "... may result in physical symptoms, or emotional distress, and require the services of a sacred folk healer" (Helman, 1994: 69).

The final health sector is the professional sector. It is primarily associated with biomedical or allopathic medicine. Western medical doctors are organized and legally sanctioned healing professionals. Unlike popular and folk health sectors, the patient is separated from his/her family while under treatment. The emphasis, unlike the folk sector, is the individual, not the family or the community. Another distinction between the professional sector and folk/popular is its use of high tech gadgetry to diagnose and treat the patient. The basic premises of the biomedical perspective are scientific rationality; an emphasis on objective, numerical measurement, as well as physiochemical data; reductionism; a mind-body dualism; and "disease" is viewed as an entity (Helman. 1994). These basic premises have an impact on the way that physicians and the patient interact. Helman (1994) describes this impact as a shift away from allowing the "patient" to give the doctor information about the underlying disease process to one in which the doctor relies exclusively on diagnostic technology to collect and measure clinical 'facts.' 
However, in recent years there has been an increasing recognition of the need for "cultural competence" by physicians, especially with respect to the care of older pationts (Yeo, 1996).

\section{DETERMINANTS OF HEALTH AND ILLNESS BEHAVIORS}

\section{Caring and Curing: The Therapeutic Process and Networks}

The therapeutic process is culturally located, i.e., deciding when an individual is sick, who to consult initially, and who to consult next if the initial treatment does not help. In many cultures, it has been stated that individuals who are ill will receive treatment (of a variety of kinds) in each of the three sectors, sometimes even simultaneously. Usually, the first method of care is self-treatment. However as Helman (1994) wrote,

People who become ill, and who are not helped by self-treatment, make choices about whom to consult in the popular, folk, or professional sectors for further help (p. 84)

The decisions in this process can only be understood within the cultural context of the "sick" individual. However, the therapeutic process can be described as comprising four common dimensions. These four dimensions are (1) the course of a treatment event, (2) a sequence of experiential or intrapsychic phenomena, (3) the course of an illness episode and (4) social and ideological control exercised through healing practice. Further, within these four dimensions there is a common process occurring. It can be generally described as, (1) lay consultation and referral; (2) the treatment action; (3) adherence or compliance to the treatment action; and (4) barriers to health care access. 
The first step in this process usually occurs within the domestic or popular sphere. Patterns of consultation and referral are highly dependent upon their socio-cultural networks or lay consultation networks, particularly for older individuals. Usually it is a female relative that is expected to monitor the symptoms exhibited by the older individual. The female relative is also expected to direct the course of action of the ill person. However for a variety of reasons, the support network available may not be what the older individual expected when they were younger. For example, familism is an important part of Cuban culture. However, immigration to the United States and the economic blockades against the island by the U.S. government have made a gap between the expected support networks and the actual support networks available to Cuban elders. Further familism makes it difficult for Cuban elders with limited social networks in the United States to reach out for help from non-family members, since they do not want to "dishonor" their family.

The varieties of lay consultation networks are dependent on the openness of discussion of illness with extended family members and friends. Sokolovsky (1985) found that African-American elders were much more open in their discussions than Anglo-Americans. A variety of reasons can influence this greater openness in discussion and more extensive utilization of lay consultation networks. In the African-American community the reciprocity of these networks can act as a buffer against stressful situation, such as historical discrimination. In the case of Cuban immigrants, the stressful situation is the forced fleeing from their homeland. 
Once consultation of the lay network has taken place, a treatment plan or action is developed. Regardless of ethnic group, the treatment action may come from formal health professionals, licensed health practitioners, alternative or native health practitioners, lay consultation, or self-treatment. However, in most instances, selftreatment or utilization of a "home-remedy" is usually taken prior to medical consultation. The treatment action develops and is refined with time and further consultations. Kleinman (1980) describes this process in his explanatory model. He conceptualizes the doctor (healer)-patient relationship as a transaction between the doctor's explanatory model and the patient's explanatory model and/or the family's explanatory model. More explicitly, there is,

a process of elicitation, followed by processes of analysis, transfer, restructuring, ... and feedback (Klcinman, 1980: 111).

Many reasons may contribute to the difficulty that physicians educated in western biomedical medicine may have in dealing with individuals from other cultures. Most importantly is with respect to the treatment process, physicians may not know or realize that the patient is also getting treatment from other sources/spheres, such as the use of herbs, salves, or teas. This may complicate prescribed medicine in terms of effective treatments. With respect to the care of the ethnic elders (ethnogeriatrics), Yeo (1996) also mentions other issues that can affect interaction between the physician and older patient and clinical care of the elder patient. These issues are intercultural dynamics; ethnic and racial differences in epidemiology of disease; use of interpreters; showing 
respect; assessment instruments; treatment; working with families; and ethical decisionmaking, to name a few.

Effective treatment is only as good as the compliance or adherence to the treatment plan by the patient. Yet, compliance is not just a matter of "doing" what the doctor ordered. Complications arise in several areas. First, compliance entails a cooperation of cultural norms of the patient with the biomedical model. For example, the African-American male given the diagnosis of hypertension, the physician will tell the patient to lower his salt intake. Yet, many African-American males do not control the cooking of food in their families. Further, there are many types of food that have significant cultural importance in African American culture, or sometimes it is the old nemesis of poverty that forces certain food choices. Regardless of the reason, many of these food choices may be very high in salt. Thus, compliance or adherence to a treatment action is not always possible for an individual of a particular racial/ethnic group.

Second, the orientation of the explanations of ill-health between the physician/healer and the patient needs to be compatible. Kleinman (1980) states that,

... generally speaking, the explanatory model of professional practitioners are oriented toward disease, while those of the laity arc oriented toward the illness.

This difference in orientation and explanatory models between doctor and patient creates difficulties for compliance. The physician trained in the biomedical model forgets that the individual he/she is treating is not just a "body part," but a "whole" human being that lives in a network of other "whole" human beings. 
Another aspect that may hinder compliance or adherence to prescribed treatment

is in the area of the patient's understanding of how his/her body works, which is very dependent upon culture. Young \& Garro (1994) in their examination of health care decision-making in a small rural community in Mexico found that informants did not have the same view of the human body, i.e., how it worked compared to the biomedical sphere view. These differences in understanding may create illnesses that are not recognized by the biomedical sphere, or the individual may not recognize the need for treatment of other biomedically-defined diseases. Young \& Garro (1994: 44) wrote, "many illness beliefs are not comprehensible without inquiry into their ethnoanatomical and ethnophysiological basis."

These differences in discourse and understanding are just some of the ways that different cultural groups of individuals are "barred" from health care access. Language and religion are also important barriers to health care access. DeSantis \& I Ialberstein (1992) wrote that,

Numerous cultural barriers, such as language, religion, and ethnomedical (folk) beliefs and practices are typically built into the migration process... Likewise, massive influxes of refugees and immigrants into the United States over the past two decades have severely stressed health care delivery and social services agencies in many major metropolitan areas simply because of the sheer numbers they must serve.

It is clear from the above discussion that the understanding of the relationships between patient and healer is critical for a full appreciation of local/community health care systems. Further, it also promotes an understanding of the factors that contribute to the utilization of particular types of health care, i.e., folk medicine or biomedical. To 
fully understand the impact on health care system utilization there must be an indepth investigation of the system of health care beliefs and behaviors within the community. The next section of this chapter will examine the literature on health care utilization in the general population, the elder population, and the minority elder population.

\section{Health Care Utilization}

Health-related behaviors are outcomes of a variety of determinants. The literature can be categorized into five broad classifications of determinants: personal, family, social, institutional and cultural (Gochman, 1988). A brief description of these five classifications will follow. Following this, the focus of the discussion will be on personal characteristics and social determinants that have been found in research to play vital roles in determining health care service utilization.

Personal determinants research focuses on two aspects: cognitive characteristics, i.e., health beliefs and personal characteristics, i.e., age, gender, marital status, and educational attainment. Also included in the category of personal characteristics are life style, such as common habits and social networks of the individual. An example with respect to gender is osteoporosis. Eighty percent of those with severe bone loss are women ("In Brief: Gender Differences in Disease" 1999). Heart disease is usually thought to be most prevalent among men. Ilowever, recent research has found that women are more prone than men to having a second heart attack within a year of the first heart attack ("In Brief: Gender Differences in Disease" 1999). 
Family determinants focus on aspects of behaviors that are socially learncd within the family environment because "... families are powerful and lasting determinants of preventive health behaviors" (Sallis \& Nader, 1988). In a general population study of health care service utilization behaviors, family determinants will be important since "family plays a critical role in the socialization of children" (Sallis \& Nader, 1988). Ilowever, in a study of health care service utilization and elders, family determinants may not be as directly significant for elders as for younger individuals in determining health care utilization. Yet, family characteristics may play important roles in other ways, such as caregiving and health monitoring.

There are two sub-categories of social determinants: group and network characteristics. Group characteristics include "research on group membership, group norms, social status or stratification" (Gochman, 1988). On the other hand, network characteristics include "research on the characteristics of social ties or linkages" (Gochman, 1988). Both are important; however, the operationalization of group structures and social networks is "more diverse and complicated than originally recognized" (Geertsen, 1988).

There are three focuses of institutional determinants: organizational characteristics, environmental characteristics and systems barriers. Organizational characteristics include both policies and task organization, while environmental characteristics include size, location and space. The cultural context in which health care service utilization is explored is important because: 
The cultural context shapes prevailing heliefs regarding the cause of illness and appropriate forms of treatment. Whether a person is sensitive to feelings of discomfort, how symptoms are perceived and evaluated, and whether or not the individual decides to seek medical care are largely a matter of cultural prescriptions (Segall, 1988).

The operationalization of cultural determinants is complicated. Gochman (1988) notes that: "much research that has ben labeled or indexed as cultural or sociocultural is simply demographic the term 'race/ethnicity"." Race/ethnicity by itself, however, does not explain the differences in health-related behavior. Some of these differences may be due to the educational attainment differences that are in part due to earlier historical discrimination in educational opportunities for certain groups of individuals.

\section{EIder Population}

Research has shown that the elderly are heavy users of health care services (Evashwick, et al., 1984; Soldo \& Manton, 1985). A U.S. Senate Special Committee on Aging (1988) reported that while the elderly make up approximately 12 percent of the total population in the United States, they account for approximately 33 percent of health care expenditures. Furthermore, health care dollars are not equally distributed by services for elders. The Administration on Aging (1988) found that in 1984 hospitalization costs accounted for the largest percentage of total health care dollars spent for older persons (45 percent), compared to physician visits ( 21 percent) and nursing home care (21 percent).

With respect to gender, women are more likely to use health services overall (Marcus \& Siegel, 1982); and have more chronic complaints compared to men (Aday, 
1993). Verbrugge (1989) found that women have a higher morbidity rate, while men have a higher mortality rate. With respect to hospitalization, women are much more likely to be hospitalized than men are (Aday, 1993). However, men are more likely to have acute problems requiring hospitalization (Aday, 1993), which may in turn explain why men tend to have longer average stays in the hospital than women (Aday, 1993). With respect to preventive care services, the relationship between utilization and gender is inconsistent (Aday, 1993).

Marital status has been shown to be associated with health and survival outcomes, not only in the population as a whole (Joung, et al., 1998; Ren, 1997; Waldron, et al., 1996; Kisker, 1990), but also in the older population (Goldman, et al., 1995). Nonmarried individuals are substantially more likely to suffer from illness than married individuals. Specifically, Goldman, et al., (1995) found an unanticipated result; anong older non-married there were "variations in health outcomes across the three [nonmarried] groups." However, results from research investigating the relationship between widowhood, gender and labor force participation with ambulatory physician service utilization suggests that "the effects of widowhood and labor force participation are spurious" (Homan, et al., 1986). The researchers based this conclusion on the fact that more widows live alone and are less likely to work compared to widowers. Further, the researchers found that an individual will use more health scrvices if they live alone and are not in the labor force compared to an individual who lives with others and is in the labor force (Homan, et al., 1986). In other research on self-rated health assessment, Choi (1996) found that marital status was not a significant determinant of self-assessment of 
health status, all other things being equal. However, in terms of engagement in positive health behaviors, such as physical activity, eating breakfast, wearing seat belts and not smoking, Schone \& Weinick (1998) found that, "marriage encourages [positive] health behaviors in the older population."

Education affects utilization behavior in a number of ways. In particular, it seems to be an important determinant of preventive health care utilization (Aday, 1993). Overall, individuals with more education are more likely to use discretionary health care services that are preventive (National Center for Health Statistics, 1991). Examples of discretionary health care services that are preventive are general physical exams, health screening and immunizations. However, the directional effect of education on general health care utilization is unpredictable. Highly educated individuals may be more aware of the utility and the limitations of health care. However, "ignorance can lead to either too little or too much care" (Pauly, 1974). Not only does education effect an individual's awareness of health care, education may effect the value that individuals place on their health (Pauly, 1974).

It has been pointed out in other research that education and income are highly correlated. People with more education are also more likely to have higher income, although education is "better correlated with permanent income than with present income" (Pauly, 1974). This is especially important in the study of elders because many individuals 60 years or older are retired or not participating in the labor force currently. Historical discrimination of minorities in the work force, however, may have forced many 
minority elders with similar educational backgrounds to not have similar income levels. compared to majority elders.

With respect to race and ethnicity, Cockerham (1992) found across group differences with respect to: (1) response to symptoms of illness; (2) knowledge of disease; and (3) health related behavior. The National Center for Health Statistics (1991) found that whites have a generally higher utilization of physician office visits than blacks. On the other hand, blacks are much heavier users of clinics and emergency rooms for health care. However, race and ethnicity, by itself, does not explain these differences. Some of these differences may be due to the educational attainment differences that are in part due to earlier historical discrimination in educational opportunities. Income also plays a part in the differences in usage by minority elders.

Race/ethnicity ... may provide a social and cultural context for social networks in seeking health care (Cockerham, 1992)

An examination of the theoretical perspectives on aging is important to the understanding of health care utilization behavior of elders. There will be a more detailed examination of the evolution of theoretical perspectives on aging in Chapter 4 .

\section{Minority Elder Population}

The literature in the field of medical sociology lends weight to the supposition that different factors for each racial/ethnic group will be important in determining whether and how an individual will use health care services. However this has not been proven adequately. However of the research available on specific racial/ethnic groups, substantial differences have been found with respect to morbidity (Manuel \& Reid, 1982; 
Solis, et al,, 1990), mortality (Elo \& Preston, 1994; Greene \& Siegler, 1984), health and illness behavior (Higginbotham, et al., 1990), access (Haynes, et al., 1990), and utilization of health services (Lacayo, 1980; Solis, et al., 1990; Trevino \& Moss, 1984; Wallace, et al., 1994).

Although there has been research on specific minority elder groups (i.e., AfricanAmerican), the comparison of these specific minority elder groups has, for the most part, been with whites or white non-Hispanic elders. Very few have compared differences of access and utilization with other groups of minority elders. One reason may be that the percentage of elders who are of minority status, with respect to all elders, has been very small. $^{2}$ For example, in $1990,3.8$ percent of the national population aged 60 years or older was of Hispanic-origin. In a national study of racial/ethnic groups of elders, one would have to over-sample minority elders of each specific racial/ethnic group that are being considered and this could be extremely costly. However, this research is extremely important for local communities. There are large geographically local pockets of minorities throughout the country, i.e., Mexican-Americans in the Southwest, AsianAmericans in San Francisco, Polish-Americans in Chicago, as well as Cuban-Americans in Miami.

Most of the literature on minority aging "lacks integration and shares no common theoretical base" (Chapleski, 1994; Riley, Hess \& Bond, 1983). Demographic trends 
show the older population is becoming more heterogeneous. In particular, the number of minority elders is projected to grow at a faster rate than that of white non-IHispanic elders by the year 2050 (U.S. Bureau of the Census, 1992): the number of black elders will almost quadruple, while the number of Hispanic elders will more than double. The multicultural elder community of Miami-Dade County, Florida offers a unique opportunity to study the similarities and differences between racial/ethnic groups of elders.

Minority elders have had different life experiences, in some cases suffering economic and social deprivation that have continued into their present lives. These different experiences may create differences in perception of health. Thus, health care utilization behavior may be different. Therefore, there is a need to analyze and explore the issues impacting the lives and utilization behaviors of minority elders. As aging policies are developed, we must ensure that all elders have access to necessary health care that is culturally sensitive to their needs.

Much of the original theory of aging was developed pre-1960's, and took place when race and ethnicity in the elder population was insignificant (Markides \& Mindel, 1987). However, beginning in the 1960 's research on black elders began to appear in literature, and it was not until the 1970's that research on the Hispanic elder population

2 "Of the total elderly population in 1990 , about 28.0 million were White; 2.5 million, Black; 116,000 . American lndian, Eskimo, and Aleut (AIEA); 450,000, Asian and Pacific Islander (API); and 1.1 million were of Hispanic origin" (U.S. Bureau of the Census, 1992) or approximately 1 in 10 elderly persons in 1990 is non- White (U.S. Bureau of the Census, 1992).

${ }^{3}$ An overview of the evolution of theoretical perspectives on aging will take place in Chapter 3 . 
began to appear. The aging experiences of other ethnic minorities such as Asian and Native American elders, are just beginning to be explored.

Minority groups (i.e., Blacks, Hispanics, Asians, and Native Americans) are said to share a common experience of "discrimination and subordination in society" (Markides \& Mindel, 1987); in turn, this may affect health care service utilization. Ethnic minorities may not, in certain locales, be numerical minorities, but they are still minorities due to their relative lack of power and/or control over their lives. However, the notion of discrimination and subordination is hard to define for some groups of ethnic minorities. For example, in the case of Cubans, it is not long-term discrimination and subordination in the United States that most of these elders have suffered. Their "suffering" is internal through their status as a "political refugee" and being forced to flee their homeland. Some other immigrant elder groups may not have suffered discrimination and subordination in the United States, but experienced it in their homeland, as in the case of many other Latin American immigrants. Some may argue that racial/ethnic minority elders exhibit specific problems of aging that are common to all low-income groups, including low-income whites. However, this approach to minority aging is naive and simplistic because it does not take into account cultural differences in such areas as help-seeking behavior.

An important concept in the social theory of aging and race is "double jeopardy" which theorizes the disadvantages that blacks have compared to whites within each age group (i.e., lower education and socioeconomic status) (Dowd \& Bengtson, 1978). McNeely \& Cohen (1983) note that aging among racial/ethnic minorities and the majority 
white non-Hispanic population is not a uniform experience. Later theoretical developments in minority aging focused on "triple jeopardy," which adds gender differences to the mix. This was further expanded to "multiple jeopardy" which considers socioeconomic factors. The "multiple jeopardy" hypothesis seems to be given credence by the fact that blacks have, on average, a shorter life expectancy, as well as higher rates of morbidity and disability. However, after age 80 , there appears to be a "crossover effect" in which blacks tend to show an increased life expectancy and lower mortality rates compared to whites. Many researchers have tried to explain this phenomenon. Whatever the explanation, it is clear that the aging experience for blacks and other ethnic minorities is not the same as that of majority white non-Hispanic elders, which in turn, demands a health care utilization model that addresses these issues.

Hendricks \& Hendricks (1986) found that the experience of aging by many minority elders more closely resembles aging in Third World countries in terms of inadequate financial resources, ill-health, shorter life expectancy, limited access to health care, etc. Many white non-Hispanic elders in the United States, although they may not currently have adequate financial resources, have had throughout their life-course, in general, better access to economic and educational opportunities than many minority elders. There is a need for a model that is more responsive to racial/ethnic diversity. Unfortunately, it appears that in the literature, "Gerontologists have tended to downplay differences and emphasize similarities among the aged, consequently ignoring life experiences, and cultural and demographic differences among subpopulations" (Chapleski, 1991). This idea of emphasizing similarities accompanies the hypothesis of 
"age as a leveler." This hypothesis states that as individuals age the relative disadvantages of minority persons will decline. The presumption is that all elders expericnce similar hardships (i.e., declining health, increasing difficulty with activities of daily living and other functional limitations, as well as social isolation and depression). regardless of racial/ethnic background. This is also a naive presumption In their extremes, these two hypotheses, "multiple jeopardy" and "age as a leveler," present a paradox for the study of the minority aging experience (Hendricks and Hendricks, 1986). If research focuses too heavily on differences, this can lead to the notion that minorities are somehow inferior; paradoxically, if similarities are stressed too heavily then researchers may overlook some very real differences and problems that minority elders face. This research proposes an integrated perspective that combines both individual-level factors as well as structural constraints to gain perspective on the health care utilization behaviors of racial/ethnic groups of elders in a multicultural urban environment of Miami-Dade County, Florida.

A unique aspect of using Miami-Dade County as an area of study is the large percentage of elders that are not white non-Hispanic. In particular, the 1990 U.S. Census of Miami-Dade County, Florida shows that almost two-fifths of elders were Cuban (38.2\%, Ruggles, Sobek, et al., 1997). Cuban elders do not fit the standard research model of "ethnic minority" elders because they have re-created in Miami-Dade county, "their (pre-Castro) system of privately owned and operated health maintenance organizations (clinics). With emphasis on preventive and health maintenance, these 
clinics provide a full range of culturally sensitive relatively low-cost outpatient and ambulatory services" (Queralt, 1983).

While there has been an increasing amount of research on the effect of cultural heritage on health care in the lives of elders (Jackson, 1980), there have been fewer studies that focus on utilization and access to health care among the Hispanic population (Trevino \& Moss, 1983). Most of these studies have focused on Hispanics of Mexican descent. Nationally, Mexican-Americans are the largest group of Hispanics in the United States (61.2 percent of all Hispanics). The next largest group is Puerto Rican (12.1 percent of all Hispanics), and finally Cubans comprise the third largest group (4.8 percent of all Hispanics). However, among those aged 60 years or older, Cuban elders are the second largest Hispanic elder population after Mexicans (i.e., 14.8 percent and 50.6 percent, respectively). In 1990, 22.4 percent of all Cubans in the U.S. were 60 years or older. In comparison, 6.0 percent of all Mexicans and 6.7 percent of all Puerto Ricans were 60 years or older (U.S. Bureau of the Census, 1992). Further, it is projected that the number of Hispanic elders will account for 16 percent of all clders nationally by 2050 (Burnett \& Mui, 1999).

Another complication in many previous studies of Hispanics has been the tendency to treat all Hispanics as a single ethnic group thus masking important culture specific characteristics. Although Hispanic sub-populations may share a common language, there are significant variations within and across sub-populations of Hispanic elders that have particular relevance to health care access and use. Siddharthan \& 
Sowers-Hoag (1989) noted that, "little information exists on the Cuban-American elderly population to asccrtain health care needs or access and use of health care."

Cuban elders in the United States are, for the most part, immigrants who arrived in the United States between 1960 and 1969 due to the political upheaval in Cuba. They are highly concentrated in Florida, particularly Miami-Dade County, "with the majority residing in Miami (particularly in the Little Havana area) and in Hialeah" (Queralt, 1983). Most Cuban elders have been ineligible for Medicare because "few have accumulated the necessary employment credits to qualify for Social Security benefits ... to be entitled to Medicare" (Queralt, 1983). Medicare coverage may be purchased by individuals 65 years or older if they have resided in the United States for a minimum of 5 years, regardless of their employment status. However, the cost of this insurance coverage for many Cuban elders (as well as other immigrant elders) is too expensive. Recent Medicare reforms have made access even more difficult for immigrant elders, as well as native-born elders living on the edge of poverty (Zaldivar \& Rogers, 1995). In a recently reported study using data from the 1988 National Survey of Hispanic Elderly People, it was found that Cuban and Puerto Rican elders "were 2.3 and 2.6 times more likely ... to have seen a physician than Mexican American elders" (Burnett \& Mui, 1999). Further, they found that "enabling factors, especially insurance coverage and adult children had the greatest impact..." on the likelihood of a physician visit for all three groups of Hispanic elders (Burnett \& Mui, 1999).

Medicaid, as a federal-state cooperative health insurance plan for individuals with low incomes, "is available to those unable to receive Medicare and who meet 
Supplemental Security Income (SSI) eligibility requirements. But in various states, this translates into ineligibility for certain medical services, notably home health services" (Queralt, 1983). Home health care services are important because these services allow frail elders to reside within the family setting. The Cuban culture, specifically, as well as the Hispanic culture in general, places a high value on the care of frail elders within the family setting. Therefore, utilization of health care services available under Medicaid may be a "last resort" for Cuban elders, as well as other Hispanic elders, because it is not culturally sensitive. In a recent study, Lum \& Chang (1998) using data from AHEAD ${ }^{4}$ concluded that the Medicaid program does not "work equally well for low-income elderly people from racial, ethnic, and gender groups" to improve affordability and use of health services. They found significant race effects on the probability of physician visits and hospital visits. Both African-American and I Hispanic elders were more likely to visit physicians than white elders. However, Hispanic elders "were 37 percent less likely to use hospital services."

A growing body of academic literature suggests that cultural background has an important impact on utilization of health services, as well as long-term care services. For example, recent research profiling populations in three long-term care settings in Florida found that Hispanic elders were least likely to be found in nursing homes, were somewhat more likely in assisted living facilities and most likely using home and community based services (Dunlop, Brooks \& Polivka, 1995). While in another study on utilization of

\footnotetext{
${ }^{4}$ Asset and Health Dynamics Among the Oldest Old is a national representative sample of communitybased elders aged 70 years and older and their spouses. It is funded by the National Institute on Aging.
} 
community services for elders, ${ }^{5}$ Netzer, et al. (1999) found that there were significant differences across race in the use of senior centers and special transportation services. For the two home based services, no race differences were found.

\section{Impact of Community Characteristics}

Socio-Behavioral Model of health care utilization (SBM) developed by Andersen (1968) was originally conceptualized to include both personal and community characteristics that could be involved in influencing the health care utilization behavior of an individual. However, most of the research previously done has focused on the personal characteristics and has left out or ignored environmental characteristics (Andersen, 1995). Wilkinson and King (1989) noted that race, as a social concept, "cannot be interpreted apart from its environmental context." However, the environmental context has not been viewed as an important factor. Phillips, et al. (1998) undertook a systematic literature review of published articles between 1975 and 1995 that cited the socio-behavioral model to examine health care utilization with respect to environmental and provider-related variables. They found among the one hundred thirty nine articles examined that less than half included environmental variables and just 50 percent included provider-related variables. Further, they found that,

... few studies examined specific measures of the health care system or provider characteristics or used methods other than simple regression analysis with hierarchical entry of variables (Phillips, et al., 1998: 571).

\footnotetext{
"The four community services were senior centers, special transportation services, homemaker services, and in-home nursing care scrvices. Senior centers and special transportation services were considered indicators of community based services, while homemaker and nursing care services were considered indicators of home-based services.
} 
By focusing on Miami-Dade County, Florida, this research will address the issue of the environmental and provider-related variables of health care utilization behavior of elders in a multicultural environment. Further, this research will also include an examination of qualitative data from six focus groups of elders residing in Miami-Dade County.

Health care in the United States is typically provided at the local level. Unlike many other goods and services, individuals cannot shop around nationally for health care services to "find the best deal." Many view the health care system as a "non-system" due to its poor integration, lack of centralized control and regulation, fragmentation, discontinuity, and duplication (Pol \& Thomas, 1993). Variations in available services under Medicaid, and even Medicare, exist by state, as well as within states by county (Harel, et al., 1985). Therefore, studying the health care service delivery system at the local level provides a clearer picture of the environmental factors that influence individual health care utilization behavior. Further, since only one agency distributes state and federal funds for aging services, conflicting criteria or inconsistencies in programs will not hamper the research across jurisdictions. The distribution of services around the county, however, may not be equally or equitably distributed mainly due to historical residential patterns of elders in the county. Our nation's complex health care system can best be understood by understanding the local health care market's affect on the individual's utilization/health care choices. 


\section{Health Care Belief Systems: A Case Study of Cuban Elders in Miami-Dade County, Florida}

Utilization of health care services by a particular group of elders requires a holistic understanding of the ways in which individuals within the culture pattern their health care utilization. Meaning and life experience are ascribed to illness experiences. There is an intricate inter-relationship between the meanings and the individual's behavior towards illness. As discussed earlier in this chapter, culture plays an important role in understanding behaviors involved in health related situations. Many cultures are blends of other cultures. For example, Cuban culture is a blend of Spanish, African and Amerindian cultural patterns (Suarez, 1997). Unfortunately, much of the literature on health care and minority elders lumps Cuban elders with all other Hispanics, which is in large part Mexican. While Mexican culture is also a blend of cultural patterns, there are significant differences between Mexican and Cuban cultures. Further, socio-demographic attributes of the Cuban elder population play an important role in understanding the health care beliefs. For example, the wave of Cuban immigrants coming between 1960 and 1969 were disproportionately from Cuba's middle, upper, and professional classes. While the island of Cuba experienced a brain-drain, the United States, particularly Miami, received Cuba's best and brightest. Boswell (1995) wrote that "one consequence is that the Hispanic population of Miami is not associated with poverty to the same degree that it is in many other U.S. metropolitan areas" (p. 4). Thus, it is always important to be aware of socio-demographic characteristics of the cultural group with respect to their health beliefs and practices. 


\section{Historical Experiences of Cuban Medicine and Health}

Most of the literature on Cuban medicine and its health care system focus on the health care system developed post-1959. The Cuban government under Castro put health care into "a prominent place in the Cuban government's domestic and foreign policy" (Grossman, 1998: 199-200). However, the health care system that has developed under Castro had important precursors in its pre-Revolutionary period. Grossman (1998) describes the health care system before the 1959 revolution as, "an organized government-supported health program that provided medical care to the majority of citizens."

Like other colonial-established nations of the New World. the historical control of the island of Cuba impacted the medicine and health care system that was available in Cuba. Boswell \& Curtis (1984) describe the history of Cuba as "characterized by foreign domination" (p. 11). Between 1511 and 1898, Cuba was ruled by Spain. In the period up to 1790 , Danielson (1979) described Cuba's early health system as "a frail transplant of the Spanish system. Its parts were simple and few" (p. 21). While it may have been a frail transplant, Danielson (1979) also wrote that the period betwcen 1521 and 1790, "[must be] regarded as the real base of the health system that emerged in future years" ( $p$. 37).

The first medical/health revolution in Cuba occurred between 1790 and 1830 . along with economic developments in which Cuba was "transformed [from] the rather placid paternalism of early urban and agricultural Cuba [to] a brutal and cosmopolitan slavocracy of huge proportions" (Danielson, 1979: 41). In this period of economic 
transformation, the health care system had to respond to the new domestic conditions.

Danielson (1979) wrote that,

... while the ensuing changes were not earthshaking, they were highly significant precursory events to the really earthshaking changes in the health system that occur at the end of the nineteenth century (p. 41).

Between 1898 and 1902, Cuban was occupied by a United States military government. Political independence as a capitalistic state came in 1902 and remained until Castro came to power in 1959. In 1909, Cuba established a cabinet-level health department and thus became the first country in North America to do so (Hemmes, 1994 :

53). However, like other less developed countries, the health ministry in this period was "ineffective, under-funded, and corrupt" (Nayeri, 1995: 323). Feinsilver (1993) wrote that,

Perhaps the most important pre-Revolutionary organization was mutualism, a prepaid health plan much like the health maintenance organizations that became prevalent in the United States in the $1970 \mathrm{~s}$ (p. 31).

Nayeri (1995), stated that "the Mutualistas controlled most resources including about $40 \%$ of hospital beds" (p. 323). Approximately half of the population of Havana was served by these mutualistic clinics in 1958 (Feinsilver, 1993).

When Castro came to power in 1959 , many of the physicians that practiced in Havana fled the country (Feinsilver, 1993). Further, all ties to the United States for the support of the Cuban health care system in general were cut. Danielson (1979) wrote of this transition period under the socialist revolution as one in which,

physicians ... were hurt or confused by the spced of events, which made life difficult for everyone, [and] had little time to comprehend the 
revolution or find their own compromise before they were recruited en masse to the United States (p. 129).

As time went on and Castro's regime solidified its power, the health care system in post-revolutionary Cuba gained a new sponsor - Soviet Union.

The first wave of Cubans fleeing the turmoil in their homeland primarily came from the middle, upper and professional sectors. They did not see themselves as permanently situated in Miami (or the United States); nor did they think they would spend their so-called "golden years" in Miami. While they may have come to the United States with little more than the clothes on their backs, they had human capital of education and past political clout that allowed them to develop enclaves in Miami with a health care system similar to pre-Castro Cuba. This has helped Cuban immigrants maintain many of their health care beliefs and utilization patterns, such as the high use of preventive measures. However, in a working paper "Elderly Cubans in Exile" presented to the U.S. Senate's Special Committee on Aging in November 1971, it was stated that,

Because of limited ability to speak English, many older Cubans ... are unable to participate in programs which could help them immeasurably. In addition, the language barrier may make it impossible for Spanish speaking persons to understand the nuances of existing programs" (p. 7).

Lack of English-speaking skills and poverty are still significant problems for

Cuban elders in the 1990s and possibly could create barriers for utilization of a variety of community programs.

\section{Health Beliefs and Practices of Cuban Elders in Miami-Dade County, FL}

As an historical example, discrimination and poverty among native black communities in the United States made mainstream medical care, more often than not, 
inaccessible to the majority of black Americans. Immigrants to the United States who do not speak English also have experienced barriers to mainstream medical care. In more recent times, these historical barriers to mainstream medical care have been gradually eliminated.

While other minority groups may delay treatment, Cuban immigrants "exhibit high levels of preventive health behaviors..." (Grossman, 1998: 200). Even before Castro came to power, Cubans were "socialized into a strong health ideology and successful primary care system in Cuba" (Grossman, 1998: 207). This type of socialization allows Cuban immigrants to pursue both traditional and biomedical care when they encounter a health problem (Grossman, 1998). Health care providers in the Cuban-American community, particularly in Miami, are advised that "when Cuban clients consult a health care provider, in all likelihood they have already tried some folk remedies advised by older women in their family or obtained from a botanica" (Grossman, 1998: 207). The implications for older Cubans residing in Miami-Dade County are somewhat indeterminant. Little research on health beliefs has been published on Cuban elders.

The value orientation study of the various immigrant groups living in MiamiDade County, Florida done in the early to mid-1970s found that Cubans (all age groups) were similar to Americans on several value modalities. Table 2.1 shows a comparison of racial/ethnic value orientation profiles from the Health Ecology Project with core American value orientation profile (Egeland, 1978). 
From this analysis, researchers were able to examine points of conflict betwcen dominant American core medical values and the various potential patient groups in Miami-Dade County, most notable is the difference in the time modality. Table 2.1 illustrates the typical Cuban value model as: (1) doing orientation; (2) belief in man's ability to achieve mastery-over his environment; (3) an emphasis on present time; and (4) the individualistic mode of interaction. However, Egeland (1978) states that there is "a common lack of statistical significance [in the overall findings]...” (p. 116).

Points of overlap with the American medical value model are in the activity modality and the relational modality. Man-nature modality is not an exact overlap in the second preference for Cuban value model is "Subjugated to Nature," while the American medical value model is "Harmony with Nature." Again, it must be noted that the firstorder choice for "Mastery" by Egeland (1978) was not a statistically significant ranking. Therefore, the characterization in other studies describing the typical Cuban as "fatalistic," and "feeling that they lack control over circumstances influencing their lives" (Grossman, 1998: 206-207) is not in opposition to the findings in the Miami Health Ecology Project. However, it must be noted that this research is for the general population and is not specifically focusing on elders.

Because of the multiple losses that Cuban elders in Miami-Dade County, Florida have experienced (i.e., "leaving their homeland and the difficulties associated with adaptation to a new culture and environment" (Grossman, 1998: 209)), Cuban elders are at an increased risk of suffering from loneliness, insecurity and depression. 
The cultural response among Cuban-Americans to illness is an acceptance of dependency as an acceptable sick role (Grossman, 1998). It has been pointed out that "sick family members are showered with attention and support" (Grossman, 1998: 210). In general, research has shown that the experience of pain for Cubans is a "signal of physical disturbance that would warrant consultation with a traditional or biomedical healer" (Grossman, 1998: 210). Further, Cuban-Americans" response to pain tends to be expressed through "verbal complaints, moaning, crying and groaning" (Grossman, 1998: 210). In turn, these responses to pain "may serve a pain relieving function and may not necessarily signify a need for administration of pain medication" (Grossman, 1998: 210).

In general, barriers to health care for many racial/ethnic minority groups in the United States are poverty, as well as language barriers, transportation, time lag and bureaucratic red tape of health care coverage such as Medicaid. However, within the Cuban immigrant community of Miami-Dade County, Florida, there is not the same degree of association with poverty as in other Hispanic immigrant communities. Another general barrier for immigrant elders to health care utilization that has been mentioned is eligibility for Medicare. The recent linking of access to public assistance with citizenship requirements makes this an even greater issue for immigrant elders.

For individuals who must rely on Medicaid services for health care, this usually entails utilizing public health clinics. It has been well documented that individuals who use public clinics and/or public hospitals encounter at least one or more of the above mentioned difficulties. For immigrants from non-English speaking countries, language is 
a major difficulty in using most health services, whether they are public or private.

Grossman (1998: 209) states that Cuban-Americans who have been hospitalized, either in a public or private hospital, will "name language as a major difficulty in using health services." Results from the 1990 U.S. Census of Miami-Dade County, FL show that 41.7 percent of Cuban elders did not speak English at all. Thus, it would appear that Cuban elders could have significant difficulty using health care services. However, findings from the Miami Health Ecology Project's ethnic value orientation analysis (Egeland, 1978) show that typical minority barriers in communication and behavioral expectation should not be as problematic for Cuban immigrants since there appears to be a match in preferences of individualism over collaterality and over lineality in the relational modality, and doing over being in the activity modality. However, these apparent matches mask the fact that there is a lack of statistical significance in the ranking.

In the Cuban enclaves in Miami-Dade County, FL, Cuban families have access to primary health care services through the "extensive network of privately owned and operated health clinics..." (Grossman, 1998: 211). This extensive network developed primarily because of the early wave of Cuban immigrants to the United States were primarily from the middle, upper and professional classes in Cuba. Further, the high concentration of Cuban immigrants in the ethnic enclaves of Little Havana and Hialeah was also important to the development of these networks. Grossman (1998) and others note that,

The private health clinics are believed to be popular among the Cubans because they provide the services that are culturally sensitive to Cuban 
needs, such as emphasis on the family, use of the Spanish language, focus on preventive health-care behaviors and low cost (Grossman, 1998: 211). Information on the ethnicity of health care practitioners is only available generally, i.e., Hispanic-origin. With respect to health care practitioners ${ }^{6}$ of Hispanicorigin, there tends to be an under-representation of Hispanic health care practitioners in the United States, as a whole, and Florida (Ginzberg, 1991; Rojas, 1994; Grossman, 1998). Whether this is true for Miami-Dade County, in particular, has not been examined.

Up to this point, the discussion of health care beliefs and practices has focused on biomedical services available. However, as has been mentioned previously, Cuban culture equally uses biomedical and traditional health care. In the Cuban ethnic enclaves of Miami-Dade County, there are numerous stores called botanicas. Such stores sell traditional medicinal plants in the forms of teas, potions, salves, or poultices, as well as oils, ointments, powders, incenses, religious figurines, Santeria necklaces and animals used for ritual sacrifices. All are used in some fashion to "relieve maladies, bring luck, drive away evil spirits, or break curses" (Grossman, 1998: 208).

The majority of Cubans are Roman Catholic from its Spanish cultural traditions. The Roman Catholic Church plays an important role in the life of Cuban immigrants of all ages, but particularly Cuban elders (Suarez, 1998). However, Cuban culture is not just of Spanish origin. It is a blend of Spanish, African, and Amerindian cultural patterns.

\footnotetext{
${ }^{6} \mathrm{Hea}$ th caré practitioners include registered nurses, dentists, pharmacists, therapists, as well as physicians.
} 
This creates a pluralism of beliefs in which Roman Catholic beliefs co-exist with a belief in spiritism and santeria ${ }^{7}$ (Rogg \& Cooney, 1980). Danielson (1979) wrote that, ... the medicine of early Cuba long survived in the folk medicine practiced among the rural and less privileged sectors (though not limited to them) of Cuba" (p. 34).

The practice of santeria is especially prevalent and growing in the United States among the lower economic strata of Cuban immigrants. Suarez (1998: 183) believes this growth in santeria is due to their particular "need to have a system that [helps] them to cope with the problems of migration." However, many Cubans will not admit to their practice of these non-Western healing and spiritual practices because of negative mainstream attitudes toward these "spiritual systems" (Suare\%, 1998). Thus, it is suspected by many researchers in the field that the actual degree of practice may be highly under-reported due to the sensitive nature of the question (Grossman, 1998).

\section{Summary and Future Research Needs}

Medical anthropological and sociological literature notes that cultural factors determine what an individual perceives to be an illness and where/who that individual will go to for healing and or curing of the 'illness.' The increasing cultural diversity of elders will have important implications for the health care system within a community. Thus an understanding of meaning of illness and the discourse regarding the social construction of health beliefs is important to understanding health care utilization

\footnotetext{
? "Santeria is an Afro-Cuban religious and folk-healing complex that evolved from the syncretization of Spanish colonialists' Catholic beliefs and of African world-view rituals; it is 'the product of an identification between the gods of the slaves and the Catholic saints of their masters'..." (Suarez, 1998: 183).
} 
behavior of diverse cultural groups of elders. This discussion has begun this dialogue with respect to Cuban elders residing within Miami-Dade County, Florida. Further research should be done with respect to the native-born Southern black, white nonHispanic, and non-Cuban Hispanic elder communities within Miami-Dade County, since none of these communities exist within a vacuum. The public funding of health care and services for individual communities come through many of the same agencies. Thus through an exploration of the discourse found in a multicultural urban environment of Miami-Dade County, research should be able to elucidate and better represent an overall understanding of these socially constructed terms of health and illness in a multicultural urban environment. In turn, the better overall understanding of health and illness will allow for policy makers to better understand how barriers to access confront particular racial/ethnic/cultural groups of elders, as well as the possible solutions for overcoming these barriers. An examination of discourse on health and health care by racial/ethnic groups of elders will take place in Chapter 6.

Another factor that will be explored is how older individuals talk about the process of "aging." Western philosophy and theoretical perspectives tends to set up contrasting conceptual categories, i.e., old vs. young. Yet, what is "old"? How is "old" operationalized in society? How does a particular individual operationalize "old"? The expectations of aging are culturally constructed. Current research is just beginning to examine these issues. This literature will be examined in depth in Chapter 4 . What is evident from the early research is that most elders are experiencing many of the same problems with the aging process, at least biologically. However, there are different 
interpretations of the information that depend on the elder's previous life experiences and current socio-economic and familial situation. Most of the so-called knowledge concerning meaning of aging comes from the non-elders (the unmarked category). If there is a discussion from older individuals, it is primarily derived from somewhat homogeneous groups of elders, mostly white non-Hispanic. Previous research and discussion of these issues with respect to minority elders has been hampered by small population sizes. Miami-Dade County with its large minority elder population has allowed and will continue to allow for a better examination of the interplay between culture and the aging process within one geographic community. This understanding of the links within the cultural communities within a given geographical community is part of what Marcus \& Fischer (1986) define as good ethnography, i.e., must link the community under study to the outside, as well as telling the inside story of everyday experiences of its residents, not only currently, but historically. Better knowledge of the community, or "good ethnography," will heed the call by Marcus \& Fischer (1986) and others, for a more complete contemporary anthropology.

\section{APPROACHES TO HEALTH AND UTILIZATION OF SERVICES}

The relationships linking health care service utilization to individual characteristics are not easy or neat to analyze. A variety of approaches or models have been developed to analyze the determinants of health behaviors (McKinlay, 1972, 1985; Ward, 1978; Wolinsky, et al., 1987; Suchman, 1965a, 1965b, 1966; Andersen, 1968). These perspectives can be classified into four approaches: (1) cultural; (2) social; (3) 
social-psychological; and (4) sociodemographic models. A brief description of these approaches and some of the results from research and models will be presented.

Following this will be a more detailed description of the conceptual framework that will be used in the proposed research.

\section{Cultural Approach}

The cultural approach brings together two related behaviors. First, there is "the relationship between cultural content and cultural life styles" (Mechanic, 1968). Second, there is the influence of culture on an individual's response to illness. This approach explores "... health services utilization as part of a cultural complex related to other social institutions in a society or sub-culture..." (Gochman, 1988).

Numerous studies have shown the important impact that culture has on how an individual perceives, expresses and reacts to an illness (Mechanic, 1968; Fox, 1989). Parsons (1951) was a pioneer in orienting the study of health behavior towards a sociocultural approach. Previously health had primarily been analyzed solely as a physiological malfunctioning. In early work done by Zaborowski (1952), it was found that there were different responses to pain across ethnic groups. In another study, Zola (1966) also found differences across ethnic groups while studying responses to health problems. Suchman (1965b) found ethnic differences "in the extent of the belief in and acceptance of modern medicine" (Bradsher, 1992: 18). 


\section{Social Approach}

The social approach overlaps the cultural approach in some respects. However, the social approach focuses more upon the family life, and social and work activities. This perspective is, "concerned with the manner in which human qualities and responses fit social demands and the physical and economic environment" (Mechanic, 1968). In particular, studies with this approach are concerned with explaining the relationship between personal characteristics and social environment and the ability of successful adaptation to a specific social environment. With respect to health, the social perspective is important in discussion of norms of behavior in sickness and the response to sickness.

\section{Social-Psychological Approach}

In the social-psychological approach, there is a joining of the fields of psychology and sociology. The primary concern of this perspective is with, "the ways individuals interact, communicate and influence one another" (Mechanic, 1968). Several models have been developed using this perspective; the predominate one being the Health Belief Model (HBM).

It was originally developed "to explain and predict why people engage in specific preventive behaviors, such as accepting a vaccine..." (Gochman, 1988). Becker, et al., (1977) write that;

The theory argues that whether or not an individual will undertake a recommended health action is dependent upon that individual's perception of:

1) level of personal susceptibility to the particular illness or condition; 
2) degree of severity of the consequences (organic and/or social) which might result from contracting the condition ;

3) the health actions potential benefits or efficacy in preventing or reducing susceptibility and/or severity;

4) physical, psychological, financial, and other barriers or costs related to initiating or continuing the advocated behavior.

...also stipulated that a cue to action or stimulus must occur to trigger the appropriate behavior by making the individual consciously aware of ... health threat.

Becker, et al., (1979) go on to state that, “... prospective investigations generally have not found correlations between health beliefs at the beginning of a course of therapy and subsequent compliance as strong as correlations between health beliefs and concurrent compliance." They suggest that, "the relationship between health beliefs and compliance is at least partly bi-directional with health beliefs becoming congruent with actual compliance as well as the reverse of this" (Becker, et al., 1979).

There are four main criticisms of this model. The four criticisms are: (1) "no evidence exists that attitudes and beliefs cause behaviors..."; (2) "the model works only if all other factors that might affect the taking of preventive action are equal..."; (3) although the Health Belief Model has been successful in predicting behavior to prevent disease, it has questionable utility in explaining positive health behavior, that is behavior that is motivated by the pursuit of good health rather than by the threat of disease..."; and (4) while the model is intuitively appealing, it is not practical..." (Gochman, 1988).

\section{Social Demographic Approach}

This approach focuses on "personal and social attributes of individuals that affect the equity of health services" (Gochman, 1988). The most notable one and the one that 
will be used in this research is the Andersen (1968) conceptual framework. This model has been revised with help of colleagues over the years. It is known as the SocioBehavioral Model of health care utilization (SBM).

\section{Socio-Behavioral Model of Health Carc Utilization}

\section{Conceptual Framework}

The framework of Andersen's (1968), Socio-Behavioral Model (SBM) is the most widely used approach. Andersen with other colleagues further refined the original framework (i.e., Andersen \& Newman, 1973; Aday \& Andersen, 1974, 1975; Aday, et al., 1980, 1984). In SBM, health care service utilization "is seen as a joint function of the 'need,' 'predisposing,' and 'enabling' characteristics of an individual" (Soldo \& Manton, 1985). Health care utilization measures used in Andersen's initial research were physician contact, overnight hospital stay, and dental contact. With refinements, utilization measures included volume of utilization, i.e., the actual number of physician visits within a 12-month period and the number of days spent in the hospital per visit.

\section{Predisposing Factors}

Predisposing factors tend to increase the propensity of an individual to use health services. Andersen (1968) originally defined these as "... chatacteristics which exist prior to the onset of specific episodes of illness." Predisposing factors represent the sociocultural element of the behavior model (Wolinsky, et al., 1989). Further predisposing factors can be broken down as being demographic, social structural, or health beliefs. Each of these types of factors takes into account a different feature that 
may predispose an individual to use health services. For example, demographic characteristics "tap the individual's relative life cycle position" (Wolinsky, et al., 1989), while social structural characteristics "tap the individual's location in the social structure and behavioral patterns (i.e., lifestyles) to which people in such positions become socialized" (Wolinsky, et al., 1989). Health belief characteristics tap into the individual's attitudes toward physicians, disease and medical care. The demographic characteristics typically studied are age, gender, marital status and household size. The social structural characteristics typically studied are employment, educational attainment and race/ethnicity.

Cultural attitudes and beliefs of an ethnic group will influence an individual's perception of illness as well as the response to the illness. Originally, the framework placed race/ethnicity as a predisposing factor. However, this strategy assumes the relationships between the racial/ethnic groups are strictly additive. Wolinsky, et al., (1989) in a national study of elders analyzed selected racial/ethnic groups separately. Separate analysis for each racial/ethnic group facilitates the examination of both the additive and interactive effects of race/ethnicity on each of the health care utilization measures specified. Wolinsky, et al., (1989) interpreted differential predictability of the separately estimated models as an indication of inequitable access to the health care delivery system.

\section{Enabling Factors}

Enabling factors reflect the ability of an individual to obtain health care services. Most enabling characteristics "represent the economic element of the behavioral model" 
(Wolinsky, et al., 1989). However, not all enabling characteristics are economic in nature. For example, having a regular source of health care is not strictly an economic characteristic. However, if an individual has a regular source of medical care they may be more prone to using the source of care for non-acute or preventive health care. As described earlier in the case of ability to speak English, the separation of enabling characteristics and predisposing characteristics is not so clear cut. An individual who does not speak English in a predominantly English-speaking community may be less able to use available community health care services because they are not aware of them. In this context, the ability to speak English is also an enabling characteristic.

Like predisposing factors, there are two components of enabling factors: family and community. Family characteristics tap into "the individual's ability to provide for him or herself' (Wolinsky, et al., 1989). The family characteristics typically studied are household income, health insurance, and having a regular source of medical care. The community component is important because if health services are not available in the community, then it does not matter whether the individual can pay for health care services. The community characteristics typically studied are the availability of physicians and hospital beds. However, barriers to access or lack of availability of support services are not always directly available. Thus, indirect measures must be used to gain information on whether a neighborhood (service environment) is "service rich" or "service poor." Items that have been found to be indirect measures include median household, percentage of the population living below poverty, per capita income, as well as percentage elder, black and Hispanic. 


\section{Need Factors}

Individuals who perceive a need to use health care services due to an illness are most likely to use these services. Need factors "tap the individual's recognition that a health problem either exists or is in the making" (Wolinsky, et al., 1989). Again, need factors can be broken down into two components: self-perceived and professionally diagnosed. The self-perceived characteristic is usually one question in which the respondents are asked to assess their overall health status. With respect to the professionally diagnosed need factors, many studies do not have access to this information. However, it has been shown that information on activity limitations due to health reasons provides an objective assessment of need (Liang, 1986; Wolinsky, et al., 1989). Activities of daily living (ADLs) and instrumental activities of daily living (IADLs) have become "the accepted index by which to assess an older individual's ability to function independently" (Monroe, et al,, 1991). Information on difficulties with ADLs and IADLs is gathered from a fairly standardized set of questions.

\section{Health Care Service Utilization}

Utilization of health care services is the outcome of intercst. However, health care services of particular interest may have different determinants and range greatly due to a number of reasons. Originally Andersen (1968) had two types of utilization behaviors: discretionary and non-discretionary. Andersen (1968) defined usage of discretionary health services as a function of individual choice. Dental visits are typically classified as the most discretionary utilization behavior, while hospitalization is at the other end of the continuum and physician visits fall somewhere in between. Hospitalization is classified 
as a non-discretionary utilization behavior because the choice of entering the hospital is usually made by a physician rather than the individual.

Andersen and Newman (1973) expanded the dimensions that health care services could be classified by to include not only type, as in the original framework, but also unit of analysis and purpose. The types of health care services were: physician visit; hospitalization; dental visit; drug and medications; nursing home and other. In his original research, Andersen (1968) found that all of the health care services except dental care were highly correlated with physician usage. The physician is the "gatekeeper" for medical care and may in part explain this high correlation, while dentistry falls "outside of [the] medical regimen" (Andersen, 1968).

In much of the research, contact has been the primary unit of analysis. Contact is particularly important if the event is highly discretionary, such as the case in visiting the dentist, or does not occur for the majority of the population, as in the case of hospitalization. However, in the case of physician visits, contact only tells part of the story. Volume of physician contact or services used may have different determinants. Andersen and Newman (1973) write that,

The importance of volume of resources is based on the rather obvious assumption that as the resource/population ratio increases, the medical care consumed by the population will also increase.

Finally, the purpose of health care service utilization is an important consideration. Andersen and Newman (1973) distinguished four categories: primary care; secondary care; tertiary care; and custodial care. Primary care is characterized by its preventive nature, or in the fact that an itlness has not yet occurred. Other purposes of 
health care services assume that an illness has occurred. Secondary care is treatment that will return the patient to his or her previous functional ability; while tertiary care is treatment that "provides stabilization for long term itreversible illnesses..." (Andersen \& Newman, 1973); and custodial care "provides for personal needs of the patient but makes no effort to treat the underlying illness condition" (Andersen \& Newman, 1973). Much of the literature has focused on the latter three purposes. In the case of primary care, however, the determinants of service utilization may be significantly difference, since the individual does not have any outward signs of an illness.

The behavior to seek preventive care is gaining in the United States. Aday (1993) notes that while the percentage of individuals seeking an annual general physical exam has stayed relatively the same between 1963 and 1976, there has been a shift in "the most frequently cited reason for having a general physical exam from concern about symptoms of illness to preventive care in the absence of illness per se" (Aday, 1993).

\section{Application of the Socio-Behavioral Model}

\section{General Population}

The literature documents the theoretical and practical importance of studying the use of health care services. SBM has been used widely to study utilization of health care services (Andersen, 1968; Andersen \& Newman, 1973). As with most conceptual frameworks there are both positives and negatives in using a particular framework. The benefits of this framework are, its: (1) eclectic approach by combining perspectives from 
many disciplines; (2) straightforward application to survey data; and (3) ability to analyze the "equitable" distribution of health care resources.

There are also criticisms in the usage of the model. Due to the variety of levels of analysis included and the range of variables needed, several researchers criticize the framework for its difficulty to gather data for testing the complete model (Wolinsky, 1990; Becker \& Maiman, 1983). Mechanic (1979) points out further that multivariate studies of health service utilization have failed to confirm the importance of certain organizational and psycho-social factors found in other studies.

In general, studies using the SBM framework have found that: (1) need characteristics are the major determinants of the use of health services; (2) the amount of variance explained is not large; and (3) regression coefficients obtained for the predisposing and enabling characteristics are modest if not substantively unimportant.

\section{Elder Population}

There are also particular criticisms of SBM's usage with the sub-population of elders. Ward (1978) mentions several shortcomings of the framework when studying elders. First, it does not consider organizational factors associated with the bureaucratic nature of health care service delivery settings. These organizational factors may serve as a barrier to utilization. In part this may be due to the fact that older people may be more cautious and fatalistic and therefore it may be harder for them to penetrate the fragmented health care delivery system bureaucracy. A second shortcoming is the absence of social 
networks in the SBM framework which other research has suggested contribute significantly to the decision to use health care services.

Exploration of the role of social support on elders utilization of health services has largely been ignored until recently. Nelson (1993) states that "social supports play a significant role in the lives of elderly individuals." In other research it has been found that "supportive relationship[s] are associated with lower illness rates, faster recovery rates and higher levels of health care behavior" (Nelson, 1993). For instance, "widowhood significantly alters both eating behaviors and dietary quality" for the worse (Rosenbloom \& Whittington, 1993). Further in a survey of Alameda County residents, Berkman and Syme (1979) found that fewer social contact were significantly correlated with lower preventive health behaviors, as indicated by having health insurance or routine dental, medical checkups. Hagdrup (1997) stated that, "lack of health insurance is important in determining ease of access to care..."

With respect to the amount of variance explained, it is even less for the elders population than that found for the general population in the United States. Wolinsky and Coe (1984) suggested that federal entitlement programs "may have leveled much of the discriminating ability of socioeconomic status" (Wolinsky, 1990). Most elders ate covered by Medicare and thus, the importance of predisposing and enabling characteristics may have declined (Wolinsky, 1990). 
However, the literature shows that there are significant differences in the socioeconomic status of different racial/ethnic groups of elders. ${ }^{8}$ In addition, there has been some indication that there are differences in health insurance coverage by racial/ethnic groups. ${ }^{9}$ In part, this is due to socioeconomic status, but also may be related to cultural differences in defining illness and help-seeking behavior. Health insurance coverage may play an important role in whether or not an individual seeks medical care promptly or waits until there is a serious medical problem..$^{10}$ Pauly (1974) notes that there may be a further bias in that individual can choose the level of insurance coverage." However, this concern may be more important in the working age population, since there is little choice in health insurance for the retired elder population. Medicare is a federally mandated entitlement. Elders do not face the same choices in price and quantity that face working age individuals. ${ }^{2}$ However, recent research by Hagdrup (1997) and others suggests that including level of coverage will have an important impact on health care utilization, especially preventive health care services.

\footnotetext{
${ }^{3}$ Minorities generally have higher proportions of their members living below the poverty level. Further, research shows that both higher prevalence of diseases, as well as higher age-specific death rates are closely related to lower socio-economic status (Tripp-Reimer \& Sorofman, 1988; Shanas \& Maddox, 1985).

${ }^{9}$ In particular, research has shown that Hispanics have significantly higher proportion without medical insurance at all ages (Andersen, et at., 1986).

${ }^{10}$ Further, the mere fact that an individual has health insurance may not guarantee adequate access to health care. Anecdotally, many individuals are finding out too late that their health care insurance has important gaps in coverage. One important area of non-coverage for Medicare is prescription drugs.

"Bias may arise in one of two ways, either through adverse selection or "moral hazard." In the case of adverse selection, Pauly (1974) writes this "occurs when premiums are not tailored to the expected losses of individuals." Pauly (1974) further writes, "moral hazard will ... affect the quantity of insurance bought." ${ }^{12}$ Even with the enactment of Medicare, research has shown that elders with lower incomes will use certain services more often than elders with higher incomes. However, insurance coverage may not only be an economic issue, it may be cultural issue as well (Andersen, et al., 1986).
} 
The majority of research studying utilization of health care services has used SBM to analyze the determinants of utilization. However, research using this framework has shown small coefficients for the race and ethnicity variables. This, in turn, has been interpreted that health care utilization between racial/ethnic groups of elders is not substantially different. Yet, most previous research with SBM has used a dummy variable approach to modeling race and ethnicity, (i.e., a linear construct). The inclusion of dummy variables for race and ethnicity will only show differences in the intercept of the regression model, but it does not provide "any information regarding the potential differences in the [interaction] effects of the independent variables [with race/ethnicity] on the dependent variable" (LaVeist, 1994). The relationship within these two categorical variables (race and ethnicity) has been assumed to be linear. However, that may not be the case. The interaction effect of race/ethnicity with other independent variables, (i.e., poverty status, age structure, etc.) has not been taken into account by using this approach. The small differences in racial/ethnic dummy variables may be occurring because the direct effect of race and ethnicity is only being measured. However, race and ethnicity may also have indirect effects on other independent variables (i.e., education, age, and socioeconomic status). Therefore, due to the complexity of issues regarding health care utilization, simply including a dummy variable for race (i.e.. black/white) and ethnicity (i.e., Hispanic/non-Hispanic) in the regression model may not be adequate for an analysis of health care utilization behaviors. One solution that has been suggested is to analyze each racial/ethnic group separately to find those factors that 
are important for determining health care utilization behavior by racial/ethnic group (Wolinsky, et al., 1989; LaVeist, 1994).

This research will modify existing models of health care utilization to take into account the shortcomings of previous research using SBM (i.e., race/ethnicity, provider availability and other environmental characteristics). Once SBM has been specified, the model will be run for each racial/ethnic group of elders using data from the Dade County Needs Assessment (DCNA) conducted in 1994 (Rothman, et al., 1994). The differences in the parameter estimates will be tested for statistical significance within the racial/ethnic groups of elders. Using this type of analysis, one can determine whether there are racial/ethnic differences in utilization of health care services affecting enabling, predisposing, and need factors. Individual variables within each factor include insurance status, health status, marital status, and various measures of access to health care, as well as environmental variations in availability of health care services. This knowledge can aid in developing programs and/or policies to reduce racial/ethnic disparities of health care utilization by elders.

The benefits of using this framework outweigh the criticisms. The eclectic approach will be useful for analyzing health care utilization by four different racial/ethnic groups of elders within one county. Further, the data being used comes from a telephone survey. As stated earlier this framework can be applied to survey data in a straightforward manner. Finally, an important aspect of this study is analyzing the distribution of health care resources within the county (community). The criticisms to 
this framework may in some part be fixed and for others would be a criticism for most frameworks in general. An example of a criticism that can be fixed and included into the model is with respect to social support. A social support index can be constructed from other information gained from the survey and included into the framework.

Before analyzing the data to answer the research questions and hypotheses, the attention of this dissertation will turn to a theoretical examination of the terms "aging" and "old" in the United States. For many individuals, being "old" is synonymous with being frail or of ill health. Western society's focus on medicine and science, i.c., the biomedical model, has created biomedicine into "a leading institution of industrialized society." Yet, Kleinman (1995) wrote that there is,

... no essential medicine. No medicine that is independent of historical context. No timeless and place-less quiddity called medicine. (p. 23)

Further, Kleinman (1995) wrote that,

Medicine ... like religion, ethnicity and other key social institutions is a medium through which the pluralities of social life are expressed and recreated. (p. 23)

Thus, an examination of the literature and research on aging will be important to the final discussion, since it is

[the] choice of a particular diagnosis and treatment [that] becomes an idiom for negotiating ethric and social class position for claiming access to nonmedical resources, such as land, jobs, urban residence ... (Kleinman, 1995: 24). 


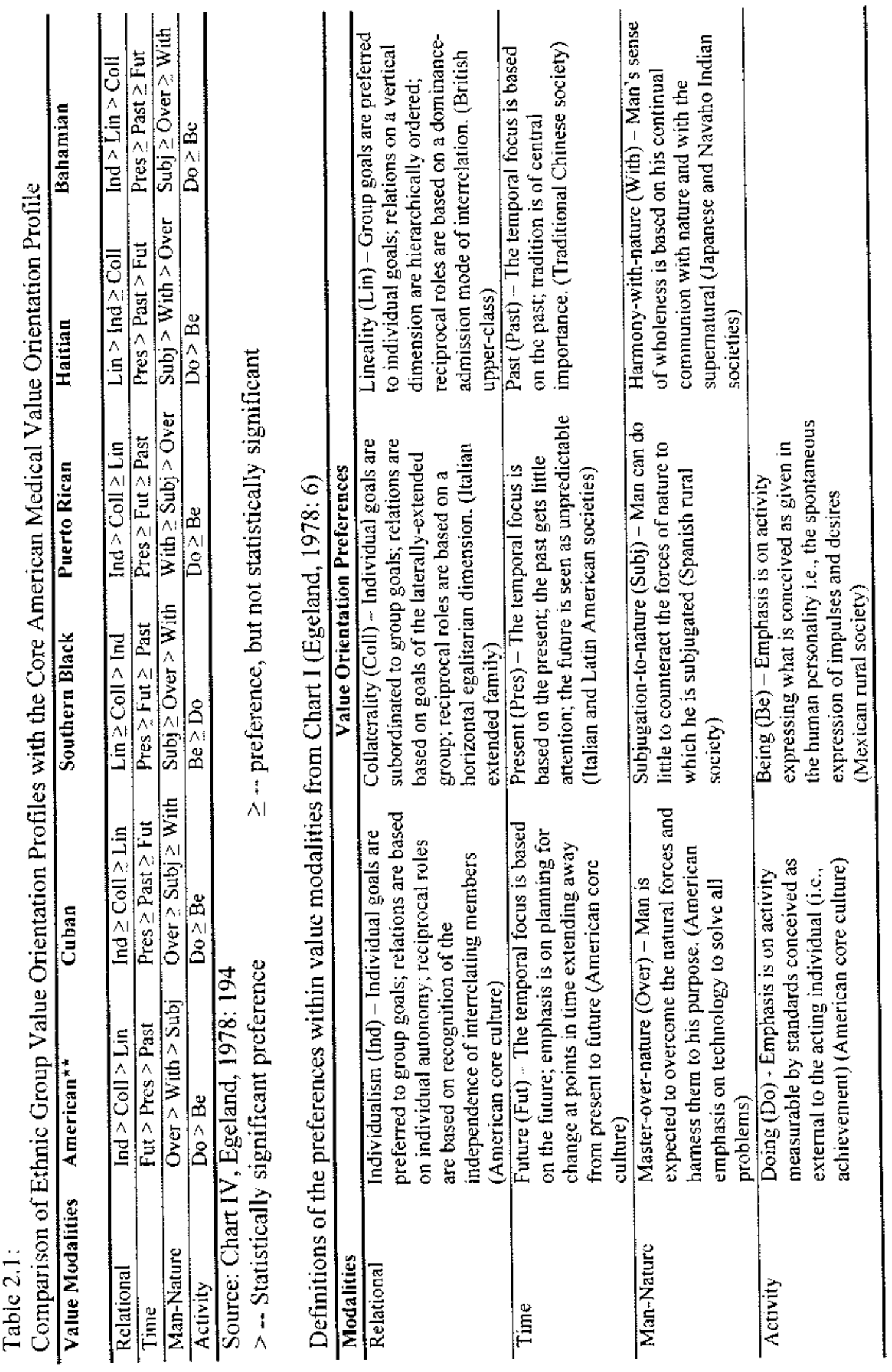




\section{CHAPTER 3: LITERATURE REVIEW ON AGING}

\section{IN THE UNITED STATES}

Two intertwined demographic trends are occurring in the United States. Both are and will have profound influences on American culture. First, the number of individuals aged 60 years and over has increased and will continue to increase into the next century. It is projected that by 2050 , the percentage of individuals aged 60 years or over will be one-quarter of the total population (25.2\%), compared to 16.5 percent as of July 1,1996 (U.S. Bureau of the Census, 1996). Primarily, this will be due to the aging of the babyboomers. Just like the baby-boom in the 1940s and 1950s influenced American culture through a more exclusive focus on youthfulness, as well as its impact on resource distribution, there will be growing demands for resources focused on older individuals. A discussion of older American interest groups and their impact on a variety of political, economic and local community issues is discussed in Day (1990). The second demographic trend within the older population is the increasing diversity of the elder population. It is projected that by 2050 , the number of black elders will quadruple, while the number of Hispanic elders will double (U.S. Bureau of the Census, 1996). White non-Hispanic elders will also increase and will continue to be the largest group of elders (U.S. Bureau of the Census, 1996).

Western theoretical perspective tends to set up contrasting conceptual categories. The most salient for this research is old vs. young. Yet, what is "old?" How is "old" operationalized or socially constructed in society? How does the individual who is 
experiencing the aging process operationalize "old"? How do we as researchers operationalize "old?" Recent research suggests that "old" is socially constructed and not just a universally clear biological process. For most federal programs, "old" is usually operationalized with respect to the retirement age of 65 years, which was arbitrarily picked in the 1930 s when Social Security was implemented as a federal assistance program (Frank, et al. 2000). In comparison, many "Senior Citizen" discounts start at 60 years. I Iowever, for the individual person, there is still a question, what is "old?" Is it over 60 years old? Is it over 70 years old? Is it over 80 years old? Betty Friedan (1993) in the preface of her book The Fountain of Age describes her own reaction to turning 60 . Her friends threw her a surprise birthday party. She wrote that, ...their toasts seemed hostile, insisting as they did that I publicly acknowledge reaching sixty, pushing me out of life, as it seemed, out of the race (Friedan, 1993: 13).

Kaufman (1986) described her elder respondents as, "not [perceiving] meaning in aging itself; rather they perceive meaning in being themselves in old age" (p. 6).

An early researcher of gerontology, Robert Butler (1974) identified a variety of stereotypes that he referred to as "myths about aging." These myths are unproductivity, disengagement, inflexibility, senility, and serenity. The literature examined in this chapter will show that these so-called traits of elders are myths. While certainly some older individuals may exhibit any number of these traits in old age, however many of these traits for the particular individual in question probably were there when the individual was younger. 
Given the two projccted demographic trends of increasing number of and greater heterogencity within the "old" population, understanding of the meaning of "aging" will become more important. The boundaries between "old" and "non-old" will become even more blurred. Like other socially constructed terms, "aging" and "old" depend upon,

...the cognitive frames in which they are placed and these in turn are products of prior social interactions, .... common meaning are arrived at when relevant audiences agree to stress certain aspects of given phenomenon and interpret them on the basis of shared past experiences" (Portes \& Stepick, 1993).

However, there is a technical difference in the usage of the two terms, "aging" and "old." "Aging" is a continuous process that we all experience regardless of years until we die. "Old" usually refers to the number of years we have already lived. While we can dichotomize between old and the unmarked category of non-old, it does not make sense to dichotomize between aging and non-aging (O'Reilly, 1997). By living day after day, everyone is aging.

Theory in general is important in the study of aging and age in that it is "the construction of explicit explanations in accounting for empirical findings" (Bengtson, Burgess \& Parrott, 1997: S72). Thus, the first step in this examination of the meaning of aging will be to examine and critique the various theoretical perspectives that have been developed over time. Following this will be a discussion of the general area of discourse on aging, as well as a more thorough examination of research of community dwelling elders' construction of their own life stories. 


\section{SOCIAL THEORETICAL PERSPECTIVES ON AGING RESEARCH}

This issue of differences of meaning in the term "aging", (i.e., social construction of aging) has only recently begun to be explored with greater depth. Fundamentally, the process of aging is biological in nature. As noted at the end of the previous chapter, the influence of biomedicine with its increasing influcnce on all sectors of human experience is ripe for focusing on aging. Estes and Binney (1989) wrote that,

[it] has become a powerful and pervasive force in the definition and treatment of aging. (p. 587)

More recently, Guo (2000) in a study of elderly Chinese in New York wrote that,

... the 'biomedicalization of aging' or the recent power of the U.S.

medical establishment to define and 'treat' aging, has exerted the most powerful influence on the meaning of aging at all levels of American society ... medicine as a social institution, a system of knowledge and a practice together with its definition of old age as discase has become the dominant framework for the lives and problems of many old people in the United States (p. 46-47).

However, by focusing only on the biological aspect of aging or aging as a "medical problem", many other important aspects are missed. Further, just counting how many individuals there are in a given location, or "nose counting", is not enough. The characteristics of the older individual, such as, demographic, socio-economic, and familial characteristics, make a difference in many arenas. ${ }^{1}$ The complex nature of the terms "aging" and "old" makes theory construction an important tool for research.

Hendricks \& Achenbaum (1999) wrote that,

\footnotetext{
${ }^{1}$ Chapter 4 will give a historical demographic description of elders in Miami-Dade County.
} 
Theorizing is a reflexive activity shaping empirical investigations; it is a way to organize what we think we know about aging; it is critical activity. (p. 36)

Thus, there are a number of theoretical perspectives that have been developed in the study of aging and social gerontology. Yet, Berman (1994) wrote in his introduction,

Despite the enormous growth of research ... what we understand about day-to-day experience in later life is still very limited. In many respects, the inner lives of older people continue to constitute a kind of terra incognita.

Berman (1994) attributes this limitation of our knowledge to the dominance of early twentieth century positivism, which has focused on finding a grand all encompassing theory to explain aging. Further, Bengtson, Burgess \& Parrott, 1997

... theory plays a critical role in research on aging. While it is not longer worthwhile to attempt a grand, all-encompassing 'theory of aging' as was the goal in the 1950s and 1960s, we now have multiple theories representing various aspects of the aging process that provide different lenses through which to view and explain phenomena of aging. (p. S72)

Beginning in the 1970 s and 1980 s, there has been an evolution of intellectual paradigms from modernism to post-modernism. The conversations between the two intellectual paradigms of modernism and post-modernism are significant.

The period of modernism generally refers to the years just after World War I through the mid-1970s. McGee \& Warms (1996) in their overview of the period state that,

the attributes of modernists writing ... were detachment, the assumption of a position of scientific neutrality, and rationalism.... (p. 480).

However, post-modernists assert that such claims of detachment, scientific neutrality of the researcher, and rationalism are virtually impossible. They extend many of the modernist arguments of symbolic interactionist who have written that, "culture 
does not exist apart from individuals but rather lies in their interpretation of events and the things around them" (McGee \& Warms, 1996). The post-modernist challenge has led to a re-examination of even the most basic assumptions in many disciplines. This reevaluation established a break from ethnoscience in which there was a notion that culture could be modeled like mathematics or logic. Geertz. (1986) has argued that culture is not so much a model within a person's head, but rather the public symbols and actions and how individuals respond to these symbols and actions. In particular, he wrote that, "Whatever sense we have of how things stand with someone else's inner life, we gain it through their expressions, not through some magical intrusion into their consciousness" (Geertz, 1986: 373).

Thus, with respect to theory development, there are two paradigms. The first is positivistic and the second is an interpretive epistemological framework. The doctrine of positivism formulated by Comte stressed that

"the only true knowledge is scientific knowledge, that is, knowledge that describes and explains the coexistence and succession of observable phenomena, including both physical and social phenomena. Comte's positivism had two dimensions: (a) methodological ... and (b) social and political, in which positive knowledge of social phenomena was expected to permit a new scientifically grounded intervention in politics and social affairs that would transform social life. (Jary \& Jary, 1991)

In contrast, the foundation of the second is interpretive sociology. Interpretive sociology includes symbolic interactionism and sociological phenomenology, as well as the approach of Weber (Jary \& Jary, 1991). Further, these forms of sociology are, ...united by an emphasis on the necessity of sociologists to understand or interpret actor's meanings .... In this argument all social reality is preinterpreted, in that it only has form as (and is constituted by) the outcome of social actors' beliefs and interpretations. Thus it is, or ought to be, a 
truism that no form of sociology can proceed without at least a preliminary grasp of actors' meanings" (Jary \& Jary, 1991).

Positivistic theory building "is based on a hierarchical structure of observations and concepts" (Bengtson, Rice \& Johnson, 1999). In contrast, post-modernist thenry building uses ethnography. The self-reflexive nature of ethnographic study is an important aspect for the study of culture as it continually evolves and the people continually experience life. Marcus \& Fischer (1986) have written that,

Ethnography is ... the sensitive register of change at the level of experience, and it is this kind of understanding that seems critical when the concepts of systems perspectives are descriptively out of joint with the reality to which they are meant to refer. (p. 82)

They go further that the methodology of writing ethnography has "followed some basic literary conventions..." (Marcus \& Fischer, 1986: 481),

Figurc 4.1 displays the evolution of theories on aging as developed by Bengtson, Burgess \& Parrott (1997). At least five general sociological perspectives have spawned a variety of sociological theories of aging. These five perspectives are structural functionalism, economic rationalism, symbolic interactionism, conflict theory (Marxism), and social phenomenology. Structural functionalism influence on aging focuses on "the ways in which societal norms structure the roles available to different age groups" (Passuth \& Bengtson, 1988). The three major theories that it has influenced in some fashion are disengagement, modernization, and age stratification. The theoretical perspective of economic rationalism has been the intellectual source for social exchange 
theory (micro-macro level), as well as political economy of aging (macro-level) ${ }^{2}$.

Exchange theory is "the conceptualization of social interaction, social structure and social order in terms of exchange relations" (Abercrombie et al., 1994). With respect to the experiences of elderly, it "has been directly applied" to study of relationships between and the experiences of the elderly (Passuth \& Bengtson, 1988). The third theoretical perspective, symbolic interactionism conceives of society as,

....an exchange of gestures which involves the use of symbols.... [It is] the study of the self-society relationship as a process of symbolic communication between social actors (Abercrombie et al., 1994).

It has had a major influence on a variety of theoretical perspectives at both the microlevel and micro-macro level. At the micro-level, there was the first generation theoretical perspective of activity theory. In the second-generation theoretical perspectives, there was continuity theory and social breakdown/labeling theory. The third generation influence of symbolic interactionism combines with hermeneutics to create social constructionism. At the micro-macro level, symbolic interactionism has influenced both subculture theory, as well as combined with Marxism (conflict theory) to influence feminist theories of aging. The conflict theory perspective examines the constraining features of social order. It "argues that the social distribution of power and resources in a capitalist society is embedded within the context of the social relations of production" (Passuth \& Bengtson, 1988). The political economy of aging has been influenced by this tradition. Finally, the theoretical perspective of social phenomenology is concerned with

\footnotetext{
2 I will only mention in passing those theoretical perspectives that do not focus on the individual (or the macro-level only theoretical perspectives).
} 
"systematic investigation of consciousness" (Marshall, 1994). The primary assumption is that, "our experience of the world including everything from our perception of objects through to our knowledge of mathematical formulae, is constituted in and by consciousness" (Marshall, 1994). Passuth \& Bengtson (1988) state that, "this approach is like symbolic interactionism in that it is concemed with the definitional nature of social life, social phenomenology has a more serious concern with the use of language and knowledge as constitutive elements in everyday realities." Gubrium \& Holstein (1999) aggregate the perspectives of phenomenology, symbolic interactionism and ethnomethodology under the theoretical perspective of "social constructionism." They wrote about these three theoretical perspectives, "social constructionism," as follows.

They are not conceived as causal explanations of the social world but instead focus attention on problems of meaning in everyday life. As analytic perspectives ... they provide broadly sensitizing orientations to the social constructed features of experience, including aging. (p. 287).

Jacobs, Gubrium and Hochschild (1975) were one of the first to directly apply the phenomenology perspective to the study of the meaning of life and growing old by those who are doing the aging (O'Reilly, 1997; Decker, 1980).

\section{Disengagement Theory}

Disengagement theory takes a macro-level approach to analyze issues on aging. It is attractive to many social scientists because it goes to the heart of "explaining the social aspects of aging" (Cockerham, 1997). It was put forth by Cumming \& Henry (1961) and can be described as "the most explicit application of structural functionalism to the condition of the elderly in terms of their social and psychological reactions to aging" 
(Passuth \& Bengtson, 1988). Further, because it is linked to classical sociological theory of structural-functionalism and the works of Durkheim's The Division of Labor in Socit'y and Parson's The Social System, there is a "strong claim to academic legitimacy" (Cockerham, 1997). The three basic propositions to disengagement theory are: (1) the "process of mutual withdrawal of aging individuals and society from each other is natural; (2) the "process of withdrawal is inevitable" and (3) "it is ... necessary for 'successful' aging" (Cockerham, 1997). Cumming \& Henry (1961) support their theory with empirical data that shows there is a negative correlation between age and the frequency of social interaction, as well as emotional involvement. Disengagement of older individuals allows society to make room for younger individuals.

Criticisms of this theory have also emerged. Conflict theorists focused on the failure of the disengagement theory to explain adequately the "processes in society as social change, conflict, and alienation" (Cockerham, 1997). While symbolic interactionists focused on "small group processes, [objecting] to the functionalist view that individual human behavior is largely determined by society" (Cockerham, 1997). Hochschild $(1975,1976)$ critique of the theory focused on three logical flaws: "(a) ...the overlooked flaws in its underlying logic - the escape clause problem, (b) ... the overly inclusive nature of its central variables - the omnibus variable problem and (c) ... the level of reality it selects for study - the assumption of meaning problem" (Hochschild, 1975: 553). Passuth \& Bengtson (1988) summarize Hochschild's (1975, 1976) critique of disengagement theory as: (1) "the theory is unfalsifiable," (2) "overinclusiveness of the 
variable age and disengagement in ... [the] model of aging" and (3) "the theory's preference for imputing compliance without measuring it."

\section{Modernization Theory}

Like disengagement theory, modernization theory is also macro-level sociological theory. It was adapted by Cowgill $(1986,1974)$ to explain aging. With this theory there is an attempt "to explain variations in age status both historically and across societies" (Passuth \& Bengtson, 1988). It "focuses on the macro-structural conditions of the elderiy in varied sociocultural settings" (Passuth \& Bengtson, 1988). The functionalist perspective of this theory "suggests that the status of the elderly derives from their relationship to evolving systems of social roles which vary across societies depending on the degree of industrialization (or 'modernization')" (Passuth \& Bengtson, 1988). Modernization theory explains aging as "a systematic relationship between aging and modernization ... [and] that the concept of old age is relative to a society's degree of modernization" (Cockerham, 1997). Further, "it highlights important cultural variables that affect the perception of the aged in contemporary societies" (Guo, 2000:46). In particular, Guo (2000) notes that it is particularly "relevant for understanding how social and cultural change affects elderly immigrants' health and well-being" (p. 46).

However, there are three main criticisms of this theory. The first, is a challenge to one of its basic presumptions of a "golden age of aging" in pre-industrial societies (Quadagno, 1982). Along with this is a criticism that the "concept of retirement for the aged worker is not new and can be found in some premodern societies..." (Cockerham, 1997). Finally, a third criticism of this theory is that it "does not adequately account for 
differences in gender and socioeconomic status in the aging of modern populations" (Cockerham, 1997).

\section{Age Stratification Theory}

Based primarily on the work of Matilda White Riley $(1971,1987)$, the theoretical perspective of age stratification is a relatively recent theoretical perspective. The basic assumptions are: (1) society is stratified by age cohorts; (2) "each age cohort has both life course and historical dimensions" (Cockerham, 1997). In sum, "the model examines the movement of successive birth cohorts across time" (Passuth \& Bengtson, 1988). Individuals belong to particular age groups depending on how long they have lived. Through their current age group they share with others of that age group at other times. similar social roles and experiences, i.e., first marriage, first birth of a child. However, there is also an historical dimension to this model that states that regardless of age, people alive at a particular time, share the experience of particular historical events. Riley, et al., (1999) wrote,

Its central theme is that, against the backdrop of history, changes in people's lives influence and are influenced by changes in social structures and institutions. These reciprocal changes are linked to the meanings of age, which vary over time. (p. 327)

There in lies the difficulty and complexity of this theoretical model. This complexity has spawned criticism from Streib \& Bourg (1984). Cockerham (1997) summarizes their criticism as questioning "whether age stratification theory actually qualifies as a theory of stratification, because it does not account for social inequality." 


\section{Exchange Theory}

In general, this is a theoretical perspective that focuses on Simmel's observation that "all contacts among men rest on the schema of giving and returning the equivalence" (Blau, 1964). As stated earlier, exchange theory "applies a rational, economic model to the study of social behavior" (Passuth \& Bengtson, 1988). Individuals are viewed as "seeking to maximize rewards from their interactions with others" (Jary \& Jary, 1991). With respect to aging, Dowd (1975) uses the research tradition of exchange theory "to reconceptualize the relationship between age and social structure as ... a process of exchange" (p. 584). Passuth \& Bengtson (1988) summarize Dowd's work as trying "to explain why older people tend to withdraw from social interaction." Thus, instead of focusing on a macro-level understanding of the experiences of older people, exchange theory focuses on the interactions of older people with other age groups. With respect to participation in special interest groups, Day (1990) writes that,

Exchange theory helps to explain how lower class or disadvantaged persons, with few resources and little time to contribute to political organizations, might still be induced to join and participate. Such people would be most likely to respond to material incentives "that are immediately and directly available and of high value to the recipicnts.... However, much of exchange theory's perspective is based on purely economic terms, which results in a limitation of its usage. Criticism of this theoretical perspective are three-fold: (1) assumption that ALL interactions between individuals involve exchange; (2) "failure to deal adequately with such phenomena as traditional action or general values;" and (3) "the great variety of human emotions" (Jary \& Jary, 1991). According to Weber (1978), 
... the definition of economic action ... must bring out the fact that all

"economic" processes and objects are characterized as such entirely by the meaning they have for human action in such roles as ends, means, obstacles, and by-products (p. 64).

\section{Activity Theory}

Robert Havighurst (1963) developed activity theory, with three basic premises. The first is that, "...the majority of normally aging people will maintain fairly constant levels of activity." The second is that, "... the amount of engagement or disengagement will be influenced by past lifestyles and socioeconomic factors, rather than by some inevitable process." Finally, the third premise is that, "... it is necessary to maintain or develop substantial levels of social, physical and mental activity if the aging experience is to be successful" (Cockerham, 1997). In direct contrast to disengagement theory, activity theory argues that "the more active elderly persons are, the greater their satisfaction with life" (Passuth \& Bengtson, 1988). There will be role loss (e.g., retirement, widowhood) that comes with age, however for well-being in late life an individual must continue acquiring new roles, while discarding old ones no longer appropriate.

However, there are also difficulties with this theory too. Cockerham (1997) has summarizes several of the difficulties, "[first] it rests on the assumption that older people judge themselves according to norms common to middle-aged activity and behavior, but does not explain what happens when the elderly do not subscribe to such norms. [Secondly,] it does not explain what happens to those older people who cannot, for physical, mental or socioeconomic reasons maintain a middle-aged standard of living regardless of how they judge themselves." 


\section{Political Economy of Aging}

This is a fairly new theoretical perspective applied to the study of aging. Bengtson, Burgess \& Parrott (1997) in their overview of the next generation of theoretical perspectives view political economy perspective as more of a framework for the examination of old age in a larger social context, rather than a formal theoretical perspective. Quadagno \& Reid (1999) state that this theoretical perspective,

...highlights how socioeconomic institutions affect individuals over the life course and how they continue to influence their social and economic well-being in old age. [Further, it] ... has shifted the focus of gerontological research from the individual's ability to adapt to aging to an examination of broader social processes that determine how resources are distributed.... (p. 355).

The recent focus of research within this perspective has been with regard to "distributional issues of welfare state provisions" (Quadagno \& Reid, 1999). The theoretical perspective of political economy of aging is promising in that it looks "at the political and economic conditions that give rise to problems of growing old ... and [asks] whose intcrests are really served in the efforts to help the elderly" (Passuth \& Bengtson, 1988). Further, this theoretical perspective explains not only "the experience of old age but also to determine why there are patterned inequalities in the experience of old age" (Quadagno \& Reid, 1999). As a result, there is a better understanding of how public policy can "ameliorate or exacerbate such inequalities" (Quadagno \& Reid, 1999).

\section{Social Phenomenology}

In general, the theoretical perspective of social phenomenology is associated with Alfred Schutz. It "examines the processes involved in, the constitution of social knowledge and social life" (Jary \& Jary, 1991). It has influenced the work of Berger and 
Luckman (1967), as well as been influential in the development of ethnomethodology.

However, social phenomenology has only recently been applied to the study of aging.

Previously, social gerontologists assigned meaning to individuals growing old. However, using this theoretical perspective, researchers are not concerned with the meaning of life assigned by social gerontologists, rather they are concerned with the meaning that elders give to aging and the meaning of life (Decker, 1980). In contrast to disengagement theory, "the phenomenological method individualizes the aging process; it sees persons as assigning their own meaning to aging, not that everyone has a unique view of aging" (O'Reilly, 1997). Passuth \& Bengtson (1988) summarized Giddens (1976) perspective on social phenomenology as emphasizing the construction of social emergent meanings.

This, in turn,

encourages the researcher to pay close attention to ongoing social circumstances. Yet, at the same time, like symbolic interactionism, phenomenology's emphasis on microsocial processes overlooks the structural features of social life. As a result, social phenomenologists tend to minimize the role of power in their analysis of everyday social behaviors....

However, the understanding of meaning attributed to an action is not always clcar-cut, since as Weber (1978) wrote,

...'one need not have been Caesar in order to understand Caesar.' 'Recapturing an experience' is important for accurate understanding, but not an absolute precondition for its interpretation. Understandable and non-understandable components of a process are often intermingled and bound up together. (p. 5) 


\section{Recent Combining of Theoretical Approaches}

In a recent essay, Laz (1998) developed a new framework to understand age and

aging. While many of the ideas in this essay were not new, what was striking was the

reconfiguration of these idcas by drawing parallels to existing scholarship in the field of sociology of gender.

The author described this framework as "age as accomplished." The foundation of the framework used a combination of theoretical perspectives: symbolic interactionism, ethnomethodology, life course traditions, and phenomenology, as well as some very recent scholarship of West and Zimmerman (1987) and Lorber (1997).

In the study of gender, like the study of aging, there is a "distinction between 'objective fact' and social and cultural meaning [which] is analogous to the distinction made in the 1960s and 1970s between sex and gender" (Laz, 1998: 91). Laz states that there is a similar process of understanding taking place between the study of age (chronological age) and age category (the social and cultural meaning of age). Laz. (1998) wrote that, "embedded in sociology is the assumption that age is an objective chronological fact" (p. 91). In building this new framework of "age as accomplished," this framework does not tear down all previous work, but is a new way of organizing all the work that has come before on age and aging. In her conclusion, Laz (1998) stated that

...the idea of age-as-accomplished can make explicit what often goes unrecognized in the sociology of age: the performative, interactive work of accomplishing age, the emotion of work involved in "becoming" and "being" an age, and the strategies people develop and use as they create and display themselves as aged (that is, as being of a particular age). To understand "age" as situated, contingent, and negotiated, and as continually constituted in interaction, provides the foundation for a sociology of age. (p. 110) 


\section{LITERATURE REVIEW OF AGING RESEARCH}

How older individuals are viewed in society and the available roles for older individuals is important. Research in the late 1960s by Clark (1967) and Clark \& Anderson (1967) documented the connection between culture, aging and mental health outcomes. Kaufman (1986) summarized their discovery as follows,

... old people in American society are thwarted in their expression of continuity of self by a society that makes rigid and contradictory demands for profound change among its oldest citizens. ...individuals who could not alter, reinterpret, or teevaluate their orientation to both self and social world in old age had more likelihood of psychiatric hospitalization than those who were able to shift orientations.

The increasing focus on work and productivity in modern society has tended to, as Keith (1983) wrote,

... [strand older individuals] in the liminal. Exit signs are clearly marked but reincorporation is not on the map. Their choice is between cultural bushwacking and clinging to the crumbling cliff of midlife roles.

Vesperi (1985) wrote that "The cultural construction of old age is a process; it is the concretization of abstract, unexamined assumptions within the context of everyday interaction."

Still, how do we structure our language in discussing "aging" and becoming "old?" The theories that have been developed help in this endeavor. However, more needs to be done as documented by Laz (1998). Little has been done in this area since, "[t]he study of aging may be unique in that while, for the most part, our subjects are not ourselves, they are indeed our 'selves' as we will become" (Vesperi, 1985).

Serious students of old age cannot avoid the realization that they are separated from their informants not by language and belicf, but by the successful internalization of a shared belief system (Vesperi, 1985). 
Unlike the study of other cultures or groups, the researcher as an individual will be, if successful, part of the study group in the long term, i.e., eventually there will be no "exotic other." The realization is, as Vesperi (1985) put it, ... that at some point I will be called upon to discard the identity I have developed over a lifetime and confront a social self that is essential different, essentially old. There is no "return home" for anthropologists who study their own aged, no resumption of the comforting distances that help us rationalize our place in informants' lives.

Hazan (1994) discussed aging research in the following manner. He wrote that, "[unlike class, ethnic, racial or even gender-based distinctions,] the boundary between life and death are a perennial human preoccupation. Ageing, as one of the major markers of that fine existential line, calls for constant clarification and reclassification" (IIazan, 1994).

Berger and Luckman (1966) wrote with respect to language and knowledge in everyday life that,

Human expressivity is capable of objectivation, that is, it manifests itself in products of human activity that are available both to their producers and to other men as elements of a common world. Such objectivations serve as more or less enduring indices of the subjective processes of their producers, allowing their availability to extend bcyond the face-to-face situation in which they can be directly apprehended.

Berger and Luckman (1966) go further to state that,

Language, which may be defined here as a system of vocal signs, is the most important sign system of human society. .... An understanding of language is thus essential for any understanding of the reality of everyday life.

Yet, what happens when the intrinsic meaning or values of certain ideals are not equally shared within the community? With growing cultural diversity within the United States 
population and in particular the older population, these "intrinsic meanings" and "values of certain ideals" may not be "equally shared" within a local community.

Further, one other problem that Hazan identified with aging research and discourse was that,

Communication about ageing does not necessarily rely on communication with the ageing, much less communication amongst the aged.

In general, aging is part of living. Thus, how an individual lives during the "younger" part of one's life, or composed one's life earlier, is important in composing one's life in old age. Bateson (1990) wrote, "Composing a life involves a continual reimagining of the future and reinterpretation of [the] past to give meaning to the present, remembering best those events that prefigured what followed, forgetting those that proved to have no meaning within the narrative" (p. 29-30).

Thus, the focus of this chapter will now turn to the examination of six works which examine and give voice to non-institutionalized, community-dwelling elders. The six texts that will be examined are not the full extent of the literature in this area.

However, these texts appear to encompass the breadth of the area of research on noninstitutionalized, community dwelling elders. While some of the research is dated, due to the increasing cultural diversity of the aging population, there has been more research focusing on these issues recently. Further, since only 5 percent of all elders in the United States lived in nursing homes in 1990 (U.S. Bureau of the Census, 1996), therefore, the litcrature that will be examined here will stay away from "health-focused" ethnographic studies of nursing home populations. 
Similar to the changing perspective in research on the black family, research on the aging population in terms of sociological meaning and cultural discourse will only become clearer with the input of individuals who are older. Thus, it is expected that academic research on aging will increase not only in amount, as well as coming closer to "getting it right" as to the meaning of "aging" and "age."

\section{Vesperi's City of Green Benches (1985)}

\section{Research Sample}

Vesperi's (1985) research focused on culture's view of old age and how it was "communicated to the low-income elderly and how the individual older person attempts to modify, adapt to, or reject this social construction." City of Green Benches is an ethnographic study of low-income elderly living in St. Petersburg, FL in the late 1970s and early $1980 \mathrm{~s}$. The research sample consists of 90 low-income older individuals with a racial distribution that has equal numbers of black and white elders.

\section{Text Construction and Themes}

The book is divided into two parts. The first part is "concerned with symbolic processes whereby older people apprehend and subsequently accept, reject, or renegotiate a variety of messages about aging received from an urban community..." (Vesperi, 1985). This first part focuses $\mathrm{n}$ the individual as agent within a highly structured environment of public services and limited informal support. The second part of the book explores the question of whose needs are being serviced. Specifically, do "the goals of specific social programs [that are] aimed at low-income retirees reflect more accurately the needs of those who are served or the perceptions of those who serve them" (Vesperi, 
1985). A 70-year-old man most succinctly communicates what Vesperi (1985) is attempting to examine in this research,

Who is that is giving us the feeling that we are alien? The greatest danger that we face is believing any of the garbage that society is trying to lay on us.

Vesperi succeeded at giving her readers another view of elders within our midst. Many of the non-old that Vesperi talked to while doing this research seemed to have ideas of what elders wanted, but in fact were wrong. One example was at the beginning of her research study. Vesperi was looking for a place to rent in the community she was studying. Many of the potential landlords that she contacted over the telephone to rent a place for herself and young daughter, would ask her how old she (Vesperi) was. Upon hearing her age, Vesperi recounted potential landlords saying, "You don't really want to live there." When they found out that she had a small child, they would say "Forget it. The neighbors don't want any kids around." Yet, Vesperi recounted that the presence of her daughter, "often facilitated the establishment of friendships with older people, who seemed hungry for contact with the young" (Vesperi, 1985: 46).

Vesperi's conclusions suggest that the theoretical perspectives that have been suggested previously are ones that are constructed by the non-old alone, not in conjunction with the old. In particular, the flaws of the theoretical perspective of disengagement theory are self-evident. 


\section{Research Sample}

Myerhoff's revered classic ethnographic work examined the patterns and behaviors of older Jewish immigrants living in Southern California and who were members of the Aliyah Senior Citizens' Center. Most of these elders spent their formative years "in one of the small, predominantly Jewish, Yiddish-speaking villages ... located within the Pale of Settlement of Czarist Russia..." (Myerhoff, 1979: 3).

\section{Text Construction and Themes}

The text is the transcribed conversations that Myerhoff has had with this group of elders, or has been party to. Through the transcription of conversations with this group of elders, Myerhoff gave these elders a voice. Further, this ethnographic work described in rich detail Jewish culture and language. Myerhoff connected the cultural heritage of these elders with their current coping strategies. The elders at the Center "fight" to be heard. Myerhoff documented numerous examples of these elders continuous "struggle" to be autonomous and independent. They may have their little physical aches and pains, but they "fight" to maintain their dignity and autonomy. Most poignant is at the beginning of the book with a description of Basha.

Basha wants to remain independent above all. Her life at the beach depends on her ability to perform a minimum number of basic tasks. She must shop and cook, dress herself, care for her body and her one-room apartment, walk, take the bus to the market and the doctor, be able to make a telephone call in case of emergency. Her arthritic hands have a difficult time with the buttons on her dress. Some days her fingers ache and swell so that she cannot fit them into the holes of the telephone dial. Her hands shake as she puts in her eyedrops for glaucoma. Fortunately she no longer has to give herself injections for her diabetes. Now it is controlled by pills,..." 
These elders are also very sensitive to other "outsiders" respect for their autonomy and being counted publicly, i.e., non-invisibility. While this is a universal trait in most cultures, how it is arranged depends greatly on the cultural environment. Myerhoff noted that there appeared to be an "inverse relationship between people"s actual effective power and their passion for publicly enacting their honor." This group of elders had been oppressed both in their youth and limited in their present situations. Thus, "honor" and "face" were important concepts in the lives of these elders. ${ }^{3}$ Myerhoff noted that the opposite of honor for these elders was invisibility, not shame. Myerhoff described her own experience with respect to honoring indjvidual elders. She gave them recognition by walking a narrow line. She wrote that,

For a long time I was puzzled when none of the gifts I had given to the individuals were brought into the Center. They were gifts that I specifically intended to be available for display, thinking that this would enhance their value to the recipients. I didn't realize that the embarrassment aroused by having 'received' overrode anyone's desire to bring into the Center a public mark of friendship with an outsider. But if I failed to display gift that one of them had given me - a scarf or piece of jewelry or book-I was sharply criticized by the giver. I had denied him or her the opportunity of making visible their act of having given to me. In another example, the elders drove out an individual who did not honor and respect them. This individual came to the Center with grand ideas of saving it, and yet he overstepped, or stepped upon the honor that these elders craved. Myerhoff wrote that,

Kominsky left behind a few lasting achievements, a few lasting blows to individuals, but his most significant heritage was giving the old people an opportunity to resist him and, in that way, renew their commitment to their beliefs, their ties to each other, and clarify their understanding of their identity by having once more performed it.

\footnotetext{
${ }^{3}$ This was also evident in Vesperi's work in her examination of "reciprocity."
} 
Like Vesperi's ethnography, this is a powerful documentation that society's perception of "old" is not always in step with the older individual's perception of himself/herself. It is the individual who must fight against the structure and stereotypes that are out there.

\section{Erikson, Erikson and Kivnick's Vital Involvement in Old Age (1986)}

\section{Research Sample}

Erikson et al. (1986), in Vital Involvement in Old Age, takes a slightly different approach in the examination of "old age," focusing on the final psychosocial stages of life. This book is part of a larger body of work in which the researchers developed eight stages of life. The individuals interviewed have been observed and interviewed since 1928/1929. The population of 29 men and women were originally chosen back in 1928/1929. The original sample design took every third birth that took place in Berkeley, CA between January 1, 1928 and June 30, 1929. This resulted in an original sample of 248 individual. The 29 men and women interviewed for this last stage are the living parents of these 248 individuals. The research informants at the time of the examination were between the ages of 75 and 95 years old.

\section{Text Construction and Themes}

The strength of this work is that it is based on a long-longitudinal study. While the majority of other longitudinal studies of adult development end in middle or late middle age, this study goes beyond and explores development into old age. If the individuals selectively remember certain events or pieces of their past lives, as we all are prone to do; earlier interviews were available for the researchers to examine. The 
researchers used these discrepancies as proof that "person's subjective reality is not a static collection of 'true' memories, but active construction that is continually revised as long as the person lives" (Frank, 1987).

For each stage in the life cycle, the authors developed a purpose or stage of development in which there were opposites and from which there developed an "adaptive strength." They refer to these opposites as "syntonic" and "dystonic." In old age, the opposites were identified as "integrity vs. despair" and the adaptive strength was "wisdom." Erikson et al. (1986) also studied maladaptive behaviors. There can be a tendency to overdo or overdevelop one or the other of these predispositions. With respect to the old age stage, when there is an overdevelopment of "integrity" this leads to the maladaptive tendency of presumption, while an overdoing of despair leads to the malignant tendency of disdain. Thus, the sociological theoretical perspective that this work could be categorized under is age structuralism.

The bulk of the book is Chapter II entitled, "The Voice of Our Informants." All eight stages or themes are developed and discussed by informants. However, the text starts with the last stage and goes back to infancy, and the lessons learned. The informants were actively involved in a balancing act between present and past through reinterpreting and re-inventing their lives in their present circumstances. Erikson et al. (1986) described the life cycle as, ...more than [an extension] into the next generation. It curves back on the life of the individual, allowing ... a reexperiencing of earlier stages in a new form. This retracing might be described as a growth toward death.... 
Just as at other stages of life, some of the informants were able to "adapt" better compared to others. Erikson et al. (1986) examined not only the individuals who were "successfully" aging, but also those who were not for a variety of reasons.

In their conclusion, Erikson et al. (1986) brought together the threads of all the conversations and took a step back. While Chapter II mainly focused on the individual (agent), the conclusion focused on society's (structure) influence on how elders are perceived and the options that are available for elders. It is also interesting to note that two of the authors were in their $90 \mathrm{~s}$ at the writing of this text and thus were also able to bring personal insight into this final chapter. They were able to pinpoint the barriers that older individuals face while on their quest for continuing development. With these insights, the authors were able to make suggestions for ways in which society's perception of "old" and "aging" should be changed.

Much of their conclusions regarding the balancing act that elders perform in reworking their life stories in Erikson et al. (1986) work, is also prevalent in Mycrhoff's elder population. Myerhoff described the process as follows,

Personal continuity was an especially important concern for these elderly for several reasons: their extreme age; their proximity to death, for the drive to shape one's life into an orderly story often grows more intense as people prepare for death; and because of the drastic discontinuity caused by emigration. Reviewing one's life and reminiscing, much practiced by the very old are expressions of their attempt to find themselves to be the same person throughout the life cycle. (Myerhoff, 1979: 108)

Throughout the text it was evident from the discourse of these clders that disengagement theory was not helping in the construction of meaning for the older person. However, Erikson's theory of psychosocial development overall has been 
criticized for its single developmental pathway. This single developmental pathway "does not account adequately for the life-span process of interpersonal attachment..." (Franz \& White, 1985: 225). Instead, Franz \& White (1985) extend Erikson's theory by proposing a tentative two-path model of life-span of individuation and attachment. Individuation is primarily the focus of males, while the attachment pathway is primarily the focus of females. The two paths are most divergent, in their extension, at the younger ages (not "Old Age"). In their modification of Erikson's epigenetic chart, there is no difference for the end stage of old age. Both proposed pathways (individuation and attachment) end up with the same syntonic/dystonic opposites, i.e., "integrity vs. despair." However, given the differences in the previous stages leading up to this last stage of life, it would seem that this might be somewhat simplistic. Thus, it appears that there needs to be more research done on this line of study.

\section{O'Reilly's Decoding the Cultural Stereotypes About Aging (1997)}

\section{Research Sample}

O'Reilly (1997) collected data from five settings. These settings were: (1) "meeting with a group of friends sharing a holiday dinner at home;" (2) "conversation with an 83-year old woman who lives alone at home;" (3) "a dialogue with a 92-year old woman who currently maintains her own apartment;" (4) "age-talk in an Adult Community Residence;" and (5) "an interview about a daughter's dilemma related [to placing her father in a nursing home]." For each of these settings, $O$ 'Reilly described both the verbal and non-verbal means of communication that were taking place. 


\section{Text Construction and Themes}

Decoding the Cultural Stereotypes About Aging (1997) is the newest monograph.

The author stated that her premise is that "Aging is a label, a symbol or a set of myths which is part of the conditions of growing old in American Society." Her goal in writing this book was "to identify the markers that demarcate the boundaries of aging." The author's background was as a nurse educator. She stated that she "noticed that many members of the health team talked about [older] people in ways that did not always appear grounded in direct experience." Coming from the background of the biomedical tradition, $O$ 'Reilly noted that health team members tended to examine the older individual only in terms of biological deterioration of the body. However, it became apparent to O'Reilly, "that the myths and labels used by members of the health team differed from those that were not part of it." O'Reilly's research focused on the organization of talk; that is the cultural construction of aging.

The book has five parts. There is an introduction in which O'Reilly delineates the reasons for the research and what she hopes to accomplish. The second chapter is a literature review that discusses and examines the major theories of aging in the fields of sociology, anthropology and communication. She also examines the theories on the social patterns of retirement. The third chapter describes the methods that she used to examine her questions that she stated in her introduction. The majority of the research relied on interviews with individuals. However, the fourth chapter steps back from people and looked at the images of aging in the media and other places. O'Reilly used not only popular television shows, as well as descriptions of Nelson Mandela's release 
from prison after 28 years. There was this paradox that was best exemplified by a comment from Nelson Mandela in which he stated that as a child, "he envied his elders. their nice grey hair," however now as an elder himself with grey hair, he docs not like his own grey hair. The final chapter of the book discussed the day-to-day language of aging that is encountered and used between all individuals.

Again, as in Myerhoff's and Vesperi's research, O'Reilly found that the older individuals she interviewed "did not fit the cultural construct of aging, nor did they want to be classified as old" (p. 110). What she found that demarcated the old from the nonold is independence, which is usually heavily reliant on the individual's health. She wrote,

What upset their world is ill-health, which places their independence in jeopardy. Impaired health inevitably puts a strain on their financial resources, and on the family or significant others.

Finally as a concluding remark, $O^{\prime}$ Reilly stated that none of her respondents were able to define the term "aging."

\section{Berman's Interpreting the Aging Self (1994)}

\section{Research Sample}

Unlike the previous works examined, Berman uses published personal journals of individuals in later life. These elders have written about their concept of self as they are experiencing life. Berman has chosen the written works of 5 older journal writers: Elizabeth Vining, May Sarton, Doris Grumbach, Alan Olmstead and Florida ScottMaxwell. The five journal writers have only one common life thread, each of these journal writers have been writers in their earlier years. Otherwise, Berman chose these 
journal writers because they focused on the writer's life, rather than "on topics such as travel or gardening" (p. xxiv).

\section{Text Construction and Themes}

Berman's intention in interpreting these texts was, "to examine their themes in light of concepts and theories from gerontology and developmental psychology" ( $p$. xxiii). He begins his book with an examination what he calls "the idea of human science." He writes that this perspective arises out of a line of criticism of the positivistic philosophy of science, "from the philosophical tradition known as hermeneutic phenomenology or, more simply human science...." He goes on to describe the central tenet of this perspective as "that humans are self-interpreting animals." However, this is not a purely late $20^{\text {th }}$ century idea. Geertz (1973) refers back to Weber's description of man "as an animal suspended in webs of significance he himself has spun." Geertz. (1973) takes "culture to be those webs and the analysis of it to be therefore not an experimental science in search of law but an interpretive one in search of meaning" (p. 5). This distinction is an important one in that it allows for the topic of "experiencing of aging" to be approached "scientifically" and therefore worthy of consideration.

All the previous works examined in this chapter have focused, in their own ways, on the "experience of aging." However, Berman takes a slightly different approach by examining "personal documents in which people create texts that capture their own lived experience" (Berman, 1994: 2). ${ }^{4}$ Berman analyzes the personal journals of his sample,

\footnotetext{
${ }^{4}$ It was not documented whether Berman bad any personal contact with his sample members. However, given the description of methods it is highly unlikely that he had any other contact than their text.
} 
"through the selection and highlighting of statements in the journals that pertain to the experience of becoming an older person" (p. 7). He believes that there is value in the examination of personal documents because,

When frail older people set down a personal interpretation of personal experience, they are, in effect, declaring their autonomy and demonstrating the dominance of mental life despite the physical losses they have endured. (p. 46)

Each of the texts examined, give a rich description of the lives of these older individuals with their ups and downs. The purpose of recording their thoughts and revelations on their self-concept were varied and even changed over time. For example, Berman writes that Doris Grumbach started her journaling at a time in her life when she is experiencing a period of emotional distress. Berman summarizes the purpose of the journal writing for Grumbach as giving her "the hope that in the recording process she would find positive value to living so long." However, as the journal unfolds, there is a shift in Grumbach's outlook from despair of her own value to one in which she has a sense of purpose. She acquires new roles and new interests that connect her and give her life purpose. This example of increased activity by Grumbach is a clear example that at the micro-level disengagement theory does not explain the experience of aging, while activity theory which argues that "the more active elderly persons are, the greater their satisfaction with life" (Passuth \& Bengtson, 1988) appears to come closer to an explanation. In all of the journals, the writers relate role losses (e.g., retirement for Olmstead, lost loves or widowhood) that come with age. However, for each of the writers there is a realization that well-being in late life an individual must continue acquiring new roles, while discarding old ones no longer appropriate. 


\section{SUMMARY AND DISCUSSION}

What is evident from the examination of these texts is that most elders are experiencing many of the same problems with the aging process. The difference between elders is in their interpretation of the information. However, their interpretations depend upon their previous life experiences and their current socio-economic and familial situations. Individuals bring to the aging process their previous life experiences to cope with their changing needs, i.e., getting the help/support they need; dealing with the formal care system, availability of family for informal support when needed, general coping skills in stressful situations. With respect to meaning of aging, these different frames and discourses do not necessary oppose one another, rather "slide past each other as if moving on different planes" (Portes \& Stepick, 1993).

Through the examination of the literature reviewed, it is evident that the theoretical perspectives in the fields of gerontology and sociology have not yet fully elucidated, represented or brought an overall understanding of what it means to be "old" in the United States. Most elders in the United States are not institutionalized. They live amongst other individuals of varying chronological ages and are usually actively involved in their lives. They are not passive agents as theorized with disengagement theory. While important on the macro-level, modernization theory does not appear to be relevant for the examination of discourse of elders in the United States. Age stratification theory is helpful in understanding and "examining the movement of successive birth cohorts across time" (Passuth \& Bengtson, 1988). However, differences between racial/ethnic/cultural groups of elders are not explained. While exchange theory is 
helpful in examining the interactions of older people with other age groups, it is also limited in it is approach. Activity theory and social phenomenology show the greatest promise in terms of helpfulness in understanding meaning of and discourse on aging by elders in the United States. Laz (1998) uses these two theoretical perspectives (as well as the life course tradition) to build a new framework which shows promise for the future of the field of sociology of age and aging. Finally, the political economy of aging is somewhat difficult to apply because it implies that all older individuals are "impoverished and disenfranchised" which is an over-statement. Empirical data show that regardless of racial/ethnic group, the percentage of children (under 18 years) living in poverty is approximately twice that of elders (U. S. Bureau of the Census, 1996).

Most of our knowledge concerning meaning of aging comes from the non-elderly (the unmarked category). If there is discussion from older individuals, it has been primarily derived from somewhat homogeneous groups of elders, mostly white nonHispanic. ${ }^{5}$ While white non-Hispanic elders have been the predominant cultural group among those age 60 years and older, in the future this may not be the case. Previous research and discussion of these issues with respect to minority elders has been hampered by small population sizes. However, in the future it is projected that the elder population will become more racially and ethnically diverse. Further, if we have good fortune, we too shall join the ranks of this "marked category" we are so intent on studying.

\footnotetext{
${ }^{5}$ This is a somewhat broad racialethnic category. Yet the meanings of these categories are also socially constructed.
} 
The ethnographic data to date has been limited by the small population size, as well as personal inexperience of aging by the researchers, and possibly the fear of the process of aging itself. Ethnographic work in the future needs to focus on other subpopulations of elders. First and foremost, there needs to be more examination of elders living in a variety of urban communities and not just focusing on the poor elder, but also elders of other socio-economic classes. Also there needs to be more of an examination of aging in America with respect to other cultural traditions, such as Hispanic, African American, and Asian, as well as the variety of white European cultural heritages (i.e., Italian, Irish, Polish), to name a few. For example, cultural heritage allowed Myerhoff's elders to remain connected with their past and make sense of their present. Further, within these socio-economic, cultural groups, there needs to be a greater examination of the gendered life-experiences' impact on meaning of aging. Finally, the majority of elders live inside metropolitan areas rather than outside metropolitan areas, thus making elders more visible and more easily accessible to the researchers (U.S. Bureau of the Census, 1996). However, in terms of total population, elders comprise a larger percentage of the outside metropolitan arca populations compared to elders inside metropolitan areas (U.S. Bureau of the Census, 1996). Living outside of metropolitan areas places a greater burden on the elder to maintain their independence, since many of the services available to elders living in a metropolitan area are not available to those living outside of a metropolitan area. These services range from public transportation to meals on wheels and senior citizen centers. These suggestions are not meant to say that there is no research in these areas, just that there needs to be more research and more 
analysis. The elder population is changing and what was true about the older individuals in the past may not be true for elders currently or in the future.

The next chapter will lay the groundwork for the eventual analysis of health care utilization behavior of elders by describing the historical demographic trends of elders within Miami-Dade County in the past 50 years. An in-depth description of the community and its development will provide a solid foundation for the quantitative and qualitative analysis of health care utilization of elders in a multicultural urban environment, i.e., what Geertz (1973) defines as "thick description." 
Figure 3.1: The generation of theories in social gerontology' as organized by Bengtson, Burgess \& Parrott (1997)

$\begin{array}{cccc}\text { Intellectual Origins } & \begin{array}{c}\text { First Generation } \\ \text { Theories }\end{array} & \begin{array}{c}\text { Second Generation } \\ \text { Theories }\end{array} & \begin{array}{c}\text { Third Generation } \\ \text { Theories }\end{array}\end{array}$

MICRO-LEVEL:
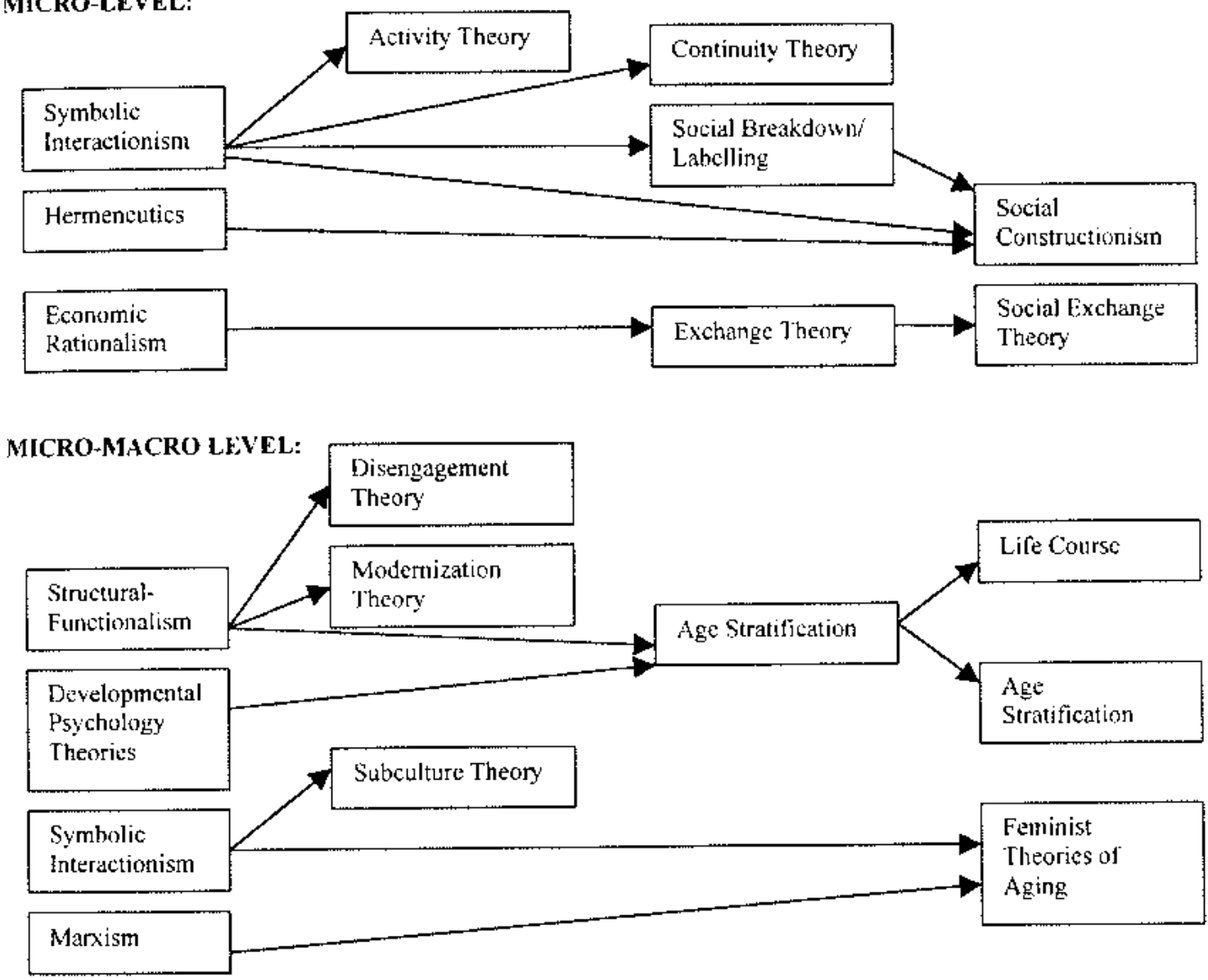

\section{MACRO-LEVEL:}

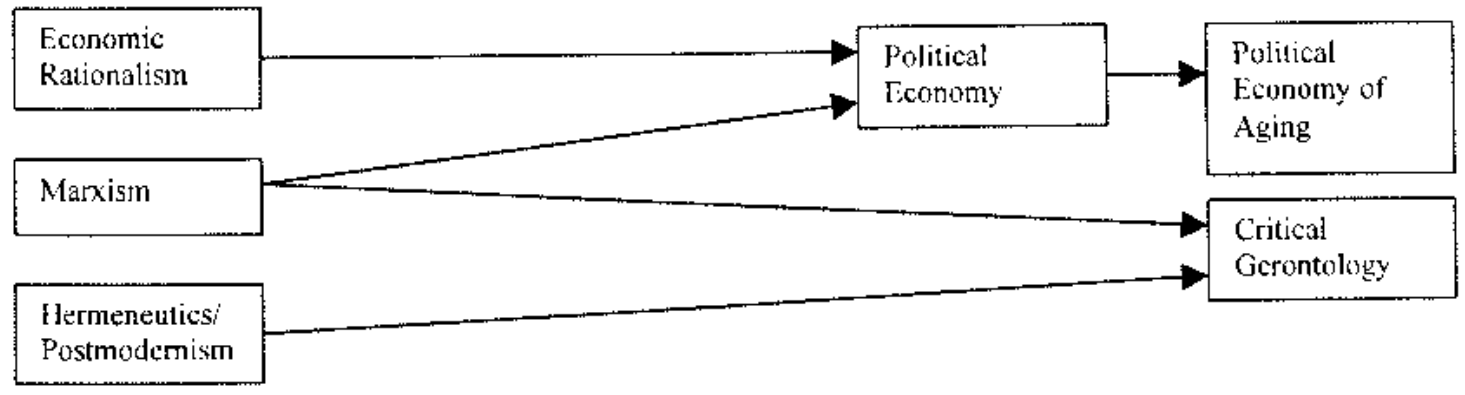

${ }^{1}$ Source: Bengtson, Burgess \& Parrott (1997): S75. 


\section{CHAPTER 4: HISTORICAL DEMOGRAPHIC TRENDS OF}

\section{ELDERS IN MIAMI-DADE COUNTY, FLORIDA, 1950-1990}

Florida ranked fourth among the states in total population size in the 1990 census (U.S. Census, 1996a) behind California $(29,760,021)$, New York $(17,990,455)$ and Texas $(16,986,510)$. Of Florida's population of $12,937,926$ in the 1990 census, a little less than one-quarter (23.5\%) were 60 years or older (U.S. Census, 1995). ${ }^{1}$ Within the state of Florida, Miami-Dade County in 1990 had 359,089 individuals age 60 years or older (BEBR, 1998) or 18.5 percent of its total population was elderly. While the percentage of elders in the total population in Miami-Dade County ${ }^{2}$ has declined to 17.8 percent in 1999, the actual number of elder residents in Miami-Dade County has increased to 377,433 (Consensus Estimating Conference, 1999). By the year 2010, it is projected that Miami-Dade County will have a total population of $2,362,130$ and of that 19.2 percent will be 60 years or older (BEBR, 1998). However, this growing number of elders will not be culturally homogeneous. In 1990, almost the same percent of elders were of Cuban descent as elders identified as white non-Hispanic. Nationally, it is projected that there will be a trend of increasing heterogeneity (Angel \& Hogan, 1992). Already, the elders of Miami-Dade County, FL are already quite diverse. Thus, the trends among the

\footnotetext{
' With respect to its elder population, Florida ranks second with $3,397,366$, behind California $(4,648,953)$ in 1998 (U.S. AOA, 1998).

${ }^{2}$ Originally the name was Dade County, however in November 1997 a ballot proposition passed to change the name to Miami-Dade County. Other names used before the end of 1997 include "Metropolitan Miami" or "Metro-Dade." In this paper, all four terms (Dade County, Miami-Dade County, Metropolitan Miami and Metro-Dade) will be used inter-changeably, regardless of time period.
} 
older residents of Miami-Dade County can be regarded as indicators of future trends for the state of Florida and nationally as the population ages.

In the 1990s there have been frequent references to Miami as a "city of the future" or "the prototypical city of the $21^{\text {st }}$ century." However, there has been only limited documentation of the demography of Miami to back up these claims. This chapter will ultimately trace the growth of the elder population in Miami-Dade County since the 1950s. There will be an examination within the elder population of their socio-economic and demographic characteristics over time using data from published census reports for 1960 , as well as public use microdata samples for $1950,1970,1980$, and 1990 produced and available on the internet by IPUMS (Ruggles, Sobek, et al., 1997). ${ }^{3}$ However, this is only one thread of the investigation. The growth of the elder population in Miami-Dade County does not occur in a vacuum. Thus, this examination will also focus on the structural development of the community of Miami-Dade County, as well as examine the literature on urban development in sociology with specific focus on Miami-Dade County within this literature. Thus, this chapter will be a more comprehensive analysis of elders residing in Miami-Dade County than has previously been undertaken. In the conclusion, population projections will be examined to see what the future might hold for elders residing in Miami-Dade County.

\footnotetext{
${ }^{3}$ The 1960 Public Use Microdata Set available from IPUMS does not have a County indicator; thus only published census information can be used for 1960 .
} 


\section{DATA}

Where not otherwise stated, elders will be defined as individuals residing in the community who are age 60 years or older. However, as Longino \& McNeal (1991), as well as others have pointed out,

... there is no universal age of admission to the elderly in the rescarch that is surveyed...

Much of this discrepancy is dependent on the field from which the research on aging is coming from. Again, citing Longino \& McNeal (1991) who state that,

Demographers have preferred 65 as an entry age, and gerontologists have often preferred 60 because that is the entitlement age used by the Administration on Aging (p. 181).

Further, for most native born citizens of the United States, turning 60 years in chronological terms is not "old," however, what "old" is depends upon the culturally specified norms and previously experienced life events of the individual (Sec Chapter 4). Yet, to remain consistent, the age at entry into elder status will be 60 years for this chapter. Data on elders that is divergent from the 60 years entry point will be documented.

The data that will be used in the analysis of trends of elders residing in MiamiDade County will come from a variety of sources. As stated earlier, population data for $1950,1970,1980$ and 1990 census for the overall county will come from IPUMS (Ruggles, Sobek, et al., 1997). Data for 1960 census will come from published census materials only. More recent population estimates and population projections will come from BEBR (1998) and official state of Florida estimates (Consensus Estimating Conference, 1999). Data for individual census tracts within the county to identify population distribution of selected characteristics throughout the county will come from 
the 1990 U.S. Census Summary Tape File $3 \mathrm{~A}$ and published data from Metro-Dade

Planning (1996). Gcographical distribution of specifically elder characteristics use census tract and block and comes from the 1990 Census of Population and Housing Special Tabulation on Aging (U.S. Census Bureau, 1994). Maps showing these geographic distributions were created using Arcview GIS software. The demographic data was joined to the Miami-Dade County shape file through tract identification numbers. The maps were exported as a bitmap file and inserted into a Word file.

Economic data will come from a variety of published sources, such as the South Florida Regional Planning Council, as well as Metro-Dade Department of Planning, Development and Regulation. The purpose of the data will be to give a contextual understanding of the environment that elders are facing.

\section{INTRODUCTION TO MIAMI: CITY AND REGION}

The importance of metropolitan Miami (Miami-Dade County) is many fold. It has variously been described as:

- “... a frontier city in search of a destination" (Bowie \& Stepick, 1998: 20).

- "... a throwback to a nineteenth century British empire colonial city: a small Anglo population dominating the 'natives"' (Beverley \& Houston, 1996: 29).

- "... is not a microcosm of the American city" (Portes \& Stepick, 1993; xi)

- "... an arena of fierce competition among ethnic groups, competition that frequently translates into social conflict ..." (Croucher, 1997: 22)

- "...riven by two fundamental divisions: black versus white and U.S.-born Americans versus immigrants" (Grenier \& Stepick, 1992: 14). 
It has been part of one of the largest continuously urbanized areas in the state of Florida that are collectively referred to as "South Florida.", Yet, Miami-Dade is unique from its neighboring counties. By itself, metropolitan Miami (Miami-Dade County) is the cighth largest county in the United States (Becker \& Dluhy, 1998). To experience metropolitan Miami is the ultimate postmodernist experience, through the coming together of many factors (and voices) from its geography and environment, to its people and the many diverse cultures' contradictory impact on the economy, class structure, political structure and history, to name a few areas. What follows can only briefly summarize the complicated nature of what we call "metropolitan Miami." However, what I hope to accomplish with this discussion is to give a background for the socio-demographic and economic trends of elders living in metropolitan Miami which will follow this section.

\section{Setting the Stage}

The city of Miami was officially incorporated in 1896 at the southern tip of the Florida peninsula and on the edge of the Everglades. Physically locating the origin of metropolitan Miami is important as research has pointed out that "geography has a long tradition of attending to how certain social groups come to experience the city in ways different to others" (Jacobs \& Fincher, 1998:3). Croucher (1997) notes that,

... the geographic area at the edge of the Everglades has always been the uneasy meeting ground of people of strikingly different cultural backgrounds (p. 25).

\footnotetext{
4 This area also includes the counties of Broward, Monroe and Palm Beach. Thus, the discussion in the following sections on population characteristics will include comparisons (where possible) of the trends in these other South Florida counties and the state of Florida.
} 
Thus, it is the geography as well as the individuals that have created the unique and diverse community of metropolitan Miami.

At the turn of the century, Miami and the County of Dade had few residents compared to northern part of the state of Florida. Most of the urban areas in the state of Florida were located in the northern part of the state; the largest urban center in 1900 was Jacksonville with a population of 28,429 . In comparison the City of Miami had a population of only 1.681. Initially the growth of Miami was dependent on tourism, as well as a haven for retirees from the north (Beverley \& Houston, 1996). However, beginning in the 1920s the area of South Florida began rapidly growing due to a land boom. In 1930 there were almost 200,000 residents living in Dade, Broward, and Monroe counties (SFRPC, 1996). Dade County alone had a resident population of 142,955 (Metro-Dade, 1997). This despite the first of two hurricane disasters in 1926, followed by the second in 1935. Miami was also hit hard by the depression and World War Il due to the economy's heavy reliance on tourism. Yet after the war, tourism did return to Miami. The population of Miami-Dade County had grown to 935,047 by 1960 (Metro-Dade, 1997). By 1990, the population had doubled to 1,937,094 (BEBR, 1998). It is expected that by the year 2010 , the total population of Miami-Dade County will be almost 2.5 million (BEBR, 1998). Boswell (1995) writes that,

No other metropolitan area in the United States has undergone as much ethnic change, as rapidly, as Miami has since 1960. (p. 2)

Figure 4.1 shows the growth of population within Miami-Dade County, FL between 1970 and 1995 . In 1970 , most of the population in the county was located in the northern and coastal areas of the county. Ten years later in 1980 , the county population was more spread out north to south along the coast. During the 1980 s, the growth in 
population started moving west into the everglades. This trend appears to be continuing in the 1990s. Figure 4.2 shows the projected population distribution between 2000 and 2015. The projected population distribution within the county suggests that growth will be primarily concentrated in the western portion of the county.

\section{Demographic Characteristics}

With respect to the demographic make-up of Miami-Dade County, prior to the 1960 s the county was similar to that of other southern United States cities (Croucher, 1997). Using the 1950 IPUMS data, the majority of residents in the county ( $82.2 \%$ ) were white non-Hispanic. The next largest racial/ethnic group was black non-Hispanic, primarily southern native-born (15.5\%). Only 2.4 percent of the total population were of Latin or Hispanic origin, identified through a Spanish surname. Primarily this small Hispanic population were of Cuban origin who had fled to Miami after the 1933 Cuban Revolution (Croucher, 1997). Further, only one-fifth (10.8\%) of the population were foreigners (either non-citizens or naturalized citizens) in 1950. This all changed beginning in 1959 with the rise of Castro and Communism in Cuba. By 1990, almost half $(49.4 \%)$ of the total population residing in Metropolitan Miami was of Hispanic origin (U.S. Bureau of the Census, 1998). ${ }^{5}$

The phenomenal growth of Miami-Dade County is an unusual confluence of many factors. For instance with respect to migratory patterns, Miami-Dade County has become a mecca of a variety of migratory groups. Prior to the 1960 s, the majority of

\footnotetext{
'Latest figures from the Bureau of the Census show that in 199755.7 percent of the total population in Miami-Dade County is of Hispanic-origin (http:/www.census.gov/population/estimates icounty/casrh/casrh12.txt)
} 
individuals migrating to Miami-Dade County were from the northern United States and Canada. Usually they came to South Florida as a temporary escape from the cold winters of the north (i.e., "snow-birds") or for retirement. This occurred especially after World War II (Croucher, 1997). For snow-birds, as time went on, usually their "temporary" stays became longer; until finally they were more permanently residing in South Florida and only occasionally visited relatives up north in the summer to escape the heat and humidity of Miami-Dade County.

While this migratory flow has continued into the 1990 s, population growth also came from the south, i.e., Latin America and the Caribbean. This flow of individuals was quite small prior to 1959 . Since 1959 , this flow of migration has exploded with each the international political events/crises since 1959. With the rise of Castro and Communism in Cuba, Metropolitan Miami again became a refuge for Cubans fleeing the revolution. Yet, not only has Metropolitan Miami become the refuge of Cubans, it has become the refuge for many individuals fleeing turmoil, both political and economic, in other Latin American and Caribbean nations since the 1960s. However, not all immigrants to Miami are refugees. There are individuals seeking a better life for themselves and their family, much like the immigrants who originally came to the United States.

Immigration is a double-edged sword. While it has been a driving force in the economy of Metropolitan Miami, it has also placed "a burden on the welfare programs, housing, health services, and public schools" (Garcia-Zamor, 1998: 186). Thus, an investigation of the economic development of Miami-Dade County will be discussed in the next section. 
Demographic characteristics are not uniformly distribution throughout the county. Using census tracts as the geographic unit of analysis, Figure 4.3 shows the proportion of blacks by census tract and Figure 4.4 shows the proportion of Hispanics by census tract. The majority of the census tracts with the highest proportion of blacks are located in the northern part of the county and away from the coastal areas. Likewise with Hispanic residents, there are blocks of census tracts with high proportions of Hispanics. It is interesting to note that those census tracts with the highest proportions of blacks are distinctly different than those with the highest proportion of Hispanics. Finally, the number of elders $(60+)$ in each census tract is shown in Figure 4.5. Census tracts with the highest numbers of elders are located in the northern sections of the county and are more likely to be located along the coast. It is interesting to note that most of the census tracts with the highest percentages of elders are distinctly different than those census tracts with the highest proportion of blacks (Figure 4.3), yet there is some overlap with census tracts with the highest proportion of Hispanics (Figure 4.4).

\section{Economic Development}

The original influx of Cuban refugees into Miami-Dade County has not only had important impacts on the racial/ethnic composition and migration patterns of the county, but also has influenced the econonic environment of the county as well. Stack \& Warren (1992) wrote that:

The successful establishment of the Cuban community served as a magnet, attracting Latin Americans who could pursue business endeavors in a hospitable environment in which Spanish language and culture were encouraged. Miami's progressive internationalization was accompanied by high levels of economic growth in the 1970 s (p. 165). 
Thus, the original influx of Cuban refugees into Miami-Dade County has brought about a distinct change in the economy, as well as more direct contacts with the political and economic events in Latin America and the Caribbean (Stack \& Warren, 1992). The economy of Metropolitan Miami has become more dependent (both positively and negatively) upon Latin American "banking, real estate development, manufacturing, tourism and drug trafficking industries" (Stack \& Warren, 1992: 165). In terms of advantages, Becker \& Dluhy (1998) state that Miami-Dade County, “...has proved itself remarkably capable of reinventing its economy..." primarily focusing on tourism and foreign trade, particularly international trade with Latin America. ${ }^{6}$ However, Becker \& Dluhy (1998) also state that there are three weaknesses in the economy of Miami-Dade County. First, the city of Miami (not the county) "is $\$ 68$ million in the hole..." Further, those outside of the South Florida do not know the distinction between the county and the city -- likely this lack of distinction will jncrease with the name change of the county in November 1997. Thus, the economic woes of the city of Miami have been "erroneously identified as synonymous with those of all of Metropolitan Miami" (Becker \& Dluhy, 1992: 8). Finally, the demographic trends of the increasing influx of immigrants and exodus of native white non-Hispanics has impacted on the economy, i.e., many companies have followed the exodus of white non-Hispanics to more northern counties of Broward and Palm Beach Countics.

\footnotetext{
5 " Tourism is strong because of the subtropical climate and because of a somewhat glamorous image.... The Port of Miami ... and Miami International Airport are the hubs of the bellweather foreign trade industry. The high number of Spanish speaking professional and technical workers in the area is a definite advantage for international trade with Latin America" (Becker \& Dluhy, 1998: 8).
} 
Figure 4.6 shows the growth of per capita income for Broward, Miami-Dade and Monroe Counties between 1969 and 1997 in 1995 dollars. It is interesting to note that Miami-Dade County per capita income has remained relatively stable over this period. compared to Broward and Monroe Counties. From 1969 to approximately 1976, the per capita income in Broward and Miami-Dade Counties were very similar. However beginning in 1976, the per capita income in Dade County remained relatively flat, while Broward County's per capita began to climb. Per capita income in Miami-Dade County was still above Monroe County until 1987. Since 1987, per capita income in MiamiDade has been the lowest in the South Florida region. This is pattern of growth in per capita income is an important indicator of the ability of a particular county to provide social services, such as public schools, health care services, housing, and welfare assistance. Interestingly, in a recent study of the health system in South Florida, it was found that the health care market has an excess of providers, with over half of the insured market enrolled in managed care (Lipson \& Da Sa, no date). On the downside, South Florida has a high uninsured rate (around 24 percent). Lipson \& Da Sa (no date) point to the fact that 95 percent of the firms employing individuals are small (fewer than 50 employees) and that almost half (47\%) of them not offering insurance to their employees.

Available data from the 1990 census shows the distribution of percentage of the total population living below poverty (Figure 4.7 ) throughout the county. The areas of highest poverty are primarily concentrated around the downtown Miami, Liberty City, and Little Haiti areas. 


\section{Political Development}

As in any urban area, the massive influx of population overall usually leads to periods of severe growing pains. In these periods, the infrastructure and support services usually becomes stressed beyond the original capacity, as well as eruption of violent clashes between newcomers and old-timers as to how development should proceed and resource allocation. Miami-Dade County has been no exception. However, there are the added elements of longstanding racism with its curfews and segregated neighborhoods for blacks, as well as the influx of culturally diverse foreign immigrants. This has lead to violent clashes as witnessed in the 1970s and early 1980s, as well as extreme neighborhood segregation. Some of this neighborhood segregation was acceptable as in the case of the "cthnic enclave" know as Little Havana. However, some of the segregation was a continuation of previous trends with respect to blacks, such as Overtown and Liberty City. One of the ways in which Miami-Dade County has tried to reduce the political strife was actually enacted prior to much of the explosion in diversity. In 1957, a two-tier metropolitan form of government was created. Stack \& Warren (1992) write that this type of government for Metro-Dade was:

...based on the progressive reform tradition of urban politics. The reform model embraced the assumptions of the Good Government movement that arose in the first three decades of the twentieth century. Like other reform movements of this period Good Government movement was motivated by a moralistic, if simplistic, view of the cause of urban decay. This movement had as its focal point a powerful missionary zeal. (p. 161)

It was thought that a two-tier metropolitan form of government would "deemphasize such perceived parochial allegiances as neighborhood, political party, and ethnic and racial interests" (Stack \& Warren, 1992: 161). 
Certainly this brief description of the development and growth of Miami-Dade County has only scratched the surface. The next section will examine the growth of the elder population in Miami-Dade County in more detail. This previous section was important as it established the framing of the many factors involved in overall historical, cultural and economic developments in Miami-Dade County. Portes \& Stepick (1993) wrote that,

Social facts are not self-intelligible. Their interpretation depends on the cognitive frames in which they are placed, and these in turn are products of prior social interactions. (p. 9)

Thus, it was necessary to give this overall description in order to be able to understand the current and future trends of elders and their experiences within this multicultural urban environment of Miami-Dade County.

\section{GROWTH OF ELDER POPULATION IN MIAMI-DADE COUNTY}

In the 1990 census, 18.5 percent of Miami-Dade County's total population was age 60 years or older (BEBR, 1998). Table 4.1 examines the percentage of the total population that is 60 years or older residing in Miami-Dade County since 1950. Up until the 1980 census there was an increasing percentage of the population that was 60 years or older residing in Miami-Dade County. Miami-Dade County, particularly the coastal areas, has been a mecca for the retiree crowd since the 1950s. The geographical distribution of elders throughout the county in 1990 is shown in Figure 4.4.

Table 4.2 compares the percentage of $60+$ population in the four South Florida counties in 1980 and 1990 . With respect to the state of Florida's percentage of elders, 
there appears to be little change between 1980 and 1990 (23.1\% in 1980 and $23.5 \%$ in 1990). However when examined by county, different trends seem to be occurring. Only Monroe County shows an increase in the percentage of elders in its population since 1980 (20.7\% in 1980 to $22.1 \%$ in 1990), while Broward, Miami-Dade and Palm Beach Counties show decreases in the percentage of elder population. Further, the elder population as a percentage of the total population living in Miami-Dade County is certainly not the largest of the four South Florida counties. However, because MiamiDade County is the largest of the four counties, the numeric numbers of elders residing in Miami-Dade are the largest of the four counties.

Growth in Miami-Dade County is not only dependent upon elders, but other segments of the population. In general, there are two ways in which the elder population within a community can numerically increase. First, elders can migrate to a community (i.e., retirement migration) which has been the primary way Miami-Dade County's elder population has grown in the past. A second type of growth in elders populations is through the aging of individuals who already reside in the community i.e., aging in place.

Figure 4.8 shows the comparison of the growth in the total population compared to the elder population between 1950 and 1990. It is interesting to note that between 1980 and 1990; the elder population does not appear to have grown as significantly as the overall total population of Miami-Dade County. Primarily the most recent growth of the population in Miami-Dade County appears to be driven by the influx of younger Latin American immigrants. In gencral, immigrants tend to be younger so they can withstand the rigors of moving. This lack of growth in the elder population compared to the overall total population is also evident in Table 4.3 which examines the percentage change in 
population both for the $60+$ population and the total population. Before the 1990 Census, the percentage change in the $60+$ population was much greater than the percentage change in the total population. However, in the 1990 Census, there is a dramatic shift. The percentage change of the total population between the 1980 and 1990 censuses is significantly greater than the percentage change for the elder population in the same period. Figure 4.9 shows the distribution of the proportion of the census tract population that is elder $(60+)$ in 1990 . The majorily of the census tracts with the highest percentage of elders ( $41.3 \%$ or more) are primarily concentrated in the northern coastal section of the county.

While the growth in the overall elder population has been significant, the total numbers and the changes in these numbers of elders only tells part of the story with regard to Miami-Dade County. Thus, the next section will analyze basic demographic characteristics of elders residing in Miami-Dade County.

\section{Basic Demographic Characteristics of Elders}

Table 4.4 shows selected demographic characteristics of elders in Miami-Dade County from 1950 to 1990 . With respect to age distribution, the growth of the $85+$ in the county has been increasing since 1950 , from 2.0 percent of the $60+$ population in 1950 to 9.0 percent in 1990. It is estimated, as of April 1,2000, that 10.1 percent of the elder population living within Miami-Dade County will be 85 years or older (Consensus Estimating Conference, 1999). With respect to gender distribution, elders in Miami-Dade County are predominantly female. This is consistent with national figures and is not surprising given that women outlive men by approximately 7 years. While age and 
gender characteristics of elders are consistent with national characteristics of elders, however, with respect to race and ethnicity distribution, Miami-Dade County elders are unique.

To show how unique Miami-Dade County's elders are compared to elders in neighboring South Florida counties, Table 4.5 compares the selected demographic characteristics of elders in Miami-Dade County of elders in Miami-Dade County with other South Florida counties and the state of Florida. Most notable is the difference in the racial/ethnic composition of the elder population in Miami-Dade County and the other South Florida counties. Less than half of elders $(42.8 \%)$ are white non-Hispanic in Miami-Dade County compared to 91.5 percent in Broward County, 95.3 percent in Monroe/Collier Counties, and 93.7 percent in Palm Beach County. Further, almost half $(47.0 \%)$ of the elders are Hispanic in Miami-Dade County, while in the other three South Florida counties, less than 5 percent of elders are Hispanic. Almost two-fifths (38.2\%) of the elders are Cuban in Miami-Dade County, compared to 2 percent or less in the other South Florida counties.

\section{Poverty and Economic Status of Elders}

Poverty and the socio-economic status of elders have been a national concern for a long time. This concern spurred the passage of federal assistance programs of Medicare and Social Security. Further, research has shown that elders living below poverty are at greater risk of frailty than those not living below poverty are. Pampel (1998) wrote, "Problems during old age strike those with fewer resources more scriously and make retirement less rewarding and satisfying" (p. 75). 
In Miami-Dade County in 1950, half of the elders lived below the Federal Poverty Line (FPL). Since then there has been a decline in the percentage of clders living below $100 \%$ FPL (See Table 4.6). While it may be expected that growing numbers of minority elders, as evidenced in Table 4.4, would lead to more elders in the County living in poverty, this does not appear to be happening. Another measure of socio-economic status in Table 4.6 is the percentage of elders with less than 12 years of education. In 1950 , 86.8 percent of elders had less than 12 years of education, this declined to 51.8 percent in 1980. However, in 1990 the percentage had risen slightly to 52.7 percent.

Table 4.7 compares elders in Miami-Dade County with those in other South Florida counties. The percentage of elders living in poverty is the highest in Miami-Dade County $(18.0 \%)$. It is almost twice that of Broward County $(9.1 \%)$ and approximately three times that found in Palm Beach and Monroe/Collier counties $(6.6 \%$ and $6.0 \%$, respectively). Consistent with the percentage of elders living in poverty, the percentage of elders with less than 12 years education in 1990 is significantly higher in Miami-Dade County $(52.7 \%)$ compared to other South Florida counties (34.3\% for Broward, $21.2 \%$ for Monroe/Collier counties, and $27.7 \%$ for Palm Beach), as well as the State of Florida $(37.8 \%)$.

\section{SOCIAL SUPPORT FOR ELDERS}

Social support can ameliorate some of the problems of poverty and low economic status of elders living within a community. From a service provision planning perspective, an elder living with others implies:

...that an older person is being supported, at least residentially, and perhaps financial by family members or others. Housing and supportive 
services originating from private relationships mean that the public sector requires fewer resources to meet the needs of the indigent and disabled elderly (Longino \& McNeal, 1991: 185).

Further, the diverse cultural and economic differences within the elder population of Miami-Dade County will also have significant impact on the demand for long-term care services (Mills, 1998).

Table 4.8 shows selected measures of social support and household characteristics of elders in Miami-Dade County between 1970 and 1990. The measures show somewhat contradictory signs for the future in terms of formal service needs. The percentage living alone has remained fairly constant between one-fifth to one-quarter, despite the growing percentage of oldest-old $(85+)$. However, the percentage of elders who are married has declined slightly since 1970. This is not surprising given the growing percentage of $85+$ in the elder population. Interestingly, the percentage of elders with no phones has declined from 12.1 percent in 1970 to 2.1 percent in 1990 . The percentage of foreignborn elders has been increasing since $1970(40.8 \%)$. In 1990 , over half $(53.8 \%)$ of elders were foreign-born. With changes in the availability of public assistance for non-citizens, this may impact the economic status of elders residing in Miami-Dade County in the future. Elders without U.S. citizenship in Miami-Dade County have increased from 14.0 percent in 1970 to 29.2 percent in 1990.

Table 4.9 compares these selected social support and household characteristics for each of the counties in South Florida in 1990. Elders in Miami-Dade County are similar with respect to the percentage living alone and percentage with no phone. Fewer elders in Miami-Dade County are currently married. The percentage of elders living with disability (either mobility limitation or personal care limitation) is somewhat higher in 
Miami-Dade County. This is not surprising given the higher levels of poverty and the greater number of oldest-old elders living in Miami-Dade County compared to the other South Florida counties. However, the most distinct difference between Miami-Dade County elders and elders in the other South Florida counties is with respect to the percentage foreign-born, the percentage who are not U.S. citizens, the percentage who cannot speak English and the percentage who are linguistically isolated. All of these indictors complicate the delivery of social support services within Miami-Dade County, through the necessary availability of language translation for elders who cannot speak English.

In terms of power that the elders have to the household wealth, an examination of the elder's relationship to the head of household may be used as an indicator of power that the elder holds within the household. Table 4.10 shows the distribution of elders not living alone and not living in group quarters by the gencral relationship to the head of household. The distribution of household relationships of elders appears to be relatively consistent since 1970. Approximately three-quarters of all elders not living alone and not in group quarters in Miami-Dade County in each of the last three censuses have indicated that they were either the head of household or the spouse of the head of household. This would appear to indicate that the majority of elders not living alone and not in group quarters had significant control over the distribution of the household wealth.

Table 4.11 shows the distribution of elders not living alone and not living in group quarters by general relationship to the head of household for each of the South Florida counties. While it was noted that a majority of elders in Miami-Dade County are head of household or spouse of the head of household (75.0\%). However, compared to 
elders in the other South Florida counties, fewer elders in Miami-Dade County have such power. Almost 9 in 10 of Broward's elders are listed as head of household or spouse of the head of household $(88.5 \%$ ). In Palm Beach and Monroe/Collier counties, it is slightly more than 9 out of 10 elders ( $93.5 \%$ and $92.3 \%$, respectively)

\section{Geographic Residency of Elders within Miami-Dade County}

Previously with respect to the total population, demographic characteristics were not uniformly distributed throughout the county. Likewise, for the elder population, selected demographic, economic and social support characteristics are not uniformly distributed throughout the county. Figure 4.10 shows the distribution of elders who are 85 years or older (oldest-old) by census tract in 1990. None of the census tracts in Miami-Dade County in 1990 had over 50 percent of its elder population in this oldest-old age category. Census tracts with the highest proportion of $85+$ were scattered throughout the county in 1990.

Racial/ethnic distribution of the elder population shows similar patterns as was found in the total population. Figure 4.11 shows the proportion of black elders in the elder population. As was found in Figure 4.3 for the total population, there are two areas of census tracts with higher proportions ( $28.8 \%$ or more) of black elders. There is a corridor in the northern part of the county around Liberty City and a smaller group of census tracts in the southem area of the county, particularly in the Richmond Heights area. Figure 4.12 shows the proportional distribution of Hispanic-origin elders within the county. Census tracts showing $45.2 \%$ or more Hispanic elders are primarily concentrated in a corridor following Okeechobee Road (US 27). There appears to be very little 
overlap in the census tracts with high concentrations of black elders with those of high concentration of Hispanic elders.

As for economic characteristic concentration, Figure 4.13 shows the distribution of percentage of elders living below poverty by census tract. The majority of the census tracts with the highest percentage of elders living below poverty $(39.7 \%$ or more) are primarily located in the areas that show the highest concentration of black and Hispanic elders, as well as a small area located around Homestead Air Reserve Base.

With respect to social support characteristics, Figure 4.14 shows the distribution of the percentage of elders living alone by census tract. The geographical distribution of this characteristic for elders could be important for policy planners, since elders living alone are more at risk for needing support services such as Meals on Wheels or transportation services to get to doctors appointments or other places they need to go. Placing necessary services in areas of the county that have higher concentration of elders living alone could allow planners to more cost-effectively use county dollars for services. Unfortunately with respect to elders living alone, there does not appear to be one area of the county with the highest concentration of census tracts, such as was found with respect to black and Hispanic elders.

\section{Racial/Ethnic Differences of Elders in Miami-Dade County}

As noted in Chapter 3, characteristics within racial/ethnic categories of elders may play a significant role in the health care needs of elders. This chapter's examination of demographic trends of elders in Miami-Dade County proposes an integrated perspective. The data so far presented has not distinguished elders by racial/ethnic categories, i.e.; it 
has aggregated all elders residing in Miami-Dade County at the time of the census together in an overall trend analysis. In this section, there will be a breakout of the four major racial/ethnic groups of elders: white non-Hispanic, black non-Hispanic, Cuban and non-Cuban Hispanic with a smaller trend analysis between 1970 and 1990 .

Table 4.12 shows the age and gender characteristics within each of racial/ethnic groups of elders in Miami-Dade County between 1970 and 1990. Gender distribution does not appear to be significantly different among the racial/ethnic groups of elders in 1990. However, age distribution is significantly different. The percentage of the white non-Hispanic elder population that is 85 years or older $(12.8 \%$ in 1990$)$ is the highest of all four racial/ethnic groups of elders in Miami-Dade County. It is almost twice that found in the black non-Hispanic (6.4\%) and Cuban (6.8\%) elder populations. This can be seen in either a positive or a negative light. A larger percentage of elders able to survive beyond age 85 years would appear to suggest that these elders have had better life experiences. However, it has been documented that those over the age of 85 years are at greater risk of becoming frail and in need of greater health care.

Table 4.13 shows selected socio-economic characteristics of elders by racial/ethnic group since 1970. As was previously expected, significantly fewer white non-Hispanic elders live below poverty, as well as have less than 12 years of education. It would appear that federal assistance programs, such as Medicare and Social Security, have had a dramatic impact on the lives of white non-Hispanic elders, compared to black non-Hispanic, Cuban, and non-Cuban Hispanic elders. In 1970, 18.6 percent of white non-Hispanic elders lived below poverty. By 1990 , the percentage of white non-Hispanic elders living below poverty had declined to 9.3 percent. Black non-Hispanic and Cuban 
elders also show a decline in the percentage living below poverty between 1970 and 1990, but certainly not as dramatic as white non-Hispanic. Non-Cuban Hispanic elders are the only racial/ethnic group of elders that show little change in the percentage living below poverty since 1970 (i.e., $23.7 \%$ in $1970,22.4 \%$ in 1980 , and $24.1 \%$ in 1990 ).

Selected social support characteristics of elders by racial/ethnicity between 1970 and 1990 are shown in Table 4.14. Cuban elders consistently have reported the lowest percentage living alone $(5.8 \%$ in $1970 ; 14.4 \%$ in 1980 ; and $18.7 \%$ in 1990$)$. A close second is found within the non-Cuban Hispanic elder population $(23.4 \%$ in $1970 ; 19.0 \%$ in 1980; and $20.1 \%$ in 1990). The highest percentage of elders living alone is found within the white non-Hispanic elder population, particularly in 1990 with 35.4 percent of white non-Hispanic elders living alone.

With respect to marital status, Cuban and white non-Hispanic elders have the highest percentage of elders who are married in 1990 (49.6\% and 54.3\%, respectively). In comparison black non-Hispanic elders have the lowest percentage currently married in $1990(37.5 \%)$.

Another indicator of social support is the ability to speak English. In 1990, over two-fifths $(41.7 \%)$ of Cuban elders in the 1990 Census indicated that they were not able to speak English, compared to one-quarter (24.9\%) of non-Cuban Hispanic elders. As discussed earlier, the ability to speak English is not necessary for Hispanics to live and work in Miami-Dade County. However, the ability of Hispanic elders to live and to navigate the social service arena can be seriously compromised, especially with respect to the legislated reforms in such national programs as Social Security and Medicare. Elders who are not able to speak English and in need of social services face another intervening 
need - an "ethical" individual to translate program rules and regulations from English to Spanish.

With the reforms legislated in federal assistance programs; U.S. citizenship has become another important indicator for access to federal assistance programs. Between the 1980 and 1990 Censuses, the percentage of black non-Hispanic elders who identified themselves as "not U.S. citizen" has increased dramatically from 2.5 percent in 1980 to 13.3 percent in 1990). A similar dramatic increase in the percentage of non-Cuban Hispanic elders who identified themselves as "not U.S. citizen" happened between 1970 and $1980(12.6 \%$ and $44.9 \%$, respectively). However, the largest racial/ethnic group of elders who identify themselves as "not U.S. citizens" can be found among Cuban elders. While the percentage of Cuban elders who are not U.S. citizens has declined since 1970 (85.1\%), the percentage of Cuban elders who are not U.S. citizens in 1990 is still almost three-fifths (58.5\%) of ALL Cuban elders. Thus, these legislated reforms in federal assistance programs will likely have a great impact on the Hispanic elder population, primarily the Cuban elder population.

Need for social support is dependent upon physical and cognitive functioning of the elder. Unfortunately, the measures available from any of the censuses are very generic. The most specific measures of functioning can be found in the 1990 U.S. Census with three questions - work disability, mobility limitation and personal care limitation. With respect to the latter two limitations, black non-Hispanic elders have the highest percentage reporting that they experience limitations in mobility and personal care $(26.8 \%$ and $28.0 \%$, respectively). Among white non-Hispanic, Cuban and nonCuban Hispanic elders, the percentages are approximately between one-seventh and one- 
fifth of the respective elder populations. Further, the percentages are relatively similar in distribution for these three racial/ethnic groups of elders.

As noted earlier, household composition is an indication of control over the distribution of the wealth of household. The distribution of household composition of elders not living alone and not living in group quarters (not institutionalized) by racial/ethnic groups is shown in Table 4.16. Almost seven-eighths of white non-Hispanic elders indicated in 1990 that they were either the head of the household or the spouse of the head of houschold ( $86.8 \%$ ). In comparison, only 69.1 percent of black non-Hispanic, 69.2 percent of Cuban elders and $55.9 \%$ percent of non-Cuban Hispanic elders indicated in 1990 that they were either household heads or spouse of household heads. The most predominant distinction between households of racial/ethnic groups of elders is in the large percentage of minority elders living as "other family members" to the head of household compared to white non-Hispanic elders $(9.6 \%$ for white non-Hispanic, $24.6 \%$ for black non-Hispanic, $27.7 \%$ for Cuban, and $37.6 \%$ for non-Cuban Hispanic in 1990). The percentage of elders who are "not family members" to the designated head of household is less than one-tenth in each of the racial/cthnic groups $(3.6 \%$ for white nonHispanic, $6.4 \%$ for black non-Hispanic, 3.1\% for Cuban, and $6.4 \%$ for non-Cuban Hispanics in 1990).

\section{SUMMARY AND DISCUSSION}

As stated in the introduction this chapter brings together several threads of research. The ultimate focus of this chapter has been the historical demographic trends of elders in Miami-Dade County, FL since 1950. However, elders do not live in a vacuum 
and thus the structural development of Miami-Dade County as a whole must also be examined. As time goes by, there are new interpretations of trends that are occurring or have occurred. For example, Longino \& McNeal (1991) did not believe that the trends and characteristics of the older population in South Florida were national indicators in the 1980s. However in the 1990s I believe that there are lessons that other communities should find useful in dealing with the competing demands faced in a multicultural urban environment regardless of the racial/ethnic composition of the community. In this chapter I have tried to document the intertwining threads that have and will continue to have on the communities within Miami-Dade County as a whole and the competing demands that are and will be faced in the future with respect to resources and services. Thus, in this respect Metropolitan Miami is not unique.

In the future, the elder population in Miami-Dade County will continue to grow and the competing demands will increase. The diversity of the elder population will also increase which in turn will place added pressure on finding culturally sensitive solutions to the increasing demand for health care in the community. 
Table 4.1: Total Elder Population Reșiding in Miami-Dade County, FL 1950-1999 (Number and Percentage of Total Population)

\begin{tabular}{lrrrrrr}
\hline & 1950 & 1960 & 1970 & 1980 & 1990 & 1999 \\
\hline $60+$ Population & 59,182 & 135,272 & 237,521 & 334,589 & 359,089 & 405,973 \\
Total Population & 495,084 & 935,047 & $1,267,792$ & $1,625,781$ & $1,937.094$ & $2,175,634$ \\
$\% 60+$ & $12.0 \%$ & $14.5 \%$ & $18.7 \%$ & $20.6 \%$ & $18.5 \%$ & $18.7 \%$ \\
\hline
\end{tabular}

Data Sources: U.S. Census (2000) for 1998; BEBR (1998) for 1990 elder population and Longino \& McNeal (1991) and U.S. Bureau of the Census (1995) for earlier periods.

Table 4.2: Comparison of Elder Populations Residing in Florida, South Florida, and the individual counties of South Florida 1980 and 1990

\begin{tabular}{lrr}
\hline & & $\begin{array}{r}\% \text { of } \\
\text { Total }\end{array}$ \\
\hline Florida & $3,429,451$ & $22.7 \%$ \\
1999 & $3,036,447$ & $23.5 \%$ \\
1990 & $2,253,437$ & $23.1 \%$ \\
1980 & & \\
Broward & 321,428 & $20.9 \%$ \\
1999 & 318,089 & $25.3 \%$ \\
1990 & 289,716 & $28.4 \%$ \\
1980 & & \\
Miami-Dade & 405,973 & $18.7 \%$ \\
1999 & 359,089 & $18.5 \%$ \\
1990 & 334,589 & $20.6 \%$ \\
1980 & & \\
Monroe & 18,864 & $23.6 \%$ \\
1999 & 17,254 & $22.1 \%$ \\
1990 & 13,064 & $20.7 \%$ \\
1980 & & \\
Palm Beach & 302,263 & $28.8 \%$ \\
1999 & 256,828 & $29.7 \%$ \\
1990 & 173,746 & $30.1 \%$ \\
1980 &
\end{tabular}

Data Sources: U.S. Census Bureau (2000) for 1998 figures; BEBR (1998) for 1990 clder population and Longino \& McNeal (1991) and Florida Consensus Estimating Conference, Book 3, Summer 1997 for 1980 elder population.

Table 4.3: Percentage Change in the Elder Population and Total Population between Censuses 1950-1998 in Miami-Dade County, Florida

\begin{tabular}{lrrrrr}
\hline & $1950-60$ & $1960-70$ & $1970-80$ & $1980-90$ & $1990-98$ \\
\hline $60+$ Population & $128.6 \%$ & $75.6 \%$ & $40.9 \%$ & $7.3 \%$ & $13.1 \%$ \\
Total Population & $88.9 \%$ & $35.6 \%$ & $28.2 \%$ & $19.2 \%$ & $12.3 \%$ \\
\hline
\end{tabular}

Data Source: U.S. Census Bureau (2000) for 1998 population; BEBR (1998) for 1990 elder population and Longino \& McNeal (1991) and U.S. Bureau of the Census (1995) for earlier periods. 
Table 4.4: Demographic Characteristics of Elders in Miami-Dade County, FL. 1950-1999

\begin{tabular}{lrrrrrr}
\hline & 1950 & 1960 & 1970 & 1980 & 1990 & 1999 \\
\hline $\begin{array}{l}60+\text { population } \\
\text { Age Distribution (\% of 60+) }\end{array}$ & 59.182 & 135,272 & 237.521 & 334,589 & 359,089 & 405,973 \\
85+ population & $2.0 \%$ & -- & $4.6 \%$ & $6.4 \%$ & $9.0 \%$ & $10.0 \%$ \\
Gender Distribution (\% of 60+) & & & & & & \\
Female population & $52.0 \%$ & -- & $56.2 \%$ & $57.9 \%$ & $58.9 \%$ & $58.3 \%$ \\
Racial/Ethnic Distribution (\% of 60+) & & & & & & \\
White non-Hispanic & $92.6 \%$ & -- & $67.1 \%$ & $65.1 \%$ & $42.8 \%$ & $32.9 \%$ \\
Black non-Hispanic & $6.4 \%$ & -- & $3.6 \%$ & $5.9 \%$ & $9.7 \%$ & $9.7 \% *$ \\
Hispanic & $1.0 \%$ & -- & $29.2 \%$ & $28.4 \%$ & $47.0 \%$ & $58.2 \%$ \\
Cuban & $\mathrm{N} / \mathrm{A}$ & -- & $11.8 \%$ & $23.7 \%$ & $38.2 \%$ & $\mathrm{~N} / \mathrm{A}$ \\
\hline
\end{tabular}

Data Source: Ruggles, Sobeck, et al., (1997) for 1950, 1970, 1980, and 1990 figures; U.S. Census Bureau (2000) for 1998 figures.

Table 4.5: Comparison of Demographic Characteristics of Elders in South Florida, 1990

\begin{tabular}{lrrrrr}
\hline & Broward & Miami-Dade & Monroe Collier & Palm Beach & Florida \\
\hline 60+ Population & 318,069 & 359,089 & 62,246 & 256,828 & $3,036.447$ \\
Age Distribution (\% of $60+$ ) & $8.4 \%$ & & & & \\
$85+$ years & $9.0 \%$ & $4.7 \%$ & $6.4 \%$ & $7.0 \%$ \\
Gender Distribution (\% of $60+$ ) & & & & \\
Female & $59.0 \%$ & $58.9 \%$ & $52.5 \%$ & $57.4 \%$ & $57.2 \%$ \\
Racial/Ethnic Distribution (\% of $60+)$ & & & & \\
White Non-Hispanic & $91.5 \%$ & $42.8 \%$ & $95.3 \%$ & $93.7 \%$ & 85.7 \\
Black Non-Hispanic & $5.0 \%$ & $9.7 \%$ & $1.6 \%$ & $3.5 \%$ & 6.1 \\
Hispanic & $3.2 \%$ & $47.0 \%$ & $3.0 \%$ & $2.5 \%$ & 7.8 \\
Cuban & $1.2 \%$ & $38.2 \%$ & $1.9 \%$ & $1.0 \%$ & 5.2 \\
\hline
\end{tabular}

Data Source: Ruggles, Sobeck, ct al., 1997.

Table 4.6: Selected Socio-Economic Characteristics of Elders in Miami-Dade County, $1950-1990$

\begin{tabular}{lrrrr}
\hline & 1950 & 1970 & 1980 & 1990 \\
\hline Living Below Poverty & $50.0 \%$ & $21.7 \%$ & $16.4 \%$ & $18.0 \%$ \\
Less than HS education & $86.8 \%$ & $64.9 \%$ & $51.8 \%$ & $52.7 \%$ \\
\hline
\end{tabular}

Source: Ruggles, Sobeck, et al., 1997.

Table 4.7: Comparison of Sclected Socio-Economic Characteristics of Elders in South Florida, 1990

\begin{tabular}{lrrrrr}
\hline & Broward & Dade & Monroe/Collier & Palm Beach & Florida \\
\hline Living Below Poverty & $9.1 \%$ & $18.0 \%$ & $6.0 \%$ & $6.6 \%$ & $10.6 \%$ \\
Less than HS education & $34.3 \%$ & $52.7 \%$ & $21.2 \%$ & $27.7 \%$ & $37.8 \%$ \\
\hline
\end{tabular}

Data Source: Ruggles, Sobeck, et al., 1997. 
Table 4.8: Selected Social Support and Household Characteristics of Elders in MiamiDade County, 1970-1990

\begin{tabular}{lrrr} 
& 1970 & 1980 & 1990 \\
\hline Living Alone & $22.0 \%$ & $26.3 \%$ & $26.2 \%$ \\
Married, spouse present & $56.2 \%$ & $55.2 \%$ & $50.3 \%$ \\
Employed & $23.8 \%$ & $22.0 \%$ & $23.7 \%$ \\
Foreign-born & $40.8 \%$ & $47.1 \%$ & $56.7 \%$ \\
Not US Citizen & $14.0 \%$ & $20.9 \%$ & $29.2 \%$ \\
Cannot speak English & not available & $13.9 \%$ & $18.7 \%$ \\
No Phone & $12.1 \%$ & $5.0 \%$ & $2.1 \%$ \\
Disabilities & & & \\
Prevents Wotking & $15.2 \%$ & $24.5 \%$ & $24.6 \%$ \\
Mobility Limitation & not available & not available & $18.5 \%$ \\
Personal Care Limitation & not available & not available & $16.0 \%$ \\
\hline
\end{tabular}

Data Source: Ruggles, Sobeck, et al., 1997.

Table 4.9: Comparison of Selected Social Support and Household Characteristics of Elders in South Florida, 1990

\begin{tabular}{lrrrrr}
\hline & Broward & Dade & Monroe/Collier & Palm Beach & Florida \\
\hline Living Alone & $29.3 \%$ & $26.2 \%$ & $19.1 \%$ & $25.5 \%$ & $25.4 \%$ \\
Married, spouse present & $57.6 \%$ & $50.3 \%$ & $69.9 \%$ & $64.8 \%$ & $60.9 \%$ \\
Employed & $17.3 \%$ & $23.7 \%$ & $19.3 \%$ & $15.4 \%$ & $16.7 \%$ \\
Foreign-born & $19.5 \%$ & $56.7 \%$ & $6.3 \%$ & $14.8 \%$ & $16.0 \%$ \\
Not a US citizen & $4.1 \%$ & $29.2 \%$ & $1.9 \%$ & $2.4 \%$ & $5.4 \%$ \\
Cannot speak English & $0.9 \%$ & $18.7 \%$ & $0.3 \%$ & $0.4 \%$ & $2.6 \%$ \\
No Phone & $1.0 \%$ & $2.1 \%$ & $2.2 \%$ & $1.1 \%$ & $1.8 \%$ \\
Disabilitics & & & & & \\
Prevents Working & $20.9 \%$ & $24.6 \%$ & $16.1 \%$ & $18.4 \%$ & $24.0 \%$ \\
Mobility Limitation & $13.3 \%$ & $18.5 \%$ & $9.7 \%$ & $11.9 \%$ & $14.0 \%$ \\
Personal Care & $14.6 \%$ & $16.0 \%$ & $7.8 \%$ & $12.5 \%$ & $12.3 \%$ \\
Limitation & & & & & \\
\hline
\end{tabular}

Data Source: Ruggles, Sobeck, et al., 1997.

Table 4.10: Selected Household Composition Characteristics of Elders Not Living Alone in Miami-Dade County, 1970-1990

\begin{tabular}{lrrr}
\hline & 1970 & 1980 & 1990 \\
\hline Head of Household & $46.0 \%$ & $47.5 \%$ & $45.8 \%$ \\
Spouse of Head of Household & $30.2 \%$ & $31.5 \%$ & $29.2 \%$ \\
Other family to Head of Household & $20.2 \%$ & $18.1 \%$ & $21.0 \%$ \\
Non-family to Head of Household & $3.6 \%$ & $2.8 \%$ & $4.0 \%$ \\
\hline
\end{tabular}

Data Source: Ruggles, Sobeck, et al., 1997. 
Table 4.11: Selected Household Composition Characteristics of Elders Not Living Alone or in Group Quarters in South Florida, 1990

\begin{tabular}{lrrrrr}
\hline & Broward & Dade & $\begin{array}{r}\text { Monroe } \\
\text { Collier }\end{array}$ & Palm Beach & Florida \\
\hline Head of Household & $49.6 \%$ & $45.8 \%$ & $50.7 \%$ & $51.2 \%$ & $51.0 \%$ \\
Spousc of Head of Housebold & $38.9 \%$ & $29.2 \%$ & $41.6 \%$ & $42.3 \%$ & $38.5 \%$ \\
Other family to Head of Household & $8.9 \%$ & $21.0 \%$ & $3.6 \%$ & $4.7 \%$ & $8.3 \%$ \\
Non-family to Head of Houschold & $2.6 \%$ & $4.0 \%$ & $4.0 \%$ & $1.8 \%$ & $2.2 \%$ \\
\hline
\end{tabular}

Data Source: Ruggles, Sobeck, et al., 1997.

Table 4.12: Selected Demographic Characteristics of Elders in Miami-Dade County by Racial/Ethnic Group, 1970-1990

\begin{tabular}{lrrrr}
\hline & White non-Hispanic & Black non-Hispanic & Cuban & Non-Cuban Hispanic \\
\hline$\%$ Age $85+$ & & & & \\
1990 & $12.8 \%$ & $6.4 \%$ & $6.8 \%$ & $9.1 \%$ \\
1980 & $7.4 \%$ & $2.5 \%$ & $4.8 \%$ & $6.3 \%$ \\
1970 & $4.8 \%$ & $2.2 \%$ & $4.1 \%$ & $5.1 \%$ \\
$\%$ Female & & & & \\
1990 & $60.3 \%$ & $60.4 \%$ & $58.3 \%$ & $56.0 \%$ \\
1980 & $58.4 \%$ & $61.3 \%$ & $56.8 \%$ & $53.8 \%$ \\
1970 & $54.8 \%$ & $52.8 \%$ & $65.4 \%$ & $55.9 \%$ \\
\hline
\end{tabular}

Data Source: Ruggles, Sobeck, et al., 1997.

Table 4.13: Selected Socio-Economic Characteristics of Elders in Miami-Dade County by Racial/Ethnic Group, 1970-1990

\begin{tabular}{lrrrr}
\hline & $\begin{array}{r}\text { White non- } \\
\text { Hispanic }\end{array}$ & $\begin{array}{r}\text { Black non- } \\
\text { Hispanic }\end{array}$ & Cuban & $\begin{array}{r}\text { Non-Cuban } \\
\text { Hispanic }\end{array}$ \\
\hline $\begin{array}{l}\text { Living Below Poverty } \\
1990\end{array}$ & $9.3 \%$ & $32.9 \%$ & $22.3 \%$ & $24.1 \%$ \\
1980 & $11.7 \%$ & $35.7 \%$ & $24.4 \%$ & $22.4 \%$ \\
1970 & $18.6 \%$ & $47.7 \%$ & $28.1 \%$ & $23.7 \%$ \\
Less than HS diploma & & & & $80.9 \%$ \\
1990 & $62.5 \%$ & $89.2 \%$ & $82.2 \%$ & $66.5 \%$ \\
1980 & $44.4 \%$ & $81.9 \%$ & $61.8 \%$ & $70.8 \%$ \\
1970 & $62.0 \%$ & $88.8 \%$ & $65.3 \%$ & \\
\hline
\end{tabular}

Data Source: Ruggles, Sobeck, et al., 1997. 
Table 4.14: Selected Social Support and Household Characteristics of Elders in MiamiDade County by Racial/Ethnic Group, 1970-1990

\begin{tabular}{|c|c|c|c|c|}
\hline & $\begin{array}{l}\text { White non- } \\
\text { Hispanic }\end{array}$ & $\begin{array}{l}\text { Black non- } \\
\text { Hispanic }\end{array}$ & Cuban & $\begin{array}{r}\text { Non-Cuban } \\
\text { Hispanic }\end{array}$ \\
\hline \multicolumn{5}{|l|}{ Living Alone } \\
\hline 1990 & $35.4 \%$ & $25.6 \%$ & $18.7 \%$ & $20.1 \%$ \\
\hline 1980 & $30.3 \%$ & $26.6 \%$ & $14.4 \%$ & $19.0 \%$ \\
\hline 1970 & $24.3 \%$ & $26.7 \%$ & $5.8 \%$ & $23.4 \%$ \\
\hline \multicolumn{5}{|l|}{ Maried, spouse present } \\
\hline 1990 & $49.6 \%$ & $37.5 \%$ & $54.3 \%$ & $45.6 \%$ \\
\hline 1980 & $56.6 \%$ & $43.2 \%$ & $55.8 \%$ & $48.7 \%$ \\
\hline 1970 & $59.5 \%$ & $47.2 \%$ & $47.5 \%$ & $51.0 \%$ \\
\hline \multicolumn{5}{|l|}{ Employed } \\
\hline $1990^{\circ}$ & $18.5 \%$ & $19.5 \%$ & $24.7 \%$ & $22.5 \%$ \\
\hline 1980 & $20.0 \%$ & $27.6 \%$ & $26.9 \%$ & $20.9 \%$ \\
\hline 1970 & $22.1 \%$ & $29.2 \%$ & $27.8 \%$ & $26.7 \%$ \\
\hline \multicolumn{5}{|l|}{ Foreign born } \\
\hline 1990 & $17.7 \%$ & $23.8 \%$ & $98.9 \%$ & $72.1 \%$ \\
\hline 1980 & $28.2 \%$ & $13.1 \%$ & $97.6 \%$ & $91.8 \%$ \\
\hline 1970 & $33.0 \%$ & $16.9 \%$ & $98.0 \%$ & $37.4 \%$ \\
\hline \multicolumn{5}{|l|}{ Cannot speak English } \\
\hline 1990 & $0.3 \%$ & $3.3 \%$ & $41.7 \%$ & $24.9 \%$ \\
\hline 1980 & $0.3 \%$ & $0.0 \%$ & $50.6 \%$ & $33.5 \%$ \\
\hline \multicolumn{5}{|l|}{ Not a U.S. citizen } \\
\hline 1990 & $2.8 \%$ & $13.3 \%$ & $58.5 \%$ & $46.5 \%$ \\
\hline 1980 & $3.4 \%$ & $2.5 \%$ & $68.1 \%$ & $44.9 \%$ \\
\hline 1970 & $2.4 \%$ & $4.5 \%$ & $85.1 \%$ & $12.6 \%$ \\
\hline \multicolumn{5}{|l|}{ Disability Status } \\
\hline \multicolumn{5}{|l|}{ Disability prevents working } \\
\hline 1990 & $24.7 \%$ & $36.9 \%$ & $23.7 \%$ & $23.2 \%$ \\
\hline 1980 & $24.6 \%$ & $35.7 \%$ & $21.5 \%$ & $22.2 \%$ \\
\hline 1970 & $14.4 \%$ & $23.5 \%$ & $17.5 \%$ & $13.6 \%$ \\
\hline Mobility limitation (1990) & $18.3 \%$ & $26.8 \%$ & $18.0 \%$ & $20.1 \%$ \\
\hline $\begin{array}{l}\text { Public Transportation limitation } \\
\text { (1980) }\end{array}$ & $15.7 \%$ & $21.6 \%$ & $12.4 \%$ & $10.8 \%$ \\
\hline Personal Care Jimitation (1990) & $14.5 \%$ & $28.0 \%$ & $16.5 \%$ & $13.8 \%$ \\
\hline
\end{tabular}

Data Source: Ruggles, Sobeck, et al., 1997. 
Table 4.15: Selected Household Composition Characteristics of Elders Not Living Alone in Miami-Dade County by Racial/Ethnic Group. 1970-1990

\begin{tabular}{lrrrr}
\hline & $\begin{array}{r}\text { White non* } \\
\text { Hispanic }\end{array}$ & $\begin{array}{r}\text { Black non- } \\
\text { Hispanic }\end{array}$ & $\begin{array}{c}\text { Cuban } \\
\text { Hen-Cuban } \\
\text { Hispanic }\end{array}$ \\
\hline 1990 & $50.7 \%$ & $48.3 \%$ & $41.8 \%$ & $38.0 \%$ \\
1980 & $51.1 \%$ & $52.4 \%$ & $40.6 \%$ & $39.7 \%$ \\
1970 & $51.0 \%$ & $52.4 \%$ & $30.9 \%$ & $38.1 \%$ \\
Spouse of Head of Household & & & & \\
1990 & $36.1 \%$ & $20.8 \%$ & $27.4 \%$ & $17.9 \%$ \\
1980 & $37.4 \%$ & $25.2 \%$ & $22.8 \%$ & $18.2 \%$ \\
1970 & $34.1 \%$ & $20.6 \%$ & $15.3 \%$ & $29.7 \%$ \\
Other family to Head of & & & & \\
Household & & & & \\
1990 & $9.6 \%$ & $24.6 \%$ & $27.7 \%$ & $37.6 \%$ \\
1980 & $9.0 \%$ & $15.4 \%$ & $34.2 \%$ & $38.9 \%$ \\
1970 & $12.4 \%$ & $19.0 \%$ & $49.1 \%$ & $25.9 \%$ \\
Non-family to Head of & & & & \\
Household & & & & \\
1990 & $3.6 \%$ & $6.4 \%$ & $3.1 \%$ & $6.4 \%$ \\
1980 & $2.5 \%$ & $7.0 \%$ & $2.4 \%$ & $3.2 \%$ \\
1970 & $2.5 \%$ & $7.9 \%$ & $4.7 \%$ & $6.3 \%$ \\
\hline
\end{tabular}

Data Source: Ruggles, Sobeck, et al., 1997. 
Figure 4.1: Population Distribution in Miami-Dade County, FL 1970-1995
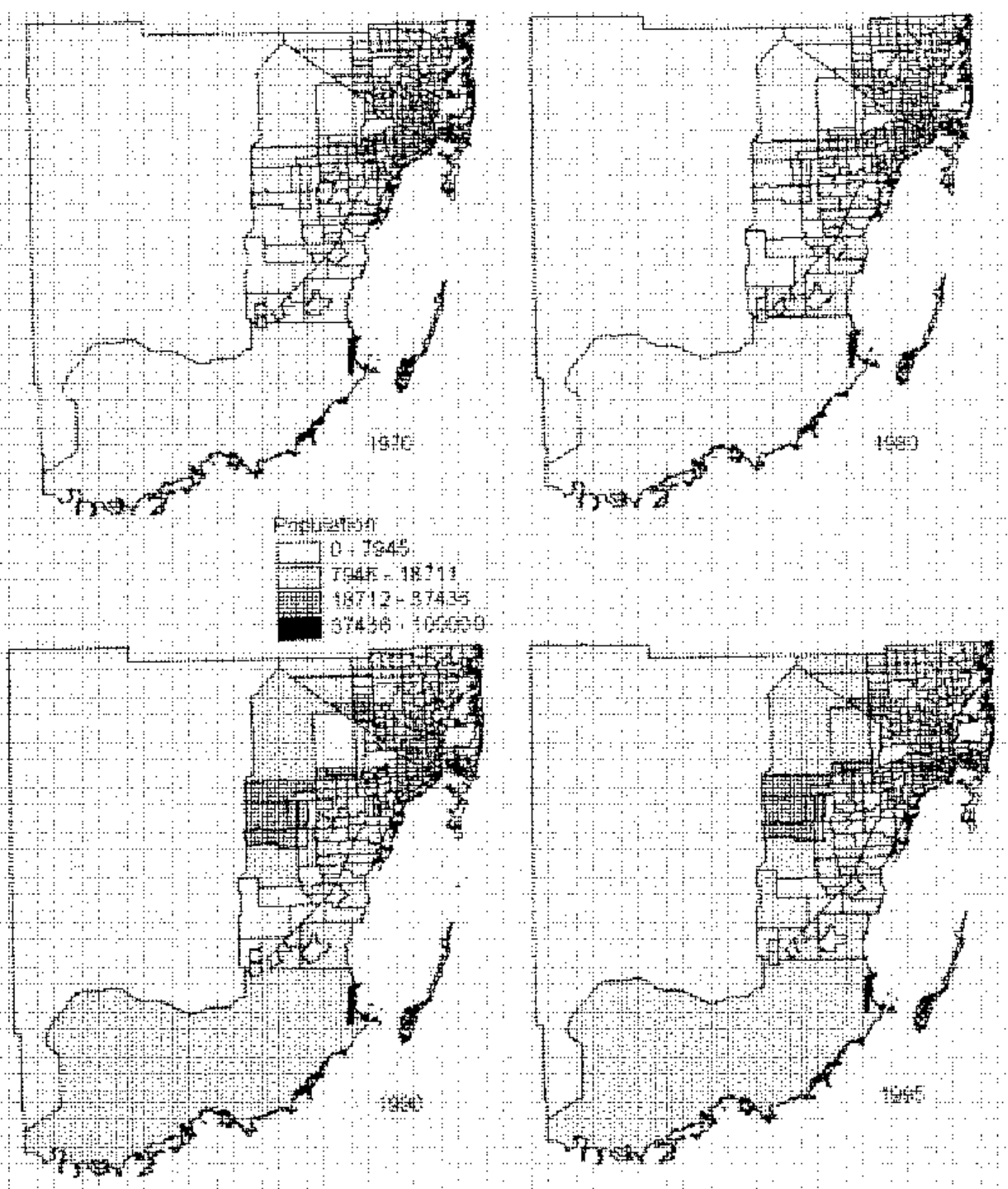
Figure 4.2: Population Distribution in Miami-Dade County, FL 2000-2015
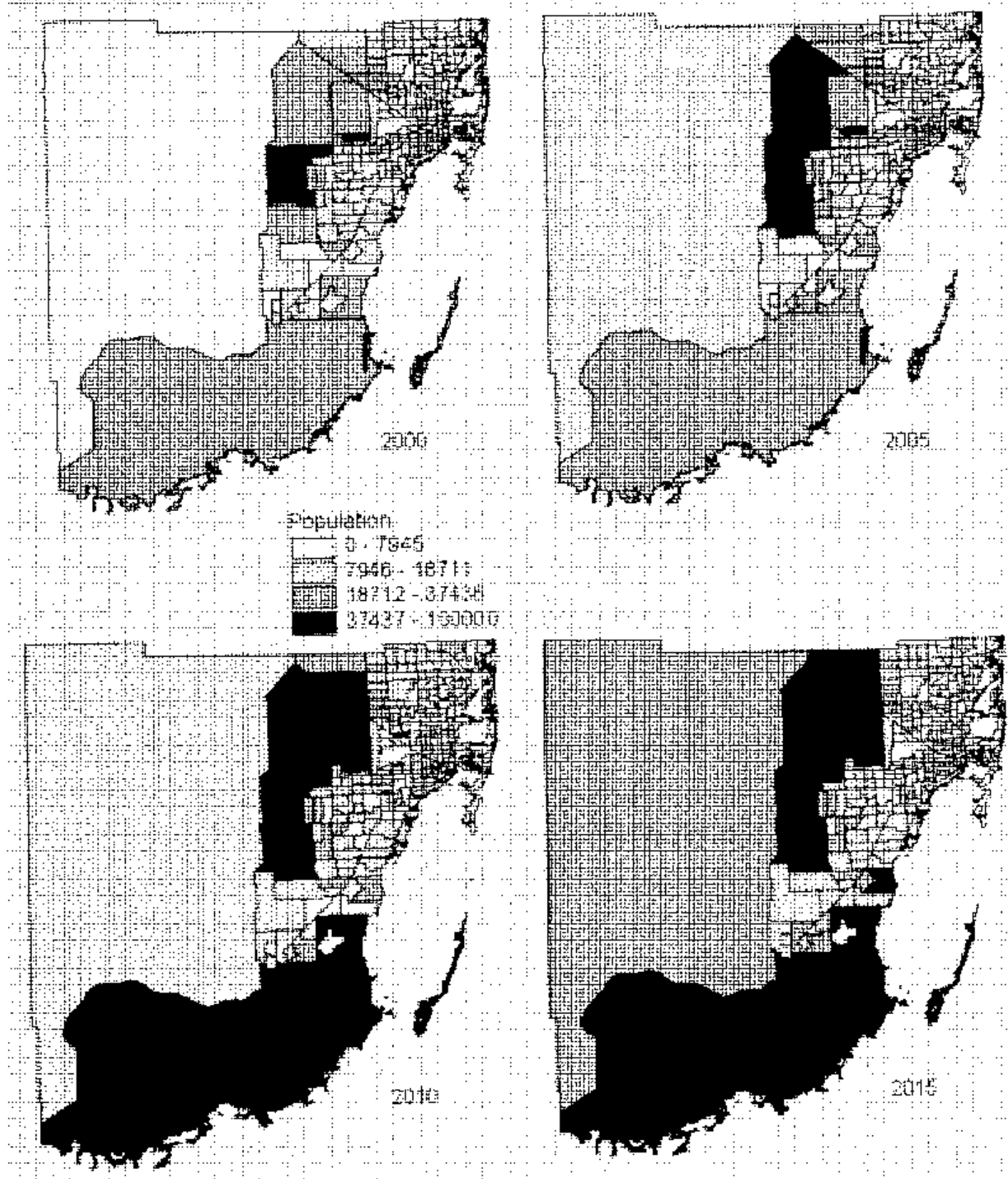
Figure 4.3: Distribution of Proportion Black in Total Population, 1990 Miami-Dade County by Census Tracts

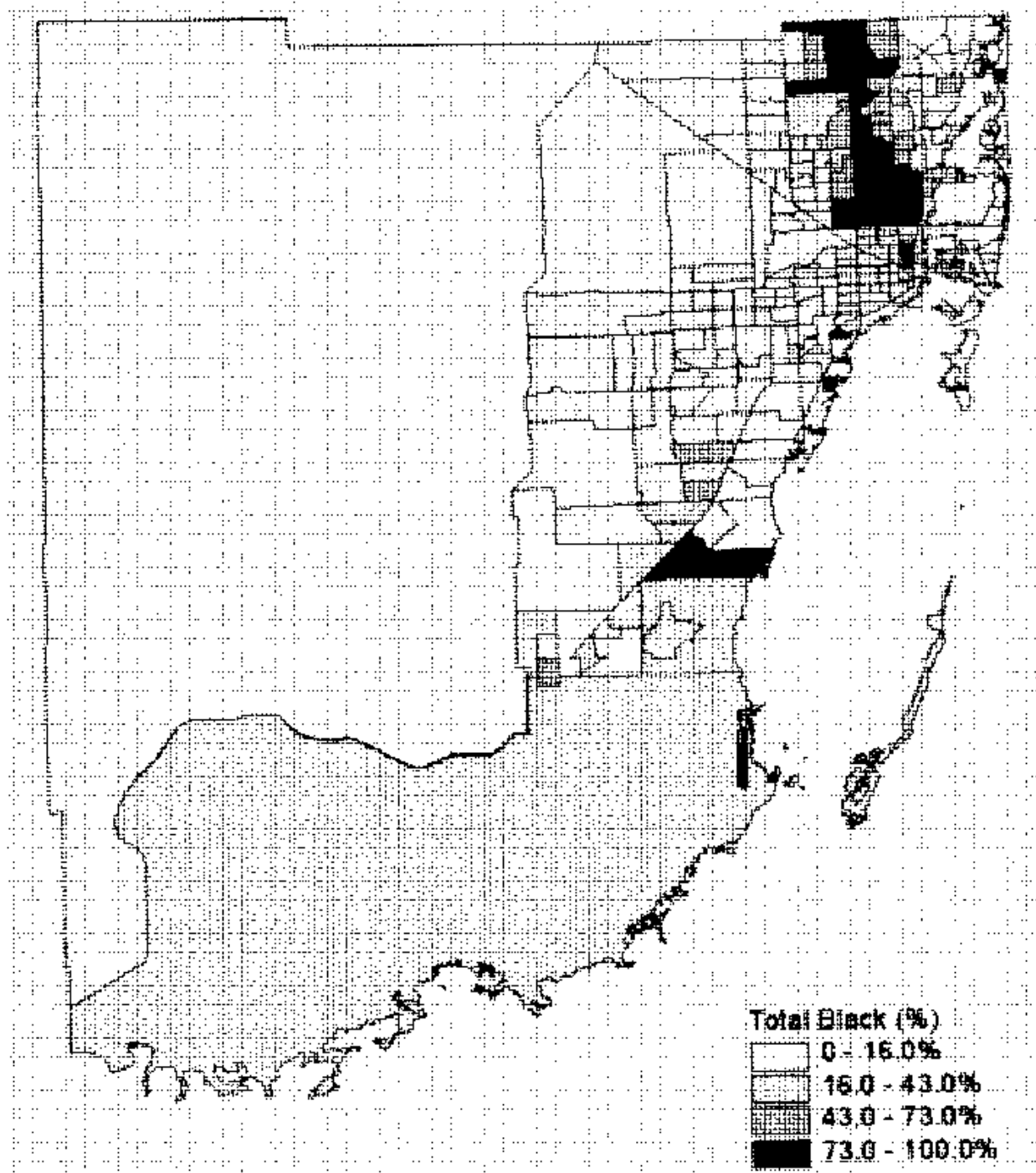


Figure 4.4: Distribution of Proportion Hispanic in Total Population, 1990 Miami-Dade County by Census Tracts

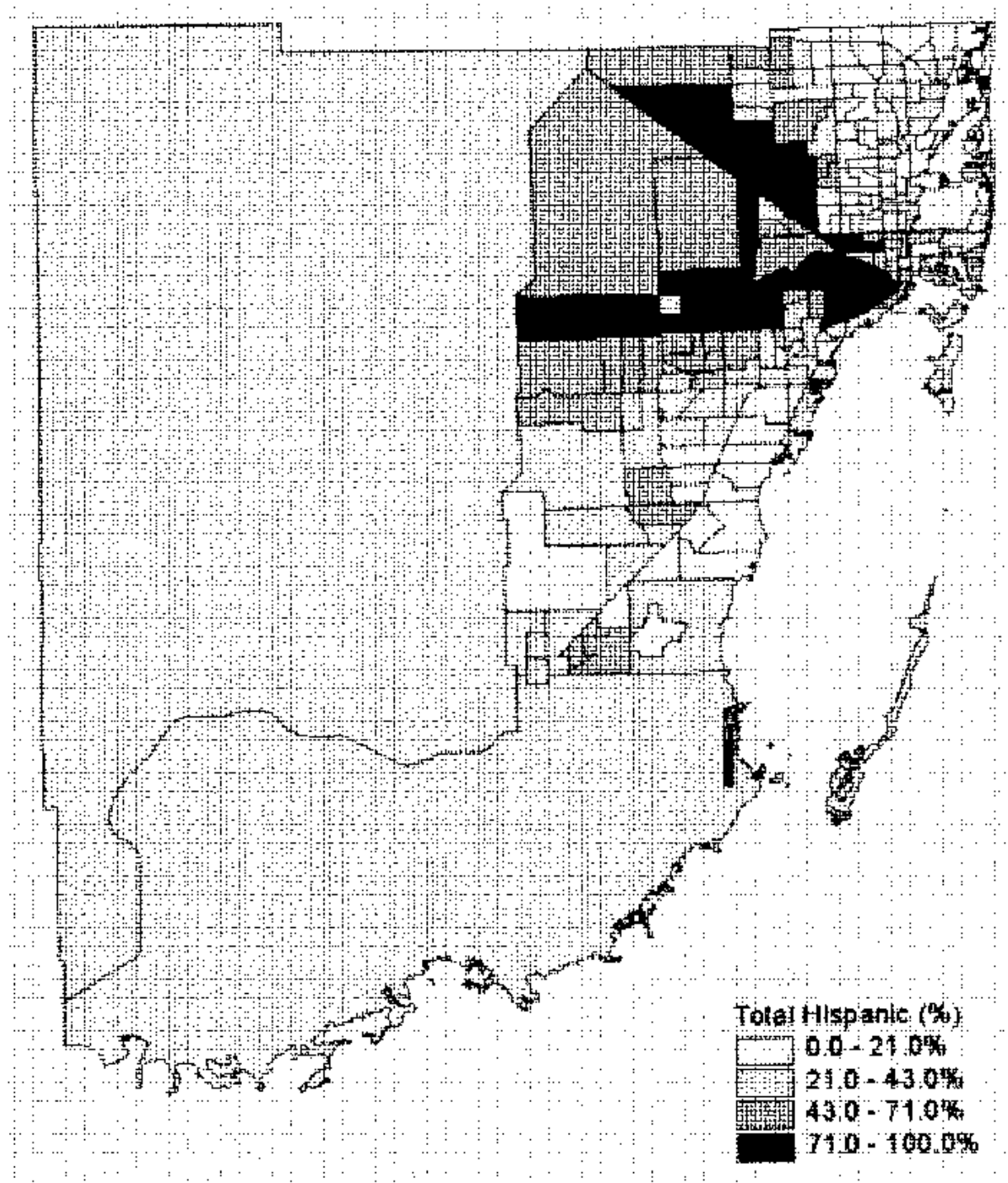


Figure 4.5: Elders (60+) in Miami-Dade County, Florida by Census Tracts (1990)

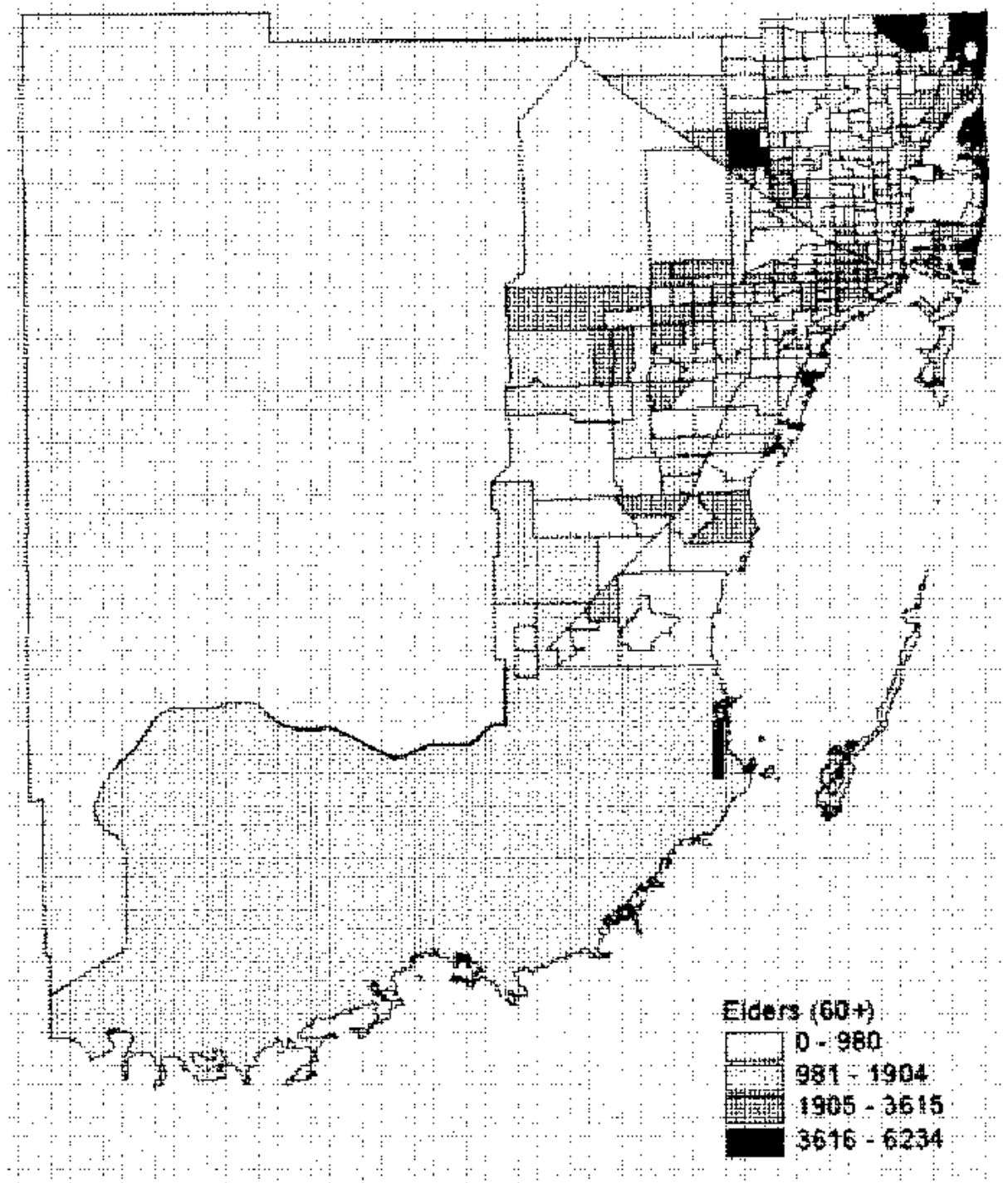


Figure 46:

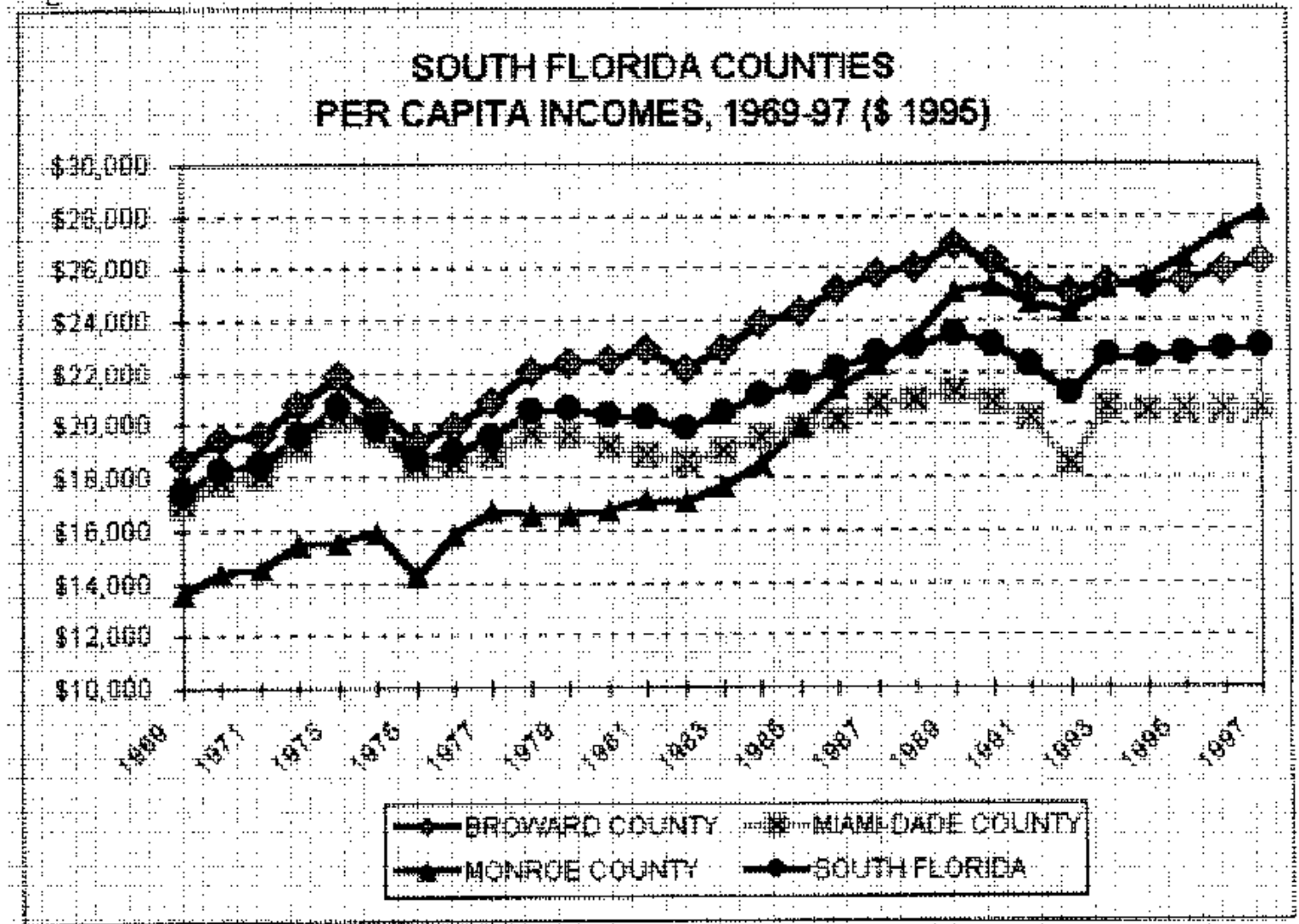

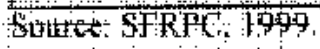


Figure 4.7: Distribution of Percentage of Total Population Living Below Poverty, 1990 Miami-Dade County by Census Tracts

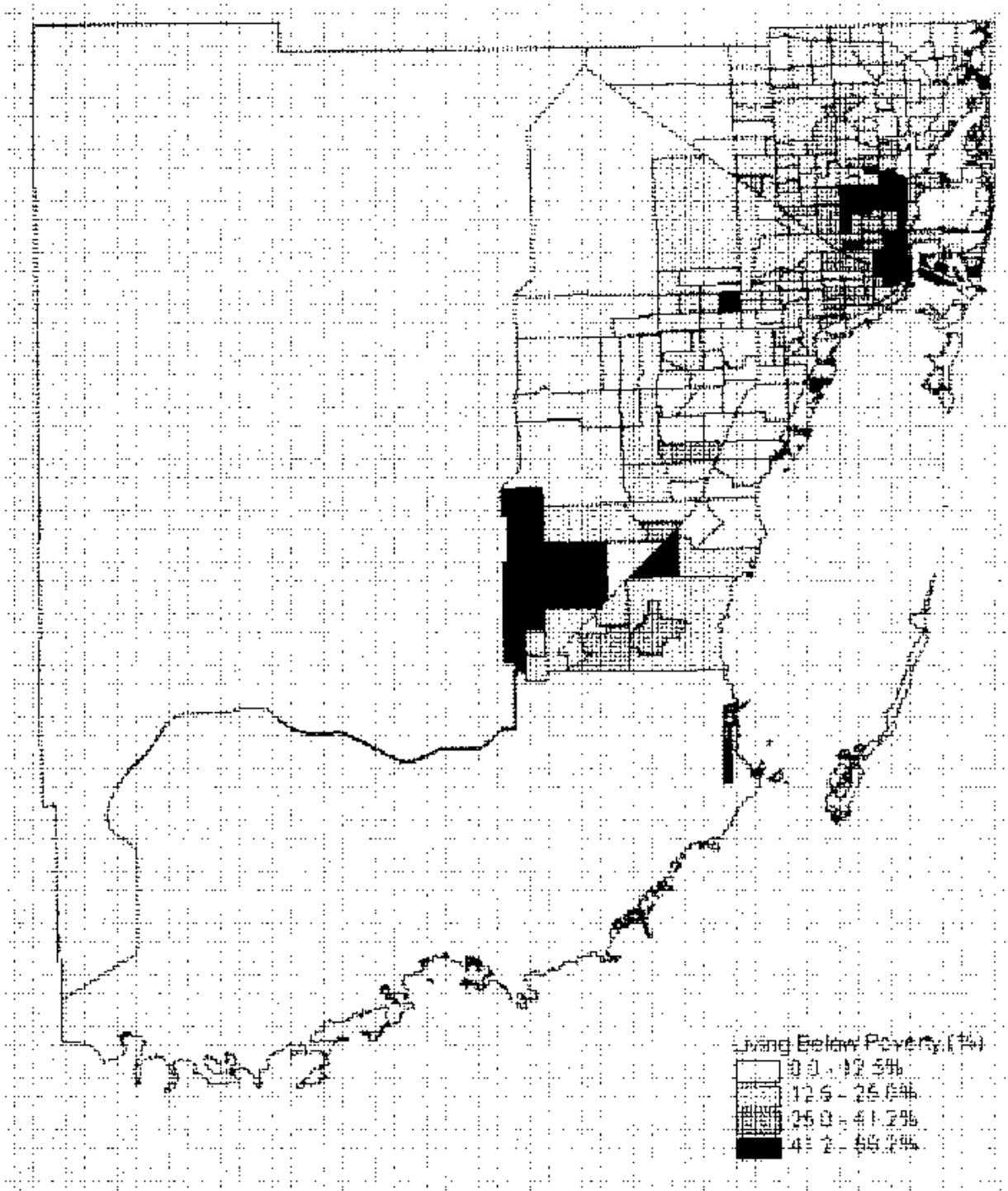


Figure 4.8: Elder and Total Population in Miami-Dade County, FL (1900-1990)

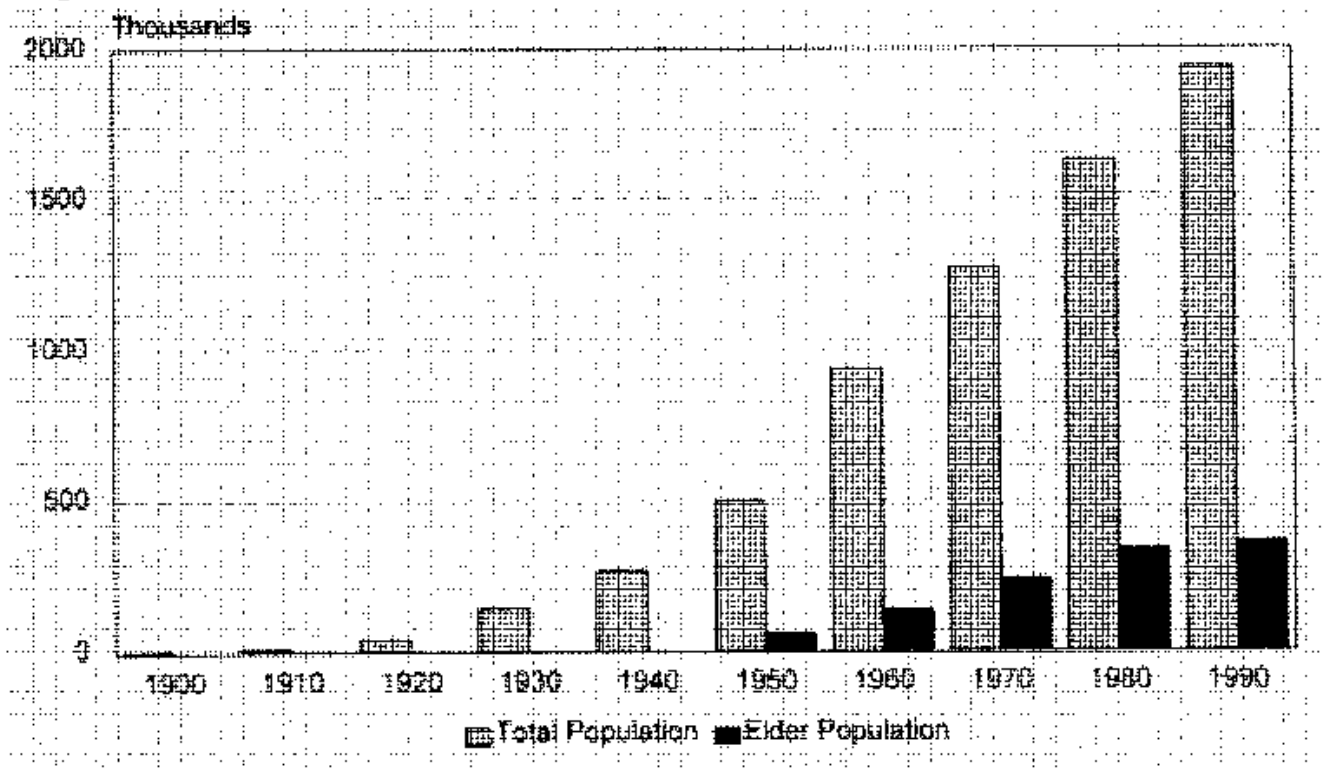


Figure 4.9: Distribution of Percentage Elders (60+) in Total Population, 1990 MiamiDade County by Census Tracts

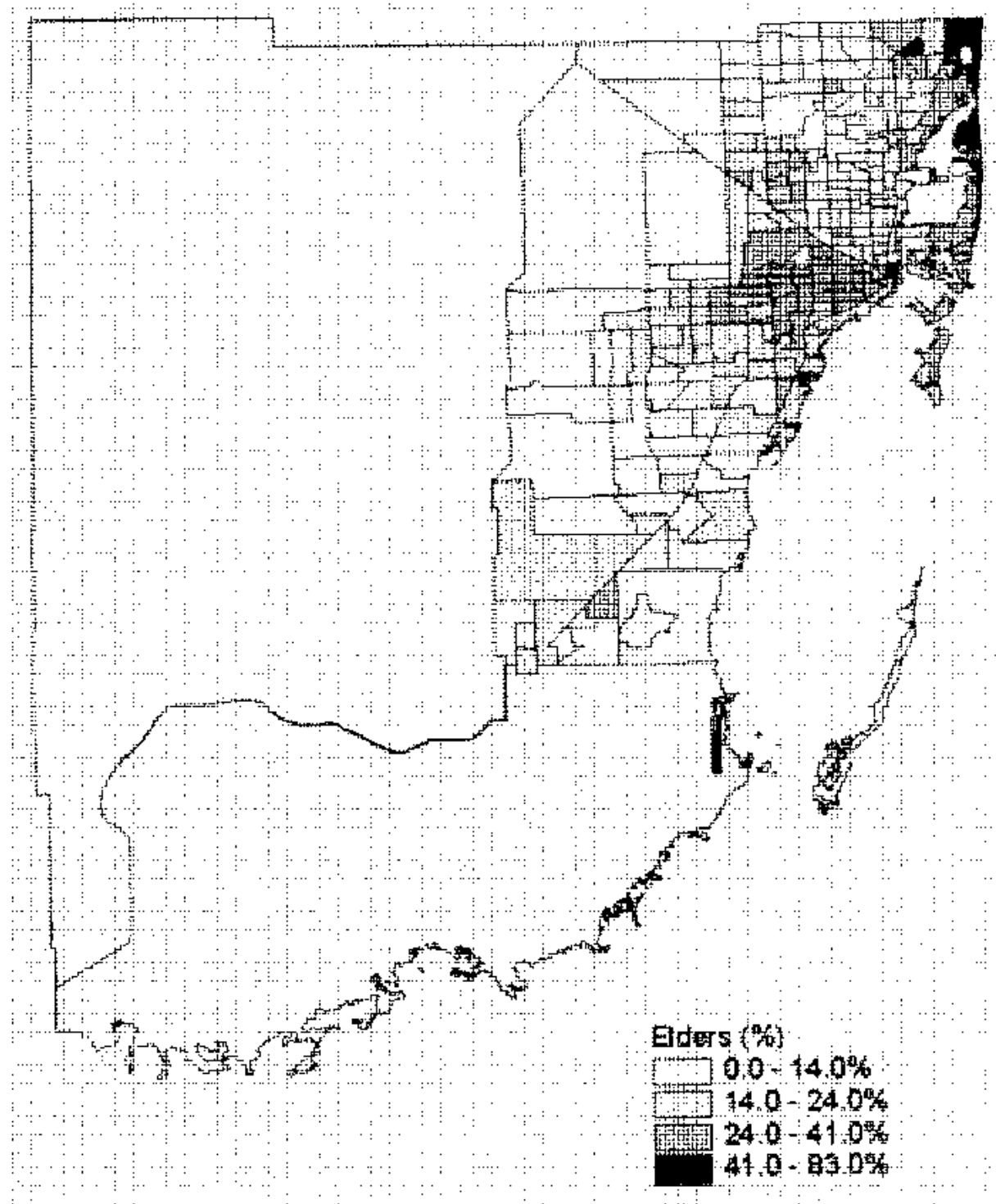


Figure 4.10: Distribution of Proportion 85+ of Elder Population, 1990 Miami-Dade County by Census Tracts

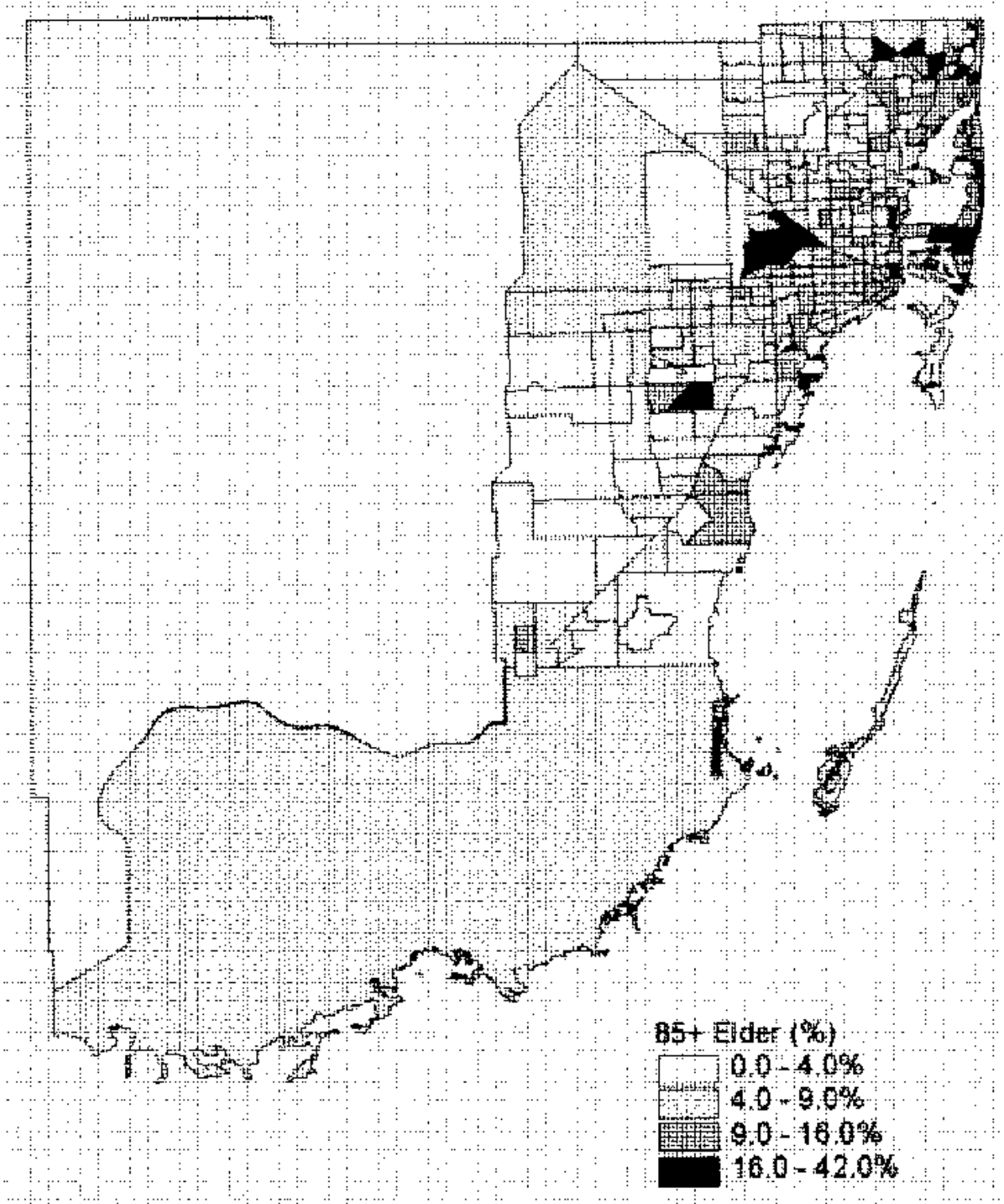


Figure 4.11: Percentage of Blacks in the Elder Population in Miami-Dade County, FL 1990

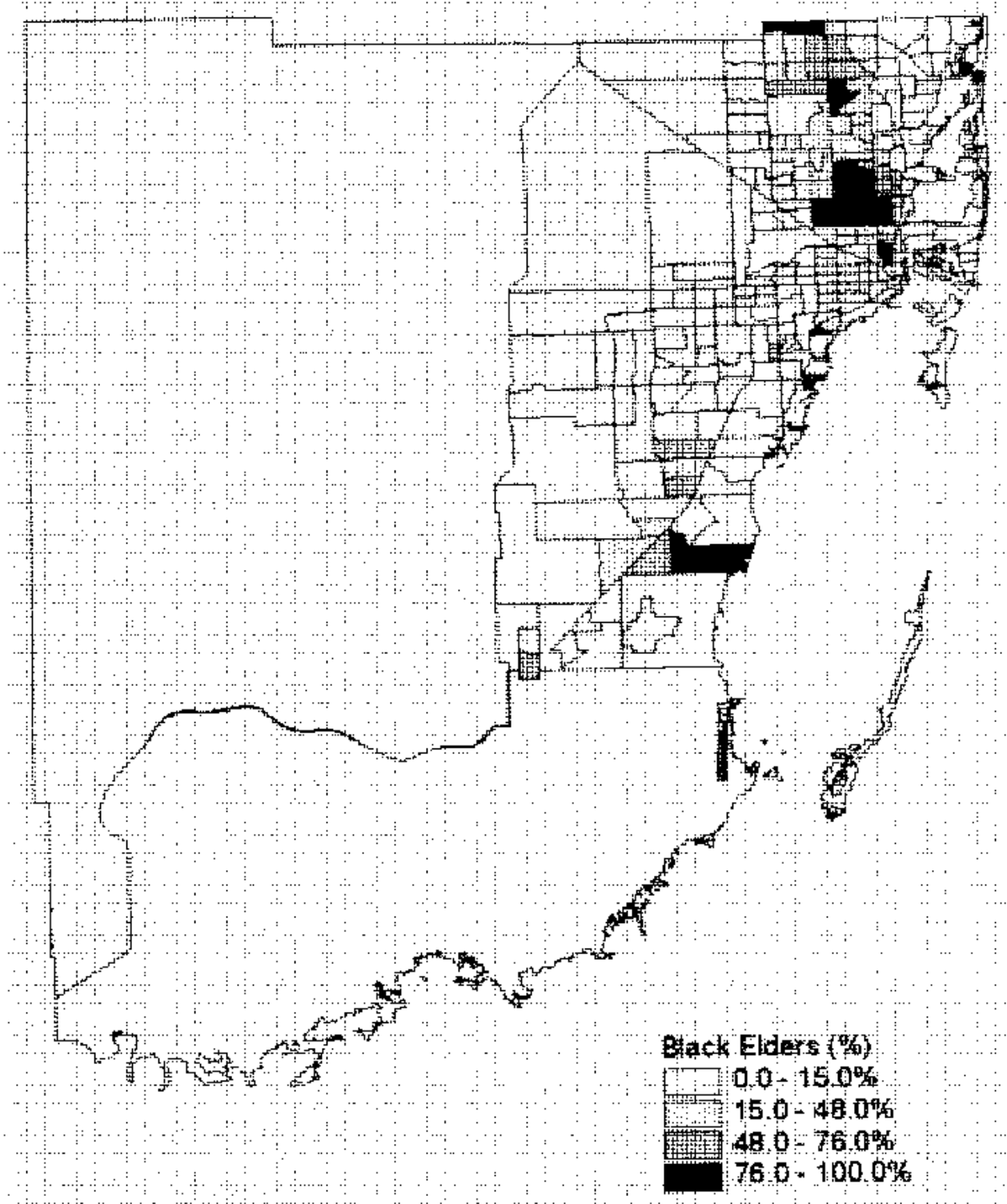


Figure 4.12: Proportion of Hispanic Origin in Elder Population in Miami-Dade County. FL 1990

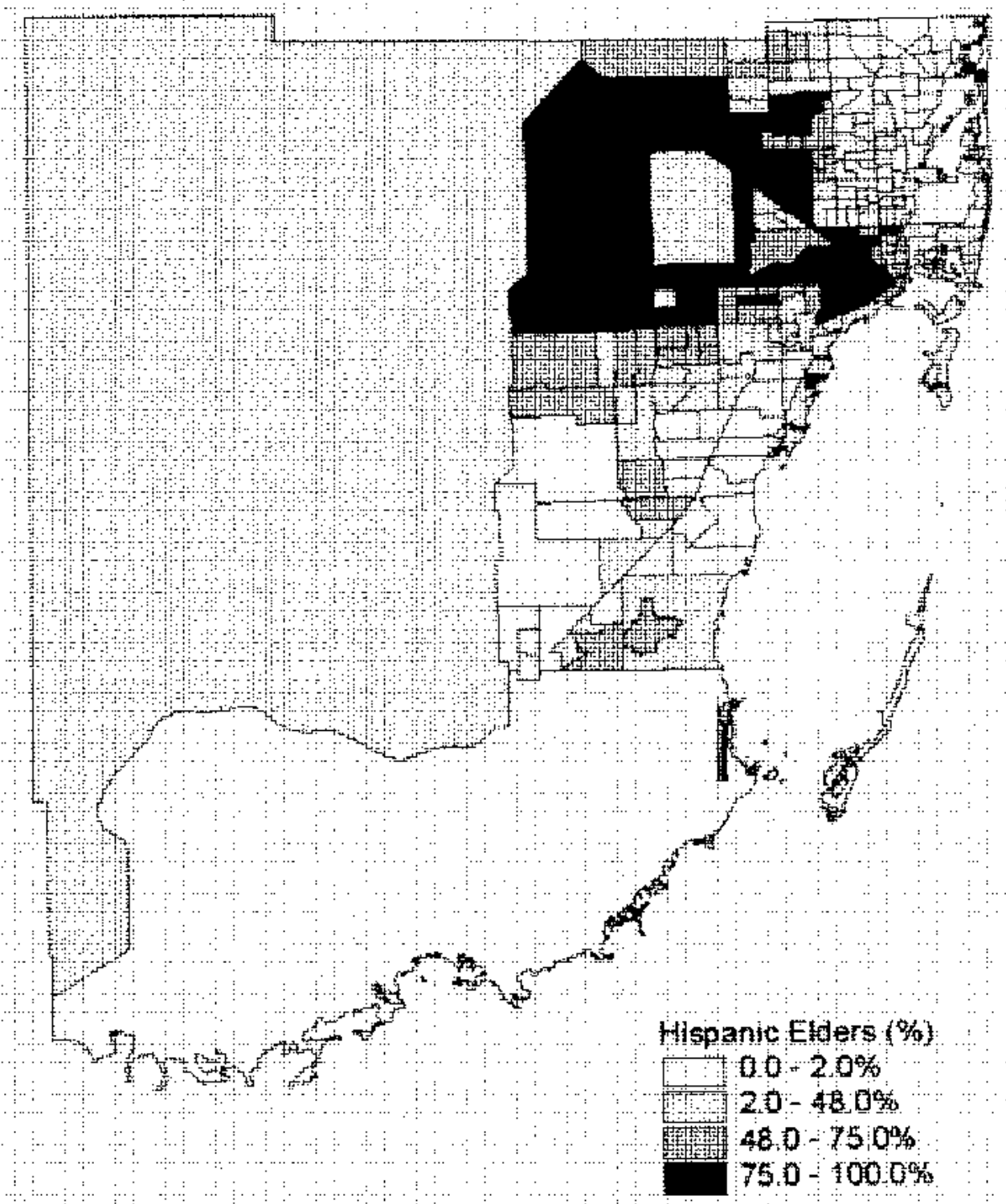


Figure 4.13: Distribution of Proportion Living Below Poverty of Elder Population, 1990 Miami-Dade County by Census Tracts

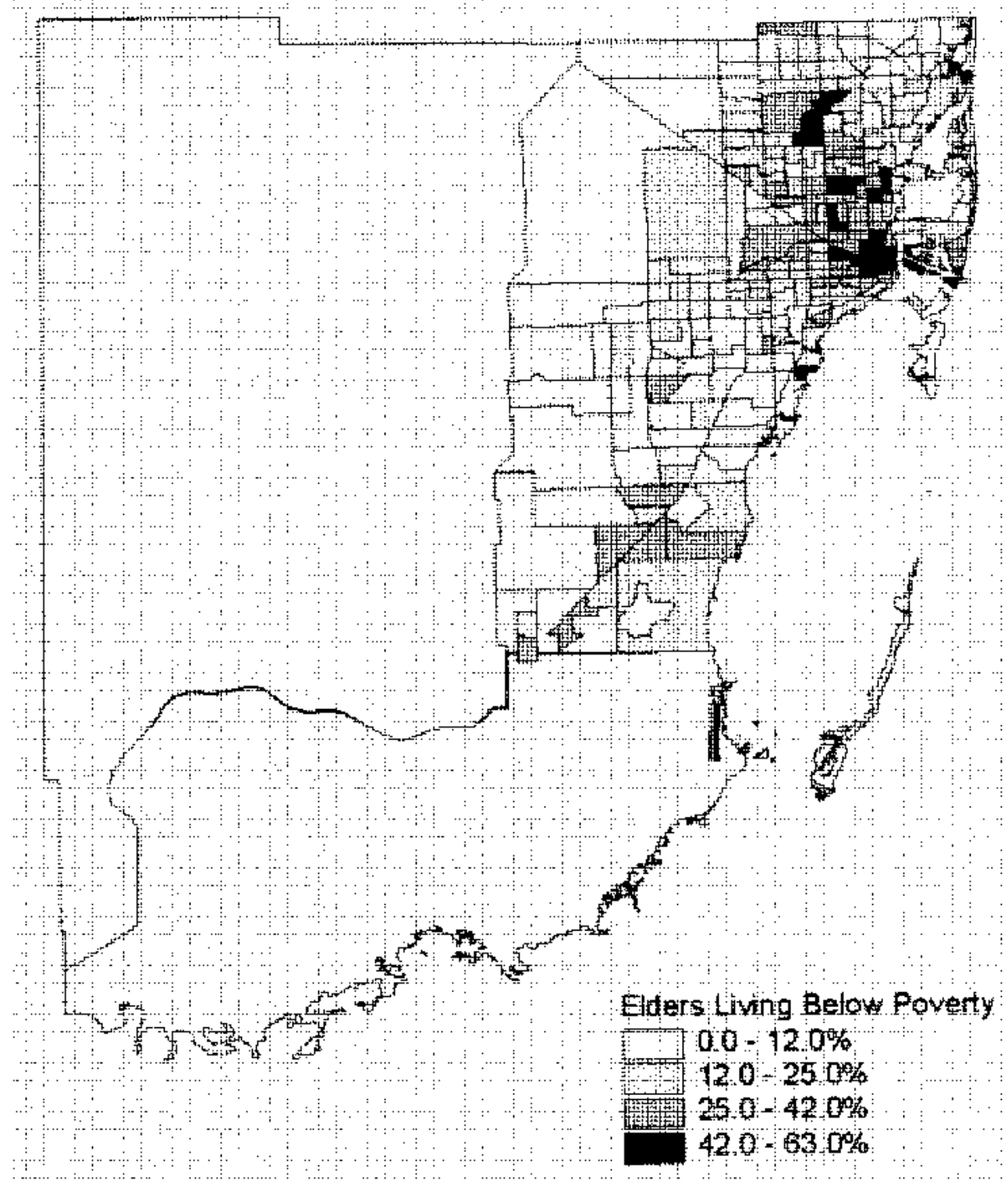


Figure 4.14: Distribution of Proportion Living Alone of Elder Population, 1990 MiamiDade County by Census Tracts

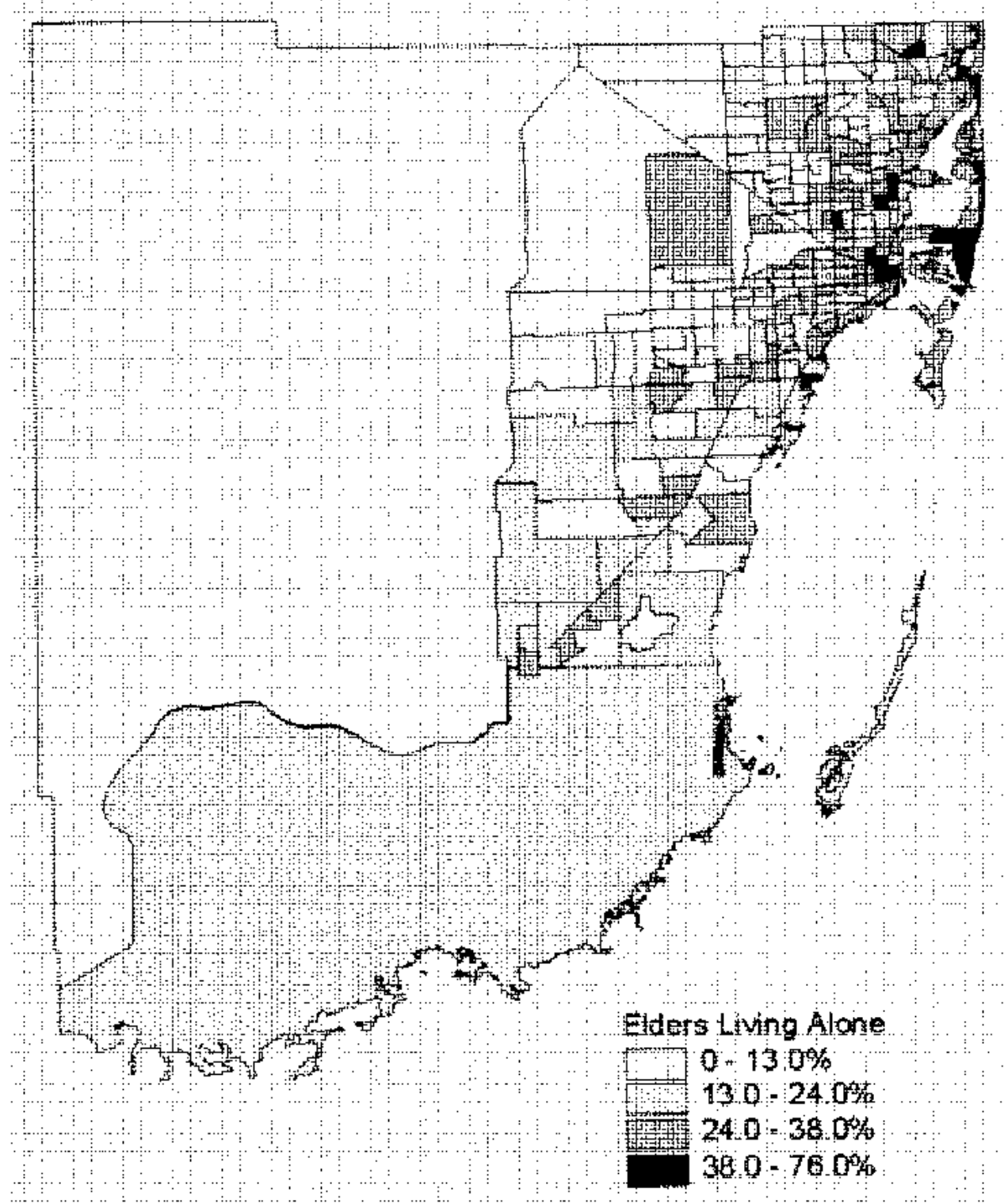




\section{CHAPTER 5: DATA AND METHODOLOGY}

This chapter will describe the sources of data to be examined and will explain the methods used to carry out the study. While the data had been collected prior to the dissertation and could not be changed, the methodology used in the analysis was, to a certain extent, reflexive. The research strategy combined both qualitative and quantitative data analysis strategies to gain a more complete understanding of health care utilization behavior of elders in a multicultural urban environment, i.e., triangulation of methodology.

While this research must be defined as secondary analysis in terms of the dissertation, it must be pointed out that the author played an integral role in the collection of the data that is analyzed in this dissertation (Rothman, et al., 1994). The primary data set used to examine the research hypotheses has not previously been analyzed for these purposes. Further, while the primary focus of this research is as a quantitative study, the research will also include a qualitative perspective.

\section{RESEARCH CONTEXT}

This study of health care service utilization of elders took place in the multicultural urban environment of Miami-Dade County, FL. A description of the historical demographic trends of elders in this county was in Chapter 4.

\section{DATA SOURCES}

This study of health care service utilization among four racial/ethnic groups of elders in Dade County, Florida used data gathered from multiple sources. The primary 
source of data to be used in this project was collected as part of a needs assessment for the Alliance for Aging, Inc. the Area Agency on Aging for Dade and Monroe Counties (Alliance), by the Southeast Florida Center on Aging of Florida International University, to document the needs of elders living in Dade County (Rothman, et al., 1994). ${ }^{\prime}$

Community contextual variables were gathered from the census publications and sampled census data from several agencies.

\section{Dade County Needs Assessment}

The needs assessment data was drawn from two sources primarily: telephone survey and focus groups of elders. Contextual community data will also be gathered by zip code and/or census tract level and then aggregated to Area Agency on Aging subareas (PSAs). The research activities of the needs assessment covered the period from September 1993 through June 1994. There were two specific purposes for the needs assessment:

- To provide data that would assist the Alliance for Aging, Inc., the Area Agency on Aging for Dade and Monroe Counties (Alliance) in its comprehensive planning of services for elders in Dade County for the next 5 to 10 years.

- To provide post-hurricane ${ }^{2}$ data for the Alliance.

Three types of data were collected for the needs assessment:

\footnotetext{
' The Dade County Needs Assessment (DCNA) was deemed necessary in the aftermath of Hurticane Andrew on August 24, 1992.

${ }^{2}$ Hurricane Andrew hit South Florida, particularly southern Dade County, on August 24, 1992. The storm destroyed or seriously damaged approximately 130,000 homes and displaced over 200,000 individuals.
} 
- ten focus groups of elders, informal caregivers, volunteers, and service providers throughout the county;

- a survey of approximately 1,900 elders throughout the county, of which approximately 1,800 interviews were conducted by telephone and approximately 100 were conducted face-to-face with individual who are low-income and/or minority; and

- a mail survey of 48 key informants who were felt to be particularly knowledgeable about the needs, services and policy regarding elders within the county. ${ }^{3}$

\section{Telephone Survey Sample}

In the telephone survey component, 1.992 elders were interviewed during the period between January and March 1994. This represents approximately 0.5 percent of the senior residents in Miami-Dade County, FL. The sample was designed to be directly representative of non-institutionalized elders living within Miami-Dade County with either a listed or unlisted telephone number. The margin of sampling error countywide was $(+/-) 2.3$ percent.

The zip code of the respondent's home identified the respondent's location within the county. Using the Alliance Planning and Services Sub-areas (sub-area), respondents

\footnotetext{
${ }^{3}$ Data from the 100 face-to-face interviews and key informant surveys will not be used in this present research.
} 
were allocated into one of the ten sub-areas (Figure 5.1). ${ }^{4}$ This will allow a more detailed documentation of health care service available at the neighborhood level.

Due to the possibility that an older person may be physically unable to complete the interview, a close family member or individual who cared for the older person was asked to complete the interview as a proxy respondent if the elder was unable to do so. One hundred seventy-one interviews used a proxy. As would be expected, the mean age of elders who had a proxy is older than elders who do not have a proxy $(75.0$ years and 71.0 years, respectively).

In the 1990 Census, it was found that 18.7 percent of all elders (Table 4.8 ) could not speak English. While this may appear to be a small percentage of the elder population, elders who cannot speak English are not distributed evenly across racial/ethnic groups. Of the Cuban elders, 41.7 percent could not speak English, while 24.9 percent of non-Cuban Hispanic elders could not speak English in the 1990 U.S. Census (Table 4.14). With so many elders reporting that they are not comfortable speaking English in Dade County, the interview instrument was professionally translated into Spanish. Overall, 34.4 percent of interviews were conducted in Spanish. There was further concern regarding the cultural nuances in interviewing older Hispanics.

\footnotetext{
"Originally the Alliance allecated the PSAs by census tract. However since most individuals do not know the census tract number they live in, and are more familiar with the zip code they live in, the Center on Aging translated PSAs from their original census tract definition to a zip code based definition. The Alliance has since switched over to using the zip code based definitions for their PSAs. Appendix A has brief descriptions of each of the PSAs, as well as the how each PSA is defined by zip codes and census tract.
} 
Therefore, Spanish interviewers received special training, in addition to the formal training provided for all interviewers.

A modified proportional sampling frame was used because the distribution of elders throughout the county was not equal as shown in Figure $4.5 .^{6}$ The margin of sampling error for individual sub-areas is approximately $(+/-) 8$ percent. Table 5.1 show's the population of elders, the number of actual interviews by sub-area, both actual and weighted for each sub-area.

\section{Limitations of Survey}

Regardless of how well thought out, all research can encounter problems with data collection. This needs assessment was no exception. Three problem issues were encountered either prior to beginning of the telephone survey or during the telephone survey data collection. These problems were non-representation of elders without telephones, low representation in PSA \#6, and low representation of Hispanic elders throughout the county.

While the telephone survey sample was designed to be directly representative of elders with either a listed or unlisted telephone number within Miami-Dade County, this did not take into account for elders without telephones. The researchers were not willing to automatically assume that any findings from the telephone survey could be

\footnotetext{
${ }^{5}$ Significantly more Cuban elders were interviewed in Spanish than non-Cuban Hispanics ( 85.2 percent of Cubans and 63.2 percent of non-Cuban Hispanics).

'The proportional sampling frame used the Alliance's sub-area population cstimates of elders (derived from the 1990 U.S. Census of Dade County). However, the sampling frame was modificd so that each subarea had at least 100 interviews. For the county-wide analysis, those sub-areas that proportionately would be expected to have less than 100 elders were weighted down to be proportional to their relative population size county-wide.
} 
extrapolated to elders without telephones. Thus, the first issue was with respect to elders without phones and the validity of extrapolating telephone survey data to elders without phones. 1990 U.S. Census data for Miami-Dade County shows that of units with at least one elder in residence and not defined as "Group Quarters," only 2.1 percent did not have telephones in their residential units (Table 4.8).

However, this 2.1 percent is not evenly distributed through the population of elders. While there are important racial/ethnic differences in distribution of elders without telephones, poverty status is a much more significant indicator of having a phone or not. Rothman, et al. (1994) found that almost seven times as many elders living below poverty as those living above poverty did not have a phone $(6.8 \%$ and $1.0 \%$, respectively). The researchers were concerned that telephone survey techniques would "miss some elders who potentially are in the greatest need of services because they are the poorest or the most socially isolated" (Rothman, et al., 1994).

To deal with this issue, researchers tried to find service agencies throughout the community that might give aid or support to elders without phones. None of the organizations contacted were able to distinguish those elder clients without telephones. Instead, researchers decided to do 100 face-to-face interviews of poor and/or minority elders with a similar closed-ended survey instrument used in the telephone survey with an additional question on phone availability. A comparison of these face-to-face interviews with telephone survey interviews controlled for poverty and racial/ethnic background found little difference between the two samples. 
During the telephone survey process, two more issues arose. The first was low representation in PSA \#6, which in turn led to an examination of low representation of Hispanic elders throughout the county.

Interviews lagged considerably behind in PSA \# 6 compared to other sub-areas. Further investigation determined that proportionately fewer phone numbers had been generated for this sub-area. In part, this was due to the fact that this sub-area includes the central business district of Miami. Business exchanges had been eliminated prior to phone number generation for this survey. Not only is PSA \#6 important because it includes the central business district of Miami, PSA $\# 6$ includes an important ethnic enclave known as Little Havana.

In the course of the investigation of PSA $\# 6$, low overall tallies, it was further observed that representation of Hispanic elders in PSA \#6 and in the county overall was low. Professionals familiar with elders in the Hispanic community were consulted to try to understand the reasons for the low response rate of Hispanic elders. This pattern of low response rate of Hispanic elders had previously been observed in the pilot study in three North Dade sub-areas. At that time it was suggested that due to the hurricane migration of South Dade County residents to northern parts of the county less impacted by Hurricane Andrew that 1990 Census data would not reflect the racial/ethnic composition post-hurricane. However, given the overall undercount of Hispanics within the county this suggestion was discounted. Focus groups of Hispanic elders suggested that the time of day that phone surveys were placed would be important determinants to whether or not an elder would answer the phone. Hispanic elders stated overwhelmingly in focus groups that interviewers should not call during "soap operas." Unfortunately, 
they failed to mention that the most popular Spanish soap operas are aired in the evening hours between 7:30 and 9:30pm. When interviewers began calling houscholds in predominantly Hispanic areas in the mid-to-late aftcrnoon, rather than in the evening hours, completion rates rose substantially for Hispanic interviews.

\section{Focus Groups of Elders}

Under the Dade County Needs Assessment, a series of 2-hour focus group discussions with 10 groups of elders, informal caregivers, volunteers, and scrvice providers throughout Dade County were held in October 1993. Six of the ten focus group discussions will be used to place issues of health and health care in the lives of elders residing in Miami-Dade County. In the Dade County Needs Assessment, the ten focus groups are described as follows:

(1) working-class Cubans primarily between the ages of 65 to 69 years and organized through Little I lavana Activities and Nutrition Centers, Inc., conducted in Spanish (Little Havana);

(2) Hispanic older caregivers, no age restriction and organized through an Alzheimer's Support Group, conducted in Spanish (Caregivers);

(3) Cubans of lower socioeconomic status, primarily over the age of 70 years and organized through the Hialeah Housing Authority, conducted in Spanish (Hialeah Housing); 
(4) Haitian elders over the age of 50 years, organized through Project Good Help located at St. Mary's Cathedral, in Little Haiti, conducted in Creole (Haitian);

(5) African-American elders of lower socioeconomic status between the ages of 60 and 69 years, organized through the James E. Scott Community Association (JESCA);

(6) African Americans in the Richmond Heights area of south Dade County, no restrictions on age or on socioeconomic status, organized through the Glendale Missionary Baptist Church (Richmond Heights);

(7) middle and upper-middle class white, non-Hispanics, over the age of 75 years, organized through the Northeast Dade Coalition (Northeast Dade);

(8) elders living in and around Homestead, the area most damaged by Hurricane Andrew, jointly organized by Musselwhite Senior Center and the Metro-Dade Community Action Agency, no restriction on age or socioeconomic status (Homestead), ${ }^{7}$

(9) elders involved as volunteers and members of the Advisory Council for the Alliance (Volunteers); and

(10) professionals from provider agencies in the County (Providers). 
In planning meetings between the Alliance and the Center on Aging, it was decided that there should be a total of 10 focus groups. Initially, it was decided that there should be two special focus groups. One would be comprised of service providers for elders in Dade County, and a second would be comprised of Haitian elders. Later on two more special focus groups were added; volunteers on the Alliance's Advisory Council, and informal caregivers of elders diagnosed with Alzheimer's Disease or other dementia syndromes. The remaining six focus groups were identified in order to provide a mix of participants according to ethnicity, age, socio-economic status and geographic locale. It was acknowledged that the groups assembled would be essentially "samples of convenience." This was primarily due to the recognition that many of the ideal focus group participants may have limited access to transportation. Further, all available information indicates that elders in Miami-Dade County are very fearful of crime, particularly being victims of con-artists, and thus, be suspicious of strangers. It was felt that working through known community agencies would address these potential barriers to assembling appropriate focus groups.

For the purpose of this dissertation only six of the ten focus groups will be used. The six that will be examined are Little Havana, Hialeah Housing, JESCA. Richmond Heights, Northeast Dade and Homestead. $\Lambda$ ppendix B has the protocols used for the focus groups of elders. The importance of this aspect of the research will be to place the

\footnotetext{
${ }^{7}$ Participants from Musselwhite Senior Center were "Anglo", while participants identified by Metro-Dade Community Action Agency (CAA) were a mix of blacks and Hispanics of varying national origin.
} 
issues of health and health care utilization in the lives of elders living in Miami-Dade County.

\section{Contextual Data Sources}

Contextual community data was gathered from a number of sources. Information on the number of hospital beds per 1000 elders in each of the 10 sub-areas of the county will be gained from the AHA Hospital Guide (1994) for number of hospital beds in Dade County. Using the zip codes of the hospitals, hospital beds were assigned to specific subareas. Information on the physicians was collected from Dade County Medical Association, Florida Medical Association and the American Medical Association directories of members, as well as the Alliance's list of Medicaid approved physicians in Dade County. Again, the zip codes of the physician's office (multiple listings will be included) were used to assign physician offices to specific sub-areas. Further, the Alliance compiled health care related services available in each of the sub-areas. This information was transformed into computer readable data for analysis.

Data from the 1990 U.S. Census for Dade County were examined by zip code. This data gave an overall neighborhood context. Information from the 1990 Census included:

- Proportion of total population in zip code who are $60+$

- Proportion of total population in zip code who live below poverty

- Proportion of total population in zip code who are black

- Proportion of total population in zip code who are Hispanic 
Census data was also used to examine historical demographic trends of elders in Miami-Dade County, FL 1950-1990 (Chapter 4). Depending on the level of analysis and time period the data will come from three particular sources. For aggregate county level data, the data will come from samples created by IPUMS (Ruggles, Sobek, et al., 1997). With respect to geographic distribution of characteristics of elders, the data will come from the Special Tabulation on Aging data file (U.S. Bureau of the Census, 1994). While characteristics of the population as a whole over time will come from published data of the Metro-Dade Planning (1996), as well as U.S. Census Summary Tape File 3A (U.S. Census Bureau, 1992b).

\section{ANALYSIS PLAN}

This dissertation combines a variety of methodologies, including telephone survey data, focus group discussions, as well as archival data sources. Combining these methods gave more confidence to the results; weaknesses in one method were counter-balanced by the strengths of another.

The analysis plan for examining and answering the research questions put forth in Chapter 1 on the health care service utilization by elders in Miami-Dade County will involve four different stages. The first stage of analysis examining Research Question 1 used qualitative data, primarily. As an introduction to this stage of the analysis, there was an examination in Chapter 3 of the evolution of theoretical perspectives on age and aging, as well as an examination of previous ethnographic literature. The discussion of Hypothesis 1 is placed in Chapter 6 of this dissertation. Examination of Hypothesis 1 will use data from six of the ten focus groups collected for the Dade County needs 
assessment, as well as information from the telephone survey data on elders current concerns and future worries of elders (for shaping of the text discussion only). The analysis will examine other issues that elders brought up in focus group discussions for the purpose of better understanding both the differences and similarities in the lives of elders from each of the four racial/ethnic groups residing in Miami-Dade County. Thus, health and health care issues will not be examined within a vacuum, as has been the case in many previous research endeavors.

The quantitative aspect of this dissertation will be analyzed in three stages. The first step in the quantitative analysis will examine the univariate distribution of dependent and independent variables for the four racial/ethnic groups. This analysis will be included in the latter part of this chapter along with the discussion of variables that were included in the analysis from the survey questionnaire. As a further introduction to this stage of the research, the historical demographic trends of elders in Miami-Dade County, Florida 1950-1990 using sampled census data was documented in Chapter 4 . The next stage of the quantitative analysis will test the existence of bivariate relationships between each predictor variable and each of the variables for health care service utilization. Chisquare statistics will be performed to provide detailed descriptive information on the relationships of health care service utilization within the four racial/ethnic groups. Zeroorder correlations among variables using Pearson correlation coefficients will measure the extent to which predictor variables are related to one another.

The final stage of the analysis will focus on Research Questions 2 and 3 through the analysis of logistic transformation (Logit regression) since the dependent variables for health care service utilization are dichotomous. Ordinary least squares (OLS) regression 
could have been used for dichotomous dependent variables if the distribution of the dichotomous dummy variable fell within the acceptable distribution of 0.25 to 0.75 . In this range OLS and logistic transformation tend to yield similar results (Cox, 1970; Cleary and Angel, 1984). However, at least one of the dichotomous dependent variables does not fall in this range (overall physician contact in the last 12 months). The examination of Research Questions 2 and 3 will use quantitative data, which was primarily gathered from the needs assessment telephone survey as documented earlier in this chapter.

The two hypotheses under Research Question 2 will be addressed individually in three-step process. Hypothesis $2 \mathrm{~A}$ will be answered by an analysis of the explained variances resulting from separately inputting the three major blocks of variables.

The method of entry of the blocks of variables was at question. Wolinsky (1978) used a hierarchical design adding the blocks by "the most 'recent' (temporally) causal determinants of the dependent variables" (p. 392) or (1) need (2) enabling (3) predisposing. However, in more recent literature on utilization of health care services another approach has been adopted in adding blocks of variables, i.e., with respect to order of pre-existing conditions, or (1) predisposing, (2) enabling and (3) need. Thus, it was decided to first examine the blocks of variables separately. Importance of the block of variables will be defined in terms of the greatest amount of explained variance $\left(R^{2}\right)$. Within the predisposing and enabling blocks, there are subgroups of factors. For predisposing there are demographic, social structure and health belief subgroups, while enabling factors can be broken down into two groups: community resources and personal/family resources. 
Separate analysis of each block of variables solved another complication to the inclusion of all predictor variables into a full-model. With respect to the smaller racial/ethnic groups of elders (i.e., black non-Hispanic and non-Cuban Hispanic elder groups), an initial examination of the coefficients in the full-model (i.e., when all the independent variables were included in the model) indicated that the coefficients were unstable, particularly with respect to overall physician contact. This result indicated a difficulty with the degrees of freedom when all variables were included in the model and would hinder the analysis. Thus, the next stage of the analysis was to examine best-fit models for each racial/ethnic group of elders and each formal health care service dependent variable. This will be done since the guiding principle of regression modelling is parsimony. The "best-fit models" for each racial/ethnic group of elders will include only those measures whose beta coefficients were statistically significant at 0.10 or less. The importance of each of these subgroups of factors will be determined by an analysis of the increment change of explained variance using the best-fit model for each racial/ethnic group of elders. Hypothesis $2 \mathrm{~B}$ and Hypothesis $2 \mathrm{C}$ will first examine the increment of change of explained variance of both the separate block entry and best-fit models using logit regression techniques, similarly to Hypothesis $2 \mathrm{~A}$. However, there will also be an examination of the coefficient results from the best-fit models to answer these specific hypotheses.

The hypotheses under Research Question 3 will follow similar pattern of analysis as Research Question 2. However, since this research question is particularly focused on racial/ethnic differences in health care utilization behavior, the attention will be focused 
on differences (and similarities) between racial/ethnic groups of elders in the analysis of increments in explained variance and the regression coefficients.

\section{INSTRUMENT USED IN DATA COLLECTION AND MEASUREMENT OF \\ VARIABLES}

The determinants and characteristics found in the review of the literature need to be related to measures found in the DCNA data set. Table 5.2 shows the coding algorithms of the predisposing, enabling, need, and utilization measures. The telephone survey instruments in English and Spanish are located in Appendix C. More detailed statistics of the sampled population will follow.

\section{Dependent Variables}

Four measures of health care utilization were examined. Two involved physician utilization. The first of which was an overall physician contact in the last 12 months and the second was a measure of volume of physician contact for those who had at least one visit in the last 12 months (high-volume with 6 or more visits). The other three measures of health care utilization were hospitalization in the last 12 months (hospital), and complete physical exam in last 12 months (genexam). The analysis focused on these measures because they have differences in the three units of analyses: volume, contact and purpose. With respect to contact, hospitalization is the least discretionary, while physician visits and general physical exam are more discretionary. With respect to purpose, complete physical exam is primary care, characterized by its preventive nature. Physician visits, on the other hand, can have a variety of purposes and in most cases 
assume that an illness has occurred. Individual's who have been hospitalized overnight were asked to not count physician contacts while they were hospitalized.

All dependent variables were measured as dummy variables. Respondents were asked to recall if in the previous 12 months they had: (a) visited with a physician; (b) been hospitalized overnight; and (c) had a general physical exam. In order to determine whether volume of contact with a physician had different determinants, respondents were asked to recall whether the number of visits was 6 or more visits in the last 12 months. Table 5.3 shows the distribution of the health care service utilization measures for each of the racial/ethnic groups of elders in Miami-Dade County, FL.

With respect to the utilization of services, the majority of elders in each of the racial/ethnic groups had contact with a physician within the last 12 months, i.e., less than 10 percent of elders indicated no contact with a physician in the last 12 months. There is also information available about the volume of contact that the respondents had with physicians in the last 12 months. While only one-third (33.1\%) of white non-Hispanic elders had 6 or more physician visits in the last 12 months, almost half ( $48.0 \%)$ of Cuban elders had 6 or more physician visits in the last 12 months. The percentage of black nonHispanic elders and non-Cuban Hispanic elders that had 6 or more physician visits was approximately two-fifths (39.0\% and 39.9\%, respectively). Respondents were asked whether they had needed to see a physician in the last 12 months but were unable to see one. The variation across the racial/ethnic groups was considerable. While less than onetenth $(7.5 \%)$ of white non-Hispanic elders indicated that they had not been able to see a physician when they needed to see one, almost one-fifth (19.3\%) of non-Cuban Hispanic elders indicated an inability to see a physician. It is interesting to note that when 
respondents were asked for the reasons that they couldn't see a physician, none of the elders indicated that language was a barrier to seeing a physician. However, reasons for not being able to see a physician for those who indicated that they had been unable to see a physician differed by racial/ethnic group. Two-fifths (40.6\%) of white non- Hispanic elders reported that they just couldn't get an appointment. In comparison, less than onefifth of all other groups of elders reported this as a problem. Reasons that black nonHispanic elders gave were primarily split between no transportation available (39.3\%) and no money $(32.1 \%)$. The primary reason that Cuban elders gave for not being able to see a physician was also no transportation $(33.3 \%)$. In contrast, over half $(51.4 \%)$ of the non-Cuban Hispanic elders indicated that money was the reason that they could not see a physician. Having no transportation was the second most frequently given reason for non-Cuban Hispanic elders (34.3\%). Almost one-third (31.4\%) of non-Cuban Hispanic elders indicated that the reason they weren't able to see a doctor was that they didn't have health insurance. Other health care services available for examination in the survey are: volume of physician visits, overnight hospital stay, and having a complete physical examination. However, since physicians are seen as the "gatekeeper" into the formal health care system in the United States, primary attention in this chapter will be given to physician visits (both contact and volume or contact) and overnight hospital stay.

\section{Independent Variables}

Most of the independent variables in the study were included because they were proposed in the original conceptualization and subsequent revisions of Socio-Behavioral Model (SBM). Others of the independent variables were included because some concept 
of them has been shown to be important predictors of health care utilization. The independent variables will be discussed in three classifications: predisposing; enabling; and need. Within each of the characteristics there is a discussion of univariate descriptive statistics for each of the four racial/ethnic groups of elders.

\section{Predisposing Factors}

There are thirteen measures of predisposing characteristics found in the DCNA dataset that will be used in this study. As originally defined by Andersen (1968), predisposing factors are those "... characteristics which exist prior to the onset of specific episodes of illness." They represent the sociocultural element of the behavior model (Wolinsky, et al., 1989). Further predisposing factors can be broken down as being demographic, social structure, or health beliefs.

Another component of predisposing characteristics is social structure. This is measured in the DCNA dataset in two ways. First there are a series of questions that examine the presence of a social support network. Four items will be used to tap the social support network: whether the respondent talks on the phone at least once a week with (a) family and (b) friends; whether the respondent visits at least once a month with (a) family and (b) friends. Religion has been shown in the literature to be an important indicator of social connectedness for elders. However, factor analysis did not indicate high correlation with the previous four items, thus religious affiliation and importance of religion will be entered separately into the model.

Finally the last component of predisposing characteristics is health beliefs. There are two measures of health beliefs found in the DCNA dataset. First, respondents were 
asked if health for elders is a very/somewhat or not a serious problem. Second,

respondents were asked if in the future they worried about losing their independence due to physical or mental deterioration.

Table 5.4 shows the descriptive statistics on the predisposing characteristics for each of the four racial/ethnic groups of elders. The sample of 1967 respondents consisted of 895 white non-Hispanic elders, 201 black non-Hispanic elders ${ }^{8}, 671$ Cuban elders, and 188 non-Cuban Hispanic elders. ${ }^{9}$ The mean age of the respondents ranges from 69.16 years for black non-Hispanics to 72.18 years for white non-Hispanics. Interesting to note is that non-Cuban Hispanic elders have the highest percentage of elders age 85 or more years $(9.1 \%)$ with white non-Hispanic elders a close second with 8.7 percent. For all racial/ethnic groups, the majority of respondents are women, ranging from a high of 71.8 percent for black non-Hispanics to a low of 62.5 percent for white non-Hispanics.

Education affects utilization behavior in a number of ways. In particular, it seems to be an important determinant of preventive health care utilization (Aday, 1993). As noted in Chapter 2, individuals with more education are more likely to use discretionary health care services that are preventive (National Center for Health Statistics, 1991 ). ${ }^{10}$ In the sample, white non-Hispanic respondents had the highest percentage of elders with more than high school education (58.4\%), while black non-Hispanic respondents had the lowest percentage (19.1\%). In comparison one-quarter (25.3\%) of Cuban and one-third

\footnotetext{
${ }^{B}$ There were 12 black non-Hispanic respondents who were removed from the anaiysis because they indicated they spoke another language other than English, particularly Creole. This was done to create cultural homogeneity for the analysis.

${ }^{y}$ There were also 22 other non-Hispanic elders and 3 elders that were unclassifiable with respect to theit racial/ethnic background. These 25 respondents will not be analyzed in this chapter.
} 
$(32.8 \%)$ of non-Cuban Hispanic elders in the sample had more than a high school education.

As noted in Chapter 2, marital status has been shown to be associated with health and survival outcomes in the older population (Goldman, et al., 1995). The percentage of elders in this sample who reported themselves as currently married range from 48.5 percent for Cubans to 39.0 percent for black non-Hispanic.

The two main languages that elders in the sample reported speaking were English or Spanish. The majority of white non-I lispanic reported English as the language spoken in the home $(95.9 \%)$. In comparison, the majority of Cuban and non-Cuban Hispanic elders do not reside in English-only speaking households. The majority reported Spanish as the language spoken in the home $(97.0 \%$ for Cuban elders and $83.5 \%$ for non-Cuban Hispanic elders). It is interesting to note that Cuban and non-Cuban Hispanic elders are somewhat dissimilar in the percentage living in non-English speaking households. Other specified languages reported spoken in the home were Creole (particularly black nonHispanic elders but were removed from the analysis in this research) and Yiddish (particularly white non-Hispanic elders).

As noted in Chapter 2, social structural sub-factors "tap the individual's location in the social structure and behavior patterns (i.e., lifestyles) to which people in such positions become socialized" (Wolinsky, et al., 1989). The dataset has information on household size (whether living alone or not), employment status, and a set four questions that will be combined to create an index on the intensity of social contact with family \&

\footnotetext{
${ }^{10}$ Examples of discretionary health care services that are preventive are general physical exams, health
} 
friends, as well as religious affiliation and self-reported importance of religion in the respondent's life. With respect to living alone, over one-third (36.7\%) of white nonHispanic elders live alone, compared to 25.1 percent for black non-Hispanic, 28.5 percent for Cuban and 24.3 percent for non-Cuban Hispanic. Approximately one-fifth of all respondents reported being employed (either full-time or part-time), ranging between 22.9 percent for black non-Hispanic elders and 15.2 percent for Cuban elders.

To measure the intensity of social contact that elders have with family and friends a series of four questions asking about how often they visit with family and friends and how often the talk on the phone with family and friends. The variables were recoded so that never visiting was coded as zero and the most intense contact (i.e., at least once a week) was coded as three. The possible range for this index was between 0 and 12 . The average score on this index ranged from 9.59 for Cuban and 9.55 for white non-Hispanic elders to 8.60 for non-Cuban Hispanic elders. The Cronbach alpha, a test of reliability of the index, was 0.5423 for the whole sample.

Religious affiliation and religion's importance in the life of the elder respondent are also important social structural predisposing factors for health utilization. White nonHispanic elders are almost equally distributed between the three major religious groups of Protestant, Catholic and Jewish. Less than one-tenth (7.9\%) of white non-Hispanic elders reported that they had no religious affiliation. In comparison, the majority of black nonHispanic elders reported themselves as Protestant (84.8\%), while the majority of Cuban and non-Cuban Hispanic elders reported themselves as Catholic $(79.0 \%$ and $71.7 \%$, 
respectively). Over one-fifth (22.1\%) of white non-Hispanic elders reported that religion was not very important in their lives. In contrast, less than one-tenth of minority elders reported that religion was not very important in their lives.

In general, health belief characteristics tap into the individual's attitudes toward physicians, disease and medical care. While the survey did not ask specific questions on the respondent's attitudes toward physicians, disease and medical care, it did ask general questions on how serious a problem area the respondent thought that health was for most elders. The percentage of respondents who stated that health was not a serious problem area ranged from 68.0 percent for white non-Hispanic to 51.5 percent for Cuban and 51.6 percent for non-Cuban Hispanic respondents. Respondents were also asked about their worries for the future regarding loss of independence due to physical deterioration or mental deterioration. Over half of white non-Hispanic and black non-Hispanic respondents responded that they were not very worried about loss independence due to physical deterioration (52.3\% and $57.4 \%$, respectively). In contrast, Cuban and nonCuban Hispanic respondents were less likely to respond that they were not very worried about loss of independence due to physical deterioration $(35.4 \%$ and $28.0 \%$, respectively. Loss of independence due to mental deterioration ranged from 72.8 percent for black non-Hispanic respondents to 35.1 percent for non-Cuban Hispanic respondents. Finally, respondents were asked about their satisfaction with life in the past 6 months. The majority of all respondents in each of the racial/ethnic groups responded that they were very or mostly satisfied. 


\section{Enabling Factors}

The second set of factors that Andersen (1968) documented were enabling factors. These are factors/conditions that "make health service resources available to the [individual]" (Andersen, 1968). Enabling factors include both personal and community factors. Primarily, previous research has focused on the personal enabling factors and left out community enabling factors. Personal enabling factors document the individual or family's "ability to obtain services" and "is assessed largely by the extent of their economic resources and their sources of medical care" (Andersen, 1968). In contrast, community resources are those characteristics of the community that the individual resides in that "enable the use of services" (Andersen, 1968). Community resources document availability of services in the neighborhood, both directly and indirectly.

With respect to personal enabling factors, the dataset has information on health insurance coverage, duration of residence within the Miami-Dade County, self-reported household income, and full-time residency within Miami-Dade County. With respect to health insurance coverage, there will be nine dichotomous variables indicating (1) whether the respondent has Medicare Part A only coverage, (2) Medicare Part A regardless of Part B coverage; (3) Medicare Part A \& B; (4) no Medicare coverage, regardless of current age status; (5) no Medicare coverage and less than 65 years; (6) no Medicare coverage and age 65+ years; (7) Medicaid coverage; (8) Health care coverage under an HMO plan; and (9) no health care coverage of any type indicated.

With respect to household income, the initial examination of this data showed that 30.8 percent or 613 respondents refused to answer this question or did not know. This 
high "missing" response was expected given focus group results. In focus group discussions with elders and community service agency personnel, there was a general understanding that elders might feel threatened by questions on income. In order not to lose these 613 respondents in the study, data from the 1990 U.S. Census Special Tabulation on Aging (U.S. Census, 1994) was examined with respect to the distribution of household income of elders by their educational attainment and racial/ethnic classification was performed. The distribution of the household income results in the 1990 Census for Dade County elders and DCNA survey show that the percentage of elders reported household incomes under $\$ 10,000$ was similar. Those who did not report household income in the DCNA survey probably had higher household incomes given their higher educational attainment and primary concentration within the white nonHispanic elder group. Thus, the variable for household income was aggregated the "No Answer" responses into the "Not less than $\$ 10,000 . "$

Duration of residence of the respondent and whether the respondent is a full-time or part-time resident of Dade County may be important determinants of knowledge of health service availability, which in turn may effect utilization. Therefore, these measures will be included in the model. Duration of residence was measured as a continuous variable. Full-time/part-time residency in Dade County was measured as a dichotomous variable. Respondents were asked how long they had lived in South Florida and how many months out of the year they lived in South Florida.

Finally, utilization of services is dependent upon availability of services in the neighborhood. Therefore, there will be measures of service availability for each subarea. There will be measures of hospital bed ratios per 1000 population in each sub-area, 
as well as physician ratios per 1000 population in each sub-area. The number of physicians and hospital beds available are not the only health care services that individuals use. Overall, services in Dade County, as in most other counties, are not equally distributed throughout the county due to many different factors. However, measuring access to services can not always be directly measured. Thus, data was collected from the 1990 U.S. Census on the proportion of blacks, Hispanics, elders $(60+)$, people living below poverty, as well as median household income and per capita income in Miami-Dade County zip codes.

Table 5.5 shows the descriptive statistics for enabling measures used in the analysis for each of the racial/ethnic groups of elders. Slightly over one-fifth (12.1\%) of white non-Hispanic elder respondent reported having a household income of less than $\$ 10,000$, compared to slightly over one-third $(34.6 \%)$ of non-Cuban Hispanic respondents, two- fifths (40.3\%) of black non-Hispanic respondents and slightly over two-fifths $(42.2 \%)$ of Cuban respondents.

Another important set of personal enabling factors are health care coverage. The majority of elders age 65 years or older should be eligible for Medicare. However, there are a surprisingly large percentage of elders $\left(65^{+}\right)$who do not have Medicare coverage. The percentage of $65+$ elders who do not have Medicare coverage ranges from 11.6 percent of non-Cuban Hispanics to 6.3 percent of Cuban Hispanics. The percentage of elders with no health insurance coverage of any kind ranges from 4.5 percent for white non-Hispanic elders to 17.0 percent for non-Cuban Hispanic elders.

Length of time lived in the county may give the elder a better understanding of the services available in the community. Given the historical migration patterns, it is not 
surprising that the average number of years lived in Miami-Dade County is much greater for both white and black non-Hispanic elders ( 31.14 years and 39.62 years, respectively), compared to Cuban and non-Cuban Hispanic elders (19.27 years and 16.44 years, respectively).

Having a private physician as a regular source of health care may allow an elder to get better continuity of care. Further, the elder may feel more comfortable talking to their private physician about sensitive health issues. There is significant difference in the percentage of elders reporting that they have a private physician as a regular source of health care across the four racial/ethnic groups under investigation. Almost threequarters $(70.1 \%)$ of white non-Hispanic elders report having a private physician as a regular source of health care, compared to 47.2 percent for black non-Hispanic, 49.8 percent for Cuban and 54.7 percent for non-Cuban Hispanic.

Community resources for health care services are both directly and indirectly measured. There are two available direct measurements of health care services in MiamiDade County. These are hospital bed ratios and primary care physician ratios per 1000 population in the district. Average hospital bed ratios per 1000 population in a district range from 4.92 for black non-Hispanic respondents to 4.15 for white non-Hispanic respondents. Average primary care physician ratios per 1000 population in a district range from 2.30 for Cuban respondents to 1.22 for black non-Hispanic respondents. Thus, direct measures of access to health care services would appear to indicate that black non-Hispanic elders have less access to preventive health care/early stage illness care services (physician ratios) compared to other racial/ethnic categories of elders. 
As discussed earlier, measuring access to all health care services can not always be directly measured. Thus, indirect measures of community resources for health care service usage were also collected. These indirect measures were from the 1990 U.S. Census broken down by zip code measuring the proportion of blacks, Hispanics, elders $(60+)$, people living below poverty, as well as median household income and per capita income in Miami-Dade County zip codes. Miami-Dade County is a highly segregated community. This becomes obvious from the ranges in these indircct measures of community contextual variables across the racial/ethnic categories of elder respondents. On average, white non-Hispanic elder respondents primarily live in zip codes where the population is approximately one-eighth black, just over one-third Hispanic, one-quarter elder (60+ years), and one-seventh of the total population lives below poverty. In contrast, the average black non-Hispanic elder respondent lives in zip codes where the population is one-half black, slightly over one-quarter Hispanic, one-seventh elder and slightly over one-quarter of the total population lives below poverty. The average Cuban elder respondent lives in a zip code where the total population is over three quarter Hispanic, less than one-tenth black, slightly less than one-quarter elder and slightly less than one-fifth of the total population lives below poverty. The average non-Cuban Hispanic elder respondent lives in a zip code where the total population is almost threefifths Hispanic, slightly less than one-seventh black, one-fifth elder and slightly less than one-fifth of the total population lives below poverty. 


\section{Need Factors}

Individuals who perceive a need to use health care services due to an illness are most likely to use formal health care services. Need factors "tap the individual's recognition that a health problem either exists or is in the making" (Wolinsky, et al., 1989). Need factors can be broken down into two components: self-perceived and professionally diagnosed. The self-perceived characteristic is usually one question in which the respondents are asked to assess their overall health status. The dataset has two questions on self-perceived health. There are four measures of need characteristics used in this study. As discussed previously, there are two components of need characteristics: perceived and evaluated health measures. With respect to perceived health status, respondents were asked to assess their own physical health status and emotional health status in comparison to others their age. With respect to evaluated health measures, there are three indicators of health. These three indicators of health are examined both separately and as an aggregated index.

The first measure of evaluated health is with respect to difficulties with Activities of Daily Living (ADL) Scale. This scale was based on the number of self-reported difficulties a respondent had with five items: bathing, dressing, eating, toileting, and getting in/out of bed/chair. The second measure of evaluated health was with respect to difficulty with Instrumental Activities of Daily Living (IADL) Scale. Similarly to the ADL Scale, this scale was based on the number of IADLs that the respondents indicated were difficult to perform: preparing meals, shopping for groceries/clothes, taking medicines, using the telephone, taking care of personal appearance, doing the laundry, 
doing other household chores, managing money and getting to places needed to go.

Finally, the third measure of evaluated health with respect to mobility, either walking or getting up and down stairs. This was constructed similarly to the ADL and IADL scales. The aggregated summary index scale included all 16 items from the ADL, IADL and mobility scale.

Descriptive statistics for each of these measures of need are shown in Table 5.6. Approximately one-tenth of white non-Hispanic, Cuban, and non-Cuban Hispanic elders reported poor overall health $(7.0 \%, 10.5 \%$, and $9.2 \%$, respectively). In contrast, 14.7 percent of black non-Hispanic elders reported poor overall health. With respect to selfreported emotional health status, the percentage of elders that reported poor emotional health ranged from 3.2 percent for non-Cuban Hispanic elders to 6.0 percent for black non-Hispanic elders.

With respect to the professionally diagnosed need factors, many studies do not have access to this information. As stated in Chapter 2, it has been shown that information on activity limitations due to health reasons provides an objective assessment of need (Liang, 1986; Wolinsky, et al., 1989). Over half of all respondents reported that they did not have difficulty with any of the activities. However, for black non-Hispanic and non-Cuban Ilispanic elders almost one-fifth reported difficulty with 4 or more of the activities specified $(18.9 \%$ and $18.1 \%$, respectively). In contrast, 11.4 percent of white non-Hispanic and 13.4 percent of Cuban elders reported difficulty with 4 or more of the activities.

In conclusion, this chapter has explained the source of the data and methods used in this research project examining health care utilization behavior of elders in a 
multicultural urban environment. Further, there has been a examination of the univariate statistics across the four racial/ethnic groups of elders in the sample. The next chapter will be a discussion of five texts that examine the lives of elders and answer the Research Question 1 and Hypothesis 1 documented in Chapter 1. 
Table 5.1: Population Estimates and Survey Sample Numbers

\begin{tabular}{lrrr}
\hline AAA Sub-Areas (PSA) & $60+$ Population & $\begin{array}{r}\text { Percent of 60+ } \\
\text { Population }\end{array}$ & $\begin{array}{c}\text { Number of Interviews } \\
\text { 1: The Beaches }\end{array}$ \\
2: Northeast Dade & 40,454 & 11.2 & 202 \\
3: North Central Dade & 60,897 & 15.8 & 284 \\
4: Northwest Dade & 45,099 & 13.0 & 226 \\
5: West Miami/Sweetwater/Westchester & 48,011 & 13.5 & 249 \\
6: Downtown Miami & 27,436 & 9.4 & 226 \\
7: Kendall & 52,892 & 16.2 & 331 \\
8: Greater Coral Gables & 30,650 & 8.8 & 171 \\
9: South Central Dade & 34,134 & 5.2 & 105 \\
10: South Dade & 14,305 & 3.9 & 99 \\
\hline
\end{tabular}


Table 5.2: Coding Algorithms for Categorical Variables and Ranges for Continuous Variables Used in the Study

\begin{tabular}{|c|c|c|}
\hline Varjable Name & Coding Scheme & Mean (SD) \\
\hline \multicolumn{3}{|c|}{ HEALTH SERVICE UTILIZATION OUTCOMES } \\
\hline DOCTOR & $\begin{array}{l}1=Y \mathrm{cs}, \text { physician contact in last } 12 \text { months } \\
0=\text { No physician contact in last } 12 \text { months }\end{array}$ & $\begin{array}{r}0.92 \\
(0.26)\end{array}$ \\
\hline \multirow[t]{3}{*}{ DOCTOR6 } & Of those with at least one physician contact in last 12 months & \\
\hline & $1=6$ or more physician contacts in last 12 months & 0.43 \\
\hline & $0=$ less than 6 physician contacts in last 12 months & $(0.49)$ \\
\hline \multirow[t]{2}{*}{ HOSPITAL } & $1=$ Yes, overnight hospital stay in last 12 months & 0.21 \\
\hline & $0=$ No overnight hospital stay in last 12 months & $(0.40)$ \\
\hline \multirow[t]{2}{*}{ GENEXAM } & $1=$ Yes complete physical exam in last 12 month & 0.79 \\
\hline & $0=$ No complete physical exam in last 12 months & $(0.40)$ \\
\hline \multicolumn{3}{|c|}{ PREDISPOSING FACTORS } \\
\hline \multicolumn{3}{|c|}{ Age } \\
\hline $\mathrm{AGE}$ & Range $=60$ to 103 years & $\begin{array}{r}71.39 \\
(8.00)\end{array}$ \\
\hline \multirow[t]{2}{*}{ AGE6064 } & $I=$ Self-reported age between 60 and 64 years & 0.23 \\
\hline & $0=$ Other age & $(0.42)$ \\
\hline \multirow[t]{2}{*}{ AGE6574 } & $1=$ Self-reported age between 65 and 74 years & 0.45 \\
\hline & $0 \approx$ Other age & $(0.50)$ \\
\hline \multirow[t]{2}{*}{ AGE7584 } & $1=$ Self-reported age between 75 and 84 years & 0.25 \\
\hline & $0=$ Other age & $(0.43)$ \\
\hline \multirow[t]{2}{*}{ AGE8599 } & $1=$ Self-reported age 85 years or more & 0.07 \\
\hline & $0=$ Other age & $(0.26)$ \\
\hline \multirow[t]{2}{*}{ FEMALE } & $1=$ Female & 0.65 \\
\hline & $0=$ Male & $(0.48)$ \\
\hline \multicolumn{3}{|c|}{ Educational Attainment } \\
\hline \multirow[t]{2}{*}{ NOHS } & $1=$ No High School $\left(<9^{\text {th }}\right.$ grade $)$ & 0.25 \\
\hline & $0=$ Other & $(0.43)$ \\
\hline \multirow[t]{2}{*}{ SOMEHS } & $1 \simeq$ Some High School, but did not graduate & 0.11 \\
\hline & $0=$ Other & $(0.31)$ \\
\hline \multirow[t]{2}{*}{ HSGRAD } & $1 \approx$ Graduated High School & 0.23 \\
\hline & $0=$ Other & $(0.42)$ \\
\hline \multirow[t]{2}{*}{ MOREHS } & $1=$ More than High School education & 0.41 \\
\hline & $0=$ Other & $(0.49)$ \\
\hline \multicolumn{3}{|l|}{ Marital Status } \\
\hline \multirow[t]{2}{*}{ MARRUED } & $l=$ Currently married $/$ Live-in partner & 0.46 \\
\hline & $0=$ Other marital status & $(0.50)$ \\
\hline \multirow[t]{2}{*}{ WIDOWED } & $1=$ Widowed & 0.34 \\
\hline & $0=$ Other marital status & $(0.47)$ \\
\hline DIVORCED! & 1 - Divorced or Separated & 0.15 \\
\hline \multirow{3}{*}{$\begin{array}{l}\text { SEPARATED } \\
\text { NEVER MARRIED }\end{array}$} & $0=$ Other marital status & $(0.36)$ \\
\hline & $1=$ Never martied & 0.05 \\
\hline & $0=$ Other marita! status & $(0.21)$ \\
\hline \multicolumn{3}{|c|}{ Racial/Ethnic Classification ${ }^{1}$} \\
\hline \multirow[t]{2}{*}{ WNH } & I = White non-Hispanic & 0.46 \\
\hline & $0=$ Other & $(0.50)$ \\
\hline \multirow[t]{2}{*}{ BNH } & $1=$ Black non-Hispanic (English-only speaking) & 0.10 \\
\hline & $0=$ Other & $(0.30)$ \\
\hline
\end{tabular}

\footnotetext{
'Models were nu separately by racial/ethnic categories to understand differences in determinants within each racialiethnic classification.
} 
Table 5.2 (cont).

\begin{tabular}{|c|c|c|}
\hline Variable Name & Coding Scheme & Mean (SD) \\
\hline \multirow[t]{2}{*}{$\mathrm{ONH}$} & $1=0$ ther non-Hispanic & 0.01 \\
\hline & $0=$ Other & $(0.10)$ \\
\hline \multirow[t]{2}{*}{ CUB } & $1=$ Cuban & 0.34 \\
\hline & $0=$ Other & $(0.47)$ \\
\hline $\mathrm{NCH}$ & $\begin{array}{l}1=\text { Non-Cuban Hispanic } \\
0=\text { Other }\end{array}$ & $\begin{array}{r}0.096 \\
(0.29)\end{array}$ \\
\hline \multicolumn{3}{|c|}{ Language Spoken in the home } \\
\hline LANGENG & $\begin{array}{l}1=\text { English Only } \\
0=\text { Other }\end{array}$ & $\begin{array}{r}0.57 \\
(0.50)\end{array}$ \\
\hline LANGSPA & $\begin{array}{l}1=\text { Spanish } \\
0 \approx \text { Other }\end{array}$ & $\begin{array}{r}0.41 \\
(0.49)\end{array}$ \\
\hline LANGCRE & $\begin{array}{l}1=\text { Creole } \\
0=\text { Other }\end{array}$ & $\begin{array}{r}0.004 \\
(0.07)\end{array}$ \\
\hline LANGYID & $\begin{array}{l}1=\text { Yiddish } \\
0=\text { Other }\end{array}$ & $\begin{array}{r}0.004 \\
(0.06)\end{array}$ \\
\hline LANGOTH & $\begin{array}{l}1=\text { Other Language spoken in the Home, not English, Spanish, } \\
\text { Creole, or Yiddish } \\
0=\text { English, Spanish, Creole, or Yiddish spoken in the I Iome }\end{array}$ & $\begin{array}{r}0.01 \\
(0.10)\end{array}$ \\
\hline ALONE & $\begin{array}{l}1=\text { Lives alone } \\
0 \approx \text { Lives with others }\end{array}$ & $\begin{array}{r}0.32 \\
(0.46)\end{array}$ \\
\hline EMPLOY & $\begin{array}{l}1=\text { Currently Employed in the Labor Force } \\
0=\text { Not in the Labor Force }\end{array}$ & $\begin{array}{r}0.19 \\
(0.39)\end{array}$ \\
\hline INTENSE $^{2}$ & $\begin{array}{l}\text { Intensity of social contact with family or friends } \\
\text { Range from } 0 \text { to } 12 \text { (Cronbach alpha } \because 0.5423 \text { ) }\end{array}$ & $\begin{array}{r}9.41 \\
(2.55)\end{array}$ \\
\hline \multicolumn{3}{|l|}{ Health Beliefs } \\
\hline HLTHPROB & $\begin{array}{l}1=\text { Health is a very } / \text { somewhat serious problem } \\
0=1 \text { Health is not a serious problem }\end{array}$ & $\begin{array}{r}0.40 \\
(0.49)\end{array}$ \\
\hline LOSSDEP & $\begin{array}{l}1=\text { Very/Somewhat worried about loss of independence due to } \\
\text { physical or mental deterioration } \\
0=\text { Not worried about loss of independence }\end{array}$ & $\begin{array}{r}0.61 \\
(0.49)\end{array}$ \\
\hline LIFESAT & $\begin{array}{l}1=\text { Very } / \text { Somewhat satisfied with life in past } 6 \text { months } \\
0=\text { Very } / \text { Somewhat dissatisfied with life in past } 6 \text { months }\end{array}$ & $\begin{array}{r}0.85 \\
(0.36)\end{array}$ \\
\hline \multicolumn{3}{|c|}{ Religious Affiliation } \\
\hline RELIGPRO & $\begin{array}{l}1=\text { Protestant religious affiliation } \\
0=\text { Other religious affiliation }\end{array}$ & $\begin{array}{r}0.29 \\
(0.45)\end{array}$ \\
\hline RELIGCAT & $\begin{array}{l}1=\text { Catholic religious affiliation } \\
0=\text { Other religious affiliation }\end{array}$ & $\begin{array}{r}0.44 \\
(0.50)\end{array}$ \\
\hline RELIGJEW & $\begin{array}{l}1=\text { Jewish religious affiliation } \\
0=\text { Other religious affiliation }\end{array}$ & $\begin{array}{r}0.16 \\
(0.36)\end{array}$ \\
\hline RELIGNOP & $\begin{array}{l}1=\text { No Preference of religious affiliation } \\
0=\text { Other religious affiliation }\end{array}$ & $\begin{array}{r}0.06 \\
(0.24)\end{array}$ \\
\hline
\end{tabular}

${ }^{2}$ Created using the following questions: (1) how often do you visit with friends, either in your home, their homes, or someplace else? (2) How often do you visit with relatives, either in your hone or theirs or someplace else? (3) How often do you talk on the telephone with family members? (5) How often do you talk on the telephone with friends? An index was created from these questions after factor analysis indicated high correlation. Further, the responses to these questions were re-ordered to indicate that lower values meant less contact. For example, with respect to the variable on visits with family members, in the recoded variable 0 meant that the respondent never visited with family members, while 3 meant that they visited with family at least once a week. Finally, with respect to telephone contact and to create consistency with visiting and telephone contact, responses "Almost every day" and "A few times a wcek" were consolidated to "At least a few times a week" or 3. 
Table 5.2 (cont).

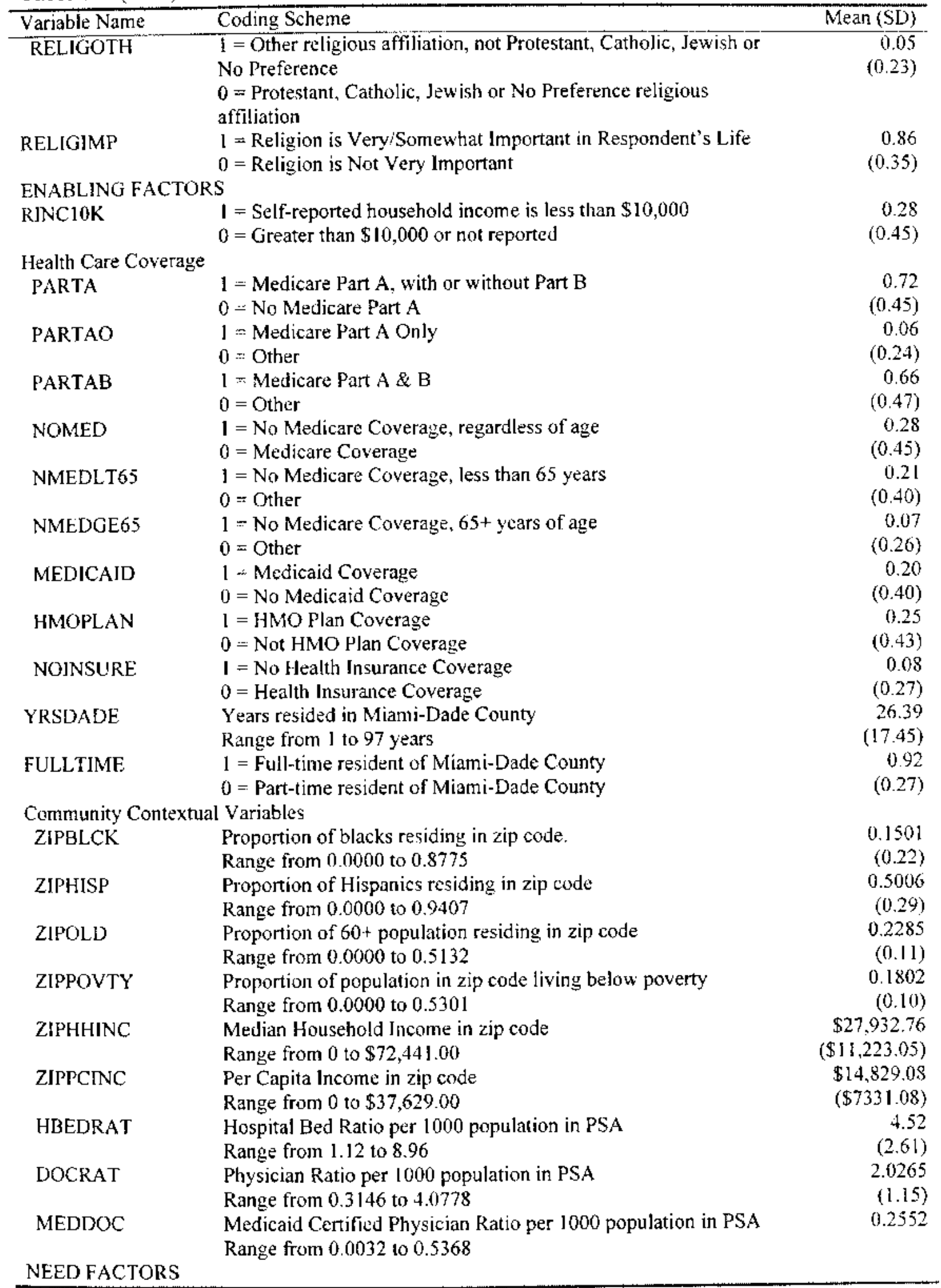


Table 5.2 (cont).

\begin{tabular}{llr}
\hline Variable Name & Coding Scheme & Mean (SD) \\
\hline PHYSHLTH & 1 = Self-reported health is excellent or good compared to others & 0.67 \\
& own age & $(0.47)$ \\
& $0=$ Self-reported health is fair or poor compared to others own age & \\
EMOTHLTH & I $=$ Self-reported emotional health is excellent or good & 0.76 \\
& $0=$ Self-reported emotional health is fair or poor & $(0.42)$ \\
NUMADL & Self-reported number of difficult Activities of Daily Living (ADLs) & 0.34 \\
& Range from 0 to 5 & $(0.85)$ \\
NUMIADL & Self-reported number of difficult Instrumental Activities of Daily & 0.62 \\
& Living (IADLs). Range from 0 to 9 & $(1.50)$ \\
NUMMOB & Self-reported number of difficult Mobility activities & 0.42 \\
& Range from 0 to 2 & $(0.72)$ \\
SUMDIFF & Combined NUMADL, NUMLADL and NUMMOB & 1.38 \\
& Range from 0 to 16 & $(2.66)$ \\
\hline
\end{tabular}


Table 5.3: Descriptive Statistics on Formal I lealth Care Service Utilization Measures for Four Racial/Ethnic Groups of Elders Residing in Miami-Dade County, FL

\begin{tabular}{|c|c|c|c|c|}
\hline & $\begin{array}{r}\text { White non- } \\
\text { Hispanic } \\
(\mathrm{N}=895)\end{array}$ & $\begin{array}{r}\text { Black non- } \\
\text { Hispanic } \\
(\mathrm{N}-201)\end{array}$ & $\begin{array}{r}\text { Cuban } \\
(N=671)\end{array}$ & $\begin{array}{r}\text { Non-Cuban } \\
\text { Hispanic } \\
(\mathrm{N}=188)\end{array}$ \\
\hline \multicolumn{5}{|c|}{ Number of Physician Visits in Last 12 months $(\%)$} \\
\hline No visits & 6.7 & 5.5 & 8.8 & 9.0 \\
\hline 1 or 2 visits & 25.6 & 24.0 & 21.2 & 26.1 \\
\hline 3 to 5 visits & 34.6 & 31.5 & 22.1 & 25.0 \\
\hline 6 or more visits & 33.1 & 39.0 & 48.0 & 39.9 \\
\hline \multicolumn{5}{|c|}{ Six or more Physician Visits in Last 12 months for those with at least one visit (\%) } \\
\hline $\mathrm{N}$ & 825 & 189 & 603 & 171 \\
\hline$<6$ visits & 64.5 & 52.6 & 47.4 & 56.1 \\
\hline $6+$ visits & 35.5 & 41.3 & 52.6 & 43.9 \\
\hline \multicolumn{5}{|c|}{ Needed to see Doctor but were unable to see one in last 12 months (\%) } \\
\hline Yes & 7.5 & 14.1 & 12.8 & 19.3 \\
\hline No & 92.5 & 85.9 & 87.2 & 80.7 \\
\hline \multicolumn{5}{|c|}{ Reason unable to see Doctor (for those who said "Yes, they were unable to see a doctor"' (\%) } \\
\hline Couldn's get appointment & 40.6 & 14.3 & 7.4 & 11.4 \\
\hline Too sick to go & 15.6 & 10.7 & 12.3 & 8.6 \\
\hline Didn't have money & 3.1 & 32.1 & 25.9 & 51.4 \\
\hline Didn't have transportation & 21.9 & 39.3 & 33.3 & 34.3 \\
\hline Language problem & 0.0 & 0.0 & 0.0 & 0.0 \\
\hline Difn't have insurance & 4.7 & 3.6 & 39.8 & 31.4 \\
\hline Didn't have enough insurance & 1.6 & 0.0 & 2.5 & 14.3 \\
\hline Other reason & 11.9 & 7.1 & 23.5 & 8.3 \\
\hline \multicolumn{5}{|l|}{ Hospitalized Overnight in Last 12 months (\%) } \\
\hline No overnight hospital stay & 79.1 & 83.1 & 77.0 & 84.6 \\
\hline One or more overnight hospital stays & 20.9 & 16.9 & 23.0 & 15.4 \\
\hline \multicolumn{5}{|c|}{ Complete Physical Exam in Last 12 months (\%) } \\
\hline No complete physical exam & 20.3 & 18.5 & 19.8 & 27.1 \\
\hline Complcte physical exam & 79.7 & 81.5 & 80.2 & 72.9 \\
\hline
\end{tabular}

\footnotetext{
${ }^{1}$ Respondent could mention more than one reason that they were unable to see a doctor in the last 12 months.
} 
Table 5.4: Descriptive Statistics on Predisposing Measures for Four Racial/Fthnic Groups of Elders Residing in Miami-Dade County, FL

\begin{tabular}{|c|c|c|c|c|}
\hline & $\begin{array}{l}\text { White non- } \\
\text { Iispanic } \\
(\mathrm{N}=895)\end{array}$ & $\begin{array}{r}\text { Black non- } \\
\text { Hispanic } \\
(\mathrm{N}=201)\end{array}$ & $\begin{array}{r}\text { Cuban } \\
(\mathrm{N}=671)\end{array}$ & $\begin{array}{r}\text { Non-Cuban } \\
\text { Hispanic } \\
(\mathrm{N}=188)\end{array}$ \\
\hline \multicolumn{5}{|l|}{ DEMOGRAPHIC FACTORS } \\
\hline Age (mean years) & 72.18 & 69.16 & 71.32 & 70.50 \\
\hline \multicolumn{5}{|l|}{ Age Distribution (\%) } \\
\hline $60-64$ years & 22.1 & 32.3 & 20.1 & 26.9 \\
\hline $65-74$ years & 40.9 & 46.5 & 49.7 & 47.3 \\
\hline $75-84$ years & 28.3 & 18.7 & 24.3 & 16.7 \\
\hline $85+$ years & 8.7 & 2.5 & 5.8 & 9.1 \\
\hline \multicolumn{5}{|l|}{ Gender (\%) } \\
\hline Male & 37.5 & 28.4 & 35.2 & 32.4 \\
\hline Female & 62.5 & 71.6 & 64.8 & 67.6 \\
\hline \multicolumn{5}{|l|}{ Educational Attainment (\%) } \\
\hline No High School $\left(<9^{\text {th }}\right.$ grade $)$ & 4.9 & 39.7 & 47.0 & 33.3 \\
\hline Some High School $\left(9^{\text {th }}-11^{\text {th }}\right.$ grade $)$ & 7.7 & 20.6 & 11.2 & 11.5 \\
\hline High School Graduate (12 ${ }^{\text {th }}$ grade) & 29.1 & 20.6 & 16.5 & 22.4 \\
\hline More than High School & 58.4 & 19.1 & 25.3 & 32.8 \\
\hline \multicolumn{5}{|l|}{ Language Spoken Most Often in Home (\%) } \\
\hline English Only & 95.9 & 100.0 & 2.8 & 16.0 \\
\hline Spanish & 1.6 & 0.0 & 97.0 & 83.5 \\
\hline Creole & 0.0 & 0.0 & 0.1 & 0.0 \\
\hline Yiddish/Hebrew & 0.9 & 0.0 & 0.0 & 0.0 \\
\hline Other & 1.7 & 0.0 & 0.0 & 0.5 \\
\hline \multicolumn{5}{|l|}{ SOCIAL STRUCTURAL FACTORS } \\
\hline \multicolumn{5}{|l|}{ Marital Status $(\%)$} \\
\hline Married/Live-in Partner & 48.0 & 39.0 & 48.5 & 39.4 \\
\hline Widowed & 35.6 & 39.5 & 29.4 & 36.2 \\
\hline Divorced/Separated & 11.7 & 17.5 & 17.3 & 18.6 \\
\hline Never Married & 4.6 & 4.0 & 4.8 & 5.9 \\
\hline Living alone $(\%)$ & 36.7 & 25.1 & 28.5 & 24.3 \\
\hline \multicolumn{5}{|l|}{ Employment Status (\%) } \\
\hline Employed & 20.0 & 22.9 & 15.7 & 20.4 \\
\hline Not Working ${ }^{2}$ & 80.0 & 77.1 & 84.3 & 79.6 \\
\hline \multicolumn{5}{|c|}{ Social Support Network Index } \\
\hline \multicolumn{5}{|c|}{ How often visit with friends, either in home or someplace else? (\%) } \\
\hline Never & 8.3 & 15.1 & 16.8 & 21.1 \\
\hline Less than once a month & 8.2 & 17.6 & 14.8 & 16.2 \\
\hline At least once a month & 16.1 & 19.1 & 16.3 & 21.6 \\
\hline At least once a week & 67.5 & 48.2 & 52.1 & 41.1 \\
\hline \multicolumn{5}{|c|}{ How often visit with relatives, either in home or someplace? (\%) } \\
\hline Never & 12.3 & 17.4 & 9.2 & 15.0 \\
\hline Less than once a month & 25.9 & 19.5 & 10.4 & 16.6 \\
\hline At least once a month & 17.2 & 14.4 & 10.7 & 9.6 \\
\hline At least once a week & 44.6 & 48.7 & 69.7 & 58.8 \\
\hline \multicolumn{5}{|c|}{ How often talk on the telephone with family members? $(\%)$} \\
\hline Never & 3.1 & 2.0 & 4.1 & 5.3 \\
\hline Less than once a week & 11.0 & 15.0 & 6.5 & 150 \\
\hline
\end{tabular}

\footnotetext{
${ }^{1}$ Includes individuals working part-time, full-time or are self-employed
}

${ }^{2}$ Includes retired, temporarily out of work, and never worked. 


\begin{tabular}{|c|c|c|c|c|}
\hline & $\begin{array}{l}\text { White non- } \\
\text { Hispanic } \\
(\mathrm{N}=895)\end{array}$ & $\begin{array}{r}\text { Black non- } \\
\text { Hispanic } \\
(\mathrm{N}=201)\end{array}$ & $\begin{array}{r}\text { Cuban } \\
(\mathrm{N}=67 !)\end{array}$ & $\begin{array}{r}\text { Non-Cuban } \\
\text { Hispanic } \\
(\mathrm{N}=188)\end{array}$ \\
\hline About once a week & 14.1 & 6.5 & 6.3 & 7.5 \\
\hline At least a few times a week & 71.8 & 76.5 & 83.2 & 72.2 \\
\hline \multicolumn{5}{|c|}{ How often talk on the telephone with friends? $(\%)$} \\
\hline Never & 4.2 & 6.5 & 7.4 & 9.8 \\
\hline Less than once a week & 8.9 & 11.1 & 11.2 & 17.9 \\
\hline About once a week & 9.2 & 11.6 & 11.2 & 10.3 \\
\hline At least a few times a week & 77.7 & 70.9 & 70.2 & 62.0 \\
\hline \multicolumn{5}{|c|}{ Intensity of Social Contact with Family \& Friends } \\
\hline Mean & 9.55 & 8.99 & 9.59 & 8.60 \\
\hline $0 \ldots 3(\%)$ & 2.5 & 4.2 & 2.8 & 5.5 \\
\hline $4-6(\%)$ & 9.8 & 14.6 & 11.8 & 15.4 \\
\hline $7-9(\%)$ & 29.9 & 32.8 & 24.1 & 37.9 \\
\hline $10-12(\%)$ & 57.8 & 48.4 & 61.3 & 41.2 \\
\hline \multicolumn{5}{|l|}{ Religious Affiliation (\%) } \\
\hline Protestant & 33.0 & 84.8 & 11.1 & 15.0 \\
\hline Catholic & 20.7 & 5.6 & 79.0 & 71.7 \\
\hline Jewișh & 33.7 & 1.0 & 0.3 & 1.1 \\
\hline No Preference & 7.9 & 2.0 & 4.6 & 4.8 \\
\hline Other $^{3}$ & 4.7 & 6.6 & 4.9 & 7.5 \\
\hline \multicolumn{5}{|l|}{ lmportance of Religion in Life? (\%) } \\
\hline Very Important & 49.4 & 89.4 & 75.5 & 81.4 \\
\hline Somewhat Important & 28.5 & 7.5 & 15.5 & 9.0 \\
\hline Not Very Important & 22.1 & 3.0 & 9.0 & 9.6 \\
\hline \multicolumn{5}{|c|}{ HEALTH BELIEFS } \\
\hline \multicolumn{5}{|c|}{ is health a very/somewhat or not serious problem area for you? $(\%)$} \\
\hline Very serious problem area & 10.8 & 15.0 & 30.7 & 31.2 \\
\hline Somewhat serious problem area & 21.2 & 26.5 & $\mathbf{1 7 . 8}$ & 17.2 \\
\hline Not Serious problem area & 68.0 & 58.5 & 51.5 & 51.6 \\
\hline \multicolumn{5}{|c|}{$\begin{array}{l}\text { In the future would you say that you are very/somewhat or not very worried about loss of independence due } \\
\text { to physical deterioration? }(\%)\end{array}$} \\
\hline Very Worried & 15.3 & 15.8 & 51.6 & 47.8 \\
\hline Somewhat Worried & 32.3 & 26.8 & 13.0 & 24.2 \\
\hline Not Very Worried & 52.3 & 57.4 & 35.4 & 28.0 \\
\hline \multicolumn{5}{|c|}{$\begin{array}{l}\text { In the future would you say that you are very/somewhat or not very worried about loss of independence due } \\
\text { to mental deterioration? }(\%)\end{array}$} \\
\hline Very Worried & 11.1 & 10.3 & 53.3 & 49.2 \\
\hline Somewhat Worried & 21.7 & 16.9 & 10.8 & 15.7 \\
\hline Not Very Worried & 67.2 & 72.8 & 35.9 & 35.1 \\
\hline \multicolumn{5}{|c|}{ Life satisfaction in the past 6 months ( $\%$ ) } \\
\hline Very Satisfied & 43.8 & 47.9 & 46.8 & 45.2 \\
\hline Mostly Satisfied & 42.0 & 40.1 & 36.6 & 38.7 \\
\hline Mostly Dissatisfied & 9.4 & 6.8 & 12.7 & 10.2 \\
\hline Very Dissatisfied & 4.8 & 5.2 & 3.9 & 5.9 \\
\hline
\end{tabular}

\footnotetext{
"Includes "Christian," "Spiritual," and "Other, not coded."
} 
Table 5.5: Descriptive Statistics on Enabling Measures for Four Racial/Ethnic Groups of Elders Residing in Miami-Dade County, FL

\begin{tabular}{|c|c|c|c|c|}
\hline & $\begin{array}{l}\text { White non- } \\
\text { Hispanic } \\
(\mathrm{N}=895)\end{array}$ & $\begin{array}{r}\text { Black non- } \\
\text { Hispanic } \\
(\mathrm{N}=201)\end{array}$ & $\begin{array}{r}\text { Cuban } \\
(\mathrm{N}=671)\end{array}$ & $\begin{array}{r}\text { Non-Cuban } \\
\text { Hispanic } \\
(\mathrm{N}=188)\end{array}$ \\
\hline \multicolumn{5}{|l|}{ Personal/Family Resources } \\
\hline \multicolumn{5}{|l|}{ Household Income (\%) } \\
\hline Less than $\$ 10,000$ per year & 12.1 & 40.3 & 42.2 & 34.6 \\
\hline$\$ 10,000$ per year or more & 58.3 & 25.4 & 27.4 & 34.0 \\
\hline No answer & 29.6 & 34.3 & 30.4 & 31.4 \\
\hline \multicolumn{5}{|l|}{ Health Insurance Coverage } \\
\hline Medicare Part A Only & 4.5 & 9.3 & 7.6 & 3.9 \\
\hline Both Medicare Part A and Part B & 67.6 & 58.2 & 68.4 & 62.4 \\
\hline No Medicare Coverage, Age 65+ years & 7.1 & 6.7 & 6.3 & 11.6 \\
\hline No Medicare Coverage, less than 65 years & 20.8 & 25.8 & 17.7 & 22.1 \\
\hline Medicaid Coverage & 3.6 & 22.4 & 35.8 & 33.3 \\
\hline No Health lnsurance of any type & 4.5 & 10.9 & 9.2 & 17.0 \\
\hline HMO Plan Coverage & 24.8 & 34.2 & 24.9 & 15.1 \\
\hline $\begin{array}{l}\text { Mean Duration of Residence in Miami-Dade } \\
\text { County (years) }\end{array}$ & 31.14 & 39.62 & 19.27 & 16.44 \\
\hline $\begin{array}{l}\text { Fuli-time Resident of Miami-Dade County } \\
(\%)\end{array}$ & 85.5 & 96.5 & 98.4 & 96.8 \\
\hline $\begin{array}{l}\text { Regular source of health care from a private } \\
\text { physician }(\%)\end{array}$ & 70.1 & 47.2 & 49.8 & 54.7 \\
\hline \multicolumn{5}{|l|}{ Community Resources } \\
\hline $\begin{array}{l}\text { Ilospital Bed Ratio per } 1000 \text { population in } \\
\text { PSA }\end{array}$ & 4.15 & 4.92 & 4.83 & 4.69 \\
\hline $\begin{array}{l}\text { Primary Care Physician Ratio per } 1000 \\
\text { population in PSA }\end{array}$ & 2.01 & 1.22 & 2.30 & 2.05 \\
\hline Proportion Black in zip code & 0.1266 & 0.5100 & 0.0668 & 0.1380 \\
\hline Proportion Hispanic in zip code & 0.3634 & 0.2693 & 0.7436 & 0.5811 \\
\hline Proportion Elder in zip code & 0.2554 & 0.1459 & 0.2288 & 0.2020 \\
\hline $\begin{array}{l}\text { Proportion Living Below Federal Poverty } 1 \text { ine } \\
\text { in zip code }\end{array}$ & 0.1448 & 0.2717 & 0.1965 & 0.1836 \\
\hline Median Household Income in zip code & $\$ 30,965.08$ & $\$ 23,798.99$ & $\$ 25,018.01$ & $\$ 27,850.77$ \\
\hline Per Capita Income in zip code & $\$ 18.276 .86$ & $\$ 10,806.96$ & $\$ 11,738.38$ & $\$ 13,646.80$ \\
\hline
\end{tabular}


Table 5.6: Descriptive Statistics on Need Measures for Four Racial/Fthnic Groups of Elders Residing in Miami-Dade County, FL.

\begin{tabular}{|c|c|c|c|c|}
\hline & $\begin{array}{l}\text { White non- } \\
\text { Hispanic } \\
(\mathrm{N}=895)\end{array}$ & $\begin{array}{r}\text { Black non- } \\
\text { Hispanic } \\
(\mathrm{N}=201)\end{array}$ & $\begin{array}{r}\text { Cuban } \\
(\mathrm{N}=671)\end{array}$ & $\begin{array}{r}\text { Non-Cuban } \\
\text { Hispanic } \\
(\mathrm{N}=188)\end{array}$ \\
\hline \multicolumn{5}{|l|}{ Perceived Physical Health Status (\%) } \\
\hline Excellent & 33.6 & 16.8 & 22.9 & 31.9 \\
\hline Good & 40.7 & 40.1 & 39.7 & 27.6 \\
\hline Fair & 18.7 & 28.4 & 26.9 & 31.4 \\
\hline Poor & 7.0 & 14.7 & 10.5 & 9.2 \\
\hline \multicolumn{5}{|l|}{ Perceived Emotiona! Health Status (\%) } \\
\hline Excellent & 35.4 & 23.5 & 33.2 & 41.6 \\
\hline Good & 47.1 & 45.0 & 38.6 & 30.8 \\
\hline Fair & 13.5 & 25.5 & 23.7 & 24.3 \\
\hline Poor & 4.0 & 6.0 & 4.5 & 3.2 \\
\hline $\begin{array}{l}\text { Mean Number of Difficulties with Activities of } \\
\text { Daily living (ADL) }\end{array}$ & 0.28 & 0.33 & 0.36 & 0.54 \\
\hline $\begin{array}{l}\text { Mean Number of Difficulties with Instrumental } \\
\text { Activities of Daily Living (IADL) })^{2}\end{array}$ & 0.51 & 0.87 & 0.62 & 0.84 \\
\hline $\begin{array}{l}\text { Mean Number of Difficulties with Mobility } \\
\text { within the home } \\
\text { 3 }\end{array}$ & 0.37 & 0.56 & 0.40 & 0.47 \\
\hline \multicolumn{5}{|l|}{ ADL/1ADL/Mobility Index (\%) } \\
\hline No Problems & 67.8 & 50.7 & 59.6 & 54.3 \\
\hline One Problem & 10.3 & 17.4 & 14.6 & $\$ 2.2$ \\
\hline 2.3 Problems & 10.5 & 12.9 & 12.4 & 15.4 \\
\hline 4-16 Problems & 11.4 & 18.9 & 13.4 & 18.1 \\
\hline
\end{tabular}

\footnotetext{
${ }^{1}$ Activitjes of Daily Living (ADL) are: Eating, Dressing, Bathing, Toileting, and Getting in/out of bed/chair.

${ }^{2}$ Instrumental Activities of Daily Living (IADL) are: Preparing meals, Shopping for groceries or clothes. Managing money and paying bills, Dialing and using the telephone, Doing laundry, Doing other housework, Getting to the places needing to go, Taking care of appearance, and Taking medicine. ${ }^{3}$ Mobility within the home includes: Walking and Getting up and down stairs.
} 
Figure 5.1: Location of Area Agency on Aging Sub-areas within Miami-Dade Counly, Florida

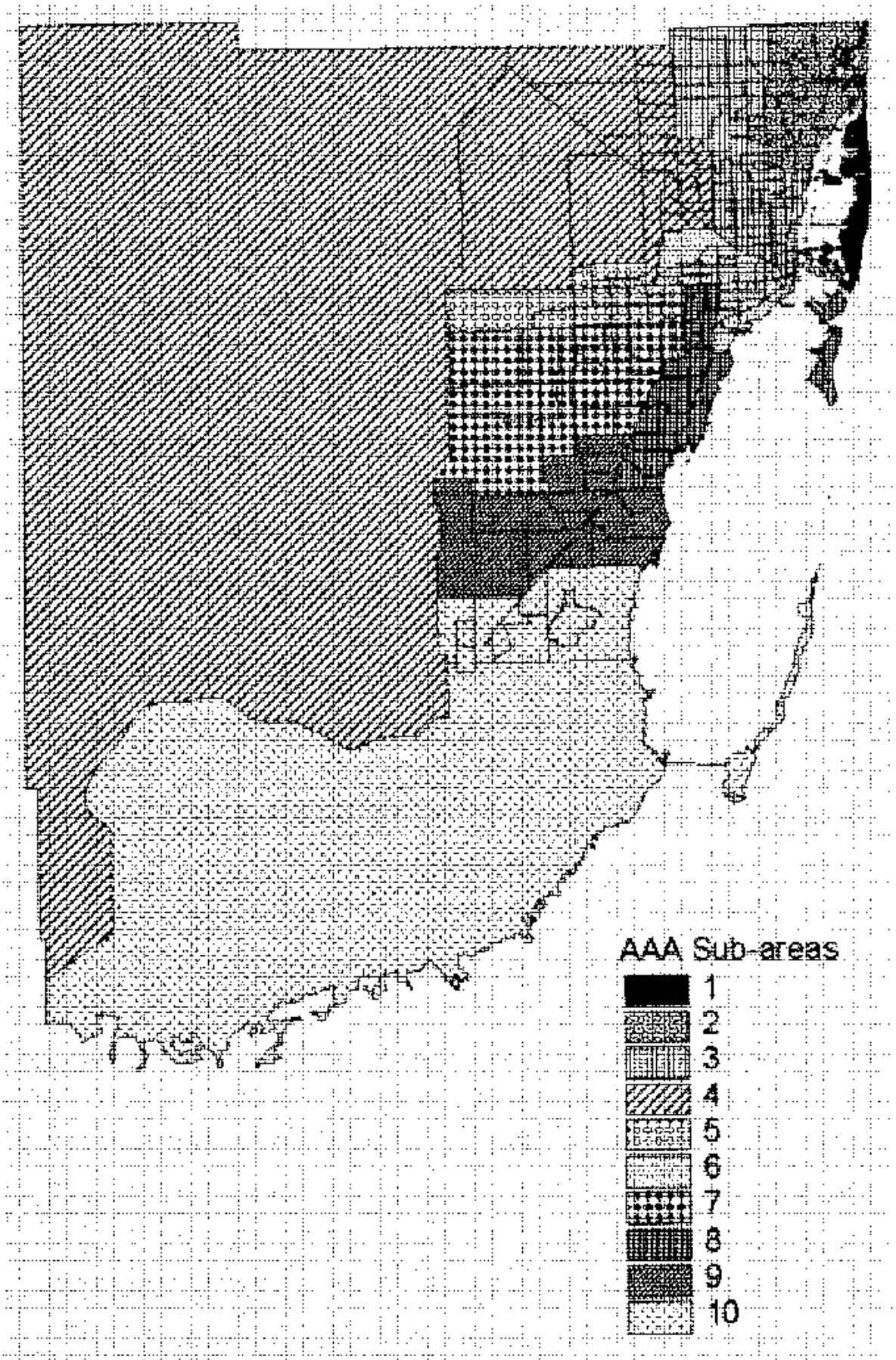




\section{CHAPTER 6: QUALITATIVE ANALYSIS OF HEALTH IN THE LIVES OF ELDERS IN MIAMI-DADE COUNTY, FL}

Given the increasing racial/ethnic diversity within the elder population, the focus of this chapter will be on examining results of the field work done in Dade County, FL. This chapter will focus on answering the first research question: "Are there differences in perception of the importance of health and health care in the lives of elders by cultural groups?" The hypothesis that will be tested qualitatively is that discourse on health and health care issues are significantly different by racial/cthnic groups because of significantly different concerns in their lives. Further, there will be an examination of other arcas of concerns in the lives of Miami-Dade County elders expressed in the focus groups. This information will allow for a better understanding of the overall importance of health and health care issues and the linkages within the lives of elders. Hcalth and health care issues will not be examined within a vacuum, rather they will be examined as one aspect in the lives of elders.

\section{DATA AND METHODOLOGY}

An overview of the data to be examined was given in Chapter 5 . In this chapter there will be a more detailed examination of the focus group data. The focus group discourse of three racial/ethnic groups of elders will be analyzed: white, black, and Hispanic, primarily Cuban. These three racial/ethnic groups of elders have had different life experiences, as well as different expectations of the aging process. Unlike other areas in the United States, Miami-Dade County already has a very heterogeneous elder 
population (60+ years). Thus, Miami-Dade County provides a unique opportunity to study in microcosm the future complex interaction of age structure and racial/ethnic diversity on health care service utilization that will come to characterize the aging population in the United States.

As documented in the previous chapter, the focus group data used in this study was originally collected for a needs assessment of elders in Dade County. Elders were defined as individuals age 60 years or older. The purpose of the needs assessment was to provide data for the Alliance for Aging Inc., the Area Agency on Aging for Dade and Monroe Counties (Alliance). The Alliance needed this information to assist in its comprehensive planning of services for elders in Dade County for the next 5 to 10 years. The needs assessment was based on three information sources: a) ten focus groups of elders, informal caregivers, volunteers, and service providers throughout Dade County; b) a survey of 1,900 elders in Dade County, of which 1,800 interviews were conducted by telephone and 100 interviews were conducted face-to-face with individuals who are lowincome and/or of minority status; and c) interviews with approximately $40 \mathrm{key}$ informants particularly knowledgeable about needs, services, and policy regarding elders in Dade County. However, for the purpose of this chapter I will be relying on six of the ten focus groups for discussion of the meaning of aging and living in a multicultural urban environment as an older person.

The focus groups were conducted during October 1993. The insights gained from the focus group made the final survey questionnaire sensitive to the cultural and economic characteristics of Dade County's multicultural elder population and, thereby. elicit the most relevant information on their needs and concerns. 
For the purpose of the needs assessment, ten focus groups were completed. Four out of the ten were special focus groups. These four groups were: service providers for elders in Dade County, Haitian elders (aged 50 years and over), elders who serve as volunteers on the Alliance's Advisory Council, and informal caregivers of elders diagnosed with Alzheimer's Disease or other dementia syndromes. The remaining six focus groups of elders were chosen in order to provide a mix of participants according to age (60-74, 75+), ethnicity (African-American, Hispanic, Anglo), socio-economic status (low-income, middle-income, upper-middle income) and geographic locale (north Dade, south Dade). The groups assembled were essentially "samples of convenience" identified by the Alliance. However, the groups assembled were adequately representative for gaining insight into how elders from these sub-populations might react to questions on topics covered by telephone and face-to-face interviews. Appendix E has the focus group interview transcripts.

\section{Identification of the Focus Groups and Entry into the Field}

Two approaches to identifying focus group participants were considered originally. The first approach was to randomly generated phone numbers to identify 10 to 12 individuais who were 60 years of age or over and fit the targeted characteristics. Further these individuals would live within each of the 10 sub-areas, and would be willing to come to a central location for the focus group meeting. The second approach was to work through community organization, such as churches and other community organizations, to identify potential participants in each of the 10 sub-areas. 
$\Lambda$ fter considering the pros and cons of these respective approaches, it was

concluded that the second approach would be more efficient and less costly overall.

Working through community organizations, it was reasoned, would also address three other considerations that could not be easily addressed by the first approach. The first consideration is that focus groups would be composed of older people some of whom may be poor, in poor health, and may have limited access to transportation. Secondly, all available research indicates that elders in Dade County are very fearful of crime and therefore, may be suspicious of strangers. Finally, centrally located venues for the group meetings would have to be solicited.

Within each focus group, to assure privacy and confidentiality of individual participants, flower names were assigned to or chosen by each participant. It was felt that participants may be more willing to talk about their concerns and needs more freely with this gesture to protect their privacy and confidentiality.

\section{Descriptions of Focus Groups}

For the purposes of this chapter I will be analyzing only six of the ten focus groups. The description of these six focus groups and host organizations are as follows:

- working class Cubans primarily between the ages of 65 to 69 years and organized through Little Havana Activities and Nutrition Centers, Inc., conducted in Spanish (Little Havana); 
- Cubans of lower socio-economic status, primarily over the age of 70 years and organized through the Hialeah Housing Authority conducted in Spanish (Hialeah Housing);

- African-American elders of lower socio-economic status between the ages of 60 and 69 years, organized through James E. Scott Community Association (JESCA);

- African-Americans in the Richmond Heights area of south Dade, no restriction on age or on socio-economic status, organized through the Glendale Missionary Baptist Church (Richmond Heights);

- middle and upper-middle class white, non-Hispanics, over the age of 75 years organized through the Northeast Dade Coalition (Northeast Dade);

- elders living in and around Homestead, the area most damaged by Hurricane Andrew, joint organized by Musselwhite Senior Center and the Metro-Dade Community Action Agency (CAA), no restriction on age or socio-economic status (participants from Musselwhite were Anglo, and participants identified by the CAA were a mix of blacks and Hispanics of varying national origin) (Homestead).

\section{Description of Dade County Elder Population}

Socio-demographic characteristics of Miami-Dade County elders using U.S.

Census data were documented in Chapter 4 . However, descriptions such as the elders perceptions on the seriousness of current problems and concerns for the future, as well as self-rated health status and functional ability results from the telephone survey portion of 
the Dade County Needs Assessment will be examined as a background to focus group discourse that will be the primary focus of this chapter.

\section{Seriousness of Current Problems and Concerns for Future ${ }^{\mathfrak{t}}$}

The four "current" problems most frequently mentioned (out of a list of seven potential problems areas) as being very or somewhat serious were: 1) fear of crime (56.2 percent); 2) money (42.2 percent); 3) health (40.5 percent); and 4) personal/family stress ( 35.1 percent). Table 6.1 shows the top four ordered responses by elders in each of the racial/ethnic groups with respect to perceived potential problem areas. Black nonHispanics were the only ethnic group that did not rank fear of crime most frequently; however, they ranked it second behind money.

Table 6.1: Top Four Ordered Responses of Elders on Perceived Potential Problem Arcas as Very or Somewhat Serious by Race/Ethnicity of Respondent (Percentage)

\begin{tabular}{|c|c|c|c|c|}
\hline Rank-order & $\begin{array}{l}\text { White non- } \\
\text { Hispanic }\end{array}$ & $\begin{array}{l}\text { Black non- } \\
\text { Hispanic }\end{array}$ & $\overline{C u b a n}$ & $\begin{array}{l}\text { Non-Cuban } \\
\text { Hispanic }\end{array}$ \\
\hline First & $\begin{array}{l}\text { Fear of Crime } \\
(45.0 \%)\end{array}$ & Money $(52.4 \%)$ & $\begin{array}{l}\text { Fear of Crime } \\
(70.6 \%)\end{array}$ & $\begin{array}{l}\text { Fear of Crime } \\
(61.4 \%)\end{array}$ \\
\hline Second & Health $(31.6 \%)$ & $\begin{array}{l}\text { Fear of Crime } \\
(45.0 \%)\end{array}$ & Money $(53.3 \%)$ & Money $(54.1 \%)$ \\
\hline Third & $\begin{array}{l}\text { Personal/Family } \\
\text { Stress }(27.1 \%)\end{array}$ & Tlealth $(39.0 \%)$ & Health $(48.5 \%)$ & $\begin{array}{l}\text { Personal/ Family } \\
\text { Stress }(48.9 \%)\end{array}$ \\
\hline Fourth & Money $(25.9 \%)$ & $\begin{array}{l}\text { Personal/ Family } \\
\text { Stress }(23.6 \%)\end{array}$ & $\begin{array}{l}\text { Personal/ Family } \\
\text { Stress }(43.5 \%)\end{array}$ & Health $(48.4 \%)$ \\
\hline
\end{tabular}

Elders were also asked about concerns for their future. The five most frequently mentioned (out of a list of nine potential concerns) as being very or somewhat serious were: 1) loss of independence due to physical deterioration $(56.6 \%) ; 2)$ being a victim of a crime, other than fraud $(56.4 \%) ; 3)$ mental deterioration $(47.7 \%) ; 4)$ going to a nursing 
home (47.1\%); and 5) becoming a financial burden (46.9\%). Table 6.2 shows that the patterns of concerns differ somewhat by racial/ethnic group. For example, the greatest percentage of Cuban elders reported being very or somewhat seriously worried about being a victim of crime other than fraud ( 73.5 percent), while loss of independence due to physical deterioration was fifth ( 64.6 percent). In comparison, the greatest percentage of white non-Hispanic elders reported loss of independence due to physical deterioration $(49.0 \%)$ and being a victim of crime other than fraud was second $(41.8 \%)$.

Table 6.2: Top Five Ordered Responses of Elders Who are Very or Somewhat Worried About Problems in Their Future by Race/Ethnicity of Respondent

\begin{tabular}{|c|c|c|c|c|}
\hline Rank-order & $\begin{array}{l}\text { White non- } \\
\text { Hispanic }\end{array}$ & $\begin{array}{l}\text { Black non- } \\
\text { Hispanic } \\
\end{array}$ & Cuban & $\begin{array}{l}\text { Non-Cuball } \\
\text { Hispanic } \\
\end{array}$ \\
\hline First & $\begin{array}{l}\text { Loss of } \\
\text { independence due } \\
\text { to physica! } \\
\text { deterioration } \\
(49.0 \%)\end{array}$ & $\begin{array}{l}\text { Loss of } \\
\text { independence due } \\
\text { to physical } \\
\text { deterioration } \\
(43.0 \%)\end{array}$ & $\begin{array}{l}\text { Being a victim of } \\
\text { crime, other than } \\
\text { fraud }(73.5 \%)\end{array}$ & $\begin{array}{l}\text { Loss of } \\
\text { independence due } \\
\text { to physical } \\
\text { deterioration } \\
(72.0 \%)\end{array}$ \\
\hline Second & $\begin{array}{l}\text { Being a victim of } \\
\text { crime, other than } \\
\text { fraud }(41.8 \%)\end{array}$ & $\begin{array}{l}\text { Being a victim of } \\
\text { crime, other than } \\
\text { fraud }(39.3 \%)\end{array}$ & $\begin{array}{l}\text { Becoming a } \\
\text { financial burden } \\
(66.5 \%)\end{array}$ & $\begin{array}{l}\text { Being a victim of } \\
\text { crime, other than } \\
\text { fraud }(69.9 \%)\end{array}$ \\
\hline Third & $\begin{array}{l}\text { Loss of } \\
\text { independence due } \\
\text { to mental } \\
\text { deterioration } \\
(34.1 \%)\end{array}$ & $\begin{array}{l}\text { Becoming a } \\
\text { financial burden } \\
(35.6 \%)\end{array}$ & $\begin{array}{l}\text { Being a victim of } \\
\text { fraud or con-artist } \\
(65.5 \%)\end{array}$ & $\begin{array}{l}\text { Becoming a } \\
\text { financial burden } \\
(67.9 \%)\end{array}$ \\
\hline Fourth & $\begin{array}{l}\text { Going to a } \\
\text { nursing home } \\
(32.9 \%)\end{array}$ & $\begin{array}{l}\text { Being a victim of } \\
\text { fraud or con-artist } \\
(29.0 \%)\end{array}$ & $\begin{array}{l}\text { Going to a } \\
\text { nursing home } \\
(65.1 \%)\end{array}$ & $\begin{array}{l}\text { Loss of } \\
\text { independence duc } \\
\text { to mental } \\
\text { deterioration } \\
(64.9 \%)\end{array}$ \\
\hline Fifth & $\begin{array}{l}\text { Becoming a } \\
\text { financial burden } \\
(26.7 \%)\end{array}$ & $\begin{array}{l}\text { Loss of } \\
\text { independence due } \\
\text { to mental } \\
\text { deterioration } \\
(27.3 \%)\end{array}$ & $\begin{array}{l}\text { Loss of } \\
\text { independence due } \\
\text { to physical } \\
\text { deterioration } \\
(64.6 \%)\end{array}$ & $\begin{array}{l}\text { Going to a } \\
\text { nursing home } \\
(61.6 \%)\end{array}$ \\
\hline
\end{tabular}




\section{Health Status and Functional Ability'}

Overall, the majority of telephone survey respondents self-rated their physical health and emotional well-being as excellent or good and their level of satisfaction with life in the last six months as very or mostly satisfied (65.8 percent, 75.6 percent, and 84.7 percent, respectively). Table 6.3 shows self-reported health status for each of the four racial/ethnic groups of elders. Black elders were the least likely to rate their physical or emotional health highly, although they were the most likely to express satisfaction with their lives in the past six months.

Table 6.3: Self-Reported Health Status of Telephone Survey Respondents by Racial/Ethnic Group

\begin{tabular}{|c|c|c|c|c|}
\hline & $\begin{array}{l}\text { White non- } \\
\text { Hispanic }\end{array}$ & $\begin{array}{l}\text { Black non- } \\
\text { Hispanic }\end{array}$ & Cuban & $\begin{array}{l}\text { Non-Cuban } \\
\text { Hispanic }\end{array}$ \\
\hline $\begin{array}{l}\text { Self-reported physical health status as } \\
\text { excellent or good }\end{array}$ & $73.6 \%$ & $55.4 \%$ & $62.6 \%$ & $59.5 \%$ \\
\hline $\begin{array}{l}\text { Self-reported emotional health status as } \\
\text { excellent or good }\end{array}$ & $81.6 \%$ & $68.5 \%$ & $71.9 \%$ & $72.4 \%$ \\
\hline $\begin{array}{l}\text { Very or somewhat satisfied with life in } \\
\text { the last six months }\end{array}$ & $84.9 \%$ & $88.7 \%$ & $83.5 \%$ & $83.9 \%$ \\
\hline
\end{tabular}

Respondents were asked whether it is difficult for them to do a series of specific tasks which are classified as activities of daily living (ADLs: bathing/showering; dressing; eating; using toilet; and getting in/out of bed/chair), instrumental activities of daily living (IADLs: taking medicine; caring for appearance; preparing meals; shopping for groceries or clothes; managing money; dialing or using telephone; doing laundry; doing other household chores; and getting places needed to go) and mobility activities (walking and getting up/down stairs). Table 6.4 shows the distribution of self-reported responses to the number of difficult ADLs, IADLs and mobility limitations by 
racial/ethnic group of elder respondent. As is the case nationally, regardless of racial/ethnic group, over half the elder respondents report having no difficulties with ADLs, IADLs or mobility activities. However, there are variations between racial/ethnic groups of elders. For example, white non-Hispanics are the most likely to report absence of difficulties with ADLs, IADLs and mobility limitations despite their older mean age (84.3 percent, 80.0 percent, and 75.1 percent, respectively). Non-Cuban Hispanics are the least likely to report no difficulty (70.2 percent) and the most likely to have trouble with three or more ADLs (6.4 percent), as well as both mobility limitations (15.4\%). Black non-Hispanics, on the other hand, are the most likely to report difficulty with three or more IADLs $(12.6 \%)$.

Table 6.4: Self-Reported Functional Ability of Telephone Survey Respondents by Racial/Ethnic Group

\begin{tabular}{|c|c|c|c|c|}
\hline & $\begin{array}{l}\text { White non- } \\
\text { Hispanic }\end{array}$ & $\begin{array}{l}\text { Black non- } \\
\text { Hispanic }\end{array}$ & Cuban & $\begin{array}{l}\text { Non-Cuban } \\
\text { Hispanic }\end{array}$ \\
\hline \multicolumn{5}{|c|}{ Self-Reported Number of Difficulties with Activities of Daily Living (ADLs)' } \\
\hline $\begin{array}{l}\text { No ADLs } \\
1 \text { to } 2 \text { ADLs } \\
3+\text { ADLs }\end{array}$ & $\begin{array}{r}84.3 \% \\
11.8 \% \\
3.9 \%\end{array}$ & $\begin{array}{r}81.5 \% \\
15.9 \% \\
2.7 \%\end{array}$ & $\begin{array}{r}78.1 \% \\
18.3 \% \\
3.6 \%\end{array}$ & $\begin{array}{r}70.2 \% \\
23.4 \% \\
6.4 \%\end{array}$ \\
\hline \multicolumn{5}{|c|}{ Self-Reported Number of Difficulties with Instrumental Activities of Daily Living (IADLs) ${ }^{2}$} \\
\hline $\begin{array}{l}\text { No IADLs } \\
1 \text { to } 2 \text { IADLs } \\
\text { 3+ IADLs }\end{array}$ & $\begin{array}{r}80.0 \% \\
12.0 \% \\
8.0 \%\end{array}$ & $\begin{array}{l}68.6 \% \\
18.7 \% \\
12.6 \%\end{array}$ & $\begin{array}{r}74.7 \% \\
17.1 \% \\
8.2 \%\end{array}$ & $\begin{array}{l}71.8 \% \\
17.0 \% \\
11.2 \%\end{array}$ \\
\hline \multicolumn{5}{|c|}{ Self-Reported Mobility Limitations ${ }^{3}$} \\
\hline $\begin{array}{l}\text { No mobility limitations } \\
1 \text { mobility limitation } \\
2 \text { mobility limitations }\end{array}$ & $\begin{array}{l}75.1 \% \\
11.2 \% \\
13.7 \%\end{array}$ & $\begin{array}{l}60.9 \% \\
24.6 \% \\
14.5 \%\end{array}$ & $\begin{array}{l}72.4 \% \\
14.8 \% \\
12.8 \%\end{array}$ & $\begin{array}{l}68.1 \% \\
16.5 \% \\
15.4 \%\end{array}$ \\
\hline
\end{tabular}

${ }^{1}$ ADLs are: Bathing/Showering; Dressing/Undressing; Eating; Using Toilet; and Getting In/Out of Bed Chair.

${ }^{2}$ LADLs are: Taking medicine; Caring for appearance; Preparing meals; Shopping for groceries or clothes; Managing money; Dialing/using telephone; Doing laundry; Doing other housework; and Getting places needed to go.

${ }^{3}$ Mobility limitations are defined as walking or getting up'down stairs. 


\section{FINDINGS FROM FOCUS GROUPS OF ELDERS IN MIAMI-DADE COUNTY}

Although from the telephone survey results, there are generally the same types of concerns expressed by elders. What is most striking are the differences that appear in the ways that these concerns were expressed. As will become apparent many of the issues, concerns and needs of elders are intertwined and depending upon the life experience of an elder, different aspects of these intertwined issues will come to the forefront, while for other elders they will stay in the background. The two broad topic areas that will be focused on from the focus group protocols are the discussion of likes/dislikes about living in Dade County, FL and second, how do elders talk about their concerns and what do they choose to see as the main problems.

In examining the results from the six focus groups, there are two aspects of the field work that I want to address. The first is, Are there racial/ethnic differences in defining aging? The second aspect is, How does community influence culturally diverse elders in the aging process? The structure for the rest of the chapter will be as follows. First, there will be an examination of how elders talked about living in Dade County and what they liked (or disliked) about living in Dade County. Following this the examination will turn to the concerns and problems that elders thought were important. Health and health care issues will be the last of the five concerns discussed in this section. In the conclusion of this chapter, there will be a discussion of results in light of the research question originally posed: "Are there differences in perception of the importance of health and health care in the lives of elders by cultural groups?" 


\section{Living in the community and Dade County}

The majority of elder participants cited, in some fashion, that weather in Dade County was an important reason why they liked living in the community. For the Homestead group, it was directly stated as "climate," while the Richmond Heights elders spoke of it as "sunshine," and the Northeast Dade elders spoke of it as "weather." Interestingly, elders at the JESCA group only mentioned weather/climate when speaking about what they liked about living in Dade County, but not when speaking about what they liked about living in their neighborhood.

There were also differences in the way elders spoke about their community. Northeast Dade elders spoke about casualness of the lifestyle and not needing to dress-up when going out for dinner. Another elder in Northeast Dade group stated,

Equals of age group, weather allows getting together, convenience to shopping, accessible to wide variety of activities.

In comparison, elders in Homestead focused on neighbors and friends are like family. Part of this difference may be attributable to the length of time that elders have lived in Dade County and part of this may be because many of the Northeast Dade elders do not have family members living in the area. The majority of Northeast Dade elders moved down to Dade County from the north post retirement. At first they came for the just the winter months, keeping up a residence in the north for the summer to be with their children. However, as time went on they become more permanently entrenched in Dade County, giving up their homes in the north. In comparison, Homestead elders are less affluent than Northeast Dade elders and thus did not have the luxury of keeping up two homes, one in Florida and one in the north. The types of homes that Homestead 
elders lived in were usually single family homes in which they were responsible for the both inside and outside upkeep.

Richmond Heights elders also talked about close-knittedness of their community.

Close knit neighborhood, well kept, well disciplined, pride in neighborhood, crime rate is lower, although still a concern. There is an investment of time and money in the neighborhood. (Marigold)

The close-knittedness of the neighborhood, ... it means a lot to the neighborhood. The church is important to the neighborhood. It's friendly. (Geranium)

Elders in Richmond Heights and Homestead lived in communities that were primarily single family homes. Thus, they seemed to value the closeness of their neighbors.

Further down on the economic scale, elders in the JESCA group in comparison lived in high-rise apartments. They were not responsible for the outside upkeep of their apartments. These elders talked about the value of security and quietness of where they lived. Like the Richmond Heights and Homestead elders, they were primarily long-time, full-time residents of Dade County.

It was also interesting to note the differences in activities that each group talked about. For Northeast Dade elders, activities centered on entertainment and activities that relieved boredom for themselves. They did not seem to be particularly community aware or community building. For example,

Theatre Performing Arts, they are here in the arca, but it is easier for me to go to New York City to see plays, by flying there and seeing them there then to go to downtown Miami to see them in Miami.

While the Richmond Heights elders talked about activities that they were

involved in that were community building and community involvement. One elder spoke of his/her involvement in working with little children. He/she is "teaching them speech 
patterns and behavioral patterns." He/she ended with a sad commentary that, "The older children have drifted too far." This concern for other generations is not evident in the discourse of elders in Northeast Dade elders.

\section{Concerns \& Problems}

Although, in general, the same types of concerns were expressed by each of the groups of elders, differences appear in the ways that these concerns were expressed. Concerns and problems expressed by elders, in general, fell into five categories: fear of crime; transportation; boredom/social isolation/loneliness; housing issues; and health/health care issues.

\section{Fear of Crime}

Most elders expressed a fear of crime and becoming a victim of crime. For elders in public housing, fear of crime was expressed in terms of their personal security within the building in which they lived.

I've lived here 29 years. I'm scared of crime and the lack of security in the buildings" (Hialeah Housing Authority)

Drug problem, crime problem, needs lights in building; management hasn't done anything about it. Elders can't open their door in the evening; can't go in the elevator. (JESCA)

In comparison, elders living in Northeast Dade, a higher-income, condominiumdominated area, expressed this fear in terms of becoming victims when outside of their residences, e.g., while shopping. When Northeast Dade elders were asked how it affects their lives, one elder participant stated "Don't go out at night." While another stated "... don't congregate outside after dark, ... calling the police isn't useful." All seemed to 
focus on crimes against their person. None of their comments on fear of crime focused on burglary within their homes, or fraud and con-artists as was talked about in other focus groups. Possibly the reason for not focusing on burglary within their homes is that they are living in high-rise condominiums which have guards in the lobby as well as sign-in procedures.

Elders in the Richmond Heights did not focus their comments on crime, except ts say that their crime rate was lower. Elders in JESCA were very concerned about crime even though in the initial questions about what they liked about their community many talked about their feeling of security.

Whether expressed initially or only after specific mention by the moderator, fear of crime emerged as a highly important concern of elders in Dade County and appears to serve as a significant deterrent to elders going out and participating in activities. In turn, this increases the sense of isolation that elders may feel. Little Havana elder participants talked about fear of crime as follows,

I live at the entrance of Coconut Grove. I'm scared. I do everything before 6PM and I don't leave my house after 6PM. (Gladiola)

Crime is the first problem. I'm afraid to leave my house to go out at night. I don't put the light on so they don't know if I'm at home. (Orchid)

The areas that were safe are now being invaded by crime. (Sunflower) Elders, especially poorer elders, expressed fear about becoming victims of fraud or con-artists. It was particularly discussed in the Hialeah Housing group of elders when asked about need for services.

I don't need any help at this time. I once was offered milk supplement, but it never came. I called to report it as fraud. When I need help, I call an agency official. I had a cleaning person once, but I stopped that because I was afraid of fraud. The person sent by the agency didn't 
always come and then she wanted me to sign for those house. Now I pay for the cleaning person myself' (Lily)

I'd call an agency but don't want to abuse the system with unnecessary requests" (Magnolia)

\section{Transportation}

Transportation plays a key role in many of the problems and concerns that elders voiced, as well as increasing sense of isolation that elders feel. The ability to drive is important for elders to be able to get around. For many elders, the loss of this ability creates the feeling of losing control over their lives. Therefore transportation is intertwined with elder participation in activities and being able to "age in place." In all focus groups, elders mentioned that there is very little public transportation. Elders in most focus groups stated that there was a need for basic transportation for shopping, health care, and going to senior centers. Elders in the Northeast Dade focus group were fairly active and able to get around. However, they talked about transportation need in terms of "other" elders. One elder said that

Transportation is the primary concern - aging sometimes forces individuals to stop driving, buses would be good but not all areas are served.

Following up on this another participant who identified himself as the President of his condo association stated that,

Even with buses, ...[the] buses need to be accessible to elder women need kneeling buses since they can't get up the stairs.

Transportation issues for JESCA elders were more basic, i.e., shopping for groceries, going to doctor's office or medical treatment. The issue of need for transportation is discussed and embedded with respect to need for health care is an important one. This needs to be further explored. This area of transportation also 
seemed to be a very important issue with respect to receiving health care in Abraham's (1993) ethnography entitled Mama might be better off dead: the failure of health care in urban America.

\section{Boredom/Social Isolation/Loneliness}

Elders identified the perceived breakdown of extended family support systems as a serious problem. The desire among elders to be needed is a growing concern and the threat of no longer being needed may be particularly painful for Hispanic elders from large families. Most Hispanic elder participants said they would have family members helping them if they needed assistance. These same elders were also aware that they could not rely on family members for everything. One Hispanic elder stated,

Family will complement help given by agency. Family cannot give assistance on a daily basis. (Little Havana)

While another stated,

Family can help on a crisis, but not with daily tasks. (Little Havana)

Elders who live alone are not always able to rely on family members to help them for a variety of reasons. A Richmond Heights participant when asked about loneliness said,

Another problem, some elders live alone. Their families are far away. Worrying is another related problem. Another senior need is mental health counseling. Frustration is a problem. Helplessness at not being able to do the things that used to be able to do or need to for daily living needs. Counselors are needed. Other things seniors, especially single seniors - the church should organize activities so that decrease frustration. Also general housekeeping things that require lifting and kneeling down, laundry.

In general, the consensus of Richmond Heights elders was that most elders don't want to go to nursing homes and don't want to live with their children, they just need 
services to help them age in place. JESCA elders also did not want to rely on their children, however for slightly different reasons. For most of the JESCA participants their adult children were also struggling to make ends meet. The elders did not want to be a burden to their adult children. When these elders did receive help from their adult children, as one elder put it,

It's an individual thing, family isn't able to give money, but rapport is important.

For elders in Northeast Dade, family is up north where they retired. When they were asked about family involvement in caregiving, the majority did not have family members living close by. One elder in the Northeast Dade group stated,

Elders don't want to take responsibility for care of other elder neighbors. Elder parents do not want to put pressure on their children.

Another Northeast Dade participants mentioned that many elders come down to Florida for somewhat "selfish" reasons. This was okay when these elders were youngold and not in need of help from family members. However, now that they are growing older, they do not want to take responsibility for other elders and yet they themselves are in need of help from family members. There scemed to be agreement with this assessment of the situation. The elder making this statement was young old and was accompanied by their mother who was $80+$ years old.

In the research literature, social isolation and lack of mental activity tends to be associated with declines in health status. Whether this is because health is failing which leads to non-participation, or that non-participation leads to failing health status, needs to be further explored. This is also a subject that is very sensitive to the individual. Thus, discussion that was heard in the focus groups is only the tip of the iceberg. 


\section{Housing Issues}

Another frequently voiced concern was the lack of affordable and adequate housing. For many elders on limited or fixed incomes, rising rents are a problem exacerbated by Hurricane Andrew and gentrification. Generally, elders on limited fixed incomes lack housing options. Elders with declining physical functioning are concerned about their ability to clean and maintain their homes. They voiced a need for affordable services to help them with heavy cleaning, chores, yard work, and general maintenance of their houses.

[I need] household help. I have a heart condition and my doctor says I shouldn't be over-exerting myself. But what can I do, the house needs vacuuming, laundry needs to get done. Also need gardening help, like lawn mowing. (Homestead)

For participants in public housing, there was much frustration about maintenance of their housing, i.e., outside the facility, absence of hall lights; trash in the hallways or that maintenance and repair work was done slowly.

Cleaning, yard work and general maintenance services need to be affordable for all income levels. One focus group noted that, because of health conditions, elders might need help with a variety of housekeeping and yard work chores.

We don't expect this help to be free but it should be subsidized so that elders who need it can afford it. (Homestead)

Another participant from Homestead elaborated that "most elders can't afford the going rates for housekeeping services." In gencral there was an underlying assumption that access to these types of services would reduce the need for institutionalization and allow elders to age in place even after a deterioration in health has occurred. 
In the Little Havana focus group, one elder said, "Rents keep increasing every

year. I'm concerned that I might not be able to afford future increases." Others seconded this in the group. The various aspects of housing issues and their affects on how elders embed them into their discussion of "aging in place" need to be further explored.

\section{Health and Health Care Issues}

Finally, the discourse that elders in the individual focus groups on issues of health and health care in the lives of elders showed that there were several different but connected issues being brought up in relation to health and long-term care. The three main topics were: the affordability of health insurance; the availability of long-term care or other services that would allow elders to "age in place"; and getting information about health care services. White non-Hispanic elders tended to discuss health issues in terms of increasing population of frail elders in the community, for which Dade County, the State of Florida and the Federal governments are not prepared. One elder said,

Most frail elders who need help have private paid nursing or a homemaker, and if they can't pay [PAUSE] I don't know what they do, I guess they are out of luck.

Black non-Hispanic elders, in comparison tended to discuss aspects of health in terms of the affordability of health insurance and being able to pay for medical services. Elders in Richmond Heights noted that seniors who don't yet qualify for Medicare (i.e., individuals less than 65 years old) need hospital coverage that can cost approximately $\$ 300$ per month in premiums.

Further, for those eligible for Medicare (65+ years), out of pocket expenses (i.e., for prescription medicines, or for services covered only in nursing homes or hospitals, but not in the home) were important concerns. Elders in Little Havana noted that doctor 
coverage isn't enough under Medicare and that many retired persons go without supplemental insurance because they can't afford it. Citizenship factors while not a factor at the time of the focus group are now becoming an issue. The majority of foreignborn elders in Dade County are Cuban, and for the most part they are ineligible for Medicare because "few have accumulated the necessary benefits ... to be entitled to Medicare" (Queralt, 1983). Medicare coverage may be purchased by individuals 65 years or older if they have resided in the United States for a minimum of 5 years, regardless of their employment status. However, the cost of this insurance coverage for many Cuban elders (as well as other immigrant elders) can be too expensive.

For those in need of health care and not able to pay the premiums, there is Medicaid. However, Medicaid does not cover certain medical services that are deemed as important for the Hispanic culture, such as home health care, which would allow many frail elders to reside within the family setting. Although there was a general attitude that most elders did not want to go to nursing homes, there was also an acceptance that nursing homes may be necessary because of increasing frailty and illness. However, it was in the elders in the Little Havana focus group in which most of the adamancy of not going to a nursing home was voiced. However, one participant from Little Havana illustrated the concern about nursing homes in general: the participant's mother "died of despair" when the participant had to go out of town. A nursing home staff member had told the mother that she [the participant] "was not coming back forever!" The participant stated that the nursing home personnel seemed to "lack human sentiment" and that the "training and behavior has a lot to be desired." Anecdotes of this nature enhance elders' 
fear of placement in a "home." However, it was also an elder in the Little Havana focus group who cares for a spouse with Alzheimer's who said,

I've lived in this country 33 years; I'm retired, but I need to work so I can survive. My [SPOUSE] has Alzheimer's and is in an ACLF, but [he/she] is in need of a nursing home. I've been trying forever to get [him/her] into a nursing home.

In comparison, black non-Hispanic focus groups made little mention of concern over going to a nursing home. For the black non-Hispanic participants, they were healthy enough not to "need" nursing home services. Most of the Northeast Dade elders could pay for services for care.

Elders in the Northeast Dade focus group only brought up the issues of nursing home placement as a last resort. For example, one participant brought a spouse to the focus group. The spouse had Alzheimer's. The elder said that he/she was able to pay for services for the care of the spouse currently, but didn't know what to do if the caretaker had a problem. However, the underlying current was that elders were fearful that if they couldn't take care of themselves or could not pay for care that their families would put them in a nursing home. Many of these elders did not have family members close by to help. What usually happens to these elders is that they stay in their condos getting more isolated until they have a stroke or break a hip and then their adult children have to step in. The adult children either put them in a nursing home in Dade County, FL, or bring them back to the Northeast. It was also mentioned that elders did not want to take responsibility for the care of another elder neighbor. One Northeast Dade elder participant mentioned that,

... many elderly came down to Florida for somewhat selfish reasons when they were young-old and not in need of help from family members, but 
now as they are growing older, they do not want to take responsibility for

other elders and they themselves are in need of help from family members.

\section{SUMMARY AND DISCUSSION}

In light of these results, it is evident that there are differences in perceptions of the importance of health and health care in the lives of elders by cultural groups, as well as socio-economic status. Most elders in the focus groups were experiencing many of the same problems with the biological aging process. The differences in the discourse by focus groups show pointedly that the differences were based on their interpretation of the information, although not always based on racial/ethnic classification. Elders' interpretation depended on their previous life experiences and current socio-economic, as well as familial situations and cultural expectations. As was pointed out in Chapter 3 , an individual brings to the aging process their previous life experiences to cope with their changing needs, i.e., getting the help/support they need; dealing with the formal care system, availability of family for informal support when needed, general coping skills in stressful situations. Thus, these previous experiences of the aging individual will affect their current interpretation and expression of concerns and needs. Again, it is worthwhile to point out that the meaning of aging is dependent upon the particular frame of reference previously and currently lived by the elder. Overall the current discourses of elders do not necessarily oppose one another, rather as Portes \& Stepick (1993) write on discourse in Miami in general, these "discourses slide past each other as if moving on different planes."

With respect to the research question: "Are differences in perceptions of the importance of health and health care in the lives of elders by cultural groups?" the answer 
is mixed. The issues of health and health care are intertwined with other aspects in the lives of elders, i.e., whether they can afford health insurance and thus be able to pay for necessary medications, or do they have to choose between food, rent and medications. Cultural differences, in some ways, may not be as important as socio-economic status with respect to the importance an elder places on health and health care. However, the expectations of life in old age are deeply embedded in the minds of elders and these expectations are culturally impacted, regardless of current socio-economic status. That elders in these focus groups did not talk about "culture" per se, may be in part a function of this embeddedness.

Using the literature review examined in Chapter 3 as a guide, the field work done in Miami-Dade County, Florida shows more clearly that the theoretical perspectives in the field of gerontology and sociology do not fully elucidate, represent or bring an overall understanding of what it means to be "old" or "aging." As noted earlier, the majority of elders in the United States live in the community and are not institutionalized. They are usually actively involved in life. They are not passive agents as is theorized with disengagement theory. Not much can be said regarding this theoretical perspective since it may be that focus group participants are more involved in activities since they were chosen because of their connection with elder community groups. However, most of the elder participants did not see themselves as unusual in their activities or life-styles. They talked of a variety of activities that they participated in with other elders. They knew that frailty may force them eventually from disengaging and isolating themselves from much of their community, however currently these elder participants were engaged. 
Age stratification theory is helpful in understanding and "examining the movement of successive birth cohorts across time" (Passuth \& Bengtson, 1988). However, the differences between racial/ethnic/cultural groups of elders, particularly within Miami-Dade County, are not explained. Immigrant elders may not have experienced the same events as native-born elders. For example, many white nonHispanic elders would not have experienced the fall of Cuba to communism in the same way that Cuban elders would have experienced. However, the interpretations and meaning of aging for Cuban elders is colored by their flight from their homeland. Thus, the movement of successive birth cohorts across time is not a satisfactory explanation of aging.

With respect to exchange theory, it is helpful in examining the interactions of older people with other age groups, it also is limited in its approach. As stated in Chapter 3 , activity theory and social phenomenology show the greatest promise in terms of helpfulness in understanding the meaning of and discourses on aging by elders, particularly in Miami-Dade County. The theoretical perspective of political economy of aging is somewhat difficult to apply because it implies that all older individuals are "impoverished and disenfranchised" which is an over-statement, particularly in MiamiDade County. While there are many elders who are "impoverished and disenfranchised," there are also many elders who have money to spend on needed services. This was documented both in the discourse analyzed in this chapter, as well as documented in Chapter 4 .

The ethnographic data to date has been limited by the small population sizes, as well as personal inexperience of aging by the researchers, and possibly the fear of the 
process of aging itself. Ethnographic research, however, gives meaning to the numbers, as well as a better understanding of the influences on American culture. To this end, the field world in Dade County, Florida that was discussed can only be an initial exploration into the different frames of discourses on aging by elders in a multicultural urban environment. It does not show the whole continuum of discourse on aging that may be found in the United States, since it is limited to the geographic area of Dade County. Miami, as Portes \& Stepick (1993) write, "holds important lessons for what the American city will be about in a changed world." This exploration into a piece of the urban milieu offers a unique opportunity to study the different frames of reference and discourse among diverse race/ethnic groups of elders that has not previously been captured. 


\section{CHAPTER 7: QUANTITATIVE ANALYSIS OF HEALTH CARE \\ UTILIZATION BEHAVIOR OF ELDERS IN MIAMI-DADE}

\section{COUNTY, FLORIDA}

It has been well documented that the elderly are disproportionately heavier users of health care services (Lubitz \& Prihoda, 1984a, 1984b; Soldo \& Manton, 1985; Waldo \& Lazenby, 1984). These studies have examined the factors affecting the use of health care services by the elderly; in general they have found that need factors are the most important predictors of elders use of health care services. The majority of these studies, however, have only looked at white non-Hispanic elders, in spite of increasing racial/ethnic diversity within the elderly population. Many factors influence the health status and use of health services by older Amcricans. "Health", and its polar-pair "illness," are socially constructed terms and depend upon the "cognitive frames in which they are placed and these in turn are products of prior social interactions" (Portes \& Stepick, 1993: 9). Further, health care services in the United States are typically provided at the local or community level. It has been pointed out in Chapter 2 that health care services are not like other goods and services because individuals cannot shop around nationally for health care services "to find the best deal." Many of the previous studies of health care utilization behavior have been limited to national studies of the elderly; thus community-level enabling factors have not been included in these studies. This chapter examines the determinants of formal health care service utilization by elders in a multicultural urban environment (Miami-Dade County, FL) using the theoretical 
framework of the Socio-Bchavioral Model (SBM) ${ }^{\mathrm{l}}$ originally developed by Andersen (1968) and further revised (Andersen \& Newman, 1975; Andersen, 1995). It is the most widely used theoretical framework for studying health service usage by the elderly (Wolinsky, 1994). In this research investigation, the utilization models will be run separately for each of the formal health service dependent variables and racial/ethnic groups of elders to examine whether differential patterns of health care use exist. This chapter will provide a quantitative examination of the intertwined threads of health, culture and aging.

The focus of this chapter will be to answer the research questions and hypotheses posited in Chapter 1.

Question \# 2: What factors predict utilization of health care services?

Hypothesis \# 2a: Need factors will not be the strongest predictors of formal health care service utilization of elders.

Hypothesis \# 2b: Among enabling factors, community resources will play an important role in inhibiting or enabling use of formal health care services.

Hypothesis \# $2 \mathrm{c}$ : Among predisposing factors, social structural variables will play an important role in inhibiting or enabling use of formal health care services.

Question \# 3: Do these factors vary among racial/ethnic sub-populations?

Hypothesis \# 3a: Factors predicting health care service utilization will vary among sub-populations. Need factors, such as health status, will be a stronger predictor for white non-Hispanics, while enabling factors will be stronger predictors for black non-Hispanics, Cubans, and non-Cuban Hispanics.

Hypothesis \# 3b: Enabling factors will be stronger predictors of certain types of health care service utilization, (i.e., physician utilization), than need or predisposing factors for minority elders compared to white nonHispanic elders.

Hypothesis \# 3c: Social structure variables will be stronger predictors of health care service utilization with Hispanic elders. For black non-Hispanic

'Chapter 2 describes this theoretical framework in more detail. 
clders, personal and family resources, (i.e., reported household income less than $\$ 10,000$ ), will be stronger predictors of health care service utilization.

\section{FINDINGS}

As stated in the analysis plan in Chapter 5 , the first step in this investigation was a descriptive univariate analysis of the variables in the sample and the population of elders in the county. The descriptive results of the variables in the sample were reported in Chapter 5, while descriptive results of the overall population of elders in the county were reported in Chapter 4. The next steps in the analysis strategy called for an analysis of the bivariate and multivariate relationships between the dependent variables (formal health care service utilization) and the independent variables. These results will be discussed in this chapter.

\section{Bivariate Relationships}

Each of the four formal health care service dependent variables will be displayed separately, but in the exact same manner. First, there will be an examination of the bivariate relationships between the dependent variable and the independent variables done separately for each of the racial/ethnic groups of elders. This will be accomplished through an examination of zero-order correlation (Pearson correlation) statistics and frequency distributions (percentages or means) between the dependent variable and the independent variables for each of the racial/ethnic groups of elders. Two sets of tables for each of the four dependent variables show that there are different bivariate relationships for each of the racial/ethnic groups of elders. Each of the factors predisposing, enabling, and need - will be discussed with respect to the four dependent 
variables in more detail later in this section. Table 7.1 shows the zero-order correlations between the independent measures and the dependent variable of overall contact with physician, while Table 7.2 shows findings from cross-tabulations between selected independent variables and the dependent variable of contact with physician in the last 12 months. The dependent variable for contact with physician represents a minimum test of contact or physician utilization in the last 12 months. Having more than five physician visits in the last 12 months represents high volume utilization of physician for those who had at least one physician visit in the last 12 months. Table 7.3 shows zero-order correlations between the independent variables and the dependent variable of high volume (6 or more visits) physician contact (for those who have at least one physician contact) in the last 12 months. Table 7.4 shows the findings from cross-tabulations between selected independent vatiables and the dependent variable of high volume physician contact in the last 12 months. The third formal health service variable examined in this research is overnight hospital stay. ${ }^{2}$ Like overall physician contact, it represents a minimum test of service usage. Table 7.5 shows zero-order correlations between the independent variables and the dependent variable of overnight hospital stay, while Table 7.6 shows the findings from the cross-tabulations between selected independent variables and the dependent variable of overnight hospital stay. The last of the four dependent measures of formal health care service utilization under examination

\footnotetext{
${ }^{2}$ Andersen (1968) defines this as the least discretionary of formal health care services. "Non-discretionary behavior is primarily dictated by the physical condition of the [individual]. Decisions in the latter instance are usually made by providers of services" (Andersen, 1968: 18).
} 
in this research is a complete physical exam in the last 12 months. ${ }^{3}$ Table 7.7 shows zero-order correlations between the independent variables and the dependent variable of having complete physical exam, while Table 7.8 shows the findings from the crosstabulations between selected independent variables and the dependent variable of having complete physical exam.

In all the tables, the magnitude of associations between the independent variables and each of the dependent variables varies across the racial/ethnic groups of elders. This result gives validation for examining each of the racial/ethnic groups of elders separately for each of the dependent variables. While the magnitude of the associations between independent variables and each of the dependent variables varies across the racial/ethnic groups of elders, it is interesting that only rarely did the direction of the association vary between a particular independent and dependent variable across the racial/ethnic groups of elders. The next sections discuss these results in more detail by each of the three factors - predisposing, enabling, and need characteristics - with respect to each of the dependent variables found in Tables 7.1 through 7.8 .

\section{Predisposing Characteristics}

As noted earlier, predisposing variables are those "... characteristics which exist prior to the onset of specific episodes of illness" (Andersen, 1968). Age is a very important predictor predisposing characteristic for all the health care service utilization variables under examination. For specific racial/ethnic groups of elders, however, age is not always significantly correlated with service utilization. For example, age is

\footnotetext{
${ }^{3}$ In Andersen (1968) typology, having a complete physical exam would be a behavior that is highly
} 
significantly correlated with overall physician contact and having a complete physician exam in the last 12 months for each of the racial/ethnic groups of elders. However, for high volume physician contact, age is significantly positively correlated only for Cubans $(r=0.22, p \leq 0.01)$ and non-Cubans $(r=0.27, p \leq 0.01)$. Further, for overnight hospital stay, age is not significantly correlated for black non-Hispanic elders at the 0.05 level. When age is significant the direction of the association is positive. Thus, older individuals are more likely to have used formal health care services than younger individuals. For example, with respect to overall physician contact the average age of black non-Hispanic elders with a physician contact in the last 12 months is older compared to black non-Hispanic elders without a physician contact in the last 12 months (69.58 years and 62.82 years, respectively, $\mathrm{p} \leq 0.01)$.

Gender is only significantly correlated with high volume physician contact for Cuban elders $(r=0.09, p \leq 0.05)$. Otherwise, there are no strong associations between the formal health care service dependent variables with gender by racial/ethnic groups of elders at the 0.05 level.

Educational attainment is only significantly associated with the dependent variable of high volume physician contact, particularly having no high school education. However, the correlation coefficient among the racial/ethnic groups of elders varies between 0.07 for white non-Hispanic elders to 0.24 for black non-Hispanic elders. Examining the bivariate associations, over half of elders without any high school education have had six or more physician visits in the last 12 months $(51.3 \%$ for white 
non-Hispanic, $56.9 \%$ for black non-Hispanic, $58.8 \%$ for Cuban, and $58.9 \%$ for nonCuban Hispanic). On the other end of the educational spectrum, having more than a high school education is significantly negatively associated with high volume physician contact for black non-Hispanics $(r=-0.18, p \leq 0.01)$ and non-Cuban Hispanics $(r=-0.20$, $\mathrm{p} \leq 0.05)$

With respect to the social structure factors, being currently employed and the intensity of social contact with family friends are usually associated with all of the health care service utilization variables. However, the patterns of association vary by racial/ethnic group of elders. For example, being currently employed is significantly negatively correlated with physician contact for both white non-Hispanics $(r=-0.113, p \leq$ $0.01)$ and Cubans ( $\mathrm{r}=-0.135, \mathrm{p} \leq 0.01)$, yet no such significant negative correlation for white non-Hispanic is documented with respect to high volume physician contact at the 0.05 level. The variable measuring intensity of social contact with family and friends is correlated with most formal health care scrvice utilization particularly for Cuban elders.

Marital status has been dichotomized into four dummy variables: currently married/live-in partner, widowed, divorced/separated and never married. Marital status appears to be more significantly associated for Hispanic elders compared to non-Hispanic elders, except in the case of having a complete physical exam. Among black nonHispanic elders, being widowed $(r=0.15, \mathrm{p} \leq 0.05)$ or divorced/separated $(r=-0.15, \mathrm{p} \leq$ 0.05 ) is significantly correlated with having a complete physical exam. Being currently married is significantly negatively correlated with physician contact in the last 12 months for non-Cuban Hispanic elders $(r=-0.24, p \leq 0.01$ ), while being widowed is significantly positively correlated with physician contact in the last 12 months for Cuban elders 
$(\mathrm{r}=0.09, \mathrm{p} \leq 0.05)$. However, when examining high volume phy sician contact and overnight hospital stays, marital status is significantly associated among Cuban elders, but not among non-Cuban Hispanic elders.

Intensity of social contact with friends and family is another social structure factor that shows significant correlation with all of the health care service utilization variables among Cuban elders. The direction of the association for Cuban elders with respect to intensity of social contact is positive for both of the physician utilization variables and having a complete physical exam in the last 12 months, while it was negative for overnight hospital stay for Cuban elders. Cuban elders with intense social contact are more likely to visit a physician and have a complete physical exam, and less likely to have had an overnight hospital stay in the last 12 months.

There were two health belief variables that showed significant correlations across all the dependent variables. The first was the variable that measured how serious a concern the area of health was for elders in general $(1=$ very serious/somewhat serious concern, $0=$ not very serious concern). The second health belief variable that showed significant correlation for all racial/ethnic groups of elders was life satisfaction during the past 6 months with respect to high volume physician contact. For each of the racial/ethnic groups of elders, the direction of the association is negative and ranges from $r=-0.15$ for Cuban elders to $r=-0.25$ for black non-Hispanic elders and significant at the 0.05 level.

\section{Enabling Characteristics}

Personal/family resources show the strongest and more significant associations of enabling factors compared to community resources for all the dependent variables. In 
particular elders with no Medicare coverage, no health coverage at all and having Medicaid coverage showed the strongest association with all the dependent variables. Again, it is not surprising that having no Medicare coverage and no health care coverage overall are usually significantly negatively correlated with health care utilization, while reported Medicaid coverage is positively correlated with health care utilization. However, there are differences in the association between Medicaid coverage and health care service utilization. This would appear to back up findings from Lum \& Chang (1998) who concluded that “...Medicaid does not work equally well in improving affordability and use of health services among different racial [and] ethnic, ... groups."

An unusual result with respect to black non-Hispanic elders and the dependent variable of high volume physician contact shows that none of the health care coverage enabling factors is significantly correlated. The only enabling factor that is significantly correlated with high volume physician contact for black non-Hispanic elders is reported household income less than $\$ 10,000(r=0.18, p \leq 0.05)$.

Community resource factors are not consistently significant across the racial/ethnic groups of elders for each of the health care service utilization variables. For example among black non-Hispanic elders, only one of the community resource variables was significantly correlated at the 0.10 level with the health service utilization variables (proportion of elders in zip code with high volume physician contact, $r=-0.14, p \leq 0.10$ ). While among the other racial/ethnic groups of elders depending upon the health care service utilization variable, several other of the community resource variables were significant. For example, hospital bed ratios per 1000 population in the PSA is significantly correlated with high volume physician contact. The direction of the 
association is negative for white non-Hispanic elders, but positive for Cuban and nonCuban Hispanic elders. However, hospital bed ratio is significantly correlated with overnight hospital stay for Cuban elders $(\mathrm{r}=0.09, \mathrm{p} \leq 0.05)$, while it is significantly correlated with complete physical exam for white non-Hispanic elders $(r=0.09, p \leq$ $0.01)$.

\section{Necd Characteristics}

Generally, most of the need variables are significantly correlated with high volume physician utilization and overnight hospital stay across the four racial/ethnic groups of elders. Having a complete physical exam in the last 12 months shows the smallest correlations with most need variables. However, with respect to overall physician contact, it is interesting to note that only one of the need variables is significantly correlated at the 0.10 level for black non-Hispanic elders. This need variable is the number of mobility limitations $(r=0.12, p \leq 0.10)$.

\section{Multivariate Regression Analysis}

Turning to the multivariate regression analysis, the first step in this part of the analysis will examine the explained variance $\left(\mathrm{R}^{2}\right)$ resulting from the inclusion of each of the three major blocks of variables separately (need, enabling and predisposing). This will allow for an examination of the strength of each block of variables unique contribution to the overall explained variance. The multivariate regression will use logistic transformation (Logit regression) since the dependent variables for health care service utilization are dichotomous. As noted in Chapter 5, OLS regression could have been used if the dichotomous dependent variables fell within the acceptable distribution 
of 0.25 to 0.75 . However, at least one of the dichotomous dependent variables did not fall within this range.

In Chapter 5 there was a discussion regarding the method of entry of the blocks of variables, i.e., whether they should be examined by "most "recent' (temporally) causal determinants..." (Wolinsky, 1978: 392) or with respect to order of pre-existing conditions. By first examining the amount of explained variation of each of the blocks separately, the amount of explained variation for each of the blocks would not be dependent upon the timing of the entry into the regression model. This issue has not been explored previously in the research literature. Separately entering the blocks also solved another issue, i.e., the stability of the estimates of coefficients in the full-model for smaller racial/ethnic groups of elders.

Examining the full-models of each of the blocks of variables is useful; however, it is difficult to interpret, as well as does not show the relationship between the blocks of variables, which is another important aspect of this research. On the other hand, throwing everything into a regression model also creates difficulty for interpretation due to issues of multicollinearity, as well as issues of possible statistical weakness of all the coefficients and small degrees of freedom. Parsimony of the regression models is important. The philosophical doctrine of Ockham's Razor will be followed, i.e., "theories and explanations should be as streamlined as possible. All other things equal, the simplest theory (e.g., the explanation with the fewest predictors) is the best" (Vogt, 1993). Thus, the next step in this analysis will be an examination of parsimonious (hestfit) models of utilization variables for each of the racial/ethnic groups of elders. To find the best-fit model logistic regression will be run using a forward step method of entry for 
each of the three blocks of variables using the "LR" option in SPSS (SPSS, 1999) for each racial/ethnic group of elders for each of the dependent variables.

The method of entry used in the logit regression procedure was forward "stepwise selection method with entry testing based on the significance of the score statistics, and removal testing based on the probability of a likelihood ratio statistics based on the maximum partial likelihood estimates" (SPSS, 1999). The probability for stepwise entry was set at 0.10 and removal was set at 0.15 . Further, more independent variables were included in this analysis, since variables will be entered in a stepwise method and only those that meet the probability criteria will be included in the model. Thus, the examination of the best-fit model for each racial/ethnic group of elders served two purposes. First, since best-fit models include only those measures whose beta coefficients were statistically significant there is parsimony in the model. Second, since all variables are examined as to their statistical significance, the relationship between blocks of variables can be examined.

Tables of results for each dependent variable within each method of analysis will be presented, i.e., separate entry of blocks and best-fit models. Both methods will be examined in a two-step process. Overall, there will four tables presented for each dependent variable. In general the process will be that the first step will examine the summary of explained variation by block (either separate entry or best-fit) and the second table will give the beta coefficients for the variables entered in the blocks. The summary of explained variation by separate entry of blocks is shown in Table 7.9 thru Table 7.12. Table 7.9 shows the explained variance results for overall physician contact; Table 7.10 presents the explained variance results for high volume physician contact; Table 7.11 
displays the explained variance results for overnight hospital stay and Table 7.12 shows the explained variance results for having a complete physical exam. Table 7.13 thru Table 7.16 show the results of the individual beta coefficients for the separate entry of blocks. This set of tables will be used to examine the effect of individual independent variables within each block on the each of dependent variables across the racial/ethnic groups of elders. The logistic regression results of the modeis within each of the major blocks (need, enabling and predisposing) for the four dependent variables are shown in Tables 7.13 (overall physician contact), Table 7.14 (high volume physician contact), Table 7.15 (overnight hospital stay) and Table 7.16 (complete physical exam). Similar tables using best-fit standards are also displayed. Table 7.17 thru Table 7.20 show summary results of explained variation across racial/ethnic groups of elders for each of the health service dependent variables. Table 7.17 shows the explained variance results for overall physician contact under best-fit standards. Table 7.18 presents the explained variance results for high volume physician contact under best-fit standards. Table 7.19 displays the explained variance results for overnight hospital stay under best-fit standards and Table 7.20 shows the explained variance results for having a complete physical exam under best-fit conditions. Finally, Table 7.21 thru Table 7.24 show the results of the individual beta coefficients under best-fit conditions across racial/ethnic groups of elders for each of the health service dependent variables: Table 7.21 - overall physician contact; Table 7.22 - high volume physician contact; Table 7.23 - overnight hospital stay; and Table 7.24 - complete physical exam. Interpretation of the results documented in the tables as they relate to the hypotheses addressed in the research will follow. However, before addressing the hypotheses, there will be a brief discussion of the basic 
interpretation of each type of table using the dependent variable of physician visit in the last 12 months (specifically, Table 7.9 and Table 7.13). Following this brief description, results will be discussed in terms of each of the research hypotheses as to whether the hypothesis was proved or disproved. While both separate block models and best-fit models were examined, the hypothesis testing will rely primarily on results of the best-fit models where not otherwise documented.

In Table 7.9 , it is found that the amount of explained variation $\left(R^{2}\right)$ resulting from need factors are significant for white non-Hispanic elders $\left(R^{2}=0.018, p \leq 0.01\right)$ and nonCuban Hispanic elders $\left(\mathrm{R}^{2}=0.055, \mathrm{p} \leq 0.05\right)$, they are not significant for black nonHispanic and Cuban elders at the 0.10 level. As a block, enabling factors were significant for all racial/ethnic groups of elders at the 0.10 level. Broken down by sub-blocks, personal/family resource factors were significant for all racial/ethnic groups of elders, while community resources were not significant for any of the four racial/ethnic groups of elders. Predisposing factors, as a block, were significant for all four racial/ethnic groups of elders. Again, broken down by sub-blocks, social structure factors were significant at the 0.05 level for all racial/ethnic groups of elders (white non-Hispanic $\mathrm{R}^{2}=$ $0.009, p \leq 0.05$; black non-Hispanic $R^{2}=0.073, p \leq 0.05 ;$ Cuban $R^{2}=0.023, p \leq 0.05$ and non-Cuban Hispanic $\mathrm{R}^{2}=0.070, \mathrm{p} \leq 0.05$ ). Demographic factors were significant at the 0.05 level for white non-Hispanic elders $\left(R^{2}=0.014, p \leq 0.05\right)$, black non-Hispanic elders $\left(R^{2}=0.071, p \leq 0.01\right)$ and Cuban elders $\left(R^{2}=0.023, p \leq 0.01\right)$, but not for nonCuban Hispanic elders. Health belief factors were only significant for Cuban elders $\left(\mathrm{R}^{2}=\right.$ $0.014, p \leq 0.05$ ). In Table 7.13 , it was found that the significant need factor variable for white non-Hispanic and Cuban elders was self-reported overall health being excellent or 
$\operatorname{good}(\operatorname{Exp}(\beta)=0.21, \mathrm{p} \leq 0.01$ and $\operatorname{Exp}(\beta)=0.52, \mathrm{p} \leq 0.10$, respectively). Among the enabling factors, the independent variable that was significant for white non-Hispanic, black non-Hispanic, and Cuban elders was not having Medicare coverage $(\operatorname{Exp}(\beta)=0.36$. $\mathrm{p} \leq 0.01, \operatorname{Exp}(\beta)=0.19, \mathrm{p} \leq 0.10$, and $\operatorname{Exp}(\beta)=0.32, \mathrm{p} \leq 0.01$, respectively). In addition for Cuban elders, Medicaid coverage $(\operatorname{Exp}(\beta)=3.54, \mathrm{p} \leq 0.01)$, HMO Plan coverage $(\operatorname{Exp}(\beta)=3.29, p \leq 0.05)$ and residing in full-time in Miami-Dade County $(\operatorname{Exp}(\beta)=$ $5.38, \mathrm{p} \leq 0.10$ ) were also significant independent variables. While none of the individual variables were significant at the 0.10 level for non-Cuban Hispanic elders, the amount of explained variance $\left(\mathrm{R}^{2}\right)$ for this block of variables was significant. Among predisposing factors, age was a significant independent variable for white non-Hispanic elders, black non-Hispanic elders and Cuban elders $(\operatorname{Exp}(\beta)=1.05, \mathrm{p} \leq 0.05, \operatorname{Exp}(\beta)=1.67, \mathrm{p} \leq 0.01$, and $\operatorname{Exp}(\beta)=1.06, p \leq 0.05$, respectively), but not for non-Cuban Hispanic elders.

Because the findings are somewhat confusing depending upon the health care service and/or racial/ethnic group of elders being examined, a summary of results is located in Table 7.25 for Research Question 2 and Table 7.26 for Research Question 3. Detailed text description will be provided with respect to each of the hypotheses Research Question 2: What factors predict utilization of health care services? Hypothesis $2 \mathrm{a}:$ Need factors will not be the strongest predictors of formal health care service utilization of elders.

Examining the individual blocks of variables with respect to the four formal health care service dependent variables, need factors while important and usually significant at the 0.01 level, did not explain as much of the variance as predisposing and/or enabling factors. This was true even for the less discretionary formal health care service of overnight hospital stay. The exception was with white non-Hispanic elders and 
overnight hospital stay in which the explained variance of need factors was the same as the amount of explained variance for predisposing factors $(7.0 \%, p \leq 0.01)$.

However, examining the best-fit regression models, need factors are usually significant at the 0.05 level. Need factors in the best-fit regression models show the largest increment of explained variance for high volume physician contact and overnight hospital stay for all racial/ethnic groups of elders except non-Cuban Hispanic elders. In contrast, need factors did not show the largest increment in explained variance for overall physician contact and self-reported complete physical exam for any of the racial/ethuic groups of elders. Examining the best-fit regression models shows only partial support for this hypothesis and is dependent upon the type of formal health care service under examination.

When the hypothesis is not supported, the increment of explained variance for need factors is usually almost half of the total explained variance. For example with respect to high volume physician contact presented in Table 7.18 shows that for Cuban elders, need factors explain exactly half of the total explained variance $-11.7 \%$ of the total variance of $23.4 \%\left(\mathrm{X}^{2}=131.30, \mathrm{p} \leq 0.01\right)$, while for white non-Hispanic elders, need factors explain a little over half of the total $-8.4 \%$ of the total variance of $15.2 \%$ $\left(\mathrm{X}^{2}=107.75, \mathrm{p} \leq 0.01\right)$. In contrast, for black non-1 lispanic elders, need factors explain less than half of the explained variance $-11.9 \%$ of the total variance of $27.3 \%\left(X^{2}=\right.$ 45.62, $\mathrm{p} \leq 0.01)$.

When the hypothesis is supported, the largest increment of explained variance was not one block of factors consistently. For example with respect to overall physician contact in the last 12 months, enabling factors showed the largest increment of explained 
variance, particularly personal and family resources, except for black non-Hispanic elders. However, for self-reported complete physical exam in the last 12 months, predisposing factors usually showed the greatest predictive power, except for white nonHispanic elders. Predisposing factors can be broken down into three sub-blocks. The largest increment in explained variance for black non-Hispanic elders with respect to having a complete physical exam in the last 12 months were demographic factors $(7.1 \%$, $\left.\mathrm{X}^{2}=26.22, \mathrm{p} \leq 0.01\right)$ as well as for non-Cuban Hispanic elders $\left(5.2 \%, \mathrm{X}^{2}=14.92, \mathrm{p} \leq\right.$ 0.01 ). In contrast, health belief factors showed the largest increment in explained variance for white non-Hispanic elders $\left(1.5 \%, \mathrm{X}^{2}=21.46, \mathrm{p} \leq 0.01\right)$.

Hypothesis 2b: Among enabling factors, community resources will play an important role in inhibiting or enabling use of formal health care services, especially for minority groups.

Within the block of enabling factors, both examined separately, as well as best-fit regression models, the enabling factors that were most predictive of formal health care service were personal/family resources. However, community resources did play an important role in inhibiting or enabling use of formal health care services for minority elder groups, as well as white non-Hispanic elders. Thus, this hypothesis was not completely supported. It was not supported particularly with respect to self-reported complete physical exam for white non-Hispanic elders. Table 7.20 shows that for white non-Hispanic elders, community resource factors incrementally explained 2.1 percent of the total 5.7 percent of explained variance compared to 0.9 percent explained by personal/family resources. Table 7.24 presents the coefficients for the best-fit model explaining self-reported complete physical exam. The community resource variable that has the largest predictive power for white non-Hispanic elders is the proportion of elders 
$(60+)$ living in the zip code $(\operatorname{Exp}(\beta)=11.53, p \leq 0.01)$. White non-Hispanic elders living in zip codes with high proportion of elders $(60+)$ appear to be much more likely to selfreport having a complete physical exam in the last 12 months than white non-Hispanic elders who live in zip codes with lower proportion of elders $(60+)$.

Community resources showed a larger significant impact for Cuban elders for high volume physician contact $\left(4.3 \%, \mathrm{X}^{2}=131.30, \mathrm{p} \leq 0.01\right.$ in Table 7.18$)$. Results displayed in Table 7.22 show that there are three key community resource variables for Cuban elders that have strong predictive power for high volume physician contact. These three variables are the proportion of blacks living in the zip code $(\operatorname{Exp}(\beta)=0.09, \mathrm{p} \leq$ $0.05)$, proportion elders $(\operatorname{Exp}(\beta)=0.002, \mathrm{p} \leq 0.0 \mathrm{i})$ and proportion of the population living below poverty in zip code $(\operatorname{Exp}(\beta)=618.59, \mathrm{p} \leq 0.01)$. Cuban elders living in zip codes with high proportions of blacks are less likely than those living in zip codes with lower proportion of blacks to have high volume physician contact. Likewise for Cuban elders living in zip codes with high proportion of elders. Cuban respondents living in zip codes with higher percentage of the population living below poverty are more likely to have high volume physician contact than those Cuban respondents living in zip codes with lower overall percentage of the population living in poverty.

Community resources for non-Cuban Hispanic elders with respect to high volume physician contact are also significant but do not have as great an impact as personal/family resources $(6.8 \%$ and $7.5 \%$, respectively of the total $35.4 \%$ explained variance, $\mathrm{X}^{2}=56.36, \mathrm{p} \leq 0.01$ in Table 7.18 ). The most predictive community resource variables for non-Cuban Hispanics were hospital bed ratio for the PSA $(\operatorname{Exp}(\beta)=1.24, p$ $\leq 0.05)$ and proportion elders in the zip code $(\operatorname{Exp}(\beta)=0.001, \mathrm{p} \leq 0.05)$. Higher hospital 
bed ratios in a PSA for non-Cuban Hispanic respondents increased the likelihood that the respondent will have six or more physician visits in the past 12 months, while high proportion of elders in the zip code of a non-Cuban Hispanic respondent decreased the likelihood of high volume physician contact.

Hypothesis 2c: Among predisposing factors, social structural variables will play an important role in inhibiting or enabling use of formal health care services.

Examining predisposing variables separately and depending upon which health carc service utilization variable under examination this hypothesis was not always supported. For example, when examining predisposing factors alone presented in Table 7.9 with respect to the dependent variable of overall physician contact in the last 12 months for minority elders, this hypothesis was supported. However for Cuban elders, demographic variables explain as much as social structural variables $(2.3 \%, \mathrm{p} \leq 0.05$, respectively). For black non-Hispanic and non-Cuban Hispanic, social structural variables have the greatest predictive ability $(7.3 \%$ and $7.0 \%$, respectively, $\mathrm{p} \leq 0.05)$. Social structure variables have the strongest predictive power for Cuban elders with respect to high volume physician contact $(7.3 \%, \mathrm{p} \leq 0.01)$ and overnight hospital stay $(3.1 \%, p \leq 0.01)$. However, social structure variables did not explain very much with respect to self-reported complete physical exam.

Similar to the separate block analysis, examining the results of the best-fit models show that the hypothesis was not always supported. Again examining overall physician contact in the last 12 months, Table 7.17 shows that social structure variables had varying predictive power by racial/ethnic groups of elders. For white non-Hispanic elders, social structure variables were the only predisposing factors that were significant in the best-fit model. For black-non-Hispanic elders, demographic factors showed greater predictive 
power than social structure factors $(7.3 \%$ and $4.7 \%$, respectively of the total $18.5 \%)$.

Demographic variables were the only predisposing factors that were significant in the best-fit model for non-Cuban Hispanic elders $(1.7 \%$ of the total $11.8 \%)$. For Cuban elders, social structure variables were a stronger predictor in the best-fit model explaining overall physician contact in the last 12 months compared to health belief variables $(3.3 \%$ and $1.1 \%$, respectively of the total $9.0 \%$ ). Social structure variables for Cuban elders that made the best-fit model and significant at the 0.01 level were being divorced/separated $(\operatorname{Exp}(\beta)=0.26, p \leq 0.01)$; and intensity of social contact with family and friends $(\operatorname{Exp}(\beta)$ $=1.24, \mathrm{p} \leq 0.01$ ). Intensity of social contact with family and friends for Cuban elders was also a significant predictor of high volume physician contact $(\operatorname{Exp}(\beta)=0.92, \mathrm{p} \leq$ $0.10)$; and overnight hospital stay $(\operatorname{Exp}(\beta)=0.88, \mathrm{p} \leq 0.05)$, but was not significant or included in the best-fit model explaining self-reported complete physical exam.

Health belief variables for particular service utilization variables also had an important impact on utilization. For example, the best-fit model with respect to overnight hospital stay (Table 7.19) shows that health belief factors are significant at the 0.01 level for white non-Hispanic elders, and are significant at the 0.10 level for black non-Hispanic and Cuban elders. Further, coefficient results in the best-fit model explaining overnight hospital stay presented in Table 7.23 show that the response to the question on whether the area of health is a serious or very serious concern/problem area for elders in general was a strong predictor for all racial/ethnic groups of elders, except non-Cuban Hispanic elders [white non-Hispanic $(\operatorname{Exp}(\beta)=2.06, \mathrm{p} \leq 0.01)$; black non $-H i s p a n i c(\operatorname{Exp}(\beta)=3.46$, $\mathrm{p} \leq 0.10)$; and Cuban $(\operatorname{Exp}(\beta)=1.55, \mathrm{p} \leq 0.10)]$. Another strong health belief predictor for overnight hospital stay for white non-Hispanic elders is life satisfaction $(\operatorname{Exp}(\beta)=$ 
$0.59, p \leq 0.10$ ). White non-Hispanic elders who responded that they were very or most satisfied with their lives in the last 6 months were over 40 percent less likely to have had an overnight hospital stay than white non-Hispanic elders who responded that they werc very or somewhat dissatisfied with their lives. Life satisfaction was also a significant negative predictor for high volume physician contact among white non-Hispanics $(\operatorname{Exp}(\beta)$ $=0.56, \mathrm{p} \leq 0.05)$ and black non-Hispanics $(\operatorname{Exp}(\beta)=0.10, \mathrm{p} \leq 0.01)$, but was not significant for either Hispanic elder group. However, life satisfaction was a significant positive predictor of having a complete physical exam in the last 12 months for Cuban elders $(\operatorname{Exp}(\beta)=2.31, \mathrm{p} \leq 0.01)$.

Research Question 3: Do these factors vary among race/ethnic sub-populations?

Hypothesis 3a: Factors predicting heallh care service utilization will vary among subpopulations. Need factors, such as self-reported health status, will be a stronger predictor for white non-Hispanics, while enabling factors, such as availability of personal/family resources, will be stronger predictors for black non-Hispanics, Cubans, and non-Cuban Hispanics.

Overall, the first part of this hypothesis is supported, i.e., factors predicting health care service utilization vary among sub-populations. As has been shown in the previous discussion, factors predicting utilization of formal health care service usage in the previous 12 months do vary by racial/ethnic groups of elder. The amount of variance explained differs widely. For example, when examining the separate blocks of independent variables with respect to overall physician contact, need variables explained 1.8 percent $(\mathrm{p} \leq 0.01)$ of the variation for white non-Hispanic elders, while need variables were not significant for either black non-Hispanic, or Cuban elders. However, for nonCuban Hispanic elders, need variables explained 5.5 percent $(p \leq 0.05)$ of the variation with respect to overall physician contact. Of the three major blocks of variables the 
explained variance was the greatest for predisposing factors for white non-Hispanics ( $3.0 \%, \mathrm{p} \leq 0.05)$, black non-Hispanics $(17.3 \%, \mathrm{p} \leq 0.01)$, and non-Cuban Hispanics $(13.3 \%, p \leq 0.05)$, while enabling factors explained 6.5 percent $(\mathrm{p} \leq 0.01)$ for Cuban elders. Similarly when examining the best-fit model explaining overall physician contact (Table 7.17), need variables for white non-Hispanic elders explain 0.9 percent $(p \leq 0.05)$, while for black non-Hispanic need variables explain 4.1 percent $(\mathrm{p} \leq 0.05)$. Need variables for Cuban elders explain 0.6 percent $(p \leq 0.10)$ and for non-Cuban Hispanic elders, need variables explain 5.0 percent $(\mathrm{p} \leq 0.01)$.

However, with respect need factors being a stronger predictor for white nonHispanics, this piece of the hypothesis was only partially supported. The explained variance for need factors has the largest increment of explained variance only with respect to the dependent variable of overnight hospital stay $(8.2 \%, \mathrm{p} \leq 0.01)$ and high volume physician contact $(8.4 \%, p \leq 0.01)$ for white non-Hispanic elders. However, need factors also show the largest increments of explained variance for black non-Hispanic and Cuban elders for high volume physician contact $(11.9 \%, \mathrm{p} \leq 0.01$ and $11.7 \%, \mathrm{p} \leq 0.01$, respectively) and overnight hospital stay $(10.6 \%, \mathrm{p} \leq 0.01$ and $4.1 \%, \mathrm{p} \leq 0.01$, respectively) in the best-fit models. Need factors are not significant for either of these dependent variable with respect to non-Cuban Hispanic elders.

Examined separately by block, availability of family/personal resources among enabling factors is consistently the strongest predictors of service utilization across racial/ethnic groups of elders and type of service. However, examined in the best-fit model, this part of the hypothesis was not supported. When personal/family resources variables met the criteria for the forward-step entering into the model were usually 
significant. However, they were not always the best predictors of utilization for minority elders. Table 7.17 presents the increment of explained variance by block for the best-fit models explaining overall physician contact. While personal/family resource variables explain 3.5 percent $(p \leq 0.01)$ for Cuban elders and 5.1 percent for $(p \leq 0.05)$ for nonCuban elders, personal/family resource variables also exhibited the largest increment in explained variance for white non-Hispanic elders $(1.5 \%, \mathrm{p} \leq 0.01)$. For black nonHispanic elders, personal/family resource variables while significant at the 0.10 level have the smallest predictive power for explaining overall physician contact.

Hypothesis 3b: Enabling factors will be stronger predictors of certain types of health care service utilization, (i.e., physician utilization), than need or predisposing factors for minority elders compared to white non-Hispanic elders.

Examined separately, enabling factors are not consistently stronger predictors for health care service utilization of minority. Further, as discussed in the previous hypothesis, when enabling factors were stronger predictors of certain types of health care utilization than need or predisposing factors, it was across the board for all elders not just minority elders. For example, examining the best-fit models explaining overall physician contact (Table 7.17), while enabling variables were the strongest predictors for nonCuban Hispanic elders $(5.1 \%, p \leq 0.05)$, they were also the strongest predictors for white non-Hispanic elders $(2.0 \%, \mathrm{p} \leq 0.10)$. With respect to the best-fit model explaining selfreported complete physical exam, enabling factors had the largest explained variance for white non-Hispanic elders $(3.0 \%, \mathrm{p} \leq 0.01)$, while for minority elders enabling factors were important but not the strongest predictor. Thus, this hypothesis was not supported.

The particular variables within the enabling block presented in Table 7.21 that were important showed variation across racial/ethnic groups of elders. With respect to 
personal/family resources on overall physician contact, not having Medicare for both white non-Hispanic and Cuban elders was significantly negative and had approximately the same impact $(\operatorname{Exp}(\beta)=0.41, \mathrm{p} \leq 0.01$ and $\operatorname{Exp}(\beta)=0.42, \mathrm{p} \leq 0.05$, respectively $)$. However, for Cuban elders both Medicaid and having an HMO plan coverage reported were also significant personal/family resource variables in explaining overall physician contact. This was not the case for any other racial/ethnic group of elders. Having Medicaid coverage for Cuban elders increased the likelihood of having a physician contact in the last 12 months by almost 3 times $(\operatorname{Exp}(\beta)=2.86, \mathrm{p} \leq 0.05)$. Similarly, having HMO coverage for Cuban elders increased the likelihood of having a physician contact in the last 12 months by almost 3 times $((\operatorname{Exp}(\beta)=2.64, p \leq 0.05)$. That HMO coverage is highly significant and positively related to physician contact for Cuban elders is not surprising given that Cuban elders have had a longer experience with health maintenance organizations compared to non-Hispanic cultural groups of elders as mentioned in Chapter 2.

Hypothesis 3c: Social structure variables will be stronger predictors of health care service utilization with Hispanic elders. For black non-Hispanic elders, personal and family resources, (i.e., reported household income less than $\$ 10,000$ ), will be stronger predictors of health care service utilization.

This hypothesis was not consistently supported across the four service utilization variables. For example in the examination of the best-fit regression model explaining overall physician contact, social structure variables were stronger predictor variables for black non-Hispanic elders compared to personal/family resource variables rather than for Cuban elders (Table 7.17). However, increment of explained variance for the best-fit model explaining high volume physician contact for Cuban elders social structure variables are stronger predictors than personal/family resources (Table 7.18), but the two 
sets of variables are tied in their predictive power with respect to overnight hospital stay (Table 7.19). For black non-Hispanic elders, enabling factors in general did not play any part in the best-fit regression model explaining high volume physician contact. However, for overnight hospital stay and self-reporting of a complete physical exam in the last 12 months, personal/family resource variables were stronger predictors compared to social structure variables.

Further, Table 7.21 shows the coefficient results of the best-fit regression models explaining overall physician contact in the last 12 months, being divorced/separated $(\operatorname{Exp}(\beta)=0.26, p \leq 0.01)$ and intensity of social contact with family and friends $(\operatorname{Exp}(\beta)$ $=1.24, \mathrm{p} \leq 0.01)$. However, given the strength of Medicaid coverage and HMO plan coverage variables for Cuban elders, social structure variables are not as strong predictors for with respect to overall physician contact. In contrast, because Medicaid and HMO coverage variables are not significant predictors for black non-Hispanic elders, social structure variables have a stronger predictive power, particularly being widowed $(\operatorname{Exp}(\beta)$ $=0.06, \mathrm{p} \leq 0.05)$ and being divorced/separated $(\operatorname{Exp}(\beta)=0.04, \mathrm{p} \leq 0.05)$.

In contrast as presented in Table 7.23 with respect to the best-fit model explaining overnight hospital stay, Medicaid coverage is a significant variable for both black nonHispanic and Cuban elders at the 0.10 level. However, for black non-Hispanic elders another important predictor variable among personal/family resource factors is having a private physician as a regular source of health care $(\operatorname{Exp}(\beta)=2.78, \mathrm{p} \leq 0.10)$. This would seem to be a paradox. However given ethnographic evidence that black non-I lispanic elders tend to wait for an acute health emergency before seeking formal health care services (Abraham, 1993; Bailey, 1987), having a private physician as a regular source of 
health care may indicate that these respondents are sicker or have had previous hospital experience. Unfortunately, the survey does not ask about the number of overnight hospital visits in the last 12 months or the number of nights that the elder spent in the hospital in the last 12 months. This measure of hospital utilization is only a minimum test of service usage.

Among the social structure factors for Cuban elders, intensity of social contact with family and friends had a significant negative impact on the likelihood of an overnight hospital stay $(\operatorname{Exp}(\beta)=0.88, \mathrm{p} \leq 0.01)$, while living alone is a significant social structure variable for black non-Hispanic $(\operatorname{Exp}(\beta)=2.94, \mathrm{p} \leq 0.10)$. Neither white nonHispanic nor non-Cuban Iispanic elders had social structure variables that were significant at the 0.10 level.

\section{SUMMARY AND DISCUSSION}

In this chapter two research questions have been examined to identify differences in predictors of utilization by type of formal health service and by racial/ethnic groups of elders. Need factors did not show up as the strongest predictors when examined separately by block. However when examined within the context of best-fit regression models, need factors were usually significant at the 0.05 level and showed the largest increment of explained variance for high volume physician contact and overnight hospital stay for all racial/ethnic groups of elders except non-Cuban Hispanic elders. Need factors did not show the largest increment of explained variance for overall physician contact or self-reported complete physical exam in the last 12 months for all racial/ethnic groups of elders. This goes against what has been documented in the literature for the overall population, as well as national samples of elders with respect to overall physician contact 
in the last 12 months. In addition, the hypothesis that need factors would be a stronger predictor for white non-Hispanic elders as compared to minority elders was supported only with respect to overnight hospital stay, but was not supported for any of the other three formal health care service utilization variables. One possible explanation for this is that the white non-Hispanic elder population residing in Miami-Dade County may be considered unique compared to other communities. Many white non-Hispanic elders migrated to South Florida from other geographic regions. White non-Hispanic elders who migrate to South Florida are a healthier and somewhat wealthier group of white nonHispanic elders. When they do become ill or too frail to care for themselves, their adult children move them back to their origin communities and/or closer to the residence of the adult children so that the frail elder may receive necessary care. Thus, this survey would not catch these frailer white non-Hispanic elders in the sample.

The most consistent predictive variable across the types of formal health care services under investigation for white non-Hispanic elders was having no Medicare, except for self-reported complete physical exam in the last 12 months. Having no Medicare was also important for Cuban elders with respect to overall physician contact and having a complete physical exam.

Comparing the impact of community resource variables with personal/family resource variables found that usually personal/family resource variables are stronger predictors of service utilization compared to community resource variables. However, community resource variables do play a significant role in inhibiting or enabling use of formal health care services, not only for minority groups of elders, but also for white nonHispanic elders. 
The predictive strength of social structure variables among predisposing factors was not consistent across service types or racial/ethnic groups of elders. Social structure variables were one of the strongest or the strongest predictors for Cuban elders across all services types except for self-reported complete physical exam in the last 12 months. In comparison with respect to black non-Hispanic elders, social structural variables were the strongest predictors among predisposing factors of overall physician contact in the last 12 months. For non-Cuban Hispanic elders, social structure variables were the strongest predictors among predisposing factors of overnight hospital stay. Otherwise, social structure variables were either superceded in predictive power or were not significant. It is also important to note that this discussion of results supports the hypothesis that factors predicting health care service utilization vary across racial/ethnic groups of elders.

These results indicate several important issues. First, examining elders as homogeneous group of users of formal health care service in a community as has been done in much previous research using the SBM model is simplistic. As indicated in Chapter 2, cultural attitudes and beliefs of an ethnic group will influence an individual's perception of illness as well as the response to the illness. The strategy of using dummy variables for racial/ethnic groups assumes that the relationships between the racial/ethnic groups are strictly additive. Separate analysis for each racial/ethnic group facilitates the examination of both the additive and interactive effects of race/ethnicity on each of the health care utilization measures specified. As documented in Chapter 2, Wolinsky, et al. (1989) examination of a nationally representative sample of elders separately analyzed each racial/ethnic group of elders. They interpreted differential predictability of the separately estimated models as an indication of inequitable access to the health care 
delivery system. Thus, previous research using dummy variables for racial/ethnic groups in a single model may not give an accurate picture of the dynamics involved between the three blocks of factors. The analysis documented in this chapter shows that formal health care service utilization is dependent upon the type of formal health care service, as well as the racial/ethnic group of elder. The racial/ethnic groups of elders differ in important ways and thus, must be examined separatefy by cultural context.

In the following final chapter of this research, there will be a bringing together of the quantitative results from this chapter with the qualitative results analyzed in Chapter 6, focus group discussions by elders residing in Miami-Dade County. Further, there will be a discussion of the implications of these results for future results, as well as a discussion on the implications these results have for policy. 
Table 7.1: Zero-Order Correlations of Selected Independent Measures with Self-Reported Physician Visit in the Last 12 Months for Four Racial/Ethnic Groups of Elders

\begin{tabular}{|c|c|c|c|c|}
\hline Physician Visit in Last 12 months & $\begin{array}{l}\text { White Non- } \\
\text { Hispanic } \\
(\mathrm{N}-884)\end{array}$ & $\begin{array}{l}\text { Black Nont- } \\
\text { Hispanic } \\
(\mathrm{N}=200)\end{array}$ & $\begin{array}{c}\text { Cuban } \\
(\mathrm{N} \cdots 661)\end{array}$ & $\begin{array}{l}\text { Non-Cuban } \\
\text { Hispanic } \\
(\mathrm{N}=188)\end{array}$ \\
\hline \multicolumn{5}{|l|}{ PREDISPOSING FACTORS } \\
\hline \multicolumn{5}{|l|}{ Demographic } \\
\hline Age (years) & $0.12 * * *$ & $0.21 * * *$ & $0.16^{* * *}$ & $0.16 * *$ \\
\hline Female & 0.01 & -0.06 & $0.07^{*}$ & 0.02 \\
\hline \multicolumn{5}{|l|}{ Educational Attainment } \\
\hline No High School & -0.004 & 0.02 & 0.03 & 0.03 \\
\hline Some High School & 0.01 & -0.10 & -0.03 & -0.06 \\
\hline High School Graduate & -0.02 & -0.04 & -0.01 & $-0.0]$ \\
\hline More than High School & 0.01 & 0.12 & -0.01 & 0.02 \\
\hline \multicolumn{5}{|l|}{ Language spoken in home } \\
\hline English only & -0.01 & All & 0.02 & -0.06 \\
\hline Spanish spoken in household & -0.04 & -- & -0.02 & 0.06 \\
\hline Creole spoken in household & -- & -- & 0.01 & -- \\
\hline Yiddish spoken in household & 0.03 & -- & -- & н \\
\hline $\begin{array}{l}\text { Other language spoken in } \\
\text { household }\end{array}$ & 0.04 & -- & -- & 0.02 \\
\hline \multicolumn{5}{|l|}{ Social Structure } \\
\hline \multicolumn{5}{|l|}{ Marital Status } \\
\hline Married/Live-in Partner & -0.02 & 0.10 & -0.03 & $-0.24 * * *$ \\
\hline Widowed & 0.05 & -0.03 & $0.09^{* *}$ & 0.12 \\
\hline Divorced/Separated & -0.03 & -0.12 & -0.06 & 0.10 \\
\hline Never Married & -0.01 & 0.05 & -0.03 & 0.08 \\
\hline Lives alone & -0.004 & -0.06 & 0.05 & 0.08 \\
\hline $\begin{array}{l}\text { Currently Employed in the Labor } \\
\text { Force? }\end{array}$ & $.0 .11 * * *$ & -0.08 & $-0.14^{* * *}$ & -0.02 \\
\hline $\begin{array}{l}\text { Intensity of Social Contact with } \\
\text { Family \& Friends } \\
\text { Religious Preference }\end{array}$ & -0.003 & 0.07 & $0.18 * * *$ & -0.11 \\
\hline Protestant & -0.04 & 0.02 & 0.02 & -0.08 \\
\hline Catholic & -0.04 & 0.06 & 0.03 & 0.05 \\
\hline Jew & $0.08^{* *}$ & 0.02 & 0.02 & 0.03 \\
\hline No Preference Stated & -0.02 & -0.12 & $-0.12 * * *$ & 0.07 \\
\hline Other Religious Preference & 0.02 & -0.02 & 0.02 & -0.05 \\
\hline $\begin{array}{l}\text { Religion is Very/Somewhat } \\
\text { Important in Life } \\
\text { Health Beliefs }\end{array}$ & -0.03 & -0.04 & $0.07^{*}$ & $0.15 * *$ \\
\hline $\begin{array}{l}\text { Health is a very serious/serious } \\
\text { concern as a elder problem area }\end{array}$ & $0.09 * *$ & 0.12 & $0.10^{* * *}$ & $0.16 * *$ \\
\hline $\begin{array}{l}\text { Very/Somewhat worried about } \\
\text { loss of independence }\end{array}$ & 0.05 & 0.03 & 0.03 & 0.09 \\
\hline $\begin{array}{l}\text { Very/mostly satisfied with life } \\
\text { during past } 6 \text { months }\end{array}$ & -0.03 & 0.12 & 0.02 & -0.09 \\
\hline
\end{tabular}


TABLE 7.1 (cont.)

\begin{tabular}{|c|c|c|c|c|}
\hline Physician Visit in Last 12 months & $\begin{array}{l}\text { White Non- } \\
\text { Hispanic } \\
(\mathrm{N}=884)\end{array}$ & $\begin{array}{l}\text { Black Non- } \\
\text { Hispanic } \\
(\mathrm{N}=200) \\
\end{array}$ & $\begin{array}{c}\text { Cuban } \\
(\mathrm{N}=661)\end{array}$ & $\begin{array}{l}\text { Non-Cuban } \\
\text { Hispanic } \\
(\mathrm{N}=188)\end{array}$ \\
\hline \multicolumn{5}{|l|}{$\begin{array}{l}\text { ENABLING FACTORS } \\
\text { Personal/Family Resources }\end{array}$} \\
\hline Household lncome $<\$ 10 \mathrm{~K}$ ? & 0.02 & -0.07 & -0.01 & -0.005 \\
\hline \multicolumn{5}{|l|}{ Medicare Coverage } \\
\hline Medicare Part A only & 0.04 & 0.002 & -0.04 & 0.06 \\
\hline Medicare Part A \& B & $0.12^{* * *}$ & $0.20^{* * *}$ & $0.19 * * *$ & $0.14 *$ \\
\hline No Medicare & $-0.14 * * *$ & $-0.21 * * *$ & $-0.18 * * *$ & $-0.17 * *$ \\
\hline \multicolumn{5}{|l|}{ Other Health Care Coverage } \\
\hline No Insurance & $-0.07 * *$ & $-0.35 * * *$ & $-0.27^{* * *}$ & $-0.25 * * *$ \\
\hline Medicaid Coverage Reported & 0.03 & $0.13 *$ & $0.15 * * *$ & $0.14 * *$ \\
\hline HMO Plan Coverage Reported & 0.02 & $0.13^{*}$ & $0.07^{*}$ & $0.13^{*}$ \\
\hline $\begin{array}{l}\text { Ycars Living in Miami-Dade } \\
\text { County, FL }\end{array}$ & -0.01 & 0.06 & 0.02 & 0.02 \\
\hline Resides Full-time in Dade County & 0.01 & -0.05 & 0.04 & -0.06 \\
\hline $\begin{array}{l}\text { Regular source of health care } \\
\text { from a private physician } \\
\text { Community Resources }\end{array}$ & 0.04 & 0.01 & 0.030 & -0.08 \\
\hline $\begin{array}{l}\text { Hospital Bed Ratio per } 1000 \\
\text { Population in PSA }\end{array}$ & -0.001 & 0.08 & 0.03 & 0.07 \\
\hline $\begin{array}{l}\text { Primary Care Pbysician Ratio per } \\
1000 \text { Population in PSA }\end{array}$ & 0.01 & -0.01 & $0.07 *$ & -0.06 \\
\hline Proportion Black in Zip Code & -0.03 & 0.08 & $-0.10 * * *$ & 0.10 \\
\hline Proportion Hispanic in Zip Code & 0.01 & -0.07 & 0.01 & -0.09 \\
\hline Proportion $60+$ in Zip Code & $0.07 * *$ & -0.06 & 0.04 & -0.02 \\
\hline $\begin{array}{l}\text { Proportion Living Below Poverty } \\
\text { in Zip Code }\end{array}$ & -0.03 & 0.05 & -0.06 & 0.11 \\
\hline \multicolumn{5}{|l|}{ NEED FACTORS } \\
\hline $\begin{array}{l}\text { Self-reported Overall Health as } \\
\text { Excellent Good }\end{array}$ & $-0.12 * * *$ & -0.03 & $-0.08 * *$ & $-0.15^{* *}$ \\
\hline $\begin{array}{l}\text { Self-reported Emotional Health as } \\
\text { Excellent'Good }\end{array}$ & -0.01 & -0.02 & 0.01 & $-0.15^{* *}$ \\
\hline Number of Reported ADLs & 0.02 & 0.04 & 0.04 & $0.13 *$ \\
\hline Number of Reported JADLs & $0.06 *$ & 0.05 & 0.04 & $0.14 * *$ \\
\hline Number of Mobility Limitations & $0.10^{* * *}$ & $0.12 *$ & $0.07^{*}$ & $0.12 *$ \\
\hline
\end{tabular}

${ }^{*} \mathrm{p} \leq 0.10$ (two-tailed) ${ }^{* *} \mathrm{p} \leq 0.05$ (two-tailed) ${ }^{* * *} \mathrm{p} \leq 0.01$ (two-tailed)

-- no cases 
Table 7.2: Bivariate Associations of Selected Independent Measures with Self-Reported Physician Visit in the Last 12 Months for Four Racial/Ethnic Groups of Elders

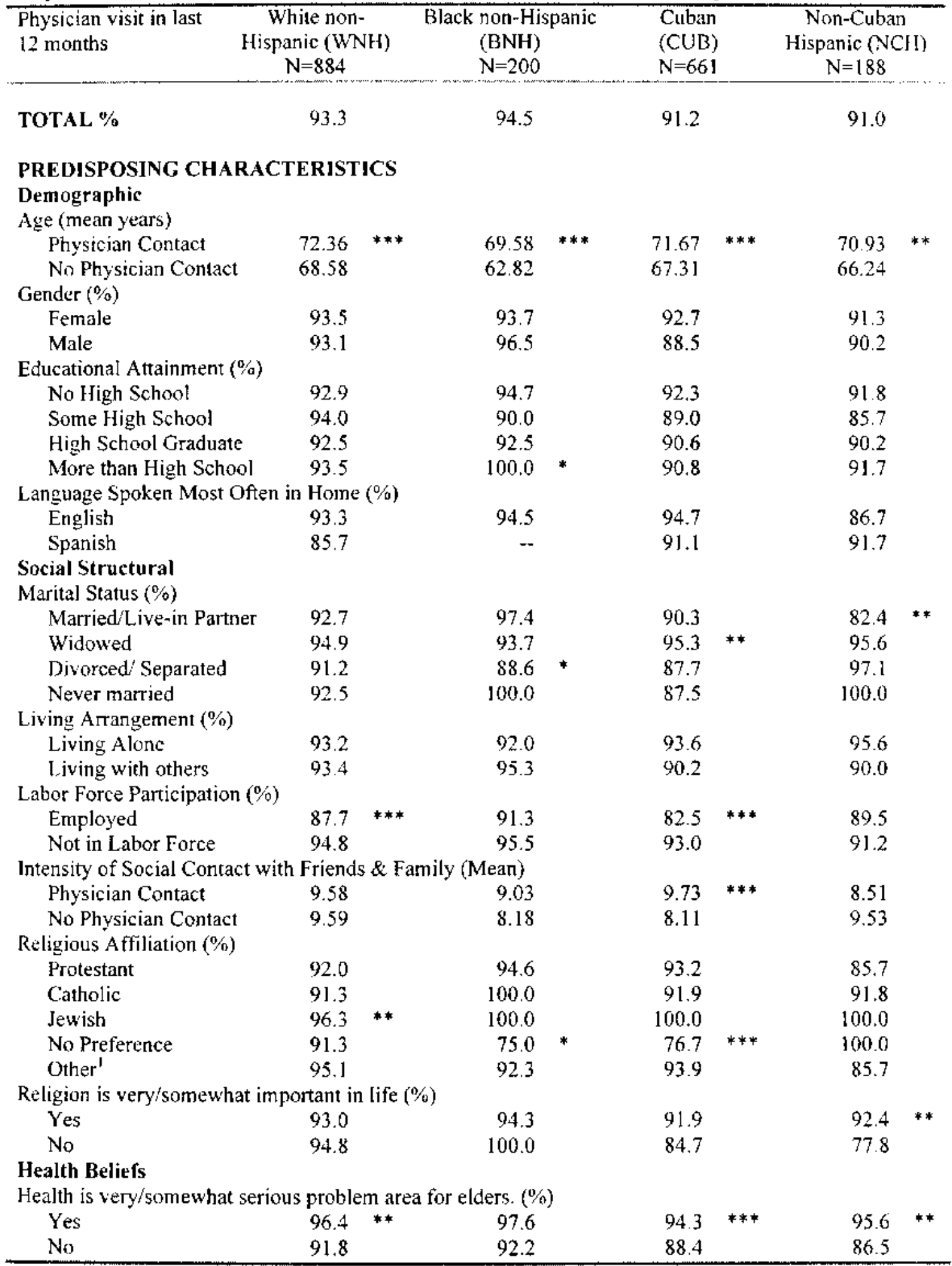

Includes "Christian," "Spiritual," and "Other, not coded." 


\begin{tabular}{|c|c|c|c|c|c|c|c|c|}
\hline $\begin{array}{l}\text { Physician visit in last } \\
12 \text { months }\end{array}$ & $\begin{array}{l}\text { White non- } \\
\text { Hispanic (WN } \\
\mathrm{N}=884\end{array}$ & & $\begin{array}{c}\text { Black non-His } \\
(\mathrm{BNH}) \\
\mathrm{N}=200\end{array}$ & panic & $\begin{array}{l}\text { Cuban } \\
\text { (CUB) } \\
N=-661\end{array}$ & & $\begin{array}{l}\text { Non-Cuban } \\
\text { Hispanic (NC) } \\
\mathrm{N}=188\end{array}$ & \\
\hline $\begin{array}{l}\text { Very/somewhat worried ab } \\
\text { future.(\%) }\end{array}$ & bout loss of in & deper & dence due to $\mathrm{ph}$ & ysical & rental de & terior: & fion in the & \\
\hline Yes & 94.6 & & 95.5 & & 91.8 & & 92.2 & \\
\hline No & 92.1 & & 94.1 & & 89.4 & & 86.4 & \\
\hline Very/Mostly Satisfied with & $h$ Life in the $p$ & ast 6 & nonths $(\%)$ & & & & & \\
\hline Yes & 92.9 & & 95.2 & & 91.9 & & 89.7 & \\
\hline No & 95.0 & & 87.0 & & 90.4 & & 96.7 & \\
\hline ENABLING CHARACTE & ERISTICS & & & & & & & \\
\hline Personal/Family Resource & & & & & & & & \\
\hline Household Income less that & an $\$ 10,000 \mathrm{pe}$ & ryear & $(\%)$ & & & & & \\
\hline Yes & 94.4 & & 92.6 & & 91.0 & & 90.8 & \\
\hline No & 93.2 & & 95.8 & & 91.4 & & 91.1 & \\
\hline Medicare Coverage (\%) & & & & & & & & \\
\hline Medicare Part A Only & 97.4 & & 94.4 & & 87.8 & & 100.0 & \\
\hline Medicare Part A and B & 95.2 & $* * *$ & 97.7 & $* * *$ & 94.8 & $* * *$ & 94.7 & \\
\hline No Medicare Coverage & 87.6 & $* * *$ & 87.9 & $* * *$ & 82.1 & $* * *$ & 85.2 & $* *$ \\
\hline Medicaid Coverage (\%) & & & & & & & & \\
\hline Medicaid Covcrage & 96.9 & & 100.0 & $*$ & 97.0 & $* * *$ & 96.8 & $* *$ \\
\hline No Medicaid Coverage & 93.2 & & 92.7 & & 88.4 & & 87.9 & \\
\hline HMO Plan Coverage $(\%)$ & & & & & & & & \\
\hline JMO Plan & 94.0 & & 98.5 & * & 95.1 & $*$ & 100.0 & $*$ \\
\hline Not HMO Plan & 93.1 & & 92.2 & & 90.3 & & 89.2 & \\
\hline Coverage & & & & & & & & \\
\hline Part-time or Full-time Resi & ident of Miam & ij-Dac & le County $(\%)$ & & & & & \\
\hline Full-time & 93.4 & & 94.6 & & 91.4 & & 90.5 & \\
\hline Part-time & 92.9 & & 100.0 & & 81.8 & & 100.0 & \\
\hline Regular Source of Health $C$ & Care $(\%)$ & & & & & & & \\
\hline Private Physician & 94.6 & & 95.7 & & 92.6 & & 90.8 & \\
\hline Other Source & 92.7 & & 95.2 & & 91.0 & & 95.1 & \\
\hline Community Resources ( & (Means) & & & & & & & \\
\hline Hospital Bed Ratio in PSA & & & & & & & & \\
\hline Physician Contact & 4.15 & & 4.98 & & 4.85 & & 4.74 & \\
\hline No Physician Contact & 4.15 & & 4.25 & & 4.59 & & 4.14 & \\
\hline Primary Care Physician Ra & atio in PSA & & & & & & & \\
\hline Physician Contact & 2.01 & & 1.22 & & 2.33 & & 2.03 & \\
\hline No Physician Contact & 1.95 & & 1.26 & & 2.08 & & 2.27 & \\
\hline Proportion Black in Zip Co & ode & & & & & & & \\
\hline Physician Contact & .1260 & & .5171 & & .0621 & $* * *$ & .1444 & \\
\hline No Physician Contact & .1468 & & .4259 & & .1107 & & .0735 & \\
\hline Proportion Hispanic in Zip & p Code & & & & & & & \\
\hline Physician Contact & .3622 & & .2658 & & .7428 & & .5737 & \\
\hline No Physician Contact & .3566 & & .3092 & & .7393 & & .6560 & \\
\hline Proportion Elder $(60+)$ in 2 & Zip Code & & & & & & & \\
\hline Physician Contact & .2577 & $* *$ & .1451 & & .2297 & & .2013 & \\
\hline No Physicjan Contact & .2214 & & .1612 & & .2196 & & .2089 & \\
\hline Proportion of Population $L$ & Living Below & Pover & ty in Zip Code & & & & & \\
\hline Physician Contact & .1438 & & .2737 & & .1943 & & .1874 & \\
\hline No Physician Contact & .1541 & & .2444 & & .2134 & & .1453 & \\
\hline
\end{tabular}


Table 7.2 (cont.)

\begin{tabular}{lcccc}
\hline $\begin{array}{l}\text { Physicjan visit in last } \\
12 \text { months }\end{array}$ & $\begin{array}{c}\text { White non- } \\
\text { Hispanic (WNH) } \\
\mathrm{N}=884\end{array}$ & $\begin{array}{c}\text { Black non-Hispanic } \\
\text { (BNH) }\end{array}$ & $\begin{array}{c}\text { Cuban } \\
\text { (CUB) }\end{array}$ & Non-Cuban \\
& $\mathrm{N}=200$ & $\mathrm{~N}=661$ & $\mathrm{~N}=188$ \\
\hline
\end{tabular}

\section{NEED CHARACTERISTICS}

Perceived Physical Health Status is Excellent or Good (\%)

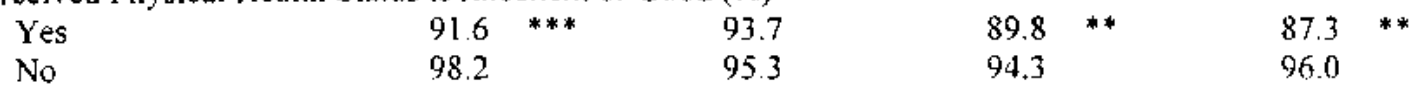

Perceived Emotional Health Status is Excellent or Good (\%)

$\begin{array}{lcccc}\text { Yes } & 93.2 & 94.1 & 90.8 & 88.8 \\ \text { No } & 94.0 & 95.2 & 91.3 & 98.0\end{array}$

ADL/IADL/Mobility Indexes, mean number of reported difficulties

ADLs

Physician Contact $\quad 0.28$

No Physician Contact $\quad 0.20$

0.34

0.37

0.58

IADLS

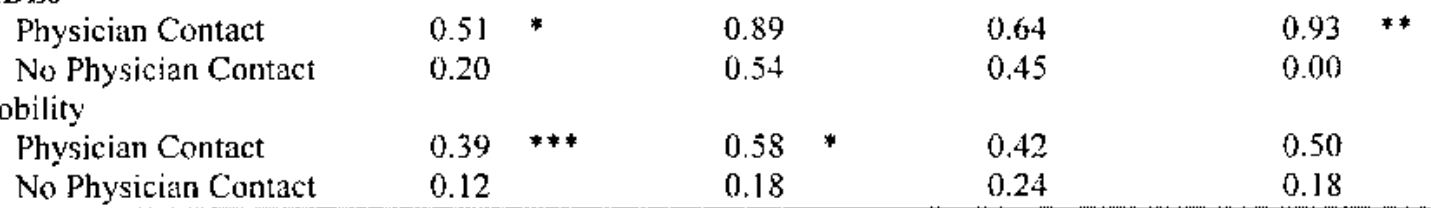

${ }^{*} p \leq 0.10$ (two-tailed) ${ }^{* *} \mathrm{p} \leq 0.05$ (two-tailed) ${ }^{* *} \mathrm{p} \leq 0.01$ (two-tailed)

-n No cases available 
Table 7.3: Zero-Order Correlations of Selected Independent Measures with Six or More Self-Reported Physician Visits in the Last 12 Months for Four Racial/Ethnic Groups of Elders

\begin{tabular}{|c|c|c|c|c|}
\hline $\begin{array}{l}\text { High volume }(6+) \text { physician visits } \\
\text { for those who had at least one visit }\end{array}$ & $\begin{array}{l}\text { White Non- } \\
\text { Hispanic } \\
(\mathrm{N}=825)\end{array}$ & $\begin{array}{l}\text { Black Non- } \\
\text { Hispanic } \\
(\mathrm{N} \text { - } 389)\end{array}$ & $\begin{array}{c}\text { Cuban } \\
(N=603)\end{array}$ & $\begin{array}{l}\text { Non-Cubail } \\
\text { Hispanic } \\
(\mathrm{N}=171)\end{array}$ \\
\hline \multicolumn{5}{|l|}{$\begin{array}{l}\text { PREDISPOSING } \\
\text { Demographic }\end{array}$} \\
\hline Agc (years) & $0.06^{*}$ & 0.04 & $0.22 * * *$ & $0.27^{* * *}$ \\
\hline Female & 0.03 & -0.01 & $0.09^{* *}$ & 0.03 \\
\hline \multicolumn{5}{|l|}{ Educational Attainment } \\
\hline No High School & $0.07 * *$ & $0.24 * * *$ & $0.12 * * *$ & $0.23^{* * *}$ \\
\hline Some High School & 0.03 & 0.02 & -0.05 & -0.07 \\
\hline High School Graduate & -0.02 & $-0.13^{*}$ & -0.06 & 0.005 \\
\hline More than 1 Iigh School diploma & -0.03 & $-0.18 * *$ & -0.05 & $-0.20 * *$ \\
\hline \multicolumn{5}{|l|}{ Language Spoken in the Home } \\
\hline English only & 0.01 & All & 0.01 & $-0.178^{* *}$ \\
\hline Spanish & 0.04 & -. & -0.02 & $0.19 * *$ \\
\hline Creole & -- & -- & 0.04 & -- \\
\hline Yiddish & -0.02 & -- & -- & -- \\
\hline Other language & -0.02 & - & - & -0.07 \\
\hline \multicolumn{5}{|l|}{ Social Structural } \\
\hline \multicolumn{5}{|l|}{ Marital Status } \\
\hline Married/Live-in Partner & -0.02 & -0.09 & $-0.15^{* * *}$ & -0.04 \\
\hline Widowed & 0.01 & 0.10 & $0.15 * * *$ & 0.11 \\
\hline Divorced/Separated & -0.01 & 0.004 & 0.03 & $-0.14 *$ \\
\hline Never Married & 0.04 & -0.02 & -0.03 & 0.10 \\
\hline Lives alone & -0.001 & 0.05 & $0.14 * *$ & -0.03 \\
\hline $\begin{array}{l}\text { Currently Employed in the Labor } \\
\text { Force }\end{array}$ & -0.06 & -0.27 & $.0 .26 * * *$ & $-0.14 *$ \\
\hline $\begin{array}{l}\text { Intensity of Social Contact with } \\
\text { Farnily and Friends } \\
\text { Self-Reported Religious Preference }\end{array}$ & -0.03 & -0.04 & $0.09 * *$ & -0.12 \\
\hline Protestant & 0.01 & 0.10 & 0.02 & -0.02 \\
\hline Catholic & 0.04 & 0.02 & 0.01 & 0.10 \\
\hline Jew & -0.05 & -0.09 & 0.06 & -0.10 \\
\hline No Preference Stated & -0.02 & .0 .11 & -0.02 & 0.05 \\
\hline Other Religious Preference & 0.02 & -0.08 & -0.02 & $-0.15 * *$ \\
\hline $\begin{array}{l}\text { Religion is Very/Somewhat } \\
\text { Important in Life } \\
\text { Health Beliefs }\end{array}$ & -0.04 & -0.03 & $-0.09 * *$ & -0.12 \\
\hline $\begin{array}{l}\text { Health is a very serious/serious } \\
\text { concern as an elder problem area }\end{array}$ & $0.31 * * *$ & $0.29^{* * *}$ & $0.25^{* * *}$ & $0.26^{* * * *}$ \\
\hline $\begin{array}{l}\text { Very/somewhat woried about loss } \\
\text { of independence }\end{array}$ & $0.07^{*}$ & 0.03 & 0.05 & $0.14^{*}$ \\
\hline $\begin{array}{l}\text { Very/mostly satisfied with life } \\
\text { during past } 6 \text { months }\end{array}$ & $-0.20 * * *$ & $-0.25 * * *$ & $-0.15 * * *$ & $-0.17^{* *}$ \\
\hline
\end{tabular}




\begin{tabular}{lcccc}
\hline High volume $(6+)$ physician visits & White Non- & Black Non- & Non-Cuban \\
for those who had at least one visit & Hispanic & Hispanic & Cuban & Hispanic \\
& $(\mathrm{N}=825)$ & $(\mathrm{N}=189)$ & $(\mathrm{N}=603)$ & $(\mathrm{N}=171)$ \\
\hline
\end{tabular}

\section{ENABLING FACTORS}

\section{Personal/Family Resources}

Household lncome $<\$ 10 \mathrm{~K}$ ?

Medicare Coverage

Medicare Part A ONLY

Medicare Part A \& B

No Medicare

\begin{tabular}{|c|c|c|c|}
\hline $0.10^{* * *}$ & $0.18^{* *}$ & $0.11^{* * *}$ & 0.03 \\
\hline-0.02 & -0.01 & -0.01 & -0.01 \\
\hline $0.09^{* *}$ & 0.12 & $0.17 * * *$ & $0.18^{* *}$ \\
\hline$-0.08^{* *}$ & -0.12 & $-0.18^{* * *}$ & $-0.19 * *$ \\
\hline 0.02 & 0.03 & $-0.13 * * *$ & $-0.15^{* *}$ \\
\hline $0.08^{* *}$ & 0.09 & $0.23 * * *$ & $0.29^{* * *}$ \\
\hline 0.02 & -0.06 & $-0.07 *$ & -0.04 \\
\hline 0.004 & 0.005 & $-0.07^{*}$ & $-0.19^{* *}$ \\
\hline $0.07^{* *}$ & -0.01 & $0.08^{*}$ & $0.17^{* *}$ \\
\hline$-0.06^{*}$ & -0.003 & 0.02 & 0.04 \\
\hline $.0 .09^{* * *}$ & -0.05 & $0.09 * *$ & $0.18 * *$ \\
\hline$-0.07 * *$ & -0.10 & 0.04 & 0.06 \\
\hline 0.02 & 0.04 & 0.03 & $0.15^{*}$ \\
\hline 0.05 & -0.06 & 0.06 & -0.02 \\
\hline-0.05 & $-0.14^{*}$ & 0.003 & -0.04 \\
\hline-0.02 & 0.004 & $0.18^{* * *}$ & $0.18^{* *}$ \\
\hline
\end{tabular}

Other Health Care Coverage

No Health Insurance

Medicaid Coverage Reported

HMO Plan Coverage Reported

Years Living in Dade County, FL

Resides Full-time in Dade County

Regular source of health care from a

private physician

Community Resources

Hospital Bed Ratio per 1000

Population in PSA

Primary Care Physician Ratio per

1000 Population in PSA

Proportion Black in Zip Code

Proportion Hispanic in Zip Code

Proportion 60+- in Zip Code

0.004

$0.18^{* * *}$

$0.18 * *$

Zip Code

\section{NEED FACTORS}

Self-reported Overall Health as

$\begin{array}{llll}-0.26^{* * *} & -0.24^{* * *} & -0.28^{* * *} & -0.22 * * * \\ -0.21^{* * *} & -0.26^{* * *} & -0.14^{* * *} & -0.14^{*} \\ 0.10^{* * *} & 0.09 & 0.19^{* * *} & 0.25 * * * \\ 0.11^{* * *} & 0.10^{* * *} & 0.22^{* * *} & 0.12 \\ 0.14^{* *} & 0.34^{* * *} & 0.24^{* * *} & 0.25^{* * *}\end{array}$

Excellent/Good

Self-reported Emotional Health as

Excellent/Good

Number of Reported ADLs

Number of Reported IADLs

Number of Mobility Limitations

* 50.10 (two-tailed)

** $\mathrm{p} \leq 0.05$ (two-tailed)

*** $\mathrm{p} \leq 0.01$ (two-tailed)

-- No cases available 
Table 7.4: Bivariate Associations of Selected Independent Measures with Six or More Self-Reported Physician Visits in the Last 12 Months for Four Racial/Ethnic Groups of Elders

\begin{tabular}{|c|c|c|c|c|c|c|}
\hline $\begin{array}{l}\text { High volume (6r) } \\
\text { physician visits in last } \\
12 \text { months }\end{array}$ & $\begin{array}{c}\text { White non- } \\
\text { Hispanic (WNH) } \\
\mathrm{N}=825\end{array}$ & $\begin{array}{c}\text { Black non- } \\
\text { Hispanic (BNI }) \\
N=189\end{array}$ & \multicolumn{2}{|c|}{$\begin{array}{l}\text { Cuban } \\
\text { (CUB) } \\
\mathrm{N}=603\end{array}$} & \multicolumn{2}{|c|}{$\begin{array}{c}\text { Non-Cuban } \\
\text { Hispanic (NCH) } \\
\mathrm{N}=171 \\
\end{array}$} \\
\hline TOTAL \% & 35.5 & 41.3 & 52.6 & & 43.9 & \\
\hline \multicolumn{7}{|c|}{$\begin{array}{l}\text { PREDISPOSING CHARACTERISTICS } \\
\text { Demographic }\end{array}$} \\
\hline \multicolumn{7}{|c|}{ Age (mean years) } \\
\hline 6+ Physician Visits & 73.01 & 69.92 & 73.27 & *** & 73.52 & *** \\
\hline$<6$ Physician Visits & 71.99 & 69.34 & 69.88 & & 68.87 & \\
\hline \multicolumn{7}{|l|}{ Gender $(\%)$} \\
\hline Female & 36.8 & 41.0 & 55.7 & ** & 44.8 & \\
\hline Male & 33.4 & 41.8 & 46.6 & & 41.8 & \\
\hline \multicolumn{7}{|l|}{ Educational Attainment \% } \\
\hline No Iligh School & $51.3 * *$ & 56.9 & 58.8 & $* * *$ & 58.9 & *** \\
\hline Some High School & 41.3 & 44.4 & 46.2 & & 33.3 & \\
\hline Iligh School Graduate & 34.0 & 29.7 & 45.8 & & 43.2 & \\
\hline More than High School & 34.3 & 24.3 & 48.6 & & 29.1 & * \\
\hline \multicolumn{7}{|c|}{ Language Spoken Most Often in Home (\%) } \\
\hline English unly & 35.6 & $\mathrm{n} / \mathrm{a}$ & 55.6 & & 23.1 & ** \\
\hline Spanish & 50.0 & n/a & 52.4 & & 47.9 & ** \\
\hline \multicolumn{7}{|l|}{ Social Structural } \\
\hline \multicolumn{7}{|l|}{ Marital Status (\%) } \\
\hline Married/Live-in Partner & 34.4 & 36.0 & 44.8 & $* * *$ & 41.0 & \\
\hline Widowed & 36.3 & 47.3 & 64.1 & $* * *$ & 50.8 & \\
\hline Divorced/Separated & 34.4 & 41.9 & 56.0 & & 29.4 & * \\
\hline Never married & 43.2 & 37.5 & 46.4 & & 63.6 & \\
\hline \multicolumn{7}{|l|}{ Living Arrangement (\%) } \\
\hline Living Alone & 35.3 & 45.7 & 63.4 & *** & 41.9 & \\
\hline Living with others & 35.4 & 39.7 & 48.1 & & 45.2 & \\
\hline \multicolumn{7}{|l|}{ Labor Force Participation (\%) } \\
\hline Employed & 29.9 & 16.7 & 20.0 & *** & 29.4 & * \\
\hline Not in Labor Force & 36.8 & 48.3 & 57.9 & & 47.4 & \\
\hline \multicolumn{7}{|c|}{ Intensity of Social Contact with Family and Friends (mean out of maximum of 12) } \\
\hline 6+ Physician Visits & 9.44 & 8.91 & 9.53 & ** & 8.12 & \\
\hline < 6 Physician Visits & 9.61 & 9.11 & 9.96 & & 8.81 & \\
\hline \multicolumn{7}{|l|}{ Religious Affiliation (\%) } \\
\hline Protestant & 36.2 & 42.7 & 55.9 & & 41.7 & \\
\hline Catholic & 40.1 & 45.5 & 52.9 & & 47.2 & \\
\hline Jewish & 32.7 & 0.0 & 0.0 & & 0.0 & \\
\hline No Preference & 33.3 & 0.0 & 47.8 & & 55.6 & \\
\hline Other $^{1}$ & 41.0 & 25.0 & 48.4 & & 16.7 & $* *$ \\
\hline \multicolumn{7}{|c|}{ Rcligion is very/somewhat important in life } \\
\hline Yes & 34,4 & 40.9 & 51.2 & ** & 42.0 & \\
\hline No & 38.7 & 50.0 & 68.0 & & 64.3 & \\
\hline
\end{tabular}

'Includes "Christian," "Spiritual," and "Other, not coded." 
Tabie 7.4 (cont.)

\begin{tabular}{|c|c|c|c|c|c|c|c|c|}
\hline $\begin{array}{l}\text { High volume }\left(6^{+}\right) \\
\text {physician visits in last } \\
12 \text { months }\end{array}$ & \multicolumn{2}{|c|}{$\begin{array}{c}\text { White non- } \\
\text { Hispanic (WNH) } \\
\mathrm{N}=825\end{array}$} & \multicolumn{2}{|c|}{$\begin{array}{c}\text { Black non- } \\
\text { Hispanic (BNH) } \\
\mathrm{N}=189\end{array}$} & \multicolumn{2}{|c|}{$\begin{array}{l}\text { Cuban } \\
\text { (CUB) } \\
N=603\end{array}$} & \multicolumn{2}{|c|}{$\begin{array}{c}\text { Non-Cuban } \\
\text { Hispanic (NCH) } \\
N=17 !\end{array}$} \\
\hline \multirow{2}{*}{\multicolumn{9}{|c|}{ Health Beliefs }} \\
\hline & & & & & & & \\
\hline Yes & 56.3 & $* * *$ & 58.0 & $* * *$ & 65.2 & **** & 55.8 & *** \\
\hline No & 25.0 & & 29.0 & & 40.1 & & 30.1 & \\
\hline \multicolumn{9}{|c|}{ Very/somewhat worried about loss of independence due to physical or mental deterioration in the future. } \\
\hline Yes & 38.7 & ** & 43.5 & & 54.1 & & 47.7 & * \\
\hline No & 32.1 & & 40.6 & & 48.4 & & 31.6 & \\
\hline \multicolumn{9}{|c|}{ Very/Mostly Satisfied with Life in the past 6 months } \\
\hline Yes & 31.8 & $* * *$ & 35.6 & $* * *$ & 48.6 & $* * *$ & 40.0 & ** \\
\hline No & 58.4 & & 75.0 & & 69.1 & & 62.1 & \\
\hline \multicolumn{9}{|c|}{ ENABLING CHARACTERISTICS } \\
\hline \multicolumn{9}{|c|}{ Personal/Family Resources } \\
\hline \multicolumn{9}{|c|}{ Household Income less than $\$ 10,000$ per year $(\%)$} \\
\hline Yes & 48.0 & $* * *$ & 52.0 & ** & 59.1 & $* * *$ & 45.8 & \\
\hline No & 33.7 & & 34.2 & & 47.9 & & 42.9 & \\
\hline \multicolumn{9}{|l|}{ Medicare Coverage (\%) } \\
\hline Medicare Part A Only & 31.6 & & 41.2 & & 51.2 & & 42.9 & \\
\hline Medicare Part A \& B & 38.4 & ** & 46.8 & & 58.4 & **** & 51.4 & ** \\
\hline No Medicare Coverage & 28.8 & ** & 33.3 & & 35.9 & $* * *$ & 30.8 & ** \\
\hline \multicolumn{9}{|l|}{ Medicaid Coverage } \\
\hline Yes & 54.8 & $* *$ & 50.0 & & 67.3 & *** & 63.3 & *** \\
\hline No & 34.6 & & 39.3 & & 43.5 & & 33.0 & \\
\hline \multicolumn{9}{|l|}{ HMO Plan (\%) } \\
\hline Yes & 36.6 & & 37.9 & & 46.1 & * & 39.3 & \\
\hline No & 34.9 & & 44.1 & & 54.6 & & 44.7 & \\
\hline \multicolumn{9}{|c|}{ Duration Living in Miami-Dade County (mcan years) } \\
\hline 6+ Physician Visits & 31.04 & & 40.07 & & 18.61 & * & 13.71 & ** \\
\hline$<6$ Physician Visits & $31.2 !$ & & 39.85 & & 20.19 & & 18.71 & \\
\hline \multicolumn{9}{|c|}{ Part-time or Full-time Resident of Miami-Dade County $(\%)$} \\
\hline Full-time & 37.0 & ** & 41.2 & & 53.2 & * & 46.3 & $* *$ \\
\hline Part-time & 27.1 & & 42.9 & & 22.2 & & 0.0 & \\
\hline \multicolumn{9}{|c|}{ Regular Source of Health Care $(\%)$} \\
\hline Private Physician & 33.9 & & 41.1 & & 53.6 & & 46.1 & \\
\hline Other Source & 39.9 & & 41.3 & & 52.0 & & 41.6 & \\
\hline \multicolumn{9}{|c|}{ Community Resources (Means) } \\
\hline \multicolumn{9}{|c|}{ Hospital Bed Ratio in I'SA (per 1000 population in PSA) } \\
\hline 6+ Physician Visits & 3.83 & $* * *$ & 4.86 & & 5.08 & ** & 5.25 & ** \\
\hline$<6$ Physician Visits & 4.32 & & 5.06 & & 4.60 & & 4.35 & \\
\hline \multicolumn{9}{|c|}{ Primary Care Physician Ratio in PSA (per 1000 population in PSA) } \\
\hline $6+$ Physician Visits & 1.89 & ** & 1.14 & & 2.37 & & 2.10 & \\
\hline$<6$ Physician Visits & 2.07 & & 1.28 & & 2.29 & & 1.98 & \\
\hline Proportion Black in Zip Co & & & & & & & & \\
\hline $6+$ Physician Visits & .1303 & & 5311 & & .0656 & & .1807 & * \\
\hline$<6$ Physician Visits & .1238 & & .5076 & & .0582 & & .1166 & \\
\hline Proportion Hispanic in Zip & Code & & & & & & & \\
\hline $6+$ Physician Visits & .3791 & & .2572 & & .7554 & & .5671 & \\
\hline$<6$ Physician Visits & .3530 & & .2716 & & .7288 & & .5787 & \\
\hline
\end{tabular}


Table 7.4 (cont.)

\begin{tabular}{|c|c|c|c|c|c|c|c|}
\hline \multirow{2}{*}{$\begin{array}{l}\text { High volume }(6+) \\
\text { physician visits in last } \\
12 \text { months } \\
\text { Proportion Elder }(60+) \text { it }\end{array}$} & $\begin{array}{c}\text { White non- } \\
\text { Hispanic (WNH) } \\
N=825\end{array}$ & \multicolumn{2}{|c|}{$\begin{array}{c}\text { Black non- } \\
\text { Hispanic (BNH) } \\
\mathrm{N}=189 \\
\end{array}$} & \multicolumn{2}{|c|}{$\begin{array}{l}\text { Cuban } \\
\text { (CUB) } \\
N=603\end{array}$} & \multicolumn{2}{|c|}{$\begin{array}{c}\text { Non-Cuban } \\
\text { Hispanic (NCH) } \\
\text { N }: 171\end{array}$} \\
\hline & ip Code & & & & & & \\
\hline $6+$ Physician Visits & .2491 & .1355 & * & .2299 & & .1964 & \\
\hline$<6$ Physician Visits & .2624 & .1518 & & .2295 & & .2051 & \\
\hline \multicolumn{8}{|c|}{ Proportion of Population in Zip Code Living Below Poverty } \\
\hline 6r Physician Visits & .1421 & .2743 & & 2108 & **** & .2110 & ** \\
\hline$<6$ Physician Visits & .1448 & .2733 & & .1760 & & .1695 & \\
\hline \multicolumn{8}{|c|}{ NEED CHARACTERISTICS } \\
\hline \multicolumn{8}{|c|}{ Perceived Physical Health Status is Excellent or Good (\%) } \\
\hline Yes & $28.0 \quad * * *$ & 30.8 & $* * *$ & 42.0 & $* * *$ & 34.4 & $* * *$ \\
\hline No & 55.9 & 54.3 & & 70.4 & & 56.9 & \\
\hline \multicolumn{8}{|c|}{ Perceived Emotional Health Status is Excellent or Good (\%) } \\
\hline Yes & $31.2 \quad * * *$ & 32.8 & $* * *$ & 48.1 & *** & 38.7 & * \\
\hline No & 57.4 & 60.0 & & 64.3 & & 54.0 & \\
\hline \multicolumn{8}{|c|}{ ADL/IADL/Mobility Indexes, mean number of reported difficulties } \\
\hline \multicolumn{8}{|c|}{ ADLs (mean number of reported difficulties out of a maximum of 5) } \\
\hline $6+$ Physician Visits & *** & .42 & & .52 & **** & .88 & ***** \\
\hline$<6$ Physician Visits & .22 & .28 & & .20 & & .34 & \\
\hline \multicolumn{8}{|c|}{ IADLs (mean number of reported difficulies out of a maximum of 9) } \\
\hline $6+$ Physician Visits & $.71 * * *$ & 1.10 & & .94 & *** & 1.20 & \\
\hline$<6$ Physician Visits & .40 & .75 & & .30 & & .72 & \\
\hline \multicolumn{8}{|c|}{ Mobility (mean number of reported difficulties out of a maximum of 2) } \\
\hline 6+ Physician Visits & $.52 * * *$ & .90 & $* * *$ & .57 & *** & .72 & $* * *$ \\
\hline$<6$ Physician Visits & 31 & .37 & & .24 & & .33 & \\
\hline
\end{tabular}

nia not applicable 
Table 7.5: Zero-Order Correlations of Selected Independent Measures with At Least One Overnight Hospital Stay in the Last 12 Months for Four Racial/Ethnic Groups of Elders

\begin{tabular}{|c|c|c|c|c|}
\hline $\begin{array}{l}\text { Overnight Hospital Stay in Last } 12 \\
\text { months }\end{array}$ & $\begin{array}{l}\text { White Non- } \\
\text { Hispanic } \\
(\mathrm{N}=890)\end{array}$ & $\begin{array}{l}\text { Btack Non- } \\
\text { Hispanic } \\
(\mathrm{N}=201)\end{array}$ & $\begin{array}{c}\text { Cuban } \\
(\mathrm{N}=670)\end{array}$ & $\begin{array}{l}\text { Non-Cuban } \\
\text { Hispanic } \\
(\mathrm{N}=188)\end{array}$ \\
\hline \multirow{2}{*}{\multicolumn{5}{|c|}{$\begin{array}{l}\text { PREDISPOSING } \\
\text { Demographic }\end{array}$}} \\
\hline & & & & \\
\hline Age & $0.10^{* * *}$ & 0.10 & $0.14^{* * *}$ & $0.15^{* *}$ \\
\hline Female & -0.03 & -0.10 & -0.02 & -0.05 \\
\hline \multicolumn{5}{|l|}{ Educational Attainment } \\
\hline No High School & 0.01 & 0.05 & 0.004 & 0.09 \\
\hline Some High School & 0.03 & +0.05 & -0.005 & -0.09 \\
\hline High School Graduate & -0.05 & -0.05 & -0.02 & -0.02 \\
\hline More than High School diploma & 0.03 & 0.04 & 0.02 & -0.01 \\
\hline \multicolumn{5}{|l|}{ Language spoken in household } \\
\hline English only & -0.02 & All & 0.04 & -0.11 \\
\hline Spanish & 0.002 & $-\cdots$ & -0.03 & 0.11 \\
\hline Creole & - & -- & -0.02 & -- \\
\hline Yiddish & -0.02 & -- & -- & -- \\
\hline Other language & 0.04 & -- & -- & -0.03 \\
\hline \multicolumn{5}{|l|}{ Social Structural } \\
\hline \multicolumn{5}{|l|}{ Marital Status } \\
\hline Married/Live-in Partner & -0.05 & -0.06 & $-0.07 *$ & -0.10 \\
\hline Widowed & 0.02 & 0.02 & 0.005 & 0.08 \\
\hline Divorced/Separated & 0.02 & 0.002 & $0.08 * *$ & 0.06 \\
\hline Never Married & 0.02 & 0.11 & 0.01 & -0.04 \\
\hline Lives alone & 0.003 & 0.05 & 0.02 & -0.11 \\
\hline $\begin{array}{l}\text { Currently Employed in the Labor } \\
\text { Force }\end{array}$ & $-0.07 * *$ & -0.09 & $-0.12 * * *$ & $-0.14 * *$ \\
\hline $\begin{array}{l}\text { Intensity of Social Contact with } \\
\text { Family \& Friends } \\
\text { Self-Reported Religious Affiliation }\end{array}$ & -0.06 & -0.10 & $-0.13 * * *$ & -0.07 \\
\hline Protestant & -0.02 & -0.08 & -0.04 & 0.03 \\
\hline Catholic & 0.04 & 0.07 & $0.00 \mathrm{I}$ & -0.03 \\
\hline Jew & 0.000 & -0.04 & -0.03 & -0.04 \\
\hline No Preference Stated & 0.02 & 0.03 & 0.01 & 0.11 \\
\hline Other Religious Preferencc & -0.06 & 0.05 & 0.06 & -0.07 \\
\hline $\begin{array}{l}\text { Religion is Very/Somewhat } \\
\text { Important in Life } \\
\text { Health Beliefs }\end{array}$ & -0.03 & -0.08 & $-0.07 *$ & 0.04 \\
\hline $\begin{array}{l}\text { Health is a very serious/serious } \\
\text { concern as an elder problem area }\end{array}$ & $0.23 * * *$ & $0.32 * * *$ & $0.19^{* * *}$ & 0.12 \\
\hline $\begin{array}{l}\text { Very/Somewhat worried about loss } \\
\text { of independence }\end{array}$ & $0.09^{* * *}$ & 0.12 & 0.03 & 0.02 \\
\hline $\begin{array}{l}\text { Very/mostly satisfied with Life } \\
\text { during past } 6 \text { months }\end{array}$ & $-0.16 * * *$ & $-0.12 *$ & -0.05 & -0.09 \\
\hline
\end{tabular}


Table 7.5 (cont.)

\begin{tabular}{|c|c|c|c|c|}
\hline $\begin{array}{l}\text { Overnight Hospital Stay in Last } 12 \\
\text { months }\end{array}$ & $\begin{array}{l}\text { White Non-" } \\
\text { Hispanic } \\
(\mathrm{N}=890)\end{array}$ & $\begin{array}{l}\text { Black Non- } \\
\text { Hispanic } \\
(\mathrm{N}-201)\end{array}$ & $\begin{array}{c}\text { Cuban } \\
(\mathrm{N}:-670)\end{array}$ & $\begin{array}{l}\text { Non-Cuban } \\
\text { Hispanic } \\
(\mathrm{N}=188)\end{array}$ \\
\hline \multicolumn{5}{|l|}{$\begin{array}{l}\text { ENABLING FACTORS } \\
\text { Personal/Family Resources }\end{array}$} \\
\hline Household Income $<\$ 10 K$ ? & 0.04 & 0.01 & $0.07 *$ & .0 .06 \\
\hline \multicolumn{5}{|l|}{ Medicare Coverage } \\
\hline $\begin{array}{l}\text { Medicare Part A-May Have Part B } \\
\text { Also }\end{array}$ & $0.10^{* * *}$ & 0.05 & 0.03 & 0.10 \\
\hline No Medicare & $-0.10 * * *$ & -0.01 & -0.03 & -0.10 \\
\hline \multicolumn{5}{|l|}{ Other Health Care Coverage } \\
\hline No Health Insurance & -0.03 & -0.07 & -0.05 & -0.12 \\
\hline Medicaid Coverage Reported & $0.08 * *$ & $0.25 * * *$ & $0.12 * * *$ & $0.14 *$ \\
\hline HMO Plan Coverage Reported & $-0.07 * *$ & -0.04 & -0.03 & -0.02 \\
\hline $\begin{array}{l}\text { Years Living in Miami-Dade County, } \\
\mathrm{FL}\end{array}$ & -0.05 & 0.01 & 0.05 & 0.01 \\
\hline $\begin{array}{l}\text { Resides Full-time in Miami-Dade } \\
\text { County, FL }\end{array}$ & 0.04 & 0.01 & -0.01 & 0.08 \\
\hline $\begin{array}{l}\text { Regular source of bealth care from a } \\
\text { private physician } \\
\text { Community Resources }\end{array}$ & 0.03 & 0.02 & $0.09 * *$ & $0.14 *$ \\
\hline $\begin{array}{l}\text { Hospital Bed Ratio per } 1000 \\
\text { Population in PSA }\end{array}$ & 0.004 & 0.02 & $0.09 * *$ & $0.13 *$ \\
\hline $\begin{array}{l}\text { Primary Care Physician Ratio per } \\
1000 \text { Population in PSA }\end{array}$ & -0.01 & 0.01 & $0.10^{* * *}$ & 0.05 \\
\hline Proportion Black in Zip Code & -0.01 & 0.03 & -0.01 & 0.08 \\
\hline Proportion Hispanic in Zip Code & $0.07^{* *}$ & -0.01 & 0.03 & -0.06 \\
\hline Proportion $60+$ in Zip Code & -0.004 & -0.02 & $0.09 * *$ & -0.02 \\
\hline $\begin{array}{l}\text { Proportion Living Below Poverty in } \\
\text { Zip Code }\end{array}$ & 0.02 & 0.06 & 0.06 & $0.13 *$ \\
\hline NEED FACTORS & & & & \\
\hline $\begin{array}{l}\text { Self-reported Overail Health as } \\
\text { Excellent Good }\end{array}$ & $-0.24 * * *$ & $-0.20 * * *$ & $-0.18 * * *$ & -0.05 \\
\hline $\begin{array}{l}\text { Self-reported Emotional IJealth as } \\
\text { Excellent/Good }\end{array}$ & $-0.11 * * *$ & $-0.16^{* *}$ & -0.06 & $-0.21 * * *$ \\
\hline Number of Reported ADLs & $0.16^{* * *}$ & $0.21 * * *$ & $0.12 * * *$ & $0.18^{* *}$ \\
\hline Number of Reported IADLs & $0.20 * * *$ & $0.28^{* * *}$ & $0.13 * * *$ & $0.20 * * *$ \\
\hline Number of Mobility Limitations & $0.15 * * *$ & $0.24^{* * *}$ & $0.18 * * *$ & 0.10 \\
\hline
\end{tabular}

${ }^{*} \mathrm{p} \leq 0.10$ (two-tailed)

** $p \leq 0.05$ (two-tailed) *** $p \leq 0.01$ (two-tailed)

- No cases available 
Table 7.6: Bivariate Associations of Selected Independent Measures with Overnight Hospital Stay in the Last 12 Months for Four Racial/Ethnic Groups of Elders

\begin{tabular}{|c|c|c|c|c|c|c|}
\hline \multirow{2}{*}{$\begin{array}{l}\text { Overnight hospital stay } \\
\text { in the last } 12 \text { months } \\
\text { TOTAL \% }\end{array}$} & $\begin{array}{c}\text { White non- } \\
\text { Hispanic (WNH) } \\
\mathrm{N}=890\end{array}$ & $\begin{array}{c}\text { Black non- } \\
\text { Hispanic (BNH) } \\
\mathrm{N}=201\end{array}$ & \multicolumn{2}{|c|}{$\begin{array}{l}\text { Cuban } \\
\text { (CUB) } \\
\mathrm{N}=670\end{array}$} & \multicolumn{2}{|c|}{$\begin{array}{c}\text { Non-Cuban } \\
\text { Hispanic (NCH) } \\
\mathrm{N}=188\end{array}$} \\
\hline & 20.9 & 16.9 & 23.0 & & 15.4 & \\
\hline \multirow{2}{*}{\multicolumn{7}{|c|}{ PREDISPOSING CHARACTERISTICS }} \\
\hline & & & & & & \\
\hline \multicolumn{7}{|c|}{ Age (mean years) } \\
\hline Overnight Hospital Stay & $73.75 * * *$ & 70.70 & 73.35 & $* * *$ & 73.45 & ** \\
\hline None & 71.71 & 68.84 & 70.70 & & 69.96 & \\
\hline \multicolumn{7}{|l|}{ Gender $(\%)$} \\
\hline Female & 20.0 & 14.6 & 22.5 & & 14.2 & \\
\hline Male & 22.5 & 22.8 & 23.8 & & 18.0 & \\
\hline \multicolumn{7}{|l|}{ Educational Attainment (\%) } \\
\hline No High School & 23.3 & 18.2 & 22.7 & & 18.0 & \\
\hline Some High School & 20.3 & 12.5 & 21.9 & & 4.8 & \\
\hline High School Graduate & 17.3 & 12.5 & 20.6 & & 12.2 & \\
\hline More than High School & 21.6 & 18.9 & 23.8 & & 13.3 & \\
\hline \multicolumn{7}{|c|}{ Language Spoken Most Often in Home (\%) } \\
\hline English & 20.7 & $\mathrm{~N} / \mathrm{A}$ & 31.6 & & 6.7 & \\
\hline Spanish & 21.4 & $\mathrm{~N} / \mathrm{A}$ & 22.8 & & 17.2 & \\
\hline \multicolumn{7}{|l|}{ Social Structural } \\
\hline \multicolumn{7}{|l|}{ Marital Status (\%) } \\
\hline Married'Live-in Partner & 19.0 & 14.1 & 20.1 & * & 10.8 & \\
\hline Widowed & 22.3 & 17.7 & 23.4 & & 19.1 & \\
\hline Divorced/Scparated & 23.1 & 17.1 & 30.2 & ** & 20.0 & \\
\hline Never married & 25.0 & 37.5 & 25.0 & & 9.1 & \\
\hline \multicolumn{7}{|l|}{ Living Arrangement (\%) } \\
\hline Living Alone & 20.9 & 20.0 & 24.2 & & 8.9 & \\
\hline Living with others & 20.6 & 15.4 & 22.7 & & 17.9 & \\
\hline \multicolumn{7}{|l|}{ Labor Force Participation (\%) } \\
\hline Employed & $15.1 * *$ & 10.9 & 11.4 & *** & 5.3 & ** \\
\hline Not in Labor Force & 22.4 & 18.7 & 24.9 & & 18.2 & \\
\hline \multicolumn{7}{|c|}{ Intensily of Social Contact with Friends \& Family (Mean) } \\
\hline Overnight Hospital Stay & 9.29 & 8.44 & 9.00 & *** & 8.14 & \\
\hline None & 9.62 & 9.11 & 9.76 & & 8.69 & \\
\hline \multicolumn{7}{|l|}{ Religious Affiliation (\%) } \\
\hline Protestant & 19.9 & 15.0 & 17.8 & & 17.9 & \\
\hline Catholic & 24.0 & 27.3 & 23.1 & & 14.9 & \\
\hline Jewish & 20.9 & 0.0 & 0.0 & & 0.0 & \\
\hline No Preference & 23.2 & 25.0 & 25.8 & & 33.3 & \\
\hline Other' & $9.8 *$ & 23.1 & 33.3 & & 7.1 & \\
\hline \multicolumn{7}{|c|}{ Religion is very/somewhat important in life (\%) } \\
\hline Yes & 20.2 & 16.1 & 22.1 & * & 15.9 & \\
\hline No & 23.2 & 33.3 & 31.7 & & 11.1 & \\
\hline \multicolumn{7}{|c|}{ Health Beliefs } \\
\hline \multicolumn{7}{|c|}{ Health is very/somewhat serious concern for elders. (\%) } \\
\hline Yes & $33.9 * * *$ & 31.3 & 31.7 & *** & 18.9 & \\
\hline No & 14.4 & 6.8 & 15.3 & & 10.4 & \\
\hline
\end{tabular}

'Includes "Christian," "Spiritual," and "Other, not coded." 
Table 7.6 (cont.)

\begin{tabular}{|c|c|c|c|c|c|c|c|c|}
\hline $\begin{array}{l}\text { Overnight hospital stay } \\
\text { in the last } 12 \text { montlss }\end{array}$ & \multicolumn{2}{|c|}{$\begin{array}{c}\text { White non- } \\
\text { Hispanic (WNH) } \\
N=890\end{array}$} & \multicolumn{2}{|c|}{$\begin{array}{c}\text { Black non- } \\
\text { Hispanic (BNH) } \\
\mathrm{N}=201\end{array}$} & \multicolumn{2}{|c|}{$\begin{array}{l}\text { Cuban } \\
(\mathrm{CUB}) \\
N=670\end{array}$} & \multicolumn{2}{|c|}{$\begin{array}{c}\text { Non-Cuban } \\
\text { Hispanic (NCH) } \\
\mathrm{N}=188\end{array}$} \\
\hline \multicolumn{9}{|c|}{$\begin{array}{l}\text { Very/somewhat wortied about loss of independence due to physical or mental deterioration in the } \\
\text { future. }(\%)\end{array}$} \\
\hline Yes & 24.4 & $* * *$ & 22.5 & & 23.7 & & 15.6 & \\
\hline No & 16.9 & & 13.6 & & 21.1 & & 13.6 & \\
\hline \multicolumn{9}{|c|}{ Very/Mostly Satisfied with Life in the past 6 months $(\%)$} \\
\hline Yes & 18.1 & *** & 16.0 & * & 21.5 & & 14.1 & \\
\hline No & 36.1 & & 30.4 & & 27.4 & & 23.3 & \\
\hline \multirow{2}{*}{\multicolumn{9}{|c|}{$\begin{array}{l}\text { ENABLING CHARACTERISTICS } \\
\text { Personal/Family Resources }\end{array}$}} \\
\hline & & & & & & & & \\
\hline \multicolumn{9}{|c|}{ Household Income less than $\$ 10,000$ per year $(\%)$} \\
\hline Yes & 25.0 & & 17.3 & & 26.2 & $*$ & 12.3 & \\
\hline No & 20.3 & & 16.7 & & 20.6 & & 17.1 & \\
\hline \multicolumn{9}{|l|}{ Medicare Coverage $(\%)$} \\
\hline Medicare Part A & 23.6 & $* * *$ & 16.8 & & 23.7 & & 17.5 & \\
\hline No Medicare Coverage & 14.3 & $* * *$ & 15.9 & & 20.4 & & 9.8 & \\
\hline \multicolumn{9}{|l|}{ Medicaid Coverage $(\%)$} \\
\hline Yes & 37.5 & ** & 34.1 & $* * *$ & 29.8 & $* * *$ & 22.6 & * \\
\hline No & 20.2 & & 11.8 & & 19.2 & & 12.1 & \\
\hline \multicolumn{9}{|l|}{ HMO Plan $(\%)$} \\
\hline Yes & 16.0 & $* *$ & 14.9 & & 21.1 & & 14.3 & \\
\hline No & 22.4 & & 17.8 & & 23.6 & & 15.8 & \\
\hline \multicolumn{9}{|c|}{ Part-time or Full-time Resident of Miami-Dade County (\%) } \\
\hline Full-time & 21.6 & & 17.0 & & 23.0 & & 15.6 & \\
\hline Part-time & 17.3 & & 14.3 & & 27.3 & & 0.0 & \\
\hline \multicolumn{9}{|l|}{ Regular Source of Health Care } \\
\hline Private Physician & 21.9 & & 18.1 & & 26.8 & ** & 20.4 & $*$ \\
\hline Other Source & 18.9 & & 16.2 & & 19.5 & & 9.9 & \\
\hline \multicolumn{9}{|c|}{ Community Resources (Means) } \\
\hline \multicolumn{9}{|c|}{ Hospital Bed Ratio in PSA (per 1000 population in PSA) } \\
\hline Overnight Hospital Stay & 4.16 & & 4.90 & & 5.27 & $* *$ & 5.43 & $*$ \\
\hline None & 4.14 & & 5.02 & & 4.71 & & 4.55 & \\
\hline \multicolumn{9}{|c|}{ Primary Care Physician Ratio in PSA (per 1000 population in PSA) } \\
\hline Overnight Hospital Stay & 1.99 & & 1.21 & & 2.50 & $* * *$ & 2.17 & \\
\hline None & 2.00 & & 1.24 & & 2.25 & & 2.03 & \\
\hline \multicolumn{9}{|l|}{ Proportion Black in Zip Code } \\
\hline Overnight Hospital Stay & .1240 & & .5252 & & .0641 & & .1797 & \\
\hline None & .1278 & & .5068 & & .0676 & & .1305 & \\
\hline \multicolumn{9}{|c|}{ Proportion Hispanic in Zip Code } \\
\hline Overnight Hospital Stay & .3952 & ** & .2667 & & .7547 & & .5461 & \\
\hline None & .3540 & & .2698 & & .7402 & & .5874 & \\
\hline \multicolumn{9}{|c|}{ Proportion Elder $(60+)$ in Zip Code } \\
\hline Overnight Hospital Stay & .2542 & & .1429 & & .2416 & ** & .1975 & \\
\hline None & .2556 & & .1465 & & .2252 & & .2028 & \\
\hline \multicolumn{9}{|c|}{ Proportion of Population Living Below Poverty in Zip Code } \\
\hline Overnight Hospital Stay & .1479 & & .2880 & & .2071 & & .2178 & * \\
\hline None & .1437 & & .2682 & & .1936 & & .1776 & \\
\hline
\end{tabular}


Table 7.6 (cont.)

\begin{tabular}{|c|c|c|c|c|c|c|c|c|}
\hline \multirow{2}{*}{$\begin{array}{l}\text { Overnight hospital stay } \\
\text { in the last } 12 \text { months } \\
\text { Yes }\end{array}$} & \multicolumn{2}{|c|}{$\begin{array}{c}\text { White non- } \\
\text { Hispanic (WNH) } \\
\text { N }=890\end{array}$} & \multicolumn{2}{|c|}{$\begin{array}{c}\text { Black non- } \\
\text { Hispanic }(\mathrm{BNH}) \\
\mathrm{N}=201\end{array}$} & \multicolumn{2}{|c|}{$\begin{array}{l}\text { Cuban } \\
\text { (CUB) } \\
\mathrm{N}=670\end{array}$} & \multicolumn{2}{|c|}{$\begin{array}{c}\text { Non-Cuban } \\
\text { Hispanic }(\mathrm{NCH}) \\
\text { N }=188\end{array}$} \\
\hline & 14.9 & ***m & 10.7 & $* * *$ & 17.1 & $* * *$ & 13.6 & \\
\hline No & 37.7 & & 25.9 & & 32.3 & & 17.3 & \\
\hline \multicolumn{9}{|c|}{ Perceived Emotional Health Stalus is Excellent or Good (\%) } \\
\hline Yes & 19.0 & *** & 12.4 & ** & 21.3 & & 10.4 & $* *$ \\
\hline No & 31.2 & & 25.4 & & 27. I & & 27.4 & \\
\hline \multicolumn{9}{|c|}{$\begin{array}{l}\text { ADL/IADL/Mobility Indexes, mean number of reported difficulties } \\
\text { ADLs }\end{array}$} \\
\hline Overnight Hospital Stay & .52 & *** & .70 & $* * *$ & .53 & *** & .97 & * \\
\hline None & .21 & & .25 & & .30 & & .46 & \\
\hline \multicolumn{9}{|l|}{ IADLs } \\
\hline Ovemight Hospital Stay & 1.05 & *** & 1.91 & **** & .96 & *** & 1.69 & $* * *$ \\
\hline None & .36 & & .66 & & .52 & & .69 & \\
\hline \multicolumn{9}{|l|}{ Mobility } \\
\hline Overnight Hospital Stay & .58 & $* * *$ & .97 & $* * *$ & .63 & **** & .66 & \\
\hline None & .32 & & .48 & & .33 & & .44 & \\
\hline
\end{tabular}

-- No cases available 
Table 7.7: Zero-Order Correlations of Selected Independent Measures with Self-Reported Complete Physical Exam in the Last 12 Months for Four Racial/Ethnic Groups of Elders

\begin{tabular}{|c|c|c|c|c|}
\hline $\begin{array}{l}\text { Complete Physical Exam in Last } \\
12 \text { months }\end{array}$ & $\begin{array}{l}\text { White Non- } \\
\text { Hispanic } \\
(\mathrm{N}=667)\end{array}$ & $\begin{array}{l}\text { Black Non- } \\
\text { Hispanic } \\
(\mathrm{N}=200)\end{array}$ & $\begin{array}{c}\text { Cuban } \\
(\mathrm{N}=667)\end{array}$ & $\begin{array}{l}\text { Non-Cuban } \\
\text { Hispanic } \\
(\mathrm{N}=188)\end{array}$ \\
\hline \multicolumn{5}{|l|}{$\begin{array}{l}\text { PREDISPOSING } \\
\text { Demographic }\end{array}$} \\
\hline Age & $0.16^{* * * *}$ & $0.25^{* * *}$ & $0.11^{* * *}$ & $0.2 ! * * *$ \\
\hline Female & 0.05 & 0.07 & -0.02 & -0.01 \\
\hline \multicolumn{5}{|l|}{ Educational Aftainment } \\
\hline No High School & -0.02 & -0.08 & 0.02 & 0.03 \\
\hline Some High School & .0 .01 & -0.03 & $-0.08 * *$ & $-0.16^{*}$ \\
\hline High School Graduate & -0.02 & -0.03 & 0.01 & 0.01 \\
\hline More than High School diploma & 0.03 & $0.16 * *$ & 0.03 & 0.07 \\
\hline \multicolumn{5}{|l|}{ Language spoken in the home } \\
\hline English only & 0.01 & All & -0.005 & 0.005 \\
\hline Spanish & -0.03 & - & $0.00 \mathrm{i}$ & -0.01 \\
\hline Creole & $\cdots$ & -- & 0.02 & -- \\
\hline Yiddish & 0.05 & -- & 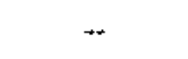 & -- \\
\hline Other language & -0.02 & -. & -- & 0.04 \\
\hline \multicolumn{5}{|l|}{ Social Structural } \\
\hline \multicolumn{5}{|l|}{ Marital Status } \\
\hline MarriedLive-in Partner & 0.02 & -0.04 & -0.03 & $-0.12 *$ \\
\hline Widowed & 0.01 & $0.15 * *$ & 0.05 & 0.11 \\
\hline Divorced/Sepirated & -0.03 & $-0.15 * *$ & 0.000 & 0.02 \\
\hline Never Married & -0.01 & 0.02 & -0.05 & -0.001 \\
\hline Lives alone & -0.02 & 0.003 & 0.01 & 0.005 \\
\hline $\begin{array}{l}\text { Currently Employed in the Labor } \\
\text { Force }\end{array}$ & $-0.10^{* * *}$ & -0.08 & $-0.07^{*}$ & -0.05 \\
\hline $\begin{array}{l}\text { Intensity of Social Contact with } \\
\text { Family \& Friends }\end{array}$ & 0.01 & $0.14 *$ & $0.11 * * *$ & 0.001 \\
\hline \multicolumn{5}{|l|}{ Self-reported Religious Affiliation } \\
\hline Protestant & $-0.13 * * *$ & -0.02 & 0.01 & -0.05 \\
\hline Catholic & -0.01 & 0.004 & 0.03 & 0.04 \\
\hline Jew & $0.36 * * *$ & 0.05 & 0.03 & 0.06 \\
\hline No Preference Stated & -0.04 & -0.02 & $-0.11 * * *$ & 0.03 \\
\hline Other Retigious Preference & -0.01 & 0.02 & 0.03 & -0.05 \\
\hline $\begin{array}{l}\text { Religion is Very/Somewhat } \\
\text { important in Life } \\
\text { Health Beliefs }\end{array}$ & -0.01 & -0.08 & 0.03 & 0.005 \\
\hline $\begin{array}{l}\text { Health is a very serious/serious } \\
\text { concern as a elder problem area }\end{array}$ & 0.04 & 0.05 & 0.06 & 0.06 \\
\hline $\begin{array}{l}\text { Very/Somewhat worried about loss } \\
\text { of independence }\end{array}$ & 0.03 & -0.10 & 0.02 & -0.06 \\
\hline $\begin{array}{l}\text { Veryimostly salisfied with life } \\
\text { during past } 6 \text { months }\end{array}$ & 0.01 & 0.07 & $0.11 * * *$ & -0.01 \\
\hline
\end{tabular}


Table 7.7 (cont.)

\begin{tabular}{|c|c|c|c|c|}
\hline $\begin{array}{l}\text { Complete Physical Exam in Last } \\
12 \text { months }\end{array}$ & $\begin{array}{l}\text { White Non- } \\
\text { Hispanic } \\
(\mathrm{N}=667)\end{array}$ & $\begin{array}{l}\text { Black Non- } \\
\text { Hispanic } \\
(\mathrm{N}=200)\end{array}$ & $\begin{array}{c}\text { Cuban } \\
(\mathrm{N}=667)\end{array}$ & $\begin{array}{l}\text { Non-Cuban } \\
\text { Hispanic } \\
(\mathrm{N}=188)\end{array}$ \\
\hline \multicolumn{5}{|l|}{$\begin{array}{l}\text { ENABLING FACTORS } \\
\text { Personal/Family Resources }\end{array}$} \\
\hline Household Income $<\$ 10 \mathrm{~K}$ ? & $-0.001 * * *$ & -0.05 & -0.01 & -0.01 \\
\hline \multicolumn{5}{|l|}{ Medicare Coverage } \\
\hline Medicare Part A only & 0.04 & 0.06 & -0.02 & -0.01 \\
\hline Medicare Part A \& B & $0.10 * * *$ & $0.21 * * *$ & $0.14 * * *$ & $0.18^{* *}$ \\
\hline No Medicare & $-0.13 * * *$ & $-0.26 * * *$ & $-0.14 * * *$ & $-0.18 * *$ \\
\hline \multicolumn{5}{|l|}{ Other Health Care Coverage } \\
\hline No Insurance & -0.04 & $-0.22^{* *}$ & $-0.26 * * *$ & $-0.20 * * *$ \\
\hline Medicaid Coverage Reported & -0.04 & 0.04 & $0.08 * *$ & $0.15^{* *}$ \\
\hline IIMO Plan Coverage Reported & -0.002 & 0.04 & $0.06 *$ & 0.12 \\
\hline Years Living in Dade County, FL & -0.05 & 0.08 & -0.002 & -0.03 \\
\hline Resides Full-time in Dade County & -0.02 & -0.02 & $-0.06^{*}$ & -0.04 \\
\hline $\begin{array}{l}\text { Regular source of health care from } \\
\text { a private physician } \\
\text { Community Resources }\end{array}$ & 0.03 & 0.02 & -0.001 & -0.01 \\
\hline $\begin{array}{l}\text { Hospital Bed Ratio per } 1000 \\
\text { Population in PSA }\end{array}$ & $0.09 * * *$ & 0.08 & 0.004 & 0.11 \\
\hline $\begin{array}{l}\text { Primary Care Physician Ratio per } \\
1000 \text { Population in PSA }\end{array}$ & $0.08 * *$ & -0.04 & 0.02 & -0.004 \\
\hline Proportion Black in Zip Code & -0.01 & 0.07 & -0.03 & 0.07 \\
\hline Proportion Hispanic in Zip Code & -0.05 & 0.02 & -0.04 & 0.02 \\
\hline Proportion $60+$ in Zip Code & $0.15^{* * *}$ & -0.01 & 0.04 & 0.05 \\
\hline $\begin{array}{l}\text { Proportion Living Below Poverty } \\
\text { in Zip Code }\end{array}$ & 0.04 & 0.07 & -0.04 & $0.15 * *$ \\
\hline \multicolumn{5}{|l|}{ NEED FACTORS } \\
\hline $\begin{array}{l}\text { Self-reported Overall Health as } \\
\text { Excellent'Good }\end{array}$ & -0.04 & 0.06 & -0.06 & 0.08 \\
\hline $\begin{array}{l}\text { Self-reported Emotional Health as } \\
\text { Excellent/Good }\end{array}$ & 0.05 & $-0.0 !$ & 0.02 & 0.01 \\
\hline Number of Reported ADLs & 0.05 & -0.01 & 0.04 & 0.05 \\
\hline Number of Reported IADLs & $0.06 *$ & 0.07 & $0.07 *$ & 0.10 \\
\hline Number of Mobility Limitations & $0.06^{*}$ & 0.05 & $0.09^{* *}$ & 0.03 \\
\hline
\end{tabular}

${ }^{*} \mathrm{p} \leq 0.10$ (two-tailed) ${ }^{* *} \mathrm{p}<0.05$ (two-tailed) ${ }^{* * *} \mathrm{p} \leq 0.01$ (two-tailed)

- No cases available 
Table 7.8: Bivariate Associations of Selected Independent Variables with a Complete Physical Exam in the Last 12 Months

\begin{tabular}{|c|c|c|c|c|c|c|c|c|}
\hline \multirow{2}{*}{$\begin{array}{l}\text { Complete Physical } \\
\text { Exam in Last } 12 \text { months } \\
\text { TOTAL \% }\end{array}$} & \multicolumn{2}{|c|}{$\begin{array}{c}\text { White non- } \\
\text { Hispanic (WNH) } \\
\mathrm{N}=892\end{array}$} & \multicolumn{2}{|c|}{$\begin{array}{c}\text { Black non- } \\
\text { Hispanic (BNH) } \\
N=200\end{array}$} & \multicolumn{2}{|c|}{$\begin{array}{l}\text { Cuban } \\
\text { (CUB) } \\
N=667\end{array}$} & \multicolumn{2}{|c|}{$\begin{array}{c}\text { Non-Cuban } \\
\text { Hispanic (NCH) } \\
N=188\end{array}$} \\
\hline & 79.7 & & 81.5 & & 80.2 & & 72.9 & \\
\hline \multicolumn{9}{|c|}{ PREDISPOSING CHARACTERISTICS } \\
\hline \multicolumn{9}{|c|}{ Demographic } \\
\hline Complete [hysical Exam & 72.83 & $* * *$ & 70.04 & $* * *$ & 71.75 & *** & 71.57 & $* * *$ \\
\hline No & 69.64 & & 65.35 & & 69.55 & & 67.69 & \\
\hline \multicolumn{9}{|l|}{ Gender $\%$} \\
\hline Female & 81.3 & & 83.2 & & 79.6 & & 72.4 & \\
\hline Male & 77.1 & & 77.2 & & 81.3 & & 73.8 & \\
\hline \multicolumn{9}{|l|}{ Educational Attainment (\%) } \\
\hline No High School & 76.7 & & 77.9 & & 80.8 & & 73.8 & \\
\hline Some High School & 77.9 & & 80.0 & & 71.2 & $* *$ & 52.4 & $* *$ \\
\hline High School Graduate & 78.5 & & 80.0 & & 81.3 & & 73.2 & \\
\hline More than High School & 80.4 & & 94.6 & ** & 82.2 & & 76.7 & \\
\hline \multicolumn{9}{|c|}{ Language Spoken Most Often in Home (\%) } \\
\hline English & 79.7 & & -- & & 78.9 & & 73.3 & \\
\hline Spanish & 71.4 & & -- & & 80.2 & & 72.6 & \\
\hline \multicolumn{9}{|l|}{ Social Structural } \\
\hline \multicolumn{9}{|l|}{ Marital Status (\%) } \\
\hline Married/Live-in Partner & 80.4 & & 79.5 & & 79.0 & & 66.2 & $*$ \\
\hline Widowed & 80.2 & & 88.6 & $* *$ & 83.5 & & 79.4 & \\
\hline Divorced/Separated & 76.2 & & 68.6 & $* *$ & 80.2 & & 74.3 & \\
\hline Never married & 77.5 & & 85.7 & & 71.9 & & 72.7 & \\
\hline \multicolumn{9}{|l|}{ Living Arrangement (\%) } \\
\hline Living Alone & 78.8 & & 82.0 & & 81.0 & & 73.3 & \\
\hline Living with others & 80.1 & & 81.8 & & 80.0 & & 72.9 & \\
\hline \multicolumn{9}{|l|}{ Labor Force Participation (\%) } \\
\hline Employed & 71.3 & $* * *$ & 76.1 & & 74.3 & $*$ & 68.4 & \\
\hline Not in Labor Force & 81.8 & & 83.1 & & 81.6 & & 73.6 & \\
\hline \multicolumn{9}{|c|}{ Intensily of Social Contact with Friends \& Family (Mean) } \\
\hline Complete Physical Exam & 9.57 & & 9.16 & $*$ & 9.73 & *** & 8.61 & \\
\hline No & 9.49 & & 8.20 & & 9.05 & & 8.60 & \\
\hline \multicolumn{9}{|l|}{ Religious Affiliation (\%) } \\
\hline Protestant & 72.3 & $* * *$ & 80.7 & & 81.1 & & 67.9 & \\
\hline Catholic & 79.1 & & 81.8 & & 81.1 & & 73.9 & \\
\hline Jewish & 88.9 & $* * *$ & 100.0 & & 100.0 & & 100.0 & \\
\hline No Preference & 74.3 & & 75.0 & & 61.3 & $* * *$ & 77.8 & \\
\hline Other' & 78.6 & & 84.6 & & 84.8 & & 64.3 & \\
\hline \multicolumn{9}{|c|}{ Religion is very/somewhat important in life (\%) } \\
\hline Yes & 79.3 & & 81.3 & & 80.7 & & 72.9 & \\
\hline No & 80.5 & & 100.0 & & 76.3 & & 72.2 & \\
\hline \multicolumn{9}{|l|}{ Health Beliefs } \\
\hline \multicolumn{9}{|c|}{ Health is very/somewhat serious concern for elders (\%) } \\
\hline Yes & 82.0 & & 84.3 & & 82.4 & & 75.6 & \\
\hline No & 78.5 & & 80.2 & & 78.0 & & 69.8 & \\
\hline
\end{tabular}

\footnotetext{
'Includes "Christian," "Spiritual," and "Other, not coded."
} 


\begin{tabular}{|c|c|c|c|c|c|c|c|c|}
\hline $\begin{array}{l}\text { Complete Physical } \\
\text { Exam in Last } 12 \text { months }\end{array}$ & \multicolumn{2}{|c|}{$\begin{array}{c}\text { White non- } \\
\text { Hispanic (WNH) } \\
\mathrm{N}=892\end{array}$} & \multicolumn{2}{|c|}{$\begin{array}{c}\text { Black non- } \\
\text { IIispanic (BNH) } \\
\mathrm{N}=200\end{array}$} & \multicolumn{2}{|c|}{$\begin{array}{l}\text { Cuban } \\
\text { (CUB) } \\
N=667 \\
\end{array}$} & \multicolumn{2}{|c|}{$\begin{array}{c}\text { Non-Cuban } \\
\text { Hispanic }(\mathrm{NCH}) \\
\mathrm{N}=188\end{array}$} \\
\hline \multirow{2}{*}{\multicolumn{9}{|c|}{$\begin{array}{l}\text { Very/somewhat worried about loss of independence due to physical or mental deterioration in the future. } \\
(\%)\end{array}$}} \\
\hline & & & & & & & & \\
\hline No & 78.6 & & 86.3 & & 78.9 & & 77.3 & \\
\hline \multicolumn{9}{|c|}{ Very/Mostly Satisfied with Life in the past 6 months $(\%)$} \\
\hline Yes & 79.7 & & 82.7 & & 82.5 & $* * *$ & 72.4 & \\
\hline No & 78.7 & & 73.9 & & 71.0 & & 73.3 & \\
\hline \multicolumn{9}{|c|}{$\begin{array}{l}\text { ENABLING CHARACTERISTICS } \\
\text { Personal/Family Resources }\end{array}$} \\
\hline \multicolumn{9}{|c|}{ Household Income less than $\$ 10,000$ per year $(\%)$} \\
\hline Yes & 79.6 & & 79.0 & & 79.8 & & 72.3 & \\
\hline No & 79.7 & & 83.2 & & 80.5 & & 73.2 & \\
\hline \multicolumn{9}{|l|}{ Medicare Coverage $(\%)$} \\
\hline Medicare Part A Only & 87.2 & & 88.9 & & 78.0 & & 71.4 & \\
\hline $\begin{array}{l}\text { Both Medicare Part A } \\
\text { and Part B }\end{array}$ & 82.3 & $* * *$ & 88.5 & $* * *$ & 83.8 & $* * *$ & 79.6 & ** \\
\hline No Medicare Coverage & 71.3 & $* * *$ & 66.7 & $* * *$ & 70.1 & $* * *$ & 62.3 & $* *$ \\
\hline \multicolumn{9}{|l|}{ Medicaid Coverage $(\%)$} \\
\hline Yes & 71.9 & & 84.1 & & 85.1 & ** & 82.3 & $* *$ \\
\hline No & 79.9 & & 80.3 & & 78.0 & & 68.5 & \\
\hline \multicolumn{9}{|l|}{ HMO Plan (\%) } \\
\hline Yes & 79.5 & & 83.6 & & 84.9 & * & 85.7 & \\
\hline No & 79.6 & & 79.8 & & 79.1 & & 70.9 & \\
\hline \multicolumn{9}{|c|}{ Part-time or Full-time Resident of Miami-Dade County (\%) } \\
\hline Full-time & 79.2 & & 81.3 & & 80.0 & * & 72.1 & \\
\hline Part-time & 82.0 & & 85.7 & & 100.0 & & 83.3 & \\
\hline \multicolumn{9}{|c|}{ Regular Source of I Iealth Care (\%) } \\
\hline Private Physician & 81.0 & & 83.0 & & 81.2 & & 73.5 & \\
\hline Other source & 78.4 & & 81.7 & & 81.2 & & 74.3 & \\
\hline \multicolumn{9}{|c|}{ Community Resources (Means) } \\
\hline \multicolumn{9}{|c|}{ Hospital Bed Ratio in PSA (per 1000 population in PSA) } \\
\hline Complete Physical Exam & 4.27 & $* * *$ & 5.00 & & 4.84 & & 4.85 & \\
\hline None & 3.69 & & 4.56 & & 4.81 & & 4.25 & \\
\hline \multicolumn{9}{|c|}{ Primary Care Physician Ratio in I'SA (per 1000 population in PSA) } \\
\hline Complete Physical Exam & 2.05 & ** & 1.20 & & 2.31 & & 2.05 & \\
\hline None & 1.81 & & 1.27 & & 2.26 & & 2.06 & \\
\hline \multicolumn{9}{|l|}{ Proportion Black in Zip Code } \\
\hline Complete Physical Exam & .1261 & & .5204 & & .0649 & & .1468 & \\
\hline None & .1298 & & .4738 & & .0762 & & .1144 & \\
\hline \multicolumn{9}{|c|}{ Proportion Hispanic in Zip Code } \\
\hline Complete Physical Exam & .3580 & & .2706 & & .7389 & & .5841 & \\
\hline None & .3858 & & .2631 & & .7588 & & .5732 & \\
\hline \multicolumn{9}{|c|}{ Proportion Elder $(60+)$ in Zip Code } \\
\hline Complete Physical Exam & .2655 & $* * *$ & .1455 & & .2300 & & .2049 & \\
\hline None & .2141 & & .1464 & & .2226 & & .1943 & \\
\hline \multicolumn{9}{|c|}{ Proportion of Population Living Below Poverty in Zip Code } \\
\hline Complete Physical Exam & .1466 & & .2768 & & .1947 & & .1938 & ** \\
\hline None & .1387 & & .2534 & & .2043 & & .1554 & \\
\hline
\end{tabular}


Table 7.8 (cont.)

\begin{tabular}{|c|c|c|c|c|c|}
\hline $\begin{array}{l}\text { Complete Physical } \\
\text { Exam in Last } 12 \text { months }\end{array}$ & $\begin{array}{c}\text { White non- } \\
\text { Hispanic (WNH) } \\
\mathrm{N}=892\end{array}$ & $\begin{array}{c}\text { Black non- } \\
\text { Hispanic (BNI) } \\
N=200\end{array}$ & \multicolumn{2}{|c|}{$\begin{array}{l}\text { Cuban } \\
\text { (CUB) } \\
\mathrm{N}=667\end{array}$} & $\begin{array}{c}\text { Non-Cuban } \\
\text { Hispanic }(\mathrm{NCH}) \\
\mathrm{N}=188\end{array}$ \\
\hline \multicolumn{6}{|c|}{ NEED CHARACTERISTICS } \\
\hline \multicolumn{6}{|c|}{ Perceived Physical J lealth Status is Excellent or Good } \\
\hline Yes & 78.7 & 83.8 & 78.5 & & 75.5 \\
\hline No & 82.0 & 78.8 & 83.5 & & 68.0 \\
\hline \multicolumn{6}{|c|}{ Perccived Emotional Ilealth Status is Excellent or Good } \\
\hline Yes & 80.3 & 81.8 & 80.9 & & 73.9 \\
\hline No & 75.3 & 82.3 & 78.7 & & 72.5 \\
\hline \multicolumn{6}{|c|}{$\begin{array}{l}\text { ADL/IADL/Mobility Indexes, mean number of reported difficulties } \\
\text { ADLs }\end{array}$} \\
\hline Complete Physical Exam & .30 & .32 & .38 & & .57 \\
\hline None & .20 & .35 & .29 & & .45 \\
\hline \multicolumn{6}{|l|}{ IALLS } \\
\hline Complete Physical Exam & .55 & .93 & .68 & $*$ & .96 \\
\hline None & .35 & .62 & .42 & & .55 \\
\hline \multicolumn{6}{|l|}{ Mobility Limitations } \\
\hline Complete Physical Exam & .40 & .58 & .44 & ** & .49 \\
\hline None & .29 & .49 & .27 & & .43 \\
\hline
\end{tabular}


Table 7.9: Explained Variance $\left(R^{2}\right)$ Resulting from Separately Entering the Three Major Blocks of Variables and Increments of Explained Variance within Major Blocks for SelfReported Physician Contact in Last 12 months for Four Racial/Ethnic Groups of Elders

\begin{tabular}{|c|c|c|c|c|}
\hline & $\begin{array}{l}\text { White non- } \\
\text { Hispanic }\end{array}$ & $\begin{array}{c}\text { Black non- } \\
\text { Hispanic }\end{array}$ & Cuban & $\begin{array}{c}\text { Non-Cubar } \\
\text { Hispanic }\end{array}$ \\
\hline Need & 0.018 & 0.007 & 0.009 & 0.055 \\
\hline Chi-square & 16.020 & 1.438 & 5.773 & 10.316 \\
\hline (Significance) & $(.001)$ & $(.697)$ & $(.123)$ & $(.016)$ \\
\hline Enabling & 0.027 & 0.104 & 0.065 & 0.110 \\
\hline Community & 0.002 & 0.018 & 0.010 & 0.026 \\
\hline Personal/Family & $0.025 * * *$ & $0.086 \quad * *$ & $0.055 \quad * * *$ & 0.084 \\
\hline $\begin{array}{l}\text { Chi-square } \\
\text { (Significance) }\end{array}$ & $\begin{array}{l}21.49 \mathrm{I} \\
(.029)\end{array}$ & $\begin{array}{l}19.237 \\
(.057)\end{array}$ & $\begin{array}{r}40.312 \\
(.000)\end{array}$ & $\begin{array}{l}18.159 \\
(.078)\end{array}$ \\
\hline Predisposing & 0.030 & .174 & 0.060 & 0.133 \\
\hline Demographic & $0.014 * *$ & $.071 \quad * * *$ & $0.023 * * *$ & 0.043 \\
\hline Social Structure & $0.009 * *$ & $.073 \quad * *$ & $0.023 * *$ & 0.070 \\
\hline Health Beliefs & 0.007 & .030 & $0.014 \quad * *$ & 0.020 \\
\hline Chi-square & 23.883 & 32.333 & 36.406 & 24.312 \\
\hline (Significance) & $(.032)$ & $(.001)$ & $(.001)$ & $(.028)$ \\
\hline
\end{tabular}

Table 7.10: Explained Variance $\left(R^{2}\right)$ Resulting from Separately Entering the Three Major Blocks of Variables and Increment of Explained Variance within Major Blocks for High Volume Physician Contact in Last 12 months for Four Racial/Ethnic Groups of Elders

\begin{tabular}{|c|c|c|c|c|c|}
\hline & $\begin{array}{l}\text { White non- } \\
\text { Hispanic }\end{array}$ & $\begin{array}{l}\text { Black non- } \\
\text { Hispanic }\end{array}$ & Cuban & \multicolumn{2}{|c|}{ Non Cuban } \\
\hline Need & 0.081 & 0.095 & 0.107 & \multicolumn{2}{|c|}{0.068} \\
\hline Chi-square & 68.422 & \multirow{2}{*}{$\begin{array}{l}18.348 \\
(.000)\end{array}$} & \multirow{2}{*}{$\begin{array}{l}67.433 \\
(.000)\end{array}$} & \multirow{2}{*}{\multicolumn{2}{|c|}{$\begin{array}{l}11.677 \\
(.009)\end{array}$}} \\
\hline (Significance) & $(.000)$ & & & & \\
\hline Enabling & 0.037 & 0.056 & 0.096 & \multicolumn{2}{|c|}{0.219} \\
\hline Community & 0.008 & $0.0 \mathrm{l} 0$ & 0.033 *** & 0.057 & $* *$ \\
\hline Personal/Family & $0.029 \quad * * *$ & 0.046 & $0.063 \quad * * *$ & 0.162 & $* * *$ \\
\hline $\begin{array}{l}\text { Chi-square } \\
\text { (Significance) }\end{array}$ & $\begin{array}{r}27.695 \\
(.004)\end{array}$ & $\begin{array}{r}9.548 \\
(.571)\end{array}$ & $\begin{array}{l}56.014 \\
(.000)\end{array}$ & \multicolumn{2}{|c|}{$\begin{array}{l}36.050 \\
(.000)\end{array}$} \\
\hline Predisposing & 0.114 & 0.279 & 0.169 & \multicolumn{2}{|c|}{0.267} \\
\hline Demographic & 0.009 & $0.068 * *$ & $0.052 \quad * * *$ & 0.125 & $* * *$ \\
\hline Social Structure & 0.006 & 0.096 & 0.073 & 0.071 & $* *$ \\
\hline Health Beliefs & 0.099 *** & $0.115 \quad * * *$ & $0.044 * *$ & 0.072 & $* * *$ \\
\hline $\begin{array}{l}\text { Chi-square } \\
\text { (Significance) }\end{array}$ & $\begin{array}{l}88.994 \\
(.000)\end{array}$ & $\begin{array}{l}52.017 \\
(.000)\end{array}$ & $\begin{array}{l}99.990 \\
(.000)\end{array}$ & \multicolumn{2}{|c|}{$\begin{array}{l}47.916 \\
(.000)\end{array}$} \\
\hline
\end{tabular}


Table 7.11: Explained Variance $\left(\mathrm{R}^{2}\right)$ Resulting from Separately Entering the Three Major Blocks of Variables and Increment of Explained Variance within Major Blocks for Overnight Hospital Stay in Last 12 months for Four Racial/Ethnic Groups of Elders

\begin{tabular}{|c|c|c|c|c|}
\hline & $\begin{array}{l}\text { White non- } \\
\text { Hispanic }\end{array}$ & $\begin{array}{l}\text { Black non- } \\
\text { Hispanic }\end{array}$ & Cuban & $\begin{array}{c}\text { Non-Cuban } \\
\text { Hispanic }\end{array}$ \\
\hline Need & 0.070 & 0.092 & 0.040 & 0.059 \\
\hline Chi-square & 63.100 & 18.814 & 26.763 & J1.224 \\
\hline (Significance) & $(.000)$ & $(.000)$ & $(.000)$ & $(.011)$ \\
\hline Enabling & 0.030 & 0.056 & 0.041 & 0.132 \\
\hline Community & 0.001 & 0.004 & $0.017 \quad * *$ & $0.041 *$ \\
\hline Personal/Family & $0.029 \quad * * *$ & 0.052 & $0.024 *$ & $0.091 *$ \\
\hline $\begin{array}{l}\text { Chi-square } \\
\text { (Significance) }\end{array}$ & $\begin{array}{l}22.624 \\
(.014)\end{array}$ & $\begin{array}{l}10.157 \\
(.427)\end{array}$ & $\begin{array}{l}25.256 \\
(.008)\end{array}$ & $\begin{array}{l}22.011 \\
(.024)\end{array}$ \\
\hline Predisposing & 0.070 & 0.167 & 0.075 & 0.069 \\
\hline Demographic & 0.007 & 0.012 & $0.025 \quad * * *$ & 0.023 \\
\hline Social Structure & 0.007 & 0.029 & 0.031 & 0.029 \\
\hline Health Beliefs & $0.056 \quad * * *$ & $0.126 * * *$ & $0.019^{* * *}$ & 0.017 \\
\hline $\begin{array}{l}\text { Chi-square } \\
\text { (Significance) }\end{array}$ & $\begin{array}{l}57.138 \\
(.000)\end{array}$ & $\begin{array}{l}31.042 \\
(.002)\end{array}$ & $\begin{array}{l}46.214 \\
(.000)\end{array}$ & $\begin{array}{l}12.212 \\
(.510)\end{array}$ \\
\hline
\end{tabular}

Table 7.12: Explained Variance $\left(\mathrm{R}^{2}\right)$ Resulting from Separately Entering the Three Major Blocks of Variables and Increment of Explained Variance within Major Blocks for SelfReported Complete Physical Exam in Last 12 months for Four Racial/Ethnic Groups of Elders

\begin{tabular}{|c|c|c|c|c|}
\hline & $\begin{array}{l}\text { White non- } \\
\text { Hispanic }\end{array}$ & $\begin{array}{l}\text { Black non- } \\
\text { Hispanic }\end{array}$ & Cuban & $\begin{array}{c}\text { Non-Cuban } \\
\text { Hispanic }\end{array}$ \\
\hline Need & 0.009 & 0.011 & 0.011 & $0.019^{\circ}$ \\
\hline Chi-square & 8.297 & 2.073 & 7.552 & 3.441 \\
\hline (Significance) & $(.040)$ & $(.557)$ & $(.056)$ & $(.328)$ \\
\hline Enabiing & 0.031 & 0.110 & 0.037 & 0.086 \\
\hline Community & $0.009 *$ & 0.014 & 0.004 & 0.033 \\
\hline PersonaliFamily & $0.022 * *$ & $0.096 * *$ & $0.033 * * *$ & 0.053 \\
\hline Chirsquare & 24.780 & 20.684 & 22.791 & 13.981 \\
\hline (Significance) & $(.010)$ & $(.037)$ & $(.0 ! 9)$ & $(.234)$ \\
\hline Predisposing & 0.046 & 0.173 & 0.035 & 0.102 \\
\hline Demographic & $0.031 \quad * * *$ & $0.133 \quad * * *$ & 0.012 & $0.058 \quad * *$ \\
\hline Social Structure & $0.014 *$ & 0.027 & 0.009 & 0.008 \\
\hline Health Beliefs & 0.001 & 0.013 & $0.014 * *$ & $0.046 *$ \\
\hline Chi-square & 37.496 & 32.288 & 21.013 & 18.419 \\
\hline (Significance) & $(.000)$ & $(.001)$ & $(.073)$ & $(.142)$ \\
\hline
\end{tabular}


Table 7.13: Regression Models Explaining Self-Reported Physician Contact in the Last 12 Months for Four Racial/Ethnic Groups of Elders When Three Major Blocks of Variables Are Separately Entered

\begin{tabular}{|c|c|c|c|c|}
\hline $\begin{array}{l}\text { Physician Contact in Last } 12 \\
\text { months }\end{array}$ & $\begin{array}{l}\text { White Non- } \\
\text { Hispanic }\end{array}$ & $\begin{array}{l}\text { Black Non- } \\
\text { Hispanic }\end{array}$ & Cuban & $\begin{array}{l}\text { Non-Cuban } \\
\text { Hispanic }\end{array}$ \\
\hline \multicolumn{5}{|c|}{ PREDISPOSING FACTORS ONLY } \\
\hline Constant & 0.53 & 0.00 & $0.0: *$ & .08 \\
\hline \multicolumn{5}{|l|}{ Demographic } \\
\hline Age (years) & $1.05^{* *}$ & $1.67 * * *$ & $1.06 * *$ & 1.07 \\
\hline Female & 0.76 & 0.19 & 1.24 & 0.56 \\
\hline \multicolumn{5}{|c|}{ Educational Attainment (reference category is high school graduate) } \\
\hline Less than High School & 0.66 & 0.33 & 1.06 & 0.45 \\
\hline \multicolumn{5}{|l|}{ Language spoken in home } \\
\hline English only & 0.50 & -- & 1.30 & 0.94 \\
\hline \multicolumn{5}{|l|}{ Social Structure } \\
\hline \multicolumn{5}{|c|}{ Marital Status (reference category is currently married $/$ live-in partner) } \\
\hline Widowed & 1.07 & $0.02 * *$ & 1.14 & $3.79 *$ \\
\hline Divorced/Separated & 0.81 & $0.07 *$ & 0.56 & $8.63 *$ \\
\hline Never Married & 0.86 & 264.12 & 0.78 & 1157.72 \\
\hline $\begin{array}{l}\text { Currently Employed in the Labor } \\
\text { Force? }\end{array}$ & $0.49^{* *}$ & 2.18 & 0.66 & 1.17 \\
\hline $\begin{array}{l}\text { Intensity of Social Contact with } \\
\text { Family \& Friends }\end{array}$ & 1.05 & 1.34 & $1.22 * * *$ & 0.89 \\
\hline $\begin{array}{l}\text { Religion is Very/Somewhat } \\
\text { Important in Life } \\
\text { Health Belicfs }\end{array}$ & 0.83 & 0.00 & 0.89 & 2.88 \\
\hline $\begin{array}{l}\text { Health is a very serious/serious } \\
\text { concern as a elder problem area }\end{array}$ & $2.13^{*}$ & $6.83 *$ & $2.74 * * *$ & 3.39 \\
\hline $\begin{array}{l}\text { Very/Somewhat worried about } \\
\text { loss of independence }\end{array}$ & 1.32 & 1.46 & 1.13 & 0.84 \\
\hline $\begin{array}{l}\text { Very/mostly satisfied with life } \\
\text { during past } 6 \text { months }\end{array}$ & 1.26 & 4.32 & 1.44 & 0.73 \\
\hline \multicolumn{5}{|l|}{ ENABLING FACTORS ONLY } \\
\hline Constant & $17.09^{* * *}$ & 25288.74 & 2.08 & 152424.33 \\
\hline \multicolumn{5}{|l|}{ Community Resources } \\
\hline $\begin{array}{l}\text { Primary Care Physician Ratio per } \\
1000 \text { Population in PSA }\end{array}$ & 0.94 & 0.45 & 1.54 & 0.69 \\
\hline $\begin{array}{l}\text { Hospital Bed Ratio per } 1000 \\
\text { Population in PSA }\end{array}$ & 1.01 & 1.42 & 0.92 & 1.12 \\
\hline $\begin{array}{l}\text { Proportion Living Below Poverty } \\
\text { in Zip Code } \\
\text { Personal/Family Resources }\end{array}$ & 0.12 & 0.49 & 0.04 & 953.95 \\
\hline Household Income $<\$ 10 \mathrm{~K}$ ? & 0.15 & 0.93 & 0.87 & 0.29 \\
\hline \multicolumn{5}{|c|}{ Medicare Coverage (reference category is Medicare Part B coverage) } \\
\hline Part A only & 430.88 & 880.13 & 0.54 & 2470.53 \\
\hline No Medicare & $0.36 * * *$ & $0.19^{*}$ & $0.32 * * *$ & 0.35 \\
\hline \multicolumn{5}{|c|}{ Other Health Care Coverage Variables } \\
\hline Medicaid Coverage Reported & 1.54 & 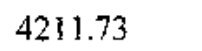 & $3.54 * * *$ & 6.48 \\
\hline HMO Plan Coverage Reported & 1.51 & 5.68 & $3.29 * *$ & 3548.96 \\
\hline
\end{tabular}


Table 7.13 (cont.)

\begin{tabular}{|c|c|c|c|c|}
\hline $\begin{array}{l}\text { Physician Contact in Last } 12 \\
\text { months }\end{array}$ & $\begin{array}{l}\text { White Non- } \\
\text { Hispanic }\end{array}$ & $\begin{array}{l}\text { Black Non* } \\
\text { Hispanic }\end{array}$ & Cuban & $\begin{array}{l}\text { Non-Cuban } \\
\text { Hispanic }\end{array}$ \\
\hline $\begin{array}{l}\text { Years Living in Miami-Dade } \\
\text { County, EL }\end{array}$ & 0.99 & 1.01 & 1.00 & 0.98 \\
\hline Resides Full-time in Dade County & 1.58 & .000 & $5.38 *$ & 0.00 \\
\hline $\begin{array}{l}\text { Regular source of health care } \\
\text { from a private physician }\end{array}$ & 1.90 & 2.62 & 1.18 & 0.38 \\
\hline NEED FACTORS ONLY & & & & \\
\hline Constant & $32.77 * * *$ & $13.13^{* * *}$ & $12.08^{* * *}$ & $6.10 * * *$ \\
\hline $\begin{array}{l}\text { Self-reported Overall Health as } \\
\text { Excelient/Good }\end{array}$ & $0.21 * * *$ & 0.91 & $0.52 *$ & 0.69 \\
\hline $\begin{array}{l}\text { Self-reported Emotional Health as } \\
\text { Excellent/Good }\end{array}$ & 1.58 & $1.1 !$ & 1.40 & 0.28 \\
\hline $\begin{array}{l}\text { Number of Reported Difficulties } \\
\text { with ADLs, IADLs, and mobility } \\
\text { activities }\end{array}$ & 1.10 & 1.19 & 1.07 & 1.52 \\
\hline
\end{tabular}


Table 7.14: Regression Models Explaining High Volume Physician Contact in the Last 12 Months for Four Racial/Ethnic Groups of Elders When Three Major Blocks of Variables Are Separately Entered

\begin{tabular}{|c|c|c|c|c|}
\hline $\begin{array}{l}\text { High Volume Physician Contact } \\
\text { in Last } 12 \text { months }\end{array}$ & $\begin{array}{l}\text { White Non- } \\
\text { Hispanic }\end{array}$ & $\begin{array}{l}\text { Black Non- } \\
\text { Hispanic }\end{array}$ & Cuban & $\begin{array}{l}\text { Non-Cuban } \\
\text { Ilispanic }\end{array}$ \\
\hline \multicolumn{5}{|c|}{ PREDISPOSING FACTORS ONLY } \\
\hline Constant & 0.33 & 3.95 & 0.65 & $0.01 * *$ \\
\hline \multicolumn{5}{|l|}{ Demographic } \\
\hline Age (years) & 1.00 & 0.99 & $1.031 * *$ & $1.09 * * *$ \\
\hline Female & 1.18 & 0.79 & 0.97 & 1.11 \\
\hline \multicolumn{5}{|c|}{ Educational Attainment (reference category is high school graduate or more) } \\
\hline Less than High School & 1.25 & $2.45 * *$ & 1.03 & 0.98 \\
\hline \multicolumn{5}{|l|}{ Language spoken in home } \\
\hline English only & 1.72 & -- & 1.34 & $0.2 .1 * *$ \\
\hline \multicolumn{5}{|l|}{ Social Structure } \\
\hline \multicolumn{5}{|c|}{ Marital Status (reference category is currently married/live-in partner) } \\
\hline Widowed & 0.87 & 1.50 & $1.69^{* *}$ & 0.65 \\
\hline Divorced/Separated & 0.82 & 1.08 & 1.39 & $0.37^{*}$ \\
\hline Never Married & 1.18 & 2.69 & 1.41 & 3.54 \\
\hline $\begin{array}{l}\text { Currently Employed in the Labor } \\
\text { Force? }\end{array}$ & 0.92 & $0.12 * * *$ & $0.25 * * *$ & 0.79 \\
\hline $\begin{array}{l}\text { Intensity of Social Contact with } \\
\text { Family \& Friends }\end{array}$ & 1.02 & 0.99 & 0.94 & 0.97 \\
\hline $\begin{array}{l}\text { Religion is Very/Somewhat } \\
\text { lmportant in Life } \\
\text { Health Beliefs }\end{array}$ & 0.74 & 3.55 & 0.45 & $0.21^{*}$ \\
\hline $\begin{array}{l}\text { Health is a very serious/serious } \\
\text { concern as a elder problem area }\end{array}$ & $3.52 * * *$ & $2.75 * *$ & $2.24 * * *$ & $3.38 * *$ \\
\hline $\begin{array}{l}\text { Very/Somewhat worried about } \\
\text { loss of independence }\end{array}$ & 0.94 & $0.48 *$ & 0.84 & 1.73 \\
\hline $\begin{array}{l}\text { Veryimostly satisfred with life } \\
\text { during past } 6 \text { months }\end{array}$ & $0.44 * * *$ & $0.07 * * *$ & 0.46 & 0.51 \\
\hline \multicolumn{5}{|l|}{ ENABLING FACTORS ONLY } \\
\hline Constant & 0.86 & 1.00 & 0.47 & 0.00 \\
\hline \multicolumn{5}{|l|}{ Community Resources } \\
\hline $\begin{array}{l}\text { Primary Care Physician Ratio per } \\
1000 \text { Population in PSA }\end{array}$ & 1.00 & 0.72 & 0.88 & 0.74 \\
\hline $\begin{array}{l}\text { Hospital Bed Ratio per } 1000 \\
\text { Population in PSA }\end{array}$ & 0.94 & 1.06 & 1.04 & $1.36 * *$ \\
\hline $\begin{array}{l}\text { Proportion Living Below Poverty } \\
\text { in Zip Code } \\
\text { Personal/Family Resources }\end{array}$ & 0.50 & 0.36 & $13.77 * *$ & 0.53 \\
\hline Household lncome $<\$ 10 \mathrm{~K}$ ? & $1.88^{* * *}$ & $1.76^{*}$ & 1.12 & 0.53 \\
\hline \multicolumn{5}{|c|}{ Medicare Coverage (reference category is Medicare Part B coverage) } \\
\hline Part A only & 0.90 & 0.78 & 0.83 & 1.56 \\
\hline No Medicare & $0.68 * *$ & 0.60 & $0.48^{* * *}$ & 0.56 \\
\hline \multicolumn{5}{|c|}{ Other Health Care Coverage Variables } \\
\hline Medicaid Coverage Reported & 1.26 & 1.53 & $1.94 * * *$ & $3.61^{* * *}$ \\
\hline IIMO Plan Coverage Reported & 0.76 & 0.85 & 0.88 & 1.19 \\
\hline
\end{tabular}


Table 7.14 (cont.)

\begin{tabular}{|c|c|c|c|c|}
\hline $\begin{array}{l}\text { High Volume Physician Contact } \\
\text { in Last } 12 \text { months }\end{array}$ & $\begin{array}{l}\text { White Non- } \\
\text { Hispanic }\end{array}$ & $\begin{array}{l}\text { Black Non- } \\
\text { Hispanic } \\
\end{array}$ & Cuban & $\begin{array}{l}\text { Non-Cuban } \\
\text { Hispanic }\end{array}$ \\
\hline $\begin{array}{l}\text { Years Living in Miami-Dade } \\
\text { County, } \mathrm{FL}\end{array}$ & 1.00 & 1.00 & $0.98 *$ & $0.96^{* *}$ \\
\hline Resides Full-time in Dade County & $1.58 *$ & 1.03 & 2.20 & 1698.73 \\
\hline $\begin{array}{l}\text { Regular source of health care } \\
\text { from a private physician }\end{array}$ & 0.69 & 1.01 & 0.94 & 1.54 \\
\hline NEED FACTORS ONLY & & & & \\
\hline Constant & $2.01^{* * *}$ & 1.47 & $1.68 * *$ & 1.04 \\
\hline $\begin{array}{l}\text { Self-reported Overall Health as } \\
\text { Excellent'Good }\end{array}$ & $0.36^{* * *}$ & 0.57 & $0.38 * * *$ & $0.54 *$ \\
\hline $\begin{array}{l}\text { Self-reported Emotional Health as } \\
\text { Excellent/Good }\end{array}$ & $0.49 * * *$ & $0.43 * *$ & 0.96 & 0.84 \\
\hline $\begin{array}{l}\text { Number of Reported Difficulties } \\
\text { with ADLs, IADLs, and mobility } \\
\text { activities }\end{array}$ & 1.01 & 1.07 & $1,207 * * *$ & $1.10^{*}$ \\
\hline
\end{tabular}


Table 7.15: Regression Models Explaining Overnight Hospital Stay in the Last 12 Months for Four Racial/Ethnic Groups of Elders When Three Major Blocks of Variables Are Separately Entered

\begin{tabular}{|c|c|c|c|c|}
\hline $\begin{array}{l}\text { Ovemight Hospital Stay in Last } \\
12 \text { months }\end{array}$ & $\begin{array}{l}\text { White Non- } \\
\text { Hispanic }\end{array}$ & $\begin{array}{l}\text { Black Non- } \\
\text { Hispanic }\end{array}$ & Cuban & $\begin{array}{l}\text { Non-Cuban } \\
\text { Hispanic }\end{array}$ \\
\hline \multicolumn{5}{|c|}{ PREDISPOSING FACTORS ONLY } \\
\hline Constant & $0.07 * *$ & 0.01 & $0.03^{* * *}$ & 0.07 \\
\hline \multicolumn{5}{|l|}{ Demographic } \\
\hline Age (years) & 1.02 & 1.04 & $1.05^{* * *}$ & 1.01 \\
\hline Female & 0.71 & 0.43 & 0.80 & $0.24 * *$ \\
\hline \multicolumn{5}{|c|}{ Educational Attainment (reference category is high school graduate or more) } \\
\hline Less than High School & 0.76 & 0.57 & 0.78 & 0.76 \\
\hline \multicolumn{5}{|l|}{ Language spoken in home } \\
\hline English only & 1.05 & -- & 1.66 & 0.84 \\
\hline \multicolumn{5}{|l|}{ Social Structure } \\
\hline \multicolumn{5}{|c|}{ Marital Status (reference category is currently married/live-in partner) } \\
\hline Widowed & 0.97 & 2.02 & 0.89 & 2.52 \\
\hline Divorced/Separated & $\mathbf{i} .03$ & 2,67 & $1.71 *$ & 2.52 \\
\hline Never Married & 0.90 & 4.86 & 1.23 & 1.65 \\
\hline $\begin{array}{l}\text { Currently Employed in the Labor } \\
\text { Force? }\end{array}$ & 0.86 & 1.02 & $0.52 *$ & $0.22 *$ \\
\hline $\begin{array}{l}\text { Intensity of Social Contact with } \\
\text { Family \& Friends }\end{array}$ & 0.98 & 0.95 & $0.91^{* *}$ & 0.99 \\
\hline $\begin{array}{l}\text { Religion is Very/Somewhat } \\
\text { Important in Life } \\
\text { Health Beliefs }\end{array}$ & 0.85 & 0.50 & 0.65 & 2.29 \\
\hline $\begin{array}{l}\text { Health is a very serious/serious } \\
\text { concern as a elder problem area }\end{array}$ & $2.80 * * *$ & $11.24^{* * *}$ & $2.03^{* * *}$ & 2.49 \\
\hline $\begin{array}{l}\text { Very/Somewhat worried about } \\
\text { loss of independence }\end{array}$ & 1.38 & 1.31 & 1.15 & 0.84 \\
\hline $\begin{array}{l}\text { Veryimostly satisfied with life } \\
\text { during past } 6 \text { months }\end{array}$ & 0.62 & 1.02 & 1.12 & 0.75 \\
\hline \multicolumn{5}{|l|}{ ENABLING FACTORS ONLY } \\
\hline Constant & $0.34 * *$ & $0.06 *$ & $0.05^{* * *}$ & 0.00 \\
\hline \multicolumn{5}{|l|}{ Community Resources } \\
\hline $\begin{array}{l}\text { Primary Care Physician Ratio per } \\
1000 \text { Population in PSA }\end{array}$ & 0.92 & 0.92 & 1.20 & 0.93 \\
\hline $\begin{array}{l}\text { Hospital Bed Ratio per } 1000 \\
\text { Population in PSA }\end{array}$ & 1.01 & 1.03 & 1.07 & 1.25 \\
\hline $\begin{array}{l}\text { Proportion Living Below Poverty } \\
\text { in Zip Code } \\
\text { Personal/Family Resources }\end{array}$ & 0.99 & 1.57 & 0.60 & 13.18 \\
\hline Household Income $<\$ 10 \mathrm{~K}$ ? & 1.13 & 0.69 & 1.45 & $0.35^{*}$ \\
\hline \multicolumn{5}{|c|}{ Medicare Coverage (reference category is Medicare Part B coverage) } \\
\hline No Medicare & $0.49^{* * *}$ & 1.07 & 1.39 & 0.64 \\
\hline \multicolumn{5}{|c|}{ Other Health Care Coverage Variables } \\
\hline Medicaid Coverage Reported & 1.44 & $4.50 * * *$ & $1.68^{* *}$ & $3.76 * *$ \\
\hline HMO Plan Coverage Reported & 0.70 & 1.10 & 1.44 & 1.74 \\
\hline Years Living in Miami-Dade & $0.99^{* *}$ & 1.00 & 1.02 & 1.00 \\
\hline
\end{tabular}


Table 7.15 (cont.)

\begin{tabular}{|c|c|c|c|c|}
\hline $\begin{array}{l}\text { Overnight Hospital Stay in Last } \\
12 \text { months }\end{array}$ & $\begin{array}{l}\text { White Non- } \\
\text { Hispanic }\end{array}$ & $\begin{array}{l}\text { Black Non" } \\
\text { Llispanic }\end{array}$ & Cuban & $\begin{array}{l}\text { Non-Cuban } \\
\text { Hispanic }\end{array}$ \\
\hline \multicolumn{5}{|l|}{ County, FL } \\
\hline Resides Full-time in Dade County & $1.65^{*}$ & 1.51 & 1.01 & 771.41 \\
\hline $\begin{array}{l}\text { Regular source of health care } \\
\text { from a private physician }\end{array}$ & 0.98 & 1.75 & $1.53^{*}$ & 3.55 \\
\hline \multicolumn{5}{|l|}{ NEED FACTORS ONLY } \\
\hline Constant & $0.48 * * *$ & $0.20 * * *$ & $0.32 * * *$ & $0.22 * * *$ \\
\hline $\begin{array}{l}\text { Self-reported Overall Health as } \\
\text { Excellent/Good }\end{array}$ & $0.34 * * *$ & $0.45^{*}$ & $0.46^{* * *}$ & 1.72 \\
\hline $\begin{array}{l}\text { Self-reported Emotional Health as } \\
\text { ExcellentGood }\end{array}$ & 0.99 & 0.91 & 1.32 & $0.30 * *$ \\
\hline $\begin{array}{l}\text { Number of Reported Difficulties } \\
\text { with ADLs, IADLs, and mobility } \\
\text { activities }\end{array}$ & $1.11^{* * *}$ & $1.21 * * *$ & $1.10 * * *$ & $1.13^{* *}$ \\
\hline
\end{tabular}


Table 7.16: Regression Models Explaining Self-Reported Complete Physical Exam in the Last 12 Months for Four Racial/Ethnic Groups of Elders When Three Major Blocks of Variables Are Separately Entered

\begin{tabular}{|c|c|c|c|c|}
\hline $\begin{array}{l}\text { Self-Reported Complete Physica! } \\
\text { Exam in last } 12 \text { months }\end{array}$ & $\begin{array}{l}\text { White Non- } \\
\text { Hispanic }\end{array}$ & $\begin{array}{l}\text { Black Non- } \\
\text { Hispanic }\end{array}$ & Cuban & $\begin{array}{l}\text { Non-Cuban } \\
\text { Hispanic }\end{array}$ \\
\hline \multicolumn{5}{|c|}{ PREDISPOSING FACTORS ONLY } \\
\hline Constant & $0.02 * * *$ & 0.00 & 0.34 & $0.01 * *$ \\
\hline \multicolumn{5}{|l|}{ Demographic } \\
\hline Age (years) & 1.07 & $1.22 * * *$ & 1.02 & $1.08 * * *$ \\
\hline Femaie & 1.26 & 2.01 & 0.77 & 0.99 \\
\hline \multicolumn{5}{|c|}{ Educational Atrainment (reference category is high school graduate or more) } \\
\hline Less than High School & 0.69 & 0.55 & 0.76 & $0.49 *$ \\
\hline \multicolumn{5}{|l|}{ Language spoken in home } \\
\hline English only & 1.15 & -- & 0.68 & 1.05 \\
\hline \multicolumn{5}{|c|}{ Social Structure } \\
\hline \multicolumn{5}{|c|}{ Marital Status (reference category is currently married Jive-in partner) } \\
\hline Widowed & $0.58 * *$ & 0.72 & 1.60 & 1.67 \\
\hline Divorced/Separated & 0.72 & 0.47 & 1.10 & 1.50 \\
\hline Never Married & 0.64 & 1.77 & 0.63 & 0.83 \\
\hline $\begin{array}{l}\text { Curtentiy Employed in the Labor } \\
\text { Force? }\end{array}$ & $0.70^{*}$ & 1.71 & 0.84 & 0.93 \\
\hline $\begin{array}{l}\text { Intensity of Social Contact with } \\
\text { Family \& Friends }\end{array}$ & 1.05 & 1.11 & 1.05 & 1.03 \\
\hline $\begin{array}{l}\text { Religion is Very/Somewhat } \\
\text { Important in Life } \\
\text { Health Beliefs }\end{array}$ & 0.90 & 0.00 & 0.93 & 0.92 \\
\hline $\begin{array}{l}\text { Health is a very serious/serious } \\
\text { concern as a elder problem area }\end{array}$ & 1.24 & 1.72 & 1.44 & $2.67 * *$ \\
\hline $\begin{array}{l}\text { Very/Somewhat worried about } \\
\text { loss of independence }\end{array}$ & 1.08 & 0.55 & 1.13 & $0.41^{*}$ \\
\hline $\begin{array}{l}\text { Very/mostly satisfied with life } \\
\text { during past } 6 \text { months }\end{array}$ & 1.17 & 1.80 & $2.14 * * *$ & 1.62 \\
\hline \multicolumn{5}{|l|}{ ENABLING FACTORS ONLY } \\
\hline \multicolumn{5}{|l|}{ Community Resources } \\
\hline $\begin{array}{l}\text { Primary Care Physician Ratio per } \\
1000 \text { Population in PSA }\end{array}$ & 1.08 & 0.67 & 1.01 & 0.88 \\
\hline $\begin{array}{l}\text { Hospital Bed Ratio per } 1000 \\
\text { Population in PSA }\end{array}$ & 1.05 & 1.21 & 1.04 & 1,11 \\
\hline $\begin{array}{l}\text { Proportion Living Below Poverty } \\
\text { in Zip Code } \\
\text { Personal/Family Resources }\end{array}$ & 3.21 & 0.45 & 0.13 & 33.05 \\
\hline Household Income $<\$ 10 \mathrm{~K} ?$ & 1.04 & 0.66 & 0.82 & 0.76 \\
\hline \multicolumn{5}{|c|}{ Medicare Coverage (reference category is Medicare Part B coverage) } \\
\hline Part A only & 1.64 & 1218.05 & 0.92 & 0.60 \\
\hline No Medicare & $0.52 * * *$ & $0.28^{* * *}$ & $0.53 * *$ & 0.59 \\
\hline \multicolumn{5}{|c|}{ Other Health Care Coverage Variables } \\
\hline Medicaid Coverage Reported & 0.56 & 1.76 & $1.82 * *$ & 1.38 \\
\hline HMO Plan Coverage Reported & 1.25 & 0.82 & 1.57 & $4.13 * *$ \\
\hline
\end{tabular}


Table 7.16 (cont.)

\begin{tabular}{|c|c|c|c|c|}
\hline $\begin{array}{l}\text { Self-Reported Complete Physical } \\
\text { Exam in last } 12 \text { months }\end{array}$ & $\begin{array}{l}\text { White Non- } \\
\text { Hispanic }\end{array}$ & $\begin{array}{l}\text { Black Non- } \\
\text { Hispanic }\end{array}$ & Cuban & $\begin{array}{l}\text { Non-Cuban } \\
\text { Hispantic }\end{array}$ \\
\hline $\begin{array}{l}\text { Years Living in Miami-Dade } \\
\text { County, FL }\end{array}$ & $0.99 *$ & 1.01 & 1.00 & 0.99 \\
\hline Resides Full-time in Dade County & 1.04 & 0.94 & 0.00 & 0.65 \\
\hline $\begin{array}{l}\text { Regular source of health care } \\
\text { from a private physician }\end{array}$ & 1.24 & 1.14 & 0.94 & 1.40 \\
\hline NEED FACTORS ONLY & & & & \\
\hline Constant & $2.60 * * *$ & $3.29 * * *$ & $3.48 * * *$ & 1.75 \\
\hline $\begin{array}{l}\text { Self-reported Overall Health as } \\
\text { ExcellentGood }\end{array}$ & 0.85 & 1.74 & 0.72 & 1.79 \\
\hline $\begin{array}{l}\text { Self-reported Emotional Health as } \\
\text { Excellent'Good }\end{array}$ & $1.69 * *$ & 0.89 & 1.46 & 0.94 \\
\hline $\begin{array}{l}\text { Number of Reported Difficulties } \\
\text { with ADLs, IADLs, and mobility } \\
\text { activities }\end{array}$ & $1.09^{*}$ & 1.07 & $1.10^{*}$ & 1.10 \\
\hline
\end{tabular}


Table 7.17: Increments of Explained Variance $\left(\mathrm{R}^{2}\right)$ Resulting from Entering the Three Major Blocks of Variables for Best-Fit Regression Models Explaining Self-Reported Physician Contact in Last 12 Months For Four Racial/Ethnic Group of Elders

\begin{tabular}{|c|c|c|c|c|c|c|c|c|}
\hline \multirow{2}{*}{ Need } & \multicolumn{2}{|c|}{$\begin{array}{l}\text { White non- } \\
\text { Hispanic }\end{array}$} & \multicolumn{2}{|c|}{$\begin{array}{c}\text { Black non- } \\
\text { Hispanic }\end{array}$} & \multicolumn{2}{|c|}{ Cuban } & \multicolumn{2}{|c|}{$\begin{array}{c}\text { Non-Cuban } \\
\text { Hispanic }\end{array}$} \\
\hline & 0.009 & $* *$ & 0.041 & $* *$ & 0.006 & * & 0.050 & $* * *$ \\
\hline Enabling & & & & & & & & \\
\hline Community & 0.005 & * & -- & & 0.005 & * & .. & \\
\hline Personal/Family & 0.015 & $* * *$ & 0.024 & * & 0.035 & *** & 0.051 & ** \\
\hline Predisposing & & & & & & & & \\
\hline Demographic & - & & 0.073 & $* * *$ & -- & & 0.017 & \\
\hline Social Structure & 0.007 & ** & 0.048 & ** & 0.033 & **** & -- & \\
\hline Health Belief & -- & & -- & & 0.011 & ** & -- & \\
\hline $\mathbf{R}^{2}$ & 0.036 & & 0.186 & & 0.090 & & 0.118 & \\
\hline Chi-square & 25.540 & **** & 30.927 & $* * *$ & 50.589 & $* * *$ & 17.390 & $* * *$ \\
\hline
\end{tabular}

Table 7.18: Increments of Explained Variance $\left(\mathrm{R}^{2}\right)$ Resulting from Entering the Three Major Blocks of Variables for Best-Fit Regression Models Explaining High Volume Physician Contact in Last 12 Months For Four Racial/Ethnic Group of Elders

\begin{tabular}{|c|c|c|c|c|c|c|c|c|}
\hline \multirow{2}{*}{ Need } & \multicolumn{2}{|c|}{$\begin{array}{l}\text { White non- } \\
\text { Hispanic }\end{array}$} & \multicolumn{2}{|c|}{$\begin{array}{c}\text { Black non- } \\
\text { Hispanic }\end{array}$} & \multicolumn{2}{|c|}{ Cuban } & \multicolumn{2}{|c|}{ Non-Cubar } \\
\hline & 0.084 & $* * *$ & 0.123 & $* * *$ & 0.117 & $* * *$ & 0.056 & $* * *$ \\
\hline Enabling & & & & & & & & \\
\hline Community & 0.021 & & -- & & 0.043 & *** & 0.068 & *** \\
\hline Personal;Family & 0.008 & ** & -- & & 0.030 & **** & 0.075 & $* * *$ \\
\hline Predisposing & & & & & & & & \\
\hline Demographic & -- & & 0.023 & * & 0.006 & ** & 0.085 & $* * *$ \\
\hline Social Structure & -- & & 0.061 & *** & 0.038 & *** & 0.051 & $* * *$ \\
\hline Health Belief & 0.039 & $* * *$ & 0.063 & *** & -- & & 0.019 & * \\
\hline $\mathrm{R}^{2}$ & 0.152 & & 0.273 & & 0.234 & & 0.354 & \\
\hline Chi-square & 107.75 & $* * *$ & 45.62 & *** & 131.30 & **** & 56.36 & **** \\
\hline
\end{tabular}


Table 7.19: Increments of Explained Variance $\left(R^{2}\right)$ Resulting from Entcring the Three Major Blocks of Variables for Best-Fit Regression Models Explaining Overnight Hospital Stay in Last 12 Months For Four Racial/Ethnic Group of Elders

\begin{tabular}{|c|c|c|c|c|c|c|c|c|}
\hline \multirow[b]{2}{*}{ Need } & \multicolumn{2}{|c|}{$\begin{array}{l}\text { White non- } \\
\text { Hispanic }\end{array}$} & \multicolumn{2}{|c|}{$\begin{array}{l}\text { Black non- } \\
\text { Hispanic }\end{array}$} & \multicolumn{2}{|c|}{ Cuban } & \multicolumn{2}{|c|}{$\begin{array}{c}\text { Non-Cuban } \\
\text { Hispanjc }\end{array}$} \\
\hline & 0.082 & $* * *$ & 0.107 & $* * *$ & 0.041 & $* * *$ & 0.036 & \\
\hline \multicolumn{9}{|l|}{ Enabling } \\
\hline Community & 0.004 & * & -- & & 0.012 & $* * *$ & -. & \\
\hline Personal/Family & 0.010 & $* *$ & 0.049 & $* *$ & 0.014 & $* *$ & -- & \\
\hline \multicolumn{9}{|l|}{ Predisposing } \\
\hline Demographic & -- & & 0.024 & $* *$ & -- & & 0.022 & * \\
\hline Social Structure & -- & & 0.015 & $*$ & 0.014 & $* * *$ & 0.024 & * \\
\hline Health Belief & 0.017 & $* * *$ & 0.019 & $*$ & 0.004 & $*$ & -- & \\
\hline $\mathrm{R}^{2}$ & 0.113 & & 0.230 & & 0.085 & & 0.082 & \\
\hline Chi-square & 84.16 & $* * *$ & 39.73 & $* * *$ & 48.04 & $* * *$ & 11.850 & $* * *$ \\
\hline
\end{tabular}

Table 7.20: Increments of Explained Variance $\left(R^{2}\right)$ Resulting from Entcring the Three Major Blocks of Variables for Best-Fit Regression Models Explaining Self-Reported Complete Physical Exam in Last 12 Months For Four Racial/Ethnic Group of Elders

\begin{tabular}{|c|c|c|c|c|c|c|c|c|}
\hline \multirow[b]{2}{*}{ Need } & \multicolumn{2}{|c|}{$\begin{array}{l}\text { White non" } \\
\text { Hispanic }\end{array}$} & \multicolumn{2}{|c|}{$\begin{array}{l}\text { Black non- } \\
\text { Hispanic }\end{array}$} & \multicolumn{2}{|c|}{ Cuban } & \multicolumn{2}{|c|}{$\begin{array}{l}\text { Non-Cuban } \\
\text { Hispanic }\end{array}$} \\
\hline & 0.017 & $* * *$ & -- & & 0.010 & $* *$ & -- & \\
\hline \multicolumn{9}{|l|}{ Enabling } \\
\hline Community & 0.021 & $* * *$ & -- & & -. & & 0.024 & * \\
\hline Personal/Family & 0.009 & $* * *$ & 0.073 & *** & 0.014 & $* * *$ & 0.026 & * \\
\hline \multicolumn{9}{|l|}{ Predisposing } \\
\hline Demographic & 0.006 & $* *$ & 0.069 & $* * *$ & $m$ & & 0.052 & $* * *$ \\
\hline Social Structure & 0.004 & $*$ & 0.017 & $*$ & - & & -- & \\
\hline Health Belief & -- & & -- & & 0.015 & $* * *$ & -- & \\
\hline $\mathrm{R}^{2}$ & 0.057 & & 0.158 & & 0.039 & & 0.102 & \\
\hline Chi-square & 41.411 & *** & 26.220 & **** & 21.459 & $* * *$ & 14.925 & *** \\
\hline
\end{tabular}


Table 7.21: Best-Fit Regression Models Explaining Self-Reported Physician Contact in the Last 12 Months for Four Racial/Ethnic Group of Elders

\begin{tabular}{|c|c|c|c|c|}
\hline $\begin{array}{l}\text { Physician Contact in Last } 12 \\
\text { months }\end{array}$ & $\begin{array}{l}\text { White Non- } \\
\text { Hispanic }\end{array}$ & $\begin{array}{l}\text { Black Non- } \\
\text { Hispanic }\end{array}$ & Cuban & $\begin{array}{l}\text { Non-Cuban } \\
\text { Hispanic }\end{array}$ \\
\hline Constant & $119.75^{* * *}$ & 0.00 & 1.80 & 128107.36 \\
\hline \multicolumn{5}{|l|}{ PREDISPOSING FACTORS } \\
\hline \multicolumn{5}{|l|}{ Demographic } \\
\hline Age (years) & & $1.759 *$ & & \\
\hline \multicolumn{5}{|l|}{ Female } \\
\hline \multicolumn{5}{|c|}{ Educational Attainment (reference category is high school graduate) } \\
\hline \multicolumn{5}{|c|}{ Less than High School } \\
\hline More than Figh School & & 17012.29 & & \\
\hline \multicolumn{5}{|l|}{ Language spoken in home } \\
\hline English only & & & & $0.29 *$ \\
\hline \multicolumn{5}{|c|}{ Social Structure } \\
\hline \multicolumn{5}{|c|}{ Marital Status (reference category is currently married/live-in partner) } \\
\hline Widowed & & $0.06^{* *}$ & & \\
\hline Divorced/Separated & & $0.04 * *$ & $0.26 * * *$ & \\
\hline \multicolumn{5}{|l|}{ Never Married } \\
\hline Lives alone & & & 2.09 & \\
\hline $\begin{array}{l}\text { Currently Employed in the Labor } \\
\text { Force? }\end{array}$ & $0.46^{* *}$ & & & \\
\hline \multicolumn{5}{|l|}{$\begin{array}{l}\text { Family \& Friends } \\
\text { Religion is Very/Somewhat } \\
\text { Important in Life } \\
\text { Health Beliefs }\end{array}$} \\
\hline \multicolumn{5}{|l|}{$\begin{array}{l}\text { concern as a elder problem area } \\
\text { Very/Somewhat worried about } \\
\text { loss of independence } \\
\text { Very/mostly satisfied with life } \\
\text { during past } 6 \text { months }\end{array}$} \\
\hline \multicolumn{5}{|l|}{$\begin{array}{l}\text { ENABLING FACTORS } \\
\text { Community Resources }\end{array}$} \\
\hline $\begin{array}{l}\text { Primary Care Physician Ratio per } \\
\text { 1000 Population in PSA } \\
\text { Hospital Bed Ratio per } 1000 \\
\text { Population in PSA }\end{array}$ & & & & \\
\hline \multicolumn{5}{|l|}{ Zip Code } \\
\hline \multicolumn{5}{|l|}{$\begin{array}{l}\text { Proportion of Hispanics Living in } \\
\text { Zip Code }\end{array}$} \\
\hline \multicolumn{5}{|l|}{$\begin{array}{l}\text { Proportion of Persons } 60+\text { Living } \\
\text { in Zip Code }\end{array}$} \\
\hline $\begin{array}{l}\text { Proportion Living Below Poverty } \\
\text { in Zip Code } \\
\text { Personal/Family Resources }\end{array}$ & $0.02 * *$ & & & \\
\hline \multicolumn{5}{|l|}{ Household Income $<\$ 10 \mathrm{~K} ?$} \\
\hline Medicare Coverage (reference cate & $\mathrm{y}$ is Medicare & It $B$ coverage) & & \\
\hline
\end{tabular}


Table 7.21 (cont.)

\begin{tabular}{|c|c|c|c|c|}
\hline $\begin{array}{l}\text { Physician Contact in Last } 12 \\
\text { months }\end{array}$ & $\begin{array}{l}\text { White Non-1 } \\
\text { Hispanic }\end{array}$ & $\begin{array}{l}\text { Black Non- } \\
\text { Ilispanic }\end{array}$ & Cuban & $\begin{array}{l}\text { Non-Cuban } \\
\text { Hispanic }\end{array}$ \\
\hline \multicolumn{5}{|l|}{ Part $A$ only } \\
\hline No Medicare & $0.41^{* * *}$ & 1.10 & $0.42^{* *}$ & 0.32 \\
\hline \multicolumn{5}{|l|}{ Other Health Care Coverage Variables } \\
\hline Medicaid Coverage Reported & & & $2.86 * *$ & \\
\hline HMO Plan Coverage Reported & & & $2.64 * *$ & 11896.76 \\
\hline \multicolumn{5}{|l|}{ Years I Living in Miami-Dade } \\
\hline \multicolumn{5}{|l|}{ County, FL } \\
\hline \multicolumn{5}{|l|}{ Resides Full-time in Dade County } \\
\hline \multirow{2}{*}{\multicolumn{5}{|c|}{$\begin{array}{l}\text { Regular source of health care } \\
\text { from a private physician }\end{array}$}} \\
\hline & & & & \\
\hline \multicolumn{5}{|l|}{ NEED FACTORS ONLY } \\
\hline $\begin{array}{l}\text { Self-reported Overall Health as } \\
\text { Excellent/Good }\end{array}$ & $0.37 *$ & & 0.57 & \\
\hline $\begin{array}{l}\text { Self-reported Emotional Health as } \\
\text { Excellent/Good }\end{array}$ & & & & 0.00 \\
\hline $\begin{array}{l}\text { Number of Reported Difficulties } \\
\text { with ADLs, IADLs, and mobility } \\
\text { activities }\end{array}$ & & 3.31 & & \\
\hline $\mathrm{N}$ & 696 & 150 & 534 & 139 \\
\hline$R^{2}$ & 0.036 & 0.186 & 0.090 & 0.118 \\
\hline Chi-square & 25.540 & 30.828 & 50.589 & 17.390 \\
\hline Significance & .000 & .000 & .000 & .002 \\
\hline
\end{tabular}


Table 7.22: Best-Fit Regression Models Explaining High Volume Physician Contact in the Last 12 Months for Four Racial/Ethnic Group of Elders

\begin{tabular}{|c|c|c|c|c|}
\hline $\begin{array}{l}\text { High Volume Physician Contact } \\
\text { in Last } 12 \text { months }\end{array}$ & $\begin{array}{l}\text { White Non- } \\
\text { Hispanic }\end{array}$ & $\begin{array}{l}\text { Black Non- } \\
\text { Hispanic }\end{array}$ & Cuban & $\begin{array}{l}\text { Non-Cuban } \\
\text { Hispanic }\end{array}$ \\
\hline Constant & $2.67 * *$ & $8.78 * *$ & 1.48 & 0.00 \\
\hline \multicolumn{5}{|l|}{ PREDISPOSING FACTORS } \\
\hline \multicolumn{5}{|l|}{ Demographic } \\
\hline Age (years) & & & 1.02 & $1.11^{* * *}$ \\
\hline \multicolumn{5}{|c|}{ Female } \\
\hline \multicolumn{5}{|c|}{ Educational Attainment (reference category is high school graduate) } \\
\hline Less than High School & & $2.06^{*}$ & & \\
\hline \multicolumn{5}{|l|}{ More than High School } \\
\hline \multicolumn{5}{|l|}{ Language spoken in home } \\
\hline English only & & & & $0.17^{* *}$ \\
\hline \multicolumn{5}{|l|}{ Social Structure } \\
\hline \multicolumn{5}{|c|}{$\begin{array}{l}\text { Marital Status (reference category is currently martied/live-in partner) } \\
\text { Widowed }\end{array}$} \\
\hline Divorced/Separated & & & & $032 *$ \\
\hline Never Married & & & & 5.01 \\
\hline Lives alone & & & $1.73 * *$ & \\
\hline $\begin{array}{l}\text { Currently Employed in the Labor } \\
\text { Force? }\end{array}$ & & $0.14^{* * *}$ & $0.25 * * *$ & \\
\hline Intensity of Social Contact with & & & $0.92 *$ & \\
\hline \multirow{2}{*}{\multicolumn{5}{|c|}{$\begin{array}{l}\text { Family \& Friends } \\
\text { Religion is Verv/Somewhat }\end{array}$}} \\
\hline & & & & \\
\hline \multicolumn{5}{|l|}{ Important in I.ife } \\
\hline \multicolumn{5}{|l|}{ Health Beliefs } \\
\hline $\begin{array}{l}\text { Health is a very serious/serious } \\
\text { concern as a elder problem area }\end{array}$ & $2.71^{* * *}$ & & & $2.72 *$ \\
\hline $\begin{array}{l}\text { Very/Somewhat worried about } \\
\text { loss of independence }\end{array}$ & & $0.49^{*}$ & & \\
\hline $\begin{array}{l}\text { Very/mostly satisfied with life } \\
\text { during past } 6 \text { months }\end{array}$ & $0.56^{* *}$ & $0.10^{* * *}$ & & \\
\hline \multicolumn{5}{|l|}{$\begin{array}{l}\text { ENABLING FACTORS } \\
\text { Community Resources }\end{array}$} \\
\hline \multicolumn{5}{|l|}{ Primary Care Physician Ratio per } \\
\hline \multicolumn{5}{|l|}{ Population in PSA } \\
\hline \multicolumn{5}{|l|}{$\begin{array}{l}\text { Proportion of Blacks Living in } \\
\text { Zip Code }\end{array}$} \\
\hline & $1.92 *$ & \multicolumn{2}{|c|}{ Zip Code } & \\
\hline $\begin{array}{l}\text { Proportion of Persons } 60+\text { Living } \\
\text { in Zip Code }\end{array}$ & $0.26 *$ & & $0.002^{* * *}$ & $0.001^{* *}$ \\
\hline \multicolumn{3}{|l|}{$\begin{array}{l}\text { Proportion Living Below Poverty } \\
\text { in Zip Code } \\
\text { Personal/Family Resources }\end{array}$} & $618.59 * * *$ & \\
\hline \multicolumn{5}{|l|}{ Household Income $<\$ 10 \mathrm{~K}$ ? } \\
\hline Medicare Coverage (reference cat & $\mathrm{y}$ is Medicart & B coverage) & & \\
\hline
\end{tabular}


Table 7.22 (cont.)

\begin{tabular}{|c|c|c|c|c|}
\hline $\begin{array}{l}\text { High Volume Physician Contact } \\
\text { in Last } 12 \text { months }\end{array}$ & $\begin{array}{l}\text { White Non- } \\
\text { Hispanic }\end{array}$ & $\begin{array}{l}\text { Black Non- } \\
\text { Hispanic }\end{array}$ & Cuban & $\begin{array}{l}\text { Non-Cuban } \\
\text { IIspanic }\end{array}$ \\
\hline \multicolumn{5}{|l|}{ Part A only } \\
\hline No Medicare & $0.61^{* *}$ & & 0.74 & \\
\hline \multicolumn{5}{|l|}{ Other Health Care Coverage Variables } \\
\hline Mcdicaid Coverage Reported & & & 1.35 & 0.98 \\
\hline \multicolumn{5}{|l|}{ HMO Plan Coverage Reported } \\
\hline \multicolumn{5}{|l|}{ Years Living in Miami-Dade } \\
\hline \multicolumn{5}{|l|}{ County, FL } \\
\hline \multicolumn{5}{|l|}{$\begin{array}{l}\text { Regular source of health care } \\
\text { from a private physician }\end{array}$} \\
\hline \multicolumn{5}{|l|}{ NEED FACTORS ONLY } \\
\hline $\begin{array}{l}\text { Self-reported Overall health as } \\
\text { Excellent } / \text { Good }\end{array}$ & $0.61^{* *}$ & 0.58 & $0.44 * * *$ & $0.38^{*}$ \\
\hline $\begin{array}{l}\text { Self-reported Emotional Health as } \\
\text { Excellent/Good }\end{array}$ & $0.54 * *$ & & & \\
\hline $\begin{array}{l}\text { Number of Reported Difficulties } \\
\text { with ADLs, IADLs, and mobility } \\
\text { activities }\end{array}$ & & 1.15 & $1.17^{* * *}$ & \\
\hline $\mathrm{N}$ & 653 & 142 & 492 & 129 \\
\hline $\mathrm{R}^{2}$ & 0.152 & 0.270 & 0.234 & 0.354 \\
\hline Chi-square & 107.748 & 44.626 & 131.297 & 56.362 \\
\hline Significance & .000 & .000 & .000 & .000 \\
\hline
\end{tabular}

${ }^{*} \mathrm{p} \leq 0.10$ (two-tailed) ${ }^{* *} \mathrm{p} \leq 0.05$ (two-tailed) ${ }^{* * *} \mathrm{p} \leq 0.01$ (two-tailed) 
Table 7.23: Best-Fit Regression Models Explaining Overnight Hospital Stay in the Last 12 Months for Four Racial/Ethnic Group of Elders

\begin{tabular}{|c|c|c|c|c|}
\hline $\begin{array}{l}\text { Overnight Hospital Stay in the } \\
\text { l.ast } 12 \text { months }\end{array}$ & $\begin{array}{l}\text { White Non- } \\
\text { Hispanic }\end{array}$ & $\begin{array}{l}\text { Black Non- } \\
\text { Hispanic }\end{array}$ & Cuban & $\begin{array}{l}\text { Non-Cuban } \\
\text { Hispanic }\end{array}$ \\
\hline Constant & $0.47^{*}$ & $0.26^{*}$ & $0.16^{* * *}$ & 0.77 \\
\hline
\end{tabular}

\section{PREDISPOSING FACTORS}

\section{Demographic}

Age (years)

Female

Educational Attainment (reference category is high school graduate)

Less than High School

More than High School

Language spoken in home

English only

\section{Social Structure}

Marital Status (reference category is currently married/live-in partmer)

Widowed

DivorcediSeparated

Never Married

Lives alone

$3.11^{*}$

Currently Employed in the Labor

Force?

Intensity of Social Contact with

Family \& Friends

Religion is Very/Somewhat

Important in Life

\section{Health Belief}

Health is a very serious/serious

concem as a elder problem area

Very/Somewhat worried about

loss of independence

Very/mostly satisfied with life

during past 6 months

\section{ENABLING FACTORS}

\section{Community Resources}

Primary Care Physician Ratio per

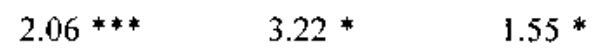

1000 Population in PSA

Hospital Bed Ratio per 1000

Population in PSA

Proportion of Blacks Living in

Zip Code

Proportion of Hispanics Living in

Zip Code

Proportion of Persons 60+ I iving

in Zip Code

Proportion Living Below Poverty

in Zip Code

PersonalFamily Resources

Household Income $<\$ 10 \mathrm{~K}$ ?

Medicare Coverage (reference category is Medicare Part B coverage) 
Table 7.23 (cont.)

\begin{tabular}{|c|c|c|c|c|}
\hline $\begin{array}{l}\text { Overnight Hospital Stay in the } \\
\text { Last } 12 \text { months }\end{array}$ & $\begin{array}{l}\text { White Non- } \\
\text { Hispanic }\end{array}$ & $\begin{array}{l}\text { Black Non- } \\
\text { Hispanic }\end{array}$ & Cuban & $\begin{array}{l}\text { Non-Cuban } \\
\text { Hispanic }\end{array}$ \\
\hline \multicolumn{5}{|l|}{ Part A only } \\
\hline No Medicare & $0.59 * *$ & & & \\
\hline \multicolumn{5}{|c|}{ Other Health Care Coverage Variables } \\
\hline Medicaid Coverage Reported & & 2.40 & $1.50^{*}$ & \\
\hline HMO PІan Coverage Reported & & & & \\
\hline $\begin{array}{l}\text { Years Living in Miami-Dade } \\
\text { County, FL } \\
\text { Resides Full-time in Dade County }\end{array}$ & $0.99 *$ & & $1.03 * * *$ & \\
\hline $\begin{array}{l}\text { Regular source of health care } \\
\text { from a private physician }\end{array}$ & & & & \\
\hline NEED FACTORS ONLY & & & & \\
\hline $\begin{array}{l}\text { Self-reported Overall Health as } \\
\text { Excellent/Good }\end{array}$ & $0.50^{* * *}$ & $0.30 *$ & $0.53^{* *}$ & \\
\hline $\begin{array}{l}\text { Self-reported Emotional Health as } \\
\text { Excellent/Good }\end{array}$ & & & $1.68 *$ & $0.26 * *$ \\
\hline $\begin{array}{l}\text { Number of Reported Difficulties } \\
\text { with ADLs, IADLs, and mobility } \\
\text { activities }\end{array}$ & $1.10 * *$ & $1.16^{*}$ & 1.06 & \\
\hline N & 699 & 151 & 538 & 139 \\
\hline $\mathrm{R}^{2}$ & .113 & 0.214 & .085 & .082 \\
\hline Chi-square & 84.163 & 36.295 & 48.044 & 11.850 \\
\hline Significance & .000 & .000 & .000 & .008 \\
\hline
\end{tabular}


Table 7.24: Best-Fit Regression Models Explaining Self-Reported Complete Physical Exam in the Last 12 Months for Four Racial/Ethnic Group of Elders

\begin{tabular}{|c|c|c|c|c|}
\hline $\begin{array}{l}\text { Complete Physical Exam in the } \\
\text { Last } 12 \text { months }\end{array}$ & $\begin{array}{l}\text { White Non- } \\
\text { Hispanic }\end{array}$ & $\begin{array}{l}\text { Black Non- } \\
\text { Hispanic }\end{array}$ & Cuban & $\begin{array}{l}\text { Non-Cuban } \\
\text { Hispanic }\end{array}$ \\
\hline Constant & $0.09^{*}$ & $0.00^{* *}$ & $2.26 * * *$ & $0.01^{* *}$ \\
\hline
\end{tabular}

\section{PREDISPOSING FACTORS}

Demographic

Age (years)

$1.04 * * \quad 1.15^{* *}$

$1.08 * * *$

Female

Educational Attainment (reference category is high school graduate)

Less than High School

More than High School

$5.06^{* *}$

Language spoken in home

English only

\section{Social Structure}

Marital Status (reference category is currently marricd/live-in partner)

Widowed

Divorced/Separated

Never Married

Lives alone

Currently Employed in the Labor

Force?

Intensity of Social Contact with

Family \& Friends

Religion is Very/Somewhat

Important in Life

Health Beliefs

Health is a very serious/serious concern as a elder problem area Very/Somewhat worried about loss of independence

Very/mostly satisfied with life

during past 6 months

\section{ENABLING FACTORS}

\section{Community Resources}

Primary Care Physician Ratio per

1000 Population in PSA

Hospital Bed Ratio per 1000

Population in PSA

Proportion of Blacks I Living in

Zip Code

Proportion of Hispanics I.iving in

Zip Code

Proportion of Persons 60+ Living

in Zip Code

Proportion Living Below Poverty

in Zip Code

Personal/Family Resources

Household Income $<\$ 10 \mathrm{~K}$ ?

Medicare Coverage (reference category is Medicare Part B coverage) 
Table 7.24 (cont.)

\begin{tabular}{|c|c|c|c|c|}
\hline $\begin{array}{l}\text { Complete Physical Exam in the } \\
\text { Last } 12 \text { months }\end{array}$ & $\begin{array}{l}\text { White Non- } \\
\text { Hispanic }\end{array}$ & $\begin{array}{l}\text { Black Non- } \\
\text { Hispanic }\end{array}$ & Cuban & $\begin{array}{l}\text { Non-Cuban } \\
\text { Hispanic }\end{array}$ \\
\hline \multicolumn{5}{|l|}{ Part A only } \\
\hline No Médicare & 0.78 & $0.38 *$ & $0.52 * * *$ & \\
\hline \multicolumn{5}{|c|}{ Other Heaith Care Coverage Variables } \\
\hline \multicolumn{5}{|c|}{ Medicaid Coverage Reported } \\
\hline HMO Plan Coverage Reported & & & & $3.40^{*}$ \\
\hline \multicolumn{5}{|l|}{ Years Living in Miami-Dade } \\
\hline \multicolumn{5}{|l|}{ County, FL } \\
\hline \multicolumn{5}{|l|}{ Resides Full-time in Dade County } \\
\hline \multicolumn{5}{|l|}{$\begin{array}{l}\text { Regular source of health care } \\
\text { from a private physician }\end{array}$} \\
\hline \multicolumn{5}{|l|}{ NEED FACTORS ONLY } \\
\hline \multicolumn{5}{|l|}{ Self-reported Overall Health as } \\
\hline \multicolumn{5}{|l|}{ Excellent/Good } \\
\hline $\begin{array}{l}\text { Self-reported Emotional Health as } \\
\text { Excellent/Good }\end{array}$ & $1.86 * *$ & & & \\
\hline $\begin{array}{l}\text { Number of Reported Difficulties } \\
\text { with ADLs, IADLs, and mobility } \\
\text { activities }\end{array}$ & $1.16^{* *}$ & & $1.16 * *$ & \\
\hline $\mathrm{N}$ & 700 & 151 & 536 & 139 \\
\hline $\mathrm{R}^{2}$ & 0.057 & 0.159 & 0.039 & 0.102 \\
\hline Chi-square & 41.411 & 26.164 & 21.459 & 14.925 \\
\hline Significance & .000 & .000 & .000 & .002 \\
\hline
\end{tabular}




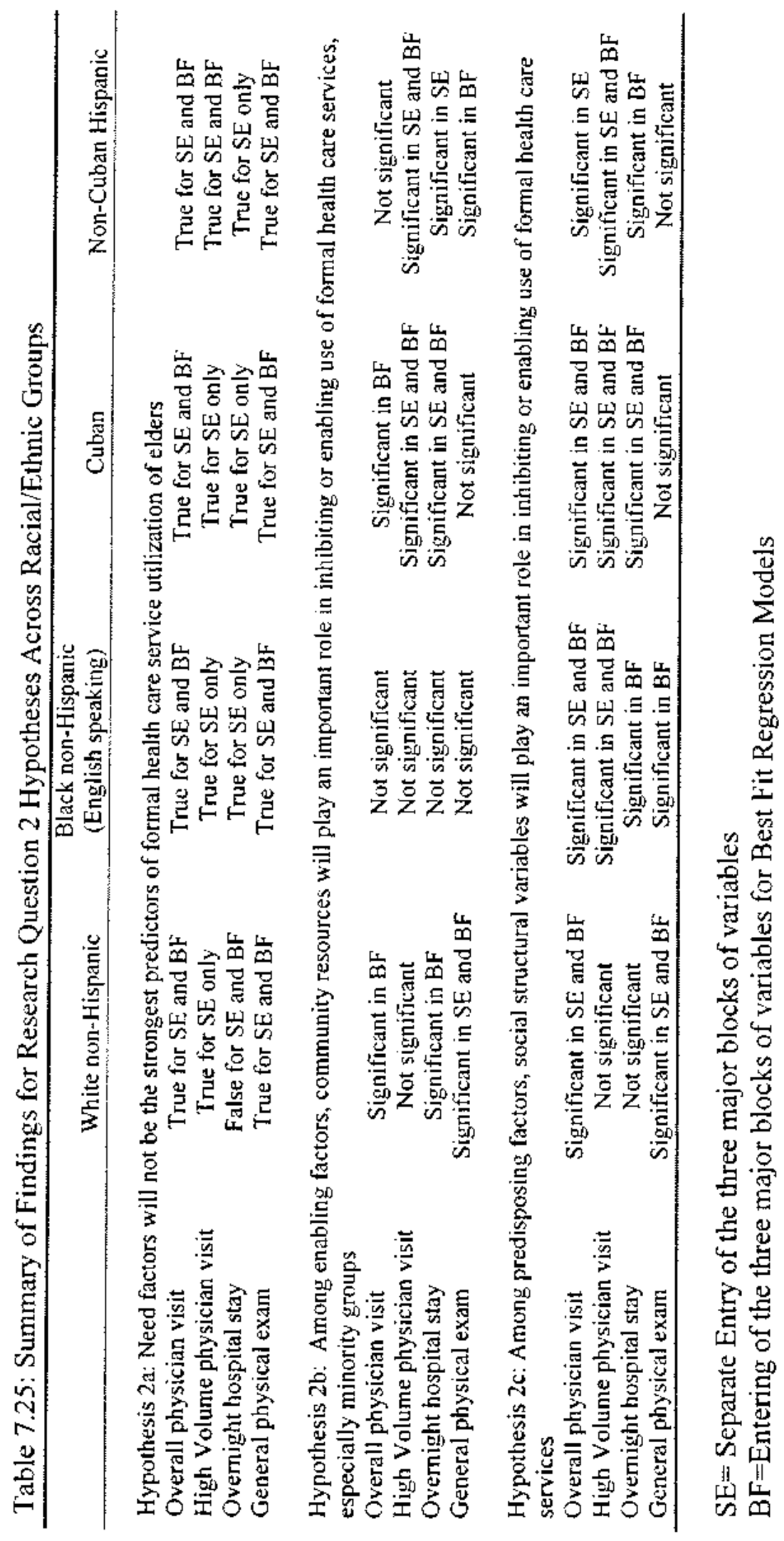




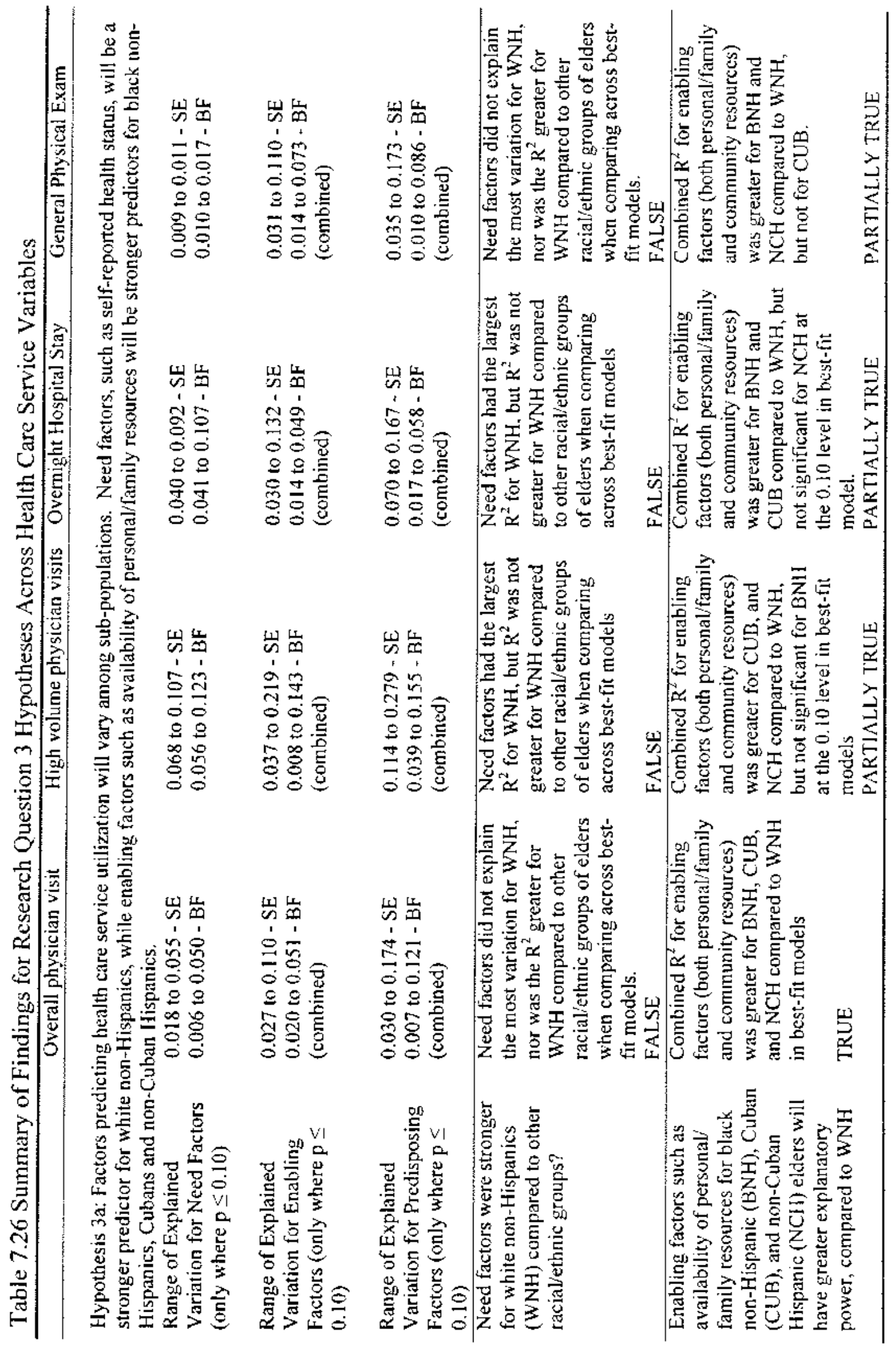




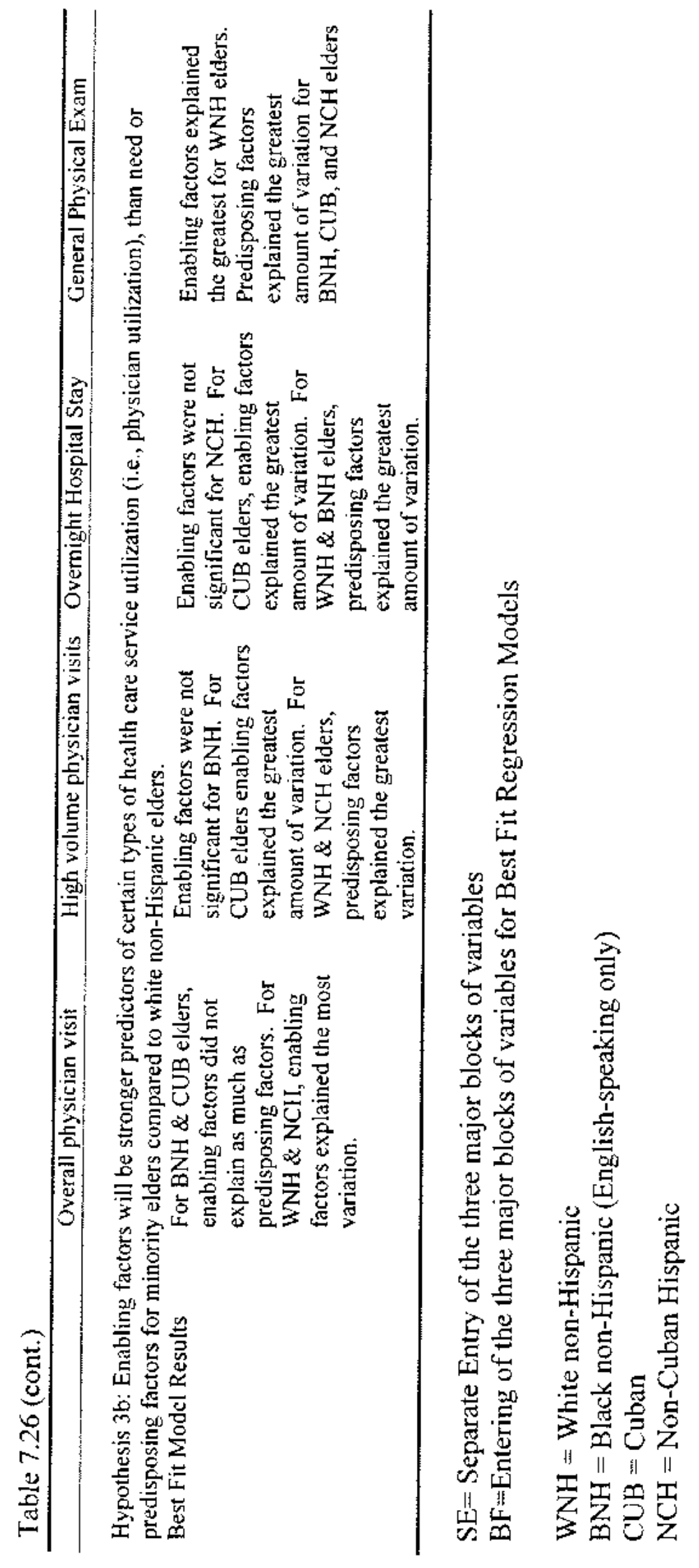




\section{CHAPTER 8: SUMMARY AND DISCUSSION}

There appears to be a disconnection in the research on health care service utilization literature. On the one hand, elders and their health were viewed as homogeneous in their need for health care services (i.e., the biological process of aging). On the other hand, there is other sociological and anthropological literature that holds that the definition of illness and health to be socially constructed, varying from individual to individual with respect to cultural background, regardless of age. In addition, I recognized from my involvement in other research projects that the community in which an individual resides might have an important impact on utilization. Community resources (or non-resources) may act as barriers to utilization, i.e., the proximity of health care services. Barriers to access, however, may not just be actual health care services in close proximity, but also cultural. The idea of cultural context being a bartier to access of health care service is not new and has recently drawn a lot of media coverage. However, the academic literature studies on health care service utilization tended not to place "aging" or "health" in a cultural context or community context. This placement, I believe, is important because, "culture does not exist apart from individuals but rather lies in their interpretation of events and the things around them" (McGee \& Warms, 1996). This goes along with Geertz (1973) statement that “... man is an animal suspended in webs of significance he himself has spun. I take culture to be those webs...."

My goal in this dissertation research was to bring together these threads of culture, health and aging in this dissertation, by examining the health care utilization behavior of 
elders within the multicultural urban environment of Miami-Dade County, Florida. However, this dissertation only scratches the surface of this "new" terrain between culture, health and aging, since as Geertz (1973) has written,

The essential vocation of interpretive [social research] is not to answer our deepest questions, but to make available to us answers that others, guarding other sheep in other valleys, have given, and thus to include them in the consultable record to what man has said (p.30).

The overall objective of this dissertation was to determine the influences of social, environmental, behavioral, and economic forces on the health care service utilization of four racial/ethnic groups of non-institutionalized elders in a multicultural urban environment. However, I did not want this dissertation research on health care utilization of elders to be just another quantitative study. I believed there was an imperative to place health and health care in the lives of elders through a qualitative analysis that focused on differences in perception of the importance of health and health care in the lives of elders by cultural groups. In conjunction, the analysis using quantitative data focused on actual health care utilization behavior of elders in a multicultural urban environment. Together these two components should give a better understanding of the complexities involved in understanding health care utilization behavior of elders in a multicultural urban environment. This chapter will summarize the results and discuss the implications of these results with respect to policy implications, as well as future research needs.

\section{STATEMENT OF THE PROBLEM}

The two major goals of this dissertation were: (1) to examine the sociodemographic, cultural, and community availability factors which affect health care 
utilization behavior; and (2) to investigate the ways in which elders of the four racial/ethnic groups talk about issues of health and health care in their lives. Further, this dissertation proposed two arguments. The first was that racial/ethnic/cultural background of elders plays an important role in how an elder uses health care services. Fitzgerald (1992) summarizes that,

Culture is an integral part of everyone's life. It is a part of every personal encounter and every interaction - including every clinical interaction.

The second argument proposed in this dissertation was that specific local environment or community context of the health care service market has an important impact on utilization decisions of elders.

The significance of this dissertation is in its ability to examine the utilization of formal health care services by elders residing in one multicultural urban environment. Most of our knowledge has been limited to national studies with relatively homogeneous cultural groups of elders. Those studies that have explored health care seeking and utilization behaviors of minority elders have been hampered by small population sizes and have usually ignored the community context of the health care service market.

This dissertation research has been unique with respect to five aspects. First, the heterogeneous nature of the elder population residing within Miami-Dade County, FL, as well as the large percentage of population considered elderly $(60+)$ has allowed this study to investigate health care utilization practices of four racial/ethnic groups of elders in one multicultural setting with its health care service market. Second, this dissertation has had a neighborhood focus within a specific county. Focusing on a county allowed the researcher to have more local community data available on actual health care services 
available to elders in their neighborhoods and not just those services available in the same

SMSA or same county. Third given this neighborhood focus, the researcher has been able to examine the equitability of access to the health care delivery system by each of the four racial/ethnic groups of elders. With this information there can be a more informed discussion of the policy implications that the equitability (or inequitability) of the distribution of health care delivery system has on elders utilization behaviors. Fourth, there has not previously been an examination of differences of preventive health care service utilization by racial/ethnic groups of elders. Finally, the data used in this research has not been previously analyzed with respect to formal health care service utilization behavior.

\section{REVIEW OF THE METHODOLOGY}

This dissertation has been designed methodologically to use both quantitative and qualitative data, in order to understand and clarify the dynamics of health care utilization of culturally diverse elders within one health care service market. To understand the overall dynamics within Miami-Dade County, FL, there was an examination of the historical demographic trends of elders in the county using sampled census data for the county since 1950 in Chapter 4 . Historical context is important, both in terms of understanding power relationships within the community (i.e., political economy), as well as for its own sake. Crapanzano (1986) wrote with respect to ethnographic research that,

Despite its frequently ahistorical ... pretense, ethnography is historically determined by the moment of the ethnographer's encounter with whomever he is studying" 
While Crapanzano was referring specifically to ethnographic research, this could also be said of all sociological research. Further, if we take Marcus \& Fisher's (1986) definition of history as "a shift in structure... [which] may occur through cataclysmic events, but more commonly it occurs incrementally, ... [then] it is the task of historically sensitive [research] to perceive structural shifts in the details of every day life..." This harkens back to the theme of culture in the lives of elders. Culture, as lived by member individuals, is a living breathing entity that is slowing being transformed by member individuals, as well as changing member individuals. Depending upon the particular instant that the social scientist comes into contact with his/her "exotic other" population will influence what the researcher "sees." To not understand that evolution and previous experiences of the individual, i.e., history, as an on-going process that we are all a part of has created an incomplete and limited picture. This is particularly important because aging is living. How individuals have lived during their "younger" years, or "composed" their earlier lives, is important in how individuals compose their current lives in old age.

Turning to the methodological analysis of the data, as stated earlier, there were two components of the research, a qualitative component and a quantitative component. The qualitative component of this dissertation was done in two parts. First, there was a literature review which examined the evolution of the theoretical perspective of "age" and "aging" in the United States; followed by an indepth examination of five texts focused on elders or were written by elders on their own aging. An understanding of the evolution of theoretical perspective on the social construction of "age" in the United States gives a solid foundation to the results found in the five texts focused on elders (Chapter 3 ). The 
second part of the qualitative component of this dissertation analyzed health and health care issues in the lives of elders residing in Miami-Dade County, FL using six focus groups conducted around the county as part of a needs assessment conducted in 1994. Further, these focus groups also shed light on the placement of health and health care in the lives of elders within a broad spectrum of issues and concerns in the lives of elders.

The quantitative component of this dissertation primarily used data from the telephone survey conducted as part of this above mentioned needs assessment. The telephone survey used a cross-sectional design. The data collection method was a closedended survey instrument designed to measure the variables at the time of the telephone survey (January thru March 1994). The survey instrument collected information on each of the three factors: predisposing, enabling and need, as well as the dependent variables measuring overall contact with physicians, high volume physician contact, overnight hospital stay, and self-reporting of a having a complete physical exam in the last 12 months. However, community contextual variables were not available from the telephone survey. Availability of primary care physicians and hospital beds were collected from other sources. It was also realized that direct measures of barriers to accessing health care services are usually unavailable, thus indirect measures of the neighborhood (service environment) that an elder lives in were collected using U.S. Census Bureau's Summary Tape File 3B. Community contextual variables, both direct and indirect measures were linked to the responding elder by the zip code of the elder.

The quantitative data analysis was conducted using the computer package SPSS for Windows using four statistical procedures: frequency distribution, reliability 
procedures, correlation analysis, and multiple regression analysis, specifically logistic transformation to develop a more comprehensive model of health care utilization behavior.

The theoretical perspective used for this research is most commonly referred to as the Socio-Behavioral Model. The Socio-Behavioral Model (SBM) is the most widely used approach and was originally developed by Andersen (1968). Andersen with other colleagues further refined it. The SBM proposes that utilization of health care services by an individual can be viewed as a behavior pattern. As such, there have been numerous attempts to explain "individual behavior as a function of characteristics of the individual... characteristics of the environment in which [the individual] lives, and/or some interaction of these individual and societal forces..." (Andersen \& Newman, 1973).

\section{SUMMARY AND DISCUSSION OF THE RESULTS}

The research questions under investigation in this dissertation were: (1) Are there differences in perception of the importance of health and health care in the lives of elders by cultural group?; (2) What factors predict utilization of health care services?; and (3) Do these factors vary among racial/ethnic sub-populations? The first research question was examined through qualitative data sources, while the second and third research questions were examined through quantitative data sources. The qualitative analysis examined the placement of health and health care in the lives of elders. However, these issues were not examined within a vacuum, rather they were examined as one aspect among others in the lives of elders. Elder participants in the focus groups were asked 
about a range of other topics, such as transportation, housing, boredom/social isolation/loncliness, and fear of crime, as well as overall living conditions in Miami-Dade County. The quantitative analysis focused on formal health care service utilization. The two research questions addressed using quantitative analysis focused on similar issues but examined them with respect to different dimensions. The second research question focused on the type of services used, while the third research question focused on racial/ethnic differences in service utilization.

Overall, the findings from both the quantitative and qualitative analysis raised several important issues. First and foremost, just because people share a common chronological age does not mean that they are the same in every other respect. Placing "aging" and "health" in a cultural context is important. The literature review on aging in Chapter 3 acknowledged that the meaning of "aging" and "old" are socially constructed. Further, it is important to note that the meaning of "aging" is NOT synonymous with ill health or frailty. Examining elders as a homogeneous group of users of formal health care services in a community is simplistic. This was a consistent finding in both the quantitative and qualitative results.

The major findings were that: (1) issues of health and health care utilization are intertwined with other aspects in the lives of elders; and (2) elders' interpretations of biological processes of aging depended on their previous life experiences and current socio-economic status, as well as familial situations and cultural expectations.

From the qualitative analysis, it was generally found that elders of different racial/ethnic/cultural groups had similar concerns. However, the differences were in how 
these concens were brought out in focus group discussions and the different perceptions of the placement of health and health care in the lives of these elders. The differences in the discourse by focus groups show pointedly that the differences were based on their interpretation of the information, although these differences were not always based on racial/ethnic classification. The interpretation that the elders brought out in the focus groups depended on their previous life experiences and current socio-economic status, as well as current familial situations and cultural expectations.

Further, it is important to stress that the results of the qualitative analysis show clearly that the theoretical perspectives identified in Chapter 3 do not fully elucidate, represent or bring an overall understanding of what it means to be "old" or "aging," particularly in Miami-Dade County, Florida. A majority of elders in the United States, regardless of racial/ethnic background, live in the community and are not frail and dependent. Most of the elder participants did not see themselves as unusual in their activities or life-styles. They were usually actively involved in life and not just passive agents as is theorized with disengagement theory. They talked of a variety of activities that they participated in with other elders. They knew that frailty might force them in the future to "disengage" from the current activities, or isolate them from much of their current community. As noted in Chapter 6, the elder participants did not talk about "culture" per se; however, this can be attributed to the embeddedness and social construction of the terms "aging" and "old."

Age-stratification theory gives an understanding of the "movement of successive birth cohorts across time" (Passuth \& Bengtson, 1988). However, this theoretical 
perspective does not explain the importance of cultural or individual life experiences of elders. In Miami-Dade County, Florida with its large Cuban elder population, the interpretation of the meaning of aging for these Cuban elders is colored by their flight from their homeland. Many of these Cuban elders did not expect to be living in United

States in their old age. Thus, the movement of successive birth cohorts across time is not a satisfactory explanation of aging.

Another theoretical perspective applied to aging is the exchange theory. While it is helpful in examining the interactions of older people with other age groups, it is also limited in its approach since it does not address the "meaning" of the exchange, just that an "exchange" takes place. White non-Hispanic elders in Miami-Dade County are generally older, but healthier, somewhat wealthier, however they have few (if any) family members residing close by. These elders tended to discuss health issues in terms of increasing population of frail elders in the community, for which all levels of government (local/county, state, and federal) are not prepared.' In comparison, minority elders tended to discuss the aspects of health and health care in terms of affordability of health insurance and being able to pay for medical services. This example also touches on the

\footnotetext{
'Again, it must be pointed out that the expectations of white non-Hispanic elders residing in Miami-Dade County, FL may not be consistent with white non-Hispanic elders in other communities across the nation. This is because many of these elders have migrated to South Florida from other geographic origins. Migration to South Florida would not take place if the elder is already sick, frail or in a poor economic situation. Those who do migrate to South Florida are usually healthier and somewhat wealthier group of white non-Hispanic elders. As they age in place in their "new" community, these elders will eventually become sick or too frail to care for themseives. However, when this happens, their adult children. usually living someplace else, will usually try to find local in-home services for their parents until it is obvious that in-home care is not enough. At this point, adult children will usually move their frail parent back to the origin community or the community of residence of the adult child caregiver, so that the frail elder may
} 
theoretical perspective of political economy of aging which implies that all older individuals are "impoverished and disenfranchised" which is definitely an over-statement with respect to elders residing in Miami-Dade County.

Turning the attention to the quantitative results specifically focusing on health care utilization, it is not surprising that the results show that there are differences in predictors of utilization by type of formal health service and by racial/ethnic group of elders. As documented in Chapter 2, previous research examining elders showed that need factors were the most important set of factors for elders. However, as noted above in the focus group discussion, as well as in Chapter 7, the frailer white non-Hispanic elders have usually moved out of the community and are not visible in the sample. Thus, it is not surprising that need factors did not show up as the strongest predictors for white non-Hispanic elders compared to other racial/ethnic groups of elders for all service types, except for overnight hospital stay. As discussed in the qualitative analysis, what it means to be "old" is not the same for all cultural groups. Thus, the interpretation of the importance of need factors for predicting formal health care utilization may be seen as a mitigating factor for the biological process of "aging," and not "aging" as a whole.

Enabling factors were important for all racial/ethnic groups of elders, even for the "mainstream" white non-Hispanic elders. This suggests that there was a lack of equity in the health care service market, regardless of minority status, for older individuals.

Enabling factors posed serious barriers to health care utilization. For example, having no

receive necessary care. Thus, Miami-Dade County's white non-Hispanic elders do not represent the whole continuum in the health status of its elder population. 
Medicare was an important predictor variable for most formal health care service utilization across most racial/ethnic groups of elders. Further, the importance of Medicaid coverage was not found to be a consistent predictor of utilization. Medicaid has generally been thought to "level the playing field" of the health care service delivery market for poorer elders. However, recent research by Lum \& Chang (1998) concluded that "... Medicaid does not work equally well in improving affordability and use of health services among different racial [and] ethnic ... groups." This result was confirmed in this research as well. Medicaid coverage was a significantly strong predictor for Cuban elders in explaining overall physician contact, but not for any other racial/ethnic group of elders.

Another important enabling factor for Cuban elders was HMO coverage. Having HMO coverage increased the likelihood of having a physician contact in the last 12 months by almost 3 times for Cuban elders. This result is not surprising given that Cuban elders have had a longer experience with health maintenance organizations compared to non-Hispanic cultural groups of elders, as discussed in case study of Cuban elder health belief systems in Chapter 2 .

Not only are there personal/family resource barriers, there are also community resources that can be important for elders in the utilization of formal health care services. While community resources are not usually perceived as important as personal/family resources, they do play an important role in inhibiting or enabling utilization of formal health care services for all racial/ethnic groups of elders, not only for minority elders. Further, the particular variable representing predictive community resources were not 
consistent across the racial/ethnic groups or service types. As shown in the maps in Chapter 4, Miami-Dade County's population is highly segregated with respect to racial/ethnic groups. Elders of one racial/ethnic group may feel marginalized if they reside in an area of the county that is dominated by another racial/ethnic group (i.e., a white non-Hispanic elder residing in a largely Hispanic neighborhood, or a native-born black non-Hispanic elder residing in a largely Hispanic neighborhood). This is in part due to the fact that health care services will be focused on the dominant group.

Differences in health belicfs systems by the "minority" individual (regardless of racial/ethnic group) may be overlooked and thus, the elder individual may decide to forego utilization of health care services in their local community until the need is critical. For example with respect to high volume physician contact, white non-Hispanic elders residing in zip codes with higher percentages of Hispanics were significantly more likely to have high volume physician contact in the last 12 months compared to those who lived in zip codes with lower percentages of Hispanics. Similarly, Cuban elders living in zip codes with higher percentages of blacks were significantly more likely to have high volume physician contact, compared to Cuban elders living in zip codes with lower percentages of blacks. However, it does not always work in the negative. For example, white non-Hispanic elders living in zip codes with high percentages of elders were significantly more likely to have had a complete physical exam in the last 12 months, compared to white non-Hispanic elders living in zip codes with lower percentages of elders. 
Specifically addressing the quantitative analysis, previous research using dummy variables for racial/ethnic groups in a single model for service utilization does not give an accurate picture of the dynamics involved between the three blocks of factors. While all aging individuals share a mutual orientation toward aging (biological process), they do not age the same way (social construction of "aging"). Thus, policy makers and those interested in serving the elder population within a community must be aware of the particular cultural context, as well as the previous life experiences of the individuals that they hope to serve. This analysis has documented the importance of culture and geographic community in understanding health care service utilization of elders.

\section{SUGGESTIONS FOR FUTURE RESEARCH}

As stated earlier in this chapter, this dissertation only scratches the surface of the work that needs to be done with respect to the three intertwined themes of culture, aging and health. While the findings and the implications of the findings are important additions to the overall knowledge base, it is also important to examine the assumptions used in this dissertation analysis that have lead to limitations in the research findings, and thus suggestions for future research. Assumptions in this research range from the selection of variables and indices, to the assumptions used in the dataset collection methodology, to the collection of additional community contextual data, and the analytical methods employed to examine the hypotheses in this dissertation.

The decision to examine formal health care service utilization of elders is by no means the only type of health care service available or used by elders residing in Miami- 
Dade County. Given the case study of health belief systems of Cuban elders documented in Chapter 2, alternative or "folk medicine" practices are used quite frequently by Cuban elders. Black non-Hispanic and non-Cuban Hispanic elders may not have the same health belief systems guiding their decision-making with respect to their choices of health care. However, similar types of behavior are probably occurring among these groups of elders. The reason that this research has focused on usage of formal health care services is that it is more amenable to policy intervention, as well as it is theoretically available to all elders, regardless of cultural background. While the research documented in this analysis shows that differentiation within formal health care service type is crucial, future research will also need to focus on the linkages between the health care sectors (formal, folk and popular). In addition, there will need to be a better understanding of the health belief systems that make individuals choose one course of treatment over another.

Another important assumption is the appropriateness of using the theoretical perspective of the Socio-Behavioral Model for measuring the use of formal health care services among cultural groups of eIders. As documented in Chapter 2, there have been criticisms regarding the usage of this theoretical perspective for the study of health care service utilization by elders. The analysis in this dissertation has modified the existing models of health care utilization under this theoretical perspective to take into account the shortcomings of previous research using SBM (i.e., race/ethnicity, provider availability and other environmental characteristics). One such modification was modeling health care service utilization separately for each racial/ethnic group of elders, thus the variables and blocks of variables were not constrained to be linearly related. As documented in 
Chapter 2, the inclusion of dummy variables for racial and ethnic identity will only show differences in the intercept of the regression model. It will not provide "any information regarding the potential differences in the [interaction] effects of the independent variables [with race/ethnicity] on the dependent variable" (LaVeist, 1994). The interaction effect of racial/ethnic origin with other independent variables, (i.e., poverty status, age structure, etc.) should be taken into account where possible. Overall, with respect to this theoretical perspective, it is believed that the benefits of using SBM outweigh the criticisms documented in the literature.

The choice of community contextual variables was somewhat arbitrary and dependent upon availability and ability to link to respondents by zip code. Further, it is recognized that community resource variables are evaluated differently across cultural groups of elders. Data collection of other community contextual variables for refinement of this block in the model is crucial for future research.

While anecdotal evidence suggests that social support plays a significant role in the lives of older individuals (Nelson, 1993), exploration of the role of social support on elderly utilization of health services has largely been ignored until recently. The measure of social contact intensity with family and friends used in this analysis is one way to measure the importance of informal social support networks without being too invasive to the privacy of the elder respondent. Kin availability has also been suggested as a measure. However, there are cultural differences in definition of kinship networks. Thus, measurement of kinship networks would need to be further investigated as a possible measure. Further, family is not the only way that elders may receive social support. 
With respect to white non-Hispanic elders, many are physically distant from their adult children and extended families because they retired to South Florida. Thus, friendships may also be important aspects of informal social support networks and should be taken into account.

The measuring of health beliefs was somewhat limited by the availability of information on the survey instrument. The measures used to indicate health beliefs (i.e., level of concern regarding hcalth as a problem for elders, future worry about loss of independence, and overall life satisfaction in the last 6 months) begin to look at the importance of health beliefs in the lives of elders. Future research should refine these measures of health belief for utilization within a telephone survey design. Further the questions on "health beliefs" need to be critically examined with respect to their equivalence across cultures.

In terms of more macro issues, it is important to realize that aging in a community should not be examined within a vacuum. The findings from this research, both qualitatively and quantitatively, suggest that issues of aging and health care utilization cannot be understood in isolation from the community that an elder resides in politically, socially, geographically, economically, and culturally. Availability of health care services is located within a specific sphere of the community where an elder resides physically, as well as belief-wise. Future research should focus on local area studies to better understand the connections that enable or inhibit elders using health care services in their community. Health care services are delivered at the local level. In the future, while other goods and services may be ordered over the Internet, formal health care 
services will still need to be delivered in person. For example, if an elder falls breaking their hip, that elder does not want to shop around nationally to find a doctor and a hospital to go to and have their hip fixed long distance. Thus, local area studies of actual available services will allow for a clearer understanding of the linkages between availability of service and utilization. 


\section{REFERENCES}

Abercrombie, N.; Hill, S.; and Turner, B. S. 1994. The Penguin Dictionary of Sociology. $3^{\text {rd }}$ edition. New York: Penguin Books.

Abraham, L. K. 1993. Mama might be better off dead: The failure of health care in Urban America. Chicago: University of Chicago Press.

Aday, L. A. 1993. "Indicators and Predictors of Health Services Utilization." In Stephen J. Williams and Paul R. Torrens (eds.) Introduction to Health Services. Albany, NY: Delmar Publishers, Inc.

Aday, L. A. and Andersen, R. M. 1974. " $\triangle$ framework for the study of access to medical care." Health Services Research. 9: 208-220.

Aday, L. A. and Andersen, R. M. 1975. Access to Medical Care. Ann Arbor, MI: Health Administration Press.

Aday, L. A.; Andersen, R. M.; and Fleming, G. V. 1980. Health care in the U.S. Equitable for whom? Beverly Hills, CA: Sage Publications.

Aday, L. A.; Fleming, G. V. and Andersen, R. M. 1984. Access to medical care in the U.S.: Who has it, who doesn 't. Chicago, IL: Pluribus Press.

Administration on Aging. 1988. A profile of older Americans. Washington, D.C.: Administration on Aging.

Andersen, R. M. 1968. A behavior model of families' use of heallh services. Chicago, IL: Center for Health Administration Studies

Andersen, R. M. 1995. "Revisiting the Behavioral Model and Access to Medical Care: Does it Matter?" Journal of Health and Social Behavior, 36(1): 1-10.

Andersen, R. M. and Newman, J. 1973. "Societal and individual determinants of medical care utilization in the United States." Milbank Memorial Fund Quarterly. 51: 95-124.

Andersen, R. M.; Giachello, and Aday, L.A. 1986. "Access of Hispanics to health care and cuts in services: A state-of-the-art overview." Public Health Reporter. 10:238-252.

Angel, J. L. and Hogan, D. P. 1992. "The Demography of Minority Aging Populations." Jounal of Family History. 17(1): 95-115.

Bailey, E. 1987. "Sociocultural Factors and Health Care-Seeking among Black Americans." Journal of National Medical Association. 79: 389-392

Bateson, M. C. 1990. Composing A Life. New York: Plume Books.

Becker, F. W. and Dluhy, M. J. 1998. "Solving Problems in Urban Areas Characterized by Fragmentation and Divisiveness: An Overview." In Research in Urban Policy: Solving Problems in Urban Areas Characterized by Fragmentation and 
Divisiveness. Fred W. Becker and Milan J. Dluhy (eds.) pp. 1-18, Vol. 7. Stamford, CT: JAI Press, Inc.

Becker, M. and Maiman, L. 1983. "Models of health-related behavior." In David Mechanic (ed.) Handbook of Health. Healthcare and Health Professions. (pp. 539-658). New York: Free Press.

Becker, M.; Hacfner, D.; Kash, S.; Kirscht, J.; Maiman, L.; and Rosenstock, I. 1977. "Selected psychosocial models and correlates of individual health-related behaviors." Medical Care, 15(5): 27-46.

Bengtson, V., Parrott, T. and Burgess, E. 1997. "Theory, explanation, and a third generation of theoretical developments in social gerontology." Journal of Gerontology: Social Sciences. 52B: S72-88.

Bengtson, V. L.; Rice, C. J.; and Johnson, M. L. 1999. "Are Theories of Aging Important? Models and Explanations in Gerontology at the Turn of the Century." In Vern L. Bengtson and K. Warner Schaie (eds.) Handbook of Theories of Aging. Pp. 3-20. New York: Springer Publishing Co.

Berger, P. L. and Luckman, T. 1966. The Social Construction of Reality: A Treatise in the Sociology of Knowledge. New York: Anchor Books.

Berkman, L. F. and Syme, S. L. 1979. "Social networks, host resistance and mortality: A nine-year follow-up study of Alameda County residents." American Journal of Epidemiology. 109: 186-204.

Beverley, J. and Houston, D. 1996. "Notes on Miami." boundary 2. 23(2): 19-46.

Blau, P. 1964. Exchange and Power in Social Life. New York: Wiley.

Boswell, T. D. 1995. The Cubanization and Hispanicization of Metropolitan Miami. (Revised). Miami, FL: Cuban American National Council, Inc.

Boswell, T. D. and Curtis, J. R. 1984. The Cuban-American Experience. Totowa, NJ: Rowman \& Allanheld.

Bowie, S. L. and Stepick, Alex, III. "Diversity and Division: Ethnicity and the History of Miami." In Research in Urban Policy: Solving Problems in Urhan Areas Characterized by Fragmentation and Divisiveness. Fred W. Becker and Milan J. Dluhy (eds.) pp. 19-32, Vol. 7. Stamford, CT: JAI Press, Inc.

Bradsher, J. E. 1992. "Physician Utilization Among the Elderly: An Analysis Using Structural Equation Modeling." Ph.D. Dissertation, University of Miami.

Bureau of Economic and Business Research (BEBR). 2000. Population Projections by Age, Sex and Race (5-year breakdown). Gainesville, FL: Bureau of Economic and Business Research, University of Florida.

Burnette, D. and Mui, A. C. 1999. "Physician utilization by Hispanic elderly persons National perspective." Medical Care. 37(4): 362 374. 
Butler, R. N. 1974. "Successful aging and the role of life review." In S. H. Zarit (ed.) Readings in aging and death: Contemporary perspectives. (2 $2^{\text {rd }} \mathrm{ed}$.) pp. 22-26. New York: Harper \& Row, Publishers.

Chapleski, E. E. 1991. "Determinants of Utilization of Services to the Elderly: A Political Economy Perspective.” Ph.D. Dissertation, University of Michigan.

Clark, M. 1967. "The Anthropology of Aging. A New Area for Studies of Culture and Personality." Gerontologist. 7:55-64.

Clark, M. and Anderson, B. 1967. Culture and Aging. Springfield: Charles Thomas.

Cleary, P. D. and Angel, R. 1984. "The Analysis of Relationships Involving Dichotomous Dependent Variables." Journal of Ilealth and Social Behavior. 25: 334-348.

Cockerham, W. 1992. Medical Sociology. Fifth edition. Englewood Cliffs, NJ: Prentice-Hall, Inc.

Cockerham, W. 1995. Medical Sociology. Sixth edition. Englewood Cliffs, NJ: Prentice-Hall, Inc.

Cockerham, W. C. 1997. This Aging Society. $2^{\text {nd }}$ edition. Upper Saddle River, NJ: Prentice IIall.

Cohen, L. 1994. "Old Age: Cultural and Critical Perspectives." Annual Review of Anthropology. 23:137-188.

Consensus Estimating Conference. 1999. "Population by Age Group, Race, and Sex for Dade County." (http://fen.state.fl.us/edr/population/dadepop.htm)

Cowgill, D. O. 1974. "Aging and modernization: A revision of the theory." In J. F. Gubrium (ed.), Late life. pp. 123-146. Springfield, IL: Charles C. Thomas.

Cowgill, D. O. 1986. Aging Around the World. Belmont, CA: Wadsworth.

Cox, D. R. 1970. The Analysis of Binary Data. London: Methuen.

Crapanzano, V. 1986. "Hermes' Dilemma: the Masking of Subversion in Ethnographic Description." In James Clifford and George E. Marcus (eds.) pp. 51-76. Writing Culture: The Poetics and Politics of Ethnography. Berkeley, CA: University of California Press.

Croucher, S. L. 1997. Imagining Miami: Ethnic Politics in a Postmodern World. Charlottesville, VA: University Press of Virginia.

Csordas, T. J. and Kleinman, A. 1996. "The Therapeutic Process." In Medical Anthropology: Contemporary Theory and Method. Carolyn F. Sargent and Thomas M. Johnson (eds.) Revised edition. (pp. 3-20). Westport, CT: Praeger Publishers.

Cumming, E and Henry, W. E. 1961. Growing old: The process of disengagement. New York: Basic Books. 
Day, C. L. 1990. What Older Americans Think: Interest Groups and Aging Policy. Princeton, NJ: Princeton University Press.

Day, J. C. 1996. Population Projections of the United States by Age. Sex, Race and Hispanic (rigin: 1995 to 2050. U.S. Bureau of the Census, Current Population Reports, P25-1130. U.S. GPO: Washington, D.C.

Decker, D. L. 1980. Social gerontology. Boston: Little Brown and Company.

Daniclson, R. 1979. Cuban Medicine. New Brunswick, NJ: Transaction, Inc.

DeSantis, I. and Halberstein, R. 1992. "The Effects of Immigration on the Health Care System of South Florida." Human Organization. 51(3): 223-234.

Dluhy, M. J, and Krebs, C. E. 1987. Dade County, Florida: a needs assessment of the 60 and over population. Miami, FL: Southeast Florida Center on Aging. Publication no. 305. Flotida International University.

Dowd, J. and Bengtson, V. 1978. "Aging in minority populations." Journal of Gerontology. 33: 427-436.

Dowd, J. J. 1975. "Aging as exchange: A preface to theory." Journal of Gerontology. 30: 584-594.

Dunlop, B. D.; Brooks, M. and Polivka, L. 1996. The Florida Long-Term Care Elder Papulation Profiles Survey. Final Report to the Commission on Long-Term Care in Florida. Tampa, FL: Florida Policy Exchange Center on Aging, University of South Florida.

Egeland, J. A. 1978. Ethnic Value Orientation Analysis: A Research Component of the Miami Health Ecology Project. Volume II of Miami Ilealth Ecology Project Report. Miami, FL: University of Miami.

Elo, I. and Preston, S. H. 1994. "Estimating African-American Mortality from Inaccurate Data." Demography. 31(3): 427-458.

Erikson, E. H.; Erikson, J. M. and Kivnick, H. Q. 1986. Vital Involvement in Old Age. New York: W. W. Norton \& Co.

Estes, C. L. and Binney, E. A. 1989. "The Biomedicalization of Aging: Dangers and Dilemmas." The Gerontologist. 29(5): 587-596.

Evashwick, C.; Rowe, J.; Diehr, P. and Branch, L. 1984. "Factors explaining the use of health care services by the elderly." Health Services Research. 19:357-382.

Feinsilver, J. M. 1993. Healing the Masses: Cuban health politics at home and abroad. Berkeley, CA: University of California Press.

Fitzgerald, M. H. 1992. "Multicultural Clinical Interactions." Journal of Rehabilitation. (April/May/June): 38-42.

Fisher, C. 1980. "Differences by age groups in health care spending." Health Care Financing Review. 1: 65-90. 
Fox, R. C. 1989. The Sociology of Medicine, a participant observer's view. Englewood Cliffs, NJ: Prentice-Hall.

Frank, J. B. 1987. "Vital Involvement in Old Age." Book Review. Journal of American Medical Association (JAMA), 258(2): 268.

Frank, H. A.; Condon, K. M.; Dunlop, B. D.; Rothman, M. B. 2000. "The Retirement Planning Gap: A View From the Public Sector." International Journal of Organization, Theory \& Behavior. 3(1\&2): 235-274.

Franz, C. E. and White, K. M. 1985. "Individuation and attachment in personality development: extending Erikson's theory." Journal of Personality. 53:224-256.

Freiman, M. P. 1998. "The Demand for Healthcare Among Racial/Ethnic Subpopulations." Health Services Research. 33(4): 867-890.

Friedan, B. 1993. The Fountain of Age. New York: Simon \& Schuster.

Fry, C. L. 1999. "Anthropological Theories of Age and Aging." In Vern L.. Bengtson and K. Warner Schaie (eds.). Handbook of Theories of Aging. New York: Springer Publishing Company.

Garcia-Zamor, J-C. 1998. "Social Service Delivery for Immigrants in Southeast Florida." In Research in Urban Policy: Solving Problems in Urban Areas Characterized by Fragmentation and Divisiveness. Fred W. Becker and Milan J. Dluhy (eds.) pp. 18-200, Vol. 7. Stamford, CT: JAI Press, Inc.

Geertsen, R. 1988. "Social Group Characteristics and Health Behavior." In D. S. Gochman, ed., Health Behavior: Emerging Research Perspectives. New York: Plenum Press.

Geertz, C. 1973. The Interpretation of Cultures. New York: Basic Books.

Giddens, A. 1976. New rules of sociological method: A positive critique of interpretive sociologies. New York: Basic Books.

Gilligan, C. 1982. In a different voice. Cambridge, MA: Harvard University Press.

Ginzberg, E. 1991. "Access to health care for Hispanics." Journal of American Medical Association. 265(2): 238-241.

Gochman, D. S. 1988. "Health Behavior: Plural Perspectives." In D. S. Gochman, ed., Health Behavior: Emerging Research Perspectives. New York: Plenum Press.

Gold, M. 1998. "Beyond coverage and supply: Measuring access to healthcare in today's market." Health Services Research. 33(3, Part 2): 625-652.

Goldman, N.; Korenman, S.; and Weinstein, R. 1995. "Marital status and Health Among the Elderly." Social Science \& Medicine. 40(12): 1717-1730.

Greene, R. L. and Siegler, I. C. 1984. "Blacks." In Erdman B. Palmore (ed.) Handbook on the Aged in the United States. Westport, CT: Greenwood Press. 
Grenier, G. J. and Stepick, A., III. 1992. "Introduction." In Miami Now!: Immigration, Ethnicity and Social Change. Guillermo Grenier and Alex Stepick, III (eds.) pp. 1-17. Gainesville, FL: University Press of Florida.

Grossman, D. 1998. "Cuban Americans." In Larry D. Purnell and Betty J. Paulanka (eds.) Transcultural Health Care: A Culturally Competent Approach. Philadelphia, PA: F. A. Davis Company.

Gubrium, J. F. and Holstein, J. A. 1999. "Constructionist Perspectives on Aging." In Vern L. Bengtson and K. Warner Schaie (eds.) Handbook of Theories of Aging. New York: Springer Publishing Company.

Guo, Z. 2000. Ginseng and Aspirin: Health Care Alternatives for Aging Chinese in New York. Ithaca and London: Cornell University Press.

Hagdrup, N. A. 1997. "Health care coverage: traditional and preventive measures and associations with chronic disease risk factors." Journal of Community Health. 22(5): 387-399.

Harel, Z.; Noelker, L. and Blake, B. F. 1985. "Comprehensive services for the aged: Theoretical and empirical perspectives." The Gerontologist. 25(6): 644-649.

Havighurst, R. A. 1963. "Successful Aging." In Processes of Aging, edited by R. Williams, C. Tibbitts, and W. Donahue. pp. 299-320. New York: Atherton.

Haynes, S. G.; Harvey, C.; Monttes, H.; Nickens, II.; and Cohen, B. H. 1990. "Patterns of Cigarette Smoking Among Hispanics in the United States: Results from HHANES 1982-84," American Journal of Public Health. 80(Supplement): 4754.

Hazan, H. 1994. Old age: constructions and deconstructions. New York, NY: Cambridge University Press.

Helman, C. G. 1994. Culture, Health and Illness: An Introduction for Health Professionals. $3^{\text {rd }}$ edition. Boston, MA: Butterworth-Heinemann, Ltd.

Hendricks, J. and Achenbaum, A. 1999. "Historical Development of Theories of Aging." In Vern L. Bengtson and K. Warner Schaie (eds.) Handbook of Theories of Aging. New York: Springer Publishing Company.

Hendricks, J. and Hendricks, C. D. 1986. Aging in mass society: Myths and realities. $3^{\text {rd }}$ edition. Boston, MA: Little-Brown.

Higginbotham, J. C.; Trevino, F. M.; and Ray, L. A. 1990. "Utilization of curanderos by Mexican-Americans: Prevalence and Predictors, Findings from HHANES 1982-

84." American Journal of Public Health. 80(Supplement): 32-35.

Hochschild, A. 1975. "Disengagement theory: A critique and proposal. American Sociological Review. 40:553-569.

Hochschild, A. 1976. "Disengagement theory: A logical, empirical, and phenomenological critique." In J. F. Gubrium (ed.) Time, roles, and self in old age. pp. 53-87. New York: Human Sciences Press. 
Homan, S. M.; Haddock, C. C.; Winner, C. A.; Coe, R. M.; and Wolinsky, F. D. 1986. "Widowhood, scx, labor force participation and the use of physical services by elderly adults." Joturnal of Gerontology. 41: 793-796.

Jackson, J. J. 1980. Minorities and Aging. Belmont, CA: Wadsworth Publishing Co. Jacobs, J. M. and Fincher, R. 1998. "Introduction." In Cities of Difference. Ruth Fincher and Jane M. Jacobs, eds. (pp1-25). New York, The Guildford Press.

Jacobs, J.; Gubrium, J.; and Hochschild, A. R. 1975. "The phenomenological theory" In D. L. Decker (ed.), Social geronotology. Boston: Little Brown and Company.

Jary, D. and Jary, J. 1991. The Harper Collins Dictionary of Sociology. New York: HarperCollins Publishers.

Johnson, R. J. and Wolinsky, F. D. 1996. "Use of community-based long-term care services by older-adults." Journal of Aging and Health. 8(4): 512-537.

Joung, 1. M. A.; Van DeMheen, H. D.; Stronks, K.; Van Poppel, F. M. A.; and Mackenbach, J. P. 1998. "A Longitudinal Study of Health Selection in Marital Transitions." Social Science \& Medicine. 46(3): 425-435.

Kasl, S. V. and Cobb, S. 1966. "Health behavior, illness behavior, and sick-role behavior: I. Health role behavior." Archives of Environmental Health. 12: 246" 266.

Katz, S. 1996. Disciplining Old Age: The Formation of Gerontological Knowledge. Charlottesville, VA: University of Virginia Press.

Kaufman, S. R. 1994. "Old Age, Disease, and the Discourse on Risk: Geriatric Assessment in U.S. Health Care." Medical Anthropology Quarterly. 8(4): 430447.

Kaufman, S. R. 1986. The Ageless Self: Sources of Meaning in Late Life. Madison, WI: The University of Wisconsin Press.

Keith, J. B. 1983. "Age and Informal Interaction." In Jay Sokolovsky (ed.) Growing Old in Different Societies. Belmont, CA: Wadsworth.

Kincaid, J. (Forthcoming). "Introduction: Federalism Values and Health Values." Scipolicy. The Journal of Science and Health Policy.

Kisker, E. E. 1990. "Marital Status Differentials in Health, Health Care Utilization and Mortality: A Review of the Literature." Unpublished manuseript. Mathematica Policy Research, Inc.: Princeton, NJ.

Kleinman, A. 1995. Writing at the Margins: Discourse Between Anthropology and Medicine. Berkeley, Los Angeles, and London: University of California Press.

Kleinman, A. 1980. Patients and Healers in the Context of Culture: An Exploration of the Borderland between Anthropolog; Medicine, and Psychiatry. Berkeley, CA: University of California Press. 
Kravitz, S. L.; Pelaez, M. B. and Rothman, M. B. 1989. "Delivering services to elders: Responsiveness to populations in need." In S. A. Bass, E. Kutza, and F. TorresGil (eds.), Diversity in aging: Challenges facing planners and policymakers in the 1990s. Pp. 47-71. Glenview, IL: Scott, Foresman, Inc.

Lacayo, C. G. 1980. A nutional study to assess the service needs of the Hispanic elderly. Asociacion Nacional Pro Personas Mayores, Los Angeles, CA. Washington, D.C.: Department of Health and Human Services.

LaVeist, T. A. 1994. "Beyond sample selection, stratification and dummy variables: What health services researchers ought to know about race as a variable." Health Services Research. 29: 1-16.

Laz, C. 1998. "Act Your Age." Sociological Forum. 13(1): 85-114.

Liang, J. 1986. "Self-reported physical health among aged adults." Journal of Gerontology. 41: 248-260.

Lipson, D. J. and Da Sa, J. (no date). "South Florida: Site Visit Report." In Community Snapshot Project: Capturing Health System Change. Paul B. Ginsburg and Nancy J. Fasciano (eds.). Princeton, NJ: Robert Wood Johnson Foundation.

Longino, C. F., Jr. and McNeal, R. B., Jr. 1991. "The Elderly Population of South Florida." In South Florida: the Winds of Change. Thomas D. Boswell (ed.). Miami, FL: Association of American Geographers.

Lorber, J. 1994. Paradoxes of Gender. New Haven, CT: Yale University Press.

Lubitz, J. and Prihoda, R. 1984a. "Use and costs of Medicare services in the last year of life." In Health, United States, 1983. DHHS Publication 84-1232. Washington, D.C.: Government Printing Office.

Lubitz, J. and Prihoda, R. 1984b. "Use and Costs of Medicare Services in the Last 2 Years of Life." Health Care Financing Review. 5:117-131.

Lum, Y. S. and Chang, H. J. 1998. "The effect of Medicaid coverage on use of health services by low-income elderly people." Social Work Research. 22(1): 31-43.

Maingot, A. P. 1992. "Immigration from the Caribbean Basin." In Miami Now!. Immigration, Ethnicity and Social Change. Guillermo Grenier and Alex Stepick, III (eds.) pp. 18-40. Gainesville, FL: University Press of Florida.

Manuel, R. C. and Reid, J. 1982. "A comparative demographic profile of the Minority and Nonminority Aged." In Ron C. Manuel (ed.) Minority Aging: Sociological and Social Psychological Issues. Westport, CT: Greenwood Press.

Marcus,A. C. and Siegel, J. M. 1982. "Sex differences in the use of physician services: A preliminary test of fixed role hypothesis." Journal of Health and Social Behavior. 23: 186-197.

Marcus, G. E. and Fischer, M. M. J. 1986. Anthropology as Cultural Critique: An Experimental Moment in the Human Sciences. Chicago, lL: University of Chicago Press. 
Markides, K. S. and Mindel, C. H. 1987. Aging and Ethncity. Beverly Hills, CA: Sage Publications.

Marshail, G. (ed.) 1994. The Concise Oxford Dictionary of Sociology. New York: Oxford University Press.

McGee, R. Jon and Warms, Richard L. 1996. Anthropological Theory: An Introductory History. Mountain View, CA: Mayfield Publishing Company.

McKinlay, J. B. 1972. "Some approaches and problems in the study of use of services: An overview." Journal of Health and Social Behavior. 13: 115-152.

McNeely, R. L. and Cohen, J. L. (eds.). 1983. Aging in minority groups. Beverly Hills, CA: Sage Publications.

Mechanic, D. 1968. Medical sociology: A Selective V'iew. New York: Free Press.

Mechanic, D. 1979. "Correlates of physician utilization: Why do multivariate studies of physician utilization find trivial psychosocial and organizational effects?" Journal of Health and Social Behavior. 20:387-396.

Metro-Dade County Department of Planning, Development and Regulation, Research Section (Metro-Dade). 1997. "Dade County Facts - 1997: A Compendium of Selected Statistics Updated Annually." Miami, FL: metro-Dade Department of Planning, Development and Regulation.

Metro-Dade County Department of Planning, Development and Regulation, Planning Division (Metro-Dade Planning). 1996. Population Projections by Minor Statistical Area and Census Tract. Dade County, Florida 1970 to 2015. Miami, FL: Metro-Dade County Department of Planning.

Mills, G. 1998. "Fragmentation and Long-Term Care of the Elderly." In Research in Urban Policy: Solving Problems in Urban Areas Characterized by Fragmentation and Divisiveness. Fred W. Becker and Milan J. Dluhy (eds.) pp. 201-213, Vol. 7. Stamford, CT: JAI Press, Inc.

Monroe, D. J.; Rothman, M. B.; Becker, N. E.; and Smith, J. M. 1991. Aging 2000: Projecting the Needs of Florida's Older Population to the Year 1995 and 2000. Miami, FL: Southeast Florida Center on Aging, Florida International University.

Myerhoff, B. 1979. Number Our Days. New York: E. P. Dutton.

National Center for Health Statistics. 1991. Health United States, 1990. Washington, D.C.: U.S. Government Printing Office.

Nayeri, K. 1995. "The Cuban Health Care System and Factors Currently Undermining It." Journal of Community Healh. 20(4): 321-334.

Nelson, M. A. 1993. "Race, Gender, and the Effect of Social Supports on the Use of Health Services by Elderly Individuals." International Journal of Aging and Human Development. 37(3): 227-246. 
Netzer, J. K.; Coward, R. T.; Peek, C. W.; Henretta, J. C.; Duncan, R. P. and Dougherty, M. C. 1997. "Race and residence differences in the use of formal services by older adults." Research on Aging. 19(3): 300-332.

O'Reilly, E. M. 1997. Decoding the Cultural Stereotypes About Aging: New Perspectives on Aging Talk and Aging Issues. New York: Garland Publishing, Inc.

Pampel, F. C. 1998. Aging, Social Inequality, and Public Policy. Thousand Oaks, CA: Pine Forge Press.

Parsons, T. 1951. The Social System. New York: Free Press.

Passuth, P. M. and Bengtson, V. L. 1988. "Sociological Theories of Aging: Current Perspectives and Future Directions." In Emergent Theories of Aging. James E. Birren and Vern L. Bengtson (eds.) pp. 333-355. New York: Springer Publishing Company.

Pauly, M. V. 1974. "Economic Aspects of Consumer Use." In Selma J. Mushkin (ed.) Consumer Incentives for Health Care. New York: PRODIST.

Phillips, K. A.; Morrison, K. R.; Andersen, R. and Aday, L. A. 1998. "Understanding the context of healthcare utilization: Assessing environmental and providerrelated variables in the behavioral model of utilization." Health Services Research. 33(3, Part 1): 571-596.

Pol, L. G. and Thomas, R. K. 1993. The Demography of Health and Health Care. New York: Plcnum Press.

Portes, A. and Stepick, A. 1993. City on the Edge: The Transformation of Miami. Berkeley, CA: University of California Press.

Pratt, G. 1998. "Grids of Difference: Place and Identity Formation." In Cities of Difference. Ruth Fincher and Jane M. Jacobs, eds. (pp. 26-48). New York, The Guildford Press.

Quadagno, J. 1982. Aging in Early Industrialized Society: work, Family and Social Policy in Nineteenth Century England. New York: Academic Press.

Quadagno, J. and Reid, J. 1999. "The Political Economy Perspective in Aging." In Vern L. Bengtson and K. Warner Schaie (eds.) Handbook of Theories of Aging. New York: Springer Publishing Company.

Queralt, M. 1983. "The elderly of Cuban origin." In R. M. McNeely and J. Colen (eds.). Aging in Minority Groups. Beverly Hills, CA: Sage Publications.

Ren, X. S. 1997. "Marital status and quality of relationships: the impact on health perception." Social Science \& Medicine. 44(2): 241-249.

Riley, M. W. 1971. "Social Gerontology and the Age Stratification of Society." Gerontologist. 11: 79-87.

Riley, M. W. 1987. "On the Significance of Age in Society." American Sociological Review. 52: 1-14. 
Riley, M. W., Foner, A. and Riley, J. W.. Jr. 1999. "The Aging and Society Paradigm." In Vern L. Bengtson and K. Warner Schaie (eds.) Handbook of Theories of Aging. New York: Springer Publishing Company.

Riley, M. W.; Hess, B. B.; and Bond, K. 1983. Aging in society: Selected reviews of recent research. Hillsdale, $\mathrm{NJ}$ : Lawrence Erlbaum Associates.

Rogg, E. M. and Cooney, R. M. 1980. "Adaptation and Adjustment of Cubans: West New York, New Jersey." Bronx, NY: Fordham University Hispanic Research Center.

Rojas, D. 1994. "Leadership in a multicultural society: A case in role development." Nursing and Health Care. 15(5): 258-261.

Rosenbloom, C. A. and Whittington, F. J. 1993. "The Effects of Bereavement on Eating Behaviors and Nutrient Intakes in Elderly Widowed Persons." Journal of Gerontology. 48(4): S223-S229.

Rothman, M. B.; Dunlop, B. D.; and Condon, K. M. 1994. The Elders of Dade County: A Needs Assessment of Persons 60 and Over. Miami, FL: Southeast Florida Center on Aging, Florida International University.

Ruggles, S., Sobek, M., et al. 1997. Integrated Public Use Microdata Series: Version 2. http://www.ipums.umn.edu. Minneapolis, MN: Historical Census Project, University of Minnesota.

Sallis, J. F. and Nader, P. R. 1988. "Family Determinants of Health Behaviors." In D. S. Gochman (ed.) Health Behavior: Emerging Research Perspectives. New York: Plenum Press.

Sandoval, M. C. 1979. "Santeria as a Mental Health Care System: An Historical Overview." Social Science and Medicine. 13b: 137-151.

Sankar, A. 1984. “" 'It's Just Old Age': Old Age as a Diagnosis in American and Chinese Medicine." In Age and Anthropological Theory. David I. Kertzer and Jennie Keith (eds.). (pp. 250-280). Ithaca, NY: Cornell University Press.

Segall, A. 1988. "Cultural Factors in Sick-Role Expectations." In D. S. Gochman (ed.) Health Behavior: Emerging Research Perspectives. New York: Plenum Press.

Shanas, E. and Maddox, G. L. 1985. "Health, Health Resources and the Utilization of Care." In Robert H. Binstock and Ethel Shanas (eds.) Handhook of Aging and the Social Sciences. Pp. 697-726. ( $2^{\text {nd }}$ edition). New York: Van Nostrand Reinhold Co.

Siddharthan, K. and Sowers-Hoag, K. 1989. "Elder's attitudes and access to health care: a comparison of Cuban immigrants and native-born Americans." Journal of Applied Gerontology. 8: 86-96.

Soldo, B. J. and Manton, K. G. 1985. "Health status and service needs of the oldest old: Current patterns and future trends." Milbank Memorial Fund Quarterly. 63: 287319. 
Solis, J. M.; Marks, G.; Garcia, M.; and Shelton, D. 1990. "Acculturation, Access to Care and Use of Preventive Services by Hispanics: Findings from HHANES 1982-84." American Journal of Public Health. 80(Supplement): 11-19.

South Florida Regional Planning Council (SFRPC). 1996. "Our Region - Regional Profile - Our People." hittp://www.sfrpc.com/region/profpeop.htm.

South Florida Regional Planning Council (SFRPC). 1999. "Economic Profile, 19701997." http://www.sfrpe,com/region/menu42la.htm.

SPSS, Inc. 1999. SPSS for Windows. Release 10.0.5. Chicago, IL (Software Program). Stack, John F., Jr. and Warren, Christopher L. 1992. "The Reform Tradition and Ethnic politics: Metropolitan Miami Confronts the 1990s." In Miami Now!: Immigration, Ethnicity and Social Change. Guillermo J. Grenier and Alex Stepick, III (eds.) pp. 160-185. Gainesville, FL: University Press of Florida.

Starr, P. 1982. The Social Transformation of American Medicine. New York: Basic Books, Inc.

State of Florida, Department of Elder Affairs. 1998a. "Demographic and County Profiles: Florida County Profiles September 1998: Broward."

hitp:/www state.fl.us/doea/countyprofiles/body county proliles Broward.htm?

State of Florida, Department of Elder Affairs. 1998b. "Demographic and County Profiles: Florida County Profiles September 1998: Dade." http:/ www state.tl.us/doea/countyprofiles/body county profiles_Dade.html

State of Florida, Department of Elder Affairs. 1998c. "Demographic and County Profiles: Florida County Profiles September 1998: Monroe." http:/www.state.fl us/doea/countyproliles/bodv county_profiles_Monroe.html

State of Florida, Department of Elder Affairs. 1998d. "Demographic and County Profiles: Florida County Profiles September 1998: Palm Beach." http:/www.state. fl.us/doea/countyprofiles/body_county protiles_PalmBeach.htm I

State of Florida, Legislative Division of Economic and Demographic Research. 1997. Florida Consensus Estimating Conference, Book 3: State of Florida Population \& Demographic Forecast. Volume 13, Summer 1997.

Streib, G. F. and Bourg, C. J. 1984. "Age Stratification Theory, Inequality and Social Change." Comparative Social Research. 7: 63-77.

Suarez, Z. E. 1998. "Cuban-American Families." In Charles H. Mindel, Robert W. Habenstein, and Roosevelt Wright, Jr. (eds.). Ethnic Families in America: Patterns and Variations. Fourth edition. Upper Saddle River, NJ: Prentice Hall.

Suarez, Z. E. 1997. "Cuban American Families." In Mary Kay DeGenova (ed.) Families in Cultural Context: Strengths and Challenges in Diversity. Mountain View, CA: Mayfield Publishing Company. 
Suchman, E. A. 1965a. "Social patterns of illness and medical care." Journal of Health and Human Behavior. 6: 2-16.

Suchman, E. A. 1965b. "Stages of illness and medical care." Journal of Health and Social Behavior. 6: 114-128.

Suchman, E. A. 1966. "Health orientation and medical care." American Journal of Public Health. 56: 97-105.

Takahashi, L. M. 1998. "Community Responses to Human Service Delivery in U.S. Cities." In Cities of Difference. Ruth Fincher and Jane M. Jacobs, eds. (pp. 120148). New York, The Guildford Press.

Trevino, F. M. and Moss, A. J. 1983. "Health insurance coverage and physician visits among Hispanic and non-Hispanic people." In Health, United States 1983. DHHS Publication No. (OHS) 84-1232. Washington, D.C.: GPO.

Trevino, F. M. and Moss, A.J. 1984. Health Indicators for Hispanic, Black, and White Americans. Vital and Health Statistics Series 10, No. 148, DHHS Publication No. (PHS) 84-1576. Washington, D.C.: GPO.

Tripp-Reimer, T. and Sorofman, B. 1988. "Minority Elderly: Health care and Policy Issues." In Winston A. Van Horne (ed.) Ethnicity and Health. Milwaukee, WI: The University of Wisconsin System Institute on Race and Ethnicity.

U.S. Senate Special Committee on Aging. 1988. Developments in Aging. Washington, D.C.: U.S. Government Printing Office.

United .States General Accounting (USGAO). 1992. Hispanic Access to Health Care: Significant Gaps Exist. ([Washington, D.C.]: Program Evaluation and Methodology Division, pp. 1-26).

United States Administration on Aging (U.S. AoA). 1998. "1998 Census Estimates of the Older Population, for States Older Population by Selected Age Group (50+, etc.) (Rank Based on Number of Older Persons in State)."

http:/www.aoa.dhhs.ogv/aoa/stats 98 pop/rankxnumber.html.

United States Bureau of the Census, Current Population Reports, Special Studies, P23190. $65+$ in the United States. Washington, D.C.: U.S. Government Printing Office. 1996.

United States Bureau of the Census. 1992a. 1990 Census of Population and Housing Summary of Social, Economic, and Housing Characteristics. Florida.

Washington, D.C.: U.S. Government Printing Office.

United States Bureau of the Census. 1992b. 1990 census of population and housing. Summary tape file 3A. Florida, Alachua-Lee computer file. [comp file] Washington, DC : U.S. Dept. of Commerce, Bureau of the Census, Data User Services Division.

United States Bureau of the Census. 1994. 1990 Census of Population and Housing: Special Tabulation on Aging (Florida). CD-rom. DC90-AOA-US. Sponsored by the Administration of Aging, U.S. Department of Health and Human Services. 
United States Bureau of the Census. 1995. Population of Counties by Decennial Census: 1900 to 1990. http:/www.census, grovipopulation/cencounts/11190090.txt.

United States Bureau of the Census. 1996. Intercensal Estimates of the Total Resident Population of States 1980 to 1990. http://www census.gov/population/estimates/state/stts/st8090ts.txt.

United States Bureau of the Census. 1998. Estimates of the Population of Counties by Race and Hispanic Origin: 1990-1997. PE-64, pkg 3. http:/www.census.gov/population/estimates/county/crh/crh9097.txt

United States Bureau of the Census. 2000. Population Estimates for Counties by Age, Race, Sex, and Hispanic-Origin: Annual Time Series July 1. 1990 to July 1, 1999. http:/www census.gov/populationjeslimates/county/casrh/casth12.txt

United States Senate Special Committee on Aging. 1971. "Elderly Cubans in Exile." Working Paper. Washington, D.C.: U.S. Government Printing Office.

Verbrugge, L. M. 1989. "The twain meet: Empirical explanations of sex differences in health and mortality." Journal of Health and Social Behavior. 30: 282-304.

Vesperi, M. D. 1985. City of green benches: growing old in a new downtown. Ithaca, NY: Cornell University Press.

Waldo, D. and Lazenby, H. 1984. "Demographic characteristics and health care use and expenditures by the aged in the U.S." Health Care Financing Review. 6:1-49.

Waldron, I.; Hughes, M. E.; and Brooks, T. L. 1996. "Marriage Protection and Marriage Selection - Prospective Evidence for Reciprocal Effects of Marital Status and Health." Social Science \& Medicine. 43(I): 113-123.

Wallace, S. P.; Campbe!l, K.; Lew-Ting, C. Y. 1994. "Structural barriers to the use of formal in-home services by elderly Latinos." Journal of Gerontology. 49(5): S253-263.

Ward, R. 1978. "Services for older people: An integrated framework for research." Journal of Health and Social Behavior. 28(4); 401-412.

Weber, M. 1978. Economy and Society: An Outline of Interpretive Sociology. Edited by Gunther Roth and Claus Wittich. Berkeley, CA: University of California Press.

West, C. and Zimmerman, D. H. 1987. "Doing gender." Gender \& Society. 1: 125-151.

Wilkinson, D. Y. and King, G. 1989. "Conceptual and Methodological lssues in the Use of Race as a Variable: Policy Implications." In David P. Willis (ed.) Health Policies and Black Americans (pp. 56-71). New Brunswick, NJ: Transaction Publishers.

Wilson, J. Q. 1973. Political Organizations. New York: Basic Books.

Wolinsky, F. D and Amold, C. L. 1988. "A different perspective on health and health services utilization." Annual Review of Gerontology and Geriatrics. 8: 71-101. 
Wolinsky, F. D. 1990. Health and Health Behavior Among Elderly Americans: An AgeStratification Perspective. New York: Springer Publishing Co.

Wolinsky, F. D. and Coe, R. M. 1984. "Physician and hospital utilization among elderly adults: An analysis of the health interview survey." Joumal of Gerontology. 39: 334-341.

Wolinsky, F. D.; Aguire, B. E.; Fann, L.; Keith, V. M.; Arnold, C. L.; Niederhauer, J. C.; and Dietrich, K. 1989. "Ethnic Differences in the Demand for Physician and Hospital Utilization Among Older Adults in Major American Cities: Conspicuous Evidence of Considerable Inequalities." The Milbank Quarterly. 67(3-4): 412 449.

Wolinsky, F. D.; Coe, R. M.; and Mosely, R. R. 1987. "The use of health services by elderly Americans: Implications from a regression-based cohort analysis." In R. Ward and S. Tobin (eds.) Health in aging: Sociological issues and policy. (pp. 106-132). New York: Springer.

Yeo, G. 1996. "Ethnogeriatrics: Cross-Cultural Care of Older Adults." Generations Journal of the American Society on Aging. 20(4): 72-77.

Young, J. C. and Garro, L. C. 1994. Medical Choice in a Mexican Village. Prospects Heights, IL: Waveland Press, Inc.

Zaborowski, M. 1952. "Cultural Components in Responses to Pain." Journal of Social Issues. 8(4): 16-30.

Zaldivar, R. A. and Rogers, P. 1995. "Cost-control drive brings Medicare to turning point." The Miami Herald. September 3, 1995. P. 1A, 20A.

Zola, I. 1966. "Culture and symptoms-An analysis of patients' presenting complaints." American Sociological Review. 31:615-630. 
APPENDIX A:

MIAMI-DADE COUNTY, FL MAP OF TEN PLANNING AND SERVICE SUB-AREAS (PSA) AS DESIGNATED BY THE ALLIANCE FOR AGING, INC., THE AREA AGENCY ON AGING FOR DADE AND MONROE COUNTIES 
In 1992, the Alliance for Aging divided Dade County into ten sub-areas for the purpose of planning and analysis. The boundaries of these areas are indicated on the appended map; many of those in the text are only approximations to orient the reader. Brief descriptions of those arcas are presented below.

Sub-Area 1: The Beaches. Extending from Bal Harbour on the north to South Beach, and from the Atlantic on the east to Biscayne Bay on the west, Area 1 ("The Beaches") includes Bay Harbor Island, Indian Creek Village, Surfside, North Bay Islands and Miami Beach.

Suh-Area 2: Northeast Dade. Area 2 forms the northeastern corner of Dade County, from the Broward county line (on the north) to $103^{\text {rd }}$ Road (south), and from the Atlantic and Intracoastal Waterway (east) to near I-95 (west). Its municipalities include Biscayne Park, North Miami, North Miami Beach and part of Miami Shores.

Sub-Area 3: North Central Dade. North Central Dade is an irregularly-shaped Area. It extends from the Broward County border (north) to a diagonal on the south, running from the southeastern tip of Hialeah to Bayfront Park. Its western boundary follows Hialeah's eastern city limits until Red Road, where it continues northward to the Broward county line. On the east, it follows Biscayne Bay up through most of Miami Shores; further north, its eastern boundary is approximated by I-95. It includes the cities of Opa-Locka and El Portal, much of Miami and most of Miami Shores.

Sub-Area 4: Northwest Dade. Bounded by the county line on its north and west, the Tamiami Trail on its south, and the Hialeah city line one its east, Northwest Dade includes the cities of Hialeah, Hialeah Gardens, Medley, Miami Springs, Pennsucco, and Virginia Gardens.

Sub-Area 5: West Miami/Sweetwater/Westchester. Area 5 is a narrow strip of land which includes the cities of West Miami and Sweetwater. It extends west to the Everglades. The region is bounded on the south by Bird Road and the Tamiami Trail runs along much of its northern border. Coral Way runs east-west through the center of it.

Sub-Area 6: Downtown Miami. The City of Miami southwest of a line running from the southern tip of Hialeah to Bayfront Park lies in Area 6. Miami International Airport, the areas just west of it to the Palmetto (S.R. 826), and just south of it in unincorporated Dade are also included.

Sub-Area 7: Kendall. The Kendall Planning and Study Area is that portion of Dade County south of Bird Road, generally north of $152^{\text {nd }}$ Street, and west of U.S. 1. It includes almost all of south Miami, but no other municipality.

Sub-Area 8: Greater Coral Gables. Area 8 is largely coastal region of southern Dade County, extending from the Miami/Coral Gables border south to $184^{\text {th }}$ Street and west to U.S. 1. Virginia Key and Key Biscayne are included. The City of Coral Gables and Village of Key Biscayne are within the Area, as is that portion of South Miami south and east of U.S. 1. 
Sub-Area 9: South Central Dade. Area 9 is a belt of land extending from the coast to county line largely bounded by SW $152^{\text {nd }}$ Street on the north and SW $272^{\text {nd }}$ Strcet on the south, though narrower near the coast.

Sub-Area 10: South Dade. Area 10 consists of all of Dade County south of $242^{\text {nd }}$ Street (east of U.S. 1) or south $272^{\text {nd }}$ Street (west of U.S. 1). It includes Homestead and Florida City.

Table $\wedge-1$. Distribution of Zip Codes for each Planning and Service Sub-area in MiamiDade County, FL

\begin{tabular}{|c|c|c|c|c|c|}
\hline \multicolumn{6}{|l|}{ SUB-AREA 1} \\
\hline 33132 & 33137 & 33139 & 33140 & 33141 & 33154 \\
\hline \multicolumn{6}{|l|}{ SUB-AREA 2} \\
\hline 33160 & 33161 & 33162 & 33168 & 33179 & 33180 \\
\hline \multicolumn{6}{|l|}{33181} \\
\hline \multicolumn{6}{|l|}{ SUB-AREA 3} \\
\hline 33054 & 33055 & 33056 & 33127 & 33136 & 33138 \\
\hline 33142 & 33147 & 33150 & 33167 & 33169 & \\
\hline \multicolumn{6}{|l|}{ SUB-AREA 4} \\
\hline 33010 & 33012 & 33013 & 33014 & 33015 & 33016 \\
\hline 33166 & 33172 & 33178 & 33182 & 33192 & \\
\hline \multicolumn{6}{|l|}{ SUB-AREA 5} \\
\hline 33144 & 33155 & 33165 & 33174 & 33175 & 33184 \\
\hline 33185 & 33194 & & & & \\
\hline \multicolumn{6}{|l|}{ SUB-AREA 6} \\
\hline 33122 & 33125 & 33126 & 33128 & 33129 & 33130 \\
\hline 33131 & 33133 & 33135 & 33145 & & \\
\hline \multicolumn{6}{|l|}{ SUB-AREA 7} \\
\hline 33143 & 33173 & 33176 & 33183 & 33186 & 33193 \\
\hline \multicolumn{6}{|l|}{33196} \\
\hline \multicolumn{6}{|l|}{ SUB-AREA 8} \\
\hline 33109 & 33134 & 33146 & 33149 & 33156 & 33158 \\
\hline \multicolumn{6}{|l|}{ SUB-AREA 9} \\
\hline 33031 & 33157 & 33170 & 33177 & 33187 & 33189 \\
\hline \multicolumn{6}{|l|}{33190} \\
\hline \multicolumn{6}{|l|}{ SUB-AREA 10} \\
\hline 33030 & 33032 & 33033 & 33034 & 33035 & 33039 \\
\hline
\end{tabular}


Figure A-1: Map of the Ten Planning and Service Sub-areas in Miami-Dade County

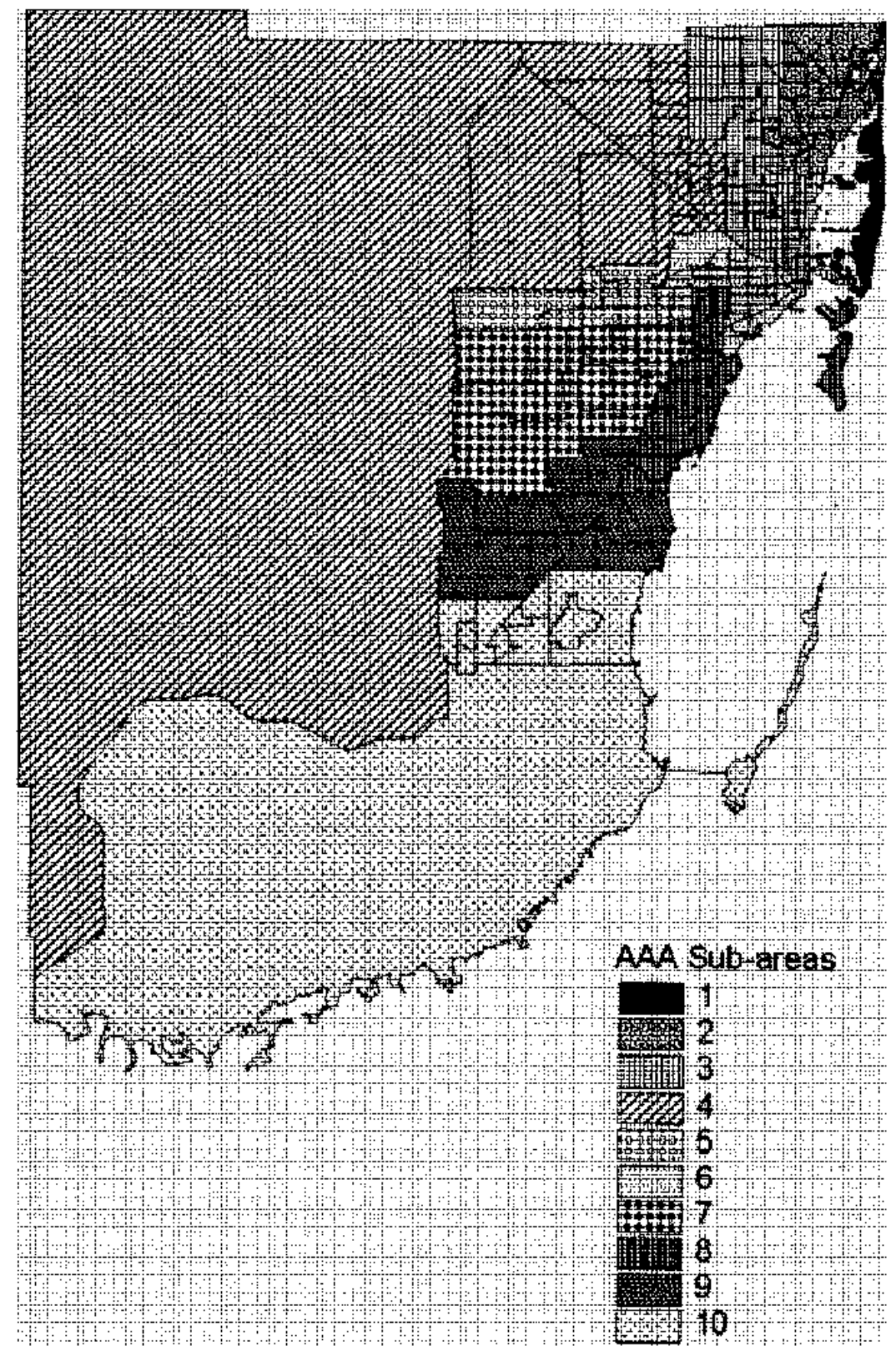




\section{APPENDIX B:}

DADE COUNTY NEEDS ASSESSMENT FOCUS GROUP INTERVIEW PROTOCOL 


\section{APPENDIX B: Dade County Needs Assessment Focus Group Interview Protocol}

1. What things about your neighborhood or community do you and others your age like best?

2. What things about living in Dade County do you and others your age like best?

Rationale for Q.1 and Q.2: After introduction of the purpose, these questions begin on a positive note and could reveal what things, including services seem to be working well.

3. What are the things about living in your neighborhood or community that most concern you and others your age?

PROBES - depending upon what was mentioned in above Q.1 and Q.2.

1. What are the major problems or difficulties that you see that make living in your neighborhood less pleasant or comfortable than it could be?

2. You mentioned FEAR OF CRIME as a concern for you or for others your age. To what extent is FEAR OF CRIME a problem for you or for others your age? How does it affect the way you lead your life?

3. You mentioned HEALTH CARE as a concern for you or for others your age, to what extent is HEALTH CARE a problem for you or for others your age? How does it affect the way you lead your life?

4. You mentioned MONEY as a concern for you or for others your age, to what extent MONEY a problem for you or for others your age? How does it affect the way you lead your life?

5. You mentioned that GETTING TO THE PL ACES ONE NEEDS TO GO TO as a concern for you or for others your age, what problems do you or others your age have in GETTING AROUND? To what extent is GETTING AROUND a problem for you or for others your age? How does it affect the way you lead your life?

6. Other older individuals mentioned that LONELINESS is a concern as one ages; what do you think causes this concern about loneliness in elders? What do you think can be done to alleviate or reduce loneliness as a concern for elders.

7. What do persons of your age in your community or neighborhood need help with in carrying out their daily activities?

PROBE: Activities such as shopping, cooking, cleaning, bathing, and so forth? 
RATIONALE: Type of service needed can be inferred from the difficulty

8. In general would you say that most elders needing assistance with these activities receive help from family members? For what types of activities do elders primarily rely on family members for assistance?

9. In what circumstances would elders turn to non-family members, such as friends or neighbors for assistance. How often do elders seek assistance from friends or neighbors?

10. In what circumstances would elders seck assistance from service agencies? How often do elders seek assistance from service agencies?

11. How many don't get all the help they really need in carrying out these daily activities?

12. What kind of additional help exactly do they need?

13. How would you describe the persons who typically do not receive enough help? PROBE: Are they older? Very disabled? Without family? Live alone? What else?

14 . What exactly don't they get the help they need?

15. Do you know of any sources of help for those needs in your neighborhood or community?

16. Do you know of any sources of this kind of help in Dade County?

17. Do you (or other older people you know) provide such help to others?

18. If yes, What problems do those you/they help have? 


\section{APPENDIX C:}

DADE COUNTY NEEDS ASSESSMENT TELEPHONE SURVEY INSTRUMENT 


\section{START TIME}

\section{1. (RECORD RESPONDENT'S SEX)}

male

female

2. First l'd [1ke to ask you about the way you usually get to the places you need to go to. Do you usually drive youraelf, fide with comeone else, use publit bransportation, or use some othet way to get around?

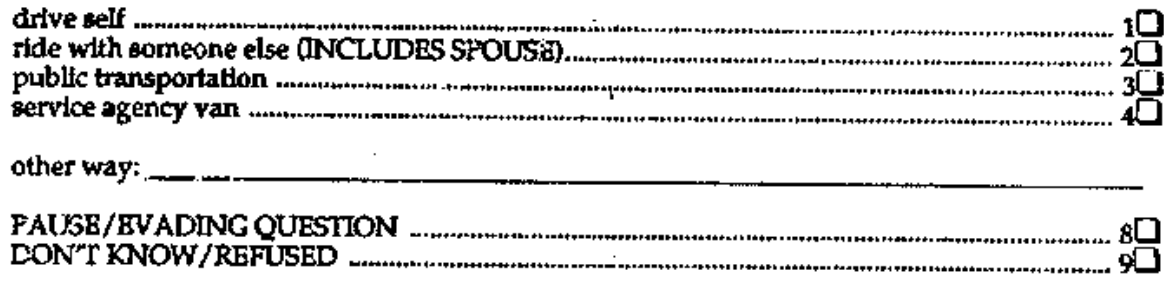

3. Have you ever heard of the Special Transportation Service or STS, a service offered for disabled or elderly incitviduals?

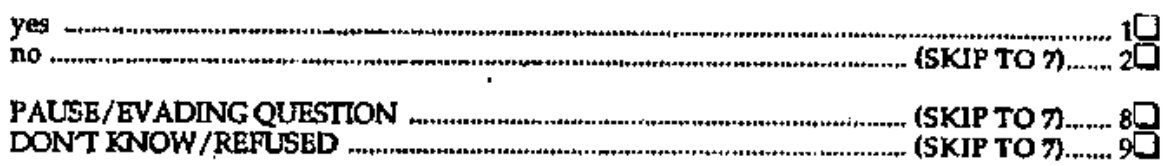

4. Have you ever used the Special Transportation Service?

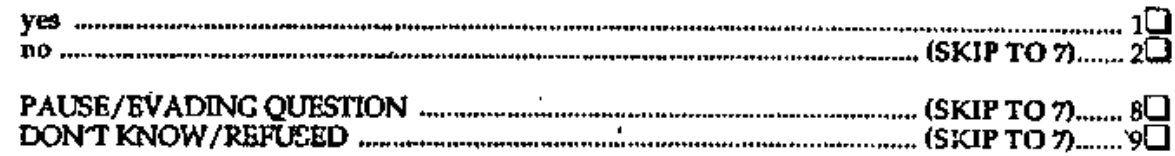

5. In general, would you say you have been satisfied or dissatisfied with this service?

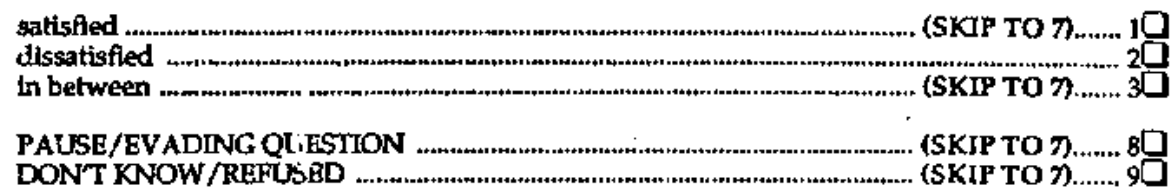

6. Why are you dissatisfied?

always late/not dependioble ......................................................................................................... $1 \square$

other:

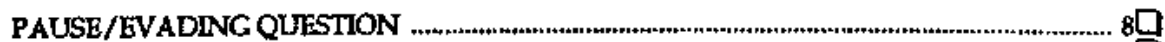

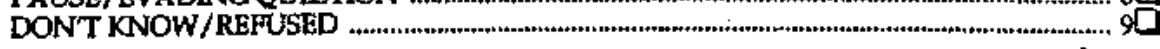

7. Now I'd like to ask ame questions about your health. Compared to others your age, how would you rate your overall health - would you say it is excelleat, good, fair, or poor?

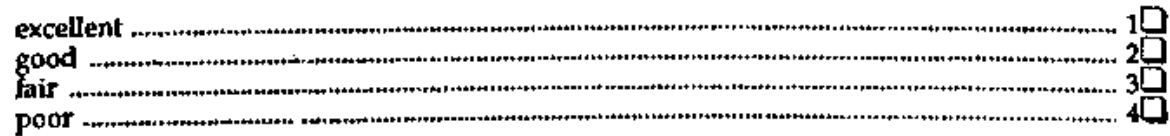

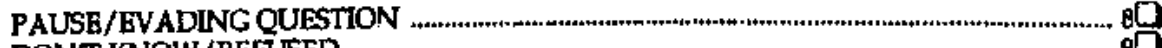

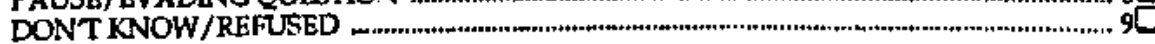


6. Where do you usually go for health care - to a private physician, an HMO, an energency room, a elinic, or do you go to someplace eloe?

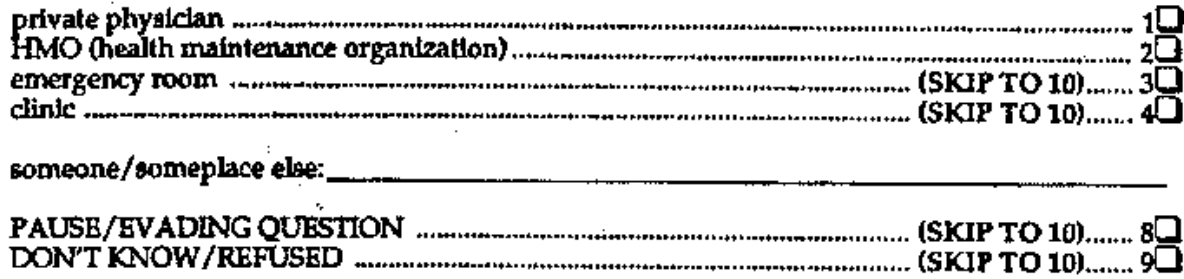

9. Is there a particular doctor that you uswally go to that you consider to be your personal or famlly doctor?

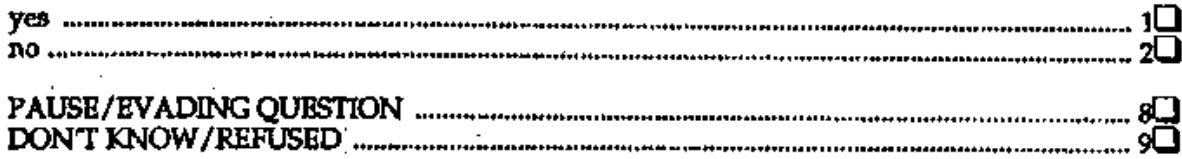

10. Have you had a complete physical examination in the last 12 months?

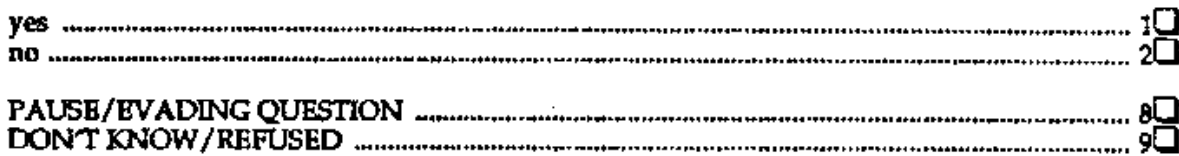

11. Other than a complete physical examination, have you had any hesith screening in the past year? By health screening I mean things like blood presaure or cholesterol tests, eye exams for glaucoma, etc?

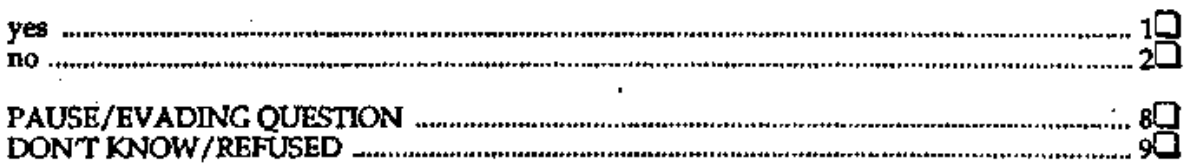

12. Have you been adrilted to the hospltal, when you alayed at least one night, at any time in the last 12 monthis?
yets
no
(SKIP TO 14)....... 20
PAUSE/EVADING QUESTION
(SKIP TO 14)...... 8
DON'T KNOW/REFUSED
(SKIP TO 14) of

13. Other than the time you were in the hospital, how many times have you seen a doctor during the past 12 months - would you say one or two times, three to five times, more than five times, or not at all?
1 or 2 times
(SKIP TO 15)...... 1
3 to $5 \mathrm{times}$
(SKTP TO 15)....... 2 [
more than 5 times
(SKIP TO 15)........ 3
not at all
(SKIP TO 15)....... 4
PAUSE/EVADING OUESTION
(SKIP TO 15)
DONT KNOW/REFUSED
(SKIP TO 15)..... 90

14. How many times have you seen a doctor during the past 12 months - would you say one of two times, three to five times, more than five times, or not at all?

1 or 2 times

1[

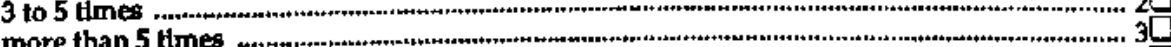

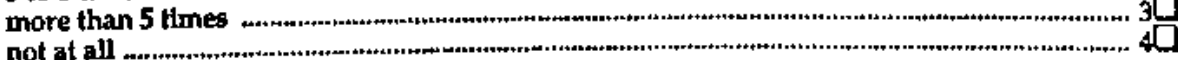

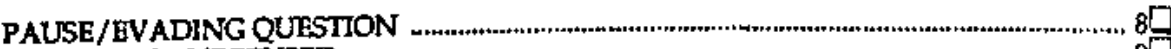

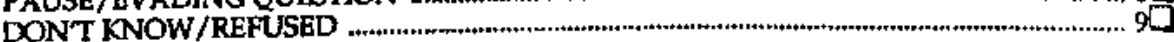


15. During the last year, was there ever o tome when you needed to see a doctor but were unable to see one?

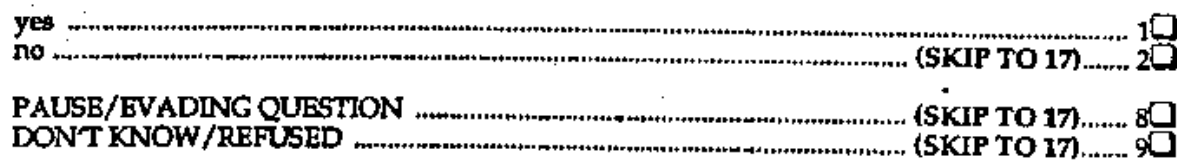

16. Why were you unable to see a doctor?

(CHECK ALI. THAT ARE MENTIONED)

couldn't get an appointment

too sick to go

didn't have the money

didn't have transportation

language problem

didn't have any insurance

didn't have enough insurance

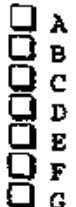

other:

PAUSE/BVADING QURSTION

DONT KNOW/REFUSED

17. Now I need to ask you some questions about the mexls that you eat. How many meals do you . usually eat every day - one, two, three, or more than three?

One .
tho
more than three . .
PAUSE/EVADNG QUESTION
DONT KNOW/REFUSED

18. In general, do you feel that you get enough to eat?

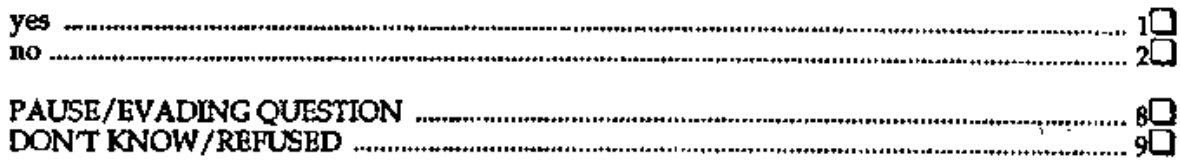

19. How often do you eat your meals alone - would you say some of the time, mast of the timk, all of the tint, or do you never eat your meals alone?

some of the time
most of the the
all of the time
neVer
PAUSE/EVADING QUESTION
DONT KNOW/REFUSED

20. Do you have any difficulty chewing meat?

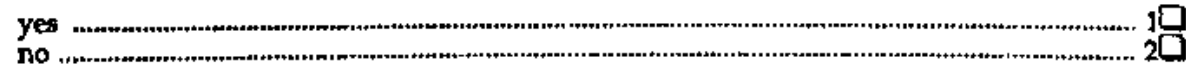

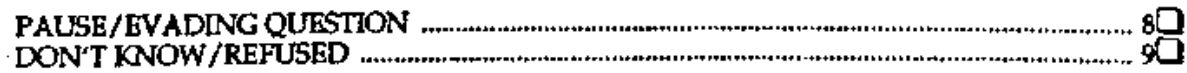

21. Do you have any medical condition that has caused you to make changes in the kind or the amount of food that you eat?

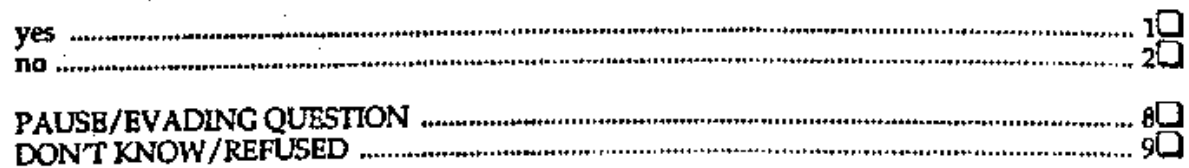


22. Have you had a aignificant weight change - either a gain or a lose - In the last eix monthe?

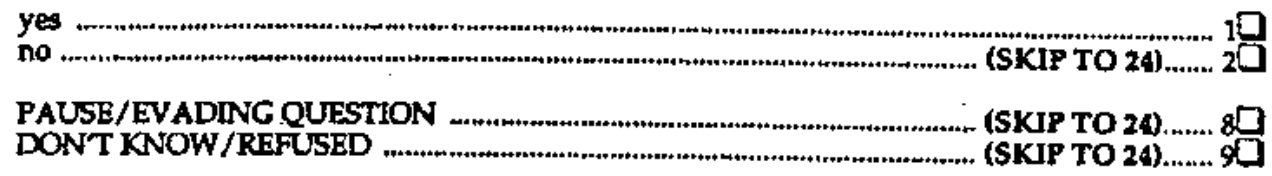

23. Was that weight change the result of a planned diet or program, or was it unplanned?

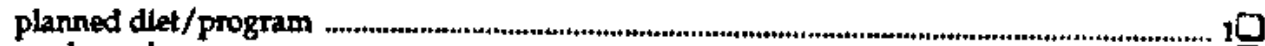

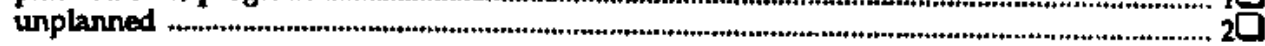

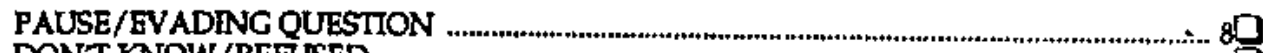

DONT KNOW/REFUSED

24. Are you presently recelving food atampa?

Ye6

PAUSE/EVADING QUESTION

DONT KNOW/REFUSED

25. I'm going to mention come supportive devices. Pleuse tell me whether you are using any of these devices. Do you use ... (READ ITEMS BELOW)

\begin{tabular}{|c|c|c|c|c|}
\hline & yes & no & PAU/EV & DKNA \\
\hline $\begin{array}{l}\text { eyeglasses or contacts } \\
\text { hearing aid } \\
\text { denture plates } \\
\text { leg brace or artificial limb } \\
\text { cane or walker } \\
\text { wheelchair }\end{array}$ & $\begin{array}{l}10 \\
10 \\
10 \\
10 \\
10\end{array}$ & $\begin{array}{l}20 \\
20 \\
20 \\
20 \\
20 \\
20\end{array}$ & $\begin{array}{l}80 \\
80 \\
80 \\
80 \\
80 \\
80\end{array}$ & 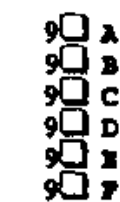 \\
\hline
\end{tabular}

26. Do you meed any supportive devices or spedal equipgent that you don't have?

(IF XES ASK $\rightarrow$ What do you need? DO NOT READ LIST - SHECK ALL MENTIONED)

eyeglagses or contacts

hearing ald

denture plates

leg brace or artificlal imb

cane or walker

wheelchatr

곡

B

C

D

other

no

(SKIP TO 28).

PAUSE/EVADINC QUESTION

(SKJP TO 28)...... 8 DONT KNOW/REFUSED

27. Why have you been unable to get this/these device(s)?

\section{(CHECK ALL THAT ARE MENTIONED)}

cost

don't know where to get them

곡

no way to get to the supplier

G

haven't gotten around to it.

$\square c$

other:

PAUSE/EVADING QUESTION

DONT KNOW/REFUSED 
28. If you were to become sick, but were able to atsy at home, is there anyone who would be able to take care of you during your illneas?

yes $\rightarrow$ ASK: Who would that person be? (WRTTE ALL, THAT ARE MENTIONED)

IF MORE THAN ONE PERSON IS NAMED ASK $\rightarrow$ Which of these people would be most responstble for taking care of you?

Most responsibl ? person:

no (SKIP TO 30) $\ldots . . . . .2 \square$

PAUSE/EVADING QURSTION (SKIP TO 30)....... 8 DONT KNOW/REFUSED (SKIP TO 30)........ 埙

29. IF "SPOUSE" IS ANSWER FOR QUESTION 28 ASK $\rightarrow$ Does you kusband/wife live in your house with you? IF ANSWER IS "NO" CONTINUE WTTH...

OTHERWISE ASK $\rightarrow$ How lung would it normally take __ (caregiver from 28)___ to get to your house from where he/she lives? Would It take 10 minutes ar less, 10 to 30 minutes, 30 gilnutes to one hour, more than one hour but lase than one day, or more than one day?

10 minutes or leas

10 to 30 minutes

$1 \mathrm{a}$

30 minutes to one hour

more than one hour bu! less than one day

more than one day

LIVES IN HOUSEHOLD

PAUSE/EVADING QUESTION

DON'T KNOW/REFUSED

30. When was the last time you went to the dentiot - was it within the last year, more than ore but less than three years ago, or mase than 3 jears ago?

within the last year

one to three years 10

more than 3 years ago 3

PAUSE/EVADING QUESTION

DONT KNOW/REFUSED

31. Have you had any serlous denbl probleme in the last year?

yes<smiles>[C]1[CH]C=C1</smiles>

no

PAUSE/EVADNGG QUESTION DON'T KNOW/REFUSED

32 Now I'm going to mention several kinds of medical coverage or insurance. Please tell me whether you have them. First, do you have Medicare?

yes $\rightarrow$ ASK: Are you covered for hospitalization only or hospitalization and doctor bills?

hospitalization only (part $A$ ).

hospltalization and doctor bills (parts $A$ and $B$ )

no 
33. Do you have ... (READ LIST BELOW - CHECK BOX ONLY IF THEY HAVE THE COVERACE)

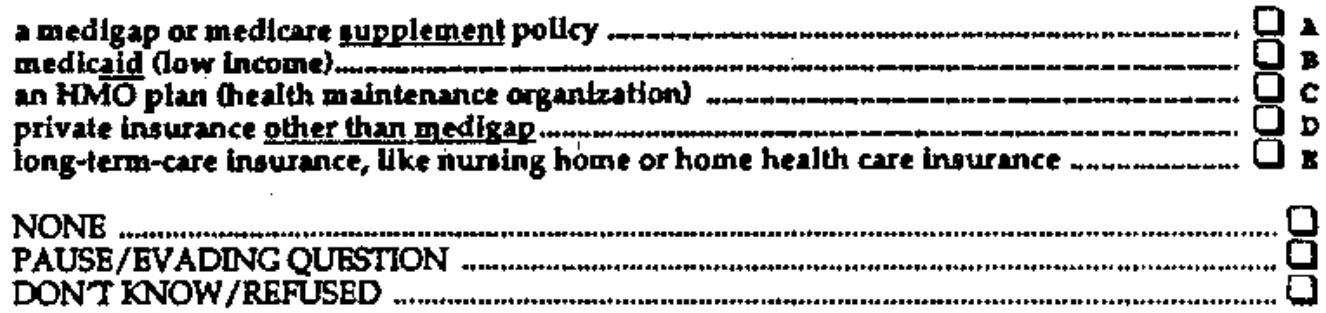

34. Now I need to ask you a few queatlons sbout your houting. First, do you live in a ningle-family houre, a townhouse, a duplex, an apartonent, a condominium, a mobile home, do you rent a room in someone tlas's home, or do you live ameplace elae?

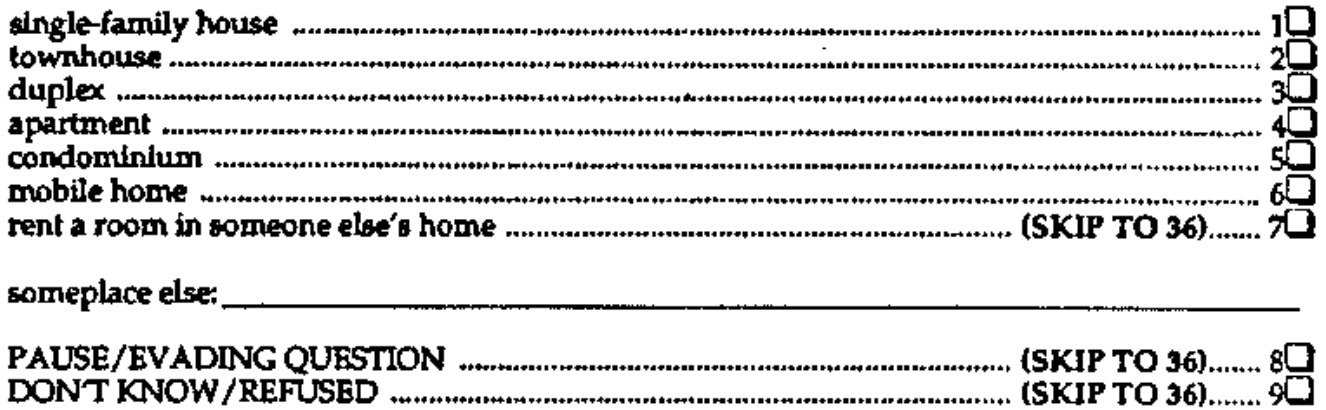

35. Do you own or rent your housing?

own (SKIP TO 37) ....... 1무

rent (SKIP TO 38)

other. (SKIP TO 377...... 8 8

PAUSE/EVADING QUESTTON (SKIP TO 37)....... 9

36. Since Hurricane Andrew, has your rent increased greatly, moderately, or not at all?

grently

moderately

not at all

PAUSE/EVADING QUESTION

DONT KNOW/REFUSED

37. Do you have home oumer's/renter's insurance?

yes 10 no 
38. Including yourself, how many people live in your househoid?

ntumber of people: (IF ONE, SXIP TO (0)

PAUSE/ FVADING QUESTION

39. Who elae liren in your hane with you?

(CHECK ALL THAT ARE MENTIONED)

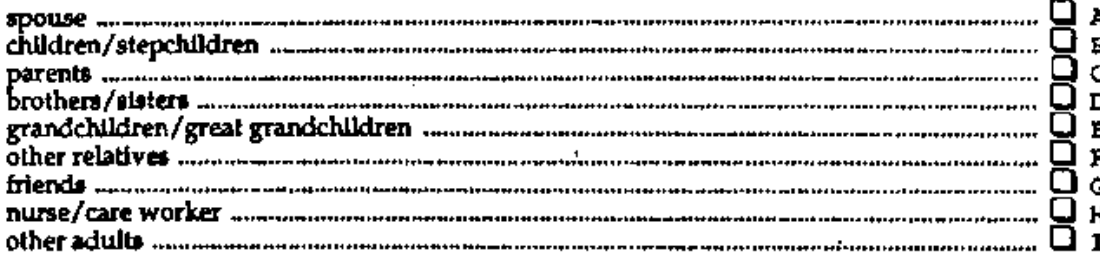

PAUSE/EVADING QUUESTION

DONT KNOW/REFUุSED

40. How satiafled are you whth your housing and living arrangements - would you say you are very satisfied, mostly astisfied, mostly dinatiffed, ar very distatisfied with your housing and living arrangementa?

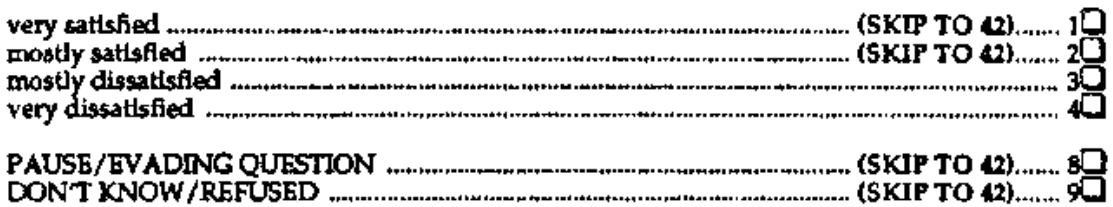

41. In what wayo are you disantiafied?

PAUSE/EVADING QUESTION

42 If you had to move from where you are liven now, how difficult do you think it would be to find housing of the arme quallty and cost as what you have now? Would you say It would be very difficult, moderately difficult, or not very difficult?

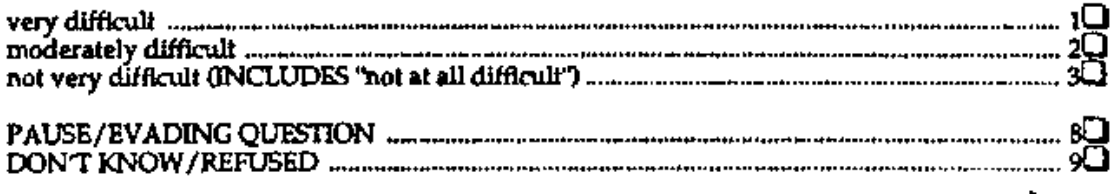

4. How difficult is it for you to poy the contu of your housing, thinge like your enortgage or rent payment and the coats of malnkining your hogne? Would you wy that paytng these costs fir very difficult, moderately difficult, or not very difficult for you?

very diffirult

moderately difficult

not very difficult QNCLUDES "not at all diffictlt") ..................................................................... 3

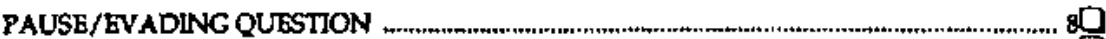

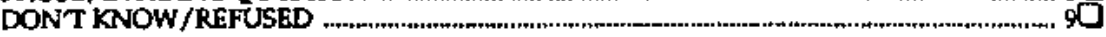

44. Are you recelving any government essislance with your housing of utility costa?

yes $\rightarrow$ ASK: What type of andistance are you recelving?

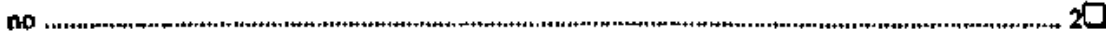

PAUSE/EVADING QUESTION 
45. Do you receive any andance with your housing coats from friends or relatives?

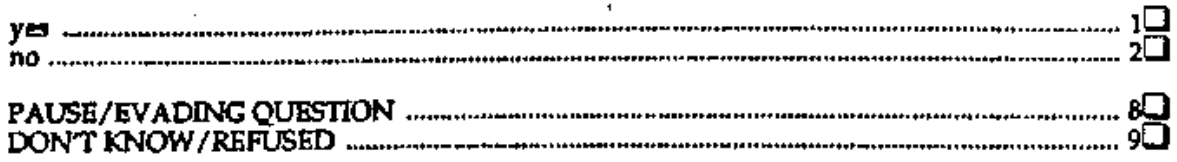

46. Naw I'd llke to ak you come question about crime. Pirat, in the neighborhood where you Itve would you eay critone is a very cerlout problem, \& moderately serious problem, or not a verious problem?

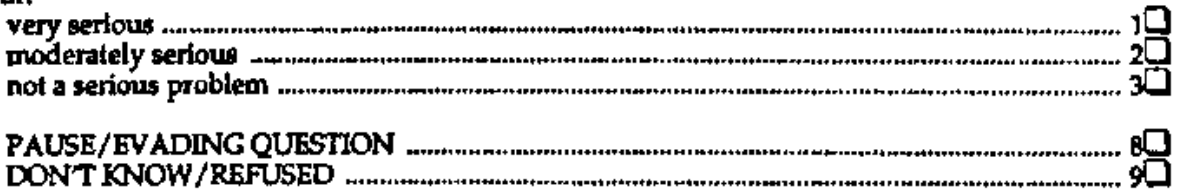

67. How concemed are you that you ar someone living in your household will be a pictim of crime - would you way you are very concerned, moderately concerned, or not very concented?

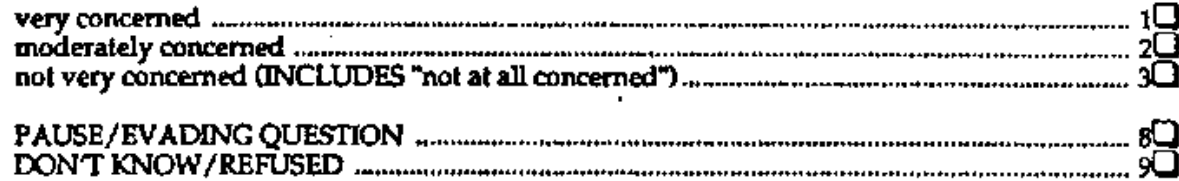

48. Have you or anyone in your household been the viction of a cime in the last year?

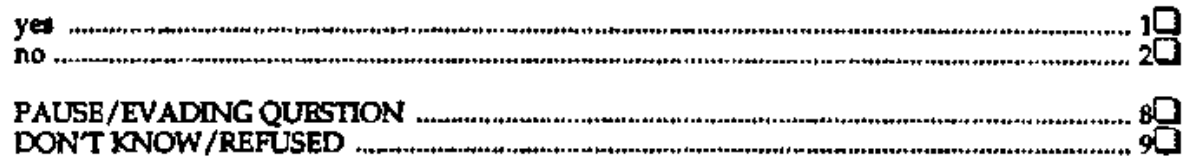

49. Now 1 need to wh you about your sctivitiea. Finc, are you retired, employed full-time, employed part-time, temporarily out of work, of comething elice?

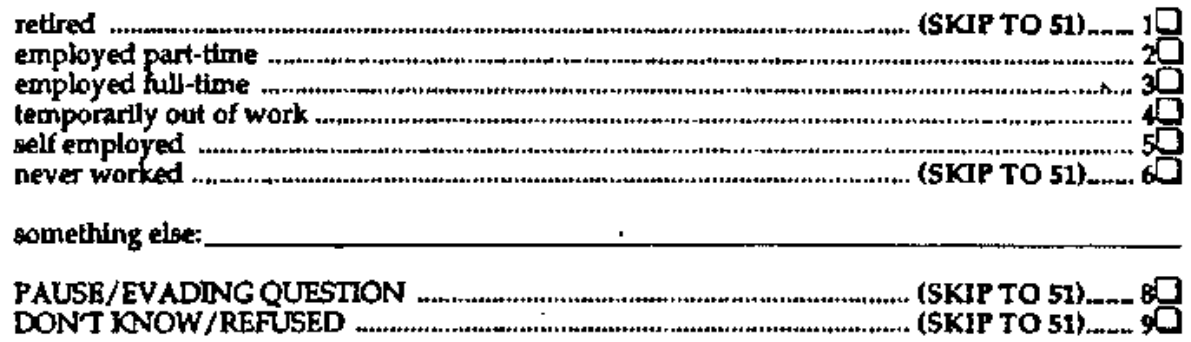

50. Do you work primarity for the money, or do you work for none other reason?

money
other reason:
DOES NOT WORK
BOTH
PAUSE/EVADING QUESTION

51. If the opportunity were avallable, would you be interested in leaning a new akjli, trade, or bustnesu?

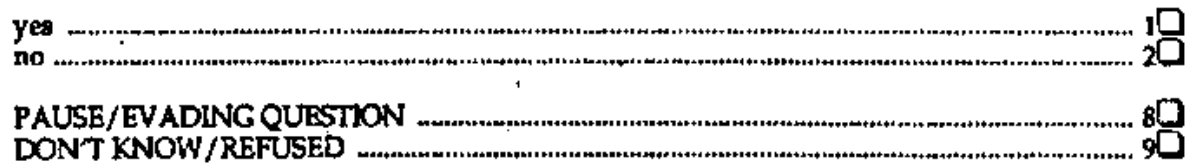


52. If the opportunity were avallable, would you be interested in taking classea to further your interests ar personal growth?

yes $\rightarrow$ ASK. What clasees would you be intereated in taking?

PAUSE/EVADING QUESTION

53. How important is it to you to have the oppostunity lo do some kind of rolunteer work - would yous say it is important or not very important?

important

not very important

PAUSE/EVADING QUESTION

DONT KNOW/REFUSED

54. Are you actlve In eny arganisations or group social activitiea?

yes $\rightarrow$ ASK. In what groups or activitien are you active?

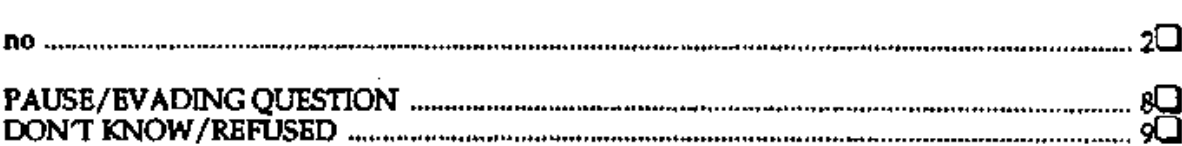

55. IF "NO" FOR QUESTION 54 ASK $\rightarrow$ Would you like to be doing more social activities or do you feel you are doing the right amount for you?

OTHERWISE ASK $\rightarrow$ Regarding your social activitien, do you feel you are involved in too many social actuvities, that you are doing about the right amount, or would you like to be doing more?
too many activities
(SKIP TO 57)
right amount
(SKIP TO 57)
would like to do more
(SKIP TO 57)...... 85
PAUSE/EVADING, QUESTION
(SKIP TO 57)..... on
DONT KNOW/REF

\section{(n)}

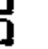
. 
58. How often do you risit with relatives, either in your home or theirs - at least once a week, at least once a month, leas than once a month, or never?

At least once w week
at least once a month
less than once a month
never.
PAUSE/EVADTNG QUESTION
DONT KNOW/REFUSED

59. How often do you attend rellglou scrvices or other religlous group activitien - at ieast once a week, at least once a month, for religious holldayl only, ot never?

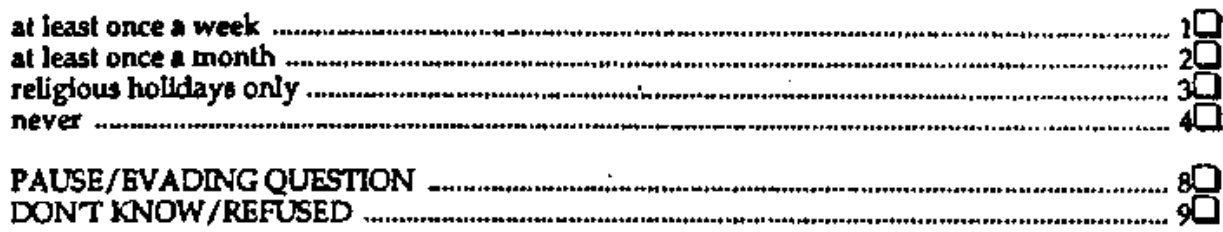

60. How often do you talk on the telephone with family members - would you say almost every day, a few times a week, about once a week, or lecs than ance a week?

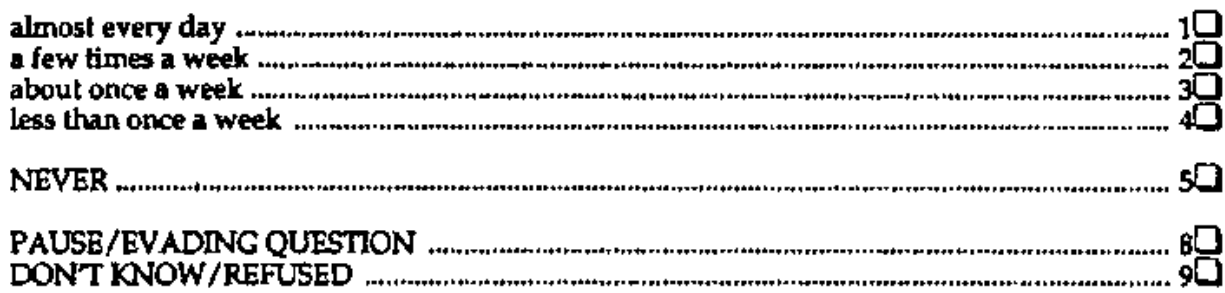

61. How often do you talk on the telephone with filends - almost every day, few tomet a week, about once week, or less than once 1 week?

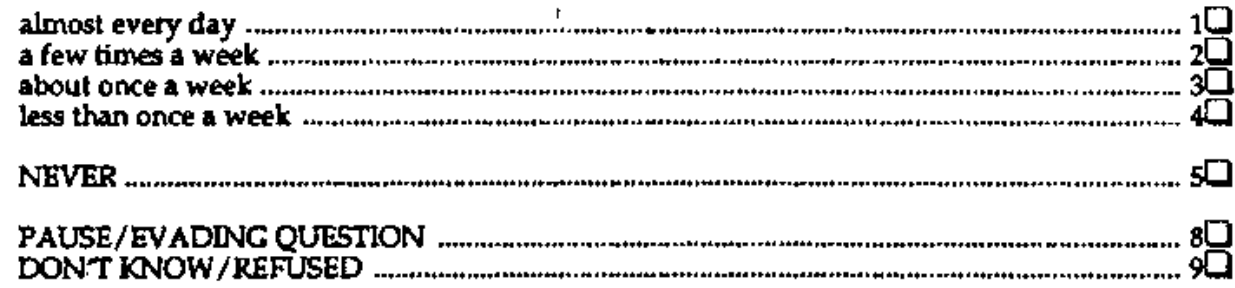

62. How often do you get out of your bouse - would you way every day, a few times a week, about once a week, or lesa than once a week?

every day ....
a few times a week
about once a week
less than once a week
NEVER
PAUSE/EVADRG QUESTION
DONT KNOW/REFUSED

63. The next questions are about how wetl you are able to do certain activlties of daily living. First because of a health or physical problem do you have any difficulty bathing or showering?

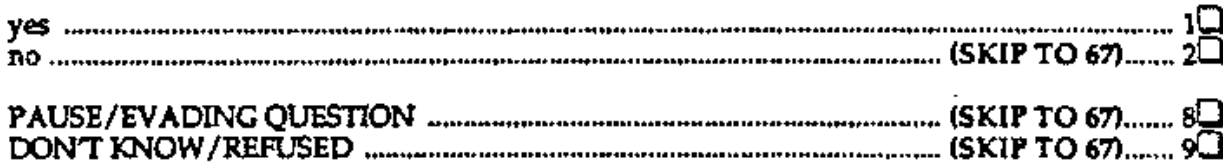


64. By yourself and without using any spedal equipment, how much difficulty do you have bathing of ahowertng - would you ay you have come diffeutty, - lot of difficulty, ar are you completely unable to do thil whout mistance from people or speclal equipment?

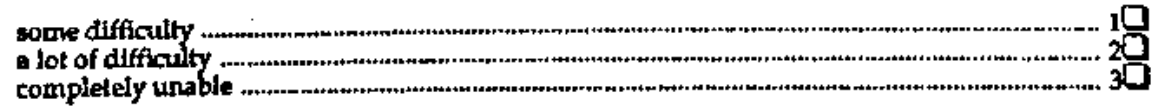

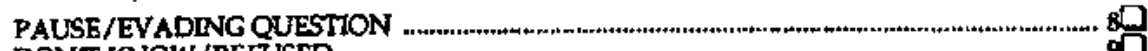
DONT KNOW/REFUSED ................................................................................................. 9

65. Do you get any help from another peraon in bathing and showering?

yes $\rightarrow$ ASK: Who helps yout

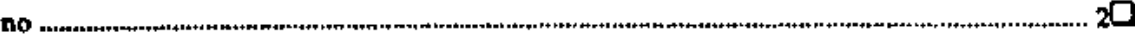

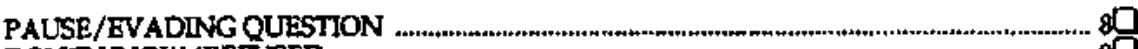

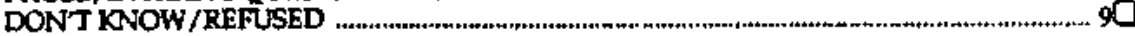

66. Do you use any apectal equipment to asiat you in bathing and thowering?

yes $\rightarrow$ ASK What equipment do you use?

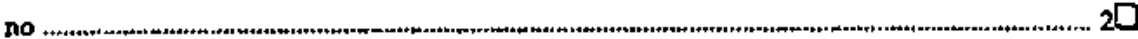

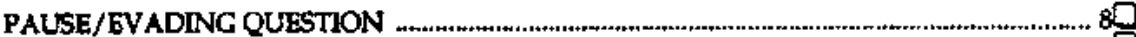

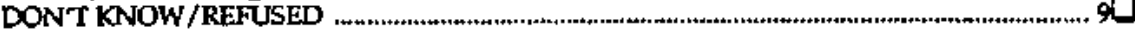

67. Becuuse of a health or phyic: problen, do you have any difflculty getting dressed?

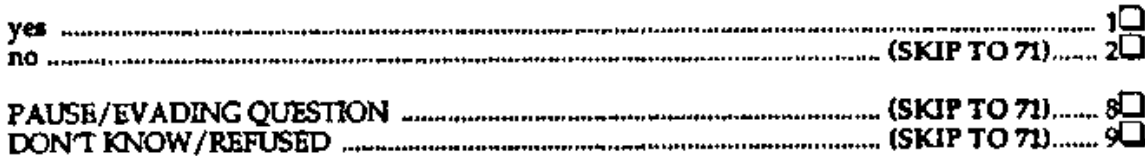

68. How much difficulby do you have getting dressed - would you any you hape some dtfficulty, a lot of difficulty, or are you completely unab!e to do this without astistance from people or apeclal equlpment?

some difficulty $1 \square$

a lot of difficulty

completely unable

PAUSE/EVADING QUESTION 9 DONT KNOW/REFUSED

69. Do you get any help from another person in getting dressed?

yes $\rightarrow$ ASK: Who helpo you?

no

PAUSE/EVADINC QUESIION 8

DONT KNOW/REFUSED

70. Do you use any speclal equipment to asoist you in getting dremed?

yes $\rightarrow$ ASK: What equipment do your use?

no

PAUSE/EVADING QUESTION

7. Because of a health or physical problem, do you have any difficulty eating?

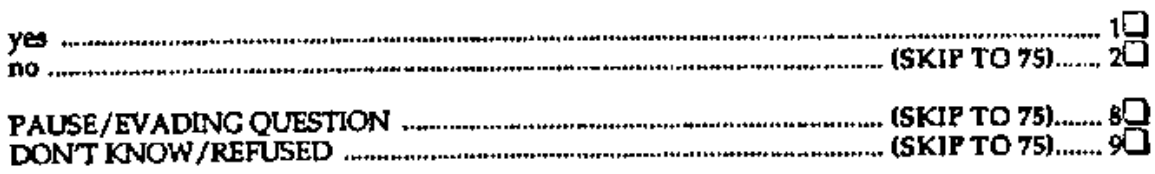


72. How much difficully do you have eating - would you suy you have come difficulty, at lot of difflculty, or are you completely unable to do this whout aseistance from people or opecial equipment?

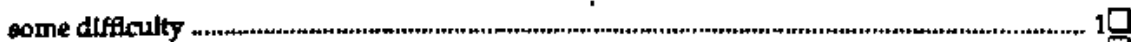

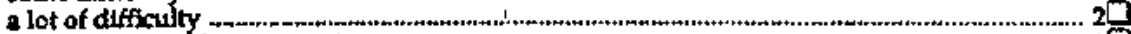

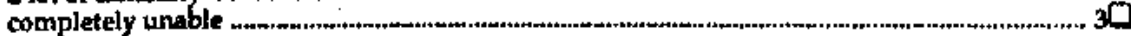

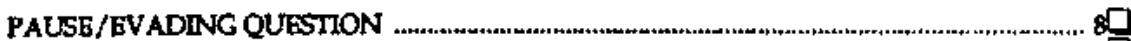

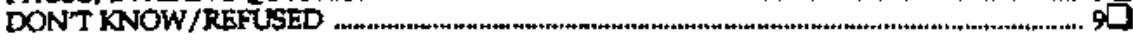

7J. Do you get any help from another perion in eating?

yes $\rightarrow$ ASK: Who helps you?

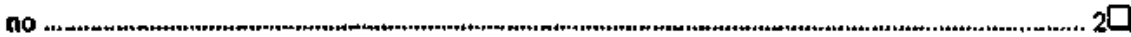

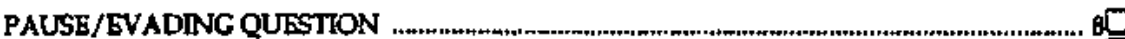

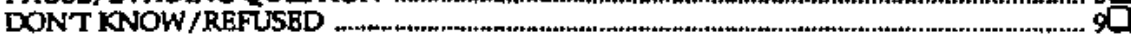

74. Do you use any apedal equiptontent to asolst you in eating?

yes $\rightarrow$ ASK. What equjpment do you tire?

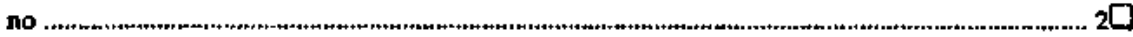

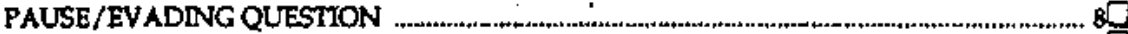

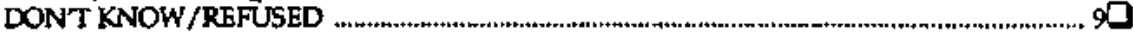

75. Becouse of a health or physical problem do you have any difficalty uking medicine?

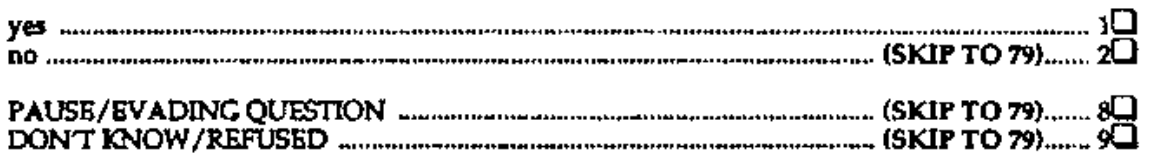

76. How much difficulty do you have taking medicine - would you any you have come difficulty, lot of difficulty, or are you completely unable to do this without assistance from people or spedal equipment?

some difficulty

lot of diffloulty

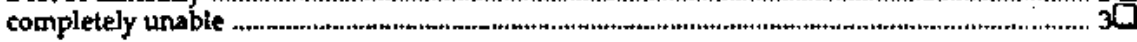

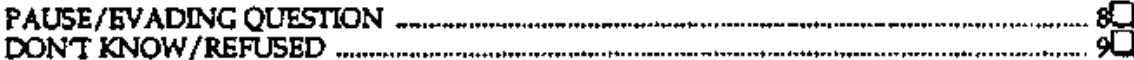

77. Do you get ery help trom another perton in taking medicine?

yes $\rightarrow$ ASK. Who helps you?

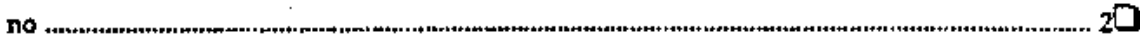

PAUSE/EVADING QUESTION ............................................................................

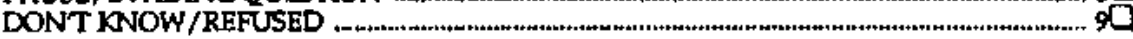

78. Do you use any opedal equipment to asial you in taking medicine?

yes $\rightarrow$ ASK: What equipment do you wee?

no ...................................................,

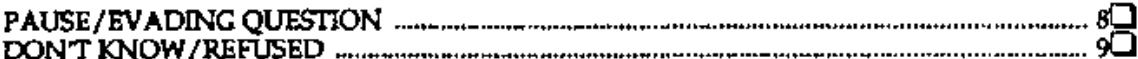

79. Because of health or phyeical problen do you have any difficulty taking care of your appearance (combing your hair, etc.)?
yes
(SKIP TO 83)
10
PAUSE/EVADNAGQUESTION
(SKIP TO 83)...... 80
DONT KNOW/REFUSED
(SKIP TO 83),..... on 
80. How much difftculty do you hove taklng care of your appearance - would you wy you have come difficulty, a lot of difficulty, or are you completely unable to do this without assistance from people or apecial equipment?

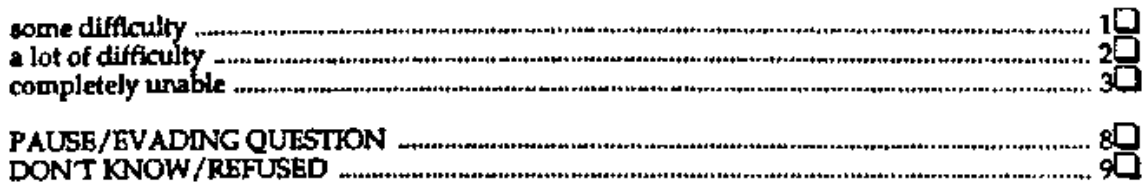

81. Do you get any help from another person in taking care of your appenance?

yes $\rightarrow$ ASK: Who helps you?

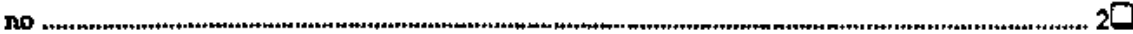

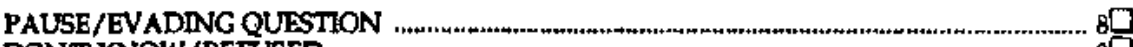

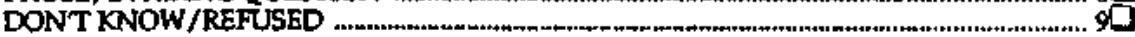

82. Do you use any special equipment to assiat you ta taking care of your appearance?

yes $\rightarrow \rightarrow$ ASK: What equipment do you use?

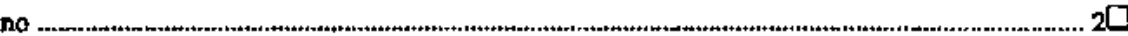

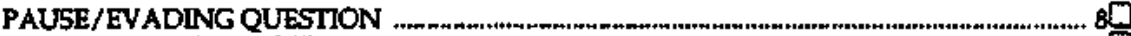

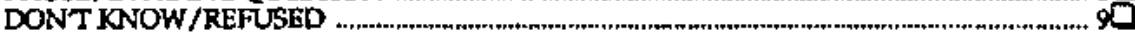

23. Becaure of a health or physleal probleon, do you have any difflculty using the tollet?

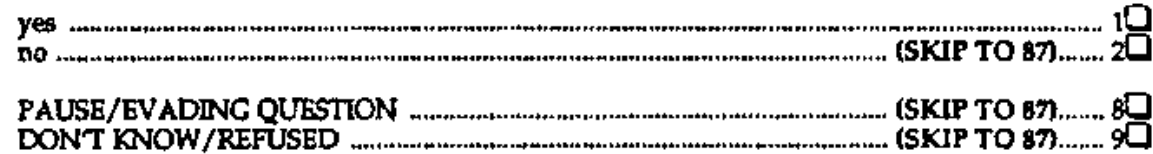

84. How much difficulty do you have uing the tollet - would you any you hare some difficulty, a lot of difficulty, or are you completely unable to do this withoul assistance from people or special equipment?

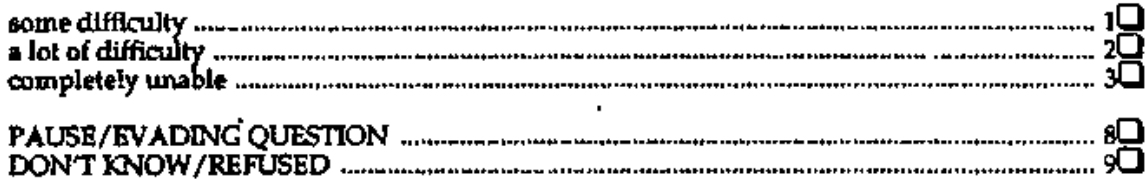

05. Do you get any help from another persan in whing the tollet?

yes $\rightarrow$ ASK: Who helpu you?

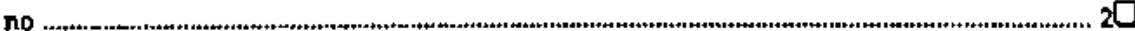

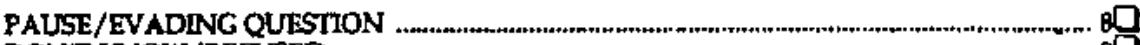

DONT KNOW/REFUSED ..............................................................................................

86. Do you use any apecial equipment to assist you in using the boliet?

yes $\rightarrow$ ASK: What equipment do you uat?

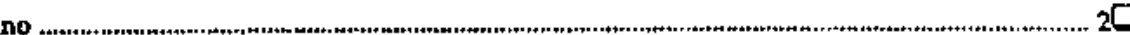

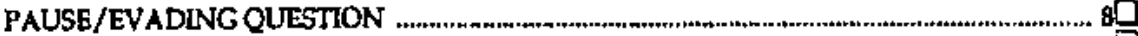

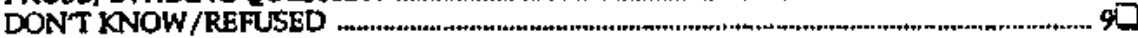

87. Because of a health or physical problen, do you have any difflculty getting in and out of bed ar a chair?

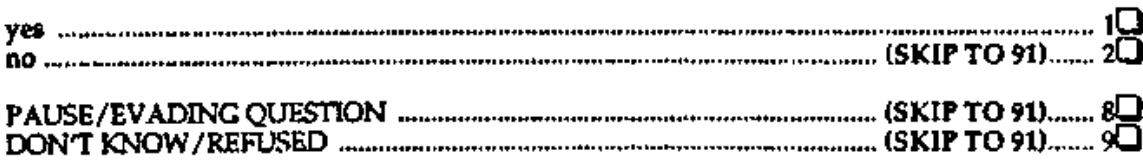


88. How much difficulty do you have getting in and out of bed or a hair - would you say you have some difficulty, lot of difficulty, or are you completely unable to do this without assistance from people or special equipment?
some difflculty
a lot of difficulty
completely umable
$1 \square$
PAUSE/EVADTNG QLESTION 3
DONT KNOW/REFUSED

49. Do you get any help from another person in getting in and out of bed or a chain?

yes $\rightarrow$ ASK who helps you?

no

PAUSE/EVADING QUESTION 8[ DONT KNOW/REFUSED 9

90. Do you use any opecial equipment to assist you in getting in and out of bed or a chair?
yes $\rightarrow$ ASK: What equipment do you ure?
no
2
PAUSE/EVADING QUESTION
DONT KNOW/REFUSED

91. Because of health or phystcy problem, do you have any difficulty getting up and down stairs?
yes (SKIP TO 95)
PAUSE/EVADING QUESTION (SKIP TO 95)....... \& DONT KNOW/REFUSED (SKIP TO 95)...... 9

92. How much diffleulty do you have getting up and down otoiru - would you tay you have come difficulty, a lot of difficulty, or are you completely unable to do thls without assistance from people or special equipment?

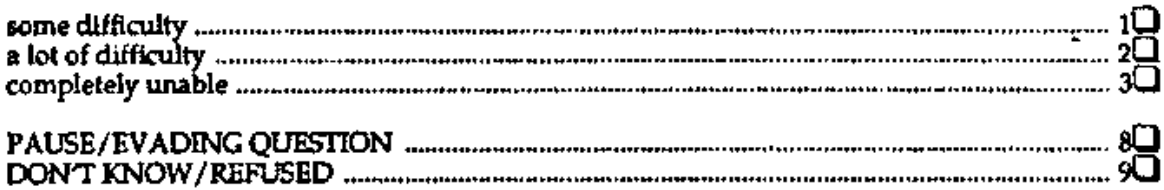

93. Do you get any help trom another person in getting up and down staits?

yes $\rightarrow$ A.SK: Who helps you?

no

PAUSE/EVADING QUESTION

DONT KNOW/REFUSED

94. Do you use any special equipenent to asiat you in getting up and down stolrs?

yes $\rightarrow$ ASK: What equipanent do you use?

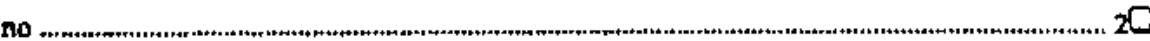

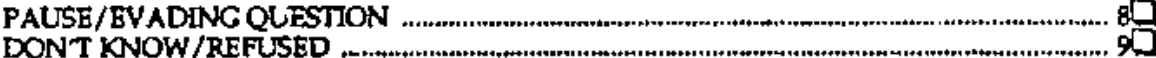

95. Becauge of genth or phyoical problem, do you have any difficulty walking?
yes (SKIP TO 99)...... 2]
PAUSE/DVADNG QUESTHON (SKIP TO 99)....... 8D DONT KNOW/REFUSED (SKIP TO 99)....... 9 
96. How much difficulty do you have walking - would you say you have wome difficulty, a lot of difficulty, or are you completely nnable to do thic without astistance from people or opecial equipment?

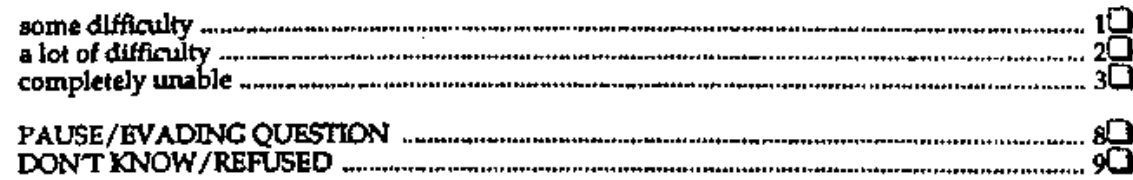

57. Do you get any help from inother peraon in walling?

yes $\rightarrow$ ASK who helpi you?

nO $\ldots \ldots+\ldots$

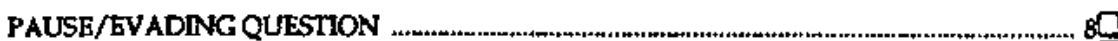

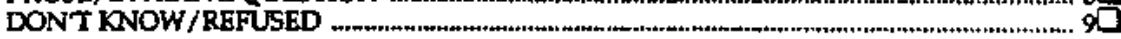

92. Do you use any apecial equipment to assits you th walking?

yes $\rightarrow$ ASK: What equipmenl do you une?

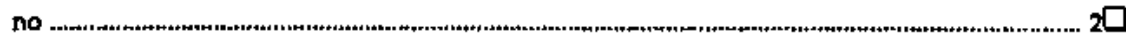

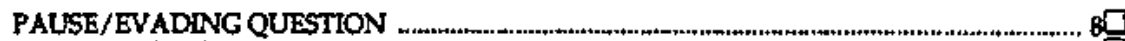

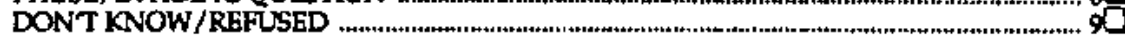

99. Now I'd like to alk you about some other ictivities. Because of a hel th or physfeal problem, do you have any difficulty preparing meat??

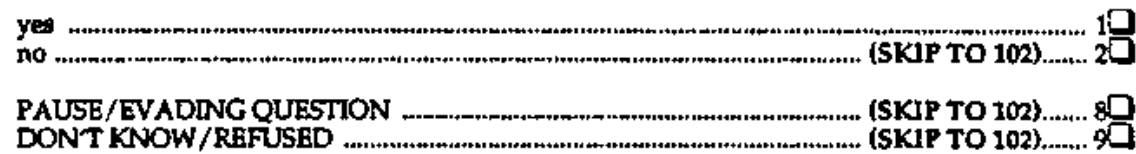

100. How much difficusty do you have preparing meale - would you way you have come difficulty, a lot of difflculty, or are you completely unable to do this without asalaturce?

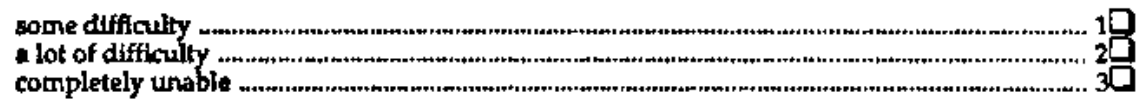

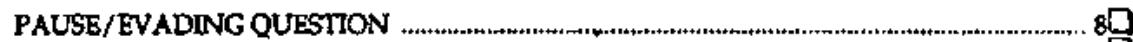

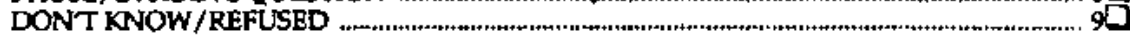

101. Do you get any help from enother perwon in preparing meals?

yes $\rightarrow$ ASK Who help' you?

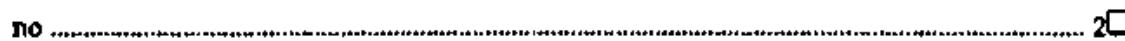

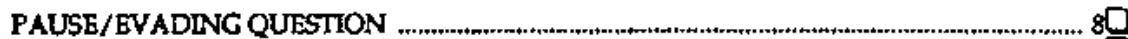

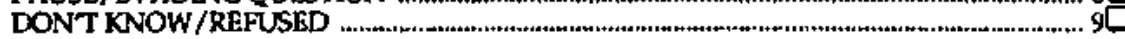

102. Because of a health or physical problem, do you have any difficulty shopping for groceries ar slollhes?

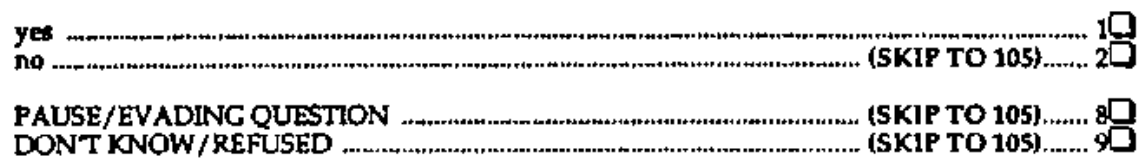

109. How much difficulty do you have whopplng for groceries or clothes - would you wy you have some difficulty, sot of difficulty, or are you completely unable to do this without assistance?

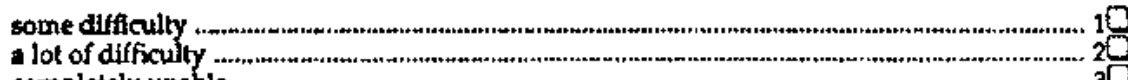

completely unable ........................................................................................................................

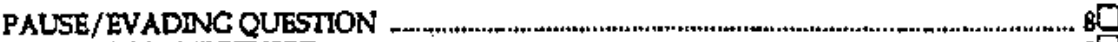

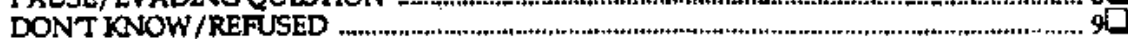


104. Do you get any help from another person in shopping for groceries or clothes?

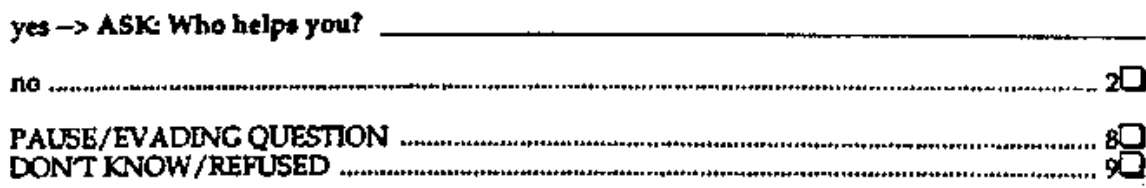

105. Becauge of a helth of physlcal problem, do you have iny difficulty managing your money and paying your bilis?

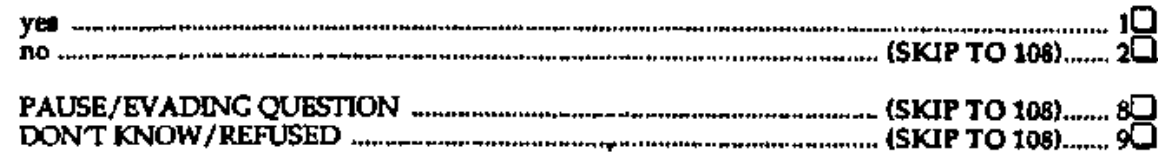

106. How much difficulty do you have managing your money and paying your bills - would you say you huve come difficulty, lot of difflculty, or are you completely unable to do thia without essistance?
some difficulty
a lot of difficulty
completely unable
. 10
PAUSE/EVADING QUESTION
DONT KNOW/REFUSED

16r. Do you get any help fom wnother person in manxging your money and paying your bills?

yes $\rightarrow$ ASK: Who helpa you?
no
PAUSE/EVADING QUESTION
DONT KNOW / REFUSED

10B. Because of a health or physical problem, da you have any difflculty dialing and uning the lelephone?
yes (SKI.......................... 19
PAUSE/BVADING OUESTION (SKIF TO 111) 1 DONT KNOW/REFUSED (SKIP TO 111)...... (SKJP TO 111)....... 90

109. How much diffeulty do you have dialing and using the telephone - would you cay you have sone difficulty, a jot of difficulty, or are you completely unable to do this without asgietance?

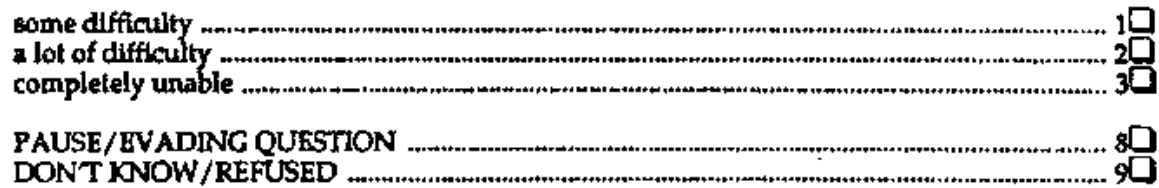

110. Do you get any help from another person in dialing and using the telephone?

yes $\rightarrow$ ASK: Who helps you?

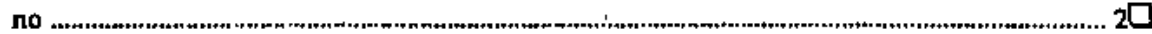

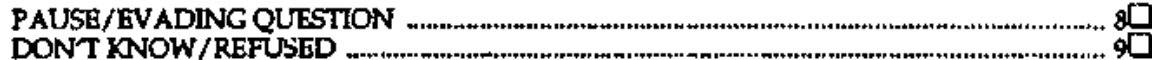

111. Because of a health of phydical problem, do you have any difficulty doing taundry?
yes (SKIP TO 110......... 20
PAUSE/EVADNNG QUESTION (SKIP TO 114),..... 8 8 DONT KNOW/REFUSED 
112. How much difficulty do you have doing laundry - would you ay you have some difficulty, a lot of difficulty, or are you completely unable to do this without asaistance?

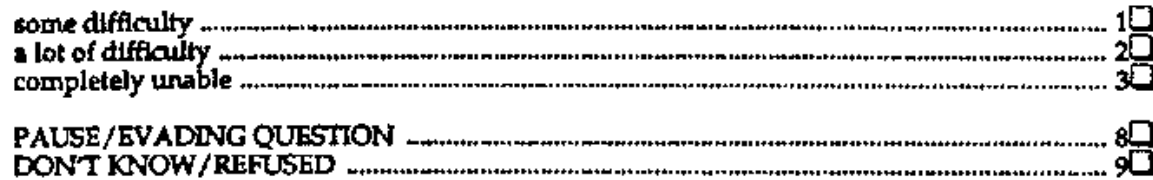

113. Do you get any help frow another peraon in dolng laundry?

yes $\rightarrow$ ASK: Who helps you?

no.

PAUSE/EVADING QUESTION DONT KNOW/REFUSED

114. Becauge of a health of phyilcal problem, do you hove any difficulty doing othes housework?

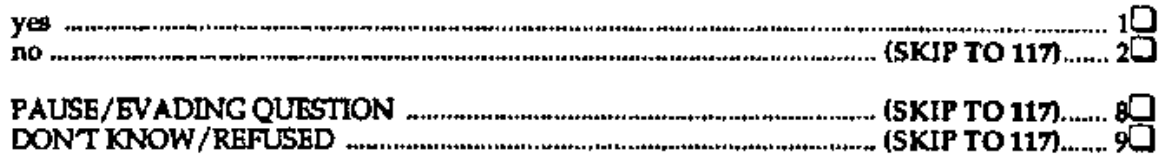

115. How much difflculty do you have daing other houeswork - would you any you have cone difficulty, a lot of difficulty, or are you completely unable to do this without assistance?

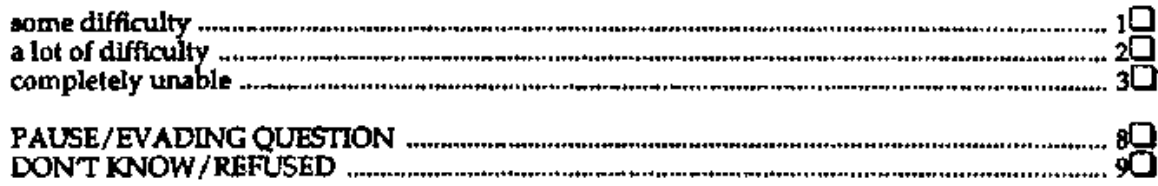

116. Do you get any help from another person in doing other housework?

yes $\rightarrow$ ASK: Who help you?

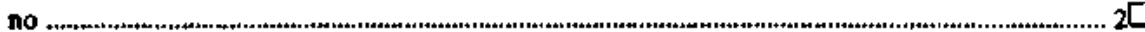

PAUSE/EVADWG QUESTION DON'T KNOW/REFUSED . 
120. Are there any important activities that I hoven't mentioned which are difficult for you to do?

no 20

PAUSE/EVADING QUESTION 80 DONT KNOW/REFUSED

122. Do you need any additional help with any of your activitiea?

:

no 20

PAUSE/EVADING QUESTION 80 DONT KNOW/REFUSED

123. If you needed information about services avallable to senjor ctizens, where would you go to get this information.

(DO NOT READ LIST - CHECK ALL THAT ARE MENTIONED)

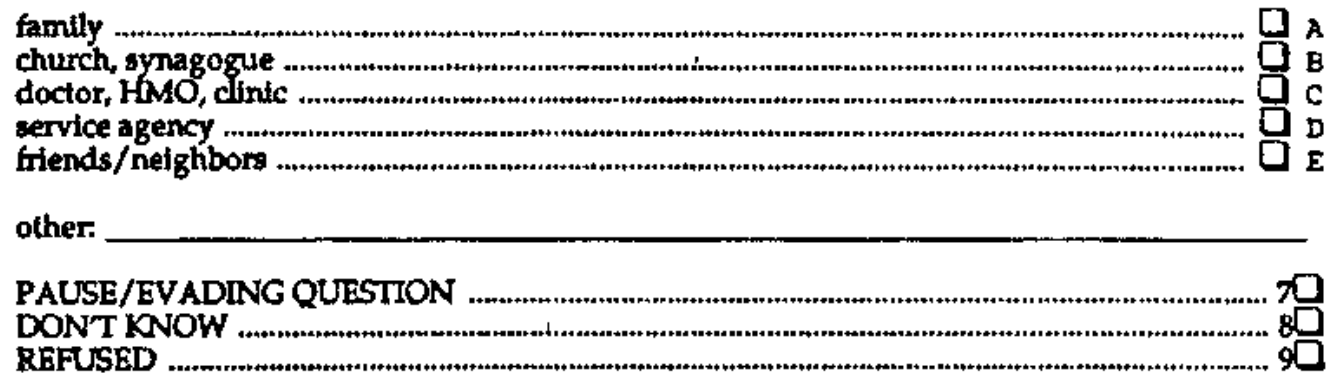

124. Now I would like to ask you about some other mattera. Flret, how would you rate your emotional health - would you say it is excellent, good, fair, or poar?

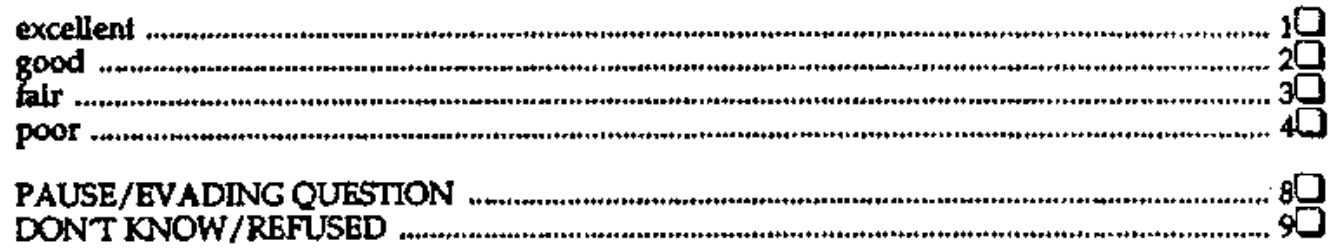

125. Overall, how satiafied have you been with your life during the past 6 months - would you say very istisfied, mostly satisfied, mostly dissatisfied, or very dissatisfied?

very satisfied

mostly satisfied

mostly dissatisfied

very dissatisfied

PAUSE/EVADRG QUESTION DONT KNOW/REFUSED 
126. I'm going to mention ceveral poenlble problem weas and I'd IIke you to telt me how serioun a problem each one it for you. Firit, money, is this * very terlous problem, womewhat serloute problem, of nat a serious problem for you? ... READ UST BELOW

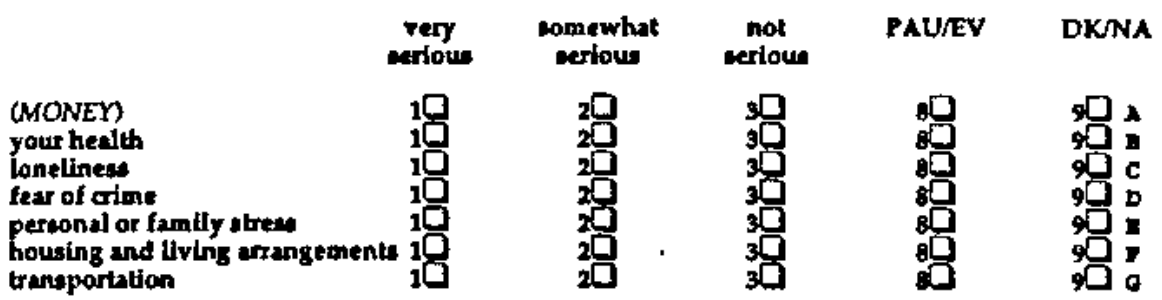

127. Now I'm going to ank you about thinge that might concern you for the future. Fint, loss of Independence becauce of general physical deterloration - would you say you we very worted, somewhat warried, ox not very worried about this for the fukure?

\begin{tabular}{|c|c|c|c|c|c|}
\hline & $\begin{array}{l}\text { Tery } \\
\text { worried }\end{array}$ & $\begin{array}{l}\text { nomewhat } \\
\text { wortied }\end{array}$ & $\begin{array}{l}\text { not very } \\
\text { worried }\end{array}$ & PAU/EV & DKNA \\
\hline \multirow{2}{*}{$\begin{array}{l}\text { (LOSS OF NDEPENDENCE) } \\
\text { mental deterioration } \\
\text { becoming \& financial burden } \\
\text { lonelinest } \\
\text { being \& viction of fraud or } \\
\text { con attigts }\end{array}$} & $\begin{array}{l}10 \\
10 \\
10\end{array}$ & $\begin{array}{l}20 \\
20 \\
20\end{array}$ & $\begin{array}{l}30 \\
30 \\
30 \\
30\end{array}$ & $\begin{array}{l}80 \\
80 \\
80 \\
80\end{array}$ & 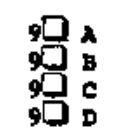 \\
\hline & 10 & 20 & مי3 & 80 & = ב \\
\hline \multirow{3}{*}{$\begin{array}{l}\text { being a victim of other } \\
\text { types of crime } \\
\text { housing and living arrangement } \\
\text { going to a nuroing home } \\
\text { getting to the places you } \\
\text { need to go to }\end{array}$} & 10 & 2[ & 3 & 80 & P \\
\hline & ש 10 & $\begin{array}{l}2 \square \\
2 \square\end{array}$ & בי3 & $\begin{array}{l}80 \\
80\end{array}$ & a \\
\hline & 10 & 20 & Dי & 口 & I \\
\hline
\end{tabular}

12. Thinking about your lffe in general, what would you say is the one thing that concerna you moet?

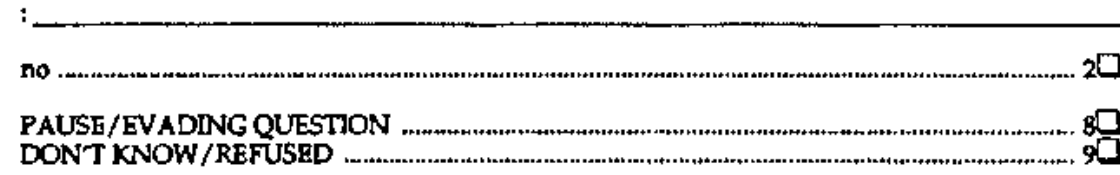

129. Now I have ane classification queations and then we'll be through First could you please tell me your age?

PAUSE/EVADING QUESTION 8 DONT KNOW/REFUSED

130. Are you married, widowed, divorced, neparated, or were you never married?

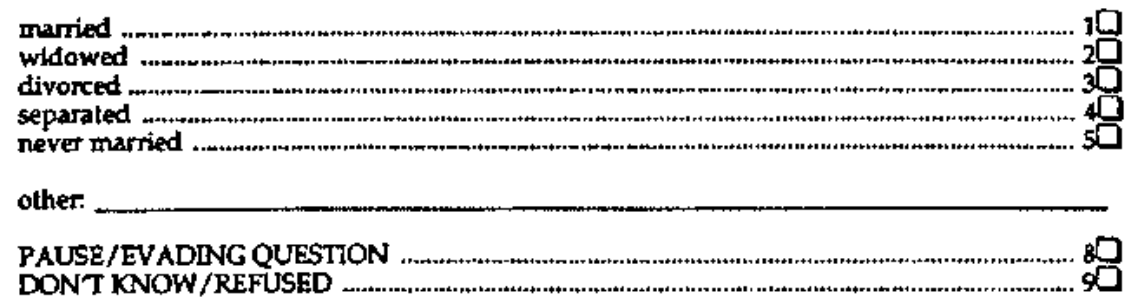

131. How long have you been llving in the home where you are now living?

PAUSE/EVADING QUESTION DONT KNOW/REFUSED 
132 What to your elp code?

:

PAUSE/EVADING QUESTION

DONT KNOW/REFUSED

133. How long have you been tiring in Dade County?

PAUSE/EVADING QUESTION 8 DONT KNOW/REFUSED

134. How many months of the year do you lve in Floridat

PAUSE/BVADING OUESTION 8 DON'T KNOW/REFUSED 997

135. What is your religious preference?

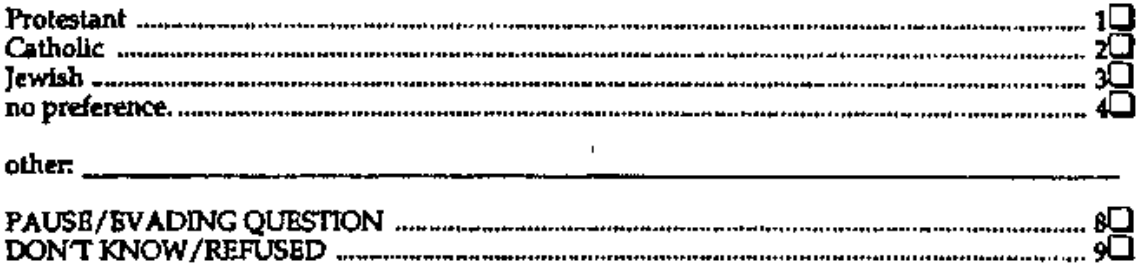

136. How important is relgion In your life? Would you wy it ie very important, comewhat important, or not very importank?

very inportant

somewhat important 10 not very important

PAUSE/EVADING QUESTION 8 DONT KNOW/REFUSED

137. What to your racial group?

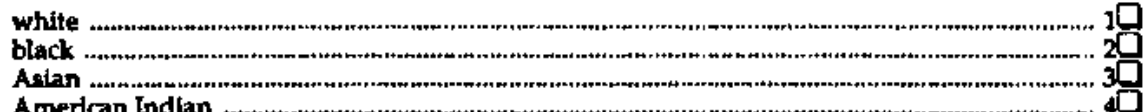
ASK: Do you conslder yourself to be white, black, or something

other. elee?

whlte 10 black $2 \square$ something else $2 \square$

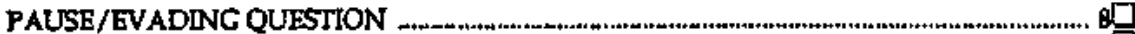
DONT KNOW/REFUSED

138. Are you Hispanic?

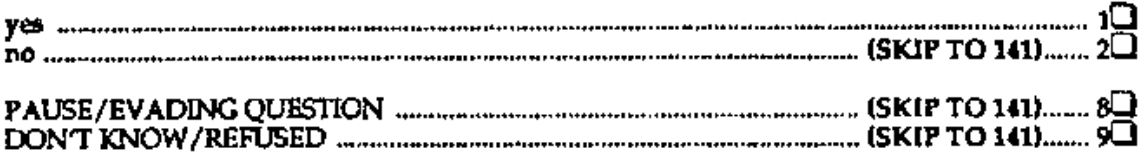

139. In what country were you bom?

United States (SKIF TO 141) ...... 10

PAUSE/EVADING QUESTION (SKIP TO 14i) ...... 8 DONT KNOW/REFUSED (SKIP TO 141)...... 9 
140. In what year did you come to the United Sutes to siay?

PAUSE/EVADING QUESTION

DON'T KNOW/REFUSED

141. What is the higheat grade of achool you have completed?

grade school (grades 1-8)

some high school (grades 9-11)

10
20
30
40
50

soune college (includes associate degree)

college graduate (four year degree only)

completed graduate degree (MA, Phd, MD, JD)

$8 \square$

PAUSE/EVADING QUESTION

142. What language do you speak most often in your home?

English

10

Spanish

Creole

other:

PAUSE/EVADING QUESTION

$8 \mathrm{D}$

DONT KNOW/REFUSED

143. Do you read the newspaper regularly?

yes $\rightarrow$ ASK: Which newepaper?:

no

PAUSE/EVADING QUESTION DON'T KNOW/REFUSED

14. If you don't mind, I need to know if your tolal annual household income, from all sources, before taxes if under $\$ 5,000, \$ 5,000$ to $\$ 10,000, \$ 10,000$ to $\$ 20,000, \$ 20,000$ to $\$ 30,000, \$ 30,000$ to $\$ 50,000$, $\$ 50,000$ to $\$ 70,000$, or over $\$ 70,000$ ?
under $\$, 000$
$\$ 5,000$ to $\$ 10,000$
$\$ 10,000$ to $\$ 20,000$
$\$ 20,000$ to $\$ 30,000$
$\$ 30,000$ to $\$ 50,000$
$\$ 50,000$ to $\$ 70,000$
over $\$ 70,000$
10
20
30
50
60
70
REFUSED/NO ANSWER
DONT KNOW
8

145. Finally, I have just a few questions sbout the effectu that Hurricane Andrew had on you and your household. First, were you a resident of South Florida when the hurrtcane hil?

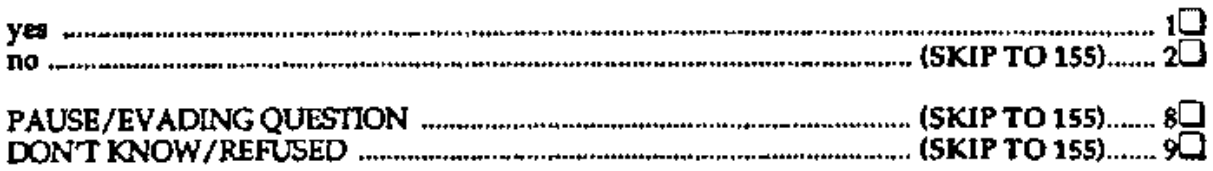

146. Did you evacuate from your home before Hurricane Andrew hit?

yes

19

no 
197. Were you forced to mone from your hame, after the hurricane, because of problems caused by the hurricane?

yes $\rightarrow$ ASK: For what reasond did you movet

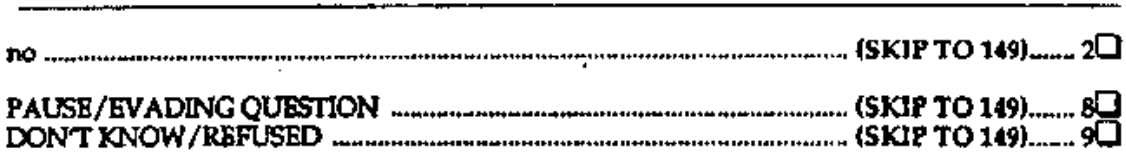

148. How long were you gone from that hone, or have you not returned to that home?

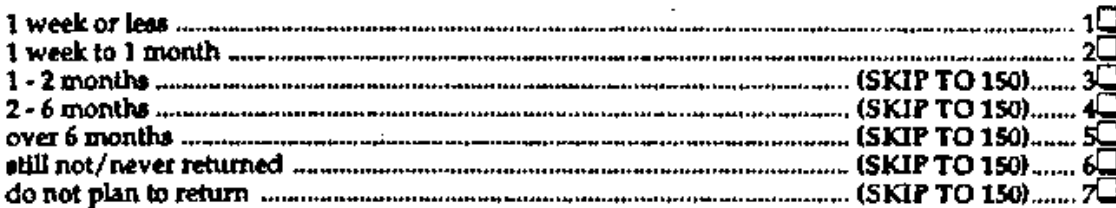

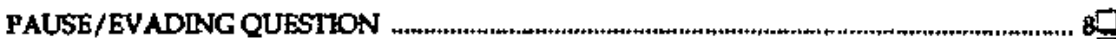

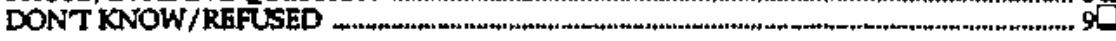

149. Did you take in anyone Into your home who was dioplaced a a result of Hurricane Andrew?

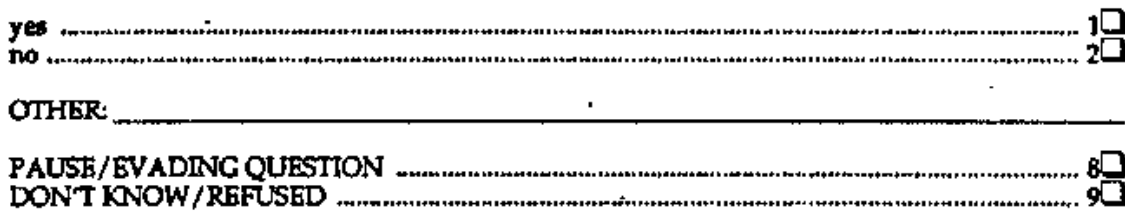

150. Would you way that Hurricane Andrew hat had a major effect, a moderate effect, minor effect, or no effect on your phyolical health?

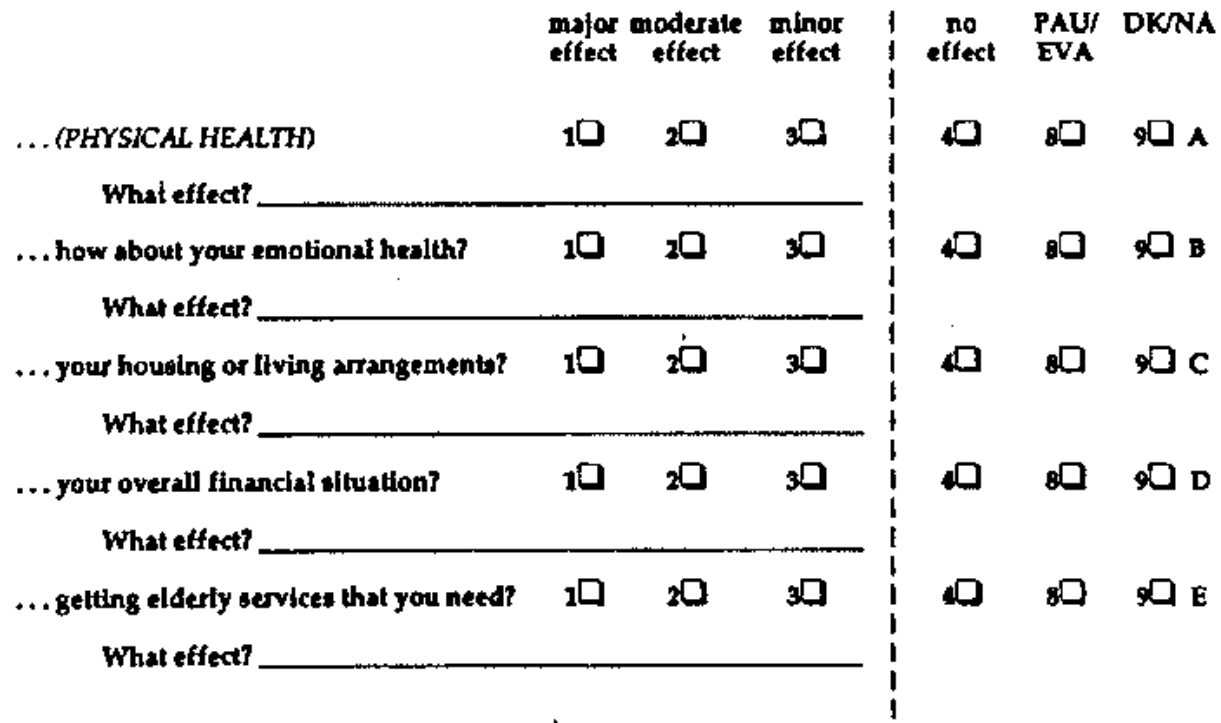

151. How prepared do you teel you would be if another hurrlane like Andrew were to hit South Florlda - very prepared, somewhat ptepared, or not at all prepared?

very prepared

(SKIP TO 153)....... 19

somewhat prepared

not at all prepared

(SKIP TO 153)...... 8D

PAUSE/EVADING QUESTION

(SKIP TO 153)...... o 
152. In what woys do you feel you are not prepared?

PAUSE/EVADINGQUESTION NONE/DONT KNOW/REFUSED

159. We will soon be conducting a study to find out how Hurricane Andrew affected the Jives of South Florldiank. Would you be willing to be called to partidpale in thile atudy?

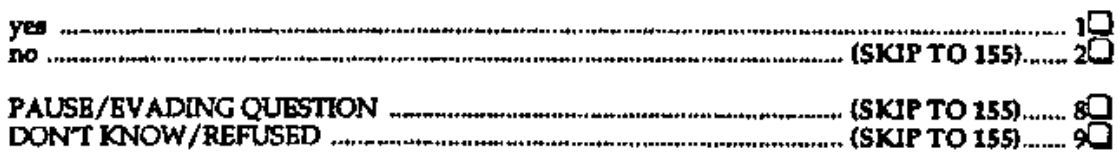

154 So that we will know whom to ank for when we call back, may I ask for your firat name?

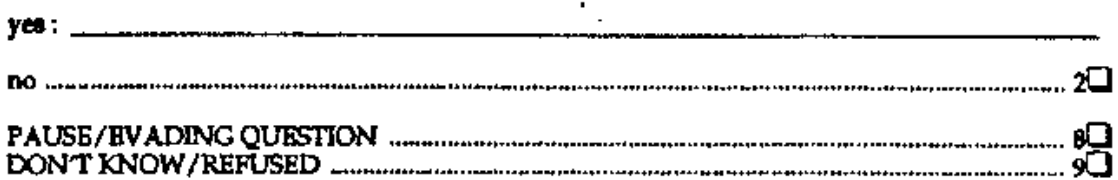

155. Well that concludes the intervew. I'd like to think you for your conperation. Do you have any comments that you would like me to write down?

ก0 -

\section{RESPONDENT WAS.}

plert and responsive

satisfactory

confused

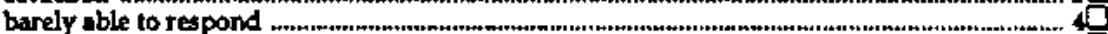

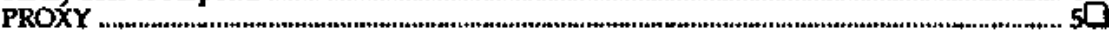

other:

\section{RATE THE QUALTY OP THS INTER VIEW FOR RESPONDENT'S UNDERSTANDING:}

very good

satisfactory

confused

barely able to respond

PROXY

other.

\section{RESPONDENT'S UNDERSTANDING OF ENGLISH:}

very good

average

poor ...

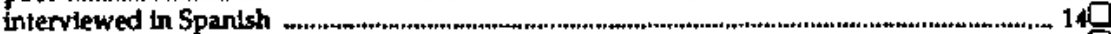

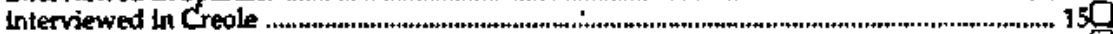

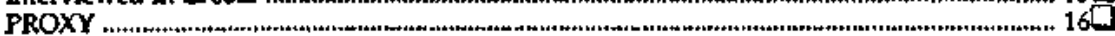

PROXY:

yes:

no.

FINISH TDME: 


\section{START TIME:}

\section{1. (RECORD RESPONDENT'S SEX)}

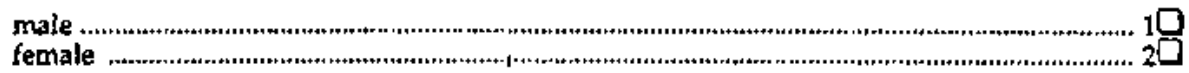

2. Quistera comenzar haciéndole unas preguntas sobre cómo va usualmente a los lugares donde necesita ir - va manejando usted mismo (a), va con alguna otra persona, usa transportación pública, va de algún otro modo?

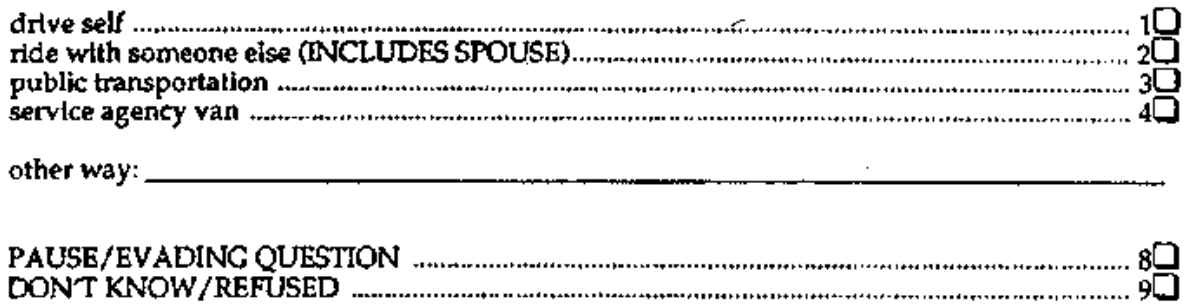

3. 2 Ha oido del Serviclo Especial de Transportación, o STS, que se ofrece para las personas mayores o personas con impedimenlos?

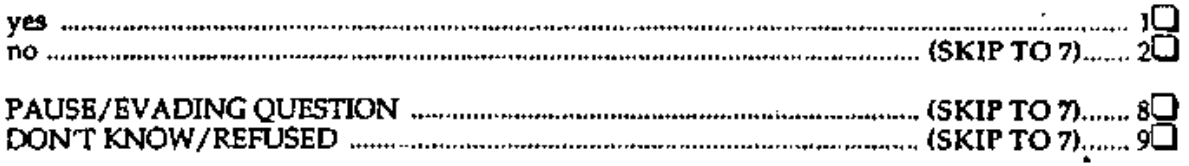

4. ¿Ha tusado alguna vez el Servicio Especial de Transportación?
yes
(SKIP TO 7) ......, 2L
PAUSE/EVADING QUESTION
(SKIP TO 7$) \ldots . ., 8$
DONT KNOW/REFUSED
(SKIP TO 7 ..... gi

5. En general, ¿điría que ẹta satisfecho (a) o que no eglá satisfecho (a) con este servicio?

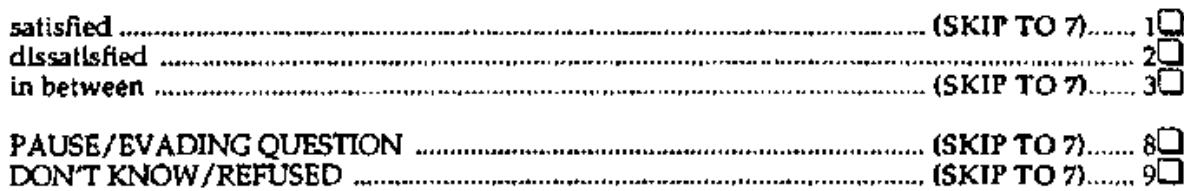

6. ¿Por qué no esth́ satisfecho?

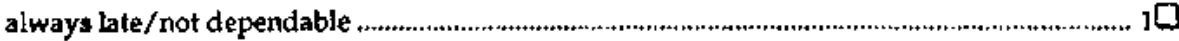

other:

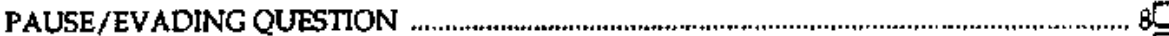

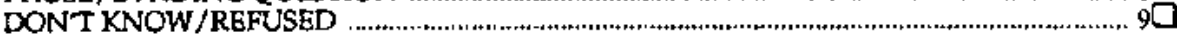

7. Ahora quisiera hacerle unas preguntas sobre su alud. ¿Comparado a otras personas de su misma edad, como calificaría su estado general de salud? Dlria que ea excelente, bueno, regular o malo?

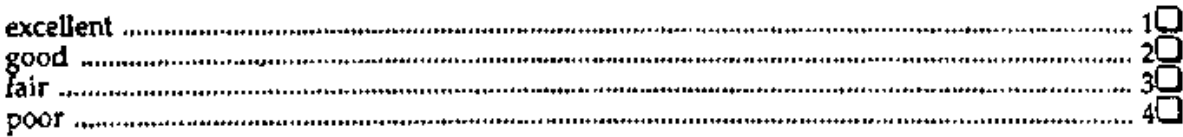

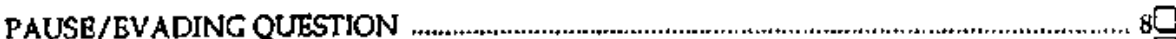

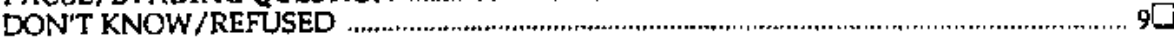


8. iA dónde va generaimente para recibir culdado de salud - a un médico privado, a un HMO, 1a ola de emergencia, una clínica, u otro lugar?

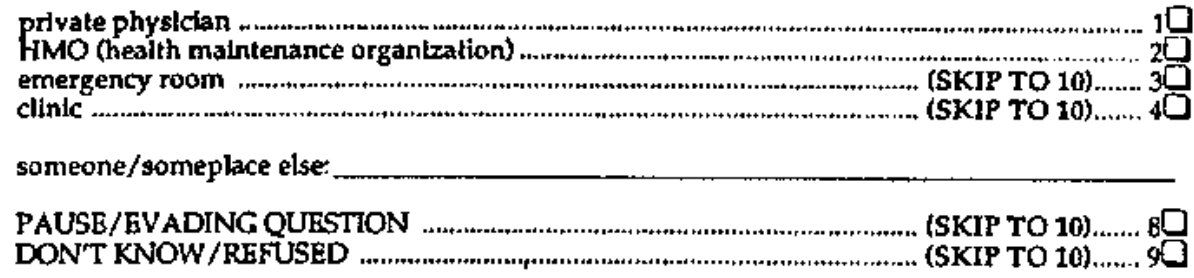

9. ¿Hay algún médico en especifico al que visita y que considera su médico personal o médico de familia?

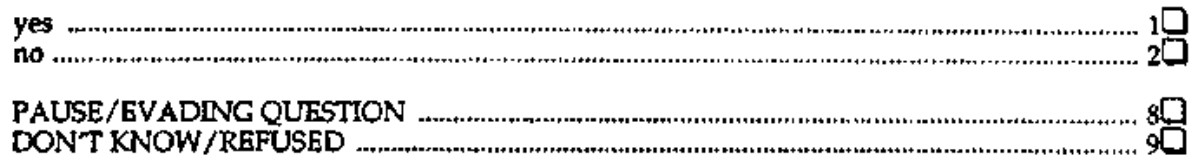

10. ¿Ha tenido un examen físico en los últimos 12 meses?

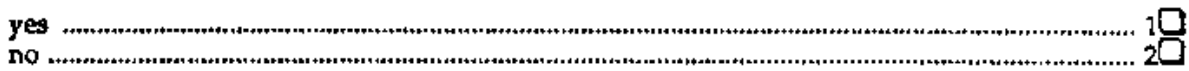

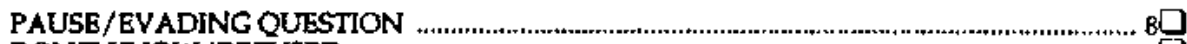

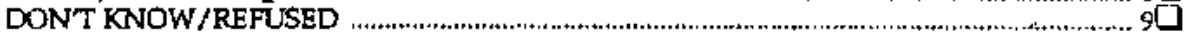

11. Ademís de un examen físico completo, ha tenjdo alguna evaluación de salud en el último año? Por evaluación de alalud qujezo decir cosas como chequeos de la presión arterial o el colesterol, examenes de la vista para glaucoma, etc.?

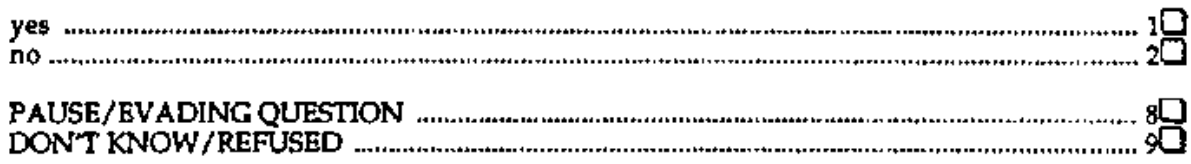

12. ¿Ha estado ingresado, y terido que quedarse por lo menos una noche en el hospltal, en los últionos 12 meses?
yes (SKIP TO 14)
2
PAUSE / EVADING QUESTION (SKIP TO 14) ...... 8D DONT KNOW/REFUSED (SKIP TO 14) ...... 9

13. Además del tiempo que estuvo hospitatizado, ¿cuśntas veces visitó al médico en los últimos 12 meses - usted diría que una o dos veces, de tres a cinco veces, mas de cinco veces, o no lo ha vuelio a ver?
1 or 2 times
(SKIP TO 15)....... 1
3 to 5 times
(SKIP TO 15)....... 2
more than 5 times
(SKIP TO 15)....... 3
not at all
(SKIP TO 15)....... 4D
PAUSE/EVADING QUESTION
(SKIP TO 15)...... 8D
DONT KNOW/REFUSED
(SKIP TO 15)...... 9

14 ¿Cuéntag veces ha ido al médico en los últimos 12 meseg - usted diría que una o dos veces, de tres a cinco veces, más de cinco veces, o no ha tdo al médico en los tiltimos 12 meses?

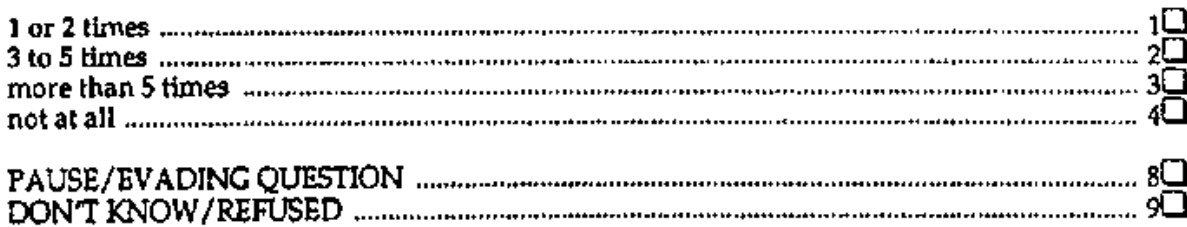


15. Durante el pasado affo, hubo alguna ocaslón en la que le hacía falta ir al médico, pero por algún motivo no le fue posible ir?

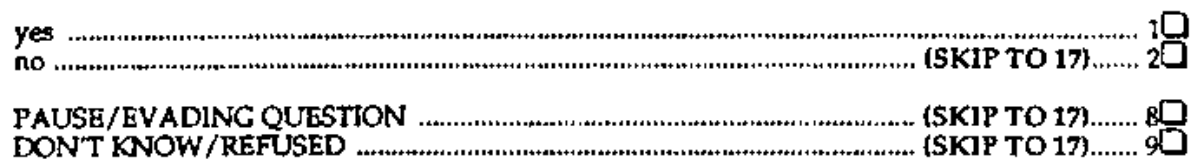

16. ¿Por qué no le fue postble ver al médico?

\section{(CHECK ALL THAT ARE MENTIONED)}

couldn't get an appolntment

too sick to go

didn't have the money ....

didn't have transportation

language problem

didn't have any Insurance

didn't have enough insurance

other.

PAUSE/EVADING QUESTION

DON'T KNOW/REFUSED

17. Ahora neceslto hacerle tunas preguntas sobre su alimentación. ¿Cuántas comidas come al dia -una, dos, tres, o más de tres?

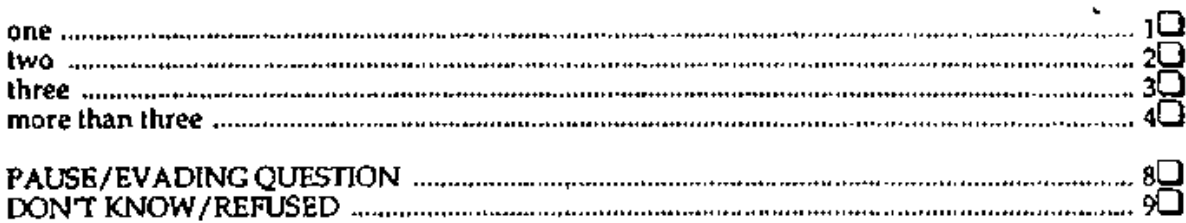

18. En general, zslente que come lo sufficiente?

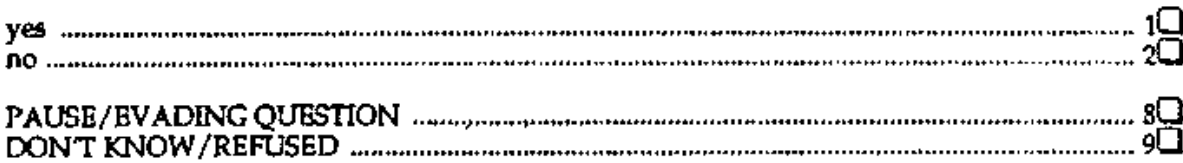

19. ¿Cutintas veces cone solo (a) - diría qué algunas veçes, la mayor parte del tiempo, todo el tiempo, o nunca come solo (a)?

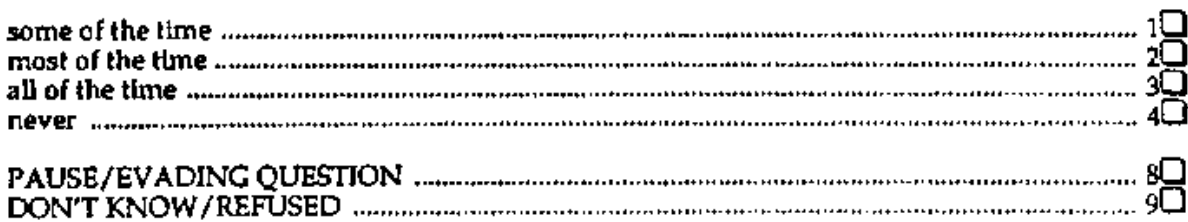

20. ¿Tiene alguna dfficultad para masticar carne?

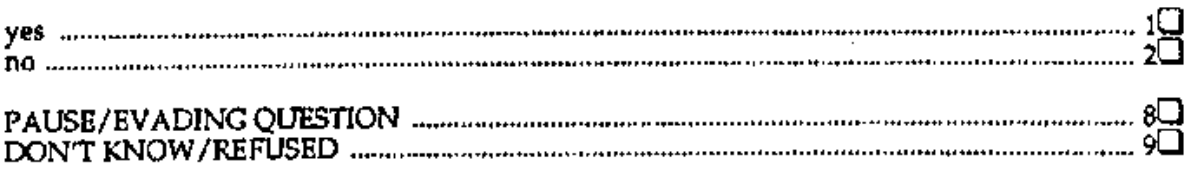

21. ¿Tiene usted alguna condleión médica por la que ha tenido que cambiar el tipo o la cantidad de comida que come?

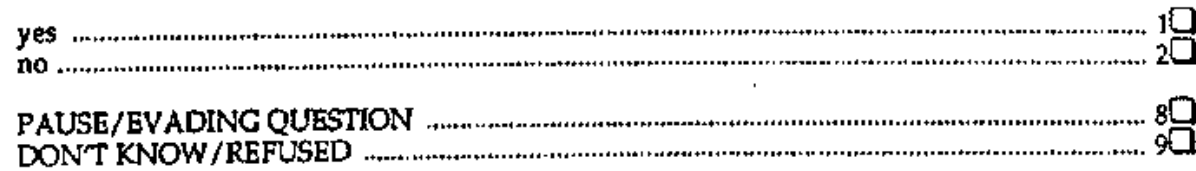


22. ¿Ha experimentado camblos signifleativos en su peso -ya sea aumento o pérdida de peso- en los últímos acls meses?

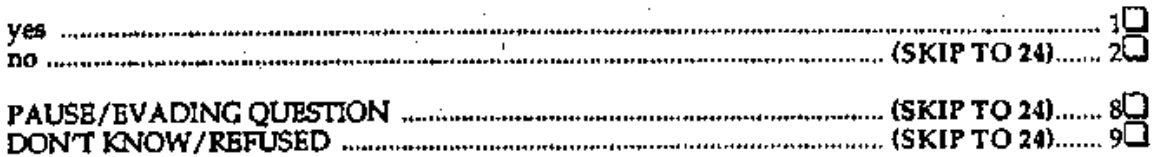

23. 2 Yeste camblo de peso, fue resultado de un plan programado o no fue intencional?

planned diet/program

1 unplanned

PAUSE/EVADING QUESTION

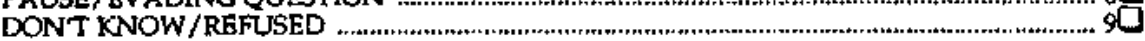

24. ¿Recibe sellos o cupones de alimentos?

yes 10 8

PAUSE/EVADING QUESTION ㅁ․․

25. LAhora le voy a menctonar vartos equipos de apoyo. Por favor dígame si usa alguno de estos equipos. ¿U $U_{62}$ (READ ITEMS BELOW)...

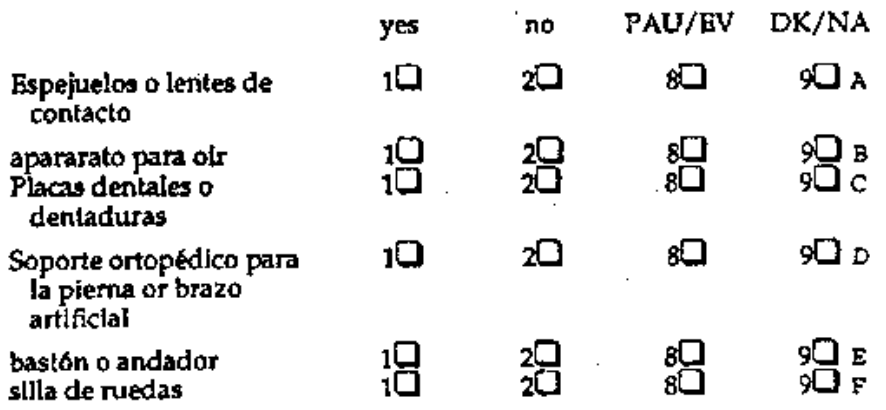

26. LNecesita algǘn equipo de apoyo que no tiene?

(IF YES ASK $\rightarrow$ Qué es lo que necesitat DO NOT READ LIST - CHECK ALL MENTIONED)

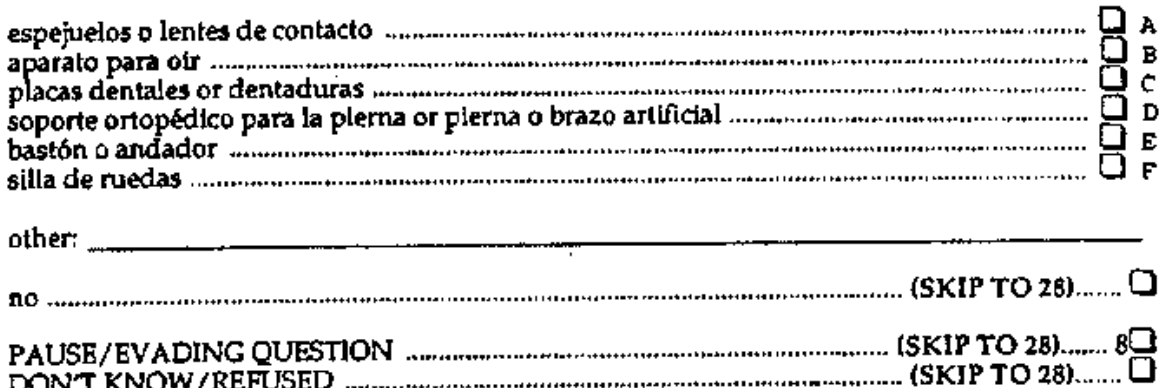

27. ¿Por qué no le ha sido posibie obtener estelestos equipo/s de apoyo?

(CHECK ALL THAT ARE MENTIONED)

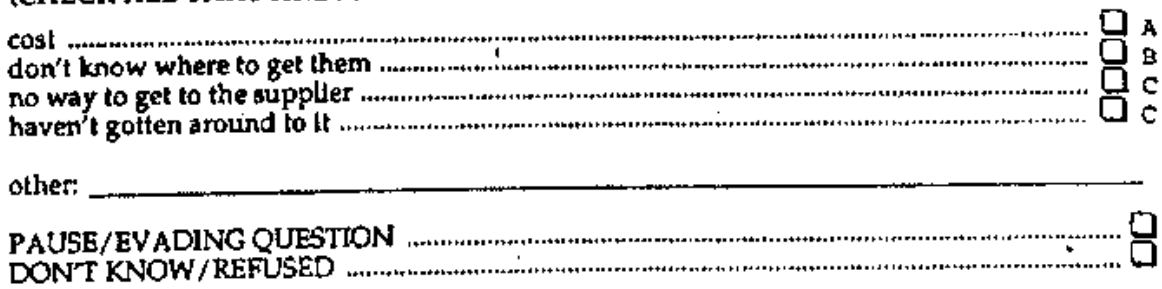


28. Sl be enferma y se queda en su casa, thay alguien que lo puede ayudar durante su enfermedad? yes $\rightarrow$ ASK: "¿Qulén sería esa persona?" (WRITE ALL THAT ARE MENTIONED)

IF MORE THAN ONE PERSON IS NAMED ASK $\rightarrow$ ¿Clál de estas personas seria mayormente responsable de culdarlo (a)?

Most responsible person:

no. (SKIP TO 30)....... 2D

PAUSE/EVADING QUESTION (SKIP TO 30)....... 8 DONT KNOW/REFUSED (SKIP TO 30)........ 9

29. IF "SPOUSE" IS ANSWER FOR QUESTION 28 ASK $\rightarrow$ ¿Su esposo(a) vive en la misma casa con usted? IF ANSWER IS "NO" CONTINUE WITH ...

OTHERWISE ASK $\rightarrow$ ¿Cuanto tiempo le tomarfa a ___ (caregiver from 28)__ para llegar a su casa desde donde élvella reside? ¿Le tomaría menos de 10 minutos, de 10 a 30 minutos, de 30 minutos a 1 hora, o más de 1 hora?

10 minutes or less
10 to 30 minutes
30 minutes to one hour .
more than one hour but less than one day
more than one day
LIVES IN HOUSEHOLD
PAUSE/EVADING QUESTION
DONT KNOW/REFUSED

30. ¿Cuśndo fue la última vez que fue al dentista - fue durante el último año, hace más de un año pero menos de tres años, o hace mís de tres años?

within the last year
one to three years
more than 3 years ago
PAUSE/EVADING QUESTION
DONT KNOW/REFUSED

31. ¿Ha tenido algún problema dental serio en el último afio?

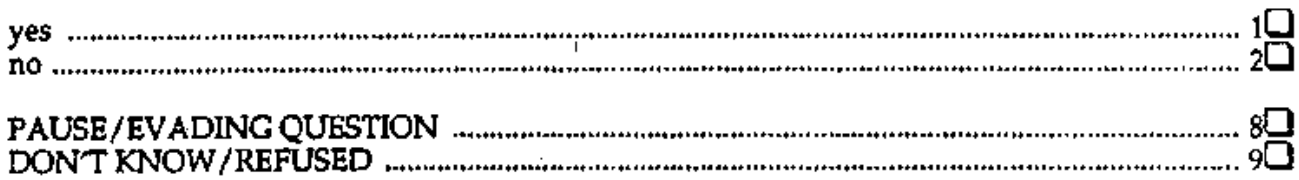

32. Ahora le voy a mencionar varios tipos de seguro médico. Por favor dígame si los tiene. Primero, tiene usted Medicare?

yes --> ASK: "2Está cubierto (a) para hospitalización solamente u hospitalización y médico?

medicare de hospltalizacion solamente (part A)

medicare de hospitalización y médico (Partes A y B).............................................................. 20

no

PAUSE/EVADING QUESTION 
33. ¿Tiene ... . 2 (READ LIST BELOW - CHECK BOX ONL $X$ IF THEY HAVE THE COVERAGE)

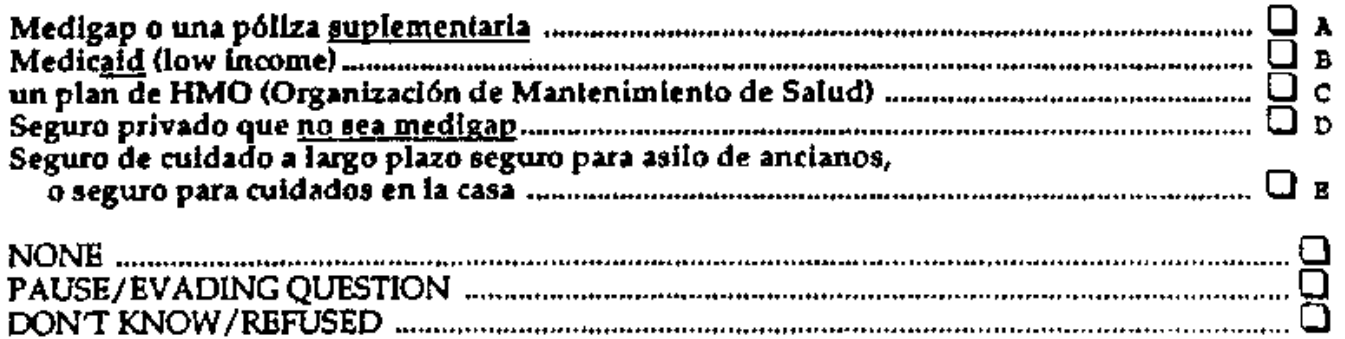

34. Ahora quiero hacerle algunas preguntas sobre el lugar donde vive. Primero, ¿vive en una casa de familla, un townhouse, un duplex, un apartamento, un condominio, una casa móvil, alquila un cuarto en casa de otra persona, o vive en otro lugar?

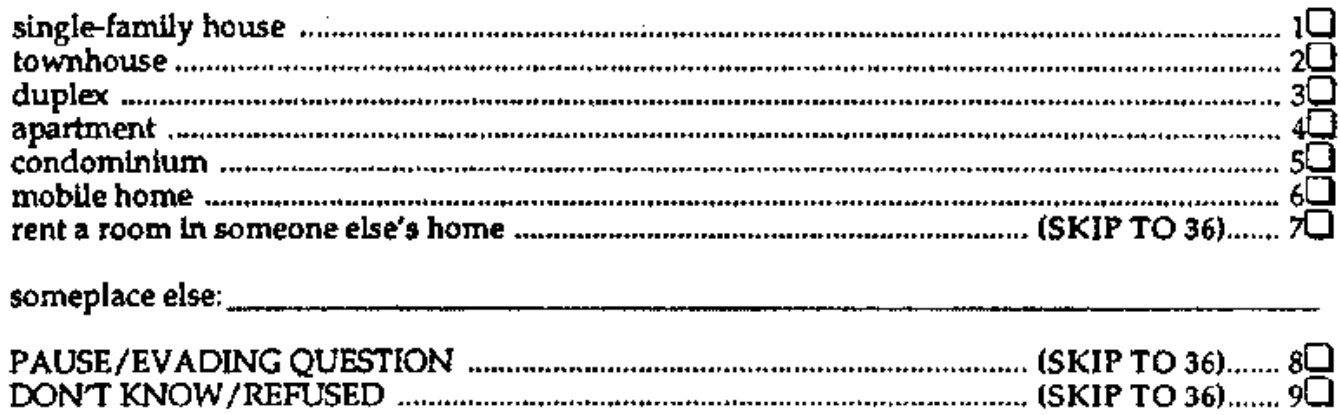

35. ¿Es el dueño (la dueña) o alquila su lugar de residencia?

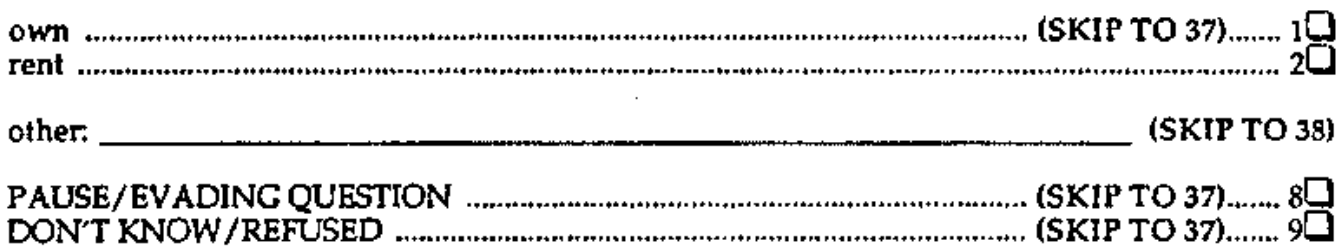

36. Después que pasó el huracán Andrés, qha aumentado su renta mayormente, moderadamente, o no ha aumentado?

greatly
moderately
not at all

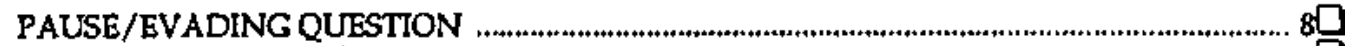

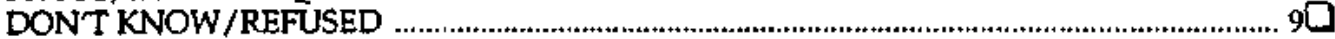

37. ¿Ttene seguro sobre su propiedad/seguro de inquilino?

yes
PAUSE/EVADING QUESTION
DON'T KNOW/REFUSED 
38. Incluyéndose a sí mismo (a) ¿cuántas personas viven en su hogar?

number of people: (IF ONE, SKIP TO 40)

PAUSE/EVADING QUESTION $8 \square$ DON'T KNOW/REFUSED

39. 2Quién más vive con usted?

(CHECK ALL THAT ARE MENTIONED)

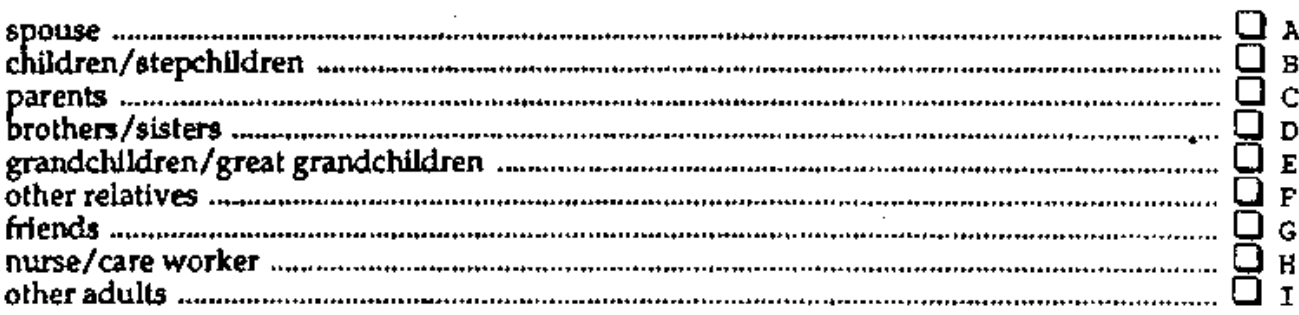

PAUSE/EVADING QUESTION

DONT KNOW/REFUSED

40. ¿Cuán sattisfecho (a) está con su vivienda - diria que está muy satisfecho, bastante satisfecho, bastante descontento, o muy descontento con su vivienda?

very satisfied

(SKIP TO \$2)....... 1

mostly satisfied

(SKIP TO 42)...... 2D

mostly dissatisfied 30

very dissatisfied

(SKIP TO 42) ....... 8D

PAUSE/EVADING QUESTION (SKIP TO 42)........ 90

41. ¿De qué forma ests descontento?

PAUSE/EVADING QUESTION 8 DON'T KNOW/REFUSED

42. Si tuviera que mudarse del lugar donde vive en estos momentos, zcuín dificil cree que seria encontrar vivienda de la misma calidad y del mismo costo que la que tiene ahora? ¿Diría que sería muy dtfícll, moderadamente difícil, o no muy difícil?

very difficult

moderately difficult

$1 \square$

not very difficult (INCLUDES "not at all difficult")

PAUSE/EVADING QUESTION

DON'T KNOW/REFUSED

43. ¿Cuán difícil le resulta pagar los gastos de su vivienda, cosas como la hipoteca o el alquiler y los gastos de mantenimiento de su casat ¿Dixía que pagar estos gastos le resulta muy dificil, moderadamente dificil, o no muy difícil?

very difftcult

moderately difficult

10

not very difficult (INCLUDES "not at all difficult")

PAUSE/EVADINGQUESTION

DONT KNOW/REFUSED 
44. 2 Rectbe algún tipo de ayuda de gobferno para sus gastos de vivienda y de servicios públicos como electrictdad y $* g u a ?$

yes $\rightarrow$ ASK: Qué típo de aslstencia esth́ recibiendo?

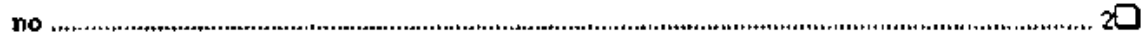

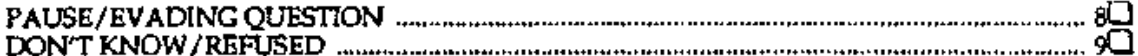

45. ¿Recibe ayuda de fanlilares o amistades para sus gastos de viviends?

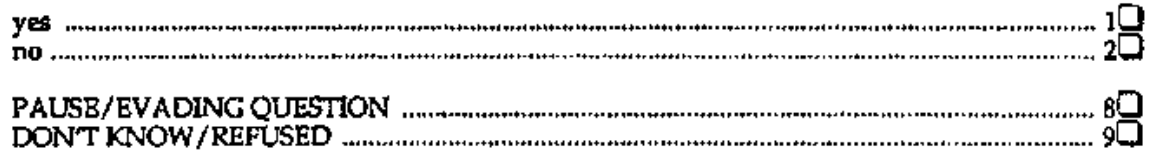

46. Ahora qulslera hacerie unas preguntas sobre el erimen. Primero, en el bartio donde vive, zdiría que el trimen es un problema muy serto, un problema moderadamente aerio, o que no es un problema serio?

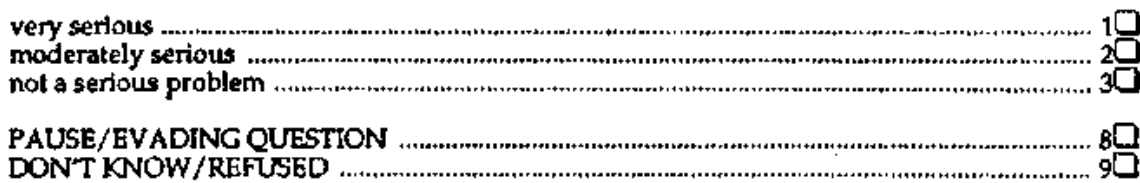

47. ¿Cún preocupado (a) eot'́ de que usted o alguien en su casa sea la victima de un crlmen - diría que está muy preocupado (a), moderadamente preocupado (a), o que no esló preocupado (a)

very concerned

moderately concerned ................................................................................................................... 2

not very concerned (INCL,UDES "not at all concerned") ......................................................... 30

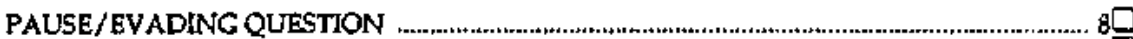

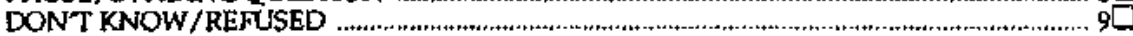

48. ¿Fue usted o alguien en su casa victima de un crimen durante el pasado año?

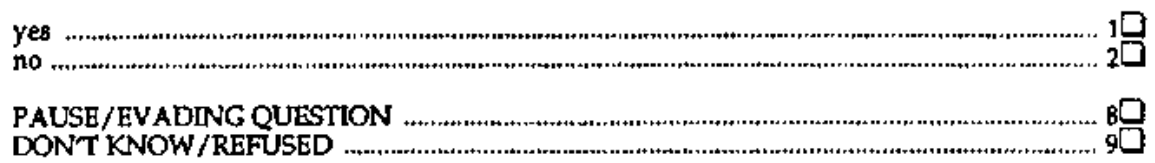

49. Ahora quisiera preguntarle sobre aus actiwdades. Primero, zesta retirado (a), trabaja a tiempo completo, trabaja a tiempo parctal (part-time), está sin trabajo temporariamente, o alguna otra cosa?

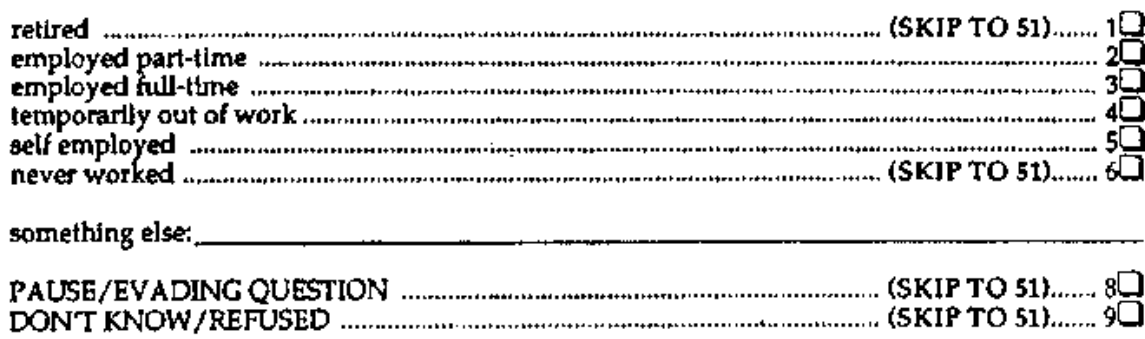

50. ¿Trabaja principalmente por el dinero, o trabaja poz alguna otra razón?

money

other reason:

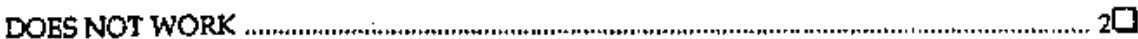

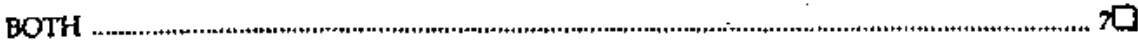

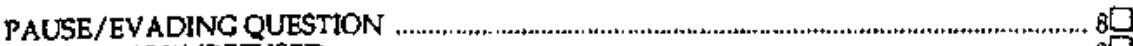

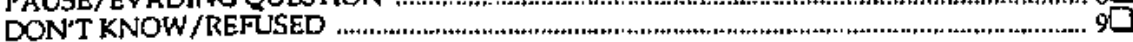


51. Si se le presentara ta oportunidad, estaria interesado (a) en aprender un nuevo oficio, o negocio?

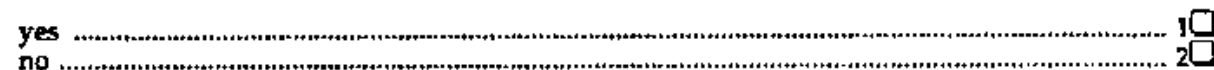

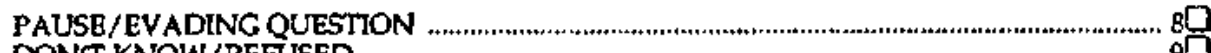
DONT KNOW/REFUSED

52. Si se le presentara la aportundidad, eslarfa interesado (a) en tomar clases para aprender unás sobre algo que le Interesa o para su desarrollo personal?

yes $\rightarrow$ ASK: ¿Qué clases eslaría interesado (a) en tomar?

no 10

PAUSE/EVADINC QUESTION DON'T KNOW/REFUSED

53. ¿Cuán importanle es para usted lenter Ia oportunidad de hacer trabajo de voluntaria - diría que muy importante o no muy importante?

important 10 not very Important

PAUSE/EVADING QUESTION

DON'T KNOW/REFUSED

54. ¿Pertenece a algún club v organtzación social?

yes $\rightarrow$ ASK 2 En qué grupos o actividades partictpa?

no $1 \square$

PAUSE/EVADING OUESTION

DONT KNOW/REFUSED

55. IF "NO" FOR QUESTION 54 ASK $\rightarrow$ ¿Le gustaría participar en más actividades sociales, 0 plensa que esta haciendo la cantidad adequada para usted?

OTHERWISE ASK $\rightarrow$ En relación a sus actividades sociales, Lpiensa que participa en demasiadas actividades sociales, que participa en la cantidad adecuada, o le gustaria participar en más actividudes sociales?

too many activitles (5KIP TO 57)...... $1 \square$

right amount (SKJP TO 57$)$

would like to do more

(SKIP TO 57)....... 8

PAUSE/EVADING QUESTION (SKIP TO 57)...... 9 DON'T KNOW/REFUSED

56. ¿Hay alguna razón por la que no participa en mbs actividades soclales de grupa?

(DO NOT READ. CHECKAS MANY Ás NEEDED)

lack of transportation .................................................................................................................

fear of crime .................................................................................................................................., activities unavailable or too fas away ....................................................................................... activity fadlity is too crowded no one to do things with don't like to go out at night health

other: 
57. ¿Con cuánla frecuencia se reúne con aus amistades, ya sea en su casa, en la casa de gus amistades o en algún otro Jugar - diría que por lo menos una vez a la semana, por lo menos una vez al mes, menos de una ver al mes, o nunca?

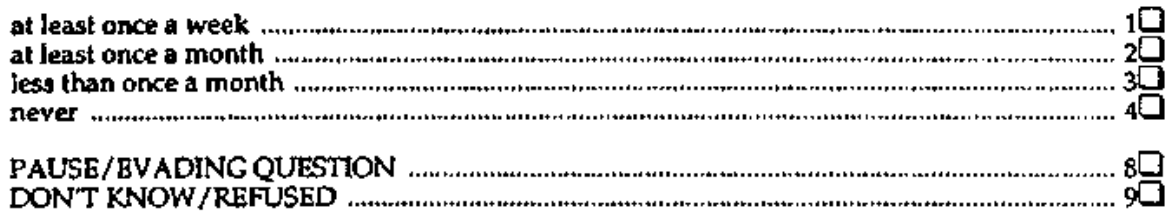

58. ¿Con cuśnta frecuencia se reúne con sus familiares, en su casa o en la casa de ellos - por lo menos una vez a la semana, por lo menos una vez al mes, menos de una vez al mes, o nunca?

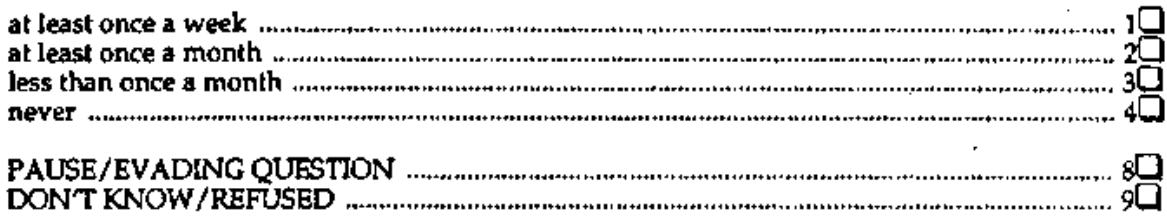

59. ¿Con euánta frecuencia va a log serviclos religlosos o a otra actividad de grupo religiosa - por lo menos una vez a la semana, por to menos una vez al mes, cuando hay alguria fiesta religiosa, 0 nunca?

at least once a week

at least once a month

religious holidays only

2

never

PAUSE/EVADING QUESTION

60. ¿Con cusinta frecuencla habla por teleffono con sua familiares - cagl todos los dlas, varias veces por semana, aproximadamente una vez a la semana, o menos de una vez la semana?

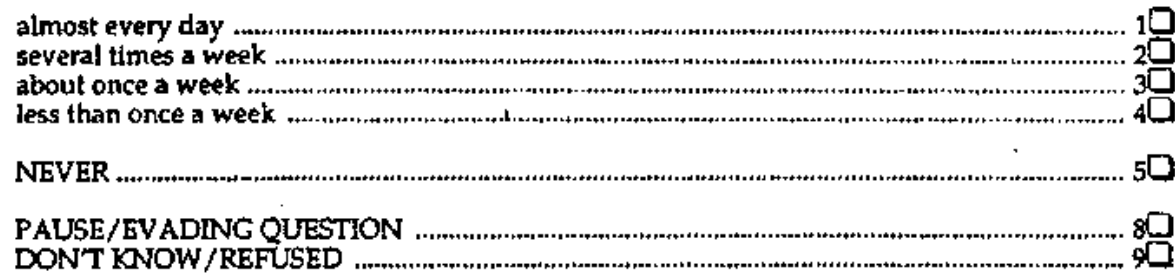

61. 2 Con cuánta frecuencia habla por teléfono con sus amistades -- casi todos los días, varias veces por semana, aproxlmadamente una vez a la semana, o menos de una vez la semana?

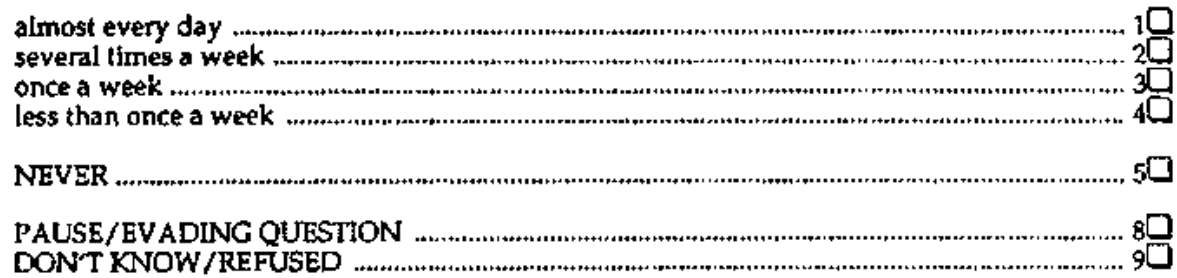

62. ¿Con cufnta frecuencia sale de su casa - dlría que todos los días, varias veces por semana, aproximadamente una vez a la semana, o menos de una vez la semana?

every day

several times a week

10

about once a week

less than once a week

NEVER 
63. Las próximas preguntas son acerca de su capacldad para reallear actividades que todos necesitumos hacer como parte de nuesta vida diaria. Primero, por algún problecna de salud o impedimento ffsteo dtiene usted alguna dlficultad para bah̆arse o darae una ducha?

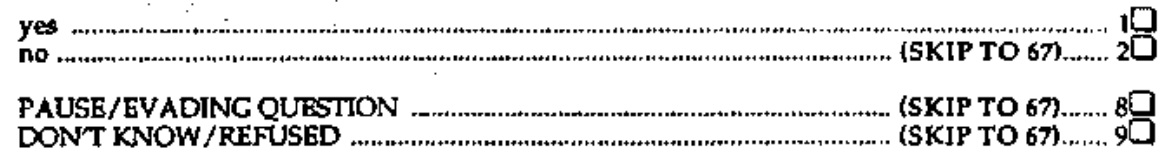

64. Usted solo (a) y sin usar ningún equipo especial, zcusinla diflcultad tiere para bañarse o darse una ducha - diria que tiene un poco de dificultad, mucha dificultad, o que no puede hacer esto sin la ayuda de otra persona o de algún equipo especisl?

some difflculty .

a lot of difficulty.

completely unabie

3.

PAUSE/EVADING QUESTION

DONT KNOW/REFUSED

65. ¿Recibe ayuda de otra persona para bañarse o darse una ducha?

yes $\rightarrow$ ASK: 2 Qulén lo ayuda?

no

PAUSE/EVADING QUESTION

DON'T KNOW/REFUSED

66. ¿Usa equipo especial de ayuda para baharse o darse una ducha?

yes $\rightarrow$ ASK: 2 Qué equjpo use?

na

PAUSE/EVADINC QUESTION

DONT KNOW/REFUSED

67. Debido a un problema de alud o físico ztiene dificultad para vestirse?

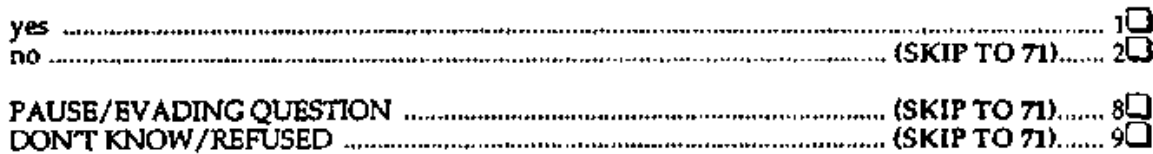

68. 2Cuínta dificultad tiene para vestirse - dirla que tiene alguna dificultad, mucha diflcultad, 0 que no puede hacer esto sin la ayuda de otra persona o de algún equipo especial?

some difficulty 1 .

a lot of difficulty

completely unable

PAUSE/EVADING QUESIION

DONT KNOW/REFUSED

69. ¿Recibe ayuda de otra persona para vestirse?

yes $\rightarrow$ ASK ¿QuIén lo ayuda?

no

PAUSE/EVADING QUESTION

DONT KNOW/REFUSED

70. ¿Usa equipo especial de ayuda para vestirse?

yes $\rightarrow$ ASK: $₫$ Que equipo usa?

PAUSE/EVADING QUESTION 80 
7. Debido un problema de salud offsico, ¿tiene dificultad para comer?

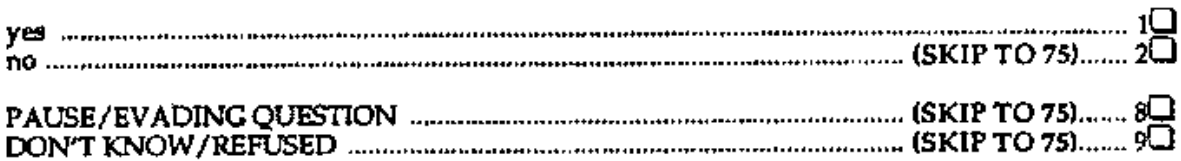

72. ¿Cuánta diftcultad tiene para comer - diris que tene un poco de dificultad, mucha dificultad, o que no puede hacer esto sin la ayuda de otra persona o de algún equipo especlal?

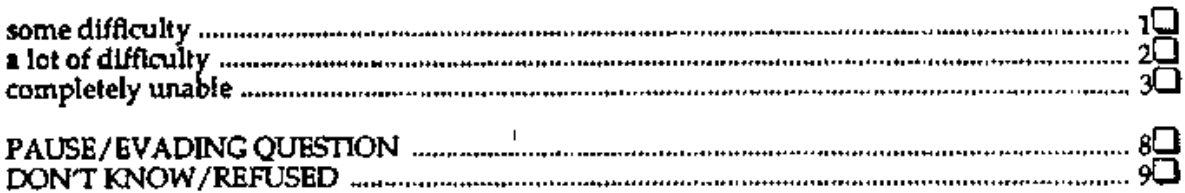

73. ¿Recibe ayuda de otra persona para courer?

yes $\rightarrow$ ASK: ¿Quíén lo ayuda?

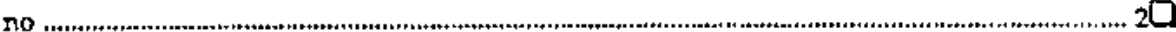

PAUSE/EVADINC QUESTION ........................................................................................ 8

DON'T KNOW/REFUSED ......................................................................................................... 9

74. ¿Usa equipo especisis de ayuda para slimentarse?

yes $\rightarrow$ ASK: $¿$ Qué equipo usa?

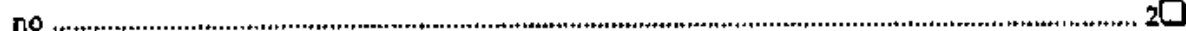

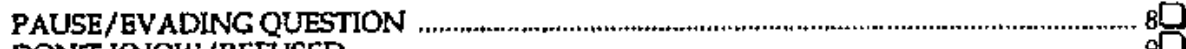

DONT KNOW/REFUSED ....................................................................................

75. Debido a un problema de salud of físico ztiene dificultad para tomar medicinas?

yes
PAUSE/EVADING QUESTION
DONT KNOW/REFUSED

76. 2 Cuánta dificultad tiene para tomar medicinas - diria que tiene un poco de dificultad, mucha dificuttad, o que no puede hacer esto sin la ayuda de otra persona o de algún equipo especialt?

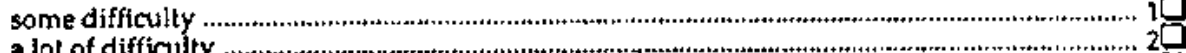

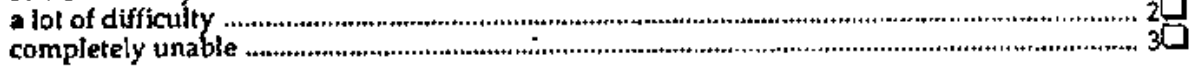

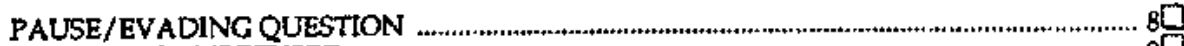

DONT KNOW/REFUSED ............................................................................................. 9

77. ¿Recibe usted ayuda de otra persona para tomar medlctnas?

yes $\rightarrow$ ASK: ¿Quién lo ayuda?

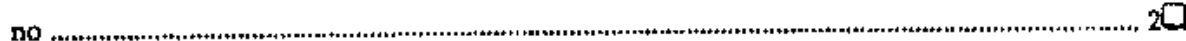

PAUSE/EVADING QUESTION ................................................................................... 8

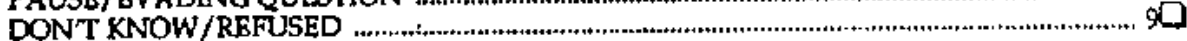

78. ¿Usn equipo especial de ayuda para poder administrarse sus medicinas?

yes $\rightarrow$ ASK ¿Qué equipo usa?

no

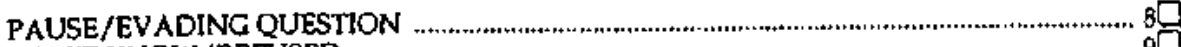

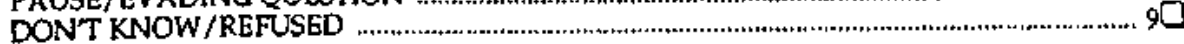


79. Debido a un problema de salud o físleo 2 tiene dificultad para cuidar de su apariencia ipeinarse el cabello, etc.)?

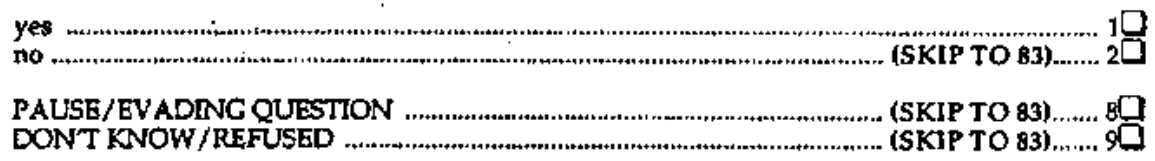

80. ¿Cuanta dificultad thene para culdar de su apariencia - diria usted que tiene un poco de diflculbod; mucha dificultad, 0 gue no puede hacer esto ain la ayuda de otra persona o de algún equipo espectal?

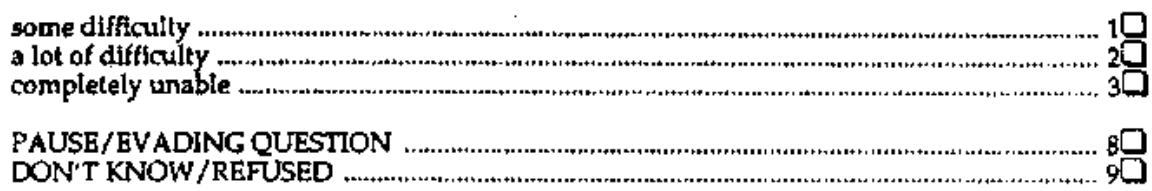

81. LRecibe ayuda de otre persona para culdar de au opariencia?

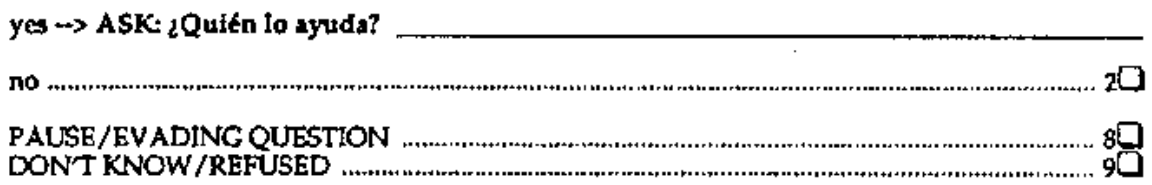

B2. ¿Usa equipo especial de ayuda para poder cuidar de su apariencla?

yes $\rightarrow$ ASK: ¿Que equipo weal

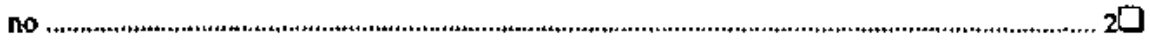

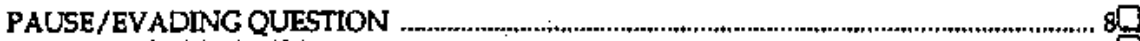

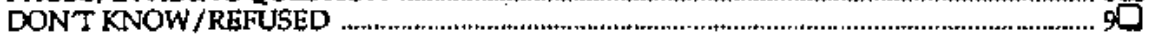

83. Debldo a un problema de salud o físico ¿tiene dificultad para usar el baño (inodorol?

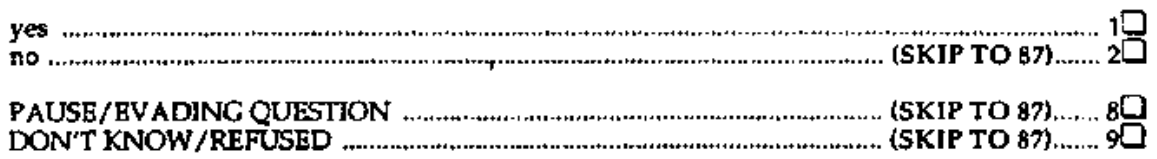

84. 2 Cuanta dificultad thene para usar el baño (lnodoro)-- diría que tiene un poto de dificultad, mucha diflcultad, o que no puede hacer esto sin la ayuda de atra pefsona o de algún equipo especial?

some difficulty

a lot of difficulty

completely unable

证

PAUSE/EVADING QUESTION

DONT KNOW/REFUSED

85. ¿Recibe ayuda de otra persona para usar el baflo (inodoro)?

yes $\rightarrow$ ASK: ¿Qulến lo ayuda?

no

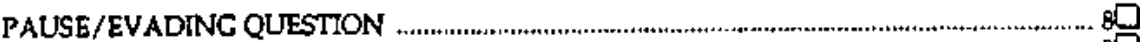

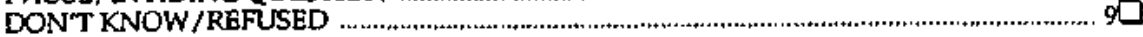

86. ¿Usa equipo especial de ayudi para usar el baño (inodorolt

yes $\rightarrow \rightarrow$ ASK: ¿Quté equipo usa?

no

PAUSE/EVADING QLESTION

DONT KNOW/REFUSED 
87. Debido a un probleme de palud o fislea ¿tiene diflcultad para sentarse y levantarse de una silla 0 acostarse y levantarse de la cama?

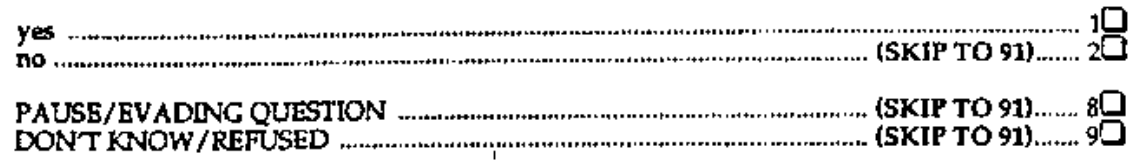

89. ¿Cuf́nta dificultad thene para centarse y levantarse de una silla o acostarse y levantarbe de la cama - dirfa que tiene un poco de dificultad, mucha dificultad, o que no puede hacer esto sin ta ayuda de otra persona o de algún equipo especial?

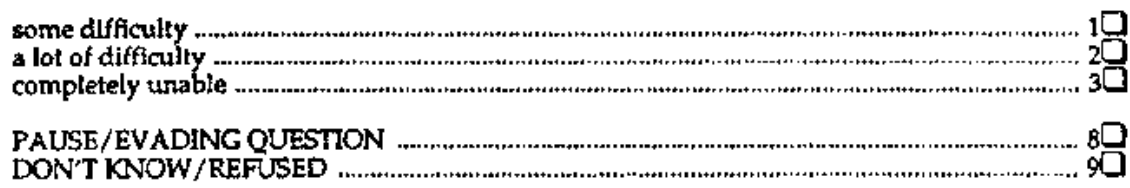

89. ¿Recibe ayuda de otra persona para sentarse y levantarse de una silla o acostarse y levantarse de la cama?

yes $\rightarrow$ ASK: ¿Quién lo ayuda?

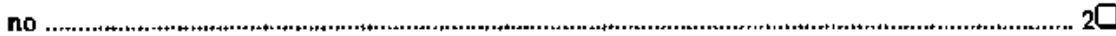

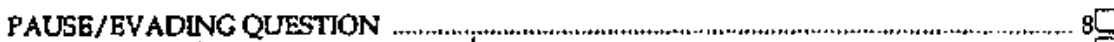

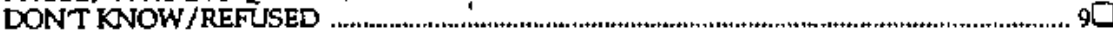

90. 2 Usa usted algún equipo espectal de ayuda para sentarse y levantarse de una silla a acostarse y levantarse de la cama?

yes $\rightarrow$ ASK: iQué equipo usa?

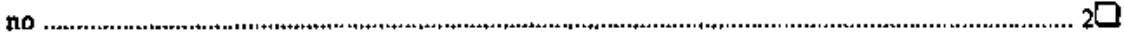

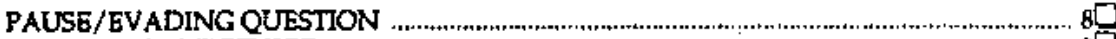

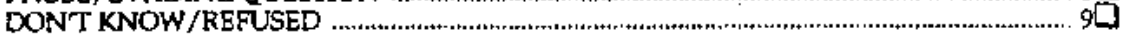

91. Debido a un problema de salud ofisico ¿liene dificullad para subir $y$ bajar escaleras?

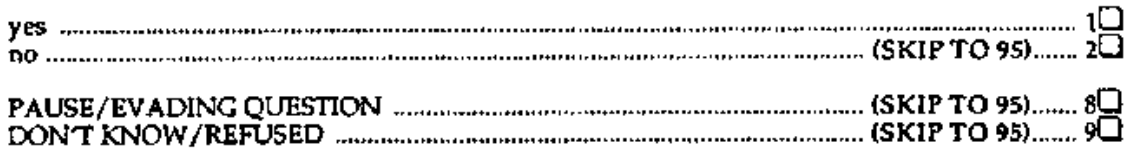

92. 2Cuśnta dificultad thene para subir y bajar escaleras - diria que tiene un poco de difieultad, mucha dificultad, o que no puede hacer egto sin la ayuda de oba persona o de algún equipo especial?

some difficulty

a Jol of difficalty

1

completely unable

2

PAUSE/EVADING OUESTION

DON'T KNOW/REFUSED ...................................................................................................

93. ¿Reclbe ayuda de olra persona para subtr y bajar escaleras?

yes $\rightarrow$ ASK: ¿Quién lo ayuda?

no.

$2 \square$

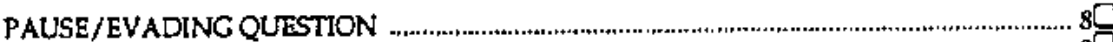

DONT KNOW/REFUSED …

94. ¿Usa equipo especial de ayuda para aubir y bajar escaleras?

yes $\rightarrow$ ASK: ¿Qué equipo usa?

no

PAUSE/EVADING OUESTION

8

DONT KNOW/REFUSED 
95. Debido a un problema de salud o físico ztiene diflcultad para caminar?

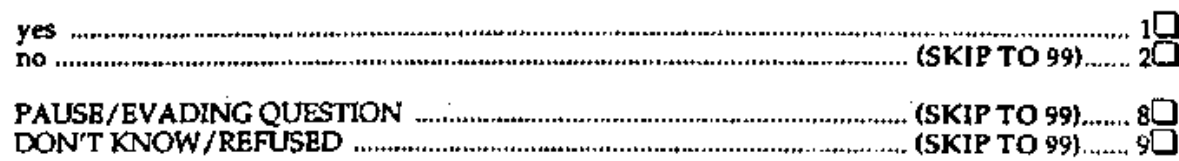

96. ¿Cuánta dificultad tlene para caminar - dirfa que tiene alguna dificulfad, mucha dificultad, o que no puede hacer ésto sin la ayuda de otra persona o de algún equipo especial?

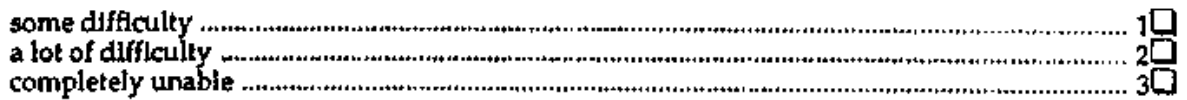

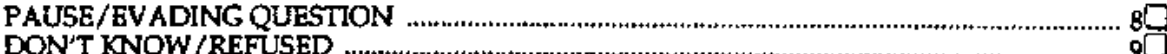

97. ¿Recibe ayuda de olra persona para caminar?

yes $\rightarrow$ ASK: ¿Quién lo ayuda?

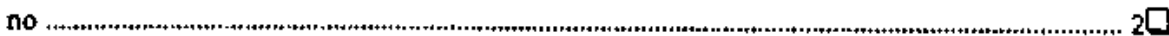

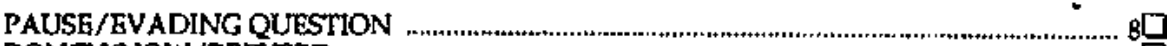

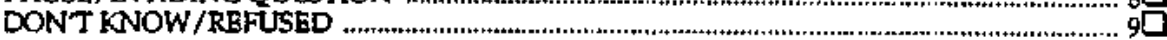

98. ¿Usa equipo especial de ayuda para caminar?

yes $\rightarrow$ ASK: ¿Qué equipo usa?

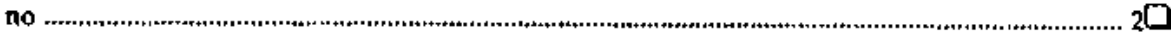

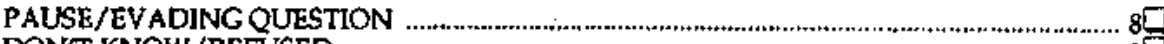

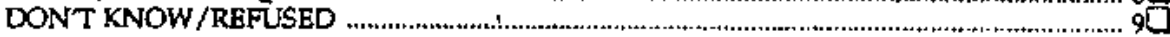

99. Ahor: quisiera preguntarle sobre otras activtdades. Debido a un problema de salud o físico ¿tiene diflecultad para preparar la comida?

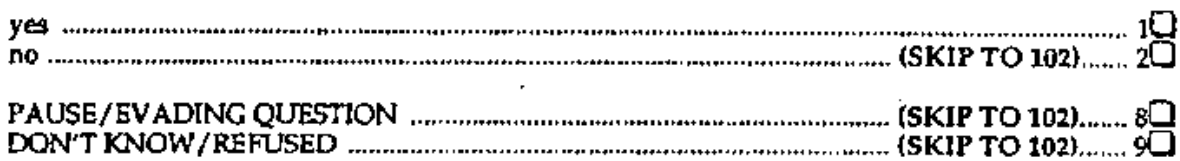

100. ¿Cuśnta dificultad tlene para preparar la comida - dirla que tlene un poco de dificultad, un ucha dificultad, o que no puede hacer esto sin ayuda?

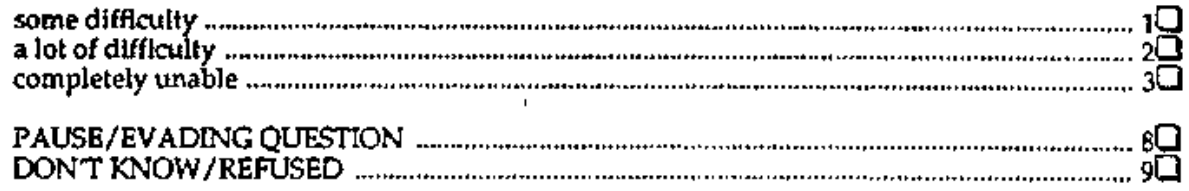

101. ¿Recibe ayuda de otra persona para preparar la comlda?

yes $\rightarrow$ ASK: 2 Quién lo ayuda?

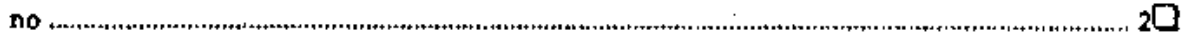

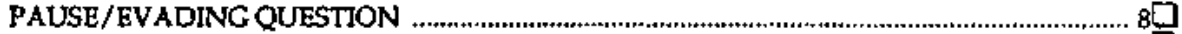

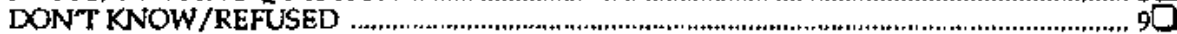

102. Debido a un problema de salud o físico ¿liene diftcultad para ir de compras de comestibles o ropa?

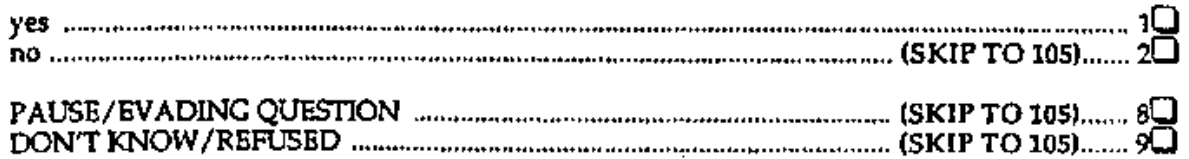


103. 2Cuśnta diffcultad tiene para tr de compra de comestiblea a ropa -- ditia que tiene un poco de dificultud, mucha difleultad, a que no puede hacer esto sin ayuda?

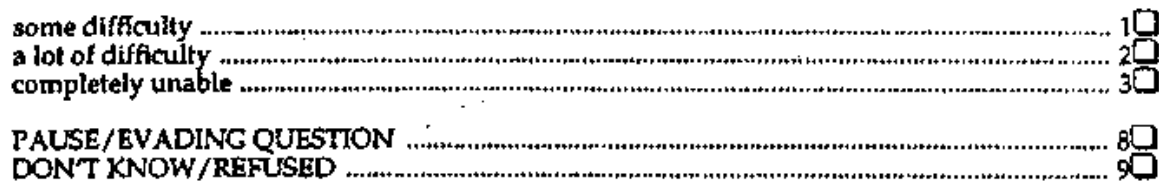

104. IRecibe ayuda de otra persona para ir de compras de comestibles o ropa?

yes $\rightarrow$ ASK: 2 Quién to ayudat

no

PAUSE/EVADING QUESTION

DON'T KNOW/REFUSED

105. Debldo an problema de spiud of fgico Ltiene dlficultad para administrar su dinero y pagar sus cuenlas?
yes (SKIP TO 108)...... 2
PAUSE/EVADING QUESTION (5KIP TO 109) ....... 8 DONT KNOW/REFUSED (SKIP TO 108).......

106. ¿Cuínta diflcultad tiene para administrar su dinero y pagar sus cuentas - diria que tiene un poco de dificultad, mucha diflcultad, o que no puede hacer esto sin ayuda?

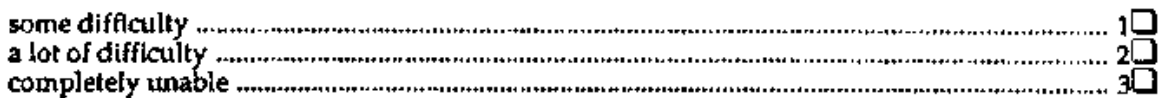

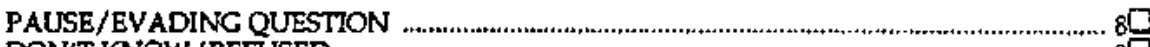

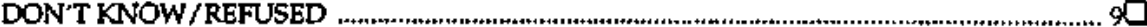

107. ¿Recibe ayuda de otra persona para administrar su dinero y pagar sus cuentas?

yes $\rightarrow$ ASK: ¿Quifn lo ayuda?

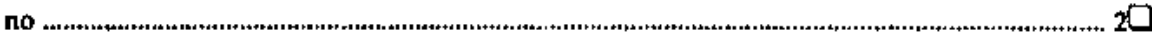

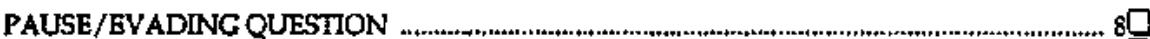

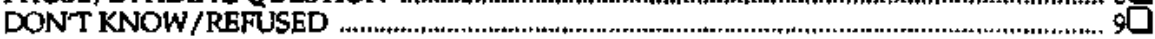

108. Debido an problema de salud ofílco \&tlene dificultad para marcar y usar el teléfono?

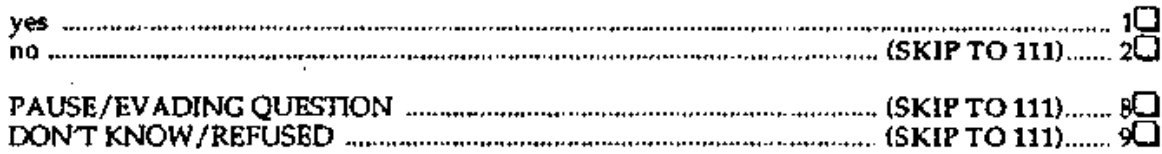

109. ¿Cuánta dificultad tiene para marcar y usar el teléfono - diría que tiene un poco de dificultad, mucha dificultad, o que no puede hacer esto sin ayuda?

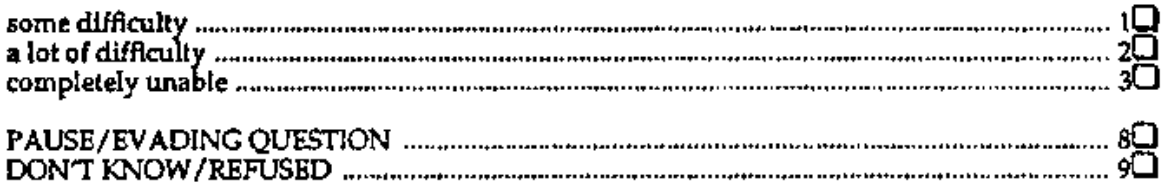

110. LRecibe ayuda de otra persona para marcar y ugar el teléfono?

yes $\rightarrow$ A.SK: ¿Qulen lo ayuda?

no .…... $2 \square$

PAUSE/EVADING QUESTION DONT KNOW/REFUSED 
11. Debido un problema de aluad o fislice tlene dificultad para lavar la ropa?

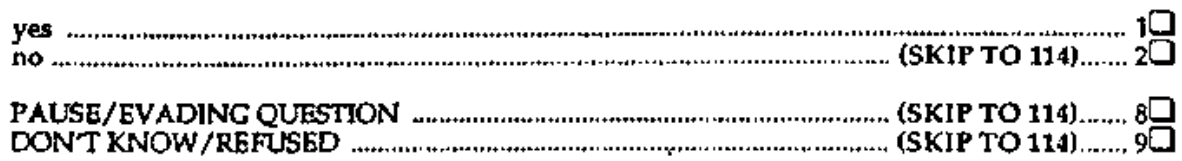

112. ¿Cuanta dificultad tiene para lavar la ropa - dinia que tiene un poco de dificultad, mucha dificultad, o qute no puede hacer esto sin ayuda?

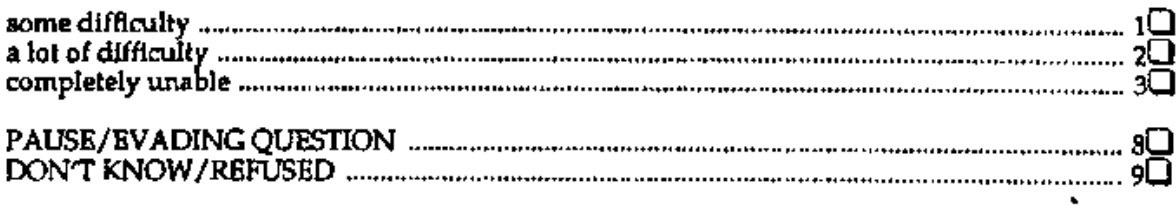

113. ¿Recibe ayuda de otra pergona para lavar la ropa?

yes $\rightarrow$ A ASK: ¿Quién lo ayuda?

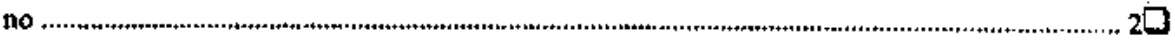

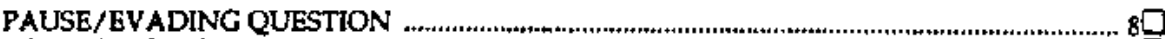

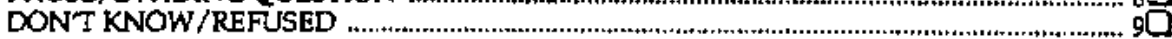

114. Debido a un problema de salud o fisico, tiene dificultad para hacer las tareas del hogar?

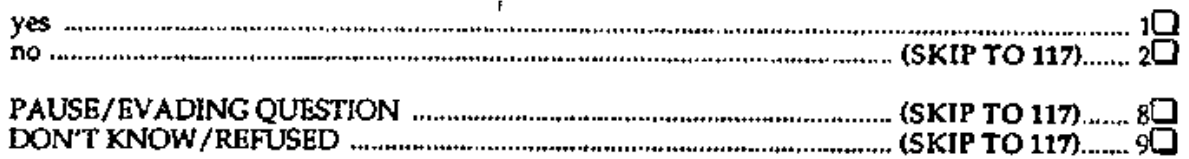

115. ¿Cuínta diflettlad tiene para hacer las tareas del hogar - diría que tiene un poso de dificultad, mucha dificultad, o que no puede hacer esto sift ayuda?

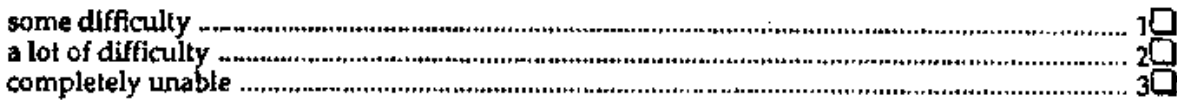

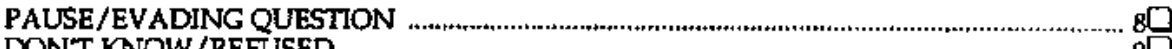

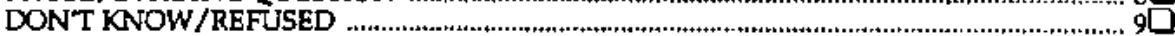

116. ZRecibe ayuda de otra persona para hacer las tareas del hogar?

yes --> ASK: ¿Qulén lo ayuda?

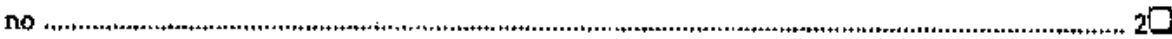

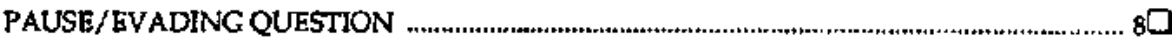

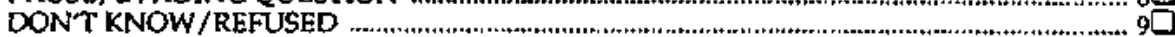

117. Debido a un problema de sajud o fisico tiene dificultad para ir a los lugares a donde la hace falta ir?
yes
(SKIP TO 120)....... 20
PAUSE/EVADING QUESTION
(SKIP TO 120)...... 8 8
DON'T KNOW/REFUSED (SKIP TO 120)...... 9

11B. ¿Cuśnta dificultad liene para ir a los lugares a donde le hace falta ir - diría que tiene un poco de dificultad, mucha dificultad, o que no puede hacer esto sin ayuda?

some difficulty

a lot of difficulty

10

completely unable 
119. ¿Reclbe ayuda de otra persona para is a los lugares a donde le hace falta ir?

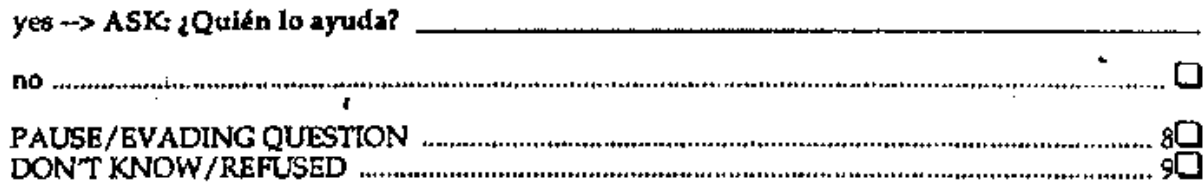

120. ¿Hay algunas otras actividades importantes que yo no he mencionado y que le resultan dificiles para usted?

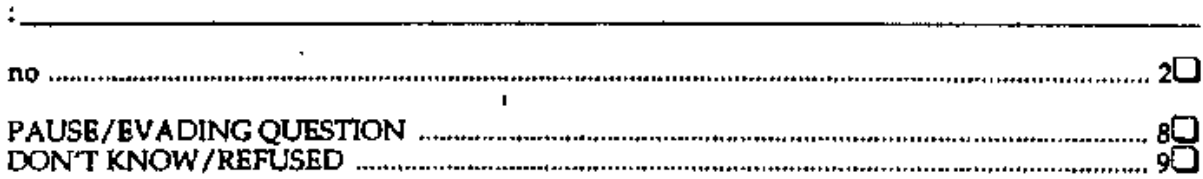

122. ¿Necesita ayuda adicional con alguna de sus actividades?

no 20

PAUSE/EVADINGQUESTION 8 DONT KNOW/REFUSED

123. Si necesitara información sobre tos servicios disponibles para personas mayores $2 a$ dónde iría a buscar esta información?

(DO NOT READ LIST $\rightarrow$ CHECK ALL THAT ARE MENTIONED)

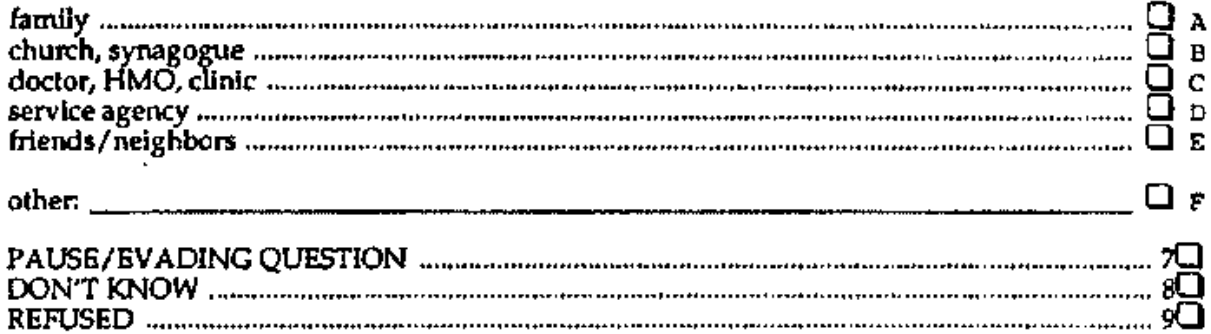

124. Ahora quiero preguntarle sobre otos asuntos. Primero, ¿Como calíficaría su salud emocional o salud mental - dirfa que es excelente, bueno, regular o mala?

excellent

good

poor

PAUSE/EVADING QUESTION

DONT KNOW/REFUSED

125. En general, zcuán satisfecho (a) está con su vlda de los pasados seis meses - điria que muy satisfecho, mayormente satisfecho, mayormente descontento o truy descontento?

very satisfled

mostly satisfied

mostly dissatisfied

30

very dissatisfied

PAUSE/EVADING QUESTION

DON'T KNOW/REFUSED 
126. Le voy a menclonar varias cosas que pueden convertirse en problemas y quisiera que me dijera cúf́r serla es cada cona para usted. Primero, el dinero. Me pudiera decir si el dinero es un problema muy serio, un problema algo serio, o no es un problema serio para usted? (READ LIST)

\begin{tabular}{|c|c|c|c|c|c|}
\hline & $\begin{array}{c}\text { very } \\
\text { serious }\end{array}$ & $\begin{array}{l}\text { somewhat } \\
\text { terious }\end{array}$ & $\begin{array}{c}\text { not } \\
\text { serious }\end{array}$ & PAU/EV & DK/NA \\
\hline $\begin{array}{l}\text { (DNERO) } \\
\text { su salud } \\
\text { soledad } \\
\text { miedo at crimen } \\
\text { estrés familiar o personal } \\
\text { arreglos de vivlenda } \\
\text { transportacion }\end{array}$ & $\begin{array}{l}10 \\
10 \\
10 \\
10 \\
10 \\
10\end{array}$ & $\begin{array}{l}2 \square \\
20 \\
20 \\
20 \\
20 \\
20 \\
20\end{array}$ & $\begin{array}{l}30 \\
30 \\
30 \\
30 \\
30 \\
30 \\
30\end{array}$ & $\begin{array}{l}80 \\
80 \\
80 \\
80 \\
80 \\
80 \\
80\end{array}$ & 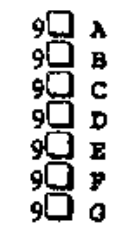 \\
\hline
\end{tabular}

127. Ahora le voy a preguntar cuínto le preocuparía a usted ciertas cosas para el futuro. Primero, la pérdtda de su independencia debido al deterioro físico - diría que le preocupa mucho, le preocupa un poco, o que no se preocupa de esto para el fuluro?

\begin{tabular}{|c|c|c|c|c|c|}
\hline & $\begin{array}{l}\text { very } \\
\text { worried }\end{array}$ & $\begin{array}{l}\text { somewhat } \\
\text { worried }\end{array}$ & $\begin{array}{l}\text { not very } \\
\text { worrled }\end{array}$ & PAU/EV & DK/NA \\
\hline $\begin{array}{l}\text { (PÉRDIDA DE INDEPEND } \\
\text { deterioro mental } \\
\text { convertirse en una carga } \\
\text { financiera }\end{array}$ & $\begin{array}{l}\text { IA) } 10 \\
10 \\
10\end{array}$ & $\begin{array}{l}20 \\
20 \\
20\end{array}$ & $\begin{array}{l}3 \square \\
3 \square \\
3 \square\end{array}$ & $\begin{array}{l}80 \\
80 \\
80\end{array}$ & 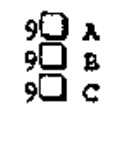 \\
\hline $\begin{array}{l}\text { soledad } \\
\text { ser victima de fraude o de } \\
\text { un estafador }\end{array}$ & $\begin{array}{l}10 \\
10\end{array}$ & 20 & $\begin{array}{l}3 \square \\
3 \square\end{array}$ & $\begin{array}{l}80 \\
80\end{array}$ & 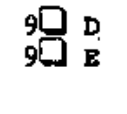 \\
\hline $\begin{array}{l}\text { ser víctima de algún otro } \\
\text { crimen }\end{array}$ & 10 & 20 & $3 \square$ & $8 D$ & $9 \mathrm{~F}$ \\
\hline $\begin{array}{l}\text { arreglos de vlvlenda } \\
\text { ir a un asilo de ancianos } \\
\text { ir a los lugares a donde }\end{array}$ & $\begin{array}{l}10 \\
10 \\
10\end{array}$ & $\begin{array}{l}2 \square \\
20 \\
2 \square\end{array}$ & $\begin{array}{l}30 \\
30 \\
30\end{array}$ & $\begin{array}{l}8 \square \\
8 \square \\
8 \square\end{array}$ & $\begin{array}{l}9 \square \\
9 \\
9 \\
9 \\
\text { I }\end{array}$ \\
\hline
\end{tabular}

128. Pensando en su vida en general, ¿Qué sería lo que a usted más le preocupa?

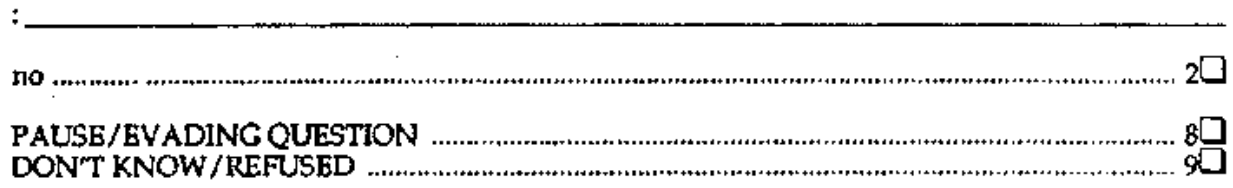

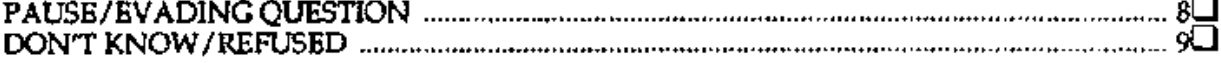

29. Ahora tengo solamente algunas preguntas de clasifieactón. Primero, ¿me podría decir su edad?

PAUSE/EVADING QUESTION

8

DONT KNOW/REFUSED.

130. ¿Está casado(a), es viudo(a), está divorclado(a), está separado(a), o nunca se caśb?

married

widowed

10

divorced

separated

never married

2

4

other:

PAUSE/EVADINC QUESTION

DON'T KNOW/REFUSED 
131. ¿Cuánto tiempo lleva viviendo en el domiclito donde vive ahora?

PAUSE/EVADING QUESTION

DON'T KNOW/REFUSED'

132. ¿Cuśl es au código postal?

PAUSE/EVADING OUESTION

133. ¿Cuánto tiempo lleva viviendo en el Condado de Dade?

PAUSE/EVADINC QUESTION

DON'T KNOW/REFUSED

134. ¿Cusntos meses del aho reside en la Florida?

PAUSE/EVADING OUESTTON $88 \square$

DONT KNOW/REFUSED

135. ¿Cuát es su religión?

Protestant

Catholic

Jewish

no preference.

... 40

other.

PAUSE/EVADING QUESTION

DONT KNOW/REFUSED

136. ¿Cujin ismportante es la relfgión en su vida - diría que es muy importante, algo Imporlante, o que no es muy importante?

very important 10

somewhat important 2

not very important 36

PAUSE/EVADING QUESTTON …............................................................................ 8

DON'T KNOW/REFUSED ......................................................................................... 9[

137. ¿Cusl es su raza?

white

black 10

Asian . 2

American Indian

other: raza? ASK: Se considera usted de la raza blanez, negra o alguna otra white 10 black $2 \square$ something else $2 \square$

PAUSE/EVADING QUESTION 8 DONT KNOW/REFUSED

138. 2Es hispano(a)?

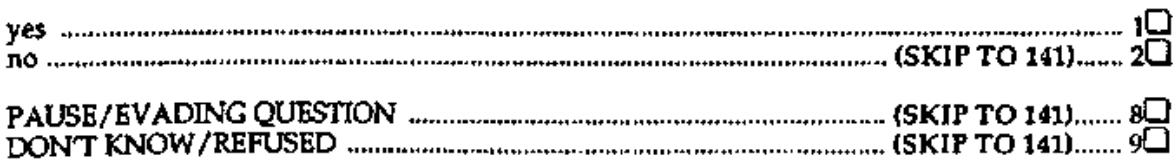


139. ¿En qué pais nact6?

United States. (SKIP TO 141)....... ]

PAUSE/EVADING QUESTION

(SKIP TO 141)...... 8 DON'T KNOW/REFUSED (SKIP TO 141)....... 9]

140. ¿En que año vino a los Estados Unidos de forma definitiva?

PAUSE / EVADING QUESTION 8 DONT KNOW/REFUSED

141. ¿Cust es el grado de escuela mis avanzado que usted ha completado?

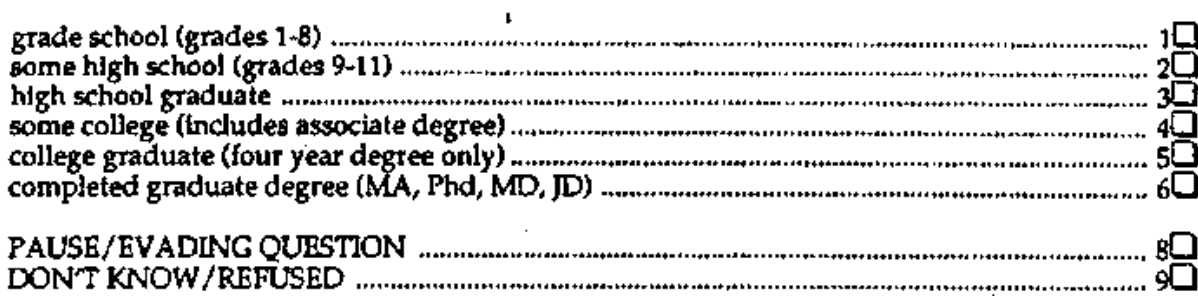

142. LQué fdiorna habla usted mayormente en su casa?

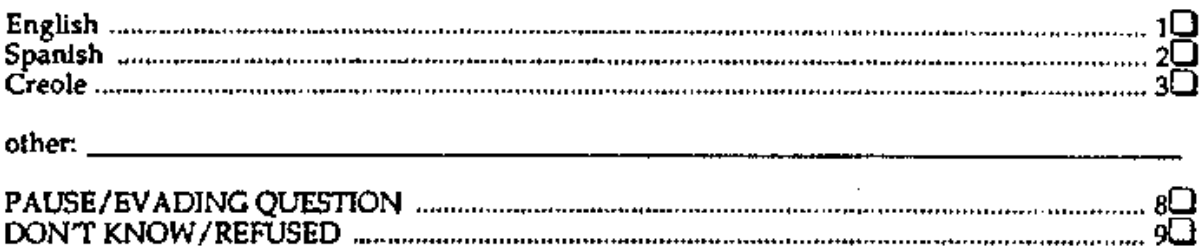

143. ¿Lee el pericdico regularmente?

yes $\rightarrow$ ASK: ¿Qué periodico?:

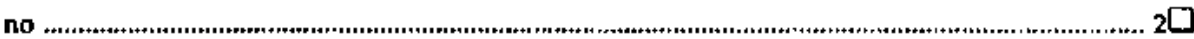

PAUSE/EVADING QZESTION .................................................................................... 8

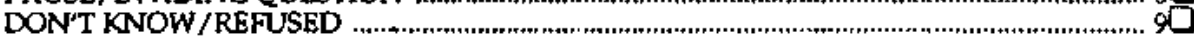

144. SI no le molesta, quistera saber el total de ingresos de su hogar, de todas las fuentes, antes de los impuestos, zes menos de $\$ 5,000, \$ 5,000$ a $\$ 10,000, \$ 10,000$ a $\$ 20,000, \$ 20,000$ an $\$ 30,000, \$ 30,000$ a $\$ 50,000, \$ 50,000$ a $\$ 70,000$, o más de $\$ 70,000 ?$

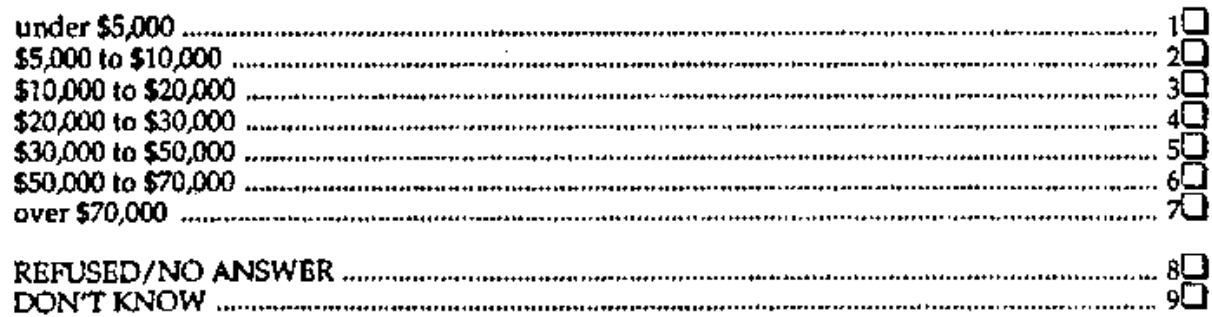

145. Finalmente, lengo unas cưantas preguntas sobre los efectos del huracón Andrés en usted y en su hogar. Primero, ¿residia en el sur de la Florida cuando pasó el huracín Andrés?

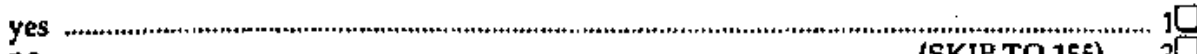
no ..................................................................................................... (SKIP TO 155)

PAUSE/EVADING QUESTION (SKIP TO 155),..... 8 DON'T KNOW/REFUSED (SKIP TO 155)...... 9 
146. ¿Evacuó su residencia antes de que e! huracian pasara?

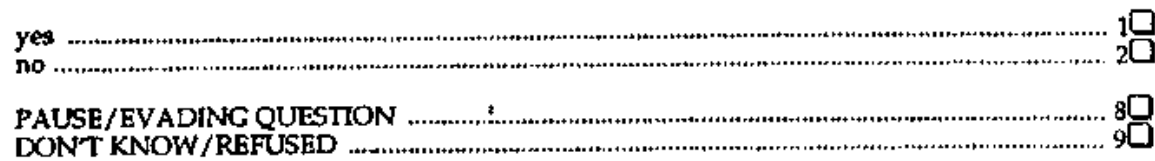

147. ¿Se vio obllgado (a) a mudarse de su casa después del huracán, debido a problemas causados por el huracón?

yes $\rightarrow$ ASK: ¿Por qué razón o razones se mud6?

no

(SKIP TO 149) ....... 2[

PAUSE/EVADINGQUESTION

(SKIP TO 149)...... 8Q

DON'T KNOW/REFUSED

(SKIP TO 149)......,

148. ¿Cuśnto hempo estuvo fuera de esta casa, o todavla no ba regresado?

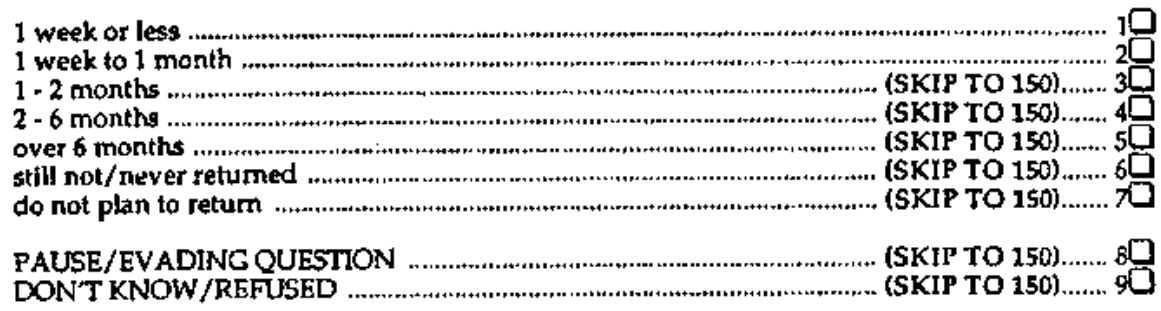

149. ¿Acogió en su casa a alguien que se quedó sin casa como resultado del huracán Andrés?

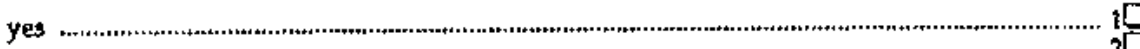

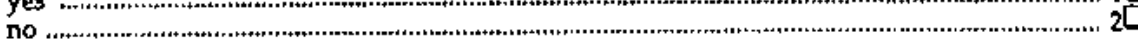

OTHER:

PAUSE/EVADING QUESTION

DONT KNOW/REFUSED

150. Diría que el huracán Andrés tuvo un gran efeclo, un efecto moderado, un efecto menos o que no tuvo ningún efecto en su galud física?

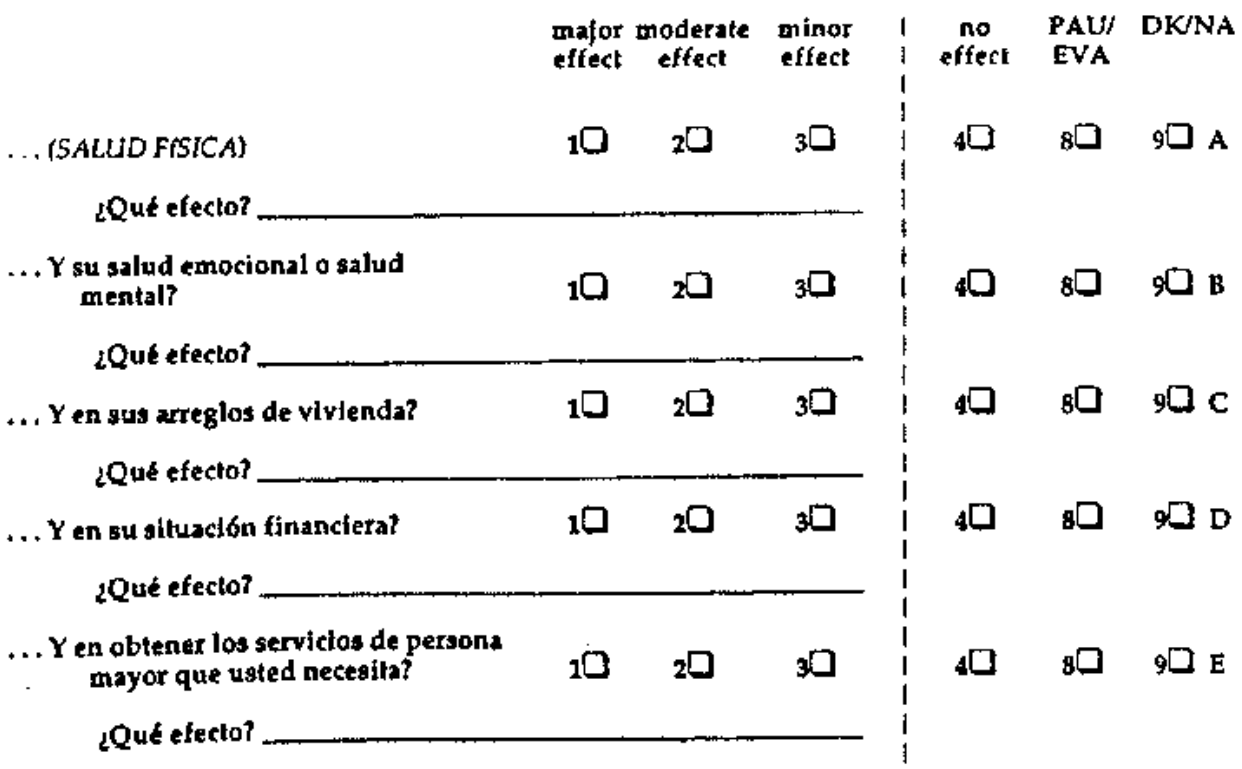


151. ¿Cuán preparado (a) se siente que está si un hurać́n como Andrés azotase de nuevo el Sur de la Florlda - muy preparado (a), algo preparado (a) o no se siente preparado (a)?
very prepared
(SKIP TO 153)....... 1
somewhat prepared
not at all prepared
PAUSE/EVADING QUESTION
(SKIP TO 153)....... 80
DONT KNOW/REFLSED
(SKIP TO 153)

152. ¿En qué forma slente que no está preparado (a)?

PAUSE/EVADING QUESTION

NONE/DON'T KNOW/REFUSED

153. Dentro de poco reallzaremos un estudio para lnvestigar cómo el huracán Andrés afectó las vidas de los residentes del Sur de la Florida. ¿Estaría dispuesto a que lo (la) llamemos para participar en este estudio?

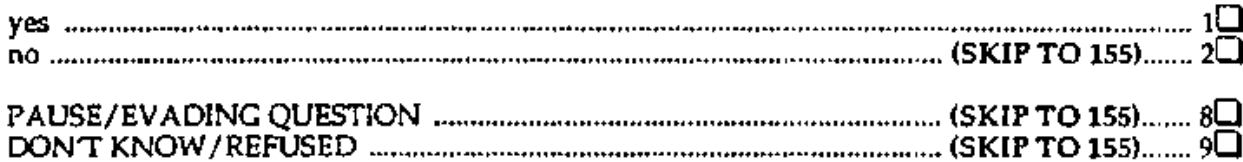

154. ¿Podríamos saber su nombre para cuando lo llamemos?

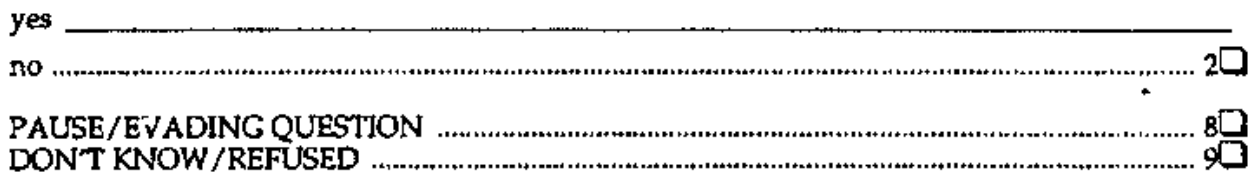

155. Bueno, esto concluye la entrevisła. Quiero agradecerle su cooperación. ¿Tiene algün comentario que le gustaria que yo anotara?

no

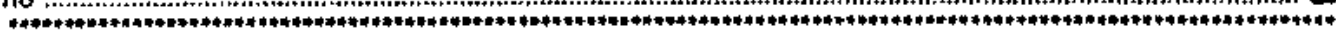

\section{RESPONDENT WAS:}

atert and responsive ......................................................................................................... 10

satisfactory ....................................................................................................................... 2 2

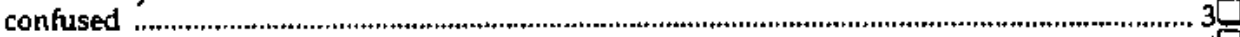

barely able to respond .......................................................................................................

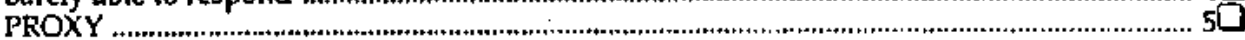

other:

RATE THE QUALITY OF THIS INTERVIEW FOR RESPONDENT'S UNDERSTANDING:

very good

satisfactory

confused

barely able to respond

PROXY

other: 


\section{RESPONDENT'S UNDERSTANDING OF ENGLISH:}

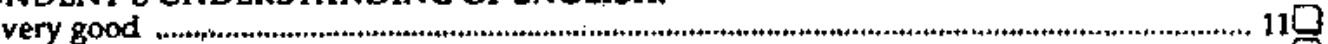

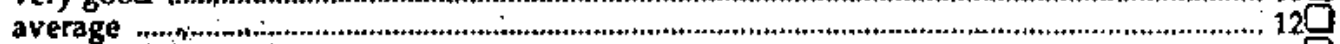

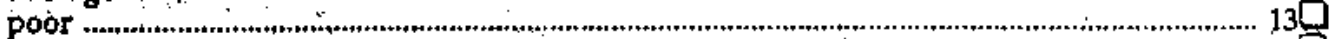

Interviewed in Spanish ........................................................................................................ 140

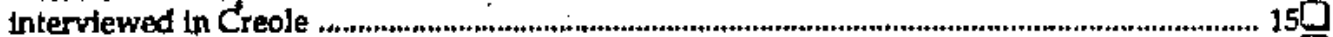

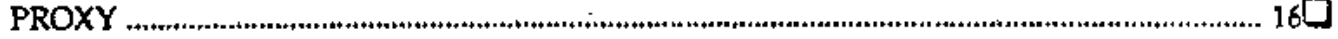

PROXY:

yes :

no

FINISH TIME: 


\section{APPENDIX D:}

TRANSCRIPTS OF SIX FOCUS GROUPS 


\section{Focus Group 1: Northeast Dade}

DESCRIPTION OF FOCUS GROUP: Middle and upper-middle class white, nonHispanic, over the age of 75 years old, organized through the Northeast Dade Coalition (Northeast Dade).

This group numbered approximately 20 participating individuals. Several of those seated in the group did not participate. One in particular did not participate because they had Alzheimer's disease and was the spouse of a participant. While this group was somewhat large for focus group, the participants had courteous exchanges and did not interrupt while someone else was talking.

What things do you like most living in the community that you do?

Weather

Equals of age group, weather allows getting together, convenience to shopping, accessible to wide variety of activities

Casualness - going out to dinner without dressing up.

What are the concerns you have of living in your community?

There is no one to speak for the elders, would like to have a group consult with seniors to tell them the "real" problems that elders face, rising taxes, they pay for everything and get nothing.

Don't have anything, can't find where to go to get information.

Crime is a problem.

Transportation is a problem, some condos have buses but other places don't have buses to go to malls.

Many residents in NE Dade, retired with many activities, however with the aging process many retirees are changing their activities because they are less mobile - aging in place is becoming a problem.

Need full-time social worker, need outreach workers so that elders can get help. 
Social isolation is a big problem in condos, even though there is activity.

Transportation - even going to FIU and JRS (Now called Pro Player Stadium) the bus stops 5 blocks away, and some elders can't walk the 5 blocks to get into the stadium.

Transportation is the primary concern - aging sometimes forces individuals to stop driving, buses would be good but not all areas are served.

Even with buses, (Older gentleman who identified himtself as President of his condo association) buses need to be accessible to elder women - need kneeling buses since they can't get up the stairs.

Metro Police calls are not responded to quickly enough regardless of the condition.

Don't know what services are available to the elderly.

(One participant gave information about Special Transportation System - STS - access).

Need lists of people with disabilities and services available.

Have the right to complain

Complaint - NE Dade Coalition has their meetings in the evening. (However, it was noted that the meeting times have recently been changed)

Participant who identified himself as part of the AARP Legal Committee said he has a list of all services available.

Theatre Performing Arts, they are here in the area, but it is easier for me to go to New York City to see plays, by flying there and seeing them there then to go to downtown Miami to see them in Miami.

Exercise, senior citizens don't exercise enough - facilities are available, but ... [FADE OFF] (Author note: I would say that it is the motivation of elders that is in questionthey need to be aware of the benefits and have the group push to get exercising) 
You mentioned FEAR OF CRIME as a concern for you or for others your age. To what extent is FEAR OF CRIME a problem for you or for others your age? How does it affect the way you lead your life?

Don't go out at night.

Not only at night is fear of crime an issue but also during the day.

Don't go out anytime alone.

Going out with another person of the same age isn't the answer, need more police, need to live defensively.

With the fear of crime high, don't congregate outside after dark, -- calling the police isn't useful.

One participant who identified himself as a member of Crime Watch stated that nothing was accomplished

Getting older, fearful of going out because don't know the area, or eyesight and hearing are poor. Some condos have put fences around and a guard house around the condo to help prevent crime.

Shopping is a problem.

Crime is a matter of chance - however the chances are increasing as age increases.

Most important thing about crime, [PAUSE] Main point is that prisoners going into hotel, not a jail, get anything they want. There needs to be a change in the prison system work to pay back the society for the crimes they committed.

No plea bargaining for criminals.

[Moderator interrupts to get back to the purpose of the focus group and moves on to the topic of frail elders with disabilities and special needs]

You mentioned FRAIL ELDERS WITH DISABILITIES and SPECIAL NEEDS, to what extent is this a problem and what can be done about helping frail elders with disabilities and special needs?

In the condos, there is an increasing number of wheel chairs. 
Most frail elders who need help have private paid nursing, homemaker, and if they can't pay, ... [PAUSE] don't know what to do. I guess they are out of luck.

A gentleman who brought his wife along, spoke up. His wife is suffering from Alzheimer's Disease. He noted that currently he is able to pay for services for care for his wife, but doesn't know what to do if the caretaker for his wife has a problem. More respite care is needed to help caretakers.

One participant state that in her condo, they have a "unique" method of dealing with help for frail elderly, have captains on every floor - alerting for handicapped needs during an emergency. Others in focus group spoke up that it wasn't unique that other buildings had a similar system. Although it appears that the system sometimes doesn't work.

One participant said that the focus group needs to address issue of what can the group advocate that the Alliance do or advocate for them. He stated two objectives: (1) What can elders/Alliance do to get around; (2) other things such as papers/legal, insurance issues in which the elder is unknowledgable.

Widows are particularly afflicted, and if they don't do anything their lives become unmanageable.

With computer technology, the Alliance should be able to give out a particular number for elders to call so that they can get information on a variety of issues.

It was also mentioned that there needs to be more individuals getting involved in calling frail or homebound elders to see if they need help - TELEPHONE REASSURANCE PROGRAMS

Need to have social services for frail elders - condo association can do so much but social services need to get more involved.

Need to have more outreach workers. 
There was a consensus about four main necessary services: 1) cooperation to get food, shopping assistance; 2) transportation - all around, but especially getting to medical appointments is difficult; 3) condo association are not social service agencies, elders need services such as chore services, etc; and 4) eldersly don't have anyone to talk to - being treated like dirt.

\section{What about family involvement in caregiving?}

Many family members do not live close by, others have family members that can't or won't care for them.

"Captain" method was brought up as a way to help those with far away family members

Buddy system, calls to elders without family members close by

Condo associations need phone numbers of places to call for help.

Elders don't want to take responsibility for care of other elder neighbors. Elder parents do not want to put pressure on their children.

What is necessary for elders to age gracefully is (1) Social Services; (2) Transportation; and (3) Feeling of Security.

It was mentioned by one participant that many elders come down to Florida for somewhat "selfish" reasons [her words] when they were young-old and not in need of help from family members, but now as they are growing older they do not want to take responsibility for other elders and they themselves are in need of help from family members. There was no argument from the group about this statement. The woman making this statement was youngold, she was accompanied by her mother who was $80+$.

The conversation moved back to transportation - someone mentioned that Eastern Shores has no transportation, NO BUSES serve the area, but there are many elders in need of transportation down there. 
Moderator moves conversation back to caregiving, but this time asks about neighbor involvement. What are neighbors responsibilities? How far can one rely on neighbors?

It was mentioned that some elders refuse to have a buddy system, they don't want it. While other condo associations and elders in those associations want and do have a buddy system.

I want to ask you about sources of information or help. You have already mentioned some when we first started this discussion. Specifically, where or who would you contact if you needed help.

\section{9-1-1}

Condo association president, but outside the condo there is a need for elders to have one central phone number to call, like 9-1-1.

It was mentioned that the Jewish Federation does have an Information and Referral Services (I\&R)

Doctor's can suggest services needed

Maintenance can be a problem, elders need to find information on chore services availability

There was another attempt to suggest establishing another 3-digit number, like 9-1-1 for non-police emergencies.

Telephone book has a number of phone numbers in the front that give information.

Another problem that was brought up was that although all the participants in this focus group live in condos, there are many elders who live in private homes - how can they get information?

This lead to a participant bringing up issues of Quality of Life - He brought up the fact that the adult education system has been lost, the classes have been cancelled. An important function of these classes was getting people out and keeping them functioning. 


\section{Focus Group 2: Homestead}

DESCRIPTION: Elders living in and around Homestead, the area most damaged by Hurricane Andrew, jointly organized by Musselwhite Senior Center and the Metro-Dade Community Action Agency (CAA). No restriction on age or socio-economic status, although participants from Musselwhite were Anglo and economically better off than participants identified by the CAA, who were a mix of blacks and Hispanics of varying national origin.

\section{SETTING:}

At the beginning of the focus group, participants were from Musselwhite. The CAA participants did not arrive until half-way through the 2 -hour session. Total participants once CAA group had arrived was 15. The room was in the back of the Musselwhite Senior Center.

\section{What do you like about living in your community?}

Zinnia: Climate

Rose: Neighbors and people feel like family. Have doctors that I can depend on.

Orchid: Don't know anywhere else.

How long have you lived in your community?

Marigold: Since June/July of this year, but I'm a native Floridian.

Orchid: 81 years

Geranium: 11 years

Daisy: 20 years

\section{What do you like about living in Dade County?}

Marigold: Family is here.

Geranium: Family

Primrose: Family

Daisy: I'm more impressed with Homestead than with Dade County. Police react faster from Homestead than from Dade County. 
Geranium: Depends on the emergency and dispatcher how fast help arrives.

\section{What concerns do you have?}

\section{Zinnia: Crime}

Generally agreed that crime is a problem. Things mentioned were muggings, beatings, break-ins and robbery. All pretty much agreed that the assailants weren't ustually caught.

How do you protect yourself?

Buttercup: I have dogs, but have had dogs beaten up and at least one died. It was a Doberman. Have fences.

Marigold: Become a Crimewatch member. Get an alarm system. Neighbors are good at watching out for one another.

Primrose: Double locks

Rose: Have dogs, a gun, and double locks.

\section{Other concerns?}

Daisy: Crime first, transportation second.

Marigold: I have a driver's license, but don't own a car. There's very little bus transportation.

Zinnia: No transportation in Homestead, if not on the beaten path then out of luck. Not even elderly services has buses.

Rose: Some HMOs provide transportation for doctor visits.

Geranium: Have to sit and wait hours in doctor office for return transportation.

Three out of the 8 belong to HMOs

Daisy: I had an elderly aunt that just recently passed away. Before she died we had been trying to find help, but couldn't get a lot of information or even help. She was put on waiting lists. After the hurricane, we brought her home 
for care and then she died. After she died, agencies started calling for placement.

Marigold: Hospice services.

\section{How about loneliness?}

Generally agree that loneliness was a problem and it was especially important after the hurricane because of the trauma.

\section{BREAK HERE - CAA participants arrive}

Carnation: There needs to be calling boxes around the community, bus services need to be expanded and the seats need to be bigger, more public toilets available where people walk.

What kind of service needs are there? What do persons your age need in carrying out activities of daily living?

Rose: Household help. I have a heart condition and my doctor says I shouldn't be over-exerting myself. But what can I do, the house needs vacuuming, laundry needs to get done. Also need gardening help, like lawn mowing.

Daisy: Agrees with Rose and adds We don't expect this help to be free but should be subsidized so that elders who need it can afford it.

Marigold: Most elders can't afford the going rates for housekeeping services.

Daisy: Transportation is an important service need.

Generally agree that transportation is important service need. Many don't have a car. They need bus service and it's too far to walk.

From Monday to Friday have some services, but on Saturday/Sunday there are NO services.

Who would you turn to for help?

Primrose: Family and friends 
Marigold: For transportation needs, need to schedule around family and friends and their schedules.

Primrose: My grand-daughter take me to my doctor and to get my medication.

Marigold: Family is usually around but friends ...

Geranium: Son, daughter-in-law both work. I do have a nurse, but otherwise nobody else. Social services didn't follow-up when I was ill.

\section{In what circumstances would you turn to Service Agency?}

Primrose: Shopping

Daisy: When I've exhausted family and friends; then I'll turn to a service agency.

\section{What about Church help?}

Marigold: Most churches only help out their own members, if don't go regularly then they don't do much. It isn't formalized help.

Carnation: [Agrees with Marigold and adds] I'm a member of a local church, but I don't get anything from them.

Hibiscus: Companion work, like 4 hours a day, mostly volunteer.

\section{What does one do if needs aren't being met?}

This seems to be a big problem, some sick, not old but are sick and no one comes to help.

Recreation is not available, need church visiting and community visiting, but if don't have health then can't do.

People are afraid because there is so much going on, i.e., crime. They close up their house and only see $t$, which makes it worse because the news sensationalizes the crime problem.

Elders need activities to keep them happy. Children move away, and many live alone. Some in apartments and some in their own homes. 
Daisy: mental problems such as depression and loneliness are a great problem.

Ilibiscus: Some don't have telephones. When sick, they need to communicate and be with friends. Without a telephone this is a problem. Apartment dwellers need to have a manager. The HUD project I'm in doesn't have no one to call if there is a problem or complaint.

Generally all participants in the group had telephones. About half of the participants had relatives in the area.

Hibiscus: Night watchman in HUD apartments are needed.

Six out of the 15 help another older person.

Generally there is a feeling that there is no central place for information.

Daisy: Even if have a telephone, it doesn't help because you're shifted around.

Generally, they don't know where to go or who to call for information. Also noted that many times the people that they are calling don't know themselves. Even if do find the right place to call, they are put on waiting lists.

Gardenia: Even before the hurricane, security was a problem.

Geranium: The language barrier is another problem in getting services and/or help.

Daisy: Cities take. There's a sense of out of sight, out of mind that is prevalent.

Hibiscus: If Andrew hadn't come through, none of the publicity of the problems we face would have been addressed. Well, ... [PAUSE] after Andrew nothing much has actually been done, but least there was some news media to pay attention to the problems.

\section{What are three to five of the things most important to seniors?}

Consensus that Transportation was number 1.

2. Fear of Crime 


\section{Employment and/or part-time activities \\ 4. Health issues \\ 5. Housing issues}

Now I'm going to ask you about where you go or who do you go to when you need information?

Marigold: Telephone book

Rose: I don't know where I got it, but I have a 2-page flyer with all sorts of helpful telephone numbers.

Buttercup: Miami Herald, the Ncighbors section. There is an elder section in the Sunday issue.

Daisy: City has phone numbers

Rose: The problem is that you can't get through. I would like to get some housework services, but I always get a busy signal or I'm told to wait on the phone.

\section{Focus Group 3: JESCA}

\section{DESCRIPTION OF FOCUS GROUP:}

African-American elders of lower socio-economic status between the ages of 60 and 69 years, organized through James E. Scott Community Association (JESCA) building on NW 54 Street in Miami. There were 16 total participants. There was only one male participant, all the rest were female.

\section{SETTING:}

The focus group was held in a large room with a long wide table, almost a conference table. The room was just off a large eating area. It was held between $10 \mathrm{AM}$ and $12 \mathrm{PM}$. There were light refreshments served.

What things do you like about where you live?

Lily: Quietness, no drug problems, safety.

Orchid: Cheap rent.

Chrsyth.: Security. 
Sunflower: Security.

Tulip: Help extended, i.e., transportation; lunch

Hibiscus: Help from social workers

Azalea: Attention and protection

Violet: Service of JESCA

Dahlia: (Lives in Claude Pepper Tower II) peaceful quiet, but there is a drug problem with younger people, need more security.

\section{What things do you like about living in Dade County?}

Dahlia: I was raised in Dade County.

Gladiola: Climate

Orchid: No snow

Violet: I'm from NJ. I came before I went into the service for a visit and then after the service came back.

Sunflower: Too old to move and was raised here.

Marigold: Came to South Florida in 1947, but need more activities available to elders. I'm 80 years old and I just call this home.

Dahlia: [Agrees with Marigold that there need to be more activities]

Sunflower: I'm 93 years old and the secret of keeping young is to keep active.

Magnolia: I like the neighborhood and the neighbors are concerned. [Lives in Batz Ctr]

What are the concerns you have of living in your community?

Daisy: Crime

Sunflower: Drug problem, crime problem, need lights in building, management hasn't done anything about it. Elders can't open their door in the evening; can't go in the elevator. 
Orchid: Crack dispensing, prostitution

Daisy: Fellowship, help being with others in the neighborhood.

\section{What about Transportation or getting places?}

Tulip: JESCA provides from CPI [Claude Pepper Tower I] ... they have a calendar and there is shopping two times a month; movides; Sadies to eat; recreation; hosiery stores.

Magnolia: Batz Ctr. there is no transportation, some seniors would like to go out.

Hibiscus: I live in Jollivette. We depend on CPI for transportation. On Monday, CPI has transportation for grocery shopping, more people could come to the center for activities if there was more transportation to the center.

Dahlia-Orchid agree that transportation is undependable on Monday, bu is more dependable on Thursday.

Daisy: Medical transportation is available to use services at the Geriatric Clinic at Cedars Medical Center, ... they have a pick-up service.

Dahlia: I walk to Jackson Memorial.

\section{What about LONELINESS?}

Dahlia: Activities are needed, loneliness is a problem.

Orchid: From Friday through Monday there are no activities available for seniors; there are no tables to sit at so they can play cards; or chairs to just sit and chat.

\section{Is there a need for help with Activities of Daily Living (ADLs)?}

Tulip: Senior companionship, someone to be able to do the odd shopping bits, all seniors aren't all the same, but some need a little bit more help than others.

Gladiola: Monitoring medication intake, timing of medication. 
Dahlia: Counselor for depression, mental and physical therapy. [It was found out after the focus group that Dahlia had tested positive for HIV]

Magnolia: Nced exercise, physical exercise.

Gladiola: Those with arthritis don't like to push themselves, but exercise is still needed.

Hibiscus: In Jollivette exercise is needed. We just need a tv with ver so can get tapes to do exercise classes. For handicapped, diabetes patients having to wait in the lines, say for food stamps, can be life threatening.

Azalea: Too often forgotten about ... my need to be happy, mentally uplifted, feeling better even with physical hardship. There isn't much out there to do activities-wise, and this is especially hard with elders on fixed incomes.

Violet: CPI since it opened, I've done volunter work and recently trained for senior aid but because a medical problem have had to cut back on activities. I've felt very depressed when $I$ had to cut back/back off the activities.

Daisy: Exercise classes are available at my center.

What about family help?:

Generally participants voiced that they didn't get much help from family members mainly because their adult children were also having a tough time to make ends meet.

If you do get help ...

Azalea: It's an individual thing, family isn't able to give money, but rapport is important.

Gladiola: I have nieces and nephews who give me help.

\section{What about neighbors and friends?}

Lily: I live in Sugarhills, there we extend help to others, like transportation. 
Tulip: In big buildings there should be a nurses station. Elders usually have a doctors visit every 2 months, but may need health stuff in the interim. Goes on to say something about "income, job, rent goes according to income charged for gross" Not sure what this means.

Dahlia: If do work/helping for money, food stamps can be cut.

Chrysth.: Copy machines are needed.

\section{What Agency in community would you go to for help?}

Hibiscus: In Jollivette, they refer to Pepper for a social worker.

Overall, most knew about JESCA, but did not know anything about Alliance for Aging.

Now I want to ask you about elders who aren't able to get all the help thcy need, what are their needs?

Hibiscus: Cleaning house

Carnation: I've worked with senior centers, community, those living with family or living alone. The most serious need is respite care, home care, transportation. Most don't know where to go and when they do they are put on waiting lists. Senior centers need more funding for agency help. A lot of seniors aren't getting help.

Lily: I have an aunt in her 80 s whose husband recently passed away [in the last 3 weeks]. She has been put on a list for other places to live, wants to get out of where she currently lives, her daughter works, she still has to pay for the rent in the old place. My aunt needs monitoring but basically can take care of herself.

Generally agreed that there is a lack of information, and then when the information is there, there is usually a waiting list.

Carnation: A lot of people don't know where to go. There needs to be more of focal point in the community for elders to go to for information. Churches are important. Get the information out to the churches and churches are good focal points. Have many church-oriented communities. 
Dahlia: Depends on others to get food, somebody to visit. monitor, need social workers.

Violet: Social workers in CPI are part-time, need more social workers. JESCA is helping.

Generally agreed that churches are good focal points for information dissemination.

\section{What are the barriers to why churches haven't done more?}

Tulip: Government has taken over.

Azalea: Resources in the church are a problem.

Gladiola: Plenty of money but doesn't go to elders.

Hibiscus: Some churches can do it, but some, like don't have cooking facilities aren't available in my church. Even though we don't have cooking facilities, we still adopted a family after Hurricane Andrew.

Carnation: Churches can do, it isn't expensive if just give out information. Within the congregation there are probably people who have skills to help elders, such as retired social workers.

Dahlia: cook food

Azalea: Organizations need to work together and stop reinventing the wheel.

Tulip: JESCA has two offices that people could go to for help.

Orchid: Elders need drama groups, they provide therapeutic [MISSING REST OF WORDS] Cleaning scams.

When you have concerns or needs that need to be met where do you go for information?

Gardenia: The phone book

Lily: The book, ... the CHOICE book was put out in 1992 by the Alliance, or was it the AARP?

How available? 
Carnation: They are in the centers, Alliance hasn't come out with 1993.

Tulip: JESCA office.

Azalea: Doctor and social worker

Daisy: JESCA and Joe Kelly's

\section{Is the Social Security Administration any help?}

Daisy: Once a year, but no other information. Problem is the rudeness of workers in government agencies.

Hibiscus: At the SS Office the waiting lines are TOO long.

Daisy: Young people get more attention than elderly. The elderly are pushed aside.

Gladiola: I've used the book from Social Security about understanding benefits.

Tulip: Social Security doesn't tell you everything unless you ask and already know what to ask.

\section{What other sources of information?}

Lily: CHOICE has helped me help other pcople in need.

Sunflower: At the county - Metro Dade County Elder Service - I turn to Fran Kramer. One of the problems is that many elders stay at home and don't try to get information. Some part of the problem is the elder not going out to get the information that IS available.

Hibiscus: Don't have transportation. Our center needs a typewriter and transportation.

Azalea: Knowledge is important. Feelings get hurt. There is a need to sit down with some elders to educate them. Elders need to be open to learning and those who are open, need to sit down and present information personally to those who may not be as open.

Violet: If information comes through mail, elders receive a lot of "junk" mail, some elders just throw it all away. Need to check mail. 


\section{Focus Group 4: Richmond Heights}

DESCRIPTION OF FOCUS GROUP:

African-American elders living in the Richmond Heights area of south Dade, no restriction on age or on socio-economic status. The majority however were better off than African American elders in the JESCA focus group. The focus group was organized through the Glendale Missionary Baptist Church. The Church building was badly damaged in Hurricane Andrew and had been razed approximately 3-4 months prior to the gathering. Many of the members had gone through Hurricane Andrew and had thought about seeking shelter at the church. The focus group had 9 participants, 7 of whom were female and 2 were males. The majority of the participants were long-standing residents of South Dade (20+ years).

\section{SETTING:}

The focus group was held in a smallish room in the outer church buildings that wasn't damaged by the hurricane. At the time of the focus group, Sunday services were being held in this building and would continue until a new church was built. The room we were using was one used for Sunday school for children younger than 10 years old. Religious art work done by the children decorated the walls of the room.

The devastation of Hurricane Andrew was still very apparent as we drove to the church compound. The demolished church site was not recognizable as having any building on it. The only reason we know that there was a church on the site was that we were told by participants.

The focus group was held between 2PM and 4PM.

How long have you lived in the neighborhood?

Daisy: 25 years in the area

Lily: 30 years

Tulip: 21 years

Snapdragon: 1 year

Poppy: 45 years

Rose: 47 years

Gardenia: 35 years

Geranium: since 1963 
Marigold: in Richmond Heights 14 years; in Dade County 67 years.

What things do you like about where you live?

Poppy: Sunshine

Marigold: Close knit neighborhood, well kept, well disciplined, pride in neighborhood, crime rate is lower, although still a concern. There is an investment of time and money in the neighborhood.

Geranium: The close-knitedness of the neighborhood, ... it means a lot to the neighborhood. The church is important to the neighborhood. It's friendly.

\section{What about Dade County?}

Geranium: Been in Dade County since December 24, 1940, people are easy to deal with/work with in Dade County.

Marigold: I agree. Dade County has a lot to offer. It has a potential to be greater.

Snapdragon: I've lived in the county since 1935 . I've seen a lot of changes, not as good as it was - changes are there, and not doing anything about it. In general, the lifestylc, young people are destroying themselves and others' property. WE need to get involved.

Poppy: Couldn't we as parents go back to the home and correct the problems. We need to have homes to help. Where do we start?

Marigold: Dade County has economic oppression [does the speaker mean "depression"?] job shortages. With the influx of people from other places, mostly foreign, the long time residents have trouble getting jobs. They are being deprived of jobs in the area by newcomers.

Rose: The environmental setup,... the social side. The social side for seniors is decreasing because of fear of crime, now economic state has passed. We were able to sightsee, grow flowers and there were other things to do. I'm currently working with little children. I'm teaching 
them speech patterns and behavioral patterns. The older children have drifted too far.

Money is generally agreed to be a problem

Health Care - AARP bulletin on cutting of health care coverage mentioned

Rose: Medicare provider is a problem, getting in a line for health care. Most elders don't want to beg.

Snapdragon: Going to the doctor, the cost of medicine is out of reach for a lot of elders.

Tulip: I couldn't afford health care/medicines without being in an IMO.

Gardenia: Seniors who don't yet qualify for Medicare need hospital coverage which can cost $\$ 300$ per month

Marigold: Transportation to doctor office is a problem.

Daisy Agreed with Marigold

Gardenia: If don't drive, what do you do when need to go to the doctor, then get prescriptions and do other errands?

Snapdragon: Special Transportation System (STS). Call-in a day ahead of when you need. It does work well. The charge used to be $\$ 1.50 / 10$ miles, it is increasing to $\$ 2.00$ straight fee. A lot of people make use of this service.

Rose: Elders don't drive at night. This poses a problem. Some can't drive at all. STS can be a solution but provided you have the money.

\section{What about LONELINESS?}

Rose: Another problem, some elders live alone. Their families are far away. Worrying is another related problem. Another senior need is mental health counseling. Frustration is a problem. Helplessness at not being able to do the things that used to be able to do or need to for daily living needs. Counselors are needed. Other things seniors, especially single seniors - the church should organize 
activities so that decrease frustration. Also general housekeeping, things that require lifting, and kneeling down, laundry.

Most elders don't want to go to nursing home and don't want to live with their children, they just need services to help them age in place.

Marigold: Elders need a place to congregate, to keep active a place where there is social activities and meals.

Tulip: Meals on wheels, a lot of people need meals to be brought to them.

Rose: After hurricane, now in trailers, families can help but some don't have families around them or near them. Senior center - JESCA is just opening one nearby. It will be providing meals, programs everyday. $A$ lot of people haven't heard about this new senior center, but word is getting out. Elders shouldn't stay inside, they need to get out and stay active.

\section{Are families providing help?}

Poppy: Not much.

Rose: I've helped my father, but haven't had to call anyone for myself.

Daisy: Children can be asked for different things.

Poppy: It really depends.

Gardenia: For transportation, ask friends.

Marigold: Friends are for companionship, telephone partners to break cycle of loneliness

Most people have a phone

What kind of things are the county agency helpful for?

Rose: Medicare, after Andrew some agencies were stranded [MY NOTES AREN'T CLEAR on what this was getting at] Don't like to beg and suffer. 
Marigold: Some elders need legal advise, legal documents, tax preparation.

Gardenia: Transportation issues ... Winn Dixie has a delivery service for a small fee.

Marigold: There need to be more senior citizen discouts.

\section{Could you describe the kind of person that doesn't get enough help.}

Rose: Individuals who make a little more than the $\$ 15,000$ cap income, those people fall into the gap - taxes are high but get ANY services.

\section{What kind of help is needed?}

Rose: Red Cross, church may help. If family has been burned out, there is a benevolence fund that is available, but not much. Social Security, welfare.

Most of these folks don't get SSI

Could get a grant after the hurricane, if didn't have insurance

Richmond Heights is an unincorporated area of Dade County

Rose: [Back to the point about help from the church] ... for elders that need help, a collection may be organized, but social security is assumed to take care of everything. The church only comes when there is a crisis.

Most participants said they really didn't know where to go for help. Basically, most said that they and other elders found things out by word of mouth. Another suggestion was to go to the local HRS office, where the food stamp place has information.

Lily: Elderly don't go because they don't get much for all the trouble they have to endure. 
Snapdragon: A lot of inconvenience to get nothing, organization is unfair to older people.

Another suggestion was the United Way. None of the participants when asked whether they receive anything in the mail said that they had, when asked about newspapers. didn't know of anything there. $7 V$ and radio was also suggested.

\section{Focus Group 5: Little Havana}

\section{DESCRIPTION:}

Working class Cubans primarily between the ages of 65 to 69 years and organized through Little Havana Activities and Nutrition Centers, Inc. The focus group was conducted in Spanish. (Little Havana) Moderated by Martha Pelaez, Associate Director of the Southeast Florida Center on Aging.

The group consisted of 16 participants: 10 females and 6 males.

After a brief introduction by the moderator:

I want to ask about the concerns elders have about living in Dade County/neighborhood. First I want you to focus on the assets/positive things about living in Dade County, and then I'll ask about obstacles or problems to quality of life that elders in Dade County face. First the benefits of living in Dade County.

Lily: Little Havana Activities Center

Orchid: Little Havana Activities Center is like a second home. Friends and neighbors have been living in the same place for 15 years.

Chrysth.: I like the activities at the center too.

Sunflower: I've got excellent neighbors, anglos, have keys to each other's houses.

Tulip: The social services

Hibiscus: Social workers are a great help and the nutrition center is a good place to come.

Azalea: The help given by social services. 
Dahlia: I've worked at the nutrition center for the past 5 years, it's my second home.

Gladiola: The activities, citizenship classes, and English classes.

Marigold: The social work department.

\section{Now I'm going to ask you to think about the obstacles or problems to quality of life that elders face living in the community.}

Gladiola: Rents keep increasing every year. I'm concerned that I might not be able to afford future increases.

Tulip, Marigold, Dahlia and Orchid: [had same concern as Gladiola]

Orchid: The center needs more income for additional transportation and better paid volunteers

[but if they are volunteers then why are they being paid?]

Sunflower: A basic problem is transportation for doctor's visits, to go get food stamps and for handicapped people.

Violet: The transportation problem affects the activities program at the centers. Activities are an important part of the program for elders. The buses are old and they require a lot of maintenance and need replacement.

Magnolia: Health insurance coverage of Medicare and Medicaid. The doctor coverage isn't enough in Medicare.

Sunflower: Medicare. There are a great number of retired persons without supplemental insurance 'cause they can't afford it. There are large numbers of persons who have to pay for prescription medicine and don't have enough money to cover other expenses

This goes to the heart of the issue of "robbing Peter to pay Paul" - Look at Mama might be better off dead: The failure of health care in urban America, about how Robert wouldn't take his high blood pressure medicine when he was feeling ok, so that they would last longer.

Azalea: I have insurance, but I don't qualify for a few dollars. I was told that I don't qualify for $\$ 2$.

Sunflower: There's misinformation regarding the procedures to apply and requirements for Medicaid. 
Orchid: Crime is the first problem. I'm afraid to leave my house to go out at night. I don't put the light on so they don't know if I'm at home.

Sunflower: The areas that were safe are now being invaded by crime.

Gladiola: I live at the entrance of Coconut Grove. I'm scared. I do everything before 6PM and I don't leave my house after $6 \mathrm{pm}$.

Daisy: Seniors need companionship. As they become frail they need a trained person to help with specific services.

Carnation: There need to be more meals [at homeimplied] for the stay at home elders.

Sunflower: There needs to be respite care for caregivers. Nursing homes - we need what and where information. Superior rated nursing homes are too expensive. There is total disinformation.

Violet: I've lived in the USA for 33 years. I'm retired now, but 1 need to work to be able to survive. My [SPOUSE] has Alzheimer's and is in an ACLF, but she is in need of a nursing home. I've been forever trying to get her into a nursing home. The person who provides information needs better training AND needs to keep information up to date.

Gladiola: I have a relative who pays $\$ 3,000$ a month for a nursing home. He is VERY WELL taken care of. Why doesn't the government supervise the quality in homes for poor people?

Magnolia: They need a gym to exercise and an exercise program. I'm a diabetic and I NEED to exercise. I can't walk in the street because I'm afraid of crime and getting mugged.

Marigold and Orchid: Both reiterate that there are statistics that say that life is prolonged by exercise. Exercise is a must for elders and that there needs to be a gym

If you nceded assistance with activities of daily living, that is eating, bathing dressing, toileting, getting in/out of a bed or chair, who would you go to for help? 
Orchid: The center

Sunflower: Family

Tulip: Family

Gladiola: Family

Gardenia, Chrysth. and Dahlia: Family

Lily: Agency

Daisy: Agency; family is too busy working.

Carnation: Family

Daffodil: I live with my daughter.

Dahlia: Very few can really turn to their family

Sunflower: Family will complement help given by agency.

Family can not give assistance on a daily basis.

Lily: Family can help on a crisis but not with daily tasks.

Tulip: I'll call 911 . I don't have any relatives.

\section{Who do you know that needs help but get nothing}

Daisy: Miami Beach nutrition center (about 180 participants) - about 20 percent need more assistance than they are getting; they have become frail and cannot take care of themselves.

Rose: About 30 percent of elders that go to the nutrition centers need Adult Day Care. There's a lack of space and personnel.

Sunflower: In Sofge Haley's nutrition center about 95 percent will need Adult Day Care. The age is very high.

Violet: There's a lack of hygiene of the participants because of no assistance or no acceptance that there is a problem.

Dahlia: Two times a week isn't enough for basic hygiene and for food needs. These people need more assistance to be able to live alone. 
Carnation: We need better nursing homes and more information. The Anglos I know are more ignorant of agency services. There is more information in the Latin community.

Sunflower: The mouth-to-mouth information is incorrect. Persons are afraid to ask for fear that they will take away what they presently have. They hear that there is abuse and they just become afraid.

We're just finishing up hurricane season, but I'd like to ask a few questions about hurricane preparedness. First, where do you get your information about hurricane preparedness?

The majority stated television or radio

How about assistance for preparing for a hurricane?

Lily: My family, especially my son.

Orchid: My family

Camation: My family. I will stay at home the next time one comes.

How about for hurricane repairs?

The consensus was that they will get assistance from their families.

Violet: I'd go to a shelter. You're more secure there. Your family helps in a crisis, but those don't have resources for long term assistance

Carnation: Family helps, but can't provide everything.

|There is a note that states that only one of the participants resided in the area most heavily damaged by Hurricane Andrew]

Now I want to turn to another topic. The Southeast Florida Center on Aging has been asked by the Area Agency on Aging to do a tclephone survey of elders, asking about their needs and concerns. What would or would not make you answer a phone survey? 
Tulip: I'm afraid. I'm afraid of who calls and for what. You need to identify yourself and state the purpose of the call.

\section{What are the best times for calling?}

Consensus was between 4 and $7 \mathrm{pm}$. Don't call during the soap opera time.

Violet: Identify yourself and tell the person the purpose of the call and how the survey will help them and other elders.

Azalea: Don't ask for social security numbers. We've been instructed to hang up!

Carnation: Don't ask my name or if I'm alone.

Tulip: Prepare the questionnaire well and make nonthreatening questions.

Sunflower: We're all afraid of crime and fraud, so don't ask information about whether we live alone.

That completes the questions I have. However, before we break up are there any comments you have that you want to share?

Lily: There is a serious problem with nursing homes. They lack human sentiment. The training and behavior of the personnel has a lot to be desired. Residents are robbed blind. There is non-humane treatment of the residents. They are physically abused in some places. My mother was 88 years old and was KILLED at the home. I had to leave town for just a few days. My mother was told by personnel that I wasn't coming back EVER! My mother gave and died of despair.

Tulip: I called 9-1-1 in an emergency. The person on the other end of the line said she didn't speak Spanish and hung up! I had to call my daughter-in-law who knows English. She called 9-1-1.

Magnolia: There's a problem with language and HUD. HUD told me that I cannot come without an interpreter.

Sunflower: At HUD, no one speaks Spanish. Also the food stamp office at 54 St., and some hospitals don't speak Spanish. 


\section{Focus Group 6: Hialeah Housing Authority}

DESCRIPTION OF GROUP: Cubans of lower socio-economic status, primarily over the age of 70 years and organized through the Hialeah Housing Authority, conducted in Spanish. (Hialeah Housing). Moderated by Martha Pelaez, Associated Director of the Southeast Florida Center on Aging.

The group consisted of 15 participants: 10 females and 5 males. Also present were 2 staff members and 2 maintenance persons (male).

After a brief introduction by the moderator:

\section{What are your concerns about where you live?}

Sunflower: Doors are broken and security is poor.

Magnolia: Elevators do not work properly. The food is no good.

Tulip: We are a big family. Neighbors help neighbors. However, the elevators need to be fixed, the food needs improvement. Less food, more quality.

Hibiscus: Need more activities

Orchid: We are a big family. We get along. Building repairs are not getting done, like the doors and awnings.

Lily: I've lived here 29 years. I'm scared of crime and the lack of security in the buildings. I don't feel secure in the streets. I'm afraid to go out alone. The building transportation doesn't operate after 4:00pm. I do use STS for transportation. I'd like to see more of a variety in the activities available. I'd like to see English classes.

Azalea: I use STS for transportation too, the building transportation ends at $4 \mathrm{pm}$. I' $\mathrm{m}$ ill and I've had three scans already.

Chrysth.: There is theft and vandalism. One night there were 5 cars vandalized or stolen!

General worries were: security, general maintenance, not enough personnel for their needs. 
Violet: There's a lack of activities after $2 \mathrm{pm}$.

\section{What kinds of service needs are there?}

Dahlia: The person sent by the agency to clean isn't effective. A sick person can't do it, then the house smells. It isn't the sick person's fault. There needs to be help with cleaning and hygiene. I have a brother, a son and 2 grandchildren. Service agencies compliment the help that I get from my family.

Gladiola: 1 don't need help. I'm ok. If my family can't help me, THEN I'll call an agency.

Lily: I've been here 21 years. I don't need any help at this time. I once was offered milk supplement, but it never came. I called to report it as fraud. When I need help, I call an agency official. I had a cleaning person once, but I stopped that because I was afraid of fraud. The person sent by the agency didn't always come and then she wanted me to sign for those hours. Now I pay for the cleaning person myself.

Azalea: I've got expensive health problems ... tumors. I need medical attention. I've called and been told that I have a tube. That's not true! I need medical equipment! I have help now with the activities of daily living but I'm afraid of becoming a burden to the system. I have two daughters that help me out.

Marigold: I need a loan to travel! I'll call my nephews.

Daisy: I'm a diabetic. I make do. I would like to have disabled person discount fares when I go on the bus. When I was dismissed from the hospital and needed help from the agency, my neighbors helped also.

Chrysth.: My family, daughter and sister help me when its absolutely necessary.

Orchid: My family helps me now. I help a little with cleaning and personal hygiene for a neighbor who doesn't want to ask for help from an agency.

Carnation: My family and neighbors help me. 
Sunflower: I drive myself. I have a daughter and a grandson.

Magnolia: I have a nephew. I'd call an agency but I don't want to abuse the system with unnecessary requests.

Hibiscus: I don't have any need now. I have two sons with families of their own.

Violet: J'll call an agency when I need it.

General problems: health, food quality, personal security, poor building repair and maintenance.

Where do you go for information?

Orchid: I'd ask my doctor or social worker.

Daisy: My doctor, Medicaid or Metropolitan.

Tulip: My doctor or social worker.

Violet: HUMANA

Carnation: My doctor or the building director

Lily: Channelling or United Home Care

Hibiscus: Social worker 


\section{VITA}

\section{KATHERINE MARIE CONDON}

Born, Princeton, New Jersey

1983

B.S., Political Economy of Natural Resources

University of California

Berkeley, California

1985-1986 Research Assistant,

Population Studies Center,

University of Pennsylvania

Philadelphia, Pennsylvania

1986-1988 Research Assistant,

Computer Core, Population Studies Center,

University of Pennsylvania

Philadelphia, Pennsylvania

1987

M.A., Demography

University of Pennsylvania

Philadelphia, Pennsylvania

1988-1989 Research Assistant,

Department of Sociology and School of Nursing,

University of Pennsylvania

Philadelphia, Pennsylvania

1990-1993 Project Analyst'Research Programmer

Mathematica Policy Research, lnc.

Princeton, New Jersey

1993-present Research Associate

Southeast Florida Center on Aging

Florida International University

Miami, Florida

Summer $1994 \quad$ Lecturer

Health Services Administration Department,

Florida Intemational University, Miami, FL

1999

Barry B. Levine Graduate Prize for the paper best using social theory in a sociological/ anthropological analysis or for the best discussion of social theory itself. Paper entitled "The Post-Modernist Challenge -The Call for a More Complete Contemporary Anthropology: An Examination of the Response in Three Ethnographies."

2000

William T. Vickers Graduate Prize for the best data-based paper analyzing a sociological/ anthropological problem. Paper entitled "Historical Demographic Trends of Elders in Miami-Dade County, Florida, 1950-1990." 


\section{PUBLICATIONS AND PRESENTATIONS}

Frank, Howard A.; Condon, Katherine M.; Dunlop, Burton D.; and Rothman, Max B. (2000). "The Retirement Planning Gap: A View From the Public Sector." International Journal of Orgonization Theory and Behovior. 3(1\&2): 235-274.

Rothman, Max B.; Dunlop, Burton D.; and Condon, Katherine M. 1997. "An Intergenerational Look at Retirement and Social Security." Presented at the Florida Aging Network Training Conference, August 27, 1997, St. Petersburg Beach, FL.

Polivka, Larry; Rothman, Max B.; Dunlop, Burton D.; Condon, Katherine M. and Ackman, Alan B. 1996. Long-Term Care in Florida: A Policy Analysis. Prepared for the Commission on Long-Term Care in Florida. Tampa, FL: Florida Policy Exchange Center on Aging, University of South Florida.

Dunlop, Burton D. and Condon, Katherine M. 1996. Results of a Health Status Suney of Medicare Plus: Program Enrollees of CAC-United Healthcare Plans of Florida, Inc. Miami. FL: Florida International University.

Condon, Carol M. and Condon, Katherine M. 1995. "Latin American Undocumented Aliens in New Jersey Who Legalized Under IRCA: A Profile." MACLAS Latin American Essays. VIl: 159-172.

Condon, Carol M. and Condon, Katherine M. 1994. "New Jersey Legalization Applicants Under IRCA 1986: A Profile." Presented at the Annual Meeting of the Eastern Economic Association, March 18-20, 1994, Boston, MA.

Condon, Katherine M. 1994. "A Portrait of Elders in Dade County." Miami Medicine, 65(7): 25-26.

Condon, Katherine M.: Dumlop, Burton D; and Rothman, Max B. 1994. Elders in Dade Cozmty, Florida: Information from the 1990 United States Census. North Miami, FL: Southeast Florida Center on Aging, Florida International University.

Rothman, Max B.; Dunlop, Burton D.; and Condon, Katherine M. 1994a. The Elders of Dade County: $A$ Needs Assessment of Persons 60 and Over. North Miami, FL: Southeast Florida Center on Aging, Florida International University.

Rothman, Max B.; Dunlop, Burton D.; and Condon, Katherine M. 1994b. "Aging in Dade County, Florida: Implications for Planning Services for Age and Ethnically Diverse Elder Populations." Presented at the Annual Meeting of the American Society on Aging, March 19-22, 1994, San Francisco, CA.

Rothman, Max B.; Dunlop, Burton D.; Condon, Katherine M. and McGovem, Patricia 1993. The Elders of Coral Gables: A Needs Assessment of Persons 65 and Over. North Miami, FL: Southeast Florida Center on Aging, Florida International University.

Dunlop, Burton D.; Condon, Katherine M. and Rothman, Max B. 1993. The Elders of North Dade: A Pilot Needs Assessment of Persons 60 and Over in Three Planning Sub-Areas. North Miami, FL:

Southeast Florida Center on Aging, Florida International University.

Condon, Katherine M; Dunlop, Burton D.; Rothman, Max B.; and Steinkohl, D. C. 1993. Initial Summary of Focus Group Findings. Prepared for The Alliance for Aging, Inc., The Area Agency on Aging for Dade and Monroe Counties under Contract No. DR-372. North Miami, FL: Southeast Florida Center on Aging, Florida International University.

Condon, Katherine M. 1992. "Female Migration in Turn-of-the Century America: Own-Children Information in the Creation of Estimated Migration Histories." Presented at the Annual Meeting of the Eastern Sociological Society, April 3-5, 1992, Arlington, VA.

Condon, Katherine M. and Pullum. Thomas. 1988. "Underenumeration for United States Censuses 1880 1980: Estimates for Age-Period-Cohort Analysis." Presented at the Annual Meeting of the Population Association of America, April 21-23, 1988, New Orleans, LA. 